\title{
National Vital Statistics Reports
}

Volume 58, Number 19

May 20, 2010

\section{Deaths: Final Data for 2007}

by Jiaquan Xu, M.D.; Kenneth D. Kochanek, M.A.; Sherry L. Murphy, B.S.; and Betzaida Tejada-Vera, B.S.; Division of Vital Statistics

\section{Abstract}

Objectives-This report presents final 2007 data on U.S. deaths, death rates, life expectancy, infant and maternal mortality, and trends by selected characteristics such as age, sex, Hispanic origin, race, marital status, educational attainment, injury at work, state of residence, and cause of death.

Methods-Information reported on death certificates, which are completed by funeral directors, attending physicians, medical examiners, and coroners, is presented in descriptive tabulations. The original records are filed in state registration offices. Statistical information is compiled in a national database through the Vital Statistics Cooperative Program of the Centers for Disease Control and Prevention's National Center for Health Statistics. Causes of death are processed in accordance with the International Classification of Diseases, Tenth Revision.

Results-In 2007, a total of 2,423,712 deaths were reported in the United States. The age-adjusted death rate was 760.2 deaths per 100,000 standard population, a decrease of 2.1 percent from the 2006 rate and a record low historical figure. Life expectancy at birth rose 0.2 year, from a 2006 value of 77.7 years to a record 77.9 in 2007. Age-specific death rates decreased for most age groups-15-24, $35-44,45-54,55-64,65-74,75-84$, and 85 and over-and remained unchanged for the age groups of under age $1,1-4,5-14$, and $25-34$. The 15 leading causes of death in 2007 remained the same as in 2006 with the exception of two causes that exchanged ranks. Alzheimer's disease, the seventh leading cause of death in 2006, became the sixth leading cause in 2007, and Diabetes mellitus, the sixth leading cause in 2006, dropped to the seventh leading cause in 2007. Heart disease and cancer continued to be the leading and second-leading causes of death, respectively, together accounting for almost one-half of all deaths (48.6 percent). The infant mortality rate in 2007 was 6.75 deaths per 1,000 live births.

Conclusions-Mortality patterns in 2007, such as the decline in the age-adjusted death rate to a record historical low, were generally consistent with long-term trends. Life expectancy reached a record high in 2007, increasing 0.2 year from 2006.

Keywords: mortality $\cdot$ cause of death $\bullet$ life expectancy • vital statistics

\section{Highlights}

\section{Mortality experience in 2007}

- In 2007, a total of 2,423,712 resident deaths were registered in the United States.

- The age-adjusted death rate, which takes the aging of the population into account, was 760.2 deaths per 100,000 U.S. standard population.

- Life expectancy at birth was 77.9 years.

- The 15 leading causes of death in 2007 were:

1. Diseases of heart (heart disease)

2. Malignant neoplasms (cancer)

3. Cerebrovascular diseases (stroke)

4. Chronic lower respiratory diseases

5. Accidents (unintentional injuries)

6. Alzheimer's disease

7. Diabetes mellitus (diabetes)

8. Influenza and pneumonia

9. Nephritis, nephrotic syndrome and nephrosis (kidney disease)

10. Septicemia

11. Intentional self-harm (suicide)

12. Chronic liver disease and cirrhosis

13. Essential hypertension and hypertensive renal disease (hypertension)

14. Parkinson's disease

15. Assault (homicide)

- In 2007, the infant mortality rate was 6.75 infant deaths per 1,000 live births.

- The 10 leading causes of infant death were:

1. Congenital malformations, deformations and chromosomal abnormalities (congenital malformations)

2. Disorders related to short gestation and low birth weight, not elsewhere classified (low birthweight)
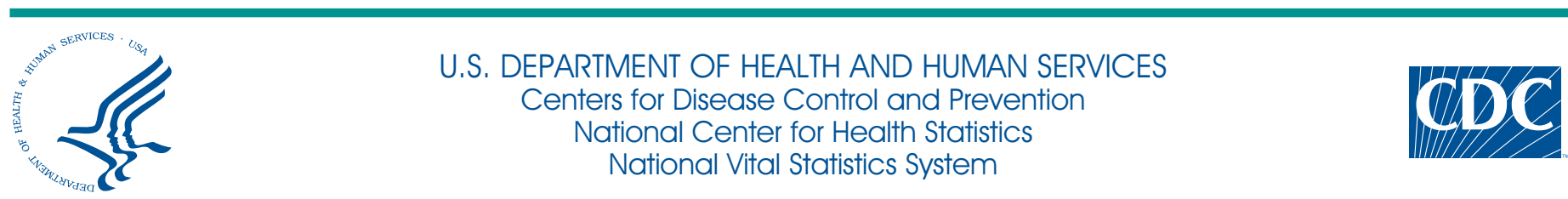
3. Sudden infant death syndrome (SIDS)

4. Newborn affected by maternal complications of pregnancy (maternal complications)

5. Accidents (unintentional injuries)

6. Newborn affected by complications of placenta, cord and membranes (cord and placental complications)

7. Bacterial sepsis of newborn

8. Respiratory distress of newborn

9. Diseases of the circulatory system

10. Neonatal hemorrhage

\section{Trends}

- The age-adjusted death rate in 2007 declined to a record low.

- Life expectancy was 77.9 years, continuing a long-term rising trend. Life expectancy increased for the total population, as well as for the black and white populations. Black males and females and white males and females experienced an increase in life expectancy in 2007 compared with 2006.

- Age-adjusted death rates decreased significantly in 2007 from 2006 for 5 of the 15 leading causes of death, and increased for 2 of the 15 leading causes. Rates for the top three leading causes-heart disease, cancer, and stroke-continued a longterm decreasing trend. Significant increases occurred for suicide and Chronic liver disease and cirrhosis.

- The differences in mortality between men and women increased slightly in 2007 from 2006. The age-adjusted death rate for men was 40.8 percent greater than that for women, up from 40.6 percent in 2006, while the difference between male and female life expectancy was 5.0 years in 2007, a slight decrease from the 2006 gap of 5.1.

- Differences in mortality between the black and white populations persisted. The age-adjusted death rate was 1.3 times greater, infant mortality rate 2.3 times greater, and maternal mortality rate 2.7 times greater for the black population than for the white population. Differences in life expectancy between the black and white populations narrowed by 0.2 year, from 5.0 years in 2006 to 4.8 in 2007.

- The postneonatal mortality rate increased 4.5 percent in 2007 from 2006.

\section{Introduction}

This report presents detailed 2007 data on deaths and death rates according to a number of social, demographic, and medical characteristics. These data provide information on mortality patterns among residents of the United States by such variables as age, sex, Hispanic origin, race, marital status, educational attainment, injury at work, state of residence, and cause of death. Information on these mortality patterns is key to understanding changes in the health and well-being of the U.S. population (1).

Preliminary data for 2007 were presented in the report "Deaths: Preliminary Data for 2007" using a 91 percent (demographic file) sample of U.S. deaths weighted to independent control totals (2). The findings of this report, based on the final mortality file, are generally consistent with those based on preliminary data; the final mortality file incorporates some modifications to the preliminary file as described in "Technical Notes." Separate companion reports will present additional details on leading causes of death and life expectancy in the United States $(3,4)$.

Mortality data in this report can be used to monitor and evaluate the health status of the United States in terms of current mortality levels and long-term mortality trends, as well as to identify segments of the U.S. population at greater risk of death from specific diseases and injuries. Differences in death rates among various demographic subpopulations, including race and ethnic groups, may reflect subpopulation differences in factors such as socioeconomic status, access to medical care, and the prevalence of specific risk factors in a particular subpopulation.

\section{Methods}

Data in this report are based on information from all resident death certificates filed in the 50 states and the District of Columbia. More than 99 percent of deaths occurring in this country are believed to be registered (5). Tables showing data by state also provide information for Puerto Rico, Virgin Islands, Guam, American Samoa, and the Commonwealth of the Northern Mariana Islands (Northern Marianas). Cause-of-death statistics presented in this report are classified in accordance with the International Classification of Diseases, Tenth Revision (ICD-10) (6). A discussion of the cause-ofdeath classification is provided in "Technical Notes."

Mortality data on specific demographic and medical characteristics except educational attainment cover all 50 states and the District of Columbia. Educational attainment data are provided separately for the 22 states and the District of Columbia that used the 2003 version of the standard death certificate, and the 26 states that used the 1989 version of the standard death certificate. Georgia and Rhode Island were excluded because the educational attainment item was not on their death certificate. Details on reporting areas for educational attainment are provided in "Technical Notes."

Measures of mortality in this report include the number of deaths; crude, age-specific, and age-adjusted death rates; infant, neonatal, postneonatal, and maternal mortality rates; life expectancy; and rate ratios. Changes in death rates in 2007 compared with 2006, and differences in death rates across demographic groups in 2007, are tested for statistical significance. Unless otherwise specified, reported differences are statistically significant. Additional information on these statistical methods, random variation and relative standard error, the computation of derived statistics and rates, population denominators, and the definition of terms is presented in "Technical Notes."

The populations used to calculate death rates for 2000-2007 and the intercensal period 1991-1999 shown in this report were produced under a collaborative arrangement with the U.S. Census Bureau and are consistent with the 2000 census. Reflecting the new guidelines issued in 1997 by the Office of Management and Budget (OMB), the 2000 census included an option for persons to report more than one race as appropriate for themselves and household members (7); see "Technical Notes." The 1997 OMB guidelines also provided for the reporting of Asian persons separately from Native Hawailan or Other Pacific Islander (NHOPI) persons. Under the prior OMB standards issued in 1977, data for Asian or Pacific Islander (API) persons were collected as a single group (8). Most death certificates currently collect 
only one race for the decedent in the same categories as specified in the 1977 OMB guidelines; that is, death certificate data do not report Asian persons separately from NHOPI persons. Death certificate data by race-the source of the numerators for death rates-are thus currently incompatible with the population data collected in the 2000 census and postcensal estimates - the denominators for the rates. To produce death rates by race for 2000-2007, and revised intercensal rates for the 1991-1999 period, the reported population data for multiple-race persons had to be "bridged" to single-race categories. In addition, the 2000 census counts were modified to be consistent with the 1977 OMB race categories; that is, to report the data for Asian and $\mathrm{NHOPI}$ persons as a combined category, API, and to reflect age as of the census reference date (9). The procedures used to produce the bridged populations are described in separate publications $(10,11)$. The bridged population data are anticipated to be used over the next few years for computing population-based rates by race. Beginning with deaths occurring in 2003, some states allowed for multiple-race reporting on the death certificate. Multiple-race data for these states are bridged to single-race categories; see "Technical Notes." Once all states are collecting data on race according to the 1997 OMB guidelines, use of the bridged-race algorithm is expected to be discontinued.

Note that the population data used to compile death rates by race in this report are based on special estimation procedures-they are not true counts. This is the case even for the 2000 populations. The estimation procedures used to develop these populations contain some error. Smaller population groups are affected much more than larger population groups (10). Over the next several years, additional information will be incorporated in the estimation procedures, possibly resulting in further revisions of the population estimates; see "Technical Notes." Data presented in this report and other mortality tabulations are available at the National Center for Health Statistics (NCHS) website, http://www.cdc.gov/nchs/deaths.htm. Availability of mortality microdata is described in "Technical Notes."

\section{Results and Discussion}

\section{Deaths and death rates}

In 2007, a total of $2,423,712$ resident deaths were registered in the United States, 2,552 fewer deaths than in 2006. The crude death rate for 2007, 803.6 deaths per 100,000 population, was 0.8 percent less than the 2006 rate (810.4) (Tables A, 1, and 3).

The age-adjusted death rate in 2007 was 760.2 deaths per 100,000 U.S. standard population, a record low value that was 2.1 percent lower than the 2006 rate of 776.5 (Tables 1 and A). Age-adjusted death rates are constructs that show what the level of mortality would be if no changes occurred in the age composition of the population from year to year. (For a discussion of age-adjusted death rates, see "Technical Notes.") Thus, age-adjusted death rates are better indicators than unadjusted (crude) death rates for examining changes in the risk of death over a period of time when the age distribution of the population is changing. Age-adjusted death rates also are better indicators of relative risk when comparing mortality across geographic areas or between sex or race subgroups of the population that have different age distributions; see "Technical Notes." Since 1980, the age-adjusted death rate has decreased every year except 1983, 1985, 1988,1993 , and 1999. Those years coincided with influenza outbreaks (12-15).The pace of decline for age-adjusted death rates during the last 7 years has been faster than for previous decades. From 1980

\section{Table A. Percentage change in death rates and age-adjusted death rates in 2007 from 2006, by age, race, and sex: United States}

[Based on death rates on an annual basis per 100,000 population, and age-adjusted rates per 100,000 U.S. standard population; see "Technical Notes." Data for specified

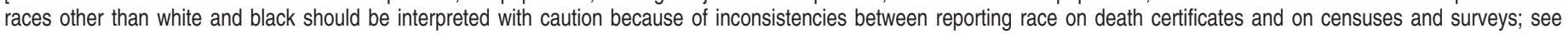
"Technical Notes." Race categories are consistent with the 1977 Office of Management and Budget (OMB) standards]

\begin{tabular}{|c|c|c|c|c|c|c|c|c|c|c|c|c|c|c|c|}
\hline \multirow[b]{2}{*}{ Age } & \multicolumn{3}{|c|}{ All races } & \multicolumn{3}{|c|}{ White $^{1}$} & \multicolumn{3}{|c|}{ Black $^{1}$} & \multicolumn{3}{|c|}{$\begin{array}{l}\text { American Indian or } \\
\text { Alaska Native }\end{array}$} & \multicolumn{3}{|c|}{ Asian or Pacific Islander ${ }^{1,3}$} \\
\hline & $\begin{array}{l}\text { Both } \\
\text { sexes }\end{array}$ & Male & Female & $\begin{array}{l}\text { Both } \\
\text { sexes }\end{array}$ & Male & Female & $\begin{array}{l}\text { Both } \\
\text { sexes }\end{array}$ & Male & Female & $\begin{array}{l}\text { Both } \\
\text { sexes }\end{array}$ & Male & Female & $\begin{array}{l}\text { Both } \\
\text { sexes }\end{array}$ & Male & Female \\
\hline All ages & \multicolumn{15}{|c|}{ Percent change } \\
\hline $\begin{array}{l}\text { Crude } \ldots \ldots \ldots \ldots \\
\text { Age adjusted } \ldots \ldots \ldots \ldots\end{array}$ & $\begin{array}{l}-0.8 \\
-2.1\end{array}$ & $\begin{array}{l}-0.6 \\
-2.1\end{array}$ & $\begin{array}{l}-1.1 \\
-2.2\end{array}$ & $\begin{array}{l}-0.8 \\
-2.0\end{array}$ & $\begin{array}{l}-0.5 \\
-1.9\end{array}$ & $\begin{array}{l}-1.0 \\
-2.1\end{array}$ & $\begin{array}{l}-1.3 \\
-2.4\end{array}$ & $\begin{array}{l}-1.4 \\
-2.6\end{array}$ & $\begin{array}{l}-1.2 \\
-2.4\end{array}$ & $\begin{array}{r}1.3 \\
-2.3\end{array}$ & $\begin{array}{r}2.3 \\
-0.4\end{array}$ & $\begin{array}{r}0.0 \\
-4.0\end{array}$ & $\begin{array}{r}0.4 \\
-3.2\end{array}$ & $\begin{array}{r}0.2 \\
-3.3\end{array}$ & $\begin{array}{r}0.6 \\
-3.3\end{array}$ \\
\hline Under 1 year $^{4}$. & -0.9 & -1.1 & -0.6 & -0.4 & -0.8 & 0.1 & -4.1 & -3.1 & -5.2 & 5.0 & -4.5 & 20.4 & 6.5 & 2.9 & 11.4 \\
\hline $1-4$ years . . & 0.7 & 2.6 & -2.3 & 1.2 & 2.9 & -1.7 & -2.5 & -3.8 & -1.0 & 0.9 & 9.5 & -8.9 & 10.7 & 39.8 & -15.2 \\
\hline $5-14$ years $\ldots \ldots \ldots \ldots \ldots$ & 0.7 & -1.1 & 2.3 & 1.4 & -1.2 & 4.2 & -1.4 & -0.8 & -2.3 & 7.7 & 34.9 & -21.7 & 1.9 & 8.0 & -4.9 \\
\hline 15-24 years. . . . . . . . . . . . . & -2.8 & -2.9 & -1.9 & -2.9 & -3.3 & -1.2 & -2.5 & -1.9 & -4.7 & -6.9 & -7.9 & -3.5 & -1.8 & -1.1 & -3.9 \\
\hline 25-34 years . . . . . . . . . . . . & -1.3 & -1.9 & -0.2 & -0.3 & -0.9 & 1.2 & -5.0 & -5.5 & -4.2 & 1.0 & 2.2 & -1.6 & -5.1 & -7.6 & -1.4 \\
\hline 35-44 years. . . . . . . . . . . . . & -3.0 & -2.9 & -3.3 & -2.5 & -2.8 & -2.2 & -4.7 & -3.4 & -6.5 & -2.6 & -1.8 & -4.2 & -1.0 & 0.5 & -3.3 \\
\hline $45-54$ years . . . . . . . . . . . . . & -1.5 & -2.0 & -0.8 & -1.0 & -1.3 & -0.4 & -3.7 & -4.9 & -2.0 & -1.5 & -3.2 & 1.2 & -3.3 & -1.5 & -6.2 \\
\hline $55-64$ years . . . . . . . . . . . . & -1.5 & -0.8 & -2.5 & -1.4 & -0.8 & -2.5 & -1.8 & -1.1 & -2.7 & 0.8 & 0.7 & 1.0 & -3.4 & -5.0 & -1.1 \\
\hline $65-74$ years . . . . . . . . . . . . . & -2.5 & -2.4 & -2.7 & -2.5 & -2.4 & -2.7 & -1.6 & -1.8 & -1.3 & -1.6 & -0.7 & -2.7 & -4.2 & -1.8 & -7.3 \\
\hline 75-84 years . . . . . . . . . . . . . & -2.0 & -2.3 & -1.9 & -1.9 & -2.2 & -1.8 & -2.7 & -3.0 & -2.5 & -4.5 & -0.1 & -8.3 & -2.1 & -1.9 & -2.2 \\
\hline 85 years and over . . . . . & -2.3 & -2.1 & -2.5 & -2.3 & -2.0 & -2.5 & -1.7 & -1.8 & -1.6 & -3.2 & 1.3 & -5.8 & -4.5 & -6.4 & -3.0 \\
\hline
\end{tabular}

${ }^{1}$ Multiple-race data were reported by 27 states and the District of Columbia in 2007. The multiple-race data for these reporting areas were bridged to the single-race categories of the 1977 OMB standards for comparability with other reporting areas; see "Technical Notes."

${ }^{2}$ Includes Aleuts and Eskimos.

${ }^{3}$ Includes Chinese, Filipino, Hawaiian, Japanese, and Other Asian or Pacific Islander.

${ }^{4}$ Death rates for "under 1 year" (based on population estimates) differ from infant mortality rates (based on live births). 
through 1989, the decline was 8.5 percent; from 1990 through 1999, 6.7 percent; and from 2000 through 2007, 12.5 percent (Figure 1 and Table 1).

Race - In 2007, age-adjusted death rates for the major race groups (Table 1) were:

- White population, 749.4 deaths per 100,000 U.S. standard population

- Black population, 958.0

- American Indian or Alaska Native (AIAN) population, 627.2

- API population, 415.0

Rates for the AIAN and API populations should be interpreted with caution because of reporting problems regarding correct identification of race on both the death certificate and in population censuses and surveys. The net effect of the reporting problems is for the AIAN rate to be approximately 30 percent understated and the API rate to be approximately 7 percent understated (16).

In 2007, the age-adjusted death rate for the black population was 1.3 times that for the white population (Table B); that is, the average risk of death for the black population was about 30.0 percent higher than for the white population. The ratio (shown to one decimal place) has remained constant since 1997. From 1960 through 1982, rates for the black and white populations declined by similar percentages-22.6 and 26.5 percent, respectively. From 1983 through 1988, rates diverged (17), increasing 5.2 percent for the black population and decreasing 1.7 percent for the white population. The disparity in ageadjusted death rates between the black and white populations reached its widest point in 1989 (1.4 times greater). Since then, the disparity between the two populations has narrowed as the age-adjusted rate for the black population declined by 24.9 percent and the rate for the white population declined by 18.6 percent. (Table 1 and Figure 2).

In 2007, decreases in age-adjusted death rates were observed for both white and black males and females compared with 2006. In order of relative magnitude of decrease, the reductions from 2006 were 2.6 percent for black males, 2.4 percent for black females, 2.1 percent for white females, and 1.9 percent for white males (Tables $A$ and 1).

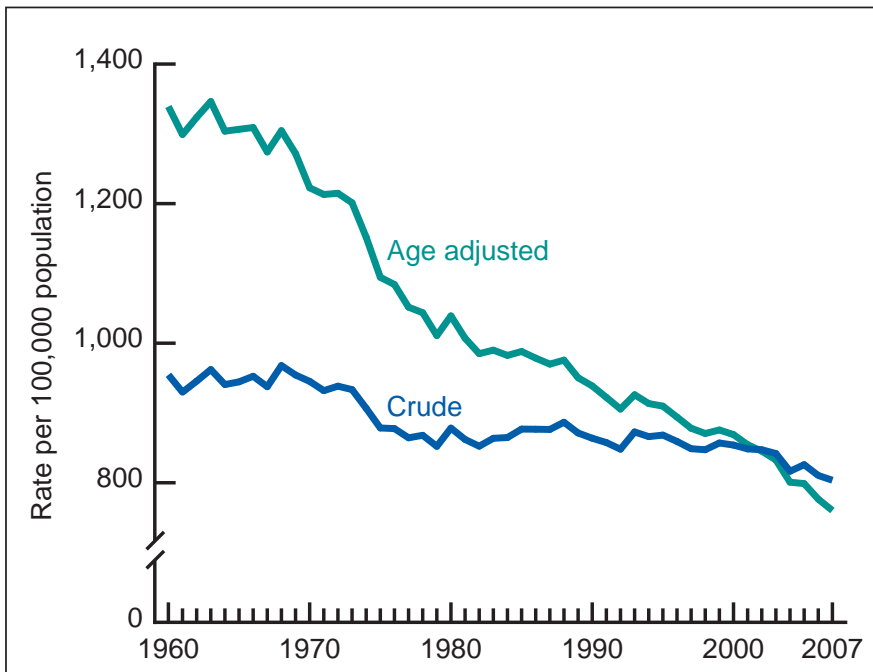

NOTE: Crude death rates are on an annual basis per 100,000 population; age-adjusted rates are per 100,000 U.S. standard population; see "Technical Notes." SOURCE: CDC/NCHS, National Vital Statistics System, Mortality.

Figure 1. Crude and age-adjusted death rates: United States, 1960-2007

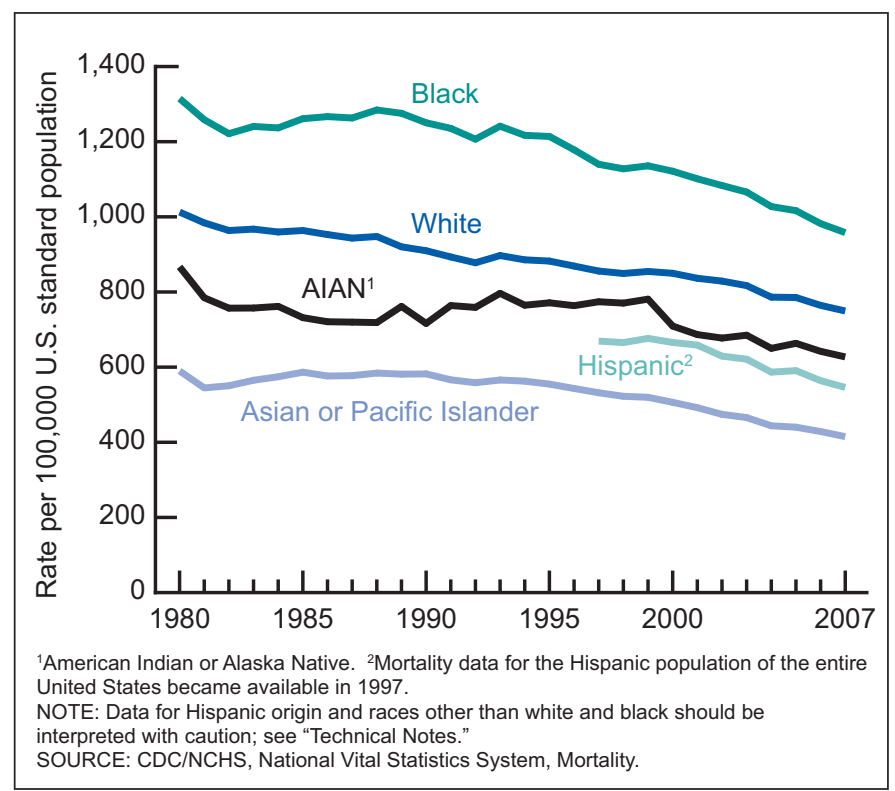

Figure 2. Age-adjusted death rates, by race and Hispanic origin: United States, 1980-2007

In general, age-adjusted death rates have declined from 1980 through 2007 for white males and females and black males and females. The rate decreased an average of 1.4 percent per year for white males, 0.8 percent for white females, 1.3 percent for black males, and 1.0 percent for black females during 1980-2007. However, increases were observed for both white males and white females in 1983, 1985, 1988, and 1993. In addition, the age-adjusted death rate for white females increased in 1995 and 1999. For black males, age-adjusted death rates tended to decrease, except for a period of increase from 1983 through 1988. Rates for black females decreased from 1980 through 2007, although with considerable variability in direction of change from year to year (Table 1).

In 2007, the age-adjusted death rate for the AIAN population was 0.8 times that for the white population (Table B); that is, the average risk of death for the AIAN population was about 20 percent lower than for the white population. Despite fluctuations over time and a trend toward convergence in rates from 1988 through 1999, the AIAN-towhite ratio has been consistently lower than 1.0 since 1980. The AIAN advantage in mortality is due in large part to the underreporting of AIAN mortality on death certificates. From 1980 through 1988, the ageadjusted rate for the AIAN population declined by 17.1 percent (Figure 2 and Table 1). However, the rate fluctuated from 1989 through 1999, peaking at 796.4 deaths per 100,000 U.S. standard population in 1993. Overall, the age-adjusted rate increased by 2.5 percent from 1989 through 1999, and has since trended downward. From 1999 through 2007, it declined by 19.7 percent. In 2007, the age-adjusted rate decreased by 4.0 percent from 2006 for AIAN females. The rate decreased by 2.3 percent from 2006 for both sexes, but the observed decrease was not statistically significant (Table A). The rate for AIAN males did not change significantly from year to year.

The age-adjusted death rate for the API population was 0.6 times that for the white population in 2007 (Table B). Some of this advantage is due to the underreporting of API mortality on death certificates. The API-to-white ratio has been consistently low over time, with a trend toward incremental divergence in rates since 1990 (Table 1 and 
Table B. Percentage of total deaths, death rates, age-adjusted death rates for 2007, percentage change in age-adjusted death rates in 2007 from 2006, and ratio of age-adjusted death rates, by race and sex for the 15 leading causes of death for the total population in 2007: United States

[Crude death rates on an annual basis per 100,000 population; age-adjusted rates per 100,000 U.S. standard population. The asterisks preceding the cause-of-death codes indicate that they are not part of the International Classification of Diseases, Tenth Revision (ICD-10), Second Edition; see "Technical Notes." Data for specified races other than white and black should be interpreted with caution because of inconsistencies between reporting race on death certificates and on censuses and surveys; see "Technical Notes." Race categories are consistent with the 1977 Office of Management and Budget (OMB) standards. Cause-of-death coding changes in 2006 and 2007 may affect comparability of data between 2007 and previous years for various causes of death; see "Technical Notes"]

\begin{tabular}{|c|c|c|c|c|c|c|c|c|c|c|c|}
\hline \multirow[b]{3}{*}{ Rank $^{1}$} & \multirow[b]{3}{*}{ Cause of death (based on ICD-10, 2004) } & \multirow[b]{3}{*}{ Number } & \multirow[b]{3}{*}{$\begin{array}{l}\text { Percent } \\
\text { of total } \\
\text { deaths }\end{array}$} & \multirow[b]{3}{*}{$\begin{array}{l}2007 \\
\text { crude } \\
\text { death } \\
\text { rate }\end{array}$} & \multicolumn{7}{|c|}{ Age-adjusted death rate } \\
\hline & & & & & & \multirow{2}{*}{$\begin{array}{c}\begin{array}{c}\text { Percent } \\
\text { change }\end{array} \\
2006 \\
\text { to } \\
2007\end{array}$} & \multicolumn{5}{|c|}{ Ratio } \\
\hline & & & & & 2007 & & $\begin{array}{c}\text { Male } \\
\text { to } \\
\text { female }\end{array}$ & $\begin{array}{c}\text { Black }^{2} \\
\text { to } \\
\text { white }\end{array}$ & $\begin{array}{c}\operatorname{AIAN}^{2,3} \\
\text { to } \\
\text { white }\end{array}$ & $\begin{array}{l}\mathrm{AP}^{2,4} \\
\text { to } \\
\text { white }\end{array}$ & $\begin{array}{c}\text { Hispanic } \\
\text { to non- } \\
\text { Hispanic white }\end{array}$ \\
\hline & All causes & $2,423,712$ & 100.0 & 803.6 & 760.2 & -2.1 & 1.4 & 1.3 & 0.8 & 0.6 & 0.7 \\
\hline 1 & $\ldots(100-109,|11| 113,,|20-| 51)$ & 616,067 & 25.4 & 204.3 & 190.9 & -4.6 & 1.5 & 1.3 & 0.7 & 0.5 & 0.7 \\
\hline 2 & Malignant neoplasms . . . . . . . . . . . . . . (C00-C97) & 562,875 & 23.2 & 186.6 & 178.4 & -1.3 & 1.4 & 1.2 & 0.7 & 0.6 & 0.6 \\
\hline 3 & Cerebrovascular diseases & 135,952 & 5.6 & 45.1 & 42.2 & -3.2 & 1.0 & 1.5 & 0.7 & 0.8 & 0.8 \\
\hline 4 & Chronic lower respiratory diseases . . . & 127,924 & 5.3 & 42.4 & 40.8 & 0.7 & 1.3 & 0.7 & 0.7 & 0.3 & 0.4 \\
\hline 5 & Accidents (unintentional injuries) . . . . (V01-X59, Y85-Y86) & 123,706 & 5.1 & 41.0 & 40.0 & 0.5 & 2.1 & 0.9 & 1.3 & 0.4 & 0.7 \\
\hline 6 & Alzheimer's disease. . . . . . . . . . . . . . . . . . . (G30) & 74,632 & 3.1 & 24.7 & 22.7 & 0.4 & 0.7 & 0.8 & 0.5 & 0.3 & 0.6 \\
\hline 7 & $\ldots \ldots \ldots \ldots(\mathrm{E} 10-\mathrm{E} 14)$ & 71,382 & 2.9 & 23.7 & 22.5 & -3.4 & 1.4 & 2.1 & 1.8 & 0.8 & 1.5 \\
\hline 8 & Influenza and pneumonia & 52,717 & 2.2 & 17.5 & 16.2 & -9.0 & 1.4 & 1.2 & 0.9 & 0.9 & 0.8 \\
\hline \multirow[t]{2}{*}{9} & Nephritis, nephrotic syndrome and nephrosis . . (NOO-N07, & & & & & & & & & & \\
\hline & N17-N19,N25-N27) & 46.448 & 1.9 & 15.4 & 14.5 & 0.0 & 1.4 & 2.2 & 1.1 & 0.7 & 0.9 \\
\hline 10 & $\ldots .(A 40-A 41)$ & 34,828 & 1.4 & 11.5 & 11.0 & 0.0 & 1.2 & 2.2 & 1.0 & 0.5 & 0.8 \\
\hline 11 & Intentional self-harm (suicide) & 34.598 & 1.4 & 11.5 & 11.3 & 3.7 & 3.9 & 0.4 & 0.9 & 0.5 & 0.4 \\
\hline 12 & Chronic liver disease and cirrhosis . . . . . (K70, K73-K74) & 29,165 & 1.2 & 9.7 & 9.1 & 3.4 & 2.2 & 0.8 & 2.6 & 0.4 & 1.6 \\
\hline \multirow[t]{2}{*}{13} & Essential hypertension and hypertensive renal & & & & & & & & & & \\
\hline & disease . . . . . . . . . . . . . . $(110,|12,| 15)^{7}$ & 23,965 & 1.0 & 7.9 & 7.4 & -1.3 & 1.0 & 2.5 & 0.9 & 1.0 & 1.0 \\
\hline 14 & Parkinson's disease. . . . . & 20,058 & 0.8 & 6.7 & 6.4 & 1.6 & 2.2 & 0.5 & 0.5 & 0.5 & 0.6 \\
\hline \multirow[t]{2}{*}{15} & Assault (homicide). . . . . . ('U01-*U02, X85-Y09, Y87.1) & 18,361 & 0.8 & 6.1 & 6.1 & -1.6 & 3.8 & 5.7 & 1.8 & 0.6 & 2.5 \\
\hline & $\ldots \ldots \ldots \ldots$ (residual) & 451,034 & 18.6 & 149.5 & & 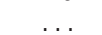 & $\ldots$ & & $\ldots$ & $\ldots$ & $\ldots$ \\
\hline
\end{tabular}

Category not applicable.

${ }^{1}$ Rank based on number of deaths; see "Technical Notes."

${ }^{2}$ Multiple-race data were reported by 27 states and the District of Columbia in 2007 . The multiple-race data for these reporting areas were bridged to the single-race categories of the 1977 OMB standards for comparability with other reporting areas; see "Technical Notes."

${ }^{3}$ AIAN is American Indian or Alaska Native; includes Aleuts and Eskimos.

${ }^{4} \mathrm{API}$ is Asian or Pacific Islander; includes Chinese, Filipino, Hawaiian, Japanese, and Other Asian or Pacific Islander.

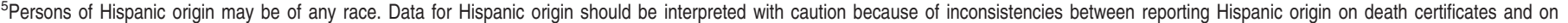
censuses and surveys.

${ }^{6} \mathrm{New}$ ICD-10 code J09 (Influenza due to identified avian influenza virus) was added to the category in 2007. No deaths occurred from this cause in 2007.

${ }^{7}$ Cause-of-death title was changed in 2006 to reflect the addition of Secondary hypertension (ICD-10 code I15).

Figure 2). From 1981 through 1985, the age-adjusted rate for the API population increased by 7.7 percent to reach a peak of 586.5 deaths per 100,000 U.S. standard population. The rate fluctuated from 1985 through 1993 before starting a persistent downward trend. From 1993 through 2007, the age-adjusted rate for the API population decreased by 26.7 percent. In 2007 , the rate for the total API population decreased by 3.2 percent from 2006 , while the rate for both API males and females decreased by 3.3 percent (Table A).

Hispanic origin-Problems of race and Hispanic-origin reporting affect Hispanic death rates and the comparison of rates for the Hispanic and non-Hispanic populations; see "Technical Notes." Mortality for Hispanics is somewhat understated because of net underreporting of Hispanic origin on the death certificate. Hispanic origin on the death certificate is underreported by an estimated 5 percent $(16,18)$; see "Technical Notes." The age-adjusted death rate for the Hispanic population in 2007 was 546.1, a decrease of 3.2 percent from the rate of 564.0 observed in 2006 (Tables $C$ and 2). The age-adjusted death rate for the total non-Hispanic population decreased by 1.9 percent relative to 2006. In 2007, the age-adjusted rate for the non-Hispanic white population decreased by 1.8 percent from 2006 , and that for the non-Hispanic black population declined by 2.3 percent.

Among Hispanic males, the age-adjusted death rate decreased by 3.1 percent in 2007 from 2006. The age-adjusted death rate for nonHispanic white males declined 1.7 percent and for non-Hispanic black males, it decreased 2.4 percent. Among Hispanic females, the ageadjusted death rate decreased by 3.4 percent. Rates also decreased for non-Hispanic white females (1.9 percent) and non-Hispanic black females (2.2 percent) (Tables $\mathrm{C}$ and 2).

In 2007, the age-adjusted death rate (Table 2) was 29.7 percent lower for the Hispanic population than for the non-Hispanic population. Similarly, the age-adjusted death rate for the Hispanic population was 28.5 percent lower than the rate for the non-Hispanic white population, and considerably lower, at 44.2 percent, than that for the non-Hispanic black population. The large differences in mortality between the Hispanic and non-Hispanic populations are partly a function of the Hispanic population's lower age-specific death rates, particularly at older ages (Table 4). Part of the difference is also attributable to underreporting of Hispanic origin on death certificates. In addition, various hypotheses 
have been proposed to explain Hispanics' favorable mortality outcomes. The most prevalent include the healthy migrant effect, which argues that Hispanic immigrants are selected for their good health and robustness, and the "salmon bias" effect, which posits that U.S. residents of Hispanic origin may return to their country of origin to die or when ill $(19,20)$.

Within the Hispanic population, the age-adjusted death rate for males was 1.4 times the rate for females (Table 2). The corresponding male-to-female ratios were 1.4 for the non-Hispanic white population and 1.5 for the non-Hispanic black population. The male-to-female ratios of age-adjusted death rate within the Hispanic population did not change in 2007 from 2006. Age-adjusted death rates in 2007 for selected Hispanic subgroups (Table 5), in order of relative magnitude, were:

- Puerto Rican population, 636.6 deaths per 100,000 U.S. standard population

- Cuban population, 596.7

- Mexican population, 568.7

- Central and South American population, 325.5

The age-adjusted death rate significantly decreased for the Puerto Rican (11.3 percent) and the Central and South American populations (12.1 percent), whereas the rate for the Cuban population increased by 4.7 percent in 2007 from 2006. Among Hispanic subgroups, the age-adjusted death rate for the Central and South American population was significantly lower than the rates for the Mexican, Puerto Rican, and Cuban populations. The differences in age-adjusted death rates among the Mexican, Puerto Rican, and Cuban populations were not statistically significant. Tests of significant differences among the Hispanic subgroups are affected by the large variation in age-specific death rates for some of the subgroups, which reflects their relatively small population sizes.

\section{Death rates by age and sex}

No statistically significant increases in age-specific death rates for the major race-sex groups were noted in 2007 (Tables A, 11, 14 and 15; Figure 3). Age-specific death rates decreased by a statistically significant margin from 2006 to 2007 for age groups 15-24, $35-44,45-54,55-64,65-74,75-84$, and 85 and over.

The death rates for males declined in 2007 from 2006 for age groups 15-24, 25-34, 35-44, 45-54, 55-64, 65-74, 75-84, and 85 and over. The observed increase of 2.6 percent for age group 1-4 in 2007 from 2006 was not statistically significant. For females, death rates declined for the age groups $35-44,55-64,65-74,75-84$, and 85 and over. The rates for the remaining age groups for females remained unchanged statistically.

Race-Age-specific death rates declined for white males in 2007 for age groups 15-24, 35-44, 45-54, 55-64, 65-74, 75-84, and 85 and over (Table A). The largest decrease, 3.3 percent, occurred for those aged 15-24. Other observed changes among white males by age group were not statistically significant. For the black male population in 2007, the rates decreased for age groups $25-34,35-44,45-54,65-74$, and $75-84$. The largest statistically significant decrease for black males was for those aged 25-34, at 5.5 percent. None of the changes in agespecific death rates for AIAN males in 2007 from 2006 were statistically significant. Rates for API males decreased for those aged 55-64 and 85 and over-the group with the largest statistically significant decrease at 6.4 percent.
For white females, the death rate decreased in 2007 for those aged 35-44, 55-64, 65-74, 75-84, and 85 and over. The largest decrease, 2.7 percent, was observed for age group 65-74. Age-specific rates for black females decreased for age groups under age 1, 35-44, 55-64, 75-84, and 85 and over, with those aged 35-44 having the largest decrease at 6.5 percent. For AIAN females, the only statistically significant change was an 8.3 percent decrease for those aged 75-84. No rate observed for API females changed statistically in 2007 from 2006.

Hispanic origin-For the Hispanic origin population in $2007 \mathrm{com}$ pared with 2006 (Table $C$ ), the age-specific death rate decreased for age groups $15-24,35-44,45-54,65-74,75-84$, and 85 and over. The largest decrease was for the age group 35-44, at 5.4 percent, and no significant increases in age-specific death rates for Hispanics occurred in 2007 from 2006. Rates for Hispanic males decreased for age groups $15-24,35-44,65-74,75-84$, and 85 and over. The largest decrease was for those aged 35-44, at 5.8 percent. For Hispanic females, age-specific rates decreased by a statistically significant amount in 2007 from 2006 for those aged 45-54, 65-74, and 85 and over. The largest decrease, 5.5 percent, was for the age group 85 and over.

\section{Expectation of life at birth and at specified ages}

Life expectancy at birth represents the average number of years that a group of infants would live if the group was to experience throughout life the age-specific death rates present in the year of birth. Life table data shown in this report for data years 2000-2007 are based on a newly revised methodology and may differ from figures previously published. The revised methodology is similar to that developed for the 1999-2001 decennial life tables; see "Technical Notes." Life tables were generated only for the total U.S., black, and white populations.

In 2007, life expectancy at birth for the U.S. population was 77.9 years, an increase of 0.2 year from 77.7 in 2006 (Tables 6-8). The trend in U.S. life expectancy since 1900 has been one of gradual improvement. In 2007, the life expectancy for females was 80.4 years, a 0.2-year increase from 2006, and the life expectancy for males was 75.4 years, a 0.3 -year increase from the previous year. From 1900 through the late 1970s, the sex gap in life expectancy widened (Figure 4; data prior to 1975 not shown), from 2.0 years to 7.8 years. Since its peak in the 1970s, the sex gap has been narrowing (Figure 4). The difference in life expectancy between the sexes was 5.0 years in 2007, a slight decrease from the 5.1-year gap in 2006.

Life expectancy increased 0.4 year for the black population in 2007 to 73.6 years compared with 2006 (73.2). Life expectancy for the white population increased 0.2 year to 78.4 years. The difference in life expectancy between the white and black populations in 2007 was 4.8 years, a 0.2-year decrease from the 2006 gap between the two races and the smallest gap ever recorded. The white-black gap has been narrowing gradually from a peak of 7.1 years in 1989 to the current record low (Figure 4). This continued a long-term decline in the whiteblack difference in life expectancy that was interrupted from 1982 through 1989 when the gap widened.

Among the major race-sex groups (Tables 7 and 8 , and Figure 5), white females continued to have the highest life expectancy at birth ( 80.8 years), followed by black females ( 76.8 years), white males ( 75.9 years), and black males ( 70.0 years). Life expectancies increased by 0.3 year for both the black male and female populations. Life expect- 


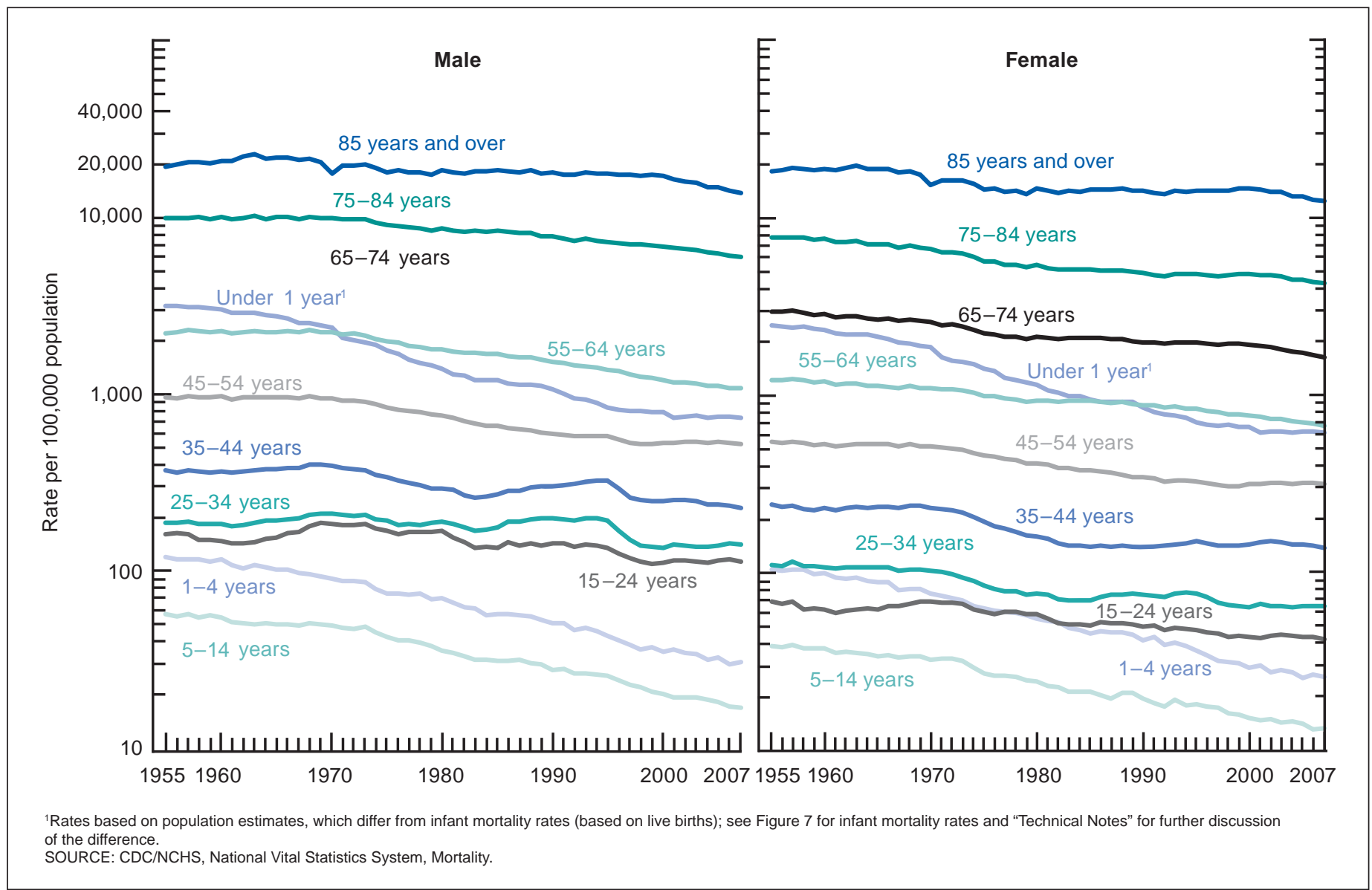

Figure 3. Death rates, by age and sex: United States, 1955-2007

Table C. Percentage change in death rates and age-adjusted death rates in 2007 from 2006, by age, Hispanic origin, race for non-Hispanic population, and sex: United States

[Based on death rates on an annual basis per 100,000 population, and age-adjusted rates per 100,000 U.S. standard population; see "Technical Notes." Race and Hispanic origin are reported separately on the death certificate. Persons of Hispanic origin may be of any race. Data for Hispanic persons are not tabulated separately by race. Data for non-Hispanic persons are tabulated by race. Data for Hispanic origin should be interpreted with caution because of inconsistencies between reporting Hispanic origin on death certificates and on censuses and surveys; see "Technical Notes"]

\begin{tabular}{|c|c|c|c|c|c|c|c|c|c|c|c|c|c|c|c|}
\hline \multirow[b]{2}{*}{ Age } & \multicolumn{3}{|c|}{ All origins ${ }^{1}$} & \multicolumn{3}{|c|}{ Hispanic } & \multicolumn{3}{|c|}{ Non-Hispanic ${ }^{2}$} & \multicolumn{3}{|c|}{ Non-Hispanic white } & \multicolumn{3}{|c|}{ Non-Hispanic black } \\
\hline & $\begin{array}{l}\text { Both } \\
\text { sexes }\end{array}$ & Male & Female & $\begin{array}{l}\text { Both } \\
\text { sexes }\end{array}$ & Male & Female & $\begin{array}{l}\text { Both } \\
\text { sexes }\end{array}$ & Male & Female & $\begin{array}{l}\text { Both } \\
\text { sexes }\end{array}$ & Male & Female & $\begin{array}{l}\text { Both } \\
\text { sexes }\end{array}$ & Male & Female \\
\hline All ages & \multicolumn{15}{|c|}{ Percent change } \\
\hline $\begin{array}{l}\text { Crude } \ldots \ldots \ldots \ldots \\
\text { Age adjusted } \ldots \ldots \ldots \ldots\end{array}$ & $\begin{array}{l}-0.8 \\
-2.1\end{array}$ & $\begin{array}{l}-0.6 \\
-2.1\end{array}$ & $\begin{array}{l}-1.1 \\
-2.2\end{array}$ & $\begin{array}{l}-0.8 \\
-3.2\end{array}$ & $\begin{array}{l}-0.6 \\
-3.1\end{array}$ & $\begin{array}{l}-0.9 \\
-3.4\end{array}$ & $\begin{array}{l}-0.6 \\
-1.9\end{array}$ & $\begin{array}{l}-0.3 \\
-1.9\end{array}$ & $\begin{array}{l}-0.8 \\
-2.0\end{array}$ & $\begin{array}{l}-0.5 \\
-1.8\end{array}$ & $\begin{array}{l}-0.2 \\
-1.7\end{array}$ & $\begin{array}{l}-0.7 \\
-1.9\end{array}$ & $\begin{array}{l}-1.1 \\
-2.3\end{array}$ & $\begin{array}{l}-1.3 \\
-2.4\end{array}$ & $\begin{array}{l}-1.0 \\
-2.2\end{array}$ \\
\hline 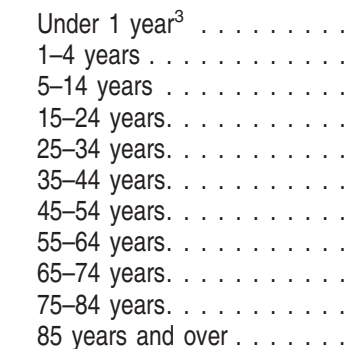 & $\begin{array}{r}-0.9 \\
0.7 \\
0.7 \\
-2.8 \\
-1.3 \\
-3.0 \\
-1.5 \\
-1.5 \\
-2.5 \\
-2.0 \\
-2.3\end{array}$ & $\begin{array}{r}-1.1 \\
2.6 \\
-1.1 \\
-2.9 \\
-1.9 \\
-2.9 \\
-2.0 \\
-0.8 \\
-2.4 \\
-2.3 \\
-2.1\end{array}$ & $\begin{array}{r}-0.6 \\
-2.3 \\
2.3 \\
-1.9 \\
-0.2 \\
-3.3 \\
-0.8 \\
-2.5 \\
-2.7 \\
-1.9 \\
-2.5\end{array}$ & $\begin{array}{l}-0.5 \\
-1.5 \\
-0.7 \\
-4.9 \\
-1.7 \\
-5.4 \\
-2.4 \\
-1.6 \\
-3.5 \\
-1.8 \\
-5.3\end{array}$ & $\begin{array}{l}-1.2 \\
-2.8 \\
-3.7 \\
-4.5 \\
-2.3 \\
-5.8 \\
-1.1 \\
-1.4 \\
-2.5 \\
-2.8 \\
-5.1\end{array}$ & $\begin{array}{r}0.3 \\
-0.8 \\
4.2 \\
-4.8 \\
0.7 \\
-5.1 \\
-5.2 \\
-2.0 \\
-5.0 \\
-0.8 \\
-5.5\end{array}$ & $\begin{array}{r}-0.7 \\
1.0 \\
0.6 \\
-2.2 \\
-1.2 \\
-2.4 \\
-1.3 \\
-1.4 \\
-2.3 \\
-1.9 \\
-2.1\end{array}$ & $\begin{array}{r}-0.8 \\
4.5 \\
0.0 \\
-2.5 \\
-1.7 \\
-2.2 \\
-1.9 \\
-0.7 \\
-2.2 \\
-2.1 \\
-1.9\end{array}$ & $\begin{array}{r}-0.5 \\
-3.0 \\
1.5 \\
-1.4 \\
-0.1 \\
-2.8 \\
-0.3 \\
-2.4 \\
-2.5 \\
-1.8 \\
-2.3\end{array}$ & $\begin{array}{r}-0.8 \\
2.0 \\
1.4 \\
-2.1 \\
0.3 \\
-1.8 \\
-0.7 \\
-1.3 \\
-2.4 \\
-1.8 \\
-2.0\end{array}$ & $\begin{array}{r}-0.8 \\
5.2 \\
-0.6 \\
-2.8 \\
-0.2 \\
-2.0 \\
-1.2 \\
-0.6 \\
-2.3 \\
-2.0 \\
-1.7\end{array}$ & $\begin{array}{r}-0.8 \\
-2.2 \\
4.2 \\
-0.5 \\
1.4 \\
-1.4 \\
0.2 \\
-2.5 \\
-2.5 \\
-1.7 \\
-2.2\end{array}$ & $\begin{array}{l}-2.2 \\
-1.4 \\
-1.4 \\
-2.6 \\
-5.1 \\
-4.4 \\
-3.4 \\
-1.8 \\
-1.4 \\
-2.6 \\
-1.5\end{array}$ & $\begin{array}{l}-1.9 \\
-1.5 \\
-1.2 \\
-2.1 \\
-5.8 \\
-3.0 \\
-4.6 \\
-1.1 \\
-1.6 \\
-3.0 \\
-1.6\end{array}$ & $\begin{array}{l}-2.7 \\
-1.2 \\
-1.7 \\
-4.4 \\
-4.2 \\
-6.4 \\
-1.7 \\
-2.7 \\
-1.2 \\
-2.4 \\
-1.5\end{array}$ \\
\hline
\end{tabular}

${ }^{1}$ Figures for origin not stated are included in "all origins" but not distributed among specified origins.

${ }^{2}$ Includes races other than white and black.

${ }^{3}$ Death rates for "under 1 year" (based on population estimates) differ from infant mortality rates (based on live births). 


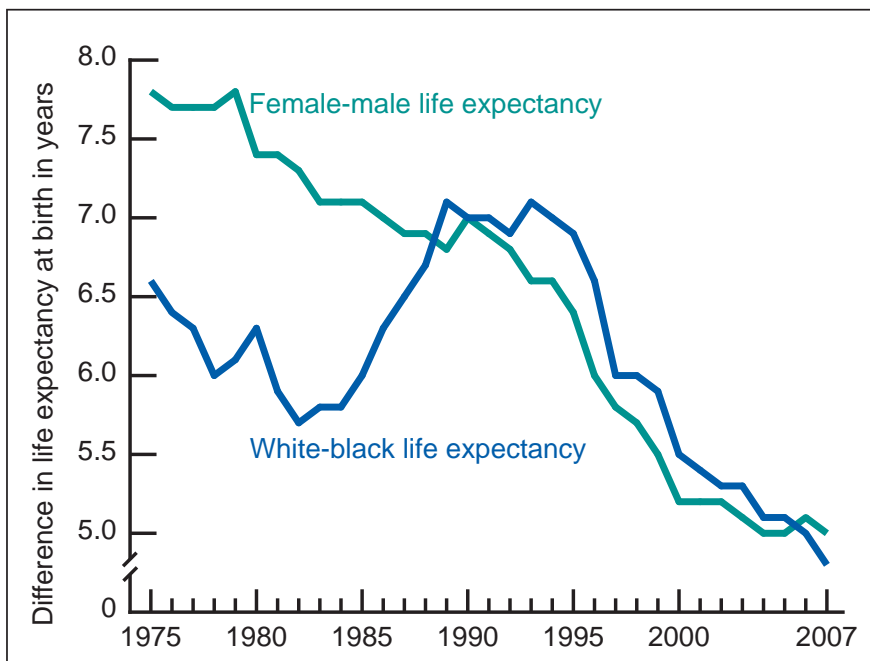

NOTE: Life expectancies for 2000-2007 were calculated using a revised methodology and may differ from those previously published; see "Technical Notes." SOURCE: CDC/NCHS, National Vital Statistics System, Mortality.

Figure 4. Differences in female-male and white-black life expectancies: United States, 1975-2007

ancies increased by 0.2 year for both white males and females. Life expectancy for black males declined every year from 1984 through 1989 , then resumed the long-term trend of increase from 1990 through 1992, 1994 through 2004, and 2005 through 2007 (Table 8). For white females, life expectancy increased most years from 1970 through 1998. In 1999, life expectancy for white females fell below 1998's record high, and it did not increase again until 2003. From 1989 through 1992, during 1994, and from 1996 through 1998, life expectancy for black females increased. In 1999, life expectancy for black females declined as it did for white females, only to begin climbing again in 2000.

Life tables shown in this report may be used to compare life expectancies at selected ages from birth to 100 years. For example, a person who has reached 65 years will live to an older age, on average,

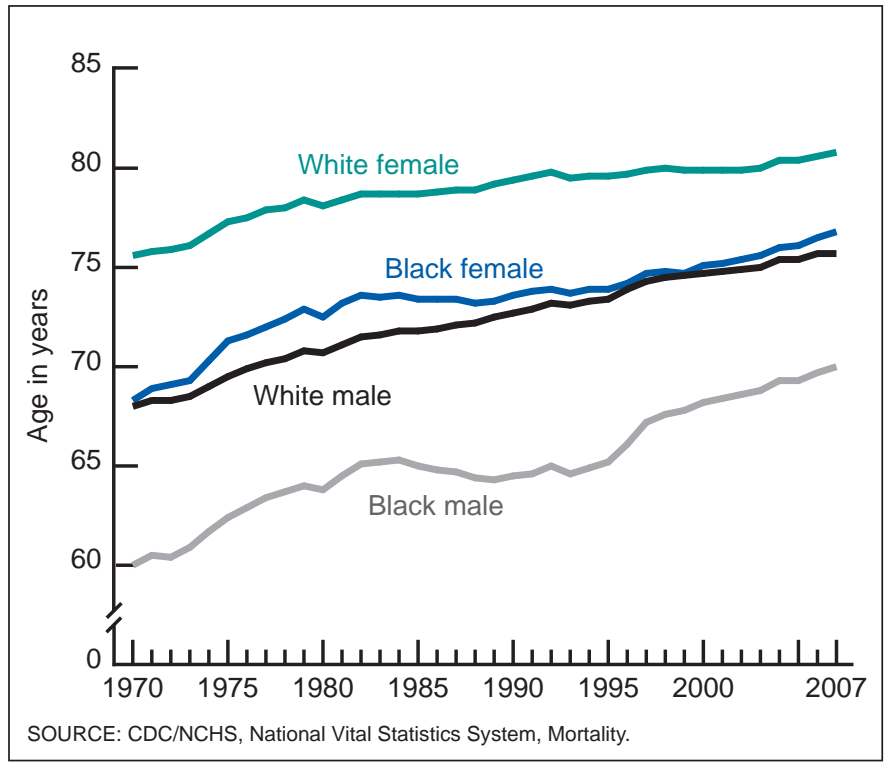

Figure 5. Life expectancy, by race and sex: United States, 1970-2007 than one who has reached 50 years. On the basis of mortality experienced in 2007, a person aged 50 could expect to live an average of 30.9 more years for a total of 80.9 years. A person aged 65 could expect to live an average of 18.6 more years for a total of 83.6 years, and a person aged 85 could expect to live an average of 6.5 more years for a total of 91.5 years (Tables 6 and 7).

\section{Leading causes of death}

The 15 leading causes of death in 2007 accounted for 81.4 percent of all deaths in the United States (Tables B and 9). Causes of death are ranked according to the number of deaths; for ranking procedures, see "Technical Notes." By rank, the 15 leading causes in 2007 were:
1. Diseases of heart (heart disease)
2. Malignant neoplasms (cancer)
3. Cerebrovascular diseases (stroke)
4. Chronic lower respiratory diseases
5. Accidents (unintentional injuries)
6. Alzheimer's disease
7. Diabetes mellitus (diabetes)
8. Influenza and pneumonia
9. Nephritis, nephrotic syndrome and nephrosis (kidney disease)
10. Septicemia
11. Intentional self-harm (suicide)
12. Chronic liver disease and cirrhosis
13. Essential hypertension and hypertensive renal disease (hypertension)
14. Parkinson's disease
15. Assault (homicide)

The 15 leading causes of death in 2007 retained the same ranking as in 2006 with the exception of Alzheimer's diseases and diabetes, which changed position relative to their 2006 placement as the age-adjusted death rate for diabetes decreased by 3.4 percent in 2007 from 2006, while the rate for Alzheimer's disease did not change significantly.

The age pattern of mortality can vary greatly by cause of death. As a result, the changing age distribution of a population can significantly influence changes in crude death rates over time. In contrast, the influence of such shifts in the population age structure is eliminated by age-adjusted death rates. Therefore, age-adjusted death rates are better indicators than crude rates for showing changes in mortality over time and among causes of death, and consequently are used in this report to depict trends for leading causes of death (Figure 6).

In 2007 , the number of deaths decreased from 2006 by 0.1 percent, or 2,552 fewer deaths (Tables 12 and 13). The age-adjusted death rate for all causes decreased by 2.1 percent. This reduction in the risk of dying has been driven mostly by net decreases in the leading causes of death such as heart disease, cancer, stroke, diabetes, and Influenza and pneumonia. Despite a more severe influenza season during 2007-2008, the drop in the death rate for Influenza and pneumonia may be due, in part, to less severe influenza seasons during 2006-2007 $(14,15)$.

Among the 15 leading causes of death, the age-adjusted death rate declined significantly for 5 of them (Table B). Long-term decreasing trends for heart disease, cancer, and stroke-the three leading causes of death-continued in 2007 , with decreases of 4.6 percent for heart 


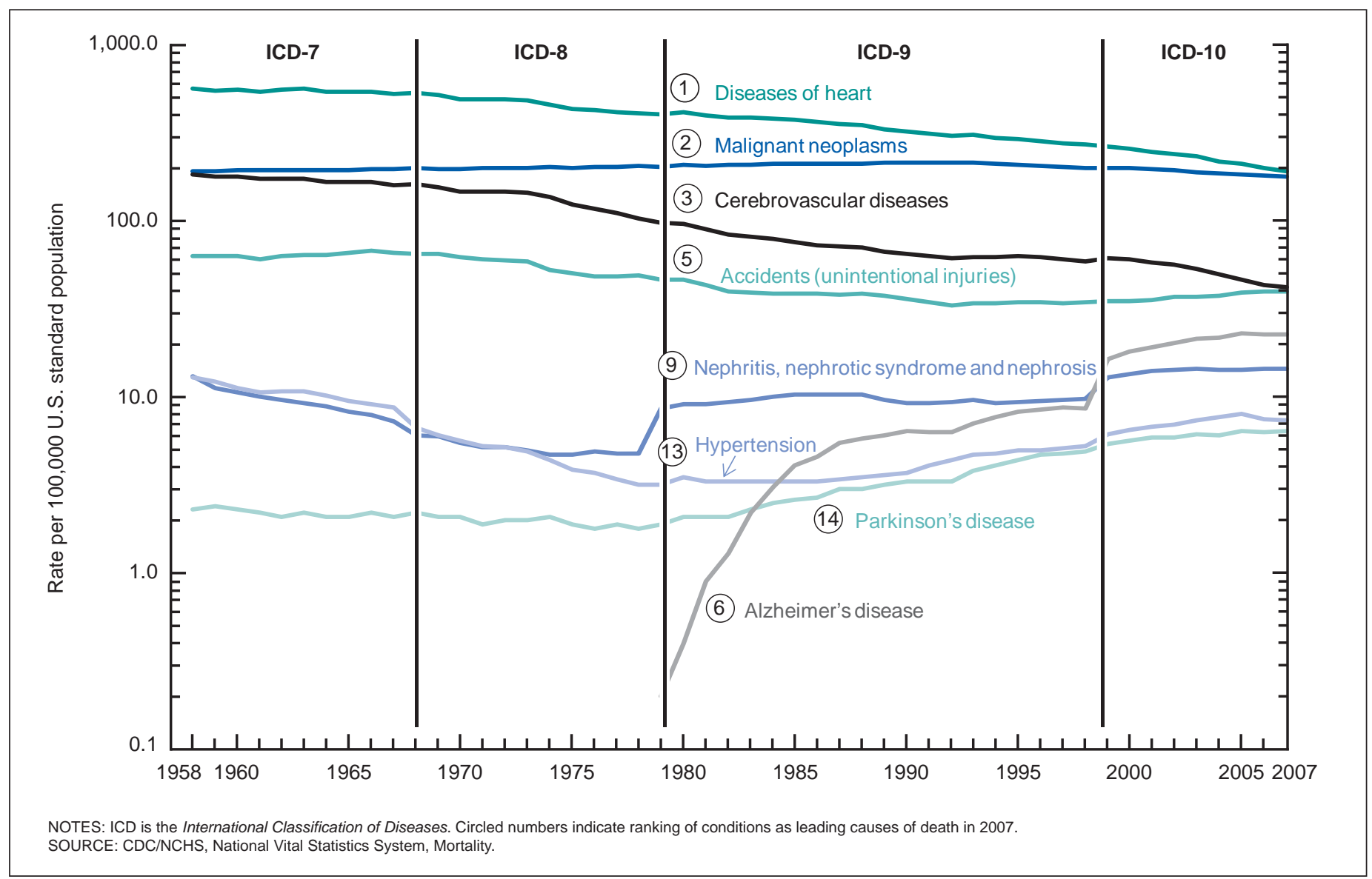

Figure 6. Age-adjusted death rates for selected leading causes of death: United States, 1958-2007

disease, 1.3 percent for cancer, and 3.2 percent for stroke compared with 2006. Except for a relatively small increase in 1993, mortality from heart disease has steadily declined since 1980 (Figure 6). The ageadjusted death rate for cancer, the second leading cause of death, has shown a gradual but consistent downward trend since 1993 (Figure 6). The rate for stroke has generally declined since 1958 , with one exception: an increase of 2.6 percent from 1992 through 1995 (Figure 6).

Additional causes with a significant decrease in the age-adjusted death rate relative to 2006 were Influenza and pneumonia (9.0 percent) and diabetes (3.4 percent). The increase in the age-adjusted death rate for Alzheimer's disease (0.4 percent) was not statistically significant. The mortality trend for Alzheimer's disease has generally been one of rapid increase (Figure 6). From 1979 through 1998, for example, the rate for Alzheimer's disease increased dramatically due to improvements in diagnosis, awareness of the condition within the medical community, and other unidentified factors (21). The transition from ICD-9 to ICD-10 also brought substantial changes to the coding and selection rules for the condition. This created a major disruption in the time series trend for Alzheimer's disease during 1998-1999 (Figure 6). The large increase in 1999 from 1998 is at least partly due to the ICD transition (22). Evaluating the observed change poses a problem because the comparability ratio (representing the net effect of the new revision on cause-of-death statistics) for Alzheimer's disease may be understated (22-25). Since 1999, the rate has trended upward through 2007.

Significant increases occurred between 2006 and 2007 in the age-adjusted death rate for suicide (3.7 percent) and Chronic liver disease and cirrhosis ( 3.4 percent). The death rate for suicide has decreased slightly from a high of 13.7 deaths per 100,000 standard population in 1977 to a low of 10.4 in 2000 . Since 2000, the ageadjusted death rate for suicide has increased by 8.7 percent. The increase in the age-adjusted death rate for Chronic liver disease and cirrhosis may be largely due to a coding change; see "Quality of reporting and processing cause of death" in "Technical Notes."

Although mortality from Human immunodeficiency virus (HIV) disease has not been on the list of 15 leading causes of death since 1997 (26), it is still of concern. HIV disease continues to be one of the five leading causes of death for specific age groups for females, and in the black population. In 2007, a total of 11,295 persons died from HIV disease (Table 10). The age-adjusted death rate (3.7 per 100,000 standard population; Table 16) declined for the 12th consecutive year, decreasing 7.5 percent in 2007 from 2006. The age-adjusted death rate for this cause reached its highest point of 16.3 per 100,000 standard population in 1995 and then declined rapidly through 1998 (decreasing 69.9 percent) (27). The rate of decline for this cause of death has slowed considerably since 1999, decreasing by 30.2 percent from 1999 through 2007.

Enterocolitis due to Clostridium difficile (C. difficile), a bacterial inflammation of the intestines, is of growing public health concern because it is often acquired in hospitals or other health care institutions with long-term patients or residents and accounts for an increasing number of deaths (28-31). In 1999, 793 deaths were due to C. difficile; by contrast, in 2007, 6,372 C. difficile deaths were recorded, an increase of 147 deaths from the 6,225 reported in 2006. Because of 
the substantial increase since 1999, beginning in 2006, C. difficile is included among the rankable causes of death and is shown appended to the List of 113 Selected Causes of Death in tables in this report; see "Technical Notes." The age-adjusted death rate for $\boldsymbol{C}$. difficile was 2.0 deaths per 100,000 standard population for both 2006 and 2007. In 2007, this cause was not among the 20 leading causes for the overall population. However, it ranked among the 20 leading causes of death for the population aged 65 and older. Approximately 92 percent of deaths due to $C$. difficile occurred in persons aged 65 and over in 2007.

Changes in mortality levels by age and cause of death have a major effect on changes in life expectancy. Life expectancy at birth increased in 2007 over 2006 by 0.2 year because of decreases in mortality from heart disease, cancer, Influenza and pneumonia, stroke, and diabetes. Decreases in mortality from these same causes of death also generated increases in life expectancy among the male and female populations, when analyzed separately. The increase in life expectancy in 2007 from 2006 for the population as a whole could have been greater than 0.2 year were it not for the increase in mortality from Chronic liver disease and cirrhosis and suicide. (For discussion of contributions to the change in life expectancy, see "Technical Notes.")

The relative risk of death in one population group compared with another can be expressed as a ratio. Ratios based on age-adjusted death rates show that males have higher rates than females for 12 of the 15 leading causes of death (Table B), with rates for males being at least two times those for females for five leading causes. The largest ratio, 3.9, was for suicide. Other large ratios were evident for homicide (3.8), Parkinson's disease and Chronic liver disease and cirrhosis (2.2 each), unintentional injuries (2.1), heart disease (1.5), and cancer, diabetes, Influenza and pneumonia, and kidney disease (1.4 each).

The difference in life expectancy between males and females decreased 0.1 year in 2007 over 2006, to 5.0 years (Table 8). The narrowing of the difference between male and female life expectancy was a result of greater improvements in mortality among males than females, particularly with respect to trends for heart disease, unintentional injuries, and HIV disease.

Age-adjusted death rates for the black population were higher than those for the white population for 9 of the 15 leading causes of death (Table B). The largest ratio was for homicide, at 5.7. Other causes for which the ratio was high include hypertension (2.5), kidney disease and Septicemia (2.2 each), diabetes (2.1), stroke (1.5), heart disease (1.3), and cancer (1.2). For six of the leading causes, age-adjusted rates were lower for the black population than for the white population. The smallest black-to-white ratios were for suicide (0.4); that is, the risk of dying from suicide is more than double for the white population than for the black population. Other conditions with a low black-to-white ratio were Parkinson's disease (0.5), Chronic lower respiratory diseases (0.7), Alzheimer's disease and Chronic liver disease and cirrhosis $(0.8$ each), and unintentional injuries (0.9).

The difference in life expectancy between the black and white populations narrowed from 5.0 years in 2006 to 4.8 years in 2007 (Table 8). The narrowing in the black-white life expectancy gap was due primarily to greater improvements in mortality for the black population than the white population. In particular, the black population gained ground due to improvements in death rates for unintentional injuries, HIV disease, homicide, and diabetes (data not shown).

Age-adjusted death rates were lower for the AIAN population than the white population for 9 of the 15 leading causes (Table B). The smallest ratios were for Alzheimer's disease and Parkinson's disease (0.5 each), then heart disease, cancer, stroke, and Chronic lower respiratory diseases ( 0.7 each). Age-adjusted rates were higher for the AIAN population than the white population for five leading causes. The largest ratio was for Chronic liver disease and cirrhosis (2.6). Other causes for which the ratio was high include homicide and diabetes (1.8 each) and unintentional injuries (1.3). Rates for the AIAN population are underestimated by about 30 percent due to underreporting on death certificates (16).

For the API population, age-adjusted death rates were lower than those for the white population for 14 of the 15 leading causes (Table B). The largest ratios were for Influenza and pneumonia (0.9), stroke and diabetes ( 0.8 each), and kidney disease (0.7). The smallest ratios were for Chronic lower respiratory diseases and Alzheimer's disease (0.3 each), and unintentional injuries and Chronic liver disease and cirrhosis (0.4 each). The risk of dying from Septicemia, suicide, or Parkinson's disease for the API population is about half that for the white population. Rates for the API population are underestimated by about 7 percent due to underreporting on death certificates (16).

Age-adjusted death rates were lower for the Hispanic population for 11 of the 15 leading causes of death compared with the nonHispanic white population (Tables B and 17). The smallest ratios were for Chronic lower respiratory diseases and suicide (0.4 each). Other causes for which the ratio was considerably smaller include cancer, Alzheimer's disease, and Parkinson's disease ( 0.6 each), heart disease and unintentional injuries ( 0.7 each), and stroke, Influenza and pneumonia, and Septicemia (0.8 each). Age-adjusted death rates for the Hispanic population were greater than for the non-Hispanic white population for 3 of the 15 leading causes of death. The largest ratio was for homicide (2.5), followed by Chronic liver disease and cirrhosis (1.6) and diabetes (1.5). Rates for the Hispanic population are underestimated by about 5.0 percent (16).

Leading causes of death for the total population and specific subpopulations are examined in more detail in a separate National Vital Statistics Report on leading causes by age, race, Hispanic origin, and $\operatorname{sex}(4)$.

\section{Injury mortality by mechanism and intent}

In 2007, a total of 182,479 deaths were classified as injury related (Table 18). Injury data are presented using the external cause-of-injury mortality matrix for ICD-10 as jointly conceived by the International Collaborative Effort (ICE) on Injury Statistics and the Injury Control and Emergency Health Services section, known as ICEHS, of the American Public Health Association $(32,33)$. The ICD codes for injuries have two essential dimensions: the mechanism of the injury and its manner or intent. The mechanism involves the circumstances of the injury (e.g., fall, motor vehicle accident, or poisoning). The manner or intent involves whether the injury was purposefully inflicted (where it can be determined) and, when intentional, whether the injury was self-inflicted (suicide) or inflicted upon another person (assault). In the List of 113 Selected Causes of Death, the focus is on manner or intent, with subcategories showing selected mechanisms. The matrix has two distinct advantages for the analysis of injury mortality data: It contains a comprehensive list of mechanisms, and data can be displayed by mechanism with subcategories of intent (Table 18) or vice versa. Four major mechanisms of 
injury in 2007-motor-vehicle traffic, poisoning, firearm, and fallaccounted for 74.9 percent of all injury deaths.

Motor-vehicle traffic-In 2007, motor-vehicle traffic-related injuries resulted in 42,031 deaths, accounting for 23.0 percent of all injury deaths (Table 18). The 4.2 percent decrease in the age-adjusted death rate for motor-vehicle traffic-related injuries-from 14.4 deaths per 100,000 U.S. standard population in 2006 (34) to 13.8 in 2007-is statistically significant.

Poisoning-In 2007, 40,059 deaths occurred as the result of poisonings, 22.0 percent of all injury deaths (Table 18). The majority of poisoning deaths were either unintentional $(74.5$ percent) or suicides (15.9 percent). However, 9.4 percent of poisoning deaths were of undetermined intent. The age-adjusted death rate for poisoning increased by 5.6 percent from 12.4 deaths per 100,000 U.S. standard population in 2006 to 13.1 in 2007. Unintentional poisoning death rates in the United States have increased each year from 1999 through 2007 (data prior to 2007 are not shown).

Firearm —In 2007, 31,224 persons died from firearm injuries in the United States (Tables 18-20), accounting for 17.1 percent of all injury deaths that year. Firearm suicide at 55.6 percent and homicide at 40.5 percent were the two major component causes of all firearm injury deaths in 2007. In 2007, the age-adjusted death rate for firearm suicide and homicide was unchanged statistically from 2006. The age-adjusted rate for all firearm injuries was the same in 2007 as in 2006-10.2 deaths per 100,000 U.S. standard population (Tables 18-20). In 2007, males had a firearm-related, age-adjusted death rate that was 6.7 times that for females. By comparison with the rate for the white population, the rate for the black population was 2.2 times higher; AIAN, 18.0 percent lower; and API, 67.4 percent lower (Table 19). The non-Hispanic white population's rate was 1.2 times that for the Hispanic population, and the rate for the non-Hispanic black population was 2.7 times that for the Hispanic population (Table 20).

Fall $-\ln 2007,23,443$ persons died as the result of falls, 12.8 percent of all injury deaths (Table 18). The overwhelming majority of fall-related deaths ( 96.5 percent) were unintentional. In 2007, the ageadjusted death rate for falls increased significantly over 2006 (34) by 5.8 percent, from 6.9 deaths per 100,000 U.S. standard population to 7.3.

\section{Drug-induced mortality}

In 2007, a total of 38,371 persons died of drug-induced causes in the United States (Tables 21 and 22). This category includes not only deaths from dependent and nondependent use of legal or illegal drugs, but also poisoning from medically prescribed and other drugs. It excludes unintentional injuries, homicides, and other causes indirectly related to drug use, as well as newborn deaths due to the mother's drug use (for a list of drug-induced causes, see "Technical Notes"). For males in 2007, the age-adjusted death rate for druginduced causes was 1.7 times the rate for females. The age-adjusted death rate for black females was 28.4 percent lower than the rate for white females, and the rate for black males was 8.9 percent lower than the rate for white males. The age-adjusted death rate for the API population was 85.3 percent lower than that for the white population (Table 21). Compared with the rate for the Hispanic population, the rate for the non-Hispanic white population was 2.3 times higher, and that for the non-Hispanic black population was 1.8 times higher (Table 22). In 2007, the age-adjusted death rate for drug-induced causes remained unchanged statistically from 2006. Among the major race-sex and race-ethnic-sex groups during the same period, the age-adjusted death rate for drug-induced causes decreased by 3.7 percent for males, 18.5 percent for black males, 22.7 percent for AIAN males, 23.3 percent for API males, 7.8 percent for Hispanic males, 12.8 percent for Hispanic females, and 18.6 percent for non-Hispanic black males (Table 21). The age-adjusted death rate increased significantly by 3.3 percent for all females, 4.1 percent for white females, and 5.6 percent for non-Hispanic white females.

\section{Alcohol-induced mortality}

In 2007, a total of 23,199 persons died of alcohol-induced causes in the United States, 1,126 more deaths than in 2006 (Tables 23 and 24). This category includes not only deaths from dependent and nondependent use of alcohol, but also accidental poisoning by alcohol. It excludes unintentional injuries, homicides, and other causes indirectly related to alcohol use, as well as deaths due to fetal alcohol syndrome (for a list of alcohol-induced causes, see "Technical Notes"). In 2007, the age-adjusted death rate for alcohol-induced causes for males was 3.2 times the rate for females. Compared with the rate for the white population, the rate for the black population was 16.0 percent lower; AIAN, 3.5 times higher; and API, 76.0 percent lower. The rate for the Hispanic population was 1.3 times the rate for the non-Hispanic white population and 1.4 times the rate for the non-Hispanic black population (Tables 23 and 24). During 2006-2007, the age-adjusted death rate for alcohol-induced causes for the total population increased by 4.3 percent, from 7.0 per 100,000 U.S. standard population in 2006 to 7.3 in 2007. The age-adjusted death rates increased significantly for white males (3.6 percent), non-Hispanic white males (3.8 percent), and nonHispanic white females ( 5.6 percent). No statistically significant changes were observed for other major race-sex and race-ethnic-sex groups.

\section{Marital status}

For those aged 15 and over, the number of deaths in 2007 among persons who were married was 917,839; widowed, 879,173; divorced, 313,863; and never married, 260,281 (Table 25); see "Technical Notes." Those who never married had the highest age-adjusted death rate $(1,780.4$ per 100,000 U.S. standard population), followed by divorced persons $(1,643.8)$, widowed persons $(1,570.7)$, and married persons (828.3). The never-married group had an age-adjusted death rate 60.5 percent higher than those who were ever married and 2.1 times the rate for the currently married. The age-adjusted death rate for widowed persons was 89.6 percent higher than that for persons who were currently married at the time of death. Divorced persons had a rate 98.5 percent higher than those who were married at the time of death.

For all age groups 15 and over, age-specific death rates for married persons were much lower than those for never-married persons. For those aged 15-24, divorced persons had the highest death rate, whereas for those aged 25-34, widowed persons had the highest death rate. Never-married persons had the highest death rate among those aged 35-44, 45-54, 55-64, 65-74, and 75 and over. 
For each marital status group in 2007, males had higher ageadjusted death rates than females, ranging from 36.6 percent greater for the never married to 72.1 percent greater for those divorced at the time of death.

\section{Educational attainment}

Age-specific and age-adjusted death rates are shown by educational attainment for age groups in the range of 25-64 years (Table 26). Figures for states that used the 2003 version of the standard death certificate are shown separately from those for states that used the 1989 version of the death certificate (see "Technical Notes"). In the District of Columbia and the 22 reporting states that used the 2003 version of the death certificate in 2007, a total of 126,184 decedents aged 25-64 had received a high school diploma or equivalent, compared with 109,248 who had completed some college or collegiate degree and 60,991 who had achieved less than a high school diploma or equivalent. For the total population, and for males and females separately, mortality is inversely associated with educational attainment; that is, the average risk of death decreases markedly with increasing educational attainment. The age-adjusted death rate for those with less than a high school diploma or equivalent was 529.5 per 100,000 U.S. standard population14.1 percent higher than the rate of 463.9 for those with a high school diploma or equivalent and 2.7 times the rate of 196.7 for those with some college or collegiate degree.

For the 26 reporting states that used the 1989 version of the death certificate, a total of 115,327 decedents aged 25-64 years had completed 12 years of education, compared with 81,214 who had completed 13 years or more and 53,458 who had completed less than 12 years. The age-adjusted death rate for those with less than 12 years of education was 664.4 per 100,000 U.S. standard population39.3 percent higher than the rate of 477.0 for those with 12 years of education and 3.4 times the rate of 195.4 for those with 13 years of education or more.

Rates are shown only for those aged 25-64 because persons under age 25 may not have completed their education. Rates are not shown for older ages because of misreporting of educational attainment on the death certificate; see "Technical Notes." Data on educational attainment must be interpreted with caution because of misreporting on the death certificate and biases that result from differences between the classification of educational attainment on the death certificate and in census surveys; see "Technical Notes."

\section{Injury at work}

For persons aged 15 and over, a total of 5,025 deaths were reported on death certificates as due to injuries at work in 2007 (Table 27). Rates were lowest for age groups 15-24 and 65 and over. The risk of work-related death was much greater for males than for females-the age-adjusted death rate for males was 3.9 deaths per 100,000 U.S. standard population compared with 0.3 for females, resulting in a mortality ratio of about 13 to 1 . The age-adjusted rate for the white population, 2.1, was slightly higher than the rate for the black population at 2.0. Male-to-female ratios were 13.3 for the white population and 9.5 for the black population.

The number of deaths due to injuries at work decreased by 273 deaths in 2007 over 2006. The age-adjusted death rate from injury at work for the population aged 15 and over decreased 4.5 percent in 2007 over the year before (Table 28). For specific sex and race groups, the age-adjusted death rate decreased for white males (7.0 percent) and did not change for white females, black males, and black females.

\section{State of residence}

Mortality patterns vary considerably by state (Table 29). The state with the highest age-adjusted death rate in 2007 was West Virginia (951.7 per 100,000 U.S. standard population), with a rate 25.2 percent above the national average (760.2). The state with the lowest age-adjusted death rate was Hawaii (607.4 per 100,000 standard population), with a rate 20.1 percent below the national average. The age-adjusted death rate for West Virginia was 56.7 percent higher than the rate for Hawaii.

Variations in mortality by state are associated with differences in socioeconomic status, race, and ethnic composition as well as differences in risk for specific causes of death (35).

\section{Infant mortality}

In 2007, a total of 29,138 deaths occurred in children under age 1 year (Table D), 611 more deaths than in 2006. The infant mortality rate was 6.75 per 1,000 live births, the neonatal mortality rate (deaths of infants aged 0-27 days per 1,000 live births) was 4.42, and the postneonatal mortality rate (deaths of infants aged 28 days -1 year per 1,000 live births) was 2.34 in 2007 (Tables 30-32 and Figure 7); see "Technical Notes" for information on alternative data sources. The year-to-year change in the infant and neonatal mortality rates during 2006-2007 was not statistically significant; however, the postneonatal mortality rate increased 4.5 percent, from 2.24 in 2006 to 2.34 in 2007, for all races combined. Rates also increased significantly for male postneonates (4.0 percent), female postneonates ( 4.5 percent), and white postneonates (5.4 percent).

The 10 leading causes of infant death in 2007 accounted for 69.0 percent of all infant deaths in the United States (Table E). By rank, the 10 leading causes were:

1. Congenital malformations, deformations and chromosomal abnormalities (congenital malformations)

2. Disorders related to short gestation and low birth weight, not elsewhere classified (low birthweight)

3. Sudden infant death syndrome (SIDS)

4. Newborn affected by maternal complications of pregnancy (maternal complications)

5. Accidents (unintentional injuries)

6. Newborn affected by complications of placenta, cord and membranes (cord and placental complications)

7. Bacterial sepsis of newborn

8. Respiratory distress of newborn

9. Diseases of the circulatory system

10. Neonatal hemorrhage

The 10 leading causes of infant death were the same in 2007 as in 2006 (34), with four leading causes changing rankings compared with 2006. Bacterial sepsis of newborn became the seventh leading cause in 2007, exchanging positions with Respiratory distress of newborn, which became the eighth leading cause. Similarly, Diseases 
Table D. Number of infant, neonatal, and postneonatal deaths and mortality rates, by sex: United States, 2006-2007

[Rates are infant (under 1 year), neonatal (under 28 days), and postneonatal (28 days-11 months) deaths per 1,000 live births in specified group]

\begin{tabular}{|c|c|c|c|c|c|c|}
\hline & \multirow[b]{2}{*}{ Infant age and sex } & \multicolumn{2}{|c|}{2007} & \multicolumn{2}{|c|}{2006} & \multirow{2}{*}{$\begin{array}{l}\text { Percent change }{ }^{1} \\
\text { from } 2006 \text { to } 2007\end{array}$} \\
\hline & & Number & Rate & Number & Rate & \\
\hline \multicolumn{7}{|c|}{ Infant } \\
\hline Total & $\ldots \ldots \ldots \ldots \ldots$ & 29,138 & 6.75 & 28,527 & 6.69 & 0.9 \\
\hline Male. & $\ldots \ldots \ldots \ldots \ldots \ldots$ & 16,293 & 7.38 & 15,980 & 7.32 & 0.8 \\
\hline Female & $\ldots \ldots \ldots \ldots$ & 12,845 & 6.09 & 12,547 & 6.03 & 1.0 \\
\hline \multicolumn{7}{|c|}{ Neonatal } \\
\hline Total & & 19,058 & 4.42 & 18,989 & 4.45 & -0.7 \\
\hline Male & & 10,587 & 4.79 & 10,564 & 4.84 & -1.0 \\
\hline Female & $\ldots$ & 8,471 & 4.02 & 8,425 & 4.05 & -0.7 \\
\hline \multicolumn{7}{|c|}{ Postneonatal } \\
\hline Total & $\ldots \ldots \ldots \ldots$ & 10,080 & 2.34 & 9,538 & 2.24 & 4.5 \\
\hline Male. & $\ldots \ldots \ldots \ldots$ & 5,706 & 2.58 & 5,416 & 2.48 & 4.0 \\
\hline Female & $\ldots \ldots \ldots \ldots \ldots \ldots$ & 4,374 & 2.07 & 4,122 & 1.98 & 4.5 \\
\hline
\end{tabular}

${ }^{1}$ Based on a comparison of the 2007 and 2006 mortality rates.

of the circulatory system became the 9th leading cause in 2007 and Neonatal hemorrhage became the 10th leading cause.

Changes in rates by cause of death among the 10 leading causes were statistically significant for only two conditions: In 2007, unintentional injuries increased by 10.8 percent and Diseases of the circulatory system increased by 14.2 percent from 2006 (Table E).

The ratio of male-to-female infant mortality rates was 1.2 in 2007-the same as in 2006. The ratio of black-to-white infant mortality rates was 2.3 in 2007. The infant mortality rate did not change significantly in 2007 from 2006 for white infants or black infants (Table 30). Race cited on the death certificate is considered to be relatively accurate for white and black infants (16). For other race groups, however, race may be misreported on the death certificate (36). A forthcoming report using data from the linked file of live births and infant deaths will provide better measures of infant mortality by race (37); see "Technical Notes."

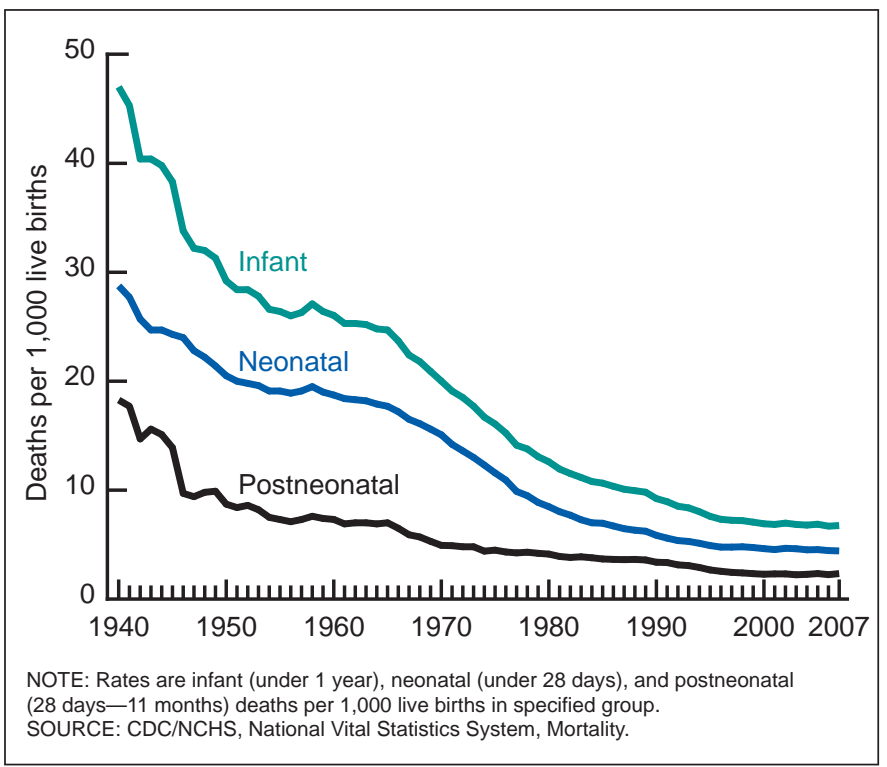

Figure 7. Infant, neonatal, and postneonatal mortality rates: United States, 1940-2007
Hispanic infant mortality —In 2007, the infant mortality rate for Hispanic infants was 5.71 deaths per 1,000 live births, and for nonHispanic white infants, 5.63 (data not shown). Among Hispanic subgroups, the infant mortality rate was 7.99 per 1,000 live births for Puerto Rican, 5.87 for Mexican, 4.59 for Cuban, and 3.24 for Central and South American populations. In 2007, the infant mortality rate for Central and South American infants increased by 14.1 percent from 2006. The postneonatal mortality rate for the Hispanic population increased by 9.3 percent. No other Hispanic infant mortality rates changed by a statistically significant amount during 2006-2007. Infant mortality rates by specified Hispanic origin and race for non-Hispanic origin are somewhat understated and better measured using data from the linked file of live births and infant deaths (36); see "Technical Notes."

\section{Maternal mortality}

In 2007, a total of 548 women were reported to have died of maternal causes (Tables 33 and 34). As in previous years, the number of maternal deaths does not include all deaths occurring to pregnant women, but only those deaths reported on the death certificate that were assigned to causes related to or aggravated by pregnancy or pregnancy management (ICD-10 codes A34, 000-095, and 098-099). Further, the number excludes deaths occurring more than 42 days after the termination of pregnancy and deaths of pregnant women due to external causes (unintentional injuries, homicides, and suicides) (6). An increasing number of states are adopting a separate item on the death certificate indicating pregnancy status of the decedent to improve measurement; see "Technical Notes." The number of areas with such an item has increased from 16 states in 1996 to 34 states and the District of Columbia in 2007. The maternal mortality rate for 2007 was 12.7 deaths per 100,000 live births. Black women have a substantially higher risk of maternal death than white women. The maternal mortality rate for black women was 26.5 , roughly 2.7 times the rate for white women (10.0 deaths per 100,000 live births).

Hispanic maternal mortality -The maternal mortality rate for Hispanic women was 8.9 deaths per 100,000 live births. The comparable rate for non-Hispanic white women was 10.5 in 2007. The difference 
Table E. Number of infant deaths, percentage of total infant deaths, and infant mortality rates for 2007, and percentage change in infant mortality rates in 2007 from 2006 for the 10 leading causes of infant death in 2007: United States

[Rates are infant deaths per 100,000 live births]

\begin{tabular}{|c|c|c|c|c|c|}
\hline Rank $^{1}$ & Cause of death (based on ICD-10, 2004) & Number & $\begin{array}{l}\text { Percent } \\
\text { of total } \\
\text { deaths }\end{array}$ & Rate & $\begin{array}{c}\text { Percent } \\
\text { change } \\
\text { in } 2007 \\
\text { from } \\
2006\end{array}$ \\
\hline$\ldots$ & All causes & 29,138 & 100.0 & 675.1 & 0.9 \\
\hline 1 & Congenital malformations, deformations and chromosomal abnormalities. . . & 5,785 & 19.9 & 134.0 & -1.8 \\
\hline 2 & Disorders related to short gestation and low birth weight, not elsewhere classified. . . (P07) & 4,857 & 16.7 & 112.5 & -0.9 \\
\hline 3 & Sudden infant death syndrome $\ldots \ldots \ldots \ldots \ldots \ldots \ldots \ldots \ldots \ldots$ (R95) & 2,453 & 8.4 & 56.8 & 4.2 \\
\hline 4 & Newborn affected by maternal complications of pregnancy . . . . . . . . . . (P01) & 1,769 & 6.1 & 41.0 & 3.8 \\
\hline 5 & 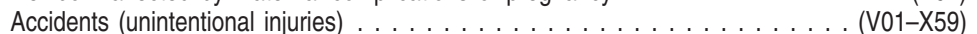 & 1,285 & 4.4 & 29.8 & 10.8 \\
\hline 6 & Newborn affected by complications of placenta, cord and membranes . . . . . . . . . (P02) & 1,135 & 3.9 & 26.3 & -1.5 \\
\hline 7 & 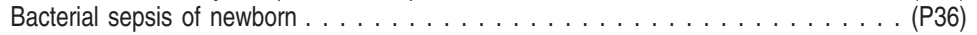 & 820 & 2.8 & 19.0 & 0.5 \\
\hline 8 & 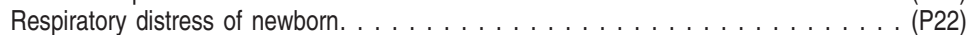 & 789 & 2.7 & 18.3 & -5.2 \\
\hline 9 & Diseases of the circulatory system . & 624 & 2.1 & 14.5 & 14.2 \\
\hline \multirow[t]{2}{*}{10} & Neonatal hemorrhage. . . . . . . . . & 597 & 2.0 & 13.8 & -4.8 \\
\hline & All other causes. ... . & 9,024 & 31.0 & 209.1 & \\
\hline
\end{tabular}

... Category not applicable.

${ }^{1}$ Based on number of deaths; see "Technical Notes."

${ }^{2}$ Based on comparison of the 2007 infant mortality rate with the 2006 infant mortality rate.

NOTE: ICD is International Classification of Diseases.

between the Hispanic and non-Hispanic white rates was not statistically significant. As with other statistics involving Hispanic origin, these should be interpreted with caution because of inconsistencies between reporting Hispanic origin on death certificates and on censuses and surveys; see "Technical Notes."

\section{References}

1. Hoyert D, Singh G, Rosenberg H. Sources of data on socioeconomic differential mortality in the United States. Journal of Official Statistics 11(3):233-60. 1995.

2. $\mathrm{Xu} \mathrm{JQ}$, Kochanek KD, Tejada-Vera B. Deaths: Preliminary data for 2007. National vital statistics reports; vol 58 no 1. Hyattsville, MD: National Center for Health Statistics. 2009. Available from: http://www.cdc.gov/nchs/data/nvsr/nvsr58/nvsr58_01.pdf.

3. Heron M. Deaths: Leading causes for 2007. National vital statistics reports. Hyattsville, MD: National Center for Health Statistics. Forthcoming.

4. Arias E. United States life tables, 2007. National vital statistics reports. Hyattsville, MD: National Center for Health Statistics. Forthcoming.

5. National Center for Health Statistics. Technical appendix. Vital statistics of the United States: Mortality. Washington, DC. Published annually. Available from:

http://www.cdc.gov/nchs/products/vsus.htm\#appendices.

6. World Health Organization. International statistical classification of diseases and related health problems, tenth revision. 2nd edition. Geneva, Switzerland. 2004.

7. Office of Management and Budget. Revisions to the standards for the classification of federal data on race and ethnicity. Federal Register 62FR58782 (58790). Washington, DC. October 30, 1997. Available from: http://federalregister.gov/a/97-28653.

8. Office of Management and Budget. Race and ethnic standards for federal statistics and administrative reporting. Statistical Policy Directive 15. Washington, DC. 1977.

9. U.S. Census Bureau. Age, sex, race, and Hispanic origin information from the 1990 census: A comparison of census results with results where age and race have been modified, 1990. CPH-L-74. Washington, DC: U.S. Department of Commerce. 1991.

10. Ingram D, Weed J, Parker J, Hamilton B, Schenker N, et al. U.S. census 2000 population with bridged race categories. National Center for Health Statistics. Vital Health Stat 2(135). 2003.

11. Schenker N, Parker J. From single-race reporting to multiple-race reporting: Using imputation methods to bridge the transition. Stat Med 22:1571-87. 2003.

12. Centers for Disease Control and Prevention. Update: Influenza activity-United States, 1998-99 season. MMWR; 48(9):177-81. Washington, DC: Public Health Service. 1999.

13. Centers for Disease Control and Prevention. Update: Influenza activity—United States, 1999-2000 season. MMWR; 49(9):173-7. Washington, DC: Public Health Service. 2000.

14. Centers for Disease Control and Prevention. Update: Influenza Activity-United States and worldwide, 2006-07 season, and composition of the 2007-08 influenza vaccine. MMWR; 56 (31):789-94. Washington, DC: Public Health Service. 2007.

15. Centers for Disease Control and Prevention. Influenza activity-United States and worldwide, 2007-08 season. MMWR; 57 (25):692-7. Washington, DC: Public Health Service. 2008.

16. Arias E, Schauman WS, Eschbach K, Sorlie PD, Backlund E. The validity of race and Hispanic origin reporting on death certificates in the United States. National Center for Health Statistics. Vital Health Stat 2(148). 2008.

17. Kochanek K, Maurer J, Rosenberg H. Causes of death contributing to changes in life expectancy: United States, 1984-89. National Center for Health Statistics. Vital Health Stat 20(23). 1994. Available from: http://www.cdc.gov/nchs/data/series/sr_20/sr20_023.pdf.

18. Arias E, Eschbach K, Schauman WS, Backlund EL, Sorlie PD. The Hispanic mortality advantage and ethnic misclassification on US death certificates. Am J Public Health 100(S1):S171-7. 2010. Available from: http://ajph.aphapublications.org/cgi/content/abstract/100/S1/S171.

19. Abraida-Lanza A, Dohrenwend B, Ng-Mak D, Turner J. The Latino mortality paradox: A test of the "salmon bias" and healthy migrant hypotheses. Am J Public Health 89(10):1543-8. 1999. Available from: http://www.ncbi.nlm.nih.gov/pmc/articles/PMC1508801/pdf/ amjph00010-0085.pdf. 
20. Palloni A, Arias E. Paradox lost: Explaining the Hispanic adult mortality advantage. Demography 41(3):385-415. 2004.

21. Hoyert D. Mortality trends for Alzheimer's disease, 1979-91. National Center for Health Statistics. Vital Health Stat 20(28). 1996. Available from: http://www.cdc.gov/nchs/data/series/sr_20/sr20_028.pdf.

22. Hoyert D, Arias E, Smith B, Murphy S, Kochanek K. Deaths: Final data for 1999. National vital statistics reports; vol 49 no 8. Hyattsville, MD: National Center for Health Statistics. 2001. Available from: http://www.cdc.gov/nchs/data/nvsr/nvsr49/nvsr49_08.pdf.

23. National Center for Health Statistics, Data Warehouse. Comparability of cause-of-death between ICD revisions [online]. 2008. Available from: http://www.cdc.gov/nchs/nvss/mortality/comparability_icd.htm.

24. National Center for Health Statistics, Data Warehouse. Updated comparability ratios (ICD-10 and ICD-9) [online]. 2004. Available from: ftp://ftp.cdc.gov/pub/Health_Statistics/NCHS/Datasets/Comparability/ icd9_icd10/Comparability_Ratio_tables.xls.

25. Anderson R, Miniño A, Hoyert D, Rosenberg H. Comparability of cause of death between ICD-9 and ICD-10: Preliminary estimates. National vital statistics reports; vol 49 no 2. Hyattsville, MD: National Center for Health Statistics. 2001. Available from: http://www.cdc.gov/nchs/data/nvsr/nvsr49/nvsr49_02.pdf.

26. Hoyert D, Kochanek K, Murphy S. Deaths: Final data for 1997. National vital statistics reports; vol 47 no 19. Hyattsville, MD: National Center for Health Statistics. 1999.

27. National Center for Health Statistics. Hist293. Age-adjusted death rates for 72 selected causes by race and sex using year 2000 standard population: United States, 1979-98 [online]. 2001. Available from: http://www.cdc.gov/nchs/data/mortab/aadr7998s.pdf.

28. Barbut F, Petit JC. Epidemiology of Clostridium difficile-associated infections. Clin Microbiol Infect 7(8):405-10. 2001.

29. Centers for Disease Control and Prevention. Severe Clostridium difficile-associated disease in populations previously at low risk-Four states, 2005. MMWR 54(47):1201-5. 2005.

30. Suneshine $\mathrm{RH}, \mathrm{McD}$ onald LC. Clostridium difficile-associated disease: New challenges from an established pathogen. Cleve Clin J Med 73(2):187-97. 2006.

31. Redelings MD, Sorvillo F, Mascola L. Increase in Clostridium difficile related mortality rates, United States, 1999-2004. Emerging infectious diseases [online series] 13(9):1417-9. 2007. Available from: http://www.cdc.gov/EID/content/13/9/1417.htm.

32. National Center for Health Statistics. Proceedings of the international collaborative effort on injury statistics; vol 1. Hyattsville, MD. 1995.

33. Fingerhut $\mathrm{L}$, Cox $\mathrm{C}$, Warner $\mathrm{M}$. International comparative analysis of injury mortality: Findings from the ICE on injury statistics. Advance data from vital and health statistics; no 303. Hyattsville, MD: National Center for Health Statistics. 1998.

34. Heron M, Hoyert DL, Murphy SL, Xu JQ, Kochanek KD, Tejada-Vera B. Deaths: Final data for 2006. National vital statistics reports; vol 57 no 14. Hyattsville, MD: National Center for Health Statistics. 2009. Available from: http://www.cdc.gov/nchs/data/nvsr/nvsr57/nvsr57_14.pdf.

35. Pamuk E, Makuc D, Heck K, Reuben C, Lochner K. Socioeconomic status and health chartbook. Health, United States, 1998. Hyattsville, MD: National Center for Health Statistics. 1998. Available from: http://www.cdc.gov/nchs/data/hus/hus98cht.pdf.

36. Mathews T, MacDorman M. Infant mortality statistics from the 2006 period linked birth/infant death data set. National vital statistics reports; vol 58 no 17. Hyattsville, MD: National Center for Health Statistics. 2010. Available from: http://www.cdc.gov/nchs/data/nvsr/nvsr58/nvsr58_17.pdf.

37. Mathews T, MacDorman M. Infant mortality statistics from the 2007 period linked birth/infant death data set. National vital statistics reports. Hyattsville, MD: National Center for Health Statistics. Forthcoming.
38. National Center for Health Statistics. 2003 revision of the U.S. Standard Certificate of Death [online]. 2003. Available from: http://www.cdc.gov/nchs/data/dvs/DEATH11-03final-acc.pdf.

39. National Center for Health Statistics. Report of the panel to evaluate the U.S. standard certificates [online]. 2000. Available from: http://www.cdc.gov/nchs/data/dvs/panelreport_acc.pdf.

40. National Center for Health Statistics. Technical appendix. Vital statistics of the United States, 1989, vol II, mortality, part A. Washington, DC. 1993.

41. Tolson G, Barnes J, Gay G, Kowaleski J. The 1989 revision of the U.S. standard certificates and reports. National Center for Health Statistics. Vital Health Stat 4(28). 1991.

42. World Health Organization. International statistical classification of diseases and related health problems, tenth revision. Geneva, Switzerland. 1992.

43. Comparability of mortality statistics for the sixth and seventh revisions, United States, 1958. Vital statistics-Special reports 51(4). Washington, DC: National Center for Health Statistics. 1965.

44. Klebba A, Dolman A. Comparability of mortality statistics for the seventh and eighth revisions of the international classification of diseases, United States. National Center for Health Statistics. Vital Health Stat 2(66). 1975.

45. Klebba A, Scott J. Estimates of selected comparability ratios based on dual coding of 1976 death certificates by the eighth and ninth revisions of the international classification of diseases. Monthly vital statistics report; vol 28 no 11. Hyattsville, MD: National Center for Health Statistics. 1980.

46. National Center for Health Statistics, Vital statistics. Instructions for classifying multiple causes of death. NCHS instruction manual; part 2b. Hyattsville, MD. Published annually.

47. National Center for Health Statistics, Vital statistics. Instructions for classifying the underlying cause of death. NCHS instruction manual; part 2a. Hyattsville, MD. Published annually.

48. National Center for Health Statistics, Vital statistics. ICD-10 ACME decision tables for classifying underlying causes of death. NCHS instruction manual; part 2c. Hyattsville, MD. Published annually.

49. National Center for Health Statistics, Vital statistics. Data entry instructions for the mortality medical indexing, classification, and retrieval system (MICAR). NCHS instruction manual; part 2g. Hyattsville, MD. Published annually.

50. National Center for Health Statistics, Vital statistics. Dictionary of valid terms for the mortality medical indexing, classification, and retrieval system (MICAR). NCHS instruction manual; part 2h. Hyattsville, MD. Published annually.

51. National Center for Health Statistics. Public-use data set documentation; control total Table 1: Mortality data set for ICD-10, 2007. Hyattsville, MD. Forthcoming.

52. Chamblee R, Evans M. TRANSAX, the NCHS system for producing multiple cause-of-death statistics, 1968-78. National Center for Health Statistics. Vital Health Stat 1(20). 1986.

53. Israel $\mathrm{R}$, Rosenberg $\mathrm{H}$, Curtin L. Analytical potential for multiple cause-of-death data. Am J Epidemiol 124(2):161-79. 1986.

54. National Center for Health Statistics. ICD-10 cause-of-death lists for tabulating mortality statistics (updated October 2007 to include WHO updates to ICD-10 for data year 2007). NCHS instruction manual, part 9. Hyattsville, MD. 2007.

55. National Center for Health Statistics. ICD-10 cause-of-death querying. NCHS instruction manual; part 20. Hyattsville, MD. Published annually.

56. National Center for Health Statistics. Vital statistics, data preparation. Computer edits for mortality data, including separate section for fetal deaths. NCHS instruction manual; part 11. Hyattsville, MD. Published annually. 
57. Miniño A, Anderson R, Fingerhut L, Boudreault M, Warner M. Deaths: Injuries, 2002. National vital statistics reports; vol 54 no 10. Hyattsville, MD: National Center for Health Statistics. 2006. Available from: http://www.cdc.gov/nchs/products/nvsr.htm\#vol54.

58. Rosenberg H, Maurer J, Sorlie P, Johnson N. Quality of death rates by race and Hispanic origin: A summary of current research, 1999. National Center for Health Statistics. Vital Health Stat 2(128). 1999.

59. Sorlie P, Rogot E, Johnson N. Validity of demographic characteristics on the death certificate. Epidemiology 3(2):181-4. 1992.

60. Mulry M. Summary of accuracy and coverage evaluation for census 2000. Research Report Series, Statistics \#2006-3. Washington, DC: Statistical Research Division, U.S. Census Bureau. 2006. Available from: http://www.census.gov/srd/papers/pdf/rrs2006-03.pdf.

61. Poe G, Powell-Griner E, McLaughlin J. Comparability of the death certificate and the 1986 national mortality followback survey. National Center for Health Statistics. Vital Health Stat 2(118). 1993. Available from: http://www.cdc.gov/nchs/data/series/sr_02/sr02_118.pdf.

62. National Center for Health Statistics. Technical appendix. Vital statistics of the United States, 1989, vol I, natality. Hyattsville, MD. 1993.

63. National Center for Health Statistics. Technical appendix. Vital statistics of the United States: Mortality, 1999. Hyattsville, MD. 2004. Available from: http://www.cdc.gov/nchs/data/statab/techap99.pdf.

64. Hoyert D. Effect on mortality rates of the 1989 changes in tabulating race. National Center for Health Statistics. Vital Health Stat 20(25). 1994. Available from: http://www.cdc.gov/nchs/data/series/sr_20/sr20_025.pdf.

65. Martin JA, Hamilton BE, Sutton PD, et al. Births: Final data for 2007. National vital statistics reports. Hyattsville, MD: National Center for Health Statistics. Forthcoming.

66. Anderson R. Method for constructing complete annual U.S. life tables. National Center for Health Statistics. Vital Health Stat 2(129). 1999. Available from: http://www.cdc.gov/nchs/data/series/sr_02/sr02_129.pdf.

67. National Center for Health Statistics. U.S. decennial life tables for 1989-91, vol 1 no 2, Methodology of the national and state life tables. Hyattsville, MD. 1998. Available from: http://www.cdc.gov/nchs/data/lifetables/life89_1_2.pdf.

68. Kestenbaum B. A description of the extreme aged population based on improved Medicare enrollment data. Demography 29:565-80. 1992.

69. Wei R, Curtin LR, Arias E, Anderson RN. U.S. decennial life tables for 1999-2001: Methodology of the United States life tables. National vital statistics reports; vol 57, no 4. Hyattsville, MD: National Center for Health Statistics. 2008. Available from:

http://www.cdc.gov/nchs/data/nvsr/nvsr57/nvsr57_04.pdf.

70. Arriaga $\mathrm{E}$. Changing trends in mortality decline during the last decades. In: Ruzicka L, Wunsch G, Kane P, editors. Differential mortality: Methodological issues and biosocial factors. Oxford: Clarendon Press. 1989.

71. Arriaga E. Measuring and explaining the change in life expectancies. Demography 21(1):83-96. 1984.

72. Kominski R, Adams A. Educational attainment in the United States, March 1993 and 1992. Current population reports, Population characteristics P20-476. Washington, DC: U.S. Bureau of the Census. 1994. Available from:

http://www.census.gov/population/www/socdemo/education/p20476.html.

73. Sorlie PD, Johnson N. Validity of education information on the death certificate. Epidemiology 7(4):437-9. 1996.

74. Rostron B, Boies J, Arias E. Education reporting and classification on death certificates in the United States. Vital Health Stat 2(151). Hyattsville, MD: National Center for Health Statistics. 2010. Available from: http://www.cdc.gov/nchs/data/series/sr_02/sr02_151.pdf.
75. MacKay A, Berg C, Duran C, Chang J, Rosenberg H. An assessment of pregnancy-related mortality in the United States. Paediatr Perinat Epidemiol 19(3):206-14. 2005.

76. Hoyert D. Maternal mortality and related concepts. National Center for Health Statistics. Vital Health Stat 3(33). Hyattsville, MD. 2007. Available from:

http://www.cdc.gov/nchs/data/series/sr_03/sr03_033.pdf.

77. National Center for Health Statistics. Vintage 2007 bridged-race postcensal population estimates of the resident population of the United States for July 1, 2000-July 1, 2007, by year, county, single-year of age, bridged race, Hispanic origin, and sex (pcen_v2007.txt). Prepared under a collaborative agreement with the U.S. Census Bureau. 2008. Available from: http://www.cdc.gov/nchs/ nvss/bridged_race/data_documentation.htm\#vintage2007.

78. U.S. Census Bureau, Housing and Household Economic Statistics Division. Population estimates for 2007 based on unpublished tabulations. 2009.

79. U.S. Census Bureau. International data base. 2008. Available from: http://www.census.gov/ipc/www/idb.

80. National Center for Health Statistics. Vintage 2006 bridged-race postcensal population estimates of the resident population of the United States as of July 1, 2006, by year, county, single-year of age, bridged race, Hispanic origin, and sex [pcen_v2006_y06.txt (ASCII)]. 2007. Available from: http://www.cdc.gov/nchs/nvss/bridged_race/ data_documentation.htm\#vintage2006.

81. National Center for Health Statistics. Vintage 2005 bridged-race postcensal population estimates of the resident population of the United States as of July 1, 2005, by year, county, single-year of age, bridged race, Hispanic origin, and sex [pcen_v2005_y05.txt (ASCII)]. 2006. Available from: http://www.cdc.gov/nchs/nvss/bridged_race/ data_documentation.htm\#vintage2005.

82. National Center for Health Statistics. Vintage 2004 bridged-race postcensal population estimates of the resident population of the United States for July 1, 2000-July 1, 2004, by year, county, single-year of age, bridged race, Hispanic origin, and sex (pcen_v2004.txt). Prepared under a collaborative agreement with the U.S. Census Bureau. 2005. Available from: http://www.cdc.gov/nchs/ nvss/bridged_race/data_documentation.htm\#vintage2004.

83. National Center for Health Statistics. Bridged-race intercensal population estimates for July 1, 1990-July 1, 1999, by year, county, 5-year age group, bridged-race, Hispanic origin, and sex (one ASCII file each per separate year). Prepared under a collaborative agreement with the U.S. Census Bureau. 2003. Available from:

http://www.cdc.gov/nchs/nvss/bridged_race.htm.

84. National Center for Health Statistics. Bridged-race population estimates for April 1, 2000, by county, single-year age group, bridged-race, Hispanic origin, and sex (br040100.txt). Prepared under a collaborative arrangement with the U.S. Census Bureau. 2003. Available from: http://www.cdc.gov/nchs/nvss/bridged_race.htm.

85. National Center for Health Statistics. Vintage 2001 bridged-race postcensal population estimates for July 1, 2001, by single-year of age, bridged-race, Hispanic origin, and sex (pcen_v2001.txt). Prepared under a collaborative arrangement with the U.S. Census Bureau. 2003. Available from: http://www.cdc.gov/nchs/nvss/bridged_race.htm.

86. National Center for Health Statistics. Vintage 2002 bridged-race postcensal population estimates for July 1, 2002, by county, single-year of age, bridged-race, Hispanic origin, and sex (pcen_v2002.txt). Prepared under a collaborative arrangement with the U.S. Census Bureau. 2003. Available from: http://www.cdc.gov/nchs/nvss/bridged_race.htm.

87. National Center for Health Statistics. Vintage 2003 bridged-race postcensal population estimates as of July 1, 2003, by county, single-year of age, bridged-race, Hispanic origin, and sex 
(pcen_v2003_y03.txt). Prepared under a collaborative arrangement with the U.S. Census Bureau. 2004. Available from: http://www.cdc.gov/nchs/nvss/bridged_race.htm.

88. Anderson $\mathrm{R}$, Rosenberg $\mathrm{H}$. Age standardization of death rates: Implementation of the year 2000 standard. National vital statistics reports; vol 47 no 3. Hyattsville, MD: National Center for Health Statistics. 1998. Available from:

http://www.cdc.gov/nchs/data/nvsr/nvsr47/nvs47_03.pdf.

89. Brillinger $D$. The natural variability of vital rates and associated statistics. Biometrics 42(4):693-734. 1986.

90. Chiang C. Introduction to stochastic processes in biostatistics. New York: Wiley. 1968.

91. DeNavas-Walt C, Proctor B, Smith J. Income, poverty, and health insurance coverage in the United States: 2007. Current Population Reports, P60-235. Washington, DC: U.S. Census Bureau. 2008. Available from: http://www.census.gov/prod/2008pubs/p60-235.pdf.

92. DeNavas-Walt C, Proctor B, Smith J. Income, poverty, and health insurance coverage in the United States: 2006. Current population reports P60-233. Washington, DC: U.S. Census Bureau. 2007. Available from: http://www.census.gov/prod/2007pubs/p60-233.pdf.

93. Fay M, Feuer E. Confidence intervals for directly standardized rates: A method based on the gamma distribution. Stat Med 16(17):791-801. 1997.

94. Schenker N, Gentleman J. On judging the significance of differences by examining the overlap between confidence intervals. The American Statistician 55(3):182-6. 2001.

95. Arnold S. Mathematical statistics. Englewood Cliffs, NJ: Prentice Hall. 1990.

\section{List of Detailed Tables}

1. Number of deaths, death rates, and age-adjusted death rates, by race and sex: United States, 1940, 1950, 1960, 1970, and 1980-2007 . . . . . . . . . . . . . . . . . . . . . . . . .

2. Number of deaths, death rates, and age-adjusted death rates, by Hispanic origin, race for non-Hispanic population, and sex: United States, 1997-2007 . . . . . . . . . . . . . . . . . . . .

3. Number of deaths and death rates, by age, race, and sex: United States, 2007 . . . . . . . . . . . . . . . . . . . . .

4. Number of deaths and death rates, by Hispanic origin, race for non-Hispanic population, age, and sex: United States, 2007 . .

5. Number of deaths and death rates by age, and age-adjusted death rates, by specified Hispanic origin, race for non-Hispanic population, and sex: United States, $2007 \ldots \ldots \ldots \ldots \ldots$. . . . .

6. Abridged life table for the total population, 2007 . . . . . . . .

7. Life expectancy at selected ages, by race and sex: United States, 2007

8. Life expectancy at birth, by race and sex: United States, 1940, 1950, 1960, 1970, and 1975-2007 . . . . . . . . . . .

9. Death rates by age and age-adjusted death rates for the 15 leading causes of death in 2007: United States, 1999-2007 . .

10. Number of deaths from 113 selected causes and Enterocolitis due to Clostridium difficile, by age: United States, 2007 . . . . .

11. Death rates for 113 selected causes and Enterocolitis due to Clostridium difficile, by age: United States, 2007 . . . . . . . . .

12. Number of deaths from 113 selected causes and Enterocolitis due to Clostridium difficile, by race and sex: United States, 2007

13. Number of deaths from 113 selected causes and Enterocolitis due to Clostridium difficile, by Hispanic origin, race for nonHispanic population, and sex: United States, 2007 . . . . . . . .

14. Death rates for 113 selected causes and Enterocolitis due to Clostridium difficile, by race and sex: United States, 2007 . . . .
15. Death rates for 113 selected causes and Enterocolitis due to Clostridium difficile, by Hispanic origin, race for non-Hispanic population, and sex: United States, $2007 \ldots \ldots$. . . . . . . .

16. Age-adjusted death rates for 113 selected causes and Enterocolitis due to Clostridium difficile, by race and sex: United States,

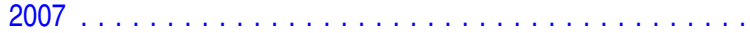

17. Age-adjusted death rates for 113 selected causes and Enterocolitis due to Clostridium difficile, by Hispanic origin, race for non-Hispanic population, and sex: United States, 2007 . . . . . .

18. Number of deaths, death rates, and age-adjusted death rates for injury deaths, by mechanism and intent of death: United States, 2007

19. Number of deaths, death rates, and age-adjusted death rates for injury by firearms, by race and sex: United States, 1999-2007.

20. Number of deaths, death rates, and age-adjusted death rates for injury by firearms, by Hispanic origin, race for non-Hispanic population, and sex: United States, 1999-2007 . . . . . . . . . .

21. Number of deaths, death rates, and age-adjusted death rates for drug-induced causes, by race and sex: United States, 1999-2007 . . . . . . . . . . . . . . . . . . . . . . . . . . . . . .

22. Number of deaths, death rates, and age-adjusted death rates for drug-induced causes, by Hispanic origin, race for non-Hispanic population, and sex: United States, 1999-2007 . . . . . . . . .

23. Number of deaths, death rates, and age-adjusted death rates for alcohol-induced causes, by race and sex: United States, 1999-2007 . . . . . . . . . . . . . . . . . . . . . . . .

24. Number of deaths, death rates, and age-adjusted death rates for alcohol-induced causes, by Hispanic origin, race for nonHispanic population, and sex: United States, 1999-2007. . . . .

25. Number of deaths, death rates, and age-adjusted death rates for ages 15 years and over, by marital status and sex: United States, 2007 . . . . . . . . . . . . . . . . . . . . . . . . . . . . . .

26. Number of deaths, death rates, and age-adjusted death rates for ages 25-64 years, by educational attainment and sex: Total of 22 reporting states and the District of Columbia using the 2003 version of the U.S. Standard Certificate of Death and total of 26 reporting states using the 1989 version of the U.S. Standard Certificate of Death, 2007 . . . . . . . . . . . . . . . . . . . . .

27. Number of deaths, death rates, and age-adjusted death rates for injury at work and ages 15 years and over, by race and sex: United States, 2007

28. Number of deaths, death rates, and age-adjusted death rates for injury at work, by race and sex: United States, 1993-2007 . . .

29. Number of deaths, death rates, and age-adjusted death rates for major causes of death: United States, each state, Puerto Rico, Virgin Islands, Guam, American Samoa, and Northern Marianas,

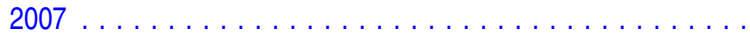

30. Infant, neonatal, and postneonatal mortality rates, by race and sex: United States, 1940, 1950, 1960, 1970, and 1975-2007. .

31. Number of infant deaths and infant mortality rates for 130 selected causes, by race: United States, 2007 . . . . . . . . . .

32. Number of infant and neonatal deaths and mortality rates, by race for the United States, each state, Puerto Rico, Virgin Islands, Guam, American Samoa, and Northern Marianas, and by sex for the United States, $2007 \ldots \ldots \ldots \ldots$. . . . . . .

33. Number of maternal deaths and maternal mortality rates for selected causes, by race: United States, 2007 . . . . . . . . .

34. Number of maternal deaths and maternal mortality rates for selected causes, by Hispanic origin and race for non-Hispanic population: United States, $2007 \ldots \ldots \ldots \ldots \ldots$. . . . . . 
Table 1. Number of deaths, death rates, and age-adjusted death rates, by race and sex: United States, 1940, 1950, 1960, 1970, and 1980-2007

[Crude rates on an annual basis per 100,000 population in specified age group; age-adjusted rates per 100,000 U.S. standard population; see "Technical Notes." Rates are based on populations enumerated as of April 1 for census years and estimated as of July 1 for all other years; see "Technical Notes." Beginning in 1970, excludes deaths of nonresidents of the United States. Data for specified races other than white and black should be interpreted with caution because of inconsistencies between reporting race on death certificates and on censuses and surveys; see "Technical Notes." Race categories are consistent with the 1977 Office of Management and Budget (OMB) standards]

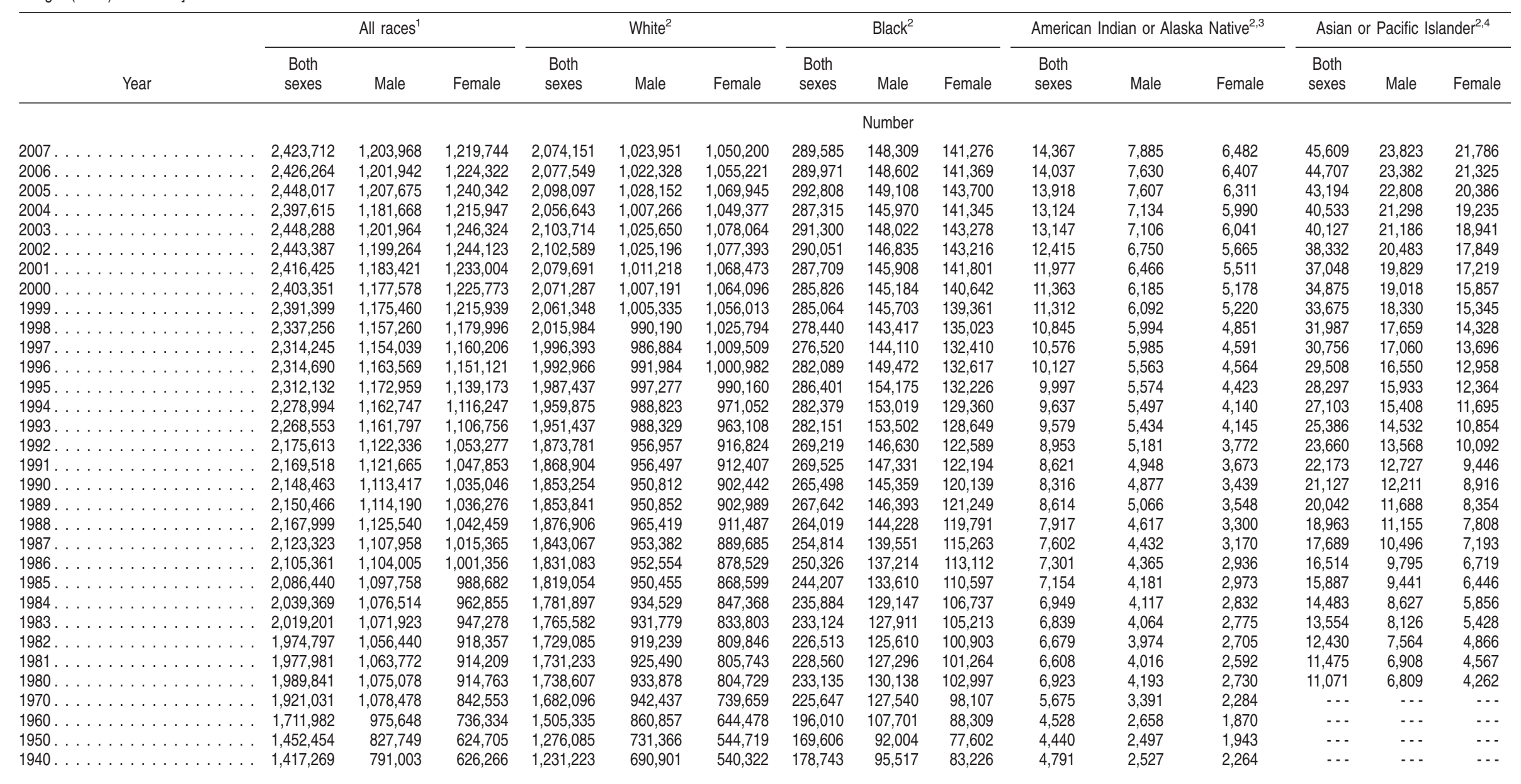


Table 1. Number of deaths, death rates, and age-adjusted death rates, by race and sex: United States, 1940, 1950, 1960, 1970, and 1980-2007-Con.

[Crude rates on an annual basis per 100,000 population in specified age group; age-adjusted rates per 100,000 U.S. standard population; see "Technical Notes." Rates are based on populations enumerated as of April 1 for census years and estimated as of July 1 for all other years; see "Technical Notes." Beginning in 1970, excludes deaths of nonresidents of the United States. Data for specified races other than white and black should be interpreted with caution because of inconsistencies between reporting race on death certificates and on censuses and surveys; see "Technical Notes." Race categories are consistent with the 1977 Office of Management and Budget (OMB) standards]

\begin{tabular}{|c|c|c|c|c|c|c|c|c|c|c|c|c|c|c|c|}
\hline \multirow[b]{2}{*}{ Year } & \multicolumn{3}{|c|}{ All races ${ }^{1}$} & \multicolumn{3}{|c|}{ White $^{2}$} & \multicolumn{3}{|c|}{ Black $^{2}$} & \multicolumn{3}{|c|}{ American Indian or Alaska Native ${ }^{2,3}$} & \multicolumn{3}{|c|}{ Asian or Pacific Islander, ${ }^{2,4}$} \\
\hline & $\begin{array}{l}\text { Both } \\
\text { sexes }\end{array}$ & Male & Female & $\begin{array}{l}\text { Both } \\
\text { sexes }\end{array}$ & Male & Female & $\begin{array}{l}\text { Both } \\
\text { sexes }\end{array}$ & Male & Female & $\begin{array}{l}\text { Both } \\
\text { sexes }\end{array}$ & Male & Female & $\begin{array}{l}\text { Both } \\
\text { sexes }\end{array}$ & Male & Female \\
\hline & \multicolumn{15}{|c|}{ Death rate } \\
\hline$\ldots \ldots \ldots$ & 803.6 & 809.9 & 797.4 & 851.5 & 848.1 & 854.9 & 723.4 & 775.6 & 675.7 & 444.0 & 488.2 & 400.0 & 308.7 & 331.4 & 287.2 \\
\hline 2006. & 810.4 & 814.8 & 806.1 & 858.1 & 852.3 & 863.9 & 733.0 & 786.7 & 684.0 & 438.5 & 477.1 & 399.9 & 307.4 & 330.6 & 285.6 \\
\hline 2005. & 825.9 & 827.2 & 824.6 & 873.7 & 864.5 & 882.8 & 749.4 & 799.2 & 703.9 & 440.3 & 481.9 & 398.8 & 307.7 & 333.9 & 282.8 \\
\hline$\ldots \ldots$ & 816.5 & 817.6 & 815.4 & 863.2 & 854.2 & 871.9 & 744.3 & 792.6 & 700.3 & 416.8 & 453.8 & 380.0 & 297.2 & 321.1 & 274.6 \\
\hline$\ldots \ldots$ & 841.9 & 840.3 & 843.4 & 890.1 & 877.6 & 902.3 & 763.6 & 813.7 & 717.9 & 422.6 & 457.6 & 387.7 & 303.9 & 330.0 & 279.2 \\
\hline 2002. & 847.3 & 846.6 & 848.0 & 895.7 & 884.0 & 907.0 & 768.4 & 816.7 & 724.4 & 403.6 & 439.6 & 367.7 & 299.5 & 331.4 & 269.7 \\
\hline 2001. & 848.5 & 846.4 & 850.4 & 895.1 & 881.9 & 907.9 & 773.5 & 823.9 & 727.7 & 392.1 & 424.2 & 360.2 & 303.8 & 335.0 & 274.4 \\
\hline 2000 . & 854.0 & 853.0 & 855.0 & 900.2 & 887.8 & 912.3 & 781.1 & 834.1 & 733.0 & 380.8 & 415.6 & 346.1 & 296.6 & 332.9 & 262.3 \\
\hline 1999. & 857.0 & 859.2 & 854.9 & 901.4 & 892.1 & 910.4 & 788.1 & 847.4 & 734.3 & 399.3 & 431.8 & 367.1 & 296.8 & 333.2 & 262.5 \\
\hline 1998. & 847.3 & 856.4 & 838.5 & 889.5 & 887.3 & 891.6 & 782.3 & 848.2 & 722.6 & 397.8 & 441.9 & 354.2 & 293.8 & 335.4 & 254.9 \\
\hline 1997. & 848.8 & 864.6 & 833.6 & 889.1 & 893.3 & 885.0 & 789.9 & 867.1 & 720.1 & 402.7 & 458.2 & 347.7 & 294.1 & 336.8 & 253.9 \\
\hline 1996. & 859.2 & 882.8 & 836.7 & 896.0 & 907.1 & 885.3 & 819.7 & 915.3 & 733.3 & 399.5 & 441.5 & 358.0 & 294.4 & 340.2 & 251.1 \\
\hline 1995. & 868.3 & 900.8 & 837.2 & 901.8 & 921.0 & 883.2 & 846.2 & 960.2 & 743.2 & 409.4 & 459.4 & 360.1 & 294.6 & 341.4 & 250.4 \\
\hline 1994. & 866.1 & 904.2 & 829.7 & 897.8 & 922.6 & 873.8 & 849.0 & 970.2 & 739.7 & 408.2 & 468.8 & 348.3 & 294.6 & 344.0 & 247.7 \\
\hline 1993. & 872.8 & 915.0 & 832.5 & 902.7 & 931.8 & 874.6 & 864.6 & 992.2 & 749.6 & 419.8 & 479.6 & 360.7 & 288.0 & 338.1 & 240.3 \\
\hline 1992. & 848.1 & 896.1 & 802.4 & 875.8 & 912.2 & 840.8 & 841.8 & 967.6 & 728.6 & 406.6 & 474.1 & 340.0 & 282.1 & 331.1 & 235.3 \\
\hline 1991. & 857.6 & 908.8 & 808.7 & 883.2 & 922.7 & 845.2 & 861.4 & 994.8 & 741.4 & 405.3 & 468.9 & 342.7 & 278.7 & 326.9 & 232.4 \\
\hline 1990. & 863.8 & 918.4 & 812.0 & 888.0 & 930.9 & 846.9 & 871.0 & $1,008.0$ & 747.9 & 402.8 & 476.4 & 330.4 & 283.3 & 334.3 & 234.3 \\
\hline 1989. & 871.3 & 926.3 & 818.9 & 893.2 & 936.5 & 851.8 & 887.9 & $1,026.7$ & 763.2 & 430.5 & 510.7 & 351.3 & 280.9 & 334.5 & 229.4 \\
\hline 1988. & 886.7 & 945.1 & 831.2 & 910.5 & 957.9 & 865.3 & 888.3 & $1,026.1$ & 764.6 & 411.7 & 485.0 & 339.9 & 282.0 & 339.0 & 227.4 \\
\hline 1987. & 876.4 & 939.3 & 816.7 & 900.1 & 952.7 & 849.8 & 868.9 & $1,006.2$ & 745.7 & 410.7 & 483.8 & 339.0 & 278.9 & 338.3 & 222.0 \\
\hline 1986. & 876.7 & 944.7 & 812.3 & 900.1 & 958.6 & 844.3 & 864.9 & $1,002.6$ & 741.5 & 409.5 & 494.9 & 325.9 & 276.2 & 335.1 & 219.9 \\
\hline 1985. & 876.9 & 948.6 & 809.1 & 900.4 & 963.6 & 840.1 & 854.8 & 989.3 & 734.2 & 416.4 & 492.5 & 342.5 & 283.4 & 344.6 & 224.9 \\
\hline 1984. & 864.8 & 938.8 & 794.7 & 887.8 & 954.1 & 824.6 & 836.1 & 968.5 & 717.4 & 419.6 & 502.7 & 338.4 & 275.9 & 336.5 & 218.1 \\
\hline 1983. & 863.7 & 943.2 & 788.4 & 885.4 & 957.7 & 816.4 & 836.6 & 971.2 & 715.9 & 428.5 & 515.1 & 343.9 & 276.1 & 339.1 & 216.1 \\
\hline 1982. & 852.4 & 938.4 & 771.2 & 873.1 & 951.8 & 798.2 & 823.4 & 966.2 & 695.5 & 434.5 & 522.9 & 348.1 & 271.3 & 338.3 & 207.4 \\
\hline 1981. & 862.0 & 954.0 & 775.0 & 880.4 & 965.2 & 799.8 & 842.4 & 992.6 & 707.7 & 445.6 & 547.9 & 345.6 & 272.3 & 336.2 & 211.5 \\
\hline 1980. & 878.3 & 976.9 & 785.3 & 892.5 & 983.3 & 806.1 & 875.4 & $1,034.1$ & 733.3 & 487.4 & 597.1 & 380.1 & 296.9 & 375.3 & 222.5 \\
\hline 1970. & 945.3 & $1,090.3$ & 807.8 & 946.3 & $1,086.7$ & 812.6 & 999.3 & $1,186.6$ & 829.2 & $\ldots$ & ... & $\ldots$ & $\ldots$ & ... & $\ldots$ \\
\hline 1960. & 954.7 & $1,104.5$ & 809.2 & 947.8 & $1,098.5$ & 800.9 & $1,038.6$ & $1,181.7$ & 905.0 & - . & $\cdots$ & $\cdots$ & $\cdots$ & $\cdots$ & $\cdots$ \\
\hline 1950. & 963.8 & $1,106.1$ & 823.5 & 945.7 & $1,089.5$ & 803.3 & $\cdots$ & $\ldots$ & . . & .. & $\ldots$ & $\ldots$ & $\cdots$ & $\ldots$ & $\cdots$ \\
\hline 1940. & $1,076.4$ & $1,197.4$ & 954.6 & $1,041.5$ & $1,162.2$ & 919.4 & $\ldots$ & $\ldots$ & $\ldots$ & $\ldots$ & .. & $\ldots$ & $\ldots$ & $\ldots$ & $\ldots$ \\
\hline
\end{tabular}

See footnotes at end of table. 
Table 1. Number of deaths, death rates, and age-adjusted death rates, by race and sex: United States, 1940, 1950, 1960, 1970, and 1980-2007-Con.

[Crude rates on an annual basis per 100,000 population in specified age group; age-adjusted rates per 100,000 U.S. standard population; see "Technical Notes." Rates are based on populations enumerated as of April 1 for census years and estimated as of July 1 for all other years; see "Technical Notes." Beginning in 1970, excludes deaths of nonresidents of the United States. Data for specified races other than white and black should be interpreted with caution because of inconsistencies between reporting race on death certificates and on censuses and surveys; see "Technical Notes." Race categories are consistent with the 1977 Office of Management and Budget (OMB) standards]

\begin{tabular}{|c|c|c|c|c|c|c|c|c|c|c|c|c|c|c|c|}
\hline \multirow[b]{2}{*}{ Year } & \multicolumn{3}{|c|}{ All races ${ }^{1}$} & \multicolumn{3}{|c|}{ White $^{2}$} & \multicolumn{3}{|c|}{ Black $^{2}$} & \multicolumn{3}{|c|}{ American Indian or Alaska Native ${ }^{2,3}$} & \multicolumn{3}{|c|}{ Asian or Pacific Islander ${ }^{2,4}$} \\
\hline & $\begin{array}{l}\text { Both } \\
\text { sexes }\end{array}$ & Male & Female & $\begin{array}{l}\text { Both } \\
\text { sexes }\end{array}$ & Male & Female & $\begin{array}{l}\text { Both } \\
\text { sexes }\end{array}$ & Male & Female & $\begin{array}{l}\text { Both } \\
\text { sexes }\end{array}$ & Male & Female & $\begin{array}{l}\text { Both } \\
\text { sexes }\end{array}$ & Male & Female \\
\hline & \multicolumn{15}{|c|}{ Age-adjusted death rate ${ }^{5}$} \\
\hline$\ldots \ldots \ldots \ldots \ldots$ & 760.2 & 905.6 & 643.4 & 749.4 & 890.5 & 634.8 & 958.0 & $1,184.4$ & 793.8 & 627.2 & 736.7 & 533.2 & 415.0 & 499.2 & 350.6 \\
\hline 2006. & 776.5 & 924.8 & 657.8 & 764.4 & 908.2 & 648.2 & 982.0 & $1,215.6$ & 813.0 & 642.1 & 739.9 & 555.7 & 428.6 & 516.0 & 362.6 \\
\hline 2005. & 798.8 & 951.1 & 677.6 & 785.3 & 933.2 & 666.5 & $1,016.5$ & $1,252.9$ & 845.7 & 663.4 & 775.3 & 567.7 & 440.2 & 534.4 & 369.3 \\
\hline 2004. & 800.8 & 955.7 & 679.2 & 786.3 & 936.9 & 666.9 & $1,027.3$ & $1,269.4$ & 855.3 & 650.0 & 758.1 & 557.9 & 443.9 & 534.7 & 375.5 \\
\hline$\ldots \ldots \ldots \ldots$ & 832.7 & 994.3 & 706.2 & 817.0 & 973.9 & 693.1 & $1,065.9$ & $1,319.1$ & 885.6 & 685.0 & 797.0 & 592.1 & 465.7 & 562.7 & 392.7 \\
\hline 2002. & 845.3 & $1,013.7$ & 715.2 & 829.0 & 992.9 & 701.3 & $1,083.3$ & $1,341.4$ & 901.8 & 677.4 & 794.2 & 581.1 & 474.4 & 578.4 & 395.9 \\
\hline 2001. & 854.5 & $1,029.1$ & 721.8 & 836.5 & $1,006.1$ & 706.7 & $1,101.2$ & $1,375.0$ & 912.5 & 686.7 & 798.9 & 594.0 & 492.1 & 597.4 & 412.0 \\
\hline 2000. & 869.0 & $1,053.8$ & 731.4 & 849.8 & $1,029.4$ & 715.3 & $1,121.4$ & $1,403.5$ & 927.6 & 709.3 & 841.5 & 604.5 & 506.4 & 624.2 & 416.8 \\
\hline 1999. & 875.6 & $1,067.0$ & 734.0 & 854.6 & $1,040.0$ & 716.6 & $1,135.7$ & $1,432.6$ & 933.6 & 780.9 & 925.9 & 668.2 & 519.7 & 641.2 & 427.5 \\
\hline 1998. & 870.6 & $1,069.4$ & 724.7 & 849.3 & $1,042.0$ & 707.3 & $1,127.8$ & $1,430.5$ & 921.6 & 770.4 & 943.9 & 640.5 & 522.4 & 646.9 & 426.7 \\
\hline 1997. & 878.1 & $1,088.1$ & 725.6 & 855.7 & $1,059.1$ & 707.8 & $1,139.8$ & $1,458.8$ & 922.1 & 774.0 & 974.8 & 625.3 & 531.8 & 660.2 & 432.6 \\
\hline 1996. & 894.1 & $1,115.7$ & 733.0 & 869.0 & $1,082.9$ & 713.6 & $1,178.4$ & $1,524.2$ & 940.3 & 763.6 & 924.8 & 641.7 & 543.2 & 676.1 & 439.6 \\
\hline 1995. & 909.8 & $1,143.9$ & 739.4 & 882.3 & $1,107.5$ & 718.7 & $1,213.9$ & $1,585.7$ & 955.9 & 771.2 & 932.0 & 643.9 & 554.8 & 693.4 & 446.7 \\
\hline 1994. & 913.5 & $1,155.5$ & 738.6 & 885.6 & $1,118.7$ & 717.5 & $1,216.9$ & $1,592.8$ & 954.6 & 764.8 & 953.3 & 618.8 & 562.7 & 702.5 & 452.1 \\
\hline 1993. & 926.1 & $1,177.3$ & 745.9 & 897.0 & $1,138.9$ & 724.1 & $1,241.2$ & $1,632.2$ & 969.5 & 796.4 & $1,006.3$ & 641.6 & 565.8 & 709.9 & 450.4 \\
\hline 1992. & 905.6 & $1,158.3$ & 725.5 & 877.7 & $1,122.4$ & 704.1 & $1,206.7$ & $1,587.8$ & 942.5 & 759.0 & 970.4 & 599.4 & 558.5 & 697.3 & 445.8 \\
\hline 1991. & 922.3 & $1,180.5$ & 738.2 & 893.2 & $1,143.1$ & 716.1 & $1,235.4$ & $1,626.1$ & 963.3 & 763.9 & 970.6 & 608.3 & 566.2 & 703.4 & 453.2 \\
\hline 1990. & 938.7 & $1,202.8$ & 750.9 & 909.8 & $1,165.9$ & 728.8 & $1,250.3$ & $1,644.5$ & 975.1 & 716.3 & 916.2 & 561.8 & 582.0 & 716.4 & 469.3 \\
\hline 1989. & 950.5 & $1,215.0$ & 761.8 & 920.2 & $1,176.6$ & 738.8 & $1,275.5$ & $1,670.1$ & 998.1 & 761.6 & 999.8 & 586.3 & 581.3 & 729.6 & 458.4 \\
\hline 1988. & 975.7 & $1,250.7$ & 781.0 & 947.6 & $1,215.9$ & 759.1 & $1,284.3$ & $1,677.6$ & $1,006.8$ & 718.6 & 917.4 & 563.6 & 584.2 & 732.0 & 451.0 \\
\hline 1987. & 970.0 & $1,246.1$ & 774.2 & 943.4 & $1,213.4$ & 753.3 & $1,263.1$ & $1,650.3$ & 989.7 & 719.8 & 899.3 & 583.7 & 577.3 & 732.4 & 448.1 \\
\hline 1986. & 978.6 & $1,261.7$ & 778.7 & 952.8 & $1,230.5$ & 758.1 & $1,266.7$ & $1,650.1$ & 994.4 & 720.8 & 926.7 & 549.3 & 576.4 & 730.5 & 445.4 \\
\hline 1985. & 988.1 & $1,278.1$ & 784.5 & 963.6 & $1,249.8$ & 764.3 & $1,261.2$ & $1,634.5$ & 994.4 & 731.7 & 926.1 & 577.2 & 586.5 & 755.4 & 456.7 \\
\hline 1984. & 982.5 & $1,271.4$ & 779.8 & 959.7 & $1,245.9$ & 760.7 & $1,236.7$ & $1,600.8$ & 976.9 & 761.7 & 946.0 & 567.9 & 574.4 & 724.7 & 443.1 \\
\hline 1983. & 990.0 & $1,284.5$ & 783.3 & 967.3 & $1,259.4$ & 763.9 & $1,240.5$ & $1,600.7$ & 980.7 & 757.3 & 945.0 & 605.5 & 565.1 & 718.8 & 428.8 \\
\hline 1982. & 985.0 & $1,279.9$ & 776.6 & 963.6 & $1,255.9$ & 758.7 & $1,221.3$ & $1,580.4$ & 960.1 & 757.0 & 940.1 & 604.4 & 550.4 & 738.2 & 410.3 \\
\hline 1981. & $1,007.1$ & $1,308.2$ & 792.7 & 984.0 & $1,282.2$ & 773.6 & $1,258.4$ & $1,626.6$ & 986.6 & 784.6 & $1,030.2$ & 588.0 & 544.7 & 710.3 & 405.3 \\
\hline 1980. & $1,039.1$ & $1,348.1$ & 817.9 & $1,012.7$ & $1,317.6$ & 796.1 & $1,314.8$ & $1,697.8$ & $1,033.3$ & 867.0 & $1,111.5$ & 662.4 & 589.9 & 786.5 & 425.9 \\
\hline 1970. & $1,222.6$ & $1,542.1$ & 971.4 & $1,193.3$ & $1,513.7$ & 944.0 & $1,518.1$ & $1,873.9$ & $1,228.7$ & $\ldots$ & $\ldots$ & $\ldots$ & $\ldots$ & $\ldots$ & $\ldots$ \\
\hline 1960. & $1,339.2$ & $1,609.0$ & $1,105.3$ & $1,311.3$ & $1,586.0$ & $1,074.4$ & $1,577.5$ & $1,811.1$ & $1,369.7$ & ... & $\ldots$ & $\ldots$ & $\ldots$ & $\ldots$ & $\ldots$ \\
\hline 1950. & $1,446.0$ & $1,674.2$ & $1,236.0$ & $1,410.8$ & $1,642.5$ & $1,198.0$ & ... & $\cdots$ & ... & $\ldots$ & $\ldots$ & $\cdots$ & $\cdots$ & $\cdots$ & $\cdots$ \\
\hline 1940. & $1,785.0$ & $1,976.0$ & $1,599.4$ & $1,735.3$ & $1,925.2$ & $1,550.4$ & ... & ... & ... & $\ldots$ & $\ldots$ & $\ldots$ & $\ldots$ & $\ldots$ & $\ldots$ \\
\hline
\end{tabular}

\footnotetext{
- - Data not available.
}

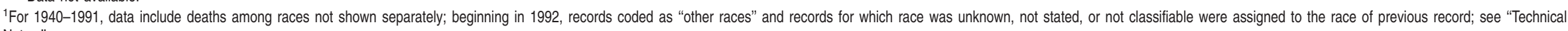
Notes.

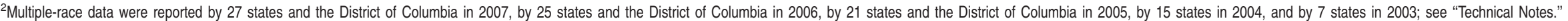
The multiple-race data for these reporting areas were bridged to the single-race categories of the 1977 OMB standards for comparability with other reporting areas; see "Technical Notes."

${ }^{3}$ Includes Aleuts and Eskimos.

"Includes Chinese, Filipino, Hawaiian, Japanese, and Other Asian or Pacific Islander.

${ }^{5}$ For method of computation, see "Technical Notes." 
Table 2. Number of deaths, death rates, and age-adjusted death rates, by Hispanic origin, race for non-Hispanic population, and sex: United States, 1997-2007

[Crude rates on an annual basis per 100,000 population in specified group; age-adjusted rates per 100,000 U.S. standard population; see "Technical Notes." Rates are based on populations enumerated as of April 1 for 2000

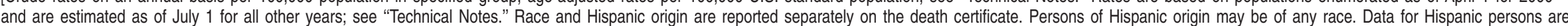
not tabulated separately by race; data for non-Hispanic persons are tabulated by race. Race categories are consistent with the 1977 Office of Management and Budget (OMB) standards. Data for Hispanic origin should be interpreted with caution because of inconsistencies between reporting Hispanic origin on death certificates and on censuses and surveys; see "Technical Notes"]

\begin{tabular}{|c|c|c|c|c|c|c|c|c|c|c|c|c|c|c|c|c|}
\hline & \multirow[b]{2}{*}{ Year } & \multicolumn{3}{|c|}{ All origins ${ }^{1}$} & \multicolumn{3}{|c|}{ Hispanic } & \multicolumn{3}{|c|}{ Non-Hispanic ${ }^{2}$} & \multicolumn{3}{|c|}{ Non-Hispanic white ${ }^{3}$} & \multicolumn{3}{|c|}{ Non-Hispanic black ${ }^{3}$} \\
\hline & & $\begin{array}{l}\text { Both } \\
\text { sexes }\end{array}$ & Male & Female & $\begin{array}{l}\text { Both } \\
\text { sexes }\end{array}$ & Male & Female & $\begin{array}{l}\text { Both } \\
\text { sexes }\end{array}$ & Male & Female & $\begin{array}{l}\text { Both } \\
\text { sexes }\end{array}$ & Male & Female & $\begin{array}{l}\text { Both } \\
\text { sexes }\end{array}$ & Male & Female \\
\hline & & \multicolumn{15}{|c|}{ Number } \\
\hline 2007 & & $2,423,712$ & $1,203,968$ & $1,219,744$ & 135,519 & 75,708 & 59,811 & $2,284,446$ & $1,125,974$ & $1,158,472$ & $1,939,606$ & 948,662 & 990,944 & 286,366 & 146,474 & 139,892 \\
\hline 2006 & & 2,426,264 & $1,201,942$ & 1,224,322 & 133,004 & 74,250 & 58,754 & $2,288,424$ & $1,124,813$ & $1,163,611$ & $1,944,617$ & 947,966 & 996,651 & 286,581 & 146,729 & 139,852 \\
\hline 2005 & & $2,448,017$ & $1,207,675$ & $1,240,342$ & 131,161 & 73,788 & 57,373 & $2,312,028$ & $1,131,013$ & $1,181,015$ & $1,967,142$ & 954,402 & $1,012,740$ & 289,163 & 147,010 & 142,153 \\
\hline 2004 & & $2,397,615$ & $1,181,668$ & $1,215,947$ & 122,416 & 68,544 & 53,872 & $2,269,583$ & $1,109,848$ & $1,159,735$ & $1,933,382$ & 938,143 & 995,239 & 283,859 & 144,022 & 139,837 \\
\hline 2003 & . & $2,448,288$ & $1,201,964$ & $1,246,324$ & 122,026 & 68,119 & 53,907 & $2,319,476$ & $1,129,927$ & $1,189,549$ & $1,979,465$ & 956,194 & $1,023,271$ & 287,968 & 146,136 & 141,832 \\
\hline 2002 & & $2,443,387$ & $1,199,264$ & $1,244,123$ & 117,135 & 65,703 & 51,432 & $2,318,269$ & $1,129,090$ & $1,189,179$ & $1,981,973$ & 957,645 & $1,024,328$ & 286,573 & 144,802 & 141,771 \\
\hline 2001. & & $2,416,425$ & $1,183,421$ & $1,233,004$ & 113,413 & 63,317 & 50,096 & $2,295,244$ & $1,115,683$ & $1,179,561$ & $1,962,810$ & 945,967 & $1,016,843$ & 284,343 & 143,971 & 140,372 \\
\hline 2000 & & $2,403,351$ & $1,177,578$ & $1,225,773$ & 107,254 & 60,172 & 47,082 & $2,287,846$ & $1,112,704$ & $1,175,142$ & $1,959,919$ & 944,781 & $1,015,138$ & 282,676 & 143,297 & 139,379 \\
\hline 1999 & & $2,391,399$ & $1,175,460$ & $1,215,939$ & 103,740 & 57,991 & 45,749 & $2,279,325$ & $1,112,718$ & $1,166,607$ & $1,953,197$ & 944,913 & $1,008,284$ & 281,979 & 143,883 & 138,096 \\
\hline 1998 & & $2,337,256$ & $1,157,260$ & $1,179,996$ & 98,406 & 55,821 & 42,585 & $2,230,127$ & $1,096,677$ & $1,133,450$ & $1,912,802$ & 931,844 & 980,958 & 275,264 & 141,627 & 133,637 \\
\hline \multirow{2}{*}{\multicolumn{2}{|c|}{1997}} & $2,314,245$ & $1,154,039$ & $1,160,206$ & 95,460 & 54,348 & 41,112 & $2,209,450$ & $1,094,541$ & $1,114,909$ & $1,895,461$ & 929,703 & 965,758 & 273,381 & 142,241 & 131,140 \\
\hline & & \multicolumn{15}{|c|}{ Death rate } \\
\hline 2007 . & & 803.6 & 809.9 & 797.4 & 297.8 & 321.8 & 272.1 & 892.0 & 899.8 & 884.5 & 964.1 & 960.4 & 967.6 & 750.7 & 805.1 & 701.0 \\
\hline 2006 & & 810.4 & 814.8 & 806.1 & 300.1 & 323.9 & 274.6 & 897.1 & 902.8 & 891.7 & 968.5 & 962.0 & 974.7 & 759.1 & 815.3 & 708.0 \\
\hline 2005 & & 825.9 & 827.2 & 824.6 & 307.3 & 334.4 & 278.2 & 911.2 & 912.6 & 910.0 & 981.8 & 970.6 & 992.6 & 774.4 & 825.7 & 727.6 \\
\hline 2004 & & 816.5 & 817.6 & 815.4 & 296.2 & 321.1 & 269.7 & 899.4 & 900.9 & 898.0 & 967.8 & 957.4 & 977.7 & 768.8 & 818.7 & 723.4 \\
\hline 2003 & & 841.9 & 840.3 & 843.4 & 305.8 & 330.7 & 279.3 & 924.4 & 922.9 & 925.9 & 993.6 & 979.1 & $1,007.6$ & 788.8 & 840.6 & 741.6 \\
\hline 2002 & & 847.3 & 846.6 & 848.0 & 302.2 & 328.7 & 274.0 & 928.8 & 928.0 & 929.5 & 997.5 & 983.9 & $1,010.6$ & 792.8 & 842.3 & 748.0 \\
\hline 2001 & & 848.5 & 846.4 & 850.4 & 306.8 & 332.9 & 279.0 & 926.2 & 923.6 & 928.6 & 991.1 & 975.6 & $1,006.1$ & 798.1 & 849.7 & 751.2 \\
\hline 2000 & & 854.0 & 853.0 & 855.0 & 303.8 & 331.3 & 274.6 & 929.6 & 928.1 & 931.0 & 993.2 & 978.5 & $1,007.3$ & 805.5 & 859.5 & 756.7 \\
\hline 1999 & & 857.0 & 859.2 & 854.9 & 305.7 & 332.6 & 277.2 & 929.9 & 932.2 & 927.8 & 990.7 & 979.6 & $1,001.3$ & 812.1 & 872.8 & 757.3 \\
\hline 1998 & & 847.3 & 856.4 & 838.5 & 303.9 & 336.0 & 270.0 & 916.0 & 925.3 & 907.1 & 972.9 & 969.2 & 976.5 & 805.6 & 873.7 & 744.1 \\
\hline \multirow[t]{2}{*}{1997} & & 848.8 & 864.6 & 833.6 & 309.0 & 343.2 & 272.9 & 913.9 & 930.4 & 898.3 & 967.4 & 970.6 & 964.3 & 813.5 & 892.9 & 741.9 \\
\hline & & \multicolumn{15}{|c|}{ Age-adjusted death rate ${ }^{4}$} \\
\hline 2007 & & 760.2 & 905.6 & 643.4 & 546.1 & 654.5 & 452.7 & 776.3 & 924.9 & 657.7 & 763.3 & 906.8 & 647.7 & 978.6 & $1,210.9$ & 810.4 \\
\hline 2006 & & 776.5 & 924.8 & 657.8 & 564.0 & 675.6 & 468.6 & 791.4 & 942.6 & 671.1 & 777.0 & 922.8 & 660.0 & $1,001.4$ & $1,241.0$ & 828.4 \\
\hline 2005 & & 798.8 & 951.1 & 677.6 & 590.7 & 717.0 & 485.3 & 812.5 & 966.7 & 690.3 & 796.6 & 945.4 & 677.7 & $1,034.5$ & $1,275.3$ & 860.5 \\
\hline 2004 & & 800.8 & 955.7 & 679.2 & 586.7 & 706.8 & 485.9 & 814.1 & 971.1 & 691.4 & 797.1 & 949.0 & 677.5 & $1,044.7$ & $1,291.5$ & 869.4 \\
\hline 2003 & & 832.7 & 994.3 & 706.2 & 621.2 & 748.1 & 515.8 & 844.5 & $1,008.0$ & 717.2 & 826.1 & 984.0 & 702.1 & $1,083.2$ & $1,341.1$ & 899.8 \\
\hline 2002 & & 845.3 & $1,013.7$ & 715.2 & 629.3 & 766.7 & 518.3 & 856.5 & $1,026.5$ & 725.8 & 837.5 & $1,002.2$ & 709.9 & $1,099.2$ & $1,360.6$ & 915.3 \\
\hline 2001 & & 854.5 & $1,029.1$ & 721.8 & 658.7 & 802.5 & 544.2 & 864.0 & $1,039.8$ & 730.9 & 842.9 & $1,012.8$ & 713.5 & $1,116.5$ & $1,393.7$ & 925.5 \\
\hline 2000 & & 869.0 & $1,053.8$ & 731.4 & 665.7 & 818.1 & 546.0 & 877.9 & $1,063.8$ & 740.0 & 855.5 & $1,035.4$ & 721.5 & $1,137.0$ & $1,422.0$ & 941.2 \\
\hline 1999 & & 875.6 & $1,067.0$ & 734.0 & 676.4 & 830.5 & 555.9 & 883.9 & $1,076.4$ & 741.9 & 859.8 & $1,045.5$ & 722.3 & $1,150.1$ & $1,449.4$ & 946.0 \\
\hline 1998 & & 870.6 & $1,069.4$ & 724.7 & 665.4 & 833.6 & 536.9 & 878.4 & $1,078.2$ & 732.4 & 854.1 & $1,046.7$ & 712.8 & $1,141.8$ & $1,448.2$ & 932.9 \\
\hline 1997 & & 878.1 & $1,088.1$ & 725.6 & 669.3 & 840.5 & 538.8 & 885.3 & $1,096.4$ & 732.6 & 859.7 & $1,063.2$ & 712.5 & $1,154.3$ & $1,476.7$ & 934.2 \\
\hline
\end{tabular}

"Figures for origin not stated are included in "all origins" but are not distributed among specified origins.

Includes races other than white and black.

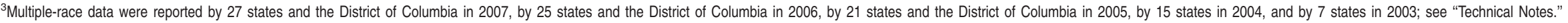
The multiple-race data for these reporting areas were bridged to the single-race categories of the 1977 OMB standards for comparability with other reporting areas; see "Technical Notes."

${ }^{4}$ For method of computation, see "Technical Notes." 
Table 3. Number of deaths and death rates, by age, race, and sex: United States, 2007

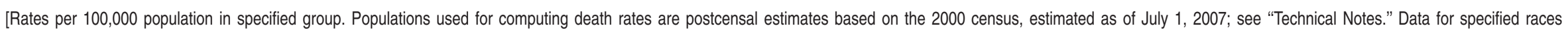
other than white and black should be interpreted with caution because of inconsistencies between reporting race on death certificates and on censuses and surveys; see "Technical Notes"]

\begin{tabular}{|c|c|c|c|c|c|c|c|c|c|c|c|c|c|c|c|c|}
\hline \multirow{2}{*}{\multicolumn{2}{|c|}{ Age }} & \multicolumn{3}{|c|}{ All races } & \multicolumn{3}{|c|}{ White $^{1}$} & \multicolumn{3}{|c|}{ Black $^{1}$} & \multicolumn{3}{|c|}{ American Indian or Alaska Native ${ }^{1,2}$} & \multicolumn{3}{|c|}{ Asian or Pacific Islander ${ }^{1,3}$} \\
\hline & & $\begin{array}{l}\text { Both } \\
\text { sexes }\end{array}$ & Male & Female & $\begin{array}{l}\text { Both } \\
\text { sexes }\end{array}$ & Male & Female & $\begin{array}{l}\text { Both } \\
\text { sexes }\end{array}$ & Male & Female & $\begin{array}{l}\text { Both } \\
\text { sexes }\end{array}$ & Male & Female & $\begin{array}{l}\text { Both } \\
\text { sexes }\end{array}$ & Male & Female \\
\hline & & & & & & & & & Number & & & & & & & \\
\hline All ages & & $2,423,712$ & $1,203,968$ & $1,219,744$ & $2,074,151$ & $1,023,951$ & $1,050,200$ & 289,585 & 148,309 & 141,276 & 14,367 & 7,885 & 6,482 & 45,609 & 23,823 & 21,786 \\
\hline Under 1 year & & 29,138 & 16,293 & 12,845 & 18,807 & 10,540 & 8,267 & 8,944 & 4,975 & 3,969 & 432 & 241 & 191 & 955 & 537 & 418 \\
\hline $1-4$ years . . & $\ldots \ldots$ & 4,703 & 2,634 & 2,069 & 3,287 & 1,847 & 1,440 & 1,137 & 621 & 516 & 97 & 57 & 40 & 182 & 109 & 73 \\
\hline $5-9$ years & $\ldots \ldots$ & 2,711 & 1,519 & 1,192 & 2,001 & 1,112 & 889 & 577 & 331 & 246 & 40 & 25 & 15 & 93 & 51 & 42 \\
\hline $10-14$ years & & 3,436 & 2,066 & 1,370 & 2,479 & 1,478 & 1,001 & 786 & 485 & 301 & 54 & 36 & 18 & 117 & 67 & 50 \\
\hline $15-19$ years & & 13,299 & 9,558 & 3,741 & 9,765 & 6,851 & 2,914 & 2,956 & 2,302 & 654 & 265 & 182 & 83 & 313 & 223 & 90 \\
\hline $20-24$ years & $\ldots \ldots \ldots$ & 20,683 & 15,758 & 4,925 & 15,372 & 11,662 & 3,710 & 4,424 & 3,449 & 975 & 357 & 259 & 98 & 530 & 388 & 142 \\
\hline $25-29$ years & $\ldots \ldots \ldots$ & 20,931 & 15,107 & 5,824 & 15,382 & 11,159 & 4,223 & 4,741 & 3,398 & 1,343 & 349 & 250 & 99 & 459 & 300 & 159 \\
\hline $30-34$ years & $\ldots \ldots \ldots$ & 21,641 & 14,685 & 6,956 & 15,799 & 10,839 & 4,960 & 4,921 & 3,250 & 1,671 & 382 & 264 & 118 & 539 & 332 & 207 \\
\hline $35-39$ years & $\ldots \ldots \ldots$ & 30,881 & 19,755 & 11,126 & 22,804 & 14,820 & 7,984 & 6,831 & 4,147 & 2,684 & 497 & 315 & 182 & 749 & 473 & 276 \\
\hline $40-44$ years & & 48,725 & 30,350 & 18,375 & 36,767 & 23,301 & 13,466 & 10,159 & 5,959 & 4,200 & 716 & 453 & 263 & 1,083 & 637 & 446 \\
\hline $45-49$ years & & 77,738 & 47,904 & 29,834 & 59,635 & 37,447 & 22,188 & 15,609 & 8,970 & 6,639 & 937 & 562 & 375 & 1,557 & 925 & 632 \\
\hline $50-54$ years & & 106,948 & 66,552 & 40,396 & 82,598 & 52,109 & 30,489 & 21,209 & 12,525 & 8,684 & 1,021 & 631 & 390 & 2,120 & 1,287 & 833 \\
\hline $55-59$ years & & 132,458 & 81,590 & 50,868 & 104,284 & 64,772 & 39,512 & 24,190 & 14,468 & 9,722 & 1,186 & 700 & 486 & 2,798 & 1,650 & 1,148 \\
\hline $60-64$ years & & 154,652 & 92,028 & 62,624 & 127,003 & 76,013 & 50,990 & 23,492 & 13,659 & 9,833 & 1,181 & 672 & 509 & 2,976 & 1,684 & 1,292 \\
\hline $65-69$ years. & & 174,991 & 100,492 & 74,499 & 145,663 & 84,199 & 61,464 & 24,555 & 13,587 & 10,968 & 1,222 & 644 & 578 & 3,551 & 2,062 & 1,489 \\
\hline $70-74$ years & 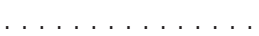 & 214,247 & 117,852 & 96,395 & 181,714 & 100,629 & 81,085 & 26,829 & 14,085 & 12,744 & 1,314 & 713 & 601 & 4,390 & 2,425 & 1,965 \\
\hline $75-79$ years & & 289,029 & 149,669 & 139,360 & 253,164 & 132,199 & 120,965 & 29,063 & 14,001 & 15,062 & 1,351 & 671 & 680 & 5,451 & 2,798 & 2,653 \\
\hline 80-84 years. & & 363,653 & 171,134 & 192,519 & 325,958 & 154,625 & 171,333 & 29,923 & 12,814 & 17,109 & 1,195 & 543 & 652 & 6,577 & 3,152 & 3,425 \\
\hline 85 years and ov & & 713,647 & 248,866 & 464,781 & 651,521 & 228,227 & 423,294 & 49,194 & 15,255 & 33,939 & 1,771 & 667 & 1,104 & 11,161 & 4,717 & 6,444 \\
\hline \multirow{2}{*}{\multicolumn{2}{|c|}{ Not stated. }} & 201 & 156 & 45 & 148 & 122 & 26 & 45 & 28 & 17 & - & - & - & 8 & 6 & 2 \\
\hline & & \multicolumn{15}{|c|}{ Rate } \\
\hline All ages ${ }^{4}$. & & 803.6 & 809.9 & 797.4 & 851.5 & 848.1 & 854.9 & 723.4 & 775.6 & 675.7 & 444.0 & 488.2 & 400.0 & 308.7 & 331.4 & 287.2 \\
\hline Under 1 year ${ }^{5}$. & & 684.5 & 747.8 & 618.1 & 573.7 & 627.8 & 516.8 & $1,250.0$ & $1,363.2$ & $1,132.2$ & 921.7 & $1,009.9$ & 830.3 & 441.8 & 483.5 & 397.6 \\
\hline $1-4$ years & & 28.6 & 31.3 & 25.7 & 25.8 & 28.3 & 23.1 & 42.2 & 45.3 & 39.0 & 54.9 & 63.6 & 46.0 & 21.7 & 25.3 & 17.9 \\
\hline $5-9$ years & & 13.7 & 15.0 & 12.3 & 12.9 & 14.0 & 11.8 & 18.0 & 20.4 & 15.6 & 16.6 & 20.4 & * & 9.8 & 10.6 & 8.9 \\
\hline $10-14$ years & $\ldots$ & 16.9 & 19.9 & 13.8 & 15.7 & 18.3 & 13.0 & 23.6 & 28.7 & 18.4 & 19.5 & 25.7 & * & 12.3 & 13.8 & 10.6 \\
\hline $15-19$ years & - & 61.9 & 86.8 & 35.7 & 58.6 & 80.0 & 36.0 & 83.4 & 128.1 & 37.4 & 86.5 & 117.4 & 54.9 & 32.7 & 45.3 & 19.4 \\
\hline 20-24 years & & 98.3 & 145.2 & 48.4 & 93.0 & 136.1 & 46.6 & 138.0 & 212.3 & 61.6 & 120.7 & 170.6 & 68.0 & 53.2 & 76.2 & 29.1 \\
\hline $25-29$ years & $\because$ & 99.4 & 140.2 & 56.6 & 93.0 & 130.6 & 52.8 & 155.4 & 227.1 & 86.4 & 129.8 & 178.6 & 76.8 & 38.2 & 50.4 & 26.2 \\
\hline $30-34$ years & - & 110.8 & 148.2 & 72.3 & 103.4 & 138.1 & 66.8 & 184.4 & 255.8 & 119.5 & 166.2 & 221.4 & 106.6 & 39.6 & 49.9 & 29.8 \\
\hline $35-39$ years & & 145.8 & 185.4 & 105.7 & 135.9 & 173.3 & 97.0 & 243.9 & 313.8 & 181.5 & 219.4 & 272.7 & 163.9 & 54.8 & 70.9 & 39.5 \\
\hline $40-44$ years & & 221.6 & 276.8 & 166.7 & 207.9 & 261.2 & 153.6 & 353.7 & 442.9 & 275.2 & 309.3 & 392.2 & 226.7 & 90.6 & 109.6 & 72.6 \\
\hline $45-49$ years & & 340.0 & 423.8 & 258.1 & 318.7 & 400.4 & 237.1 & 549.5 & 678.8 & 437.0 & 407.5 & 501.0 & 318.4 & 144.0 & 179.1 & 111.9 \\
\hline $50-54$ years & & 509.0 & 646.6 & 376.8 & 474.9 & 604.8 & 347.5 & 861.0 & $1,108.1$ & 651.5 & 512.8 & 657.2 & 378.4 & 220.9 & 286.6 & 163.1 \\
\hline $55-59$ years & & 726.3 & 922.2 & 541.8 & 682.5 & 863.5 & 508.0 & $1,215.4$ & $1,612.7$ & 889.3 & 739.0 & 906.8 & 583.5 & 347.0 & 443.4 & 264.3 \\
\hline $60-64$ years & $\ldots$ & $1,068.3$ & $1,328.4$ & 829.7 & $1,023.5$ & $1,266.7$ & 795.8 & $1,702.1$ & $2,252.6$ & $1,270.7$ & $1,024.2$ & $1,219.3$ & 845.7 & 520.1 & 634.8 & 421.0 \\
\hline 65-69 years. & & $1,627.5$ & $2,002.2$ & $1,299.4$ & $1,581.1$ & $1,937.5$ & $1,262.8$ & $2,383.5$ & $3,111.4$ & $1,848.0$ & $1,534.8$ & $1,712.7$ & $1,375.6$ & 826.6 & $1,035.6$ & 646.1 \\
\hline $70-74$ years & & $2,491.3$ & $3,046.9$ & $2,037.2$ & $2,454.7$ & $2,989.5$ & $2,008.7$ & $3,309.7$ & $4,256.1$ & $2,656.7$ & $2,298.0$ & $2,736.3$ & $1,931.0$ & $1,333.8$ & $1,674.5$ & $1,066.0$ \\
\hline $75-79$ years & & $3,945.9$ & $4,817.2$ & $3,304.0$ & $3,933.2$ & $4,786.6$ & $3,291.8$ & $4,838.5$ & $6,197.6$ & $4,019.1$ & $3,324.7$ & $3,748.8$ & $2,990.9$ & $2,207.2$ & $2,762.5$ & $1,821.1$ \\
\hline 80-84 years. & & $6,381.4$ & $7,758.7$ & $5,511.7$ & $6,423.0$ & $7,810.7$ & $5,535.5$ & $6,966.3$ & $8,650.3$ & $6,079.9$ & $4,414.2$ & $4,913.6$ & $4,069.7$ & $3,933.7$ & $4,713.6$ & $3,413.8$ \\
\hline 85 years and ov & & $12,946.5$ & $14,006.4$ & $12,442.3$ & $13,176.5$ & $14,286.4$ & $12,646.7$ & $12,281.5$ & $12,964.7$ & $11,997.4$ & $6,708.1$ & $7,638.6$ & $6,248.2$ & $7,929.2$ & $8,918.0$ & $7,334.0$ \\
\hline
\end{tabular}

* Figure does not meet standards of reliability or precision; see "Technical Notes."

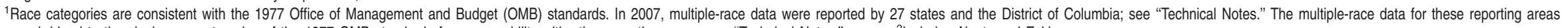
were bridged to the single-race categories of the 1977 OMB standards for comparability with other reporting areas; see "Technical Notes." ${ }^{2}$ Includes Aleuts and Eskimos.

${ }^{3}$ Includes Chinese, Filipino, Hawaiian, Japanese, and Other Asian or Pacific Islander. $\quad$ "Figures for age not stated are included in "all ages" but not distributed among age groups.

${ }^{5}$ Death rates for "under 1 year" (based on population estimates) differ from infant mortality rates (based on live births); see "Technical Notes." 
Table 4. Number of deaths and death rates, by Hispanic origin, race for non-Hispanic population, age, and sex: United States, 2007

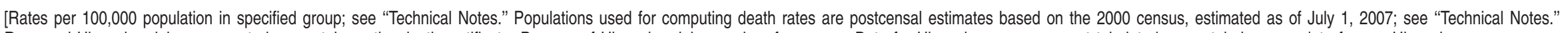
Race and Hispanic origin are reported separately on the death certificate. Persons of Hispanic origin may be of any race. Data for Hispanic persons are not tabulated separately by race; data for non-Hispanic persons are tabulated by race. Data for Hispanic origin should be interpreted with caution because of inconsistencies between reporting Hispanic origin on death certificates and on censuses and surveys; see "Technical Notes"]

\begin{tabular}{|c|c|c|c|c|c|c|c|c|c|c|c|c|c|c|c|c|}
\hline \multirow{2}{*}{\multicolumn{2}{|c|}{ Age }} & \multicolumn{3}{|c|}{ All origins ${ }^{1}$} & \multicolumn{3}{|c|}{ Hispanic } & \multicolumn{3}{|c|}{ Non-Hispanic ${ }^{2}$} & \multicolumn{3}{|c|}{ Non-Hispanic white ${ }^{3}$} & \multicolumn{3}{|c|}{ Non-Hispanic black ${ }^{3}$} \\
\hline & & $\begin{array}{l}\text { Both } \\
\text { sexes }\end{array}$ & Male & Female & $\begin{array}{l}\text { Both } \\
\text { sexes }\end{array}$ & Male & Female & $\begin{array}{l}\text { Both } \\
\text { sexes }\end{array}$ & Male & Female & $\begin{array}{l}\text { Both } \\
\text { sexes }\end{array}$ & Male & Female & $\begin{array}{l}\text { Both } \\
\text { sexes }\end{array}$ & Male & Female \\
\hline & & & & & & & & & Number & & & & & & & \\
\hline All ages & & $2,423,712$ & $1,203,968$ & $1,219,744$ & 135,519 & 75,708 & 59,811 & $2,284,446$ & $1,125,974$ & $1,158,472$ & $1,939,606$ & 948,662 & 990,944 & 286,366 & 146,474 & 139,892 \\
\hline Under 1 year. & & 29,138 & 16,293 & 12,845 & 6,068 & 3,343 & 2,725 & 22,883 & 12,839 & 10,044 & 12,998 & 7,340 & 5,658 & 8,629 & 4,794 & 3,835 \\
\hline $1-4$ years. . & & 4,703 & 2,634 & 2,069 & 1,009 & 556 & 453 & 3,680 & 2,074 & 1,606 & 2,325 & 1,316 & 1,009 & 1,102 & 609 & 493 \\
\hline $5-9$ years & $\ldots$ & 2,711 & 1,519 & 1,192 & 566 & 314 & 252 & 2,144 & 1,205 & 939 & 1,461 & 810 & 651 & 563 & 325 & 238 \\
\hline $10-14$ years & $\ldots$ & 3,436 & 2,066 & 1,370 & 591 & 349 & 242 & 2,836 & 1,709 & 1,127 & 1,901 & 1,135 & 766 & 772 & 476 & 296 \\
\hline $15-19$ years & $\ldots \ldots \ldots$ & 13,299 & 9,558 & 3,741 & 2,169 & 1,674 & 495 & 11,108 & 7,866 & 3,242 & 7,668 & 5,239 & 2,429 & 2,893 & 2,247 & 646 \\
\hline $20-24$ years & $\ldots \ldots$ & 20,683 & 15,758 & 4,925 & 3,517 & 2,832 & 685 & 17,125 & 12,893 & 4,232 & 11,939 & 8,890 & 3,049 & 4,344 & 3,388 & 956 \\
\hline $25-29$ years & $\ldots \ldots$ & 20,931 & 15,107 & 5,824 & 3,315 & 2,575 & 740 & 17,570 & 12,498 & 5,072 & 12,138 & 8,646 & 3,492 & 4,672 & 3,341 & 1,331 \\
\hline $30-34$ years & $\ldots \ldots$ & 21,641 & 14,685 & 6,956 & 3,231 & 2,381 & 850 & 18,350 & 12,259 & 6,091 & 12,619 & 8,492 & 4,127 & 4,849 & 3,197 & 1,652 \\
\hline $35-39$ years & $\ldots$ & 30,881 & 19,755 & 11,126 & 3,768 & 2,624 & 1,144 & 27,037 & 17,083 & 9,954 & 19,087 & 12,228 & 6,859 & 6,751 & 4,092 & 2,659 \\
\hline $40-44$ years & $\ldots \ldots$ & 48,725 & 30,350 & 18,375 & 4,927 & 3,413 & 1,514 & 43,670 & 26,852 & 16,818 & 31,879 & 19,911 & 11,968 & 10,046 & 5,889 & 4,157 \\
\hline $45-49$ years & $\ldots \ldots \ldots$ & 77,738 & 47,904 & 29,834 & 6,552 & 4,441 & 2,111 & 70,999 & 43,330 & 27,669 & 53,142 & 33,039 & 20,103 & 15,453 & 8,858 & 6,595 \\
\hline $50-54$ years & & 106,948 & 66,552 & 40,396 & 7,848 & 5,193 & 2,655 & 98,809 & 61,146 & 37,663 & 74,756 & 46,892 & 27,864 & 21,012 & 12,402 & 8,610 \\
\hline $55-59$ years & $\ldots \ldots$ & 132,458 & 81,590 & 50,868 & 8,464 & 5,313 & 3,151 & 123,637 & 76,027 & 47,610 & 95,806 & 59,418 & 36,388 & 23,951 & 14,320 & 9,631 \\
\hline $60-64$ years & & 154,652 & 92,028 & 62,624 & 8,960 & 5,451 & 3,509 & 145,379 & 86,358 & 59,021 & 118,070 & 70,559 & 47,511 & 23,248 & 13,503 & 9,745 \\
\hline $65-69$ years. & $\ldots \ldots$ & 174,991 & 100,492 & 74,499 & 9,806 & 5,764 & 4,042 & 164,893 & 94,533 & 70,360 & 135,914 & 78,445 & 57,469 & 24,324 & 13,452 & 10,872 \\
\hline $70-74$ years & $\ldots \ldots$ & 214,247 & 117,852 & 96,395 & 11,321 & 6,214 & 5,107 & 202,584 & 111,415 & 91,169 & 170,428 & 94,422 & 76,006 & 26,566 & 13,925 & 12,641 \\
\hline $75-79$ years & $\ldots \ldots$ & 289,029 & 149,669 & 139,360 & 14,185 & 7,280 & 6,905 & 274,482 & 142,166 & 132,316 & 239,043 & 124,921 & 114,122 & 28,763 & 13,839 & 14,924 \\
\hline 80-84 years. & 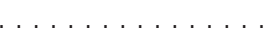 & 363,653 & 171,134 & 192,519 & 15,305 & 7,158 & 8,147 & 348,003 & 163,804 & 184,199 & 310,709 & 147,477 & 163,232 & 29,635 & 12,684 & 16,951 \\
\hline 85 years and 0 & $\ldots$ & 713,647 & 248,866 & 464,781 & 23,890 & 8,809 & 15,081 & 689,138 & 239,831 & 449,307 & 627,642 & 219,419 & 408,223 & 48,762 & 15,115 & 33,647 \\
\hline \multirow[t]{2}{*}{ Not stated... } & $\ldots \ldots$ & 201 & 156 & 45 & 27 & 24 & 3 & 119 & 86 & 33 & 81 & 63 & 18 & 31 & 18 & 13 \\
\hline & & & & & & & & & Rate & & & & & & & \\
\hline All ages ${ }^{4}$. & 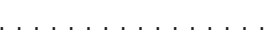 & 803.6 & 809.9 & 797.4 & 297.8 & 321.8 & 272.1 & 892.0 & 899.8 & 884.5 & 964.1 & 960.4 & 967.6 & 750.7 & 805.1 & 701.0 \\
\hline Under 1 year ${ }^{5}$ & & 684.5 & 747.8 & 618.1 & 587.4 & 632.7 & 539.9 & 709.8 & 777.9 & 638.3 & 559.7 & 616.8 & 499.6 & $1,309.1$ & $1,426.3$ & $1,187.1$ \\
\hline $1-4$ years.. & $\ldots$ & 28.6 & 31.3 & 25.7 & 26.0 & 28.0 & 23.8 & 29.2 & 32.2 & 26.1 & 25.5 & 28.1 & 22.7 & 43.7 & 47.5 & 39.8 \\
\hline $5-9$ years & $\ldots \ldots$ & 13.7 & 15.0 & 12.3 & 13.4 & 14.6 & 12.2 & 13.7 & 15.1 & 12.3 & 12.7 & 13.7 & 11.6 & 18.6 & 21.1 & 15.9 \\
\hline $10-14$ years. & $\ldots \ldots \ldots \ldots \ldots \ldots$ & 16.9 & 19.9 & 13.8 & 14.9 & 17.2 & 12.5 & 17.4 & 20.4 & 14.1 & 15.7 & 18.3 & 13.0 & 24.6 & 29.8 & 19.1 \\
\hline $15-19$ years & $\ldots \ldots$ & 61.9 & 86.8 & 35.7 & 57.9 & 86.8 & 27.2 & 62.7 & 86.7 & 37.5 & 58.0 & 77.2 & 37.8 & 85.7 & 131.4 & 38.8 \\
\hline $20-24$ years & $\ldots \ldots \ldots$ & 98.3 & 145.2 & 48.4 & 95.3 & 143.0 & 40.1 & 98.7 & 145.3 & 50.0 & 91.1 & 132.2 & 47.8 & 142.2 & 219.1 & 63.4 \\
\hline $25-29$ years & & 99.4 & 140.2 & 56.6 & 79.4 & 110.7 & 40.0 & 104.1 & 147.9 & 60.2 & 96.0 & 135.7 & 55.6 & 161.9 & 236.6 & 90.3 \\
\hline $30-34$ years & & 110.8 & 148.2 & 72.3 & 80.9 & 109.4 & 46.8 & 118.1 & 158.6 & 78.0 & 109.4 & 146.3 & 72.0 & 192.6 & 267.0 & 125.1 \\
\hline $35-39$ years & & 145.8 & 185.4 & 105.7 & 103.4 & 134.8 & 67.5 & 154.2 & 196.2 & 112.8 & 142.6 & 181.9 & 103.0 & 254.0 & 326.5 & 189.3 \\
\hline $40-44$ years & & 221.6 & 276.8 & 166.7 & 154.0 & 202.8 & 99.8 & 232.5 & 289.3 & 177.0 & 216.8 & 271.1 & 162.7 & 366.0 & 458.5 & 284.7 \\
\hline $45-49$ years & & 340.0 & 423.8 & 258.1 & 245.0 & 323.0 & 162.4 & 351.7 & 436.4 & 269.7 & 327.7 & 409.6 & 246.7 & 565.0 & 696.8 & 450.5 \\
\hline $50-54$ years & $\ldots$ & 509.0 & 646.6 & 376.8 & 378.2 & 500.0 & 256.1 & 521.7 & 660.8 & 388.9 & 483.6 & 613.1 & 356.7 & 883.6 & $1,137.9$ & 668.4 \\
\hline $55-59$ years & & 726.3 & 922.2 & 541.8 & 539.5 & 694.7 & 391.9 & 741.8 & 940.6 & 554.6 & 693.4 & 875.5 & 517.6 & $1,243.2$ & $1,651.2$ & 909.1 \\
\hline $60-64$ years & & $1,068.3$ & $1,328.4$ & 829.7 & 798.2 & $1,028.9$ & 592.1 & $1,088.7$ & $1,349.8$ & 848.6 & $1,039.6$ & $1,281.9$ & 811.8 & $1,738.9$ & $2,301.6$ & $1,298.9$ \\
\hline 65-69 years. & $\ldots \ldots$ & $1,627.5$ & $2,002.2$ & $1,299.4$ & $1,211.9$ & $1,556.5$ & 921.1 & $1,658.3$ & $2,033.5$ & $1,328.9$ & $1,608.0$ & $1,962.5$ & $1,289.9$ & $2,433.4$ & $3,178.7$ & $1,886.2$ \\
\hline $70-74$ years & & $2,491.3$ & $3,046.9$ & $2,037.2$ & $1,822.6$ & $2,278.5$ & $1,465.7$ & $2,539.1$ & $3,099.0$ & $2,079.9$ & $2,499.8$ & $3,037.2$ & $2,049.4$ & $3,373.8$ & $4,336.7$ & $2,710.8$ \\
\hline $75-79$ years & & $3,945.9$ & $4,817.2$ & $3,304.0$ & $2,959.5$ & $3,609.0$ & $2,487.6$ & $4,009.6$ & $4,893.4$ & $3,358.0$ & $3,995.4$ & $4,859.8$ & $3,344.3$ & $4,924.3$ & $6,308.6$ & $4,091.7$ \\
\hline $80-84$ years. & & $6,381.4$ & $7,758.7$ & $5,511.7$ & $4,746.9$ & $5,546.1$ & $4,213.5$ & $6,473.0$ & $7,887.9$ & $5,582.5$ & $6,515.4$ & $7,942.6$ & $5,605.4$ & $7,076.4$ & $8,795.9$ & $6,173.3$ \\
\hline 85 years and 0 & over & $12,946.5$ & $14,006.4$ & $12,442.3$ & $8,542.2$ & $8,953.7$ & $8,318.9$ & $13,170.0$ & $14,289.1$ & $12,641.5$ & $13,413.3$ & $14,588.3$ & $12,856.7$ & $12,468.1$ & $13,189.0$ & $12,169.3$ \\
\hline
\end{tabular}

${ }^{3}$ Race categories are consistent with the 1977 Office of Management and Budget (OMB) standards. In 2007, multiple-race data were reported by 27 states and the District of Columbia; see "Technical Notes." The multiple-race data for these reporting areas were bridged to the single-race categories of the 1977 OMB standards for comparability with other reporting areas; see "Technical Notes."

${ }^{4}$ Figures for age not stated are included in "all ages" but not distributed among age groups. $\quad{ }^{5}$ Death rates for "under 1 year" (based on population estimates) differ from infant mortality rates (based on live births); see "Technical Notes." 
Table 5. Number of deaths and death rates by age, and age-adjusted death rates, by specified Hispanic origin, race for non-Hispanic population, and sex: United States, 2007

[Rates are per 100,000 population in specified group; age-adjusted rates are per 100,000 U.S. standard population; see "Technical Notes." Populations used for computing death rates for "all origins," Hispanic, non-Hispanic,

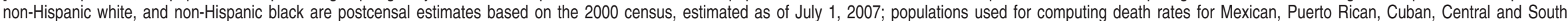
American, and other and unknown Hispanic are based on the Current Population Survey adjusted to resident population control totals. The control totals are 2000-based population estimates for the United States for July 1

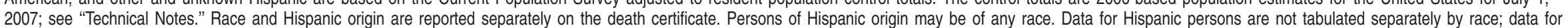
non-Hispanic persons are tabulated by race. Data for Hispanic origin should be interpreted with caution because of inconsistencies between reporting Hispanic origin on death certificates and on censuses and surveys; see "Technical Notes"]

\begin{tabular}{|c|c|c|c|c|c|c|c|c|c|c|c|c|c|c|}
\hline $\begin{array}{c}\text { Hispanic origin, race for } \\
\text { non-Hispanic population, and sex }\end{array}$ & $\begin{array}{c}\text { All } \\
\text { ages }\end{array}$ & $\begin{array}{l}\text { Under } 1 \\
\text { year }^{1}\end{array}$ & $\begin{array}{c}1-4 \\
\text { years }\end{array}$ & $\begin{array}{l}5-14 \\
\text { years }\end{array}$ & $\begin{array}{l}15-24 \\
\text { years }\end{array}$ & $\begin{array}{l}25-34 \\
\text { years }\end{array}$ & $\begin{array}{l}35-44 \\
\text { years }\end{array}$ & $\begin{array}{l}45-54 \\
\text { years }\end{array}$ & $\begin{array}{l}55-64 \\
\text { years }\end{array}$ & $\begin{array}{l}65-74 \\
\text { years }\end{array}$ & $\begin{array}{l}75-84 \\
\text { years }\end{array}$ & $\begin{array}{l}85 \text { years } \\
\text { and over }\end{array}$ & $\begin{array}{l}\text { Age not } \\
\text { stated }\end{array}$ & $\begin{array}{l}\text { Age- } \\
\text { adjusted }_{\text {rate }^{2}}\end{array}$ \\
\hline & \multicolumn{14}{|c|}{ Number } \\
\hline All origins & $2,423,712$ & 29,138 & 4,703 & 6,147 & 33,982 & 42,572 & 79,606 & 184,686 & 287,110 & 389,238 & 652,682 & 713,647 & 201 & $\ldots$ \\
\hline$\ldots \ldots$ & $1,203,968$ & 16,293 & 2,634 & 3,585 & 25,316 & 29,792 & 50,105 & 114,456 & 173,618 & 218,344 & 320,803 & 248,866 & 156 & $\ldots$ \\
\hline Female & $1,219,744$ & 12,845 & 2,069 & 2,562 & 8,666 & 12,780 & 29,501 & 70,230 & 113,492 & 170,894 & 331,879 & 464,781 & 45 & $\ldots$ \\
\hline Hispanic & 135,519 & 6,068 & 1,009 & 1,157 & 5,686 & 6,546 & 8,695 & 14,400 & 17,424 & 21,127 & 29,490 & 23,890 & 27 & $\ldots$ \\
\hline Male & 75,708 & 3,343 & 556 & 663 & 4,506 & 4,956 & 6,037 & 9,634 & 10,764 & 11,978 & 14,438 & 8,809 & 24 & $\ldots$ \\
\hline Female $\ldots \ldots \ldots$ & 59,811 & 2,725 & 453 & 494 & 1,180 & 1,590 & 2,658 & 4,766 & 6,660 & 9,149 & 15,052 & 15,081 & 3 & $\ldots$ \\
\hline Mexican & 77,274 & 4,236 & 732 & 820 & 3,917 & 4,368 & 5,289 & 8,576 & 10,086 & 11,592 & 16,033 & 11,611 & 14 & $\ldots$ \\
\hline Male & 44,389 & 2,337 & 399 & 466 & 3,119 & 3,342 & 3,694 & 5,750 & 6,209 & 6,571 & 7,934 & 4,554 & 14 & $\ldots$ \\
\hline Female . . . . . . & 32,885 & 1,899 & 333 & 354 & 798 & 1,026 & 1,595 & 2,826 & 3,877 & 5,021 & 8,099 & 7,057 & - & $\ldots$ \\
\hline Puerto Rican . . . . . . & 16,959 & 547 & 84 & 87 & 444 & 626 & 1,177 & 1,977 & 2,591 & 3,125 & 3,513 & 2,786 & 2 & $\ldots$ \\
\hline Male $\ldots \ldots \ldots$. & 9,342 & 311 & 48 & 48 & 331 & 458 & 798 & 1,327 & 1,634 & 1,744 & 1,695 & 946 & 2 & $\ldots$ \\
\hline Female . . . . . . . . & 7,617 & 236 & 36 & 39 & 113 & 168 & 379 & 650 & 957 & 1,381 & 1,818 & 1,840 & - & $\ldots$ \\
\hline Cuban. . & 12,942 & 78 & 10 & 18 & 101 & 115 & 279 & 723 & 1,094 & 2,067 & 4,107 & 4,347 & 3 & $\ldots$ \\
\hline Male & 6,644 & 48 & 6 & 12 & 72 & 87 & 202 & 524 & 727 & 1,278 & 2,116 & 1,570 & 2 & $\ldots$ \\
\hline Female & 6,298 & 30 & 4 & 6 & 29 & 28 & 77 & 199 & 367 & 789 & 1,991 & 2,777 & 1 & $\ldots$ \\
\hline Central and South American . . . . . & 14,341 & 551 & 93 & 135 & 751 & 924 & 1,113 & 1,609 & 1,880 & 2,241 & 2,755 & 2,285 & 4 & $\ldots$ \\
\hline Male $\ldots \ldots \ldots \ldots$ & 7,634 & 299 & 57 & 90 & 620 & 700 & 774 & 1,029 & 1,066 & 1,164 & 1,148 & 685 & 2 & $\ldots$ \\
\hline Female & 6,707 & 252 & 36 & 45 & 131 & 224 & 339 & 580 & 814 & 1,077 & 1,607 & 1,600 & 2 & $\ldots$ \\
\hline Other and unknown Hispanic . . . . . & 14,003 & 656 & 90 & 97 & 473 & 513 & 837 & 1,515 & 1,773 & 2,102 & 3,082 & 2,861 & 4 & $\ldots$ \\
\hline Male $\ldots \ldots \ldots$ & 7,699 & 348 & 46 & 47 & 364 & 369 & 569 & 1,004 & 1,128 & 1,221 & 1,545 & 1,054 & 4 & $\ldots$ \\
\hline Female . . . . . . . & 6,304 & 308 & 44 & 50 & 109 & 144 & 268 & 511 & 645 & 881 & 1,537 & 1,807 & - & $\cdots$ \\
\hline Non-Hispanic ${ }^{3}$. & $2,284,446$ & 22,883 & 3,680 & 4,980 & 28,233 & 35,920 & 70,707 & 169,808 & 269,016 & 367,477 & 622,485 & 689,138 & 119 & . \\
\hline Male ... & $1,125,974$ & 12,839 & 2,074 & 2,914 & 20,759 & 24,757 & 43,935 & 104,476 & 162,385 & 205,948 & 305,970 & 239,831 & 86 & $\ldots$ \\
\hline Female & $1,158,472$ & 10,044 & 1,606 & 2,066 & 7,474 & 11,163 & 26,772 & 65,332 & 106,631 & 161,529 & 316,515 & 449,307 & 33 & $\ldots$ \\
\hline White ${ }^{4} \ldots$ & $1,939,606$ & 12,998 & 2,325 & 3,362 & 19,607 & 24,757 & 50,966 & 127,898 & 213,876 & 306,342 & 549,752 & 627,642 & 81 & $\ldots$ \\
\hline Male & 948,662 & 7,340 & 1,316 & 1,945 & 14,129 & 17,138 & 32,139 & 79,931 & 129,977 & 172,867 & 272,398 & 219,419 & 63 & $\ldots$ \\
\hline Female $\ldots \ldots \ldots$ & 990,944 & 5,658 & 1,009 & 1,417 & 5,478 & 7,619 & 18,827 & 47,967 & 83,899 & 133,475 & 277,354 & 408,223 & 18 & $\ldots$ \\
\hline Black $^{4}$. & 286,366 & 8,629 & 1,102 & 1,335 & 7,237 & 9,521 & 16,797 & 36,465 & 47,199 & 50,890 & 58,398 & 48,762 & 31 & $\ldots$ \\
\hline Male & 146,474 & 4,794 & 609 & 801 & 5,635 & 6,538 & 9,981 & 21,260 & 27,823 & 27,377 & 26,523 & 15,115 & 18 & $\ldots$ \\
\hline Female & 139,892 & 3,835 & 493 & 534 & 1,602 & 2,983 & 6,816 & 15,205 & 19,376 & 23,513 & 31,875 & 33,647 & 13 & $\ldots$ \\
\hline Origin not stated ${ }^{5}$ & 3,747 & 187 & 14 & 10 & 63 & 106 & 204 & 478 & 670 & 634 & 707 & 619 & 55 & $\cdots$ \\
\hline Male & 2,286 & 111 & 4 & 8 & 51 & 79 & 133 & 346 & 469 & 418 & 395 & 226 & 46 & $\ldots$ \\
\hline Female & 1,461 & 76 & 10 & 2 & 12 & 27 & 71 & 132 & 201 & 216 & 312 & 393 & 9 & $\ldots$ \\
\hline
\end{tabular}

See footnotes at end of table. 
Table 5. Number of deaths and death rates by age, and age-adjusted death rates, by specified Hispanic origin, race for non-Hispanic population, and sex: United States, 2007-Con.

[Rates are per 100,000 population in specified group; age-adjusted rates are per 100,000 U.S. standard population; see "Technical Notes." Populations used for computing death rates for "all origins," Hispanic, non-Hispanic,

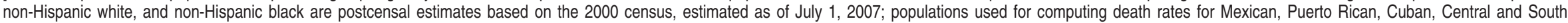
American, and other and unknown Hispanic are based on the Current Population Survey adjusted to resident population control totals. The control totals are 2000-based population estimates for the United States for July 1 ,

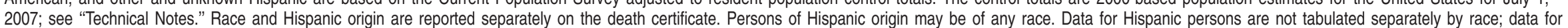
non-Hispanic persons are tabulated by race. Data for Hispanic origin should be interpreted with caution because of inconsistencies between reporting Hispanic origin on death certificates and on censuses and surveys; see "Technical Notes"]

\begin{tabular}{|c|c|c|c|c|c|c|c|c|c|c|c|c|c|c|}
\hline $\begin{array}{c}\text { Hispanic origin, race for } \\
\text { non-Hispanic population, and sex }\end{array}$ & $\begin{array}{c}\text { All } \\
\text { ages }\end{array}$ & $\begin{array}{l}\text { Under } 1 \\
\text { year }^{1}\end{array}$ & $\begin{array}{c}1-4 \\
\text { years }\end{array}$ & $\begin{array}{l}5-14 \\
\text { years }\end{array}$ & $\begin{array}{l}15-24 \\
\text { years }\end{array}$ & $\begin{array}{l}25-34 \\
\text { years }\end{array}$ & $\begin{array}{l}35-44 \\
\text { years }\end{array}$ & $\begin{array}{l}45-54 \\
\text { years }\end{array}$ & $\begin{array}{l}55-64 \\
\text { years }\end{array}$ & $\begin{array}{l}65-74 \\
\text { years }\end{array}$ & $\begin{array}{l}75-84 \\
\text { years }\end{array}$ & $\begin{array}{l}85 \text { years } \\
\text { and over }\end{array}$ & $\begin{array}{l}\text { Age not } \\
\text { stated }\end{array}$ & $\begin{array}{l}\text { Age- } \\
\text { adjusted } \text { rate }^{2}\end{array}$ \\
\hline & \multicolumn{14}{|c|}{ Rate $^{6}$} \\
\hline All origins ${ }^{7}$. & 803.6 & 684.5 & 28.6 & 15.3 & 79.9 & 104.9 & 184.4 & 420.9 & 877.7 & $2,011.3$ & $5,011.6$ & $12,946.5$ & $\ldots$ & 760.2 \\
\hline Male & 809.9 & 747.8 & 31.3 & 17.4 & 115.8 & 144.0 & 231.8 & 530.0 & $1,100.6$ & $2,456.9$ & $6,038.4$ & $14,006.4$ & $\ldots$ & 905.6 \\
\hline Female & 797.4 & 618.1 & 25.7 & 13.1 & 42.0 & 64.2 & 136.9 & 315.2 & 670.1 & $1,633.0$ & $4,304.1$ & $12,442.3$ & $\ldots$ & 643.4 \\
\hline Hispanic . & 297.8 & 587.4 & 26.0 & 14.1 & 76.5 & 80.1 & 127.1 & 303.2 & 647.4 & $1,477.1$ & $3,678.4$ & $8,542.2$ & $\ldots$ & 546.1 \\
\hline Male & 321.8 & 632.7 & 28.0 & 15.8 & 115.3 & 110.1 & 166.3 & 399.2 & 831.4 & $1,862.7$ & $4,364.8$ & $8,953.7$ & $\ldots$ & 654.5 \\
\hline Female & 272.1 & 539.9 & 23.8 & 12.3 & 33.5 & 43.4 & 82.7 & 204.0 & 476.9 & $1,162.1$ & $3,196.2$ & $8,318.9$ & $\ldots$ & 452.7 \\
\hline$\ldots \ldots \ldots \ldots \ldots \ldots$ & 260.3 & 550.5 & 26.2 & 14.4 & 80.0 & 79.2 & 122.9 & 298.5 & 669.0 & $1,473.4$ & $3,886.4$ & $9,386.9$ & $\ldots$ & 568.7 \\
\hline$\ldots \ldots \ldots \ldots \ldots$ & 284.6 & 576.7 & 28.0 & 16.2 & 120.5 & 108.3 & 157.9 & 383.2 & 809.1 & $1,742.9$ & $4,385.7$ & * & $\ldots$ & 659.6 \\
\hline Female. & 233.4 & 521.4 & 24.3 & 12.5 & 34.6 & 42.3 & 81.3 & 206.0 & 523.7 & $1,225.5$ & $3,496.4$ & $8,976.7$ & $\ldots$ & 484.5 \\
\hline Puerto Rican . & 430.2 & 800.2 & 28.6 & 11.9 & 70.1 & 99.4 & 215.2 & 434.6 & 873.8 & $1,919.7$ & $3,983.3$ & * & $\ldots$ & 636.6 \\
\hline Male... & 494.4 & * & 31.1 & 12.9 & 104.0 & 150.7 & 309.8 & 618.7 & $1,356.6$ & $2,488.0$ & * & * & $\ldots$ & 812.2 \\
\hline Female . & 371.1 & * & 25.9 & 10.8 & 35.8 & 51.6 & 131.0 & 270.3 & 543.5 & $1,489.9$ & $3,519.0$ & * & $\ldots$ & 503.7 \\
\hline$\ldots \ldots \ldots$ & 779.5 & * & * & * & 49.6 & 55.3 & 92.9 & 340.0 & 654.7 & $1,598.1$ & $4,355.7$ & * & $\ldots$ & 596.7 \\
\hline Male & 781.6 & * & * & * & 70.7 & 80.6 & 125.9 & 461.7 & 851.2 & $2,166.6$ & * & * & $\ldots$ & 704.3 \\
\hline Female & 777.3 & * & * & * & * & * & 55.1 & 200.7 & 449.2 & $1,121.5$ & $3,494.8$ & * & $\ldots$ & 507.1 \\
\hline Central and South American & 178.1 & 429.3 & 17.1 & 11.3 & 55.1 & 60.3 & 79.8 & 172.1 & 354.2 & 890.9 & $2,206.1$ & * & $\ldots$ & 325.5 \\
\hline Male .............. & 185.4 & 512.3 & 20.5 & 14.0 & 85.6 & 81.1 & 108.1 & 225.4 & 463.2 & $1,249.0$ & * & * & $\ldots$ & 408.9 \\
\hline Female & 170.5 & 360.1 & 13.5 & 8.1 & 20.5 & 33.5 & 50.0 & 121.2 & 270.8 & 680.2 & $2,000.8$ & * & $\ldots$ & 268.8 \\
\hline Other and unknown Hispanic & 645.8 & * & 55.4 & 26.6 & 140.2 & 179.6 & 280.0 & 551.1 & 936.7 & $2,103.8$ & $3,767.2$ & * & $\ldots$ & 721.8 \\
\hline Male ............. & 718.2 & * & * & 24.3 & 206.3 & 260.0 & 364.4 & 780.4 & $1,236.5$ & * & * & * & $\ldots$ & 929.5 \\
\hline Female & 575.0 & * & * & 29.1 & 67.8 & 100.2 & 187.7 & 349.3 & 657.7 & $1,568.9$ & $3,052.3$ & * & $\ldots$ & 556.1 \\
\hline Non-Hispanic ${ }^{3}$. & 892.0 & 709.8 & 29.2 & 15.6 & 80.5 & 110.8 & 194.7 & 434.0 & 896.1 & $2,050.4$ & $5,093.2$ & $13,170.0$ & $\ldots$ & 776.3 \\
\hline Male ... & 899.8 & 777.9 & 32.2 & 17.8 & 115.6 & 153.0 & 244.2 & 544.7 & $1,121.4$ & $2,498.2$ & $6,141.6$ & $14,289.1$ & $\ldots$ & 924.9 \\
\hline Female & 884.5 & 638.3 & 26.1 & 13.2 & 43.7 & 68.7 & 146.1 & 327.6 & 686.2 & $1,669.0$ & $4,371.8$ & $12,641.5$ & $\ldots$ & 657.7 \\
\hline White ${ }^{4} \ldots$ & 964.1 & 559.7 & 25.5 & 14.2 & 74.5 & 102.4 & 181.5 & 403.8 & 849.6 & $2,006.2$ & $5,113.1$ & $13,413.3$ & $\cdots$ & 763.3 \\
\hline Male & 960.4 & 616.8 & 28.1 & 16.1 & 104.6 & 140.8 & 228.4 & 508.7 & $1,057.5$ & $2,432.7$ & $6,152.7$ & $14,588.3$ & $\ldots$ & 906.8 \\
\hline Female & 967.6 & 499.6 & 22.7 & 12.3 & 42.7 & 63.4 & 134.4 & 300.5 & 651.3 & $1,634.9$ & $4,385.4$ & $12,856.7$ & $\ldots$ & 647.7 \\
\hline Black $^{4} \ldots$ & 750.7 & $1,309.1$ & 43.7 & 21.6 & 112.6 & 176.2 & 310.9 & 713.2 & $1,446.2$ & $2,847.8$ & $5,822.9$ & $12,468.1$ & $\cdots$ & 978.6 \\
\hline Male & 805.1 & $1,426.3$ & 47.5 & 25.5 & 173.1 & 250.6 & 393.4 & 900.4 & $1,913.7$ & $3,678.3$ & $7,295.1$ & $13,189.0$ & $\ldots$ & $1,210.9$ \\
\hline Female $\ldots \ldots$ & 701.0 & $1,187.1$ & 39.8 & 17.6 & 50.5 & 106.7 & 237.9 & 552.5 & $1,070.7$ & $2,255.0$ & $4,985.7$ & $12,169.3$ & $\ldots$ & 810.4 \\
\hline
\end{tabular}

Category not applicable.

- Quantity zero.

" Figure does not meet standards of reliability or precision; see "Technical Notes."

'Death rates for "under 1 year" (based on population estimates) differ from infant mortality rates (based on live births); see "Technical Notes."

${ }^{2}$ For method of computation, see "Technical Notes."

Includes races other than white and black.

${ }^{4}$ Race categories are consistent with the 1977 Office of Management and Budget (OMB) standards. In 2007, multiple-race data were reported by 27 states and the District of Columbia; see "Technical Notes." The multiple-race data for these reporting areas were bridged to the single-race categories of the 1977 OMB standards for comparability with other reporting areas; see "Technical Notes."

5 Includes deaths for which Hispanic origin was not reported on the death certificate.

${ }^{6}$ Figures for age not stated are included in "all ages" but not distributed among age groups.

${ }^{7}$ Figures for origin not stated are included in "all origins" but not distributed among specified origins. 
Table 6. Abridged life table for the total population, 2007

[For explanation of the columns of the life table, see "United States Life Tables, 2005," National Vital Statistics Reports, Volume 58, Number 10]

\begin{tabular}{|c|c|c|c|c|c|c|}
\hline & $\begin{array}{l}\text { Probability } \\
\text { of dying } \\
\text { between } \\
\text { ages } x \text { to } x+n\end{array}$ & $\begin{array}{l}\text { Number } \\
\text { surviving to } \\
\text { age } x\end{array}$ & $\begin{array}{c}\text { Number } \\
\text { dying } \\
\text { between } \\
\text { ages } x \text { to } x+n\end{array}$ & $\begin{array}{c}\text { Person-years } \\
\text { lived } \\
\text { between } \\
\text { ages } x \text { to } x+n\end{array}$ & $\begin{array}{l}\text { Total } \\
\text { number of } \\
\text { person-years } \\
\text { lived above } \\
\text { age } x\end{array}$ & $\begin{array}{c}\text { Expectancy } \\
\text { of life } \\
\text { at age } x\end{array}$ \\
\hline Age & ${ }_{n} q_{x}$ & $I_{x}$ & ${ }_{n} d_{x}$ & $n^{L} L_{x}$ & $T_{x}$ & $e_{x}$ \\
\hline$\ldots \ldots \ldots \ldots \ldots \ldots$ & 0.006760 & 100,000 & 676 & 99,406 & $7,793,477$ & 77.9 \\
\hline$\ldots \ldots \ldots$ & 0.001140 & 99,324 & 113 & 397,024 & $7,694,071$ & 77.5 \\
\hline$\ldots \ldots$ & 0.000683 & 99,211 & 68 & 495,870 & $7,297,047$ & 73.6 \\
\hline$\ldots \ldots \ldots \ldots \ldots \ldots$ & 0.000839 & 99,143 & 83 & 495,563 & $6,801,177$ & 68.6 \\
\hline$\ldots \ldots$ & 0.003089 & 99,060 & 306 & 494,626 & $6,305,614$ & 63.7 \\
\hline$\ldots \ldots \ldots \ldots \ldots \ldots$ & 0.004907 & 98,754 & 485 & 492,592 & $5,810,988$ & 58.8 \\
\hline 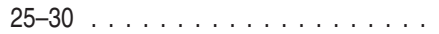 & 0.004958 & 98,269 & 487 & 490,128 & $5,318,396$ & 54.1 \\
\hline$\ldots \ldots \ldots \ldots \ldots \ldots$ & 0.005524 & 97,782 & 540 & 487,601 & $4,828,268$ & 49.4 \\
\hline $35-40$ & 0.007251 & 97,242 & 705 & 484,547 & $4,340,667$ & 44.6 \\
\hline$\ldots \ldots \ldots \ldots$ & 0.011003 & 96,537 & 1,062 & 480,216 & $3,856,120$ & 39.9 \\
\hline$\ldots \ldots \ldots \ldots$ & 0.016870 & 95,475 & 1,611 & 473,601 & $3,375,904$ & 35.4 \\
\hline$\ldots \ldots \ldots \ldots$ & 0.025217 & 93,864 & 2,367 & 463,734 & $2,902,303$ & 30.9 \\
\hline$\ldots \ldots \ldots \ldots \ldots \ldots$ & 0.035858 & 91,497 & 3,281 & 449,713 & $2,438,569$ & 26.7 \\
\hline 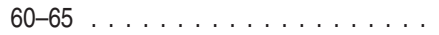 & 0.052469 & 88,216 & 4,629 & 430,150 & $1,988,856$ & 22.5 \\
\hline $65-70 \ldots \ldots \ldots \ldots \ldots \ldots$ & 0.077793 & 83,588 & 6,503 & 402,523 & $1,558,706$ & 18.6 \\
\hline$\ldots \ldots \ldots \ldots \ldots \ldots$ & 0.119029 & 77,085 & 9,175 & 363,859 & $1,156,183$ & 15.0 \\
\hline$\ldots \ldots \ldots \ldots \ldots \ldots$ & 0.191290 & 67,910 & 12,990 & 308,633 & 792,325 & 11.7 \\
\hline $80-85$ & 0.297734 & 54,919 & 16,351 & 234,721 & 483,691 & 8.8 \\
\hline $85-90$ & 0.441765 & 38,568 & 17,038 & 149,666 & 248,971 & 6.5 \\
\hline $90-95$ & 0.612438 & 21,530 & 13,186 & 72,269 & 99,305 & 4.6 \\
\hline $95-100$. & 0.778825 & 8,344 & 6,499 & 22,849 & 27,036 & 3.2 \\
\hline 100 and over . . . . . . & 1.000000 & 1,846 & 1,846 & 4,187 & 4,187 & 2.3 \\
\hline
\end{tabular}

\section{Table 7. Life expectancy at selected ages, by race and sex: United States, 2007}

[Race categories are consistent with the 1977 Office of Management and Budget (OMB) standards. Multiple-race data were reported by 27 states and the District of Columbia in 2007; see "Technical Notes." The multiple-race data for these reporting areas were bridged to the single-race categories of the 1977 OMB standards for comparability with other reporting areas; see "Technical Notes"]

\begin{tabular}{|c|c|c|c|c|c|c|c|c|c|}
\hline \multirow[b]{2}{*}{ Exact age in years } & \multicolumn{3}{|c|}{ All races $^{1}$} & \multicolumn{3}{|c|}{ White } & \multicolumn{3}{|c|}{ Black } \\
\hline & $\begin{array}{l}\text { Both } \\
\text { sexes }\end{array}$ & Male & Female & $\begin{array}{l}\text { Both } \\
\text { sexes }\end{array}$ & Male & Female & $\begin{array}{l}\text { Both } \\
\text { sexes }\end{array}$ & Male & Female \\
\hline$\ldots \ldots \ldots \ldots \ldots$ & 77.9 & 75.4 & 80.4 & 78.4 & 75.9 & 80.8 & 73.6 & 70.0 & 76.8 \\
\hline$\ldots \ldots \ldots \ldots \ldots \ldots$ & 77.5 & 74.9 & 79.9 & 77.8 & 75.4 & 80.2 & 73.6 & 70.1 & 76.8 \\
\hline$\ldots \ldots \ldots \ldots \ldots$ & 73.6 & 71.0 & 76.0 & 73.9 & 71.4 & 76.3 & 69.7 & 66.2 & 72.9 \\
\hline$\ldots \ldots \ldots \ldots \ldots$ & 68.6 & 66.1 & 71.0 & 68.9 & 66.5 & 71.3 & 64.7 & 61.3 & 67.9 \\
\hline$\ldots \ldots \ldots \ldots \ldots \ldots$ & 63.7 & 61.1 & 66.1 & 64.0 & 61.6 & 66.3 & 59.8 & 56.3 & 63.0 \\
\hline $20 \ldots \ldots \ldots \ldots \ldots$ & 58.8 & 56.4 & 61.2 & 59.2 & 56.8 & 61.5 & 55.1 & 51.7 & 58.1 \\
\hline $25 \ldots \ldots \ldots \ldots \ldots \ldots \ldots$ & 54.1 & 51.8 & 56.3 & 54.4 & 52.2 & 56.6 & 50.4 & 47.2 & 53.3 \\
\hline$\ldots \ldots \ldots \ldots \ldots$ & 49.4 & 47.1 & 51.5 & 49.7 & 47.5 & 51.7 & 45.8 & 42.7 & 48.5 \\
\hline$\ldots \ldots \ldots \ldots \ldots \ldots$ & 44.6 & 42.5 & 46.7 & 44.9 & 42.8 & 46.9 & 41.2 & 38.2 & 43.8 \\
\hline 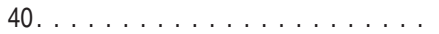 & 39.9 & 37.8 & 41.9 & 40.2 & 38.1 & 42.1 & 36.7 & 33.8 & 39.1 \\
\hline$\ldots$ & 35.4 & 33.3 & 37.2 & 35.6 & 33.6 & 37.4 & 32.3 & 29.5 & 34.7 \\
\hline$\ldots \ldots \ldots$ & 30.9 & 29.0 & 32.7 & 31.1 & 29.2 & 32.8 & 28.1 & 25.4 & 30.4 \\
\hline$\ldots \ldots \ldots \ldots \ldots \ldots$ & 26.7 & 24.9 & 28.2 & 26.8 & 25.1 & 28.4 & 24.2 & 21.7 & 26.3 \\
\hline$\ldots \ldots \ldots \ldots \ldots \ldots$ & 22.5 & 20.9 & 23.9 & 22.6 & 21.0 & 24.0 & 20.6 & 18.3 & 22.4 \\
\hline $65 \ldots \ldots \ldots \ldots \ldots$ & 18.6 & 17.2 & 19.9 & 18.7 & 17.3 & 19.9 & 17.2 & 15.2 & 18.7 \\
\hline$\ldots \ldots \ldots \ldots \ldots$ & 15.0 & 13.7 & 16.0 & 15.0 & 13.8 & 16.0 & 14.1 & 12.4 & 15.2 \\
\hline 75. & 11.7 & 10.6 & 12.5 & 11.7 & 10.6 & 12.4 & 11.2 & 9.9 & 12.1 \\
\hline 80. & 8.8 & 7.9 & 9.4 & 8.8 & 7.9 & 9.3 & 8.7 & 7.7 & 9.4 \\
\hline 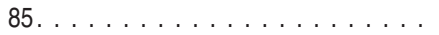 & 6.5 & 5.8 & 6.8 & 6.4 & 5.7 & 6.8 & 6.7 & 6.0 & 7.1 \\
\hline$\ldots \ldots \ldots \ldots \ldots$ & 4.6 & 4.1 & 4.8 & 4.6 & 4.1 & 4.8 & 5.1 & 4.6 & 5.3 \\
\hline$\ldots \ldots \ldots \ldots \ldots \ldots$ & 3.2 & 2.9 & 3.3 & 3.2 & 2.9 & 3.3 & 3.8 & 3.5 & 3.9 \\
\hline $100 \ldots \ldots \ldots \ldots \ldots \ldots$ & 2.3 & 2.1 & 2.3 & 2.2 & 2.0 & 2.2 & 2.8 & 2.6 & 2.8 \\
\hline
\end{tabular}

${ }^{1}$ Includes races other than white and black. 
Table 8. Life expectancy at birth, by race and sex: United States, 1940, 1950, 1960, 1970, and 1975-2007

[Race categories are consistent with 1977 Office of Management and Budget (OMB) standards]

\begin{tabular}{|c|c|c|c|c|c|c|c|c|c|}
\hline \multirow[b]{2}{*}{ Year } & \multicolumn{3}{|c|}{ All races ${ }^{1}$} & \multicolumn{3}{|c|}{ White } & \multicolumn{3}{|c|}{ Black } \\
\hline & $\begin{array}{l}\text { Both } \\
\text { sexes }\end{array}$ & Male & Female & $\begin{array}{l}\text { Both } \\
\text { sexes }\end{array}$ & Male & Female & $\begin{array}{l}\text { Both } \\
\text { sexes }\end{array}$ & Male & Female \\
\hline $2007^{2,3} \ldots \ldots \ldots \ldots \ldots$ & 77.9 & 75.4 & 80.4 & 78.4 & 75.9 & 80.8 & 73.6 & 70.0 & 76.8 \\
\hline $2006^{2,3} \ldots \ldots \ldots \ldots \ldots$ & 77.7 & 75.1 & 80.2 & 78.2 & 75.7 & 80.6 & 73.2 & 69.7 & 76.5 \\
\hline $2005^{2,3} \ldots \ldots \ldots \ldots \ldots \ldots$ & 77.4 & 74.9 & 79.9 & 77.9 & 75.4 & 80.4 & 72.8 & 69.3 & 76.1 \\
\hline $2004^{2,3} \ldots \ldots \ldots \ldots \ldots \ldots$ & 77.5 & 74.9 & 79.9 & 77.9 & 75.4 & 80.4 & 72.8 & 69.3 & 76.0 \\
\hline $2003^{2,3} \ldots \ldots \ldots \ldots \ldots \ldots$ & 77.1 & 74.5 & 79.6 & 77.6 & 75.0 & 80.0 & 72.3 & 68.8 & 75.6 \\
\hline $2002^{2} \ldots \ldots \ldots \ldots \ldots \ldots$ & 76.9 & 74.3 & 79.5 & 77.4 & 74.9 & 79.9 & 72.1 & 68.6 & 75.4 \\
\hline $2001^{2} \ldots \ldots \ldots \ldots \ldots$ & 76.9 & 74.2 & 79.4 & 77.4 & 74.8 & 79.9 & 72.0 & 68.4 & 75.2 \\
\hline $2000^{2} \ldots \ldots \ldots \ldots \ldots \ldots$ & 76.8 & 74.1 & 79.3 & 77.3 & 74.7 & 79.9 & 71.8 & 68.2 & 75.1 \\
\hline $1999 \ldots \ldots \ldots \ldots \ldots \ldots$ & 76.7 & 73.9 & 79.4 & 77.3 & 74.6 & 79.9 & 71.4 & 67.8 & 74.7 \\
\hline $1998 \ldots \ldots \ldots \ldots \ldots \ldots \ldots$ & 76.7 & 73.8 & 79.5 & 77.3 & 74.5 & 80.0 & 71.3 & 67.6 & 74.8 \\
\hline $1997 \ldots \ldots \ldots \ldots \ldots \ldots \ldots$ & 76.5 & 73.6 & 79.4 & 77.1 & 74.3 & 79.9 & 71.1 & 67.2 & 74.7 \\
\hline $1996 \ldots \ldots \ldots \ldots \ldots \ldots$ & 76.1 & 73.1 & 79.1 & 76.8 & 73.9 & 79.7 & 70.2 & 66.1 & 74.2 \\
\hline $1995 \ldots \ldots \ldots \ldots \ldots \ldots \ldots$ & 75.8 & 72.5 & 78.9 & 76.5 & 73.4 & 79.6 & 69.6 & 65.2 & 73.9 \\
\hline $1994 \ldots \ldots \ldots \ldots \ldots \ldots \ldots$ & 75.7 & 72.4 & 79.0 & 76.5 & 73.3 & 79.6 & 69.5 & 64.9 & 73.9 \\
\hline $1993 \ldots \ldots \ldots \ldots \ldots \ldots$ & 75.5 & 72.2 & 78.8 & 76.3 & 73.1 & 79.5 & 69.2 & 64.6 & 73.7 \\
\hline $1992 \ldots \ldots \ldots \ldots \ldots \ldots \ldots$ & 75.8 & 72.3 & 79.1 & 76.5 & 73.2 & 79.8 & 69.6 & 65.0 & 73.9 \\
\hline $1991 \ldots \ldots \ldots \ldots \ldots \ldots \ldots$ & 75.5 & 72.0 & 78.9 & 76.3 & 72.9 & 79.6 & 69.3 & 64.6 & 73.8 \\
\hline $1990 \ldots \ldots \ldots \ldots \ldots \ldots$ & 75.4 & 71.8 & 78.8 & 76.1 & 72.7 & 79.4 & 69.1 & 64.5 & 73.6 \\
\hline $1989 \ldots \ldots \ldots \ldots \ldots \ldots$ & 75.1 & 71.7 & 78.5 & 75.9 & 72.5 & 79.2 & 68.8 & 64.3 & 73.3 \\
\hline $1988 \ldots \ldots \ldots \ldots \ldots \ldots \ldots$ & 74.9 & 71.4 & 78.3 & 75.6 & 72.2 & 78.9 & 68.9 & 64.4 & 73.2 \\
\hline $1987 \ldots \ldots \ldots \ldots \ldots \ldots$ & 74.9 & 71.4 & 78.3 & 75.6 & 72.1 & 78.9 & 69.1 & 64.7 & 73.4 \\
\hline $1986 \ldots \ldots \ldots \ldots \ldots \ldots$ & 74.7 & 71.2 & 78.2 & 75.4 & 71.9 & 78.8 & 69.1 & 64.8 & 73.4 \\
\hline $1985 \ldots \ldots \ldots \ldots \ldots \ldots$ & 74.7 & 71.1 & 78.2 & 75.3 & 71.8 & 78.7 & 69.3 & 65.0 & 73.4 \\
\hline $1984 \ldots \ldots \ldots \ldots \ldots \ldots . \ldots \ldots$ & 74.7 & 71.1 & 78.2 & 75.3 & 71.8 & 78.7 & 69.5 & 65.3 & 73.6 \\
\hline $1983 \ldots \ldots \ldots \ldots \ldots \ldots$ & 74.6 & 71.0 & 78.1 & 75.2 & 71.6 & 78.7 & 69.4 & 65.2 & 73.5 \\
\hline $1982 \ldots \ldots \ldots \ldots \ldots \ldots \ldots$ & 74.5 & 70.8 & 78.1 & 75.1 & 71.5 & 78.7 & 69.4 & 65.1 & 73.6 \\
\hline $1981 \ldots \ldots \ldots \ldots \ldots \ldots$ & 74.1 & 70.4 & 77.8 & 74.8 & 71.1 & 78.4 & 68.9 & 64.5 & 73.2 \\
\hline $1980 \ldots \ldots \ldots \ldots \ldots \ldots$ & 73.7 & 70.0 & 77.4 & 74.4 & 70.7 & 78.1 & 68.1 & 63.8 & 72.5 \\
\hline $1979 \ldots \ldots \ldots \ldots \ldots \ldots . \ldots \ldots$ & 73.9 & 70.0 & 77.8 & 74.6 & 70.8 & 78.4 & 68.5 & 64.0 & 72.9 \\
\hline 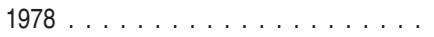 & 73.5 & 69.6 & 77.3 & 74.1 & 70.4 & 78.0 & 68.1 & 63.7 & 72.4 \\
\hline 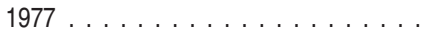 & 73.3 & 69.5 & 77.2 & 74.0 & 70.2 & 77.9 & 67.7 & 63.4 & 72.0 \\
\hline $1976 \ldots \ldots \ldots \ldots \ldots \ldots$ & 72.9 & 69.1 & 76.8 & 73.6 & 69.9 & 77.5 & 67.2 & 62.9 & 71.6 \\
\hline $1975 \ldots \ldots \ldots \ldots \ldots \ldots \ldots$ & 72.6 & 68.8 & 76.6 & 73.4 & 69.5 & 77.3 & 66.8 & 62.4 & 71.3 \\
\hline $1970 \ldots \ldots \ldots \ldots \ldots \ldots \ldots$ & 70.8 & 67.1 & 74.7 & 71.7 & 68.0 & 75.6 & 64.1 & 60.0 & 68.3 \\
\hline $1960 \ldots \ldots \ldots \ldots \ldots \ldots$ & 69.7 & 66.6 & 73.1 & 70.6 & 67.4 & 74.1 & - &.- & -- \\
\hline $1950 \ldots \ldots \ldots \ldots \ldots \ldots$ & 68.2 & 65.6 & 71.1 & 69.1 & 66.5 & 72.2 & $-\cdots$ & -- & $-\cdots$ \\
\hline $1940 \ldots \ldots \ldots \ldots \ldots \ldots . . \ldots \ldots$ & 62.9 & 60.8 & 65.2 & 64.2 & 62.1 & 66.6 & $-\cdots$ & $-\cdots$ & $-\cdots$ \\
\hline
\end{tabular}

- - - Data not available.

${ }^{1}$ Includes races other than white and black.

'Life expectancies for 2000-2007 were calculated using a revised methodology and may differ from those previously published; see "Technical Notes."

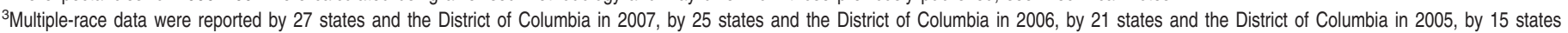

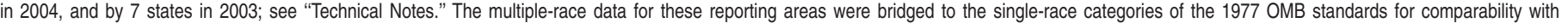
other reporting areas; see "Technical Notes." 
Table 9. Death rates by age and age-adjusted death rates for the 15 leading causes of death in 2007: United States, 1999-2007

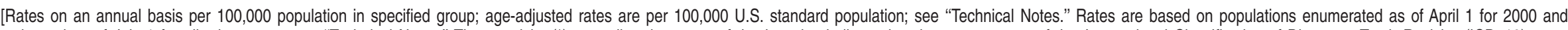
estimated as of July 1 for all other years; see "Technical Notes." The asterisks (*) preceding the cause-of-death codes indicate that they are not part of the International Classification of Diseases, Tenth Revision (ICD-10), Second Edition; see "Technical Notes"]

\begin{tabular}{|c|c|c|c|c|c|c|c|c|c|c|c|c|c|}
\hline \multirow[b]{2}{*}{$\begin{array}{l}\text { Cause of death } \\
\text { (based on ICD-10, 2004) and year }\end{array}$} & \multicolumn{12}{|c|}{ Age } & \multirow{2}{*}{$\begin{array}{l}\text { Age- } \\
\text { adjustec }^{\text {rate }}{ }^{3}\end{array}$} \\
\hline & $\begin{array}{l}\text { All } \\
\text { ages }^{1}\end{array}$ & $\begin{array}{l}\text { Under } \\
1 \text { year }^{2}\end{array}$ & $\begin{array}{c}1-4 \\
\text { years }\end{array}$ & $\begin{array}{l}5-14 \\
\text { years }\end{array}$ & $\begin{array}{l}15-24 \\
\text { years }\end{array}$ & $\begin{array}{l}25-34 \\
\text { years }\end{array}$ & $\begin{array}{l}35-44 \\
\text { years }\end{array}$ & $\begin{array}{l}45-54 \\
\text { years }\end{array}$ & $\begin{array}{l}55-64 \\
\text { years }\end{array}$ & $\begin{array}{l}65-74 \\
\text { years }\end{array}$ & $\begin{array}{l}75-84 \\
\text { years }\end{array}$ & $\begin{array}{l}85 \text { years } \\
\text { and over }\end{array}$ & \\
\hline \multicolumn{14}{|l|}{ All causes } \\
\hline $2007 \ldots$ & 803.6 & 684.5 & 28.6 & 15.3 & 79.9 & 104.9 & 184.4 & 420.9 & 877.7 & $2,011.3$ & $5,011.6$ & $12,946.5$ & 760.2 \\
\hline ․․ & 810.4 & 690.7 & 28.4 & 15.2 & 82.2 & 106.3 & 190.2 & 427.5 & 890.9 & $2,062.1$ & $5,115.0$ & $13,253.1$ & 776.5 \\
\hline$\ldots \ldots \ldots \ldots$ & 825.9 & 692.5 & 29.4 & 16.3 & 81.4 & 104.4 & 193.3 & 432.0 & 906.9 & $2,137.1$ & $5,260.0$ & $13,798.6$ & 798.8 \\
\hline$\ldots \ldots \ldots \ldots$ & 816.5 & 685.2 & 29.9 & 16.8 & 80.1 & 102.1 & 193.5 & 427.0 & 910.3 & $2,164.6$ & $5,275.1$ & $13,823.5$ & 800.8 \\
\hline$\ldots \ldots \ldots \ldots$ & 841.9 & 700.0 & 31.5 & 17.0 & 81.5 & 103.6 & 201.6 & 433.2 & 940.9 & $2,255.0$ & $5,463.1$ & $14,593.3$ & 832.7 \\
\hline 2002 & 847.3 & 695.0 & 31.2 & 17.4 & 81.4 & 103.6 & 202.9 & 430.1 & 952.4 & $2,314.7$ & $5,556.9$ & $14,828.3$ & 845.3 \\
\hline 2001 & 848.5 & 683.4 & 33.3 & 17.3 & 80.7 & 105.2 & 203.6 & 428.9 & 964.6 & $2,353.3$ & $5,582.4$ & $15,112.8$ & 854.5 \\
\hline 2000 & 854.0 & 736.7 & 32.4 & 18.0 & 79.9 & 101.4 & 198.9 & 425.6 & 992.2 & $2,399.1$ & $5,666.5$ & $15,524.4$ & 869.0 \\
\hline 1999 & 857.0 & 736.0 & 34.2 & 18.6 & 79.3 & 102.2 & 198.0 & 418.2 & $1,005.0$ & $2,457.3$ & $5,714.5$ & $15,554.6$ & 875.6 \\
\hline \multicolumn{14}{|l|}{ Diseases of heart $(100-109,|11| 113,, \mid 20-151)$} \\
\hline $2007 \ldots \ldots \ldots \ldots \ldots \ldots \ldots \ldots$ & 204.3 & 10.0 & 1.1 & 0.6 & 2.6 & 7.9 & 27.4 & 85.3 & 200.3 & 462.9 & $1,315.0$ & $4,267.7$ & 190.9 \\
\hline$\ldots \ldots \ldots$ & 211.0 & 8.4 & 1.0 & 0.6 & 2.5 & 8.2 & 28.3 & 88.0 & 207.3 & 490.3 & $1,383.1$ & $4,480.8$ & 200.2 \\
\hline 2005 & 220.0 & 8.7 & 0.9 & 0.6 & 2.7 & 8.1 & 28.9 & 89.7 & 214.8 & 518.9 & $1,460.8$ & $4,778.4$ & 211.1 \\
\hline 2004 & 222.2 & 10.3 & 1.2 & 0.6 & 2.5 & 7.9 & 29.3 & 90.2 & 218.8 & 541.6 & $1,506.3$ & $4,895.9$ & 217.0 \\
\hline 2003 & 235.6 & 11.0 & 1.2 & 0.6 & 2.7 & 8.2 & 30.7 & 92.5 & 233.2 & 585.0 & $1,611.1$ & $5,278.4$ & 232.3 \\
\hline 2002 & 241.7 & 12.4 & 1.1 & 0.6 & 2.5 & 7.9 & 30.5 & 93.7 & 241.5 & 615.9 & $1,677.2$ & $5,466.8$ & 240.8 \\
\hline 2001 & 245.8 & 11.9 & 1.5 & 0.7 & 2.5 & 8.0 & 29.6 & 92.9 & 246.9 & 635.1 & $1,725.7$ & $5,664.2$ & 247.8 \\
\hline 2000 & 252.6 & 13.0 & 1.2 & 0.7 & 2.6 & 7.4 & 29.2 & 94.2 & 261.2 & 665.6 & $1,780.3$ & $5,926.1$ & 257.6 \\
\hline 1999 & 259.9 & 13.8 & 1.2 & 0.7 & 2.8 & 7.6 & 30.2 & 95.7 & 269.9 & 701.7 & $1,849.9$ & $6,063.0$ & 266.5 \\
\hline \multicolumn{14}{|l|}{ Malignant neoplasms (CO0-C97) } \\
\hline & 186.6 & 1.7 & 2.2 & 2.4 & 3.9 & 8.5 & 30.8 & 114.3 & 315.4 & 715.5 & $1,256.3$ & $1,590.2$ & 178.4 \\
\hline $2006 \ldots \ldots \ldots \ldots \ldots$ & 187.0 & 1.8 & 2.3 & 2.2 & 3.9 & 9.0 & 31.9 & 116.3 & 321.2 & 727.2 & $1,263.8$ & $1,606.1$ & 180.7 \\
\hline$\ldots \ldots \ldots \ldots \ldots \ldots \ldots \ldots \ldots \ldots$ & 188.7 & 1.8 & 2.3 & 2.5 & 4.1 & 9.0 & 33.2 & 118.6 & 326.9 & 742.7 & $1,274.8$ & $1,637.7$ & 183.8 \\
\hline$\ldots \ldots \ldots$ & 188.6 & 1.8 & 2.5 & 2.5 & 4.1 & 9.1 & 33.4 & 119.0 & 333.4 & 755.1 & $1,280.4$ & $1,653.3$ & 185.8 \\
\hline$\ldots \ldots \ldots \ldots$ & 191.5 & 1.9 & 2.5 & 2.6 & 4.0 & 9.4 & 35.0 & 122.2 & 343.0 & 770.3 & $1,302.5$ & $1,698.2$ & 190.1 \\
\hline 2002 & 193.2 & 1.8 & 2.6 & 2.6 & 4.3 & 9.7 & 35.8 & 123.8 & 351.1 & 792.1 & $1,311.9$ & $1,723.9$ & 193.5 \\
\hline 2001 & 194.4 & 1.6 & 2.7 & 2.5 & 4.3 & 10.1 & 36.8 & 126.5 & 356.5 & 802.8 & $1,315.8$ & $1,765.6$ & 196.0 \\
\hline 2000 & 196.5 & 2.4 & 2.7 & 2.5 & 4.4 & 9.8 & 36.6 & 127.5 & 366.7 & 816.3 & $1,335.6$ & $1,819.4$ & 199.6 \\
\hline 1999 & 197.0 & 1.8 & 2.7 & 2.5 & 4.5 & 10.0 & 37.1 & 127.6 & 374.6 & 827.1 & $1,331.5$ & $1,805.8$ & 200.8 \\
\hline \multicolumn{14}{|l|}{ Cerebrovascular diseases (160-169) } \\
\hline $2007 \ldots \ldots \ldots \ldots \ldots$ & 45.1 & 3.1 & 0.3 & 0.2 & 0.5 & 1.2 & 4.9 & 14.6 & 32.1 & 93.0 & 322.3 & $1,015.5$ & 42.2 \\
\hline - & 45.8 & 3.4 & 0.3 & 0.2 & 0.5 & 1.3 & 5.1 & 14.7 & 33.3 & 96.3 & 335.1 & $1,039.6$ & 43.6 \\
\hline 2005 & 48.4 & 3.1 & 0.4 & 0.2 & 0.5 & 1.4 & 5.2 & 15.0 & 33.0 & 101.1 & 359.0 & $1,141.8$ & 46.6 \\
\hline 2004 & 51.1 & 3.1 & 0.3 & 0.2 & 0.5 & 1.4 & 5.4 & 14.9 & 34.3 & 107.8 & 386.2 & $1,245.9$ & 50.0 \\
\hline 2003 & 54.2 & 2.5 & 0.3 & 0.2 & 0.5 & 1.5 & 5.5 & 15.0 & 35.6 & 112.9 & 410.7 & $1,370.1$ & 53.5 \\
\hline 2002 & 56.4 & 2.9 & 0.3 & 0.2 & 0.4 & 1.4 & 5.4 & 15.1 & 37.2 & 120.3 & 431.0 & $1,445.9$ & 56.2 \\
\hline 2001 & 57.4 & 2.7 & 0.4 & 0.2 & 0.5 & 1.5 & 5.5 & 15.1 & 38.0 & 123.4 & 443.9 & $1,500.2$ & 57.9 \\
\hline 2000 & 59.6 & 3.3 & 0.3 & 0.2 & 0.5 & 1.5 & 5.8 & 16.0 & 41.0 & 128.6 & 461.3 & $1,589.2$ & 60.9 \\
\hline 1999 & 60.0 & 2.7 & 0.3 & 0.2 & 0.5 & 1.4 & 5.7 & 15.2 & 40.6 & 130.8 & 469.8 & $1,614.8$ & 61.6 \\
\hline
\end{tabular}

See footnotes at end of table. 
Table 9. Death rates by age and age-adjusted death rates for the 15 leading causes of death in 2007: United States, 1999-2007-Con.

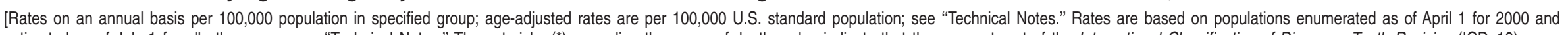
estimated as of July 1 for all other years; see "Technical Notes." The asterisks (*) preceding the cause-of-death codes indicate that they are not part of the International Classification of Diseases, Tenth Revision (ICD-10), Second Edition; see "Technical Notes"]

\begin{tabular}{|c|c|c|c|c|c|c|c|c|c|c|c|c|c|}
\hline \multirow[b]{2}{*}{$\begin{array}{c}\text { Cause of death } \\
\text { (based on ICD-10, 2004) and year }\end{array}$} & \multicolumn{12}{|c|}{ Age } & \multirow{2}{*}{$\begin{array}{l}\text { Age- } \\
\text { adjusted } \\
\text { rate }^{3}\end{array}$} \\
\hline & $\begin{array}{l}\text { All } \\
\text { ages }^{1}\end{array}$ & $\begin{array}{l}\text { Under } \\
1 \text { year }^{2}\end{array}$ & $\begin{array}{c}1-4 \\
\text { years }\end{array}$ & $\begin{array}{l}5-14 \\
\text { years }\end{array}$ & $\begin{array}{l}15-24 \\
\text { years }\end{array}$ & $\begin{array}{l}25-34 \\
\text { years }\end{array}$ & $\begin{array}{l}35-44 \\
\text { years }\end{array}$ & $\begin{array}{l}45-54 \\
\text { years }\end{array}$ & $\begin{array}{l}55-64 \\
\text { years }\end{array}$ & $\begin{array}{l}65-74 \\
\text { years }\end{array}$ & $\begin{array}{l}75-84 \\
\text { years }\end{array}$ & $\begin{array}{l}85 \text { years } \\
\text { and over }\end{array}$ & \\
\hline \multicolumn{14}{|l|}{ Chronic lower respiratory diseases (J40-J47) } \\
\hline $2007 \ldots \ldots \ldots \ldots \ldots \ldots$ & 42.4 & 1.0 & 0.3 & 0.3 & 0.4 & 0.6 & 1.8 & 9.5 & 39.1 & 148.1 & 368.9 & 596.1 & 40.8 \\
\hline $2006 \ldots \ldots \ldots \ldots \ldots \ldots$ & 41.6 & 0.7 & 0.3 & 0.3 & 0.4 & 0.6 & 1.9 & 9.1 & 39.2 & 149.3 & 363.4 & 589.1 & 40.5 \\
\hline 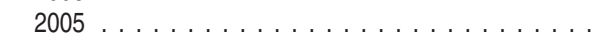 & 44.2 & 0.8 & 0.3 & 0.3 & 0.4 & 0.6 & 2.0 & 9.4 & 42.0 & 160.5 & 385.6 & 637.2 & 43.2 \\
\hline$\ldots \ldots \ldots \ldots \ldots \ldots$ & 41.5 & 0.9 & 0.3 & 0.3 & 0.4 & 0.6 & 2.0 & 8.4 & 40.4 & 153.8 & 366.7 & 601.7 & 41.1 \\
\hline$\ldots \ldots$ & 43.5 & 0.8 & 0.3 & 0.3 & 0.5 & 0.7 & 2.1 & 8.7 & 43.3 & 163.2 & 383.0 & 635.1 & 43.3 \\
\hline$\ldots \ldots \ldots$ & 43.3 & 1.0 & 0.4 & 0.3 & 0.5 & 0.8 & 2.2 & 8.7 & 42.4 & 163.0 & 386.7 & 637.6 & 43.5 \\
\hline$\ldots \ldots \ldots$ & 43.2 & 1.0 & 0.3 & 0.3 & 0.4 & 0.7 & 2.2 & 8.5 & 44.1 & 167.9 & 379.8 & 644.7 & 43.7 \\
\hline 2000 & 43.4 & 0.9 & 0.3 & 0.3 & 0.5 & 0.7 & 2.1 & 8.6 & 44.2 & 169.4 & 386.1 & 648.6 & 44.2 \\
\hline 1999 & 44.5 & 0.9 & 0.4 & 0.3 & 0.5 & 0.8 & 2.0 & 8.5 & 47.5 & 177.2 & 397.8 & 646.0 & 45.4 \\
\hline \multicolumn{14}{|l|}{ Accidents (unintentional injuries) (V01-X59,Y85-Y86) } \\
\hline$\ldots \ldots \ldots \ldots \ldots \ldots$ & 41.0 & 30.2 & 9.6 & 5.5 & 37.4 & 36.9 & 39.2 & 46.3 & 37.3 & 45.2 & 105.5 & 286.7 & 40.0 \\
\hline$\ldots \ldots \ldots \ldots \ldots$ & 40.6 & 27.8 & 9.9 & 5.6 & 38.2 & 37.0 & 40.2 & 45.5 & 36.2 & 44.5 & 105.1 & 274.9 & 39.8 \\
\hline $2005 \ldots \ldots \ldots \ldots \ldots \ldots \ldots \ldots$ & 39.7 & 26.4 & 10.3 & 6.0 & 37.4 & 34.9 & 38.6 & 43.2 & 35.8 & 46.3 & 106.1 & 279.5 & 39.1 \\
\hline$\ldots \ldots \ldots \ldots$ & 38.1 & 25.8 & 10.3 & 6.5 & 37.0 & 32.6 & 37.3 & 40.7 & 33.2 & 44.0 & 103.7 & 276.7 & 37.7 \\
\hline$\ldots \ldots \ldots \ldots \ldots \ldots$ & 37.6 & 23.6 & 10.9 & 6.4 & 37.1 & 31.5 & 37.8 & 38.8 & 32.9 & 44.1 & 101.9 & 278.9 & 37.3 \\
\hline $2002 \ldots \ldots \ldots \ldots \ldots \ldots \ldots \ldots$ & 37.0 & 23.5 & 10.5 & 6.6 & 38.0 & 31.5 & 37.2 & 36.6 & 31.4 & 44.2 & 101.3 & 275.4 & 36.9 \\
\hline$\ldots \ldots \ldots \ldots \ldots \ldots \ldots \ldots \ldots \ldots$ & 35.7 & 24.2 & 11.2 & 6.9 & 36.1 & 29.9 & 35.4 & 34.1 & 30.3 & 42.8 & 100.9 & 276.4 & 35.7 \\
\hline$\ldots \ldots \ldots \ldots \ldots \ldots$ & 34.8 & 23.1 & 11.9 & 7.3 & 36.0 & 29.5 & 34.1 & 32.6 & 30.9 & 41.9 & 95.1 & 273.5 & 34.9 \\
\hline 1999 & 35.1 & 22.3 & 12.4 & 7.6 & 35.3 & 29.6 & 33.8 & 31.8 & 30.6 & 44.6 & 100.5 & 282.4 & 35.3 \\
\hline \multicolumn{14}{|l|}{ Alzheimer's disease (G30) } \\
\hline $2007 \ldots \ldots \ldots \ldots$ & 24.7 & * & * & * & * & * & * & 0.2 & 2.2 & 20.6 & 176.7 & 849.1 & 22.7 \\
\hline $2006 \ldots \ldots \ldots \ldots \ldots \ldots \ldots$ & 24.2 & * & * & * & * & * & * & 0.2 & 2.1 & 20.2 & 175.6 & 848.3 & 22.6 \\
\hline $2005 \ldots \ldots \ldots$ & 24.2 & * & * & * & * & * & * & 0.2 & 2.1 & 20.5 & 177.3 & 861.6 & 22.9 \\
\hline 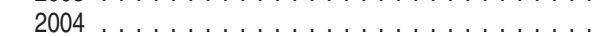 & 22.5 & * & * & * & * & * & * & 0.2 & 1.9 & 19.7 & 168.7 & 818.8 & 21.8 \\
\hline$\ldots \ldots \ldots \ldots \ldots \ldots$ & 21.8 & * & * & * & * & * & * & 0.2 & 2.0 & 20.9 & 164.4 & 802.4 & 21.4 \\
\hline$\ldots \ldots \ldots \ldots \ldots$ & 20.4 & * & * & * & * & * & * & 0.1 & 1.9 & 19.7 & 158.1 & 752.3 & 20.2 \\
\hline$\ldots \ldots \ldots \ldots \ldots \ldots$ & 18.9 & * & * & * & * & * & * & 0.2 & 2.1 & 18.7 & 147.5 & 710.3 & 19.1 \\
\hline$\ldots \ldots \ldots \ldots \ldots \ldots \ldots$ & 17.6 & * & * & * & * & * & * & 0.2 & 2.0 & 18.7 & 139.6 & 667.7 & 18.1 \\
\hline 1999 & 16.0 & * & * & * & * & * & * & 0.2 & 1.9 & 17.4 & 129.5 & 601.3 & 16.5 \\
\hline \multicolumn{14}{|l|}{ Diabetes mellitus (E10-E14) } \\
\hline 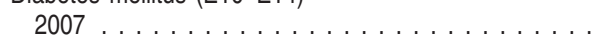 & 23.7 & * & * & 0.1 & 0.4 & 1.5 & 4.6 & 13.1 & 34.6 & 78.1 & 162.7 & 276.2 & 22.5 \\
\hline $2006 \ldots \ldots \ldots \ldots \ldots \ldots \ldots \ldots$ & 24.2 & * & * & 0.1 & 0.4 & 1.7 & 4.8 & 13.2 & 36.2 & 81.8 & 166.8 & 285.2 & 23.3 \\
\hline $2005 \ldots \ldots \ldots \ldots \ldots \ldots \ldots \ldots \ldots$ & 25.3 & * & * & 0.1 & 0.5 & 1.5 & 4.7 & 13.4 & 37.2 & 86.8 & 177.2 & 312.1 & 24.6 \\
\hline$\ldots \ldots \ldots \ldots \ldots \ldots$ & 24.9 & * & * & 0.1 & 0.4 & 1.5 & 4.6 & 13.4 & 37.1 & 87.2 & 176.9 & 307.0 & 24.5 \\
\hline$\ldots \ldots \ldots$ & 25.5 & * & * & 0.1 & 0.4 & 1.6 & 4.6 & 13.9 & 38.5 & 90.8 & 181.1 & 317.5 & 25.3 \\
\hline 2002 & 25.4 & * & * & 0.1 & 0.4 & 1.6 & 4.8 & 13.7 & 37.7 & 91.4 & 182.8 & 320.6 & 25.4 \\
\hline 2001 & 25.1 & * & * & 0.1 & 0.4 & 1.5 & 4.3 & 13.6 & 37.8 & 91.4 & 181.4 & 321.8 & 25.3 \\
\hline $2000 \ldots \ldots$ & 24.6 & * & * & 0.1 & 0.4 & 1.6 & 4.3 & 13.1 & 37.8 & 90.7 & 179.5 & 319.7 & 25.0 \\
\hline 1999 & 24.5 & * & * & 0.1 & 0.4 & 1.4 & 4.3 & 12.9 & 38.3 & 91.8 & 178.0 & 317.2 & 25.0 \\
\hline
\end{tabular}

See footnotes at end of table. 
Table 9. Death rates by age and age-adjusted death rates for the 15 leading causes of death in 2007: United States, 1999-2007-Con.

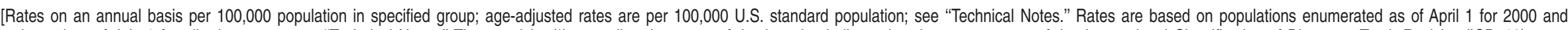
estimated as of July 1 for all other years; see "Technical Notes." The asterisks (*) preceding the cause-of-death codes indicate that they are not part of the International Classification of Diseases, Tenth Revision (ICD-10), Second Edition; see "Technical Notes"]

\begin{tabular}{|c|c|c|c|c|c|c|c|c|c|c|c|c|c|}
\hline \multirow[b]{2}{*}{$\begin{array}{l}\text { Cause of death } \\
\text { (based on ICD-10, 2004) and year }\end{array}$} & \multicolumn{12}{|c|}{ Age } & \multirow{2}{*}{$\begin{array}{l}\text { Age- } \\
\text { adjusted } \text { rate }^{3}\end{array}$} \\
\hline & $\begin{array}{l}\text { All } \\
\text { ages }\end{array}$ & $\begin{array}{l}\text { Under } \\
1 \text { year }^{2}\end{array}$ & $\begin{array}{c}1-4 \\
\text { years }\end{array}$ & $\begin{array}{l}5-14 \\
\text { years }\end{array}$ & $\begin{array}{l}15-24 \\
\text { years }\end{array}$ & $\begin{array}{l}25-34 \\
\text { years }\end{array}$ & $\begin{array}{l}35-44 \\
\text { years }\end{array}$ & $\begin{array}{l}45-54 \\
\text { years }\end{array}$ & $\begin{array}{l}55-64 \\
\text { years }\end{array}$ & $\begin{array}{l}65-74 \\
\text { years }\end{array}$ & $\begin{array}{l}75-84 \\
\text { years }\end{array}$ & $\begin{array}{l}85 \text { years } \\
\text { and over }\end{array}$ & \\
\hline \multicolumn{14}{|l|}{ Influenza and pneumonia (J09-J18) ${ }^{4}$} \\
\hline $2007 \ldots \ldots \ldots$ & 17.5 & 5.2 & 0.7 & 0.3 & 0.4 & 0.8 & 1.8 & 4.4 & 9.6 & 28.7 & 114.1 & 463.2 & 16.2 \\
\hline $2006 \ldots \ldots \ldots \ldots \ldots \ldots \ldots$ & 18.8 & 6.4 & 0.8 & 0.2 & 0.4 & 0.8 & 1.9 & 4.6 & 10.0 & 32.0 & 127.8 & 502.5 & 17.8 \\
\hline $2005 \ldots \ldots \ldots \ldots \ldots \ldots \ldots \ldots \ldots$ & 21.3 & 6.5 & 0.7 & 0.3 & 0.4 & 0.9 & 2.1 & 5.1 & 11.3 & 35.5 & 142.2 & 593.9 & 20.3 \\
\hline$\ldots \ldots \ldots \ldots \ldots$ & 20.3 & 6.7 & 0.7 & 0.2 & 0.4 & 0.8 & 2.0 & 4.6 & 10.8 & 34.6 & 139.3 & 582.6 & 19.8 \\
\hline $2003 \ldots \ldots \ldots \ldots \ldots \ldots \ldots \ldots$ & 22.4 & 8.0 & 1.0 & 0.4 & 0.5 & 0.9 & 2.2 & 5.2 & 11.2 & 37.3 & 151.1 & 666.1 & 22.0 \\
\hline $2002 \ldots \ldots \ldots \ldots \ldots \ldots \ldots \ldots \ldots$ & 22.8 & 6.5 & 0.7 & 0.2 & 0.4 & 0.9 & 2.2 & 4.8 & 11.2 & 37.5 & 156.9 & 696.6 & 22.6 \\
\hline$\ldots \ldots \ldots \ldots \ldots$ & 21.8 & 7.4 & 0.7 & 0.2 & 0.5 & 0.9 & 2.2 & 4.6 & 10.7 & 36.3 & 148.5 & 685.6 & 22.0 \\
\hline 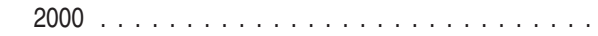 & 23.2 & 7.6 & 0.7 & 0.2 & 0.5 & 0.9 & 2.4 & 4.7 & 11.9 & 39.1 & 160.3 & 744.1 & 23.7 \\
\hline 1999 & 22.8 & 8.4 & 0.8 & 0.2 & 0.5 & 0.8 & 2.4 & 4.6 & 11.0 & 37.2 & 157.0 & 751.8 & 23.5 \\
\hline \multicolumn{14}{|l|}{$\begin{array}{l}\text { Nephritis, nephrotic syndrome and } \\
\text { nephrosis (N00-N07,N17-N19,N25-N27) }\end{array}$} \\
\hline $2007 \ldots \ldots \ldots \ldots \ldots \ldots \ldots \ldots$ & 15.4 & 3.4 & 0.1 & 0.1 & 0.2 & 0.6 & 1.7 & 5.1 & 13.6 & 40.1 & 113.0 & 290.6 & 14.5 \\
\hline 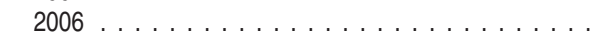 & 15.1 & 3.9 & * & * & 0.2 & 0.7 & 1.8 & 5.2 & 13.8 & 39.4 & 111.4 & 290.5 & 14.5 \\
\hline $2005 \ldots \ldots \ldots \ldots \ldots \ldots \ldots \ldots \ldots$ & 14.8 & 3.9 & * & 0.1 & 0.2 & 0.7 & 1.7 & 4.8 & 13.6 & 39.3 & 110.3 & 288.3 & 14.3 \\
\hline $2004 \ldots \ldots \ldots$ & 14.5 & 4.3 & * & 0.1 & 0.2 & 0.6 & 1.8 & 5.0 & 13.6 & 38.6 & 108.4 & 286.6 & 14.2 \\
\hline$\ldots \ldots \ldots$ & 14.6 & 4.5 & * & 0.1 & 0.2 & 0.7 & 1.8 & 4.9 & 13.6 & 40.1 & 109.5 & 293.1 & 14.4 \\
\hline $2002 \ldots \ldots \ldots \ldots \ldots \ldots \ldots \ldots \ldots$ & 14.2 & 4.3 & * & 0.1 & 0.2 & 0.7 & 1.7 & 4.7 & 13.0 & 39.2 & 109.1 & 288.6 & 14.2 \\
\hline $2001 \ldots \ldots \ldots \ldots \ldots \ldots \ldots \ldots \ldots$ & 13.9 & 3.3 & * & 0.0 & 0.2 & 0.6 & 1.7 & 4.6 & 13.0 & 40.2 & 104.2 & 287.7 & 14.0 \\
\hline$\ldots \ldots \ldots \ldots \ldots \ldots$ & 13.2 & 4.3 & * & 0.1 & 0.2 & 0.6 & 1.6 & 4.4 & 12.8 & 38.0 & 100.8 & 277.8 & 13.5 \\
\hline$\ldots \ldots \ldots$ & 12.7 & 4.4 & * & 0.1 & 0.2 & 0.6 & 1.6 & 4.0 & 12.0 & 37.1 & 97.6 & 268.9 & 13.0 \\
\hline \multicolumn{14}{|l|}{ Septicemia (A40-A41) } \\
\hline $2007 \ldots \ldots \ldots$ & 11.5 & 6.6 & 0.5 & 0.2 & 0.4 & 0.7 & 2.1 & 5.5 & 12.9 & 32.8 & 79.9 & 174.4 & 11.0 \\
\hline $2006 \ldots \ldots \ldots \ldots \ldots \ldots \ldots \ldots$ & 11.4 & 6.5 & 0.5 & 0.2 & 0.3 & 0.7 & 2.0 & 5.2 & 12.8 & 32.1 & 82.4 & 177.3 & 11.0 \\
\hline $2005 \ldots \ldots \ldots \ldots \ldots \ldots \ldots \ldots \ldots$ & 11.5 & 7.4 & 0.5 & 0.2 & 0.4 & 0.8 & 1.9 & 5.2 & 12.9 & 32.6 & 81.4 & 187.3 & 11.2 \\
\hline$\ldots \ldots \ldots \ldots \ldots \ldots$ & 11.4 & 6.6 & 0.5 & 0.2 & 0.3 & 0.8 & 1.9 & 5.4 & 12.9 & 32.4 & 81.6 & 186.7 & 11.2 \\
\hline$\ldots \ldots \ldots \ldots \ldots \ldots \ldots \ldots$ & 11.7 & 6.9 & 0.5 & 0.2 & 0.4 & 0.8 & 2.1 & 5.3 & 13.1 & 32.6 & 85.0 & 202.5 & 11.6 \\
\hline $2002 \ldots \ldots \ldots \ldots \ldots \ldots \ldots \ldots$ & 11.7 & 7.3 & 0.5 & 0.2 & 0.3 & 0.8 & 1.9 & 5.2 & 12.6 & 34.7 & 86.5 & 203.0 & 11.7 \\
\hline 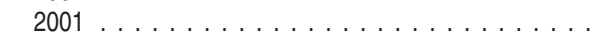 & 11.3 & 7.7 & 0.7 & 0.2 & 0.3 & 0.7 & 1.8 & 5.0 & 12.3 & 32.8 & 82.3 & 205.9 & 11.4 \\
\hline$\ldots \ldots \ldots \ldots$ & 11.1 & 7.2 & 0.6 & 0.2 & 0.3 & 0.7 & 1.9 & 4.9 & 11.9 & 31.0 & 80.4 & 215.7 & 11.3 \\
\hline 1999 & 11.0 & 7.5 & 0.6 & 0.2 & 0.3 & 0.7 & 1.8 & 4.6 & 11.4 & 31.2 & 79.4 & 220.7 & 11.3 \\
\hline \multicolumn{14}{|l|}{ Intentional self-harm (suicide) (*U03,X60-X84,Y87.0) } \\
\hline $2007 \ldots \ldots \ldots \ldots \ldots \ldots \ldots$ & 11.5 & $\ldots$ & $\ldots$ & 0.5 & 9.7 & 13.0 & 15.6 & 17.7 & 15.5 & 12.6 & 16.3 & 15.6 & 11.3 \\
\hline $006 \ldots \ldots \ldots \ldots \ldots \ldots \ldots \ldots \ldots$ & 11.1 & $\ldots$ & $\ldots$ & 0.5 & 9.9 & 12.3 & 15.1 & 17.2 & 14.5 & 12.6 & 15.9 & 15.9 & 10.9 \\
\hline $2005 \ldots \ldots \ldots \ldots \ldots \ldots \ldots \ldots$ & 11.0 & $\ldots$ & $\ldots$ & 0.7 & 10.0 & 12.4 & 14.9 & 16.5 & 13.9 & 12.6 & 16.9 & 16.9 & 10.9 \\
\hline$\ldots \ldots \ldots \ldots \ldots$ & 11.0 & $\ldots$ & $\ldots$ & 0.7 & 10.3 & 12.7 & 15.0 & 16.6 & 13.8 & 12.3 & 16.3 & 16.4 & 10.9 \\
\hline $2003 \ldots \ldots \ldots$ & 10.8 & $\ldots$ & $\ldots$ & 0.6 & 9.7 & 12.7 & 14.9 & 15.9 & 13.8 & 12.7 & 16.4 & 16.9 & 10.8 \\
\hline 2002 & 11.0 & $\ldots$ & $\ldots$ & 0.6 & 9.9 & 12.6 & 15.3 & 15.7 & 13.6 & 13.5 & 17.7 & 18.0 & 10.9 \\
\hline $2001^{5}$. & 10.8 & $\cdots$ & $\cdots$ & 0.7 & 9.9 & 12.8 & 14.7 & 15.2 & 13.1 & 13.3 & 17.4 & 17.5 & 10.7 \\
\hline$\ldots \ldots \ldots \ldots \ldots$ & 10.4 & $\ldots$ & $\ldots$ & 0.7 & 10.2 & 12.0 & 14.5 & 14.4 & 12.1 & 12.5 & 17.6 & 19.6 & 10.4 \\
\hline 1999 & 10.5 & $\ldots$ & $\ldots$ & 0.6 & 10.1 & 12.7 & 14.3 & 13.9 & 12.2 & 13.4 & 18.1 & 19.3 & 10.5 \\
\hline
\end{tabular}

See footnotes at end of table. 
Table 9. Death rates by age and age-adjusted death rates for the 15 leading causes of death in 2007: United States, 1999-2007-Con.

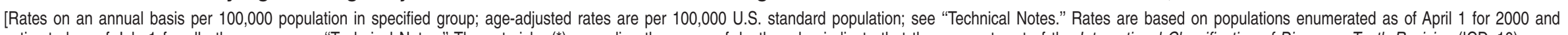
estimated as of July 1 for all other years; see "Technical Notes." The asterisks (*) preceding the cause-of-death codes indicate that they are not part of the International Classification of Diseases, Tenth Revision (ICD-10), Second Edition; see "Technical Notes"]

\begin{tabular}{|c|c|c|c|c|c|c|c|c|c|c|c|c|c|}
\hline \multirow[b]{2}{*}{$\begin{array}{c}\text { Cause of death } \\
\text { (based on ICD-10, 2004) and year }\end{array}$} & \multicolumn{12}{|c|}{ Age } & \multirow{2}{*}{$\begin{array}{l}\text { Age- } \\
\text { adjusted } \\
\text { rate }^{3}\end{array}$} \\
\hline & $\begin{array}{c}\text { All } \\
\text { ages }^{1}\end{array}$ & $\begin{array}{l}\text { Under } \\
1 \text { year }^{2}\end{array}$ & $\begin{array}{c}1-4 \\
\text { years }\end{array}$ & $\begin{array}{l}5-14 \\
\text { years }\end{array}$ & $\begin{array}{l}15-24 \\
\text { years }\end{array}$ & $\begin{array}{l}25-34 \\
\text { years }\end{array}$ & $\begin{array}{l}35-44 \\
\text { years }\end{array}$ & $\begin{array}{l}45-54 \\
\text { years }\end{array}$ & $\begin{array}{l}55-64 \\
\text { years }\end{array}$ & $\begin{array}{l}65-74 \\
\text { years }\end{array}$ & $\begin{array}{l}75-84 \\
\text { years }\end{array}$ & $\begin{array}{l}85 \text { years } \\
\text { and over }\end{array}$ & \\
\hline \multicolumn{14}{|l|}{ Chronic liver disease and cirrhosis (K70,K73-K74) } \\
\hline $2007 \ldots \ldots \ldots \ldots \ldots \ldots \ldots \ldots$ & 9.7 & * & * & * & 0.1 & 0.9 & 6.0 & 18.7 & 24.5 & 26.7 & 28.4 & 19.8 & 9.1 \\
\hline$\ldots \ldots \ldots$ & 9.2 & * & * & * & 0.1 & 0.8 & 5.8 & 17.8 & 22.8 & 26.0 & 29.0 & 19.4 & 8.8 \\
\hline$\ldots \ldots \ldots$ & 9.3 & * & * & * & 0.1 & 0.8 & 6.1 & 17.7 & 23.5 & 27.2 & 29.0 & 19.7 & 9.0 \\
\hline$\ldots \ldots \ldots \ldots \ldots \ldots \ldots \ldots \ldots \ldots$ & 9.2 & * & * & * & * & 0.8 & 6.3 & 18.0 & 22.6 & 27.7 & 28.8 & 19.7 & 9.0 \\
\hline$\ldots \ldots \ldots \ldots \ldots$ & 9.5 & * & * & * & * & 0.9 & 6.8 & 18.3 & 23.0 & 29.5 & 30.0 & 20.1 & 9.3 \\
\hline$\ldots \ldots \ldots \ldots \ldots$ & 9.5 & * & * & * & 0.1 & 0.9 & 7.0 & 18.0 & 22.9 & 29.4 & 31.4 & 21.4 & 9.4 \\
\hline$\ldots \ldots \ldots \ldots \ldots$ & 9.5 & * & * & * & 0.1 & 1.0 & 7.4 & 18.5 & 22.7 & 30.0 & 30.2 & 22.2 & 9.5 \\
\hline 2000 & 9.4 & * & * & * & 0.1 & 1.0 & 7.5 & 17.7 & 23.8 & 29.8 & 31.0 & 23.1 & 9.5 \\
\hline 1999 & 9.4 & * & * & * & 0.1 & 1.0 & 7.3 & 17.4 & 23.7 & 30.6 & 31.9 & 23.2 & 9.6 \\
\hline \multicolumn{14}{|l|}{$\begin{array}{l}\text { Essential hypertension and hypertensive } \\
\text { renal disease }(|10,| 12, \mid 15)\end{array}$} \\
\hline $2007 \ldots \ldots \ldots \ldots \ldots \ldots$ & 7.9 & * & * & * & 0.1 & 0.2 & 0.9 & 2.8 & 6.5 & 16.2 & 49.5 & 191.1 & 7.4 \\
\hline $2006 \ldots \ldots \ldots \ldots$ & 8.0 & * & * & * & 0.0 & 0.3 & 0.9 & 3.0 & 6.9 & 16.8 & 51.0 & 189.4 & 7.5 \\
\hline$\ldots \ldots \ldots$ & 8.4 & * & * & * & 0.1 & 0.2 & 0.9 & 2.7 & 6.4 & 17.7 & 55.6 & 210.0 & 8.0 \\
\hline 2004 & 7.9 & * & * & * & 0.1 & 0.3 & 0.8 & 2.7 & 6.3 & 17.1 & 52.6 & 198.5 & 7.7 \\
\hline$\ldots \ldots \ldots \ldots$ & 7.5 & * & * & * & 0.1 & 0.2 & 0.8 & 2.5 & 6.3 & 16.9 & 51.7 & 188.9 & 7.4 \\
\hline 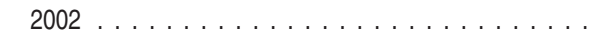 & 7.0 & * & * & * & 0.1 & 0.2 & 0.8 & 2.3 & 5.7 & 16.0 & 48.2 & 180.4 & 7.0 \\
\hline$\ldots \ldots \ldots \ldots \ldots$ & 6.8 & * & * & * & 0.1 & 0.3 & 0.7 & 2.4 & 5.8 & 15.5 & 47.7 & 171.9 & 6.8 \\
\hline$\ldots \ldots \ldots \ldots$ & 6.4 & * & * & * & * & 0.2 & 0.8 & 2.3 & 5.9 & 15.1 & 45.5 & 162.9 & 6.5 \\
\hline 1999 & 6.1 & * & * & * & * & 0.2 & 0.7 & 2.2 & 5.5 & 15.2 & 43.6 & 152.1 & 6.2 \\
\hline \multicolumn{14}{|l|}{ Parkinson's disease (G20-G21) } \\
\hline 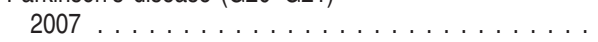 & 6.7 & * & * & * & * & * & * & 0.1 & 1.2 & 11.9 & 71.9 & 143.5 & 6.4 \\
\hline $2006 \ldots \ldots \ldots \ldots \ldots \ldots \ldots \ldots$ & 6.5 & * & * & * & * & * & * & 0.2 & 1.3 & 12.2 & 69.8 & 144.8 & 6.3 \\
\hline $2005 \ldots \ldots \ldots \ldots \ldots \ldots \ldots \ldots$ & 6.6 & * & * & * & * & * & * & 0.2 & 1.4 & 13.0 & 71.2 & 143.7 & 6.4 \\
\hline$\ldots \ldots \ldots \ldots$ & 6.1 & * & * & * & * & * & * & 0.2 & 1.2 & 12.0 & 67.5 & 135.8 & 6.1 \\
\hline 2003 & 6.2 & * & * & * & * & * & * & 0.2 & 1.3 & 12.7 & 67.8 & 138.2 & 6.2 \\
\hline 2002 & 5.9 & * & * & * & * & * & * & 0.1 & 1.2 & 12.2 & 63.9 & 135.2 & 5.9 \\
\hline 2001 & 5.8 & * & * & * & * & * & * & 0.1 & 1.2 & 11.7 & 64.6 & 134.2 & 5.9 \\
\hline$\ldots \ldots \ldots \ldots \ldots$ & 5.6 & * & * & * & * & * & * & 0.1 & 1.1 & 11.5 & 61.9 & 131.9 & 5.7 \\
\hline 1999 & 5.2 & * & * & * & * & * & * & 0.1 & 1.0 & 11.0 & 58.2 & 124.4 & 5.4 \\
\hline \multicolumn{14}{|l|}{ Assault (homicide) (*U01-*U02,X85-Y09,Y87.1) } \\
\hline $2007 \ldots \ldots \ldots \ldots \ldots \ldots \ldots$ & 6.1 & 8.3 & 2.4 & 0.9 & 13.1 & 11.7 & 7.1 & 4.9 & 3.0 & 2.1 & 2.1 & 1.5 & 6.1 \\
\hline 2006 & 6.2 & 8.1 & 2.2 & 1.0 & 13.5 & 11.7 & 6.9 & 5.1 & 3.2 & 2.1 & 2.1 & 1.9 & 6.2 \\
\hline 2005 & 6.1 & 7.5 & 2.3 & 0.8 & 13.0 & 11.8 & 7.1 & 4.8 & 2.8 & 2.4 & 2.2 & 2.1 & 6.1 \\
\hline$\ldots \ldots \ldots \ldots$ & 5.9 & 8.0 & 2.4 & 0.8 & 12.2 & 11.2 & 6.8 & 4.8 & 3.0 & 2.4 & 2.2 & 2.1 & 5.9 \\
\hline 2003 & 6.1 & 8.5 & 2.4 & 0.8 & 13.0 & 11.3 & 7.0 & 4.9 & 2.8 & 2.4 & 2.5 & 2.2 & 6.0 \\
\hline 2002 & 6.1 & 7.5 & 2.7 & 0.9 & 12.9 & 11.2 & 7.2 & 4.8 & 3.2 & 2.3 & 2.3 & 2.1 & 6.1 \\
\hline $2001^{5}$ & 7.1 & 8.2 & 2.7 & 0.8 & 13.3 & 13.1 & 9.5 & 6.3 & 4.0 & 2.9 & 2.5 & 2.4 & 7.1 \\
\hline 2000 & 6.0 & 9.2 & 2.3 & 0.9 & 12.6 & 10.4 & 7.1 & 4.7 & 3.0 & 2.4 & 2.4 & 2.4 & 5.9 \\
\hline 1999 & 6.1 & 8.7 & 2.5 & 1.1 & 12.9 & 10.5 & 7.1 & 4.6 & 3.0 & 2.6 & 2.5 & 2.4 & 6.0 \\
\hline
\end{tabular}

" Figure does not meet standards of reliability or precision; see "Technical Notes."

Category not applicable.

${ }^{1}$ Figures for age not stated included in "all ages" but not distributed among age groups.

2Death rates for "under 1 year" (based on population estimates) differ from infant mortality rates (based on live births); see "Technical Notes."

${ }^{3}$ For method of computation, see "Technical Notes."

${ }^{4}$ New ICD-10 code J09 (Influenza due to identified avian influenza virus) was added to the category in 2007. No deaths occurred from this cause in 2007.

${ }^{5}$ Figures include September 11, 2001-related deaths for which death certificates were filed as of October 24, 2002; see "Technical Notes" from "Deaths: Final Data for 2001." 
Table 10. Number of deaths from 113 selected causes and Enterocolitis due to Clostridium difficile, by age: United States, 2007

[The asterisks (*) preceding the cause-of-death codes indicate that they are not part of the International Classification of Diseases, Tenth Revision (ICD-10), Second Edition; see "Technical Notes."

\begin{tabular}{|c|c|c|c|c|c|c|c|c|c|c|c|c|c|}
\hline Cause of death (based on ICD-10, 2004) & $\begin{array}{c}\text { All } \\
\text { ages }\end{array}$ & $\begin{array}{c}\text { Under } 1 \\
\text { year }\end{array}$ & $\begin{array}{c}1-4 \\
\text { years }\end{array}$ & $\begin{array}{l}5-14 \\
\text { years }\end{array}$ & $\begin{array}{l}15-24 \\
\text { years }\end{array}$ & $\begin{array}{l}25-34 \\
\text { years }\end{array}$ & $\begin{array}{l}35-44 \\
\text { years }\end{array}$ & $\begin{array}{l}45-54 \\
\text { years }\end{array}$ & $\begin{array}{l}55-64 \\
\text { years }\end{array}$ & $\begin{array}{l}65-74 \\
\text { years }\end{array}$ & $\begin{array}{c}75-84 \\
\text { years }\end{array}$ & $\begin{array}{l}85 \text { years } \\
\text { and over }\end{array}$ & $\begin{array}{c}\text { Not } \\
\text { stated }\end{array}$ \\
\hline All causes & $2,423,712$ & 29,138 & 4,703 & 6,147 & 33,982 & 42,572 & 79,606 & 184,686 & 287,110 & 389,238 & 652,682 & 713,647 & 201 \\
\hline Salmonella infections & 30 & 2 & 2 & - & - & 2 & - & 3 & 2 & 4 & 6 & 9 & - \\
\hline Shigellosis and amebiasis. & 4 & - & - & 2 & - & - & 1 & - & - & - & 1 & - & \\
\hline Certain other intestinal infections $\ldots \ldots \ldots \ldots$ (A04,A07-A09) & 6,758 & 11 & 14 & 5 & 5 & 15 & 36 & 122 & 325 & 915 & 2,446 & 2,864 & - \\
\hline$\ldots \ldots \ldots \ldots \ldots(\mathrm{A} 16-\mathrm{A} 19)$ & 554 & 2 & - & 1 & 6 & 14 & 32 & 73 & 88 & 97 & 151 & 90 & - \\
\hline$\ldots \ldots \ldots \ldots(\mathrm{A} 16)$ & 424 & 2 & - & 1 & 3 & 10 & 27 & 53 & 62 & 73 & 119 & 74 & - \\
\hline$\ldots \ldots \ldots \ldots . .(\mathrm{A} 17-\mathrm{A} 19)$ & 130 & - & - & - & 3 & 4 & 5 & 20 & 26 & 24 & 32 & 16 & - \\
\hline Whooping cough . & 9 & 8 & - & - & - & - & - & 1 & - & - & - & - & - \\
\hline Scarlet fever and erysipelas & 3 & - & - & - & - & 1 & - & - & - & - & - & 2 & - \\
\hline Meningococcal infection & 87 & 11 & 5 & 11 & 21 & 6 & 9 & 8 & 8 & 4 & 1 & 3 & - \\
\hline 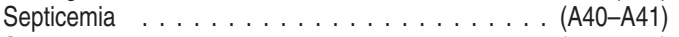 & 34,828 & 283 & 78 & 74 & 160 & 297 & 910 & 2,431 & 4,231 & 6,345 & 10,403 & 9,614 & 2 \\
\hline Syphilis . . . . . . . . . . . . . . . . . . . . . (A50-A53) & 42 & 5 & - & - & - & - & 4 & 2 & 1 & 8 & 12 & 10 & - \\
\hline$\ldots \ldots \ldots \ldots \ldots(\mathrm{A} 80)$ & - & - & - & - & - & - & - & - & - & - & - & - & 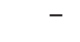 \\
\hline Arthropod-borne viral encephalitis . . . . . . (A83-A84,A85.2) & 3 & - & - & 1 & - & - & - & - & - & 1 & - & 1 & - \\
\hline$\ldots \ldots \ldots \ldots$ (B05) & - & - & - & - & - & - & - & - & - & - & - & - & - \\
\hline Viral hepatitis & 7,407 & 1 & 1 & - & 14 & 72 & 489 & 2,815 & 2,413 & 871 & 570 & 161 & \\
\hline Human immunodeficiency virus (HIV) disease & 11,295 & 5 & 4 & 10 & 160 & 1,091 & 3,572 & 4,156 & 1,721 & 448 & 109 & 16 & 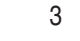 \\
\hline Malaria $\ldots \ldots \ldots \ldots \ldots \ldots \ldots$ & 5 & - & - & - & - & - & 2 & - & 1 & 1 & - & 1 & 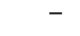 \\
\hline \multirow{2}{*}{\multicolumn{14}{|c|}{$\begin{array}{r}\text { Other and unspecified infectious and parasitic diseases and } \\
\text { their sequelae .......A00,A05,A20-A36,A42-A44,A48- } \\
\text { A49,A54-A79,A81-A82,A85.0-A85.1,A85.8,A86-B04, } \\
\text { B06-B09,B25-B49,B55-B99) }\end{array}$}} \\
\hline & 5,825 & 154 & 63 & 51 & 97 & 136 & 229 & 511 & 919 & 1,098 & 1,463 & 1,104 & - \\
\hline 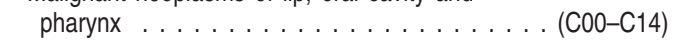 & 8,067 & - & - & 1 & 18 & 44 & 251 & 1,136 & 2,019 & 1,915 & 1,741 & 942 & - \\
\hline Malignant neoplasm of esophagus $\ldots \ldots$ & 13,592 & - & - & - & 5 & 28 & 246 & 1,452 & 3,379 & 3,726 & 3,376 & 1,380 & - \\
\hline Malignant neoplasm of stomach . . . . . . . . . . & 11,388 & 1 & - & - & 14 & 127 & 456 & 1,151 & 1,879 & 2,555 & 3,255 & 1,950 & - \\
\hline $\begin{array}{l}\text { Malignant neoplasms of colon, rectum and anus . (C18-C21) } \\
\text { Malignant neoplasms of liver and intrahepatic bile }\end{array}$ & 53,586 & - & - & 1 & 35 & 275 & 1,302 & 4,793 & 9,058 & 11,634 & 15,417 & 11,069 & 2 \\
\hline ducts $\ldots \ldots \ldots \ldots \ldots \ldots \ldots \ldots \ldots$ (C22) & 17,146 & 6 & 19 & 25 & 38 & 90 & 368 & 2,503 & 4,181 & 3,884 & 4,266 & 1,766 & 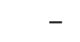 \\
\hline Malignant neoplasm of pancreas $\ldots \ldots \ldots$ & 34,117 & - & - & 2 & 5 & 52 & 538 & 2,808 & 6,507 & 8,671 & 10,317 & 5,217 & - \\
\hline Malignant neoplasm of larynx . . & 3,634 & - & - & - & 2 & 2 & 55 & 450 & 932 & 989 & 871 & 333 & - \\
\hline Malignant neoplasms of trachea, bronchus and & & & & & & & & & & & & & \\
\hline $\begin{array}{l}\text { lung ................ } \\
\text { Malignant melanoma of skin }\end{array}$ & 158,760 & 1 & 1 & 4 & 25 & 135 & 1,852 & 12,480 & 31,216 & 48,157 & 48,358 & 16,528 & 3 \\
\hline Malignant melanoma of skin . . . . . . . . . & 8,461 & - & 1 & 2 & 31 & 175 & 466 & 1,174 & 1,742 & 1,737 & 2,035 & 1,098 & - \\
\hline Malignant neoplasm of breast $\ldots \ldots \ldots$ & 40,970 & - & - & - & 15 & 344 & 2,184 & 5,990 & 8,756 & 8,179 & 9,075 & 6,426 & \\
\hline Malignant neoplasm of cervix uteri $\ldots \ldots \ldots$ & 4,021 & - & - & - & 8 & 183 & 645 & 915 & 892 & 611 & 506 & 261 & 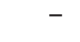 \\
\hline Malignant neoplasms of corpus uteri and uterus, part & & & & & & & & & & & & & \\
\hline$\ldots \ldots \ldots \ldots(\mathrm{C} 54-\mathrm{C} 55)$ & 7,456 & - & - & - & 2 & 31 & 168 & 584 & 1,583 & 2,021 & 1,960 & 1,107 & - \\
\hline Malignant neoplasm of ovary & 14,621 & - & - & - & 28 & 79 & 352 & 1,532 & 2,997 & 3,616 & 3,946 & 2,071 & \\
\hline Malignant neoplasm of prostate & 29,093 & - & 1 & - & 1 & 1 & 21 & 428 & 2,271 & 5,716 & 11,257 & 9,397 & \\
\hline Malignant neoplasms of kidney and renal pelvis . (C64-C65) & 12,703 & 2 & 9 & 25 & 34 & 50 & 240 & 1,243 & 2,584 & 3,159 & 3,539 & 1,818 & \\
\hline Malignant neoplasm of bladder $\ldots . . . \ldots \ldots$ & 13,843 & 1 & - & - & - & 7 & 93 & 570 & 1,564 & 2,817 & 5,009 & 3,782 & \\
\hline related tissue . . . . . . . . . . . . . . . (C81-C96) & 54,991 & 26 & 111 & 352 & 630 & 771 & 1,464 & 3,606 & 7,694 & 12,223 & 17,884 & 10,228 & 2 \\
\hline
\end{tabular}

See footnotes at end of table. 
Table 10. Number of deaths from 113 selected causes and Enterocolitis due to Clostridium difficile, by age: United States, 2007-Con.

[The asterisks ( ${ }^{*}$ ) preceding the cause-of-death codes indicate that they are not part of the International Classification of Diseases, Tenth Revision (ICD-10), Second Edition; see "Technical Notes."

\begin{tabular}{|c|c|c|c|c|c|c|c|c|c|c|c|c|c|}
\hline Cause of death (based on ICD-10, 2004) & $\begin{array}{l}\text { All } \\
\text { ages }\end{array}$ & $\begin{array}{l}\text { Under } 1 \\
\text { year }\end{array}$ & $\begin{array}{c}1-4 \\
\text { years }\end{array}$ & $\begin{array}{l}5-14 \\
\text { years }\end{array}$ & $\begin{array}{l}15-24 \\
\text { years }\end{array}$ & $\begin{array}{l}25-34 \\
\text { years }\end{array}$ & $\begin{array}{l}35-44 \\
\text { years }\end{array}$ & $\begin{array}{l}45-54 \\
\text { years }\end{array}$ & $\begin{array}{l}55-64 \\
\text { years }\end{array}$ & $\begin{array}{l}65-74 \\
\text { years }\end{array}$ & $\begin{array}{l}75-84 \\
\text { years }\end{array}$ & $\begin{array}{l}85 \text { years } \\
\text { and over }\end{array}$ & $\begin{array}{l}\text { Not } \\
\text { stated }\end{array}$ \\
\hline Hodgkin's disease & 1,271 & - & - & 3 & 65 & 120 & 135 & 145 & 176 & 209 & 286 & 132 & - \\
\hline Non-Hodgkin's lymphoma . . . . . & 20,528 & 2 & 5 & 33 & 133 & 206 & 516 & 1,392 & 2,922 & 4,476 & 6,868 & 3,975 & - \\
\hline Leukemia $\ldots \ldots \ldots \ldots \ldots \ldots \ldots$ (C91-C95) & 21,825 & 21 & 106 & 314 & 428 & 438 & 657 & 1,362 & 2,801 & 4,611 & 6,858 & 4,228 & 1 \\
\hline Multiple myeloma and immunoproliferative & & & & & & & & & & & & & \\
\hline $\begin{array}{l}\text { neoplasms ................................. (C88,C90) } \\
\text { Other and unspecified malianant neoplasms of Iymphoid. }\end{array}$ & 11,307 & - & - & - & 2 & 7 & 155 & 705 & 1,788 & 2,917 & 3,853 & 1,879 & 1 \\
\hline hematopoietic and related tissue ........... (C96) & 60 & 3 & - & 2 & 2 & - & 1 & 2 & 7 & 10 & 19 & 14 & - \\
\hline $\begin{array}{l}\text { All other and unspecified malignant } \\
\text { neoplasms } \ldots \ldots \ldots \text { (C17,C23-C24,C26-C31,C37-C41, } \\
\text { C44-C49,C51- C52,C57-C60,C62-C63,C66,C68-C69, }\end{array}$ & & & & & & & & & & & & & \\
\hline $\begin{array}{c}\text { C73-C80,C97) } \\
\text { In situ neoplasms, benign neoplasms and neoplasms of }\end{array}$ & 63,192 & 20 & 113 & 245 & 558 & 700 & 1,742 & 5,436 & 10,942 & 13,854 & 18,188 & 11,394 & - \\
\hline uncertain or unknown behavior . . . . . . . . . (D00-D48) & 14,204 & 59 & 59 & 84 & 81 & 138 & 340 & 699 & 1,416 & 2,402 & 4,717 & 4,209 & - \\
\hline Anemias $\ldots \ldots \ldots \ldots \ldots \ldots \ldots \ldots \ldots \ldots$ (D50-D64) & 4,829 & 17 & 17 & 32 & 90 & 136 & 187 & 255 & 322 & 560 & 1,224 & 1,989 & - \\
\hline$\ldots \ldots \ldots \ldots \ldots \ldots(E 10-E 14)$ & 71,382 & 7 & 5 & 21 & 168 & 610 & 1,984 & 5,753 & 11,304 & 15,112 & 21,189 & 15,227 & 2 \\
\hline Nutritional deficiencies . . . . . . . . . . . . . . . . . . (E40-E64) & 2,852 & 7 & 5 & 3 & 4 & 19 & 36 & 93 & 201 & 333 & 808 & 1,343 & - \\
\hline Malnutrition $\ldots \ldots \ldots \ldots \ldots$ & 2,644 & 6 & 2 & 3 & 4 & 18 & 35 & 87 & 188 & 314 & 755 & 1,232 & - \\
\hline Other nutritional deficiencies . . . . . & 208 & 1 & 3 & - & - & 1 & 1 & 6 & 13 & 19 & 53 & 111 & - \\
\hline Meningitis ............... & 655 & 82 & 16 & 25 & 35 & 34 & 66 & 111 & 89 & 72 & 88 & 37 & - \\
\hline Parkinson's disease & 20,058 & - & - & - & 2 & 2 & 12 & 60 & 396 & 2,310 & 9,363 & 7,911 & 2 \\
\hline Alzheimer's disease & 74,632 & - & - & - & - & 1 & 8 & 95 & 728 & 3,984 & 23,009 & 46,804 & 3 \\
\hline Major cardiovascular diseases . . . & 806,156 & 571 & 230 & 338 & 1,369 & 3,950 & 14,867 & 46,280 & 80,797 & 115,623 & 229,050 & 313,044 & 37 \\
\hline Diseases of heart $\ldots \ldots \ldots \ldots(100-109,|11| 13,, \mid 20-151)$ & 616,067 & 424 & 173 & 241 & 1,084 & 3,223 & 11,839 & 37,434 & 65,527 & 89,589 & 171,257 & 235,249 & 27 \\
\hline Acute rheumatic fever and chronic rheumatic heart & & & & & & & & & & & & & \\
\hline diseases . . . . . . . . . . . . . . . . . (100-109) & 3,201 & 2 & 1 & 1 & 12 & 28 & 70 & 177 & 337 & 529 & 1,055 & 989 & - \\
\hline Hypertensive heart disease. & 30,780 & 1 & 1 & - & 44 & 338 & 1,372 & 3,604 & 4,487 & 4,009 & 6,324 & 10,598 & 2 \\
\hline Hypertensive heart and renal disease & 2,987 & - & - & - & 5 & 32 & 77 & 178 & 293 & 379 & 792 & 1,231 & - \\
\hline Ischemic heart diseases . . . . . . . . . . . (I20-I25) & 406,351 & 24 & 8 & 21 & 151 & 1,048 & 6,219 & 24,390 & 46,164 & 63,027 & 116,152 & 149,126 & 21 \\
\hline Acute myocardial infarction. & 132,968 & 10 & 4 & 11 & 54 & 400 & 2,402 & 9,467 & 17,835 & 23,441 & 37,629 & 41,711 & 4 \\
\hline Other acute ischemic heart diseases $\ldots \ldots \ldots \ldots$ (I24) & 4,092 & 2 & - & - & 3 & 17 & 109 & 376 & 679 & 740 & 1,021 & 1,145 & - \\
\hline $\begin{array}{l}\text { Other forms of chronic ischemic heart disease } \\
\text { Atherosclerotic cardiovascular disease, }\end{array}$ & 269,291 & 12 & 4 & 10 & 94 & 631 & 3,708 & 14,547 & 27,650 & 38,846 & 77,502 & 106,270 & 17 \\
\hline $\begin{array}{l}\text { so described . . . . . . . . . . . . . . . (125.0) } \\
\text { All other forms of chronic ischemic heart }\end{array}$ & 59,051 & 1 & - & - & 17 & 211 & 1,395 & 5,910 & 10,055 & 9,884 & 14,221 & 17,347 & 10 \\
\hline disease $\ldots \ldots \ldots \ldots \ldots(120,125.1-125.9)$ & 210,240 & 11 & 4 & 10 & 77 & 420 & 2,313 & 8,637 & 17,595 & 28,962 & 63,281 & 88,923 & 7 \\
\hline Other heart diseases $\ldots \ldots \ldots \ldots \ldots \ldots$ (I26-I51) & 172,748 & 397 & 163 & 219 & 872 & 1,777 & 4,101 & 9,085 & 14,246 & 21,645 & 46,934 & 73,305 & 4 \\
\hline Acute and subacute endocarditis $\ldots \ldots \ldots \ldots$ (I33) & 1,225 & 3 & 1 & 1 & 9 & 32 & 95 & 198 & 207 & 236 & 308 & 134 & 1 \\
\hline Diseases of pericardium and acute & & & & & & & & & & & & & \\
\hline myocarditis $\ldots \ldots \ldots \ldots \ldots \ldots(130-131, \mid 40)$ & 867 & 25 & 19 & 24 & 48 & 53 & 87 & 116 & 123 & 119 & 137 & 116 & - \\
\hline $\begin{array}{l}\text { Heart failure } \ldots \ldots \ldots \text { (150) } \\
\text { All other forms of heart disease } \ldots \ldots \ldots \text { (I26-I28, }\end{array}$ & 56,565 & 21 & 11 & 12 & 43 & 87 & 317 & 1,073 & 2,758 & 5,749 & 15,935 & 30,558 & 1 \\
\hline |34-|38,|42-|49,|51) & 114,091 & 348 & 132 & 182 & 772 & 1,605 & 3,602 & 7,698 & 11,158 & 15,541 & 30,554 & 42,497 & 2 \\
\hline Essential hypertension and hypertensive renal & & & & & & & & & & & & & \\
\hline disease $\ldots \ldots \ldots \ldots \ldots \ldots \ldots(110,112,115)$ & 23,965 & 1 & 1 & - & 23 & 85 & 384 & 1,235 & 2,124 & 3,133 & 6,442 & 10,536 & 1 \\
\hline Cerebrovascular diseases $\ldots \ldots$. & 135,952 & 132 & 52 & 83 & 195 & 505 & 2,133 & 6,385 & 10,500 & 18,007 & 41,979 & 55,975 & 6 \\
\hline Atherosclerosis . . . . . . . . & 8,232 & 1 & - & - & 2 & 1 & 27 & 134 & 350 & 829 & 2,298 & 4,590 & - \\
\hline Other diseases of circulatory system & 21,940 & 13 & 4 & 14 & 65 & 136 & 484 & 1,092 & 2,296 & 4,065 & 7,074 & 6,694 & 3 \\
\hline
\end{tabular}


Table 10. Number of deaths from 113 selected causes and Enterocolitis due to Clostridium difficile, by age: United States, 2007-Con.

[The asterisks $\left({ }^{*}\right)$ preceding the cause-of-death codes indicate that they are not part of the International Classification of Diseases, Tenth Revision (ICD-10), Second Edition; see "Technical Notes."

\begin{tabular}{|c|c|c|c|c|c|c|c|c|c|c|c|c|c|}
\hline Cause of death (based on ICD-10, 2004) & $\begin{array}{c}\text { All } \\
\text { ages }\end{array}$ & $\begin{array}{l}\text { Under } 1 \\
\text { year }\end{array}$ & $\begin{array}{c}1-4 \\
\text { years }\end{array}$ & $\begin{array}{l}5-14 \\
\text { years }\end{array}$ & $\begin{array}{l}15-24 \\
\text { years }\end{array}$ & $\begin{array}{l}25-34 \\
\text { years }\end{array}$ & $\begin{array}{l}35-44 \\
\text { years }\end{array}$ & $\begin{array}{l}45-54 \\
\text { years }\end{array}$ & $\begin{array}{l}55-64 \\
\text { years }\end{array}$ & $\begin{array}{l}65-74 \\
\text { years }\end{array}$ & $\begin{array}{l}75-84 \\
\text { years }\end{array}$ & $\begin{array}{l}85 \text { years } \\
\text { and over }\end{array}$ & $\begin{array}{l}\text { Not } \\
\text { stated }\end{array}$ \\
\hline $\begin{array}{l}\text { Aortic aneurysm and dissection } \ldots \ldots \ldots \ldots \ldots \text { (171) } \\
\text { Other diseases of arteries, arterioles and }\end{array}$ & 12,986 & 1 & - & 7 & 38 & 99 & 364 & 752 & 1,483 & 2,616 & 4,346 & 3,279 & 1 \\
\hline capillaries . . . . . . . . . . . . . . . . . (172-178) & 8,954 & 12 & 4 & 7 & 27 & 37 & 120 & 340 & 813 & 1,449 & 2,728 & 3,415 & 2 \\
\hline Other disorders of circulatory system $\ldots \ldots \ldots \ldots$ (180-199) & 4,101 & 53 & 3 & 6 & 40 & 120 & 322 & 570 & 586 & 552 & 888 & 960 & 1 \\
\hline Influenza and pneumonia . . . . . . . . & 52,717 & 222 & 109 & 103 & 163 & 331 & 784 & 1,909 & 3,152 & 5,547 & 14,859 & 25,535 & 3 \\
\hline$\ldots(\mathrm{J} 09-\mathrm{J} 11)^{1}$ & 411 & 13 & 19 & 35 & 10 & 9 & 13 & 19 & 37 & 38 & 79 & 139 & - \\
\hline$\ldots .(\mathrm{J} 12-\mathrm{J} 18)$ & 52,306 & 209 & 90 & 68 & 153 & 322 & 771 & 1,890 & 3,115 & 5,509 & 14,780 & 25,396 & 3 \\
\hline Other acute lower respiratory infections $\ldots \ldots(\mathrm{J} 20-\mathrm{J} 22, \mathrm{U} 04)^{2}$ & 255 & 46 & 17 & 3 & 2 & 5 & 5 & 14 & 19 & 27 & 36 & 81 & - \\
\hline $\begin{array}{l}\text { Acute bronchitis and bronchiolitis . . . . . . . . . (J20-J21) } \\
\text { Other and unspecified acute lower respiratory }\end{array}$ & \multicolumn{12}{|c|}{ Other and unspecified acute lower respiratory } & - \\
\hline 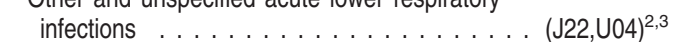 & 42 & 1 & 1 & _ & 1 & _- & - & 3 & 1 & 5 & 8 & 22 & _ \\
\hline Chronic lower respiratory diseases $\ldots \ldots \ldots \ldots \ldots$ (J40-J47) & 127,924 & 43 & 57 & 118 & 149 & 263 & 796 & 4,153 & 12,777 & 28,664 & 48,041 & 32,857 & 6 \\
\hline Bronchitis, chronic and unspecified & 667 & 24 & 10 & 2 & 1 & 5 & 14 & 24 & 52 & 88 & 181 & 266 & - \\
\hline Emphysema $\ldots \ldots \ldots \ldots$ & 12,790 & 3 & - & 1 & 1 & 10 & 60 & 486 & 1,590 & 3,294 & 4,835 & 2,509 & 1 \\
\hline$\ldots(\mathrm{J} 45-\mathrm{J} 46)$ & 3,447 & 4 & 41 & 107 & 133 & 201 & 320 & 538 & 461 & 412 & 569 & 659 & 2 \\
\hline Other chronic lower respiratory diseases & 111,020 & 12 & 6 & 8 & 14 & 47 & 402 & 3,105 & 10,674 & 24,870 & 42,456 & 29,423 & 3 \\
\hline Pneumoconioses and chemical effects . . . . . (J60-J66,J68) & 915 & - & - & - & - & 3 & 2 & 20 & 59 & 175 & 384 & 272 & - \\
\hline Pneumonitis due to solids and liquids. & 16,988 & 10 & 8 & 16 & 47 & 70 & 154 & 436 & 884 & 1,724 & 5,187 & 8,451 & 1 \\
\hline \multicolumn{14}{|l|}{ Other diseases of respiratory system . . . . (J00-J06,J30-J39, } \\
\hline Peptic ulcer . . . . . . . . . . . . . . . . . . . . (K25-K28) & $\begin{array}{r}20,045 \\
3,045\end{array}$ & 2 & - & 2 & 6 & 20 & $\begin{array}{r}490 \\
78\end{array}$ & $\begin{array}{r}1,010 \\
293\end{array}$ & $\begin{array}{r}0,050 \\
378\end{array}$ & $\begin{array}{r}0,050 \\
487\end{array}$ & $\begin{array}{r}9,000 \\
828\end{array}$ & $\begin{array}{r}1,051 \\
951\end{array}$ & - \\
\hline Diseases of appendix . . . . . & 426 & 1 & 2 & 10 & 16 & 9 & 19 & 40 & 56 & 82 & 110 & 81 & _ \\
\hline$\ldots \ldots(\mathrm{K} 40-\mathrm{K} 46)$ & 1,698 & 35 & 5 & 2 & 2 & 8 & 35 & 98 & 170 & 244 & 474 & 625 & - \\
\hline Chronic liver disease and cirrhosis & 29,165 & 4 & 4 & - & 30 & 384 & 2,570 & 8,212 & 8,004 & 5,167 & 3,694 & 1,093 & 3 \\
\hline Alcoholic liver disease . . . . . . . . . . . . . . . . (K70) & 14,406 & - & - & _- & 17 & 293 & 1,834 & 5,126 & 4,309 & 1,959 & 747 & 118 & 3 \\
\hline Other chronic liver disease and cirrhosis . . . . (K73-K74) & 14,759 & 4 & 4 & - & 13 & 91 & 736 & 3,086 & 3,695 & 3,208 & 2,947 & 975 & - \\
\hline $\begin{array}{l}\text { Cholelithiasis and other disorders of gallbladder . . (K80-K82) } \\
\text { Nephritis, nephrotic syndrome and }\end{array}$ & 3,237 & 1 & 2 & 1 & 7 & 23 & 54 & 132 & 240 & 482 & 1,011 & 1,284 & - \\
\hline $\begin{array}{c}\text { nephrosis . . . . . . . . . . . (NO0-N07,N17-N19,N25-N27) } \\
\text { Acute and rapidly progressive nephritic and nephrotic }\end{array}$ & 46,448 & 144 & 22 & 24 & 86 & 261 & 754 & 2,233 & 4,440 & 7,752 & 14,711 & 16,021 & - \\
\hline syndrome $\ldots \ldots \ldots \ldots \ldots \ldots \ldots$ (NOO-N01,N04) & 206 & 5 & 3 & 3 & 1 & 7 & 6 & 13 & 22 & 35 & 62 & 49 & _ \\
\hline \multicolumn{14}{|l|}{$\begin{array}{l}\text { Chronic glomerulonephritis, nephritis and nephropathy not } \\
\text { specified as acute or chronic, and renal sclerosis }\end{array}$} \\
\hline unspecified $\ldots \ldots \ldots \ldots \ldots$ (N02-N03,N05-N07,N26) & 2,958 & 1 & 2 & 3 & 8 & 17 & 51 & 126 & 208 & 384 & 928 & 1,230 & - \\
\hline$\ldots \ldots$ (N17-N19) & 43,263 & 138 & 16 & 18 & 77 & 237 & 696 & 2.091 & 4,205 & 7,330 & 13.718 & 14,737 & _ \\
\hline Other disorders of kidney $\ldots \ldots \ldots$ & 21 & - & 1 & - & - & - & 1 & 3 & 5 & 3 & 3 & 5 & - \\
\hline Infections of kidney . . . . . . (N10-N12,N13.6.N15.1) & 628 & 5 & 1 & 1 & 8 & 6 & 30 & 59 & 64 & 91 & 170 & 193 & _- \\
\hline Hyperplasia of prostate & 491 & _ & _ & _- & - & - & 1 & 1 & 12 & 47 & 147 & 283 & _- \\
\hline Inflammatory diseases of female pelvic organs $\ldots$ (N70-N76) & 116 & 4 & - & - & 1 & 5 & 6 & 14 & 19 & 22 & 21 & 24 & - \\
\hline Pregnancy, childbirth and the puerperium ...... (O00-099) & 769 & $\ldots$ & $\ldots$ & 1 & 160 & 326 & 220 & 59 & 2 & 1 & - & - & _- \\
\hline Pregnancy with abortive outcome .... & 31 & $\cdots$ & $\cdots$ & - & 7 & 16 & 6 & 2 & - & - & - & - & - \\
\hline Other complications of pregnancy, childbirth and the & & & & & & & & & & & & & \\
\hline puerperium ............... (010-099) & 738 & $\ldots$ & $\ldots$ & 1 & 153 & 310 & 214 & 57 & 2 & 1 & _ & _ & _- \\
\hline Certain conditions originating in the perinatal & & & & & & & & & & & & & \\
\hline period $\ldots \ldots \ldots \ldots \ldots \ldots \ldots \ldots \ldots$ (P00-P96) & 14,599 & 14,466 & 70 & 22 & 17 & 3 & 4 & 5 & 3 & 3 & 1 & _- & 5 \\
\hline Congenital malformations, deformations and chromosomal & & & & & & & & & & & & & \\
\hline abnormalities $\ldots \ldots \ldots \ldots \ldots \ldots \ldots \ldots$ (Q00-Q99) & 10,421 & 5,785 & 546 & 374 & 402 & 417 & 513 & 685 & 686 & 341 & 406 & 265 & 1 \\
\hline Symptoms, signs and abnormal clinical and laboratory & & & & & & & & & & & & & \\
\hline findings, not elsewhere classified . . . . . . . . (R00-R99) & 33,500 & 3,617 & 237 & 110 & 575 & 877 & 1,424 & 2,195 & 2,229 & 2.499 & 5,834 & 13,860 & 43 \\
\hline
\end{tabular}

See footnotes at end of table. 
Table 10. Number of deaths from 113 selected causes and Enterocolitis due to Clostridium difficile, by age: United States, 2007-Con.

[The asterisks $\left(^{*}\right)$ preceding the cause-of-death codes indicate that they are not part of the International Classification of Diseases, Tenth Revision (ICD-10), Second Edition; see "Technical Notes."

\begin{tabular}{|c|c|c|c|c|c|c|c|c|c|c|c|c|c|}
\hline Cause of death (based on ICD-10, 2004) & $\begin{array}{c}\text { All } \\
\text { ages }\end{array}$ & $\begin{array}{l}\text { Under } 1 \\
\text { year }\end{array}$ & $\begin{array}{c}1-4 \\
\text { years }\end{array}$ & $\begin{array}{l}5-14 \\
\text { years }\end{array}$ & $\begin{array}{l}15-24 \\
\text { years }\end{array}$ & $\begin{array}{l}25-34 \\
\text { years }\end{array}$ & $\begin{array}{l}35-44 \\
\text { years }\end{array}$ & $\begin{array}{l}45-54 \\
\text { years }\end{array}$ & $\begin{array}{l}55-64 \\
\text { years }\end{array}$ & $\begin{array}{l}65-74 \\
\text { years }\end{array}$ & $\begin{array}{l}75-84 \\
\text { years }\end{array}$ & $\begin{array}{l}85 \text { years } \\
\text { and over }\end{array}$ & $\begin{array}{l}\text { Not } \\
\text { stated }\end{array}$ \\
\hline All other diseases. . & 238,192 & 1,337 & 599 & 876 & 1,957 & 3,149 & 7,068 & 16,499 & 22,895 & 28,838 & 61,153 & 93,813 & 8 \\
\hline Accidents (unintentional injuries) . . . . . . (V01-X59,Y85-Y86) & 123,706 & 1,285 & 1,588 & 2,194 & 15,897 & 14,977 & 16,931 & 20,315 & 12,193 & 8,753 & 13,736 & 15,803 & 34 \\
\hline $\begin{array}{r}\text { Transport accidents } \ldots \ldots \ldots . . . . .(\mathrm{V} 01-\mathrm{V} 99, \mathrm{Y} 85) \\
\text { Motor-vehicle accidents. . . . . . V02-V04,V09.0,V09.2, } \\
\text { V12-V14,V19.0-V19.2,V19.4-V19.6,V20-V79, } \\
\text { V80.3-V80.5,V81.0-V81.1,V82.0-V82.1,V83-V86, }\end{array}$ & 46,844 & 127 & 581 & 1,374 & 10,928 & 7,452 & 6,829 & 7,199 & 4,838 & 3,194 & 2,983 & 1,326 & 13 \\
\hline $\begin{array}{r}\text { V87.0-V87.8,V88.0-V88.8,V89.0,V89.2) } \\
\text { Other land transport accidents. . . . . (V01,V05-V06, } \\
\text { V09.1,V09.3-V09.9,V10-V11,V15-V18,V19.3, } \\
\text { V19.8-V19.9,V80.0-V80.2,V80.6-V80.9,V81.2-V81.9, }\end{array}$ & 43,945 & 124 & 551 & 1,285 & 10,568 & 7,087 & 6,370 & 6,530 & 4,359 & 2,940 & 2,845 & 1,277 & 9 \\
\hline $\begin{array}{l}\text { V82.2-V82.9,V87.9,V88.9,V89.1,V89.3, V89.9) } \\
\text { Water, air and space, and other and unspecified transport }\end{array}$ & 1,083 & 1 & 19 & 53 & 174 & 134 & 181 & 235 & 123 & 81 & 61 & 18 & 3 \\
\hline accidents and their sequelae . . . . . . . . . (V90-V99,Y85) & 1,816 & 2 & 11 & 36 & 186 & 231 & 278 & 434 & 356 & 173 & 77 & 31 & 1 \\
\hline Nontransport accidents . . . . . . . . . . . (W00-X59,Y86) & 76,862 & 1,158 & 1,007 & 820 & 4,969 & 7,525 & 10,102 & 13,116 & 7,355 & 5,559 & 10,753 & 14,477 & 21 \\
\hline$\ldots \ldots \ldots \ldots$ (W00-W19) & 22,631 & 24 & 36 & 32 & 233 & 334 & 593 & 1,304 & 1,739 & 2,594 & 6,552 & 9,188 & 2 \\
\hline Accidental discharge of firearms $\ldots \ldots \ldots$. . . W32-W34) & 613 & 1 & 18 & 46 & 155 & 94 & 91 & 82 & 57 & 31 & 31 & 7 & - \\
\hline $\begin{array}{l}\text { Accidental drowning and submersion } \ldots \ldots \text { (W65-W74) } \\
\text { Accidental exposure to smoke, fire and }\end{array}$ & 3,443 & 57 & 458 & 224 & 630 & 381 & 417 & 481 & 324 & 194 & 184 & 88 & 5 \\
\hline 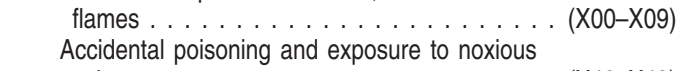 & 3,286 & 38 & 201 & 211 & 194 & 222 & 307 & 488 & 492 & 421 & 436 & 272 & 4 \\
\hline $\begin{array}{l}\text { substances .......................... X40-X49) } \\
\text { Other and unspecified nontransport accidents and their } \\
\text { sequelae .. (W20-W31,W35-W64, W75-W99,X10-X39, }\end{array}$ & 29,846 & 19 & 34 & 81 & 3,159 & 5,700 & 7,575 & 9,006 & 3,120 & 602 & 355 & 192 & 3 \\
\hline X50-X59,Y86) & 17,043 & 1,019 & 260 & 226 & 598 & 794 & 1,119 & 1,755 & 1,623 & 1,717 & 3,195 & 4,730 & 7 \\
\hline $\begin{array}{l}\text { Intentional self-harm (suicide) } \ldots \ldots \ldots \text { (*U03,X60-X84,Y87.0) } \\
\text { Intentional self-harm (suicide) by discharge of }\end{array}$ & 34,598 & $\cdots$ & $\cdots$ & 184 & 4,140 & 5,278 & 6,722 & 7,778 & 5,069 & 2,444 & 2,119 & 858 & 6 \\
\hline $\begin{array}{l}\text { firearms } \ldots \ldots \ldots \ldots \ldots \ldots \ldots \ldots \ldots \ldots \ldots \ldots \\
\text { Intentional self-harm (suicide) by other and unspecified } \\
\text { means and their sequelae } \ldots \ldots\left({ }^{*} \mathrm{U} 03, \mathrm{X} 60-\mathrm{X} 71, \mathrm{X} 75-\mathrm{X} 84 \text {, }\right.\end{array}$ & 17,352 & $\ldots$ & $\ldots$ & 53 & 1,900 & 2,306 & 2,879 & 3,531 & 2,786 & 1,700 & 1,589 & 606 & 2 \\
\hline Y87.0) & 17,246 & & & 131 & 2,240 & 2,972 & 3,843 & 4,247 & 2,283 & 744 & 530 & 252 & 4 \\
\hline $\begin{array}{l}\text { Assault (homicide) } \ldots \ldots \ldots \ldots \ldots\left({ }^{*} \cup 01-{ }^{*} \cup 02, X 85-Y 09, Y 87.1\right) \\
\text { Assault (homicide) by discharge of }\end{array}$ & 18,361 & 352 & 398 & 346 & 5,551 & 4,758 & 3,052 & 2,140 & 980 & 411 & 268 & 80 & 25 \\
\hline $\begin{array}{l}\text { firearms } \ldots \ldots \ldots \ldots \ldots \text {. . . . . . . . . . . . ( } U 01.4, X 93-X 95) \\
\text { Assault (homicide) by other and unspecified means and their }\end{array}$ & 12,632 & 15 & 48 & 201 & 4,669 & 3,751 & 2,038 & 1,159 & 446 & 185 & 88 & 23 & 9 \\
\hline X96-Y09,Y87.1) & 5,729 & 337 & 350 & 145 & 882 & 1,007 & 1,014 & 981 & 534 & 226 & 180 & 57 & 16 \\
\hline Legal intervention & 412 & - & - & 1 & 97 & 127 & 96 & 64 & 19 & 5 & 2 & 1 & - \\
\hline Events of undetermined intent & 5,381 & 88 & 49 & 49 & 579 & 872 & 1,251 & 1,501 & 599 & 183 & 132 & 70 & 8 \\
\hline Discharge of firearms, undetermined intent . . . . (Y22-Y24) & 276 & - & 3 & 12 & 82 & 44 & 36 & 40 & 35 & 17 & 6 & 1 & - \\
\hline $\begin{array}{l}\text { Other and unspecified events of undetermined intent and } \\
\text { their sequelae . . . . . (Y10-Y21,Y25-Y34,Y87.2,Y89.9) }\end{array}$ & & & & & & & & & & & & & \\
\hline $\begin{array}{c}\text { their sequelae } \ldots \ldots \ldots(\mathrm{Y} 10-\mathrm{Y} 21, \mathrm{Y} 25-\mathrm{Y} 34, \mathrm{Y} 87.2, \mathrm{Y} 89.9) \\
\text { Operations of war and their sequelae } \ldots \ldots \ldots .(\mathrm{Y} 36, \mathrm{Y} 89.1)\end{array}$ & 5,105 & 88 & 46 & $\begin{array}{r}37 \\
-\end{array}$ & $\begin{array}{r}497 \\
3\end{array}$ & $\begin{array}{r}828 \\
5\end{array}$ & $\begin{array}{r}1,215 \\
-\end{array}$ & 1,461 & $\begin{array}{r}564 \\
6\end{array}$ & 166 & $\begin{array}{r}126 \\
5\end{array}$ & 69 & $\begin{array}{l}8 \\
-\end{array}$ \\
\hline Complications of medical and surgical care ...(Y40-Y84,Y88) & 2,597 & 22 & 23 & 21 & 30 & 72 & 150 & 250 & 398 & 486 & 712 & 433 & - \\
\hline Enterocolitis due to Clostridium difficile. & 6,372 & 4 & 1 & 1 & 4 & 14 & 31 & 107 & 313 & 876 & 2,338 & 2,647 & 36 \\
\hline
\end{tabular}

- Quantity zero. … Category not applicable. $\quad{ }^{1}$ New ICD-10 code J09 (Influenza due to identified avian influenza virus) was added to the category in 2007. No deaths occurred from this cause in 2007.

${ }^{2} \mathrm{New}$ ICD-10 code U04 (Severe acute respiratory syndrome [SARS]) was added to the category in 2007. No deaths occurred from this cause in $2007 .{ }^{3}$ Cause-of-death title was changed in 2007 to reflect the addition of SARS (ICD-10 code U04).

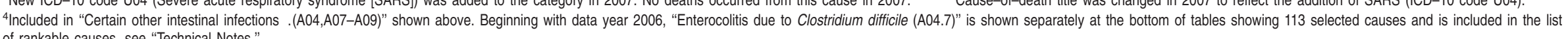
of rankable causes, see "Technical Notes."

NOTE: Complete confirmation of deaths from selected causes of death, considered to be of public health concern, was not provided by the following states: Connecticut, Florida, Indiana, Kentucky, Maryland, North Carolina, Oklahoma, Pennsylvania, Rhode

Island, South Carolina, Washington, and West Virginia; see "Technical Notes." 
Table 11. Death rates for 113 selected causes and Enterocolitis due to Clostridium difficile, by age: United States, 2007

[Rates per 100,000 population in specified group. Populations used for computing death rates are postcensal estimates based on the 2000 census, estimated as of July 1, 2007; see "Technical Notes." The asterisks (*) preceding the cause-of-death codes indicate that they are not part of the International Classification of Diseases, Tenth Revision (ICD-10), Second Edition; see "Technical Notes"]

\begin{tabular}{|c|c|c|c|c|c|c|c|c|c|c|c|c|}
\hline Cause of death (based on ICD-10, 2004) & $\begin{array}{c}\text { All } \\
\text { ages }^{1}\end{array}$ & $\begin{array}{c}\text { Under } 1 \\
\text { year }^{2}\end{array}$ & $\begin{array}{c}1-4 \\
\text { years }\end{array}$ & $\begin{array}{l}5-14 \\
\text { years }\end{array}$ & $\begin{array}{l}15-24 \\
\text { years }\end{array}$ & $\begin{array}{l}25-34 \\
\text { years }\end{array}$ & $\begin{array}{l}35-44 \\
\text { years }\end{array}$ & $\begin{array}{l}45-54 \\
\text { years }\end{array}$ & $\begin{array}{l}55-64 \\
\text { years }\end{array}$ & $\begin{array}{l}65-74 \\
\text { years }\end{array}$ & $\begin{array}{l}75-84 \\
\text { years }\end{array}$ & $\begin{array}{l}85 \text { years } \\
\text { and over }\end{array}$ \\
\hline All causes & 803.6 & 684.5 & 28.6 & 15.3 & 79.9 & 104.9 & 184.4 & 420.9 & 877.7 & $2,011.3$ & $5,011.6$ & $12,946.5$ \\
\hline$\ldots \ldots \ldots(A 01-A 02)$ & 0.0 & * & * & * & * & * & * & * & * & * & * & * \\
\hline Shigellosis and amebiasis & * & * & * & * & * & * & * & * & * & * & * & * \\
\hline Certain other intestinal infections & 2.2 & * & * & * & * & * & 0.1 & 0.3 & 1.0 & 4.7 & 18.8 & 52.0 \\
\hline Tuberculosis . . . . . . . . . & 0.2 & * & * & * & * & * & 0.1 & 0.2 & 0.3 & 0.5 & 1.2 & 1.6 \\
\hline Respiratory tuberculosis... & 0.1 & * & * & * & * & * & 0.1 & 0.1 & 0.2 & 0.4 & 0.9 & 1.3 \\
\hline$\ldots \ldots \ldots$ (A17-A19) & 0.0 & * & * & * & * & * & * & 0.0 & 0.1 & 0.1 & 0.2 & * \\
\hline Whooping cough $\ldots \ldots \ldots$ & * & * & * & * & * & * & * & * & * & * & * & * \\
\hline Scarlet fever and erysipelas . . & * & * & * & * & * & * & * & * & * & * & * & * \\
\hline 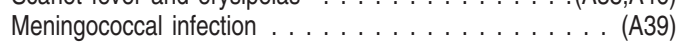 & 0.0 & * & * & * & 0.0 & * & * & * & * & * & * & * \\
\hline Septicemia . . . . . . . . . . . . . . (A40-A41) & 11.5 & 6.6 & 0.5 & 0.2 & 0.4 & 0.7 & 2.1 & 5.5 & 12.9 & 32.8 & 79.9 & 174.4 \\
\hline$\ldots \ldots \ldots$ (A50-A53) & 0.0 & * & * & * & * & * & * & * & * & * & * & * \\
\hline Acute poliomyelitis & * & * & * & * & * & * & * & * & * & * & * & * \\
\hline Arthropod-borne viral encephalitis . . . . . . (A83-A84,A85.2) & * & * & * & * & * & * & * & * & * & * & * & * \\
\hline Measles .......................... (B05) & * & * & * & * & * & * & * & * & * & * & * & * \\
\hline Viral hepatitis $\ldots \ldots \ldots$. & 2.5 & * & * & * & * & 0.2 & 1.1 & 6.4 & 7.4 & 4.5 & 4.4 & 2.9 \\
\hline Human immunodeficiency virus (HIV) disease . . . . (B20-B24) & 3.7 & * & * & * & 0.4 & 2.7 & 8.3 & 9.5 & 5.3 & 2.3 & 0.8 & * \\
\hline Malaria ............................. B50-B54) & * & * & * & * & * & * & * & * & * & * & * & * \\
\hline Other and unspecified infectious and parasitic diseases and & & & & & & & & & & & & \\
\hline
\end{tabular}

their sequelae ..... A A00,A05,A20-A36,A42-A44,A48-A49, A54-A79,A81-A82,A85.0-A85.1,A85.8,A86-B04

B06-B09,B25-B49,B55-B99

Malignant neoplasms of lip, oral cavity and

pharynx ................... (C00-C14)

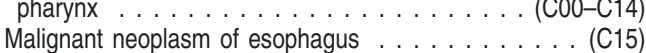

Malignant neoplasm of esophagus $\ldots \ldots \ldots \ldots \ldots$ (C15)

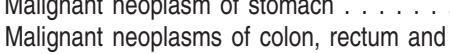

anus .................... (C18-C21)

Malignant neoplasms of liver and intrahepatic bile

ducts . . . . . . . . . . . . . . . (C22)

Malignant neoplasm of pancreas . . . . . . . . . (C25)

Malignant neoplasm of larynx

Malignant neoplasms of trachea, bronchus and

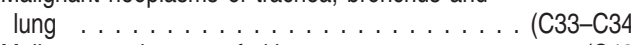

Malignant melanoma of skin . . . . . . . . (C43)

Malignant neoplasm of breast

Malignant neoplasm of cervix uteri . . . . . . . . (C53)

Malignant neoplasms of corpus uteri and uterus, part

unspecified ..................... (C54-C55)

Malignant neoplasm of ovary $\ldots \ldots \ldots \ldots \ldots$ (C56)

Malignant neoplasm of prostate ............... (C61)

Malignant neoplasms of kidney and renal pelvis . (C64-C65)

Malignant neoplasm of bladder . . . . . . . . . . (C67)

Malignant neoplasms of meninges, brain and other parts

of central nervous system ........... (C70-C72)

1.9
186.6

2.7
4.5
3.8

17.8

5.7
11.3
1.2
52.6
2.8
13.6
1.3
2.5
4.8
9.6
4.2
4.6
4.4

See footnotes at end of table. 
Table 11. Death rates for 113 selected causes and Enterocolitis due to Clostridium difficile, by age: United States, 2007-Con.

[Rates per 100,000 population in specified group. Populations used for computing death rates are postcensal estimates based on the 2000 census, estimated as of July 1, 2007; see "Technical Notes." The asterisks (*) preceding the cause-of-death codes indicate that they are not part of the International Classification of Diseases, Tenth Revision (ICD-10), Second Edition; see "Technical Notes"]

\begin{tabular}{|c|c|c|c|c|c|c|c|c|c|c|c|c|}
\hline Cause of death (based on ICD-10, 2004) & $\begin{array}{l}\text { All } \\
\text { ages }^{1}\end{array}$ & $\begin{array}{l}\text { Under } 1 \\
\text { year }^{2}\end{array}$ & $\begin{array}{c}1-4 \\
\text { years }\end{array}$ & $\begin{array}{l}5-14 \\
\text { years }\end{array}$ & $\begin{array}{l}15-24 \\
\text { years }\end{array}$ & $\begin{array}{l}25-34 \\
\text { years }\end{array}$ & $\begin{array}{l}35-44 \\
\text { years }\end{array}$ & $\begin{array}{l}45-54 \\
\text { years }\end{array}$ & $\begin{array}{l}55-64 \\
\text { years }\end{array}$ & $\begin{array}{l}65-74 \\
\text { years }\end{array}$ & $\begin{array}{l}75-84 \\
\text { years }\end{array}$ & $\begin{array}{l}85 \text { years } \\
\text { and over }\end{array}$ \\
\hline \multicolumn{13}{|l|}{ Malignant neoplasms of lymphoid, hematopoietic and } \\
\hline related tissue . . . . . . . . . . . . . . . (C81-C96) & 18.2 & 0.6 & 0.7 & 0.9 & 1.5 & 1.9 & 3.4 & 8.2 & 23.5 & 63.2 & 137.3 & 185.5 \\
\hline Hodgkin's disease & 0.4 & * & * & * & 0.2 & 0.3 & 0.3 & 0.3 & 0.5 & 1.1 & 2.2 & 2.4 \\
\hline Non-Hodgkin's lymphoma . . . . . . . . . . (C82-C85) & 6.8 & * & * & 0.1 & 0.3 & 0.5 & 1.2 & 3.2 & 8.9 & 23.1 & 52.7 & 72.1 \\
\hline Leukemia .................. (C91-C95) & 7.2 & 0.5 & 0.6 & 0.8 & 1.0 & 1.1 & 1.5 & 3.1 & 8.6 & 23.8 & 52.7 & 76.7 \\
\hline Multiple myeloma and immunoproliferative & & & & & & & & & & & & \\
\hline neoplasms $\ldots \ldots \ldots \ldots \ldots \ldots \ldots$ (C88,C90) & 3.7 & * & * & * & * & * & 0.4 & 1.6 & 5.5 & 15.1 & 29.6 & 34.1 \\
\hline $\begin{array}{l}\text { Other and unspecified malignant neoplasms of lymphoid, } \\
\text { hematopoietic and related tissue } \ldots \ldots \ldots \ldots \text { (C96) }\end{array}$ & 0.0 & * & * & * & * & * & * & * & * & * & * & * \\
\hline $\begin{array}{l}\text { All other and unspecified malignant } \\
\text { neoplasms } \ldots . . . . .(C 17, C 23-C 24, C 26-C 31, C 37-C 41 \\
\text { C44-C49,C51-C52,C57-C60,C62-C63,C66,C68-C69, }\end{array}$ & & & & & & & & & & & & \\
\hline \multicolumn{9}{|l|}{ In situ neoplasms, benign neoplasms and neoplasms of } & 33.4 & 71.6 & 139.7 & 206.7 \\
\hline uncertain or unknown behavior . . . . . . . . . . (D00-D48) & 4.7 & 1.4 & 0.4 & 0.2 & 0.2 & 0.3 & 0.8 & 1.6 & 4.3 & 12.4 & 36.2 & 76.4 \\
\hline 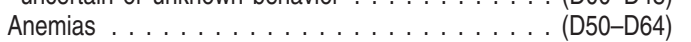 & 1.6 & * & * & 0.1 & 0.2 & 0.3 & 0.4 & 0.6 & 1.0 & 2.9 & 9.4 & 36.1 \\
\hline$\ldots \ldots \ldots \ldots \ldots(E 10-E 14)$ & 23.7 & * & * & 0.1 & 0.4 & 1.5 & 4.6 & 13.1 & 34.6 & 78.1 & 162.7 & 276.2 \\
\hline Nutritional deficiencies $\ldots \ldots \ldots \ldots$. & 0.9 & * & * & * & * & * & 0.1 & 0.2 & 0.6 & 1.7 & 6.2 & 24.4 \\
\hline$\ldots \ldots(\mathrm{E} 40-\mathrm{E} 46)$ & 0.9 & * & * & * & * & * & 0.1 & 0.2 & 0.6 & 1.6 & 5.8 & 22.4 \\
\hline Other nutritional deficiencies .... & 0.1 & * & * & * & * & * & * & * & * & * & 0.4 & 2.0 \\
\hline$\ldots(\mathrm{G} 00, \mathrm{G} 03)$ & 0.2 & 1.9 & * & 0.1 & 0.1 & 0.1 & 0.2 & 0.3 & 0.3 & 0.4 & 0.7 & 0.7 \\
\hline Parkinson's disease $\ldots \ldots \ldots \ldots \ldots \ldots$ (G20-G21) & 6.7 & * & * & * & * & * & * & 0.1 & 1.2 & 11.9 & 71.9 & 143.5 \\
\hline Alzheimer's disease . . . . . . . . . . . . . (G30) & 24.7 & * & * & * & * & * & * & 0.2 & 2.2 & 20.6 & 176.7 & 849.1 \\
\hline Major cardiovascular diseases . . . . . . . . . . . (100-178) & 267.3 & 13.4 & 1.4 & 0.8 & 3.2 & 9.7 & 34.4 & 105.5 & 247.0 & 597.5 & $1,758.7$ & $5,679.0$ \\
\hline Diseases of heart . . . . . . . (100-109,|111,I13,|20-I51) & 204.3 & 10.0 & 1.1 & 0.6 & 2.6 & 7.9 & 27.4 & 85.3 & 200.3 & 462.9 & $1,315.0$ & $4,267.7$ \\
\hline \multicolumn{13}{|l|}{ Acute rheumatic fever and chronic rheumatic heart } \\
\hline diseases . . . . . . . . . . . . . . (100-109) & 1.1 & * & * & * & * & 0.1 & 0.2 & 0.4 & 1.0 & 2.7 & 8.1 & 17.9 \\
\hline Hypertensive heart disease $\ldots \ldots \ldots \ldots \ldots \ldots$ (111) & 10.2 & * & * & * & 0.1 & 0.8 & 3.2 & 8.2 & 13.7 & 20.7 & 48.6 & 192.3 \\
\hline Hypertensive heart and renal disease . . . . . . . . (113) & 1.0 & * & * & * & * & 0.1 & 0.2 & 0.4 & 0.9 & 2.0 & 6.1 & 22.3 \\
\hline Ischemic heart diseases . . . . . . . . & 134.7 & 0.6 & * & 0.1 & 0.4 & 2.6 & 14.4 & 55.6 & 141.1 & 325.7 & 891.9 & $2,705.3$ \\
\hline Acute myocardial infarction .... & 44.1 & * & * & * & 0.1 & 1.0 & 5.6 & 21.6 & 54.5 & 121.1 & 288.9 & 756.7 \\
\hline Other acute ischemic heart diseases . . . . . . . (124) & 1.4 & * & * & * & * & * & 0.3 & 0.9 & 2.1 & 3.8 & 7.8 & 20.8 \\
\hline Other forms of chronic ischemic heart disease $\quad .(120,125)$ & 89.3 & * & * & * & 0.2 & 1.6 & 8.6 & 33.2 & 84.5 & 200.7 & 595.1 & $1,927.9$ \\
\hline $\begin{array}{l}\text { Atherosclerotic cardiovascular disease, } \\
\text { so described . . . . . . . . . . . . . . . . . . } 5.0) \\
\text { All other forms of chronic ischemic heart }\end{array}$ & 19.6 & * & * & * & * & 0.5 & 3.2 & 13.5 & 30.7 & 51.1 & 109.2 & 314.7 \\
\hline disease $\ldots \ldots \ldots \ldots \ldots \ldots(120,125.1-125.9)$ & 69.7 & * & * & * & 0.2 & 1.0 & 5.4 & 19.7 & 53.8 & 149.7 & 485.9 & 1.613 .2 \\
\hline Other heart diseases $\ldots \ldots \ldots \ldots \ldots \ldots$ (I26-151) & 57.3 & 9.3 & 1.0 & 0.5 & 2.1 & 4.4 & 9.5 & 20.7 & 43.5 & 111.8 & 360.4 & $1,329.8$ \\
\hline Acute and subacute endocarditis & 0.4 & 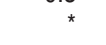 & * & * & * & 0.1 & 0.2 & 0.5 & 0.6 & 1.2 & 2.4 & 2.4 \\
\hline \multicolumn{13}{|l|}{ Diseases of pericardium and acute } \\
\hline myocarditis $\ldots \ldots \ldots \ldots \ldots \ldots(130-131, \mid 40)$ & 0.3 & 0.6 & * & 0.1 & 0.1 & 0.1 & 0.2 & 0.3 & 0.4 & 0.6 & 1.1 & 2.1 \\
\hline \multirow{2}{*}{ All other forms of heart disease . . . . . (I26-I28, } & 18.8 & 0.5 & * & * & 0.1 & 0.2 & 0.7 & 2.4 & 8.4 & 29.7 & 122.4 & 554.4 \\
\hline & & & & & & & & & & & & \\
\hline $134-|38| 142-,\mid 49,151)$ & 37.8 & 8.2 & 0.8 & 0.5 & 1.8 & 4.0 & 8.3 & 17.5 & 34.1 & 80.3 & 234.6 & 770.9 \\
\hline \multicolumn{5}{|l|}{ Essential hypertension and hypertensive renal } & 0.1 & 0.2 & 0.9 & 2.8 & 6.5 & 16.2 & 49.5 & 191.1 \\
\hline Cerebrovascular diseases . . . . . . . . . . (160-169) & 45.1 & 3.1 & 0.3 & 0.2 & 0.5 & 1.2 & 4.9 & 14.6 & 32.1 & 93.0 & 322.3 & $1,015.5$ \\
\hline
\end{tabular}


Table 11. Death rates for 113 selected causes and Enterocolitis due to Clostridium difficile, by age: United States, 2007-Con.

[Rates per 100,000 population in specified group. Populations used for computing death rates are postcensal estimates based on the 2000 census, estimated as of July 1, 2007; see "Technical Notes." The asterisks (*) preceding the cause-of-death codes indicate that they are not part of the International Classification of Diseases, Tenth Revision (ICD-10), Second Edition; see "Technical Notes"]

\begin{tabular}{|c|c|c|c|c|c|c|c|c|c|c|c|c|}
\hline Cause of death (based on ICD-10, 2004) & $\begin{array}{l}\text { All } \\
\text { ages }^{1}\end{array}$ & $\begin{array}{l}\text { Under } 1 \\
\text { year }^{2}\end{array}$ & $\begin{array}{c}1-4 \\
\text { years }\end{array}$ & $\begin{array}{l}5-14 \\
\text { years }\end{array}$ & $\begin{array}{l}15-24 \\
\text { years }\end{array}$ & $\begin{array}{l}25-34 \\
\text { years }\end{array}$ & $\begin{array}{l}35-44 \\
\text { years }\end{array}$ & $\begin{array}{l}45-54 \\
\text { years }\end{array}$ & $\begin{array}{l}55-64 \\
\text { years }\end{array}$ & $\begin{array}{l}65-74 \\
\text { years }\end{array}$ & $\begin{array}{l}75-84 \\
\text { years }\end{array}$ & $\begin{array}{l}85 \text { years } \\
\text { and over }\end{array}$ \\
\hline Atherosclerosis . & 2.7 & * & * & * & * & * & 0.1 & 0.3 & 1.1 & 4.3 & 17.6 & 83.3 \\
\hline Other diseases of circulatory system . . . . . . . (171-178) & 7.3 & * & * & * & 0.2 & 0.3 & 1.1 & 2.5 & 7.0 & 21.0 & 54.3 & 121.4 \\
\hline Aortic aneurysm and dissection $\ldots \ldots \ldots \ldots$ (171) & 4.3 & * & * & * & 0.1 & 0.2 & 0.8 & 1.7 & 4.5 & 13.5 & 33.4 & 59.5 \\
\hline \multicolumn{13}{|l|}{ Other diseases of arteries, arterioles and } \\
\hline capillaries . & 3.0 & * & * & * & 0.1 & 0.1 & 0.3 & 0.8 & 2.5 & 7.5 & 20.9 & 62.0 \\
\hline Other disorders of circulatory system . . . . . & 1.4 & 1.2 & * & * & 0.1 & 0.3 & 0.7 & 1.3 & 1.8 & 2.9 & 6.8 & 17.4 \\
\hline Influenza and pneumonia .......... & 17.5 & 5.2 & 0.7 & 0.3 & 0.4 & 0.8 & 1.8 & 4.4 & 9.6 & 28.7 & 114.1 & 463.2 \\
\hline Influenza. . . . . . . . . & 0.1 & * & * & 0.1 & * & * & * & * & 0.1 & 0.2 & 0.6 & 2.5 \\
\hline Pneumonia .. & 17.3 & 4.9 & 0.5 & 0.2 & 0.4 & 0.8 & 1.8 & 4.3 & 9.5 & 28.5 & 113.5 & 460.7 \\
\hline Other acute lower respiratory infections $\ldots \ldots(\mathrm{J} 20-\mathrm{J} 22, \mathrm{U} 04)^{4}$ & 0.1 & 1.1 & * & * & * & * & * & * & * & 0.1 & 0.3 & 1.5 \\
\hline Acute bronchitis and bronchiolitis $\ldots \ldots \ldots \ldots$ (J20-J21) & 0.1 & 1.1 & * & * & * & * & * & * & * & 0.1 & 0.2 & 1.1 \\
\hline \multicolumn{13}{|l|}{ Other and unspecified acute lower respiratory } \\
\hline Chronic lower respiratory diseases $\ldots \ldots \ldots \ldots$. $\ldots \ldots$ (J40-J47) & 42.4 & 1.0 & 0.3 & 0.3 & 0.4 & 0.6 & 1.8 & 9.5 & 39.1 & 148.1 & 368.9 & 596.1 \\
\hline Bronchitis, chronic and unspecified . ..... & 0.2 & 0.6 & * & * & * & * & * & 0.1 & 0.2 & 0.5 & 1.4 & 4.8 \\
\hline Emphysema $\ldots \ldots \ldots \ldots \ldots \ldots$ & 4.2 & * & * & * & * & * & 0.1 & 1.1 & 4.9 & 17.0 & 37.1 & 45.5 \\
\hline Asthma $\ldots \ldots \ldots \ldots \ldots \ldots \ldots \ldots$ & 1.1 & * & 0.2 & 0.3 & 0.3 & 0.5 & 0.7 & 1.2 & 1.4 & 2.1 & 4.4 & 12.0 \\
\hline Other chronic lower respiratory diseases ... & 36.8 & * & * & * & * & 0.1 & 0.9 & 7.1 & 32.6 & 128.5 & 326.0 & 533.8 \\
\hline Pneumoconioses and chemical effects . . . . . (J60-J66,J68) & 0.3 & * & * & * & * & * & * & 0.0 & 0.2 & 0.9 & 2.9 & 4.9 \\
\hline Pneumonitis due to solids and liquids . . . . . . . . . . .(J69) & 5.6 & * & * & * & 0.1 & 0.2 & 0.4 & 1.0 & 2.7 & 8.9 & 39.8 & 153.3 \\
\hline \multicolumn{13}{|l|}{ Other diseases of respiratory system . . . . (J00-J06,J30-J39, } \\
\hline Peptic ulcer . . . . . . . . . . . . . . . . . . . (K25-K28) & 1.0 & * & $*$ & * & $*$ & 0.0 & 0.2 & 0.7 & 1.2 & $\begin{array}{r}<.1 \\
2.5\end{array}$ & $\begin{array}{r}0.2 \\
6.4\end{array}$ & $\begin{array}{r}188.9 \\
17.3\end{array}$ \\
\hline Diseases of appendix & 0.1 & * & * & * & * & * & * & 0.1 & 0.2 & 0.4 & 0.8 & 1.5 \\
\hline Hernia ........ & 0.6 & 0.8 & * & * & * & * & 0.1 & 0.2 & 0.5 & 1.3 & 3.6 & 11.3 \\
\hline Chronic liver disease and cirrhosis & 9.7 & * & * & * & 0.1 & 0.9 & 6.0 & 18.7 & 24.5 & 26.7 & 28.4 & 19.8 \\
\hline Alcoholic liver disease $\ldots \ldots \ldots \ldots \ldots \ldots \ldots$ (K70) & 4.8 & * & * & * & * & 0.7 & 4.2 & 11.7 & 13.2 & 10.1 & 5.7 & 2.1 \\
\hline Other chronic liver disease and cirrhosis . . . . (K73-K74) & 4.9 & * & * & * & * & 0.2 & 1.7 & 7.0 & 11.3 & 16.6 & 22.6 & 17.7 \\
\hline Cholelithiasis and other disorders of gallbladder ... (K80-K82) & 1.1 & * & * & * & * & 0.1 & 0.1 & 0.3 & 0.7 & 2.5 & 7.8 & 23.3 \\
\hline \multicolumn{13}{|l|}{ Nephritis, nephrotic syndrome and } \\
\hline $\begin{array}{l}\text { nephrosis . . ................. (N00-N07,N17-N19,N25-N27) } \\
\text { Acute and rapidly progressive nephritic and nephrotic }\end{array}$ & 15.4 & 3.4 & 0.1 & 0.1 & 0.2 & 0.6 & 1.7 & 5.1 & 13.6 & 40.1 & 113.0 & 290.6 \\
\hline 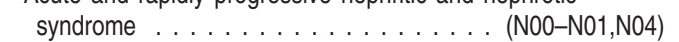 & 0.1 & * & * & * & * & * & * & * & 0.1 & 0.2 & 0.5 & 0.9 \\
\hline \multicolumn{13}{|l|}{$\begin{array}{l}\text { Chronic glomerulonephritis, nephritis and nephropathy not } \\
\text { specified as acute or chronic, and renal sclerosis }\end{array}$} \\
\hline unspecified $\ldots \ldots \ldots \ldots$ (N02-N03,N05-N07,N26) & 1.0 & * & * & * & * & * & 0.1 & 0.3 & 0.6 & 2.0 & 7.1 & 22.3 \\
\hline$\ldots \ldots \ldots \ldots \ldots(\mathrm{N} 17-\mathrm{N} 19)$ & 14.3 & 3.2 & * & * & 0.2 & 0.6 & 1.6 & 4.8 & 12.9 & 37.9 & 105.3 & 267.3 \\
\hline Other disorders of kidney $\ldots \ldots \ldots$ & 0.0 & * & * & * & * & * & * & * & * & * & * & * \\
\hline Infections of kidney $\ldots \ldots \ldots \ldots$ (N10-N12,N13.6,N15.1) & 0.2 & * & * & * & * & * & 0.1 & 0.1 & 0.2 & 0.5 & 1.3 & 3.5 \\
\hline Hyperplasia of prostate & 0.2 & * & * & * & * & * & * & * & * & 0.2 & 1.1 & 5.1 \\
\hline Inflammatory diseases of female pelvic organs & 0.0 & * & * & * & * & * & * & * & * & 0.1 & 0.2 & 0.4 \\
\hline Pregnancy, childbirth and the puerperium ... . & 0.3 & $\ldots$ & $\ldots$ & * & 0.4 & 0.8 & 0.5 & 0.1 & * & * & * & * \\
\hline Pregnancy with abortive outcome .... & 0.0 & $\ldots$ & $\ldots$ & * & * & * & * & * & * & * & * & * \\
\hline Other complications of pregnancy, childbirth and the & & & & & & & & & & & & \\
\hline puerperium . . . . . . . . . . . . . . . (010-099) & 0.2 & $\ldots$ & $\ldots$ & * & 0.4 & 0.8 & 0.5 & 0.1 & * & * & * & * \\
\hline $\begin{array}{l}\text { Certain conditions originating in the perinatal } \\
\text { period. }\end{array}$ & & 3398 & 0.4 & 01 & * & * & * & * & * & * & * & * \\
\hline
\end{tabular}

See footnotes at end of table. 
Table 11. Death rates for 113 selected causes and Enterocolitis due to Clostridium difficile, by age: United States, 2007-Con.

[Rates per 100,000 population in specified group. Populations used for computing death rates are postcensal estimates based on the 2000 census, estimated as of July 1, 2007; see "Technical Notes." The asterisks (*) preceding the cause-of-death codes indicate that they are not part of the International Classification of Diseases, Tenth Revision (ICD-10), Second Edition; see "Technical Notes"]

\begin{tabular}{|c|c|c|c|c|c|c|c|c|c|c|c|c|}
\hline Cause of death (based on ICD-10, 2004) & $\begin{array}{c}\text { All } \\
\text { ages }^{1}\end{array}$ & $\begin{array}{l}\text { Under } 1 \\
\text { year }^{2}\end{array}$ & $\begin{array}{c}1-4 \\
\text { years }\end{array}$ & $\begin{array}{l}5-14 \\
\text { years }\end{array}$ & $\begin{array}{l}15-24 \\
\text { years }\end{array}$ & $\begin{array}{l}25-34 \\
\text { years }\end{array}$ & $\begin{array}{l}35-44 \\
\text { years }\end{array}$ & $\begin{array}{l}45-54 \\
\text { years }\end{array}$ & $\begin{array}{l}55-64 \\
\text { years }\end{array}$ & $\begin{array}{l}65-74 \\
\text { years }\end{array}$ & $\begin{array}{l}75-84 \\
\text { years }\end{array}$ & $\begin{array}{l}85 \text { years } \\
\text { and over }\end{array}$ \\
\hline \multicolumn{13}{|l|}{ Congenital malformations, deformations and chromosomal } \\
\hline \multicolumn{13}{|l|}{ Symptoms, signs and abnormal clinical and laboratory } \\
\hline findings, not elsewhere classified . . . . . . . . . . (R00-R99) & 11.1 & 85.0 & 1.4 & 0.3 & 1.4 & 2.2 & 3.3 & 5.0 & 6.8 & 12.9 & 44.8 & 251.4 \\
\hline All other diseases $\ldots \ldots \ldots \ldots \ldots \ldots \ldots \ldots$ (residual) & 79.0 & 31.4 & 3.6 & 2.2 & 4.6 & 7.8 & 16.4 & 37.6 & 70.0 & 149.0 & 469.6 & $1,701.9$ \\
\hline Accidents (unintentional injuries) . . . . . . (V01-X59,Y85-Y86) & 41.0 & 30.2 & 9.6 & 5.5 & 37.4 & 36.9 & 39.2 & 46.3 & 37.3 & 45.2 & 105.5 & 286.7 \\
\hline Transport accidents $\ldots \ldots \ldots \ldots \ldots \ldots$. (V01-V99,Y85) & 15.5 & 3.0 & 3.5 & 3.4 & 25.7 & 18.4 & 15.8 & 16.4 & 14.8 & 16.5 & 22.9 & 24.1 \\
\hline \multicolumn{13}{|l|}{$\begin{array}{r}\text { Motor-vehicle accidents. . . . . . . . (V02-V04,V09.0,V09.2, } \\
\text { V12-V14,V19.0-V19.2,V19.4-V19.6,V20-V79, } \\
\text { V80.3-V80.5,V81.0-V81.1,V82.0-V82.1,V83-V86. }\end{array}$} \\
\hline V87.0-V87.8,V88.0-V88.8,V89.0,V89.2) & 14.6 & 2.9 & 3.3 & 3.2 & 24.9 & 17.5 & 14.8 & 14.9 & 13.3 & 15.2 & 21.8 & 23.2 \\
\hline \multicolumn{13}{|l|}{$\begin{array}{r}\text { Other land transport accidents. . . . . . . V01,V05-V06, } \\
\text { V09.1,V09.3-V09.9.V10-V11,V15-V18.V19.3, }\end{array}$} \\
\hline \multirow{2}{*}{\multicolumn{13}{|c|}{$\begin{array}{l}\text { V82.2-V82.9,V87.9,V88.9,V89.1,V89.3,V89.9) } \\
\text { Water, air and space, and other and unspecified transport }\end{array}$}} \\
\hline & & & & & & & & & & & & \\
\hline accidents and their sequelae. . . . . . . (V90-V99,Y85) & 0.6 & * & * & 0.1 & 0.4 & 0.6 & 0.6 & 1.0 & 1.1 & 0.9 & 0.6 & 0.6 \\
\hline Nontransport accidents . . . . . . . . . . . . (W00-X59,Y86) & 25.5 & 27.2 & 6.1 & 2.0 & 11.7 & 18.5 & 23.4 & 29.9 & 22.5 & 28.7 & 82.6 & 262.6 \\
\hline$\ldots \ldots \ldots \ldots(\mathrm{W} 00-\mathrm{W} 19)$ & 7.5 & 0.6 & 0.2 & 0.1 & 0.5 & 0.8 & 1.4 & 3.0 & 5.3 & 13.4 & 50.3 & 166.7 \\
\hline Accidental discharge of firearms. . . . . . . . (W32-W34) & 0.2 & * & * & 0.1 & 0.4 & 0.2 & 0.2 & 0.2 & 0.2 & 0.2 & 0.2 & * \\
\hline Accidental drowning and submersion. . . . . . .(W65-W74) & 1.1 & 1.3 & 2.8 & 0.6 & 1.5 & 0.9 & 1.0 & 1.1 & 1.0 & 1.0 & 1.4 & 1.6 \\
\hline Accidental exposure to smoke, fire and flames. (X00-X09) & 1.1 & 0.9 & 1.2 & 0.5 & 0.5 & 0.5 & 0.7 & 1.1 & 1.5 & 2.2 & 3.3 & 4.9 \\
\hline \multicolumn{13}{|l|}{ Accidental poisoning and exposure to noxious } \\
\hline \multicolumn{13}{|l|}{ Other and unspecified nontransport accidents and their } \\
\hline X50-X59,Y86) & 5.7 & 23.9 & 1.6 & 0.6 & 1.4 & 2.0 & 2.6 & 4.0 & 5.0 & 8.9 & 24.5 & 85.8 \\
\hline \multicolumn{13}{|l|}{$\begin{array}{l}\text { Intentional self-harm (suicide) } \ldots \ldots \ldots .\left({ }^{*} \cup 03, X 60-X 84, Y 87.0\right) \\
\text { Intentional self-harm (suicide) by discharge of }\end{array}$} \\
\hline \multirow{2}{*}{\multicolumn{13}{|c|}{ 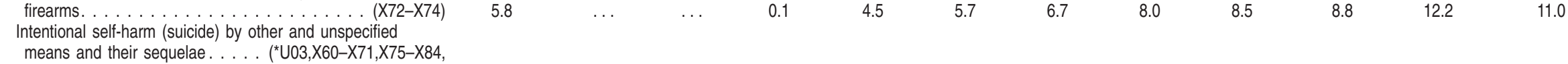 }} \\
\hline & & & & & & & & & & & & \\
\hline Y87.0) & 5.7 & & & 0.3 & 5.3 & 7.3 & 8.9 & 9.7 & 7.0 & 3.8 & 4.1 & 4.6 \\
\hline Assault (homicide) . . . . . . . . (*U01-*U02,X85-Y09,Y87.1) & 6.1 & 8.3 & 2.4 & 0.9 & 13.1 & 11.7 & 7.1 & 4.9 & 3.0 & 2.1 & 2.1 & 1.5 \\
\hline \multicolumn{13}{|l|}{ Assault (homicide) by discharge of } \\
\hline firearms. . . . . . . . . . & 4.2 & * & 0.3 & 0.5 & 11.0 & 9.2 & 4.7 & 2.6 & 1.4 & 1.0 & 0.7 & 0.4 \\
\hline \multicolumn{13}{|l|}{$\begin{array}{l}\text { Assault (homicide) by other and unspecified means and their } \\
\text { sequelae . . . ( }{ }^{*} \cup 01.0-^{*} \cup 01.3,{ }^{*} \cup 01.5-^{*} \cup 01.9,{ }^{*} \cup 02, X 85-X 92,\end{array}$} \\
\hline X96-Y09,Y87.1) & 1.9 & 7.9 & 2.1 & 0.4 & 2.1 & 2.5 & 2.3 & 2.2 & 1.6 & 1.2 & 1.4 & 1.0 \\
\hline Legal intervention... & 0.1 & * & * & * & 0.2 & 0.3 & 0.2 & 0.1 & * & * & * & * \\
\hline Events of undetermined intent. . . . . . (Y10-Y34,Y87.2,Y89.9) & 1.8 & 2.1 & 0.3 & 0.1 & 1.4 & 2.1 & 2.9 & 3.4 & 1.8 & 0.9 & 1.0 & 1.3 \\
\hline Discharge of firearms, undetermined intent. . . . (Y (Y22-Y24) & 0.1 & & & * & 0.2 & 0.1 & 0.1 & 0.1 & 0.1 & * & * & \\
\hline \multicolumn{13}{|l|}{ Other and unspecified events of undetermined intent and } \\
\hline their sequelae . . . . . . . . (Y10-Y21,Y25-Y34,Y87.2,Y89.9) & 1.7 & 2.1 & 0.3 & 0.1 & 1.2 & 2.0 & 2.8 & 3.3 & 1.7 & 0.9 & 1.0 & 1.3 \\
\hline
\end{tabular}


Table 11. Death rates for 113 selected causes and Enterocolitis due to Clostridium difficile, by age: United States, 2007-Con.

[Rates per 100,000 population in specified group. Populations used for computing death rates are postcensal estimates based on the 2000 census, estimated as of July 1, 2007; see "Technical Notes." The asterisks (*) preceding the cause-of-death codes indicate that they are not part of the International Classification of Diseases, Tenth Revision (ICD-10), Second Edition; see "Technical Notes"]

\begin{tabular}{|c|c|c|c|c|c|c|c|c|c|c|c|c|}
\hline Cause of death (based on ICD-10, 2004) & $\begin{array}{c}\text { All } \\
\text { ages }^{1}\end{array}$ & $\begin{array}{c}\text { Under } 1 \\
\text { year }^{2}\end{array}$ & $\begin{array}{c}1-4 \\
\text { years }\end{array}$ & $\begin{array}{l}5-14 \\
\text { years }\end{array}$ & $\begin{array}{l}15-24 \\
\text { years }\end{array}$ & $\begin{array}{l}25-34 \\
\text { years }\end{array}$ & $\begin{array}{l}35-44 \\
\text { years }\end{array}$ & $\begin{array}{l}45-54 \\
\text { years }\end{array}$ & $\begin{array}{l}55-64 \\
\text { years }\end{array}$ & $\begin{array}{l}65-74 \\
\text { years }\end{array}$ & $\begin{array}{l}75-84 \\
\text { years }\end{array}$ & $\begin{array}{l}85 \text { years } \\
\text { and over }\end{array}$ \\
\hline Operations of war and their sequelae & 0.0 & * & * & * & * & * & * & * & * & * & * & * \\
\hline Complications of medical and surgical care . . . (Y Y40-Y84,Y88) & 0.9 & 0.5 & 0.1 & 0.1 & 0.1 & 0.2 & 0.3 & 0.6 & 1.2 & 2.5 & 5.5 & 7.9 \\
\hline Enterocolitis due to Clostridium difficile & 2.1 & * & * & * & * & * & 0.1 & 0.2 & 1.0 & 4.5 & 18.0 & 48.7 \\
\hline
\end{tabular}

0.0 Quantity more than zero but less than 0.05 .

"Figure does not meet standards of reliability or precision; see "Technical Notes."

.. Category not applicable.

"Figures for age not stated included in "all ages" but not distributed among age groups.

2Death rates for "under 1 year" (based on population estimates) differ from infant mortality rates (based on live births); see "Technical Notes."

${ }^{3} \mathrm{New}$ ICD-10 code J09 (Influenza due to identified avian influenza virus) was added to the category in 2007. No deaths occurred from this cause in 2007.

${ }^{4} \mathrm{New}$ ICD-10 code U04 (Severe acute respiratory syndrome [SARS]) was added to the category in 2007. No deaths occurred from this cause in 2007.

${ }^{5}$ Cause-of-death title was changed in 2007 to reflect the addition of SARS (ICD-10 code U04).

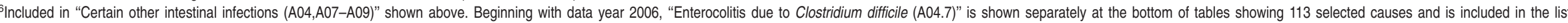
of rankable causes; see "Technical Notes."

NOTE: Complete confirmation of deaths from selected causes of death, considered to be of public health concern, was not provided by the following states: Connecticut, Florida, Indiana, Kentucky, Maryland, North Carolina, Oklahoma, Pennsylvania, Rhode Island, South Carolina, Washington, and West Virginia; see "Technical Notes." 
Table 12. Number of deaths from 113 selected causes and Enterocolitis due to Clostridium difficile, by race and sex: United States, 2007

[Data for specified races other than white and black should be interpreted with caution because of inconsistencies between reporting race on death certificates and on censuses and surveys; see "Technical Notes." The asterisks $\left({ }^{*}\right)$ preceding the cause-of-death codes indicate that they are not part of the International Classification of Diseases, Tenth Revision (ICD-10), Second Edition; see "Technical Notes"]

\begin{tabular}{|c|c|c|c|c|c|c|c|c|c|}
\hline \multirow[b]{2}{*}{ Cause of death (based on ICD-10, 2004) } & \multicolumn{3}{|c|}{ All races } & \multicolumn{3}{|c|}{ White $^{1}$} & \multicolumn{3}{|c|}{ Black $^{1}$} \\
\hline & $\begin{array}{l}\text { Both } \\
\text { sexes }\end{array}$ & Male & Female & $\begin{array}{l}\text { Both } \\
\text { sexes }\end{array}$ & Male & Female & $\begin{array}{l}\text { Both } \\
\text { sexes }\end{array}$ & Male & Female \\
\hline All causes & $2,423,712$ & $1,203,968$ & $1,219,744$ & $2,074,151$ & $1,023,951$ & $1,050,200$ & 289,585 & 148,309 & 141,276 \\
\hline Salmonella infections & 30 & 14 & 16 & 24 & 11 & 13 & 3 & 1 & 2 \\
\hline Shigellosis and amebiasis . & 4 & 2 & 2 & 3 & 2 & 1 & 1 & - & 1 \\
\hline Certain other intestinal infections . (A04,A07-A09) & 6,758 & 2,541 & 4,217 & 6,252 & 2,345 & 3,907 & 425 & 161 & 264 \\
\hline Tuberculosis . . . . . . . . . . . . . . (A16-A19) & 554 & 342 & 212 & 343 & 209 & 134 & 122 & 83 & 39 \\
\hline Respiratory tuberculosis . . . . . . . . . . . (A16) & 424 & 266 & 158 & 264 & 165 & 99 & 89 & 61 & 28 \\
\hline Other tuberculosis $\ldots \ldots \ldots \ldots \ldots$ (A17-A19) & 130 & 76 & 54 & 79 & 44 & 35 & 33 & 22 & 11 \\
\hline Whooping cough . & 9 & 1 & 8 & 9 & 1 & 8 & - & - & - \\
\hline Scarlet fever and erysipelas ..... & 3 & 1 & 2 & 2 & 1 & 1 & - & - & - \\
\hline Meningococcal infection $\ldots \ldots \ldots$. & 87 & 48 & 39 & 58 & 32 & 26 & 26 & 14 & 12 \\
\hline Septicemia $\ldots \ldots \ldots \ldots \ldots \ldots$ (A40-A41) & 34,828 & 15,839 & 18,989 & 27,750 & 12,600 & 15,150 & 6,297 & 2,835 & 3,462 \\
\hline Syphilis $\ldots \ldots \ldots \ldots \ldots$ (A50-A53) & 42 & 25 & 17 & 20 & 12 & 8 & 21 & 12 & 9 \\
\hline Acute poliomyelitis . . . . . . . . . . . . . (A80) & - & - & - & - & - & - & - & - & - \\
\hline \multicolumn{10}{|l|}{ Arthropod-borne viral } \\
\hline encephalitis . . . . . . . . . . . (A83-A84,A85.2) & 3 & 2 & 1 & 2 & 1 & 1 & 1 & 1 & - \\
\hline Measles $\ldots \ldots \ldots \ldots \ldots \ldots \ldots$ (B05) & - & - & - & - & - & - & - & - & - \\
\hline Viral hepatitis . & 7,407 & 4,910 & 2,497 & 5,959 & 4,005 & 1,954 & 1,049 & 690 & 359 \\
\hline \multicolumn{10}{|l|}{ Human immunodeficiency virus (HIV) } \\
\hline disease $\ldots \ldots \ldots \ldots \ldots \ldots$ (B20-B24) & 11,295 & 8,097 & 3,198 & 4,672 & 3,797 & 875 & 6,470 & 4,186 & 2,284 \\
\hline Malaria . . . . . . . . . . . . . . . . . . . . (B50-B54) & 5 & 3 & 2 & 3 & 2 & 1 & 1 & - & 1 \\
\hline \multicolumn{10}{|l|}{$\begin{array}{l}\text { Other and unspecified infectious and parasitic } \\
\text { diseases and their sequelae } . . . . . .(A 00, A 05 \text {, } \\
\text { A20-A36,A42-A44,A48-A49,A54-A79,A81-A82, } \\
\text { A85.0-A85.1,A85.8,A86-B04,B06-B09, }\end{array}$} \\
\hline B25-B49,B55-B99) & 5,825 & 2,982 & 2,843 & 4,850 & 2,471 & 2,379 & 767 & 399 & 368 \\
\hline Malignant neoplasms & 562,875 & 292,857 & 270,018 & 483,939 & 252,049 & 231,890 & 64,049 & 33,069 & 30,980 \\
\hline \multicolumn{10}{|l|}{ Malignant neoplasms of lip, oral cavity and } \\
\hline pharynx $\ldots \ldots \ldots \ldots \ldots \ldots$ (COO-C14) & 8,067 & 5,510 & 2,557 & 6,720 & 4,555 & 2,165 & 1,062 & 769 & 293 \\
\hline Malignant neoplasm of esophagus $\ldots . .$. (C15) & 13,592 & 10,750 & 2,842 & 11,878 & 9,493 & 2,385 & 1,453 & 1,055 & 398 \\
\hline Malignant neoplasm of stomach . & 11,388 & 6,757 & 4,631 & 8,551 & 5,099 & 3,452 & 1,998 & 1,193 & 805 \\
\hline \multicolumn{10}{|l|}{ Malignant neoplasms of colon, rectum } \\
\hline and anus $\ldots \ldots \ldots \ldots \ldots($ C18-C21) & 53,586 & 27,125 & 26,461 & 45,174 & 22,926 & 22,248 & 6,922 & 3,443 & 3,479 \\
\hline \multicolumn{10}{|l|}{ Malignant neoplasms of liver and } \\
\hline intrahepatic bile ducts $\ldots . .$. & 17,146 & 11,343 & 5,803 & 13,613 & 8,884 & 4,729 & 2,264 & 1,592 & 672 \\
\hline Malignant neoplasm of pancreas & 34,117 & 17,132 & 16,985 & 29,096 & 14,774 & 14,322 & 4,069 & 1,893 & 2,176 \\
\hline Malignant neoplasm of larynx . . . . . . . . (C32) & 3,634 & 2,890 & 744 & 2,905 & 2,283 & 622 & 659 & 547 & 112 \\
\hline \multicolumn{10}{|l|}{ Malignant neoplasms of trachea, } \\
\hline$\ldots \ldots .$. (C33-C34) & 158,760 & 88,372 & 70,388 & 138,730 & 76,502 & 62,228 & 16,494 & 9,839 & 6,655 \\
\hline Malignant melanoma of skin . . . . . . . (C43) & 8,461 & 5,506 & 2,955 & 8,258 & 5,408 & 2,850 & 136 & 59 & 77 \\
\hline Malignant neoplasm of breast . & 40,970 & 371 & 40,599 & 34,160 & 315 & 33,845 & 5,852 & 53 & 5,799 \\
\hline Malignant neoplasm of cervix uteri . . . . (C53) & 4,021 & $\ldots$ & 4,021 & 3,037 & $\ldots$ & 3,037 & 805 & $\ldots$ & 805 \\
\hline \multicolumn{10}{|l|}{ Malignant neoplasms of corpus uteri } \\
\hline and uterus, part unspecified $\ldots \ldots$ (C54-C55) & 7,456 & $\ldots$ & 7,456 & 5,962 & $\ldots$ & 5,962 & 1,295 & $\ldots$ & 1,295 \\
\hline Malignant neoplasm of ovary $\ldots \ldots \ldots$ (C56) & 14,621 & & 14,621 & 13,093 & & 13,093 & 1,160 & & 1,160 \\
\hline Malignant neoplasm of prostate . & 29,093 & 29,093 & ... & 23,666 & 23,666 & $\ldots$ & 4,908 & 4,908 & $\ldots$ \\
\hline \multicolumn{10}{|l|}{ Malignant neoplasms of kidney and } \\
\hline (C64-C65) & 12,703 & 7,964 & 4,739 & 11,142 & 6,996 & 4,146 & 1,222 & 744 & 478 \\
\hline Malignant neoplasm of bladder & 13,843 & 9,644 & 4,199 & 12,620 & 8,950 & 3,670 & 1,034 & 565 & 469 \\
\hline \multicolumn{10}{|l|}{$\begin{array}{l}\text { Malignant neoplasms of meninges, } \\
\text { brain and other parts of central }\end{array}$} \\
\hline nervous system $\ldots \ldots \ldots \ldots \ldots$ (C70-C72) & 13,234 & 7,315 & 5,919 & 12,177 & 6,759 & 5,418 & 791 & 404 & 387 \\
\hline $\begin{array}{l}\text { Mallignant neoplasms of lymphoid, } \\
\text { hematopoietic and related tissue . . (C81-C96) }\end{array}$ & 54,991 & 30,198 & 24,793 & 48,377 & 26,765 & 21,612 & 5,255 & 2.663 & 592 \\
\hline Hodgkin's disease . . . . . . . . . . (C81) & 1,271 & 706 & 565 & 1,121 & 621 & 500 & 130 & 74 & 56 \\
\hline Non-Hodgkin's lymphoma . . . . . . (C82-C85) & 20,528 & 11,004 & 9,524 & 18,581 & 9,941 & 8,640 & 1,390 & 749 & 641 \\
\hline$\ldots \ldots$ (C91-C95) & 21,825 & 12,388 & 9,437 & 19,481 & 11,147 & 8,334 & 1,831 & 951 & 880 \\
\hline Multiple myeloma and immunoproliferative & & & & & & & & & \\
\hline neoplasms $\ldots \ldots \ldots \ldots \ldots(\mathrm{C} 88, \mathrm{C}, \ldots \ldots 0)$ & 11,307 & 6,066 & 5,241 & 9,148 & 5,032 & 4,116 & 1,893 & 882 & 1,011 \\
\hline
\end{tabular}

See footnotes at end of table. 
Table 12. Number of deaths from 113 selected causes and Enterocolitis due to Clostridium difficile, by race and sex: United States, 2007-Con.

[Data for specified races other than white and black should be interpreted with caution because of inconsistencies between reporting race on death certificates and on censuses and surveys; see "Technical Notes." The asterisks $\left({ }^{*}\right)$ preceding the cause-of-death codes indicate that they are not part of the International Classification of Diseases, Tenth Revision (ICD-10), Second Edition; see "Technical Notes"]

\begin{tabular}{|c|c|c|c|c|c|c|c|c|c|}
\hline \multirow[b]{2}{*}{ Cause of death (based on ICD-10, 2004) } & \multicolumn{3}{|c|}{ All races } & \multicolumn{3}{|c|}{ White $^{1}$} & \multicolumn{3}{|c|}{ Black $^{1}$} \\
\hline & $\begin{array}{l}\text { Both } \\
\text { sexes }\end{array}$ & Male & Female & $\begin{array}{l}\text { Both } \\
\text { sexes }\end{array}$ & Male & Female & $\begin{array}{l}\text { Both } \\
\text { sexes }\end{array}$ & Male & Female \\
\hline \multicolumn{10}{|l|}{$\begin{array}{l}\text { Other and unspecified malignant neoplasms of } \\
\text { lymphoid, hematopoietic and }\end{array}$} \\
\hline $\begin{array}{l}\text { All other and unspecified malignant } \\
\text { neoplasms } \ldots \text { (C17,C23-C24,C26-C31, } \\
\text { C37-C41,C44-C49,C51-C52,C57-C60, }\end{array}$ & & & & & & & & & \\
\hline $\mathrm{C} 62-\mathrm{C} 63, \mathrm{C} 66, \mathrm{C} 68-\mathrm{C} 69, \mathrm{C} 73-\mathrm{C} 80, \mathrm{C} 97)$ & 63,192 & 32,887 & 30,305 & 54,780 & 28,674 & 26,106 & 6,670 & 3,342 & 3,328 \\
\hline \multicolumn{10}{|l|}{$\begin{array}{l}\text { In situ neoplasms, benign neoplasms and } \\
\text { neoplasms of uncertain or unknown }\end{array}$} \\
\hline behavior . . . . . . . . . . . . . . (D00-D48) & 14,204 & 7,256 & 6,948 & 12,655 & 6,470 & 6,185 & 1,190 & 594 & 596 \\
\hline Anemias . . . . . . . . . . . . . . . . (D50-D64) & 4,829 & 1,940 & 2,889 & 3,734 & 1,447 & 2,287 & 970 & 437 & 533 \\
\hline Diabetes mellitus $\ldots \ldots \ldots \ldots \ldots$ (E10-E14) & 71,382 & 35,478 & 35,904 & 56,390 & 28,744 & 27,646 & 12,459 & 5,493 & 6,966 \\
\hline Nutritional deficiencies . . . . . . . . . . . (E40-E64) & 2,852 & 1,071 & 1,781 & 2,375 & 875 & 1,500 & 393 & 164 & 229 \\
\hline Malnutrition . . . . . . . & 2,644 & 991 & 1,653 & 2,194 & 802 & 1,392 & 374 & 160 & 214 \\
\hline Other nutritional deficiencies . . & 208 & 80 & 128 & 181 & 73 & 108 & 19 & 4 & 15 \\
\hline 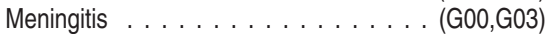 & 655 & 346 & 309 & 499 & 257 & 242 & 132 & 77 & 55 \\
\hline Parkinson's disease & 20,058 & 11,554 & 8,504 & 18,864 & 10,895 & 7,969 & 802 & 437 & 365 \\
\hline Alzheimer's disease & 74,632 & 21,800 & 52,832 & 68,933 & 20,185 & 48,748 & 4,760 & 1,301 & 3,459 \\
\hline Major cardiovascular diseases $\ldots \ldots$ & 806,156 & 388,049 & 418,107 & 691,283 & 331,560 & 359,723 & 96,051 & 46,698 & 49,353 \\
\hline Diseases of heart $\ldots \ldots(100-109,|11| 13,, \mid 20-151)$ & 616,067 & 309,821 & 306,246 & 531,636 & 266,908 & 264,728 & 71,209 & 35,669 & 35,540 \\
\hline \multicolumn{10}{|l|}{ Acute rheumatic fever and chronic } \\
\hline rheumatic heart diseases $\ldots \ldots \ldots(100-109)$ & 3,201 & 1,026 & 2,175 & 2,853 & 907 & 1,946 & 245 & 84 & 161 \\
\hline Hypertensive heart disease $\ldots \ldots \ldots \ldots$ (I11) & 30,780 & 14,249 & 16,531 & 22,938 & 10,209 & 12,729 & 7,108 & 3,669 & 3,439 \\
\hline Hypertensive heart and renal disease . . . (I13) & 2,987 & 1,318 & 1,669 & 2,064 & 864 & 1,200 & 825 & 412 & 413 \\
\hline Ischemic heart diseases . . . . . . . . (120-125) & 406,351 & 216,050 & 190,301 & 354,481 & 189,056 & 165,425 & 42,679 & 21,768 & 20,911 \\
\hline Acute myocardial infarction . . . . . (121-122) & 132,968 & 71,712 & 61,256 & 115,900 & 63,011 & 52,889 & 14,097 & 6,997 & 7,100 \\
\hline Other acute ischemic heart diseases . .(124) & 4,092 & 2,159 & 1,933 & 3,441 & 1,792 & 1,649 & 539 & 303 & 236 \\
\hline \multicolumn{10}{|l|}{ Other forms of chronic ischemic } \\
\hline heart disease $\ldots \ldots \ldots \ldots \ldots(120,125)$ & 269,291 & 142,179 & 127,112 & 235,140 & 124,253 & 110,887 & 28,043 & 14,468 & 13,575 \\
\hline $\begin{array}{l}\text { Atherosclerotic cardiovascular } \\
\text { disease, so described ..........(I25.0) } \\
\text { All other forms of chronic ischemic }\end{array}$ & 59,051 & 33,401 & 25,650 & 48,660 & 27,365 & 21,295 & 8,921 & 5,155 & 3,766 \\
\hline heart disease . . . . . . (120,|25.1-I25.9) & 210,240 & 108,778 & 101,462 & 186,480 & 96,888 & 89,592 & 19,122 & 9,313 & 9,809 \\
\hline Other heart diseases. & 172,748 & 77,178 & 95,570 & 149,300 & 65,872 & 83,428 & 20,352 & 9,736 & 10,616 \\
\hline Acute and subacute endocarditis $\ldots .$. (I33) & 1,225 & 718 & 507 & 967 & 554 & 413 & 223 & 144 & 79 \\
\hline \multicolumn{10}{|l|}{ Diseases of pericardium and acute } \\
\hline myocarditis $\ldots \ldots \ldots \ldots(|30-| 31, \mid 40)$ & 867 & 454 & 413 & 698 & 366 & 332 & 145 & 74 & 71 \\
\hline Heart failure $\ldots \ldots \ldots \ldots \ldots$ (150) & 56,565 & 22,914 & 33,651 & 50,367 & 20,262 & 30,105 & 5,497 & 2,341 & 3,156 \\
\hline All other forms of heart disease (I26-I28, & & & & & & & & & \\
\hline |34-|38,|42-|49,|51) & 114,091 & 53,092 & 60,999 & 97,268 & 44,690 & 52,578 & 14,487 & 7,177 & 7,310 \\
\hline \multicolumn{10}{|l|}{ Essential hypertension and hypertensive } \\
\hline renal disease $\ldots \ldots \ldots \ldots \ldots(110,|112| 15)$, & 23,965 & 9,417 & 14,548 & 18,583 & 7,106 & 11,477 & 4,640 & 1,979 & 2,661 \\
\hline Cerebrovascular diseases . . . . . . (160-169) & 135,952 & 54,111 & 81,841 & 114,695 & 44,714 & 69,981 & 17,085 & 7,549 & 9,536 \\
\hline Atherosclerosis $\ldots \ldots \ldots \ldots \ldots \ldots \ldots$ (170) & 8,232 & 3,220 & 5,012 & 7,444 & 2,898 & 4,546 & 647 & 259 & 388 \\
\hline Other diseases of circulatory system . . (171-178) & 21,940 & 11,480 & 10,460 & 18,925 & 9,934 & 8,991 & 2,470 & 1,242 & 1,228 \\
\hline Aortic aneurysm and dissection $\ldots . .$. (171) & 12,986 & 7,543 & 5,443 & 11,348 & 6,610 & 4,738 & 1,257 & 705 & 552 \\
\hline Other diseases of arteries, arterioles & & & & & & & & & \\
\hline and capillaries & 8,954 & 3,937 & 5,017 & 7,577 & 3,324 & 4,253 & 1,213 & 537 & 676 \\
\hline Other disorders of circulatory system & 4,101 & 1,902 & 2,199 & 3,336 & 1,523 & 1,813 & 701 & 348 & 353 \\
\hline Influenza and pneumonia $\ldots \ldots \ldots$ (J09-J18 ${ }^{4}$ & 52,717 & 24,071 & 28,646 & 45,947 & 20,720 & 25,227 & 5,155 & 2,498 & 2,657 \\
\hline Influenza . . . . . . . . . . . . . . . . . . (J09-J11) & 411 & 187 & 224 & 374 & 169 & 205 & 26 & 13 & 13 \\
\hline Pneumonia . . . . . . . . . . . . . . . (J12-J18) & 52,306 & 23,884 & 28,422 & 45,573 & 20,551 & 25,022 & 5,129 & 2,485 & 2,644 \\
\hline \multicolumn{10}{|l|}{ Other acute lower respiratory } \\
\hline infections $\ldots \ldots \ldots \ldots$ & 255 & 121 & 134 & 217 & 102 & 115 & 33 & 16 & 17 \\
\hline Acute bronchitis and bronchiolitis . . . (J20-J21) & 213 & 104 & 109 & 180 & 86 & 94 & 29 & 15 & 14 \\
\hline \multicolumn{10}{|l|}{ Other and unspecified acute lower respiratory } \\
\hline infections $\ldots \ldots \ldots \ldots($ J22,U04) $\ldots \ldots$ & 42 & 17 & 25 & 37 & 16 & 21 & 4 & 1 & 3 \\
\hline Chronic lower respiratory diseases $\ldots . .(J 40-J 47)$ & 127,924 & 61,235 & 66,689 & 118,081 & 55,934 & 62,147 & 7,901 & 4,207 & 3,694 \\
\hline Bronchitis, chronic and unspecified . . .(J40-J42) & 667 & 273 & 394 & 592 & 238 & 354 & 56 & 24 & 32 \\
\hline Emphysema $\ldots \ldots \ldots \ldots \ldots \ldots \ldots$ (J43) & 12,790 & 6,598 & 6,192 & 11,886 & 6,032 & 5,854 & 733 & 457 & 276 \\
\hline Asthma $\ldots \ldots \ldots \ldots \ldots \ldots \ldots \ldots$ (J45-J46) & 3,447 & 1,274 & 2,173 & 2,376 & 789 & 1,587 & 902 & 406 & 496 \\
\hline
\end{tabular}

See footnotes at end of table. 
Table 12. Number of deaths from 113 selected causes and Enterocolitis due to Clostridium difficile, by race and sex: United States, 2007-Con.

[Data for specified races other than white and black should be interpreted with caution because of inconsistencies between reporting race on death certificates and on censuses and surveys; see "Technical Notes." The asterisks $\left({ }^{*}\right)$ preceding the cause-of-death codes indicate that they are not part of the International Classification of Diseases, Tenth Revision (ICD-10), Second Edition; see "Technical Notes"]

\begin{tabular}{|c|c|c|c|c|c|c|c|c|c|}
\hline \multirow[b]{2}{*}{ Cause of death (based on ICD-10, 2004) } & \multicolumn{3}{|c|}{ All races } & \multicolumn{3}{|c|}{ White $^{1}$} & \multicolumn{3}{|c|}{ Black $^{1}$} \\
\hline & $\begin{array}{l}\text { Both } \\
\text { sexes }\end{array}$ & Male & Female & $\begin{array}{l}\text { Both } \\
\text { sexes }\end{array}$ & Male & Female & $\begin{array}{l}\text { Both } \\
\text { sexes }\end{array}$ & Male & Female \\
\hline $\begin{array}{l}\text { Other chronic lower respiratory } \\
\text { diseases } \ldots \ldots \ldots \ldots \ldots \ldots\end{array}$ & 111,020 & 53,090 & 57,930 & 103,227 & 48,875 & 54,352 & 6,210 & 3,320 & 2,890 \\
\hline $\begin{array}{l}\text { Pneumoconioses and chemical } \\
\text { effects }\end{array}$ & 015 & & & & & 26 & 15 & 19 & \\
\hline $\begin{array}{l}\text { Pneumonitis due to solids and liquids . . . . . (J69) } \\
\text { Other diseases of respiratory }\end{array}$ & 16,988 & 8,934 & 8,054 & $\begin{array}{r}868 \\
15,229\end{array}$ & 8,010 & 7,219 & $\begin{array}{r}45 \\
1,429\end{array}$ & $\begin{array}{r}42 \\
739\end{array}$ & 690 \\
\hline system ...... (J00-J06,J30-J39,J67,J70-J98) & 28,508 & 14,291 & 14,217 & 25,155 & 12,638 & 12,517 & 2,591 & 1,261 & 1,330 \\
\hline Peptic ulcer . . . . . . . . . . . . . (K25-K28) & 3,045 & 1,469 & 1,576 & 2,628 & 1,232 & 1,396 & 305 & 174 & 131 \\
\hline Diseases of appendix $\ldots \ldots \ldots \ldots \ldots$ (K35-K38) & 426 & 259 & 167 & 342 & 210 & 132 & 65 & 39 & 26 \\
\hline Hernia $\ldots \ldots \ldots \ldots \ldots \ldots($ K40-K46) & 1,698 & 708 & 990 & 1,504 & 608 & 896 & 167 & 86 & 81 \\
\hline Chronic liver disease and cirrhosis (K70,K73-K74) & 29,165 & 19,151 & 10,014 & 25,490 & 16,801 & 8,689 & 2,558 & 1,682 & 876 \\
\hline Alcoholic liver disease $\ldots \ldots \ldots \ldots \ldots$ (K70) & 14,406 & 10,549 & 3,857 & 12,541 & 9,267 & 3,274 & 1,203 & 851 & 352 \\
\hline $\begin{array}{l}\text { Other chronic liver disease and } \\
\text { cirrhosis } \ldots \ldots \ldots \ldots \ldots \ldots \ldots \ldots \text { K73-K74) }\end{array}$ & 14,759 & 8,602 & 6,157 & 12,949 & 7,534 & 5,415 & 1,355 & 831 & 524 \\
\hline $\begin{array}{l}\text { Cholelithiasis and other disorders of } \\
\text { gallbladder ................. (K80-K82) } \\
\text { Nephritis, nephrotic syndrome and }\end{array}$ & 3,237 & 1,441 & 1,796 & 2,839 & 1,280 & 1,559 & 299 & 118 & 181 \\
\hline nephrosis . . . . . . (N00-N07,N17-N19,N25-N27) & 46,448 & 22,616 & 23,832 & 36,871 & 18,242 & 18,629 & 8,392 & 3,772 & 4,620 \\
\hline $\begin{array}{l}\text { Acute and rapidly progressive nephritic and } \\
\text { nephrotic syndrome ..........(N00-N01,N04) } \\
\text { Chronic glomerulonephritis, nephritis and } \\
\text { nephropathy not specified as acute or } \\
\text { chronic, and renal sclerosis }\end{array}$ & 206 & 111 & 95 & 168 & 91 & 77 & 35 & 18 & 17 \\
\hline unspecified $\ldots . . .(\mathrm{N} 02-\mathrm{N} 03, \mathrm{~N} 05-\mathrm{N} 07, \mathrm{~N} 26)$ & 2,958 & 1,452 & 1,506 & 2,350 & 1,179 & 1,171 & 520 & 229 & 291 \\
\hline Renal failure $\ldots \ldots \ldots \ldots \ldots(\mathrm{N} 17-\mathrm{N} 19)$ & 43,263 & 21,038 & 22,225 & 34,337 & 16,961 & 17,376 & 7,832 & 3,521 & 4,311 \\
\hline Other disorders of kidney $\ldots \ldots \ldots(\mathrm{N} 25, \mathrm{~N} 27)$ & 21 & 15 & 6 & 16 & 11 & 5 & 5 & 4 & 1 \\
\hline Infections of kidney $\ldots \ldots(\mathrm{N} 10-\mathrm{N} 12, \mathrm{~N} 13.6, \mathrm{~N} 15.1)$ & 628 & 214 & 414 & 552 & 185 & 367 & 53 & 20 & 33 \\
\hline $\begin{array}{l}\text { Hyperplasia of prostate .............. (N40) } \\
\text { Inflammatory diseases of female pelvic }\end{array}$ & 491 & 491 & $\ldots$ & 433 & 433 & $\ldots$ & 44 & 44 & . \\
\hline $\begin{array}{l}\text { organs .................. (N70-N76) } \\
\text { Pregnancy, childbirth and the }\end{array}$ & 116 & $\ldots$ & 116 & 90 & $\ldots$ & 90 & 20 & $\ldots$ & 20 \\
\hline puerperium . . . . . . . . . . . . (000-099) & 769 & $\ldots$ & 769 & 465 & $\ldots$ & 465 & 251 & $\ldots$ & 251 \\
\hline $\begin{array}{l}\text { Pregnancy with abortive outcome ....(O00-007) } \\
\text { Other complications of pregnancy, childbirth }\end{array}$ & 31 & $\ldots$ & 31 & 12 & $\cdots$ & 12 & 18 & $\ldots$ & 18 \\
\hline $\begin{array}{l}\text { and the puerperium ..........(010-099) } \\
\text { Certain conditions originating in the perinatal }\end{array}$ & 738 & $\ldots$ & 738 & 453 & . & 453 & 233 & . & 233 \\
\hline $\begin{array}{l}\text { period .................... (P00-P96) } \\
\text { Congenital malformations, deformations and }\end{array}$ & 14,599 & 8,218 & 6,381 & 8,890 & 4,999 & 3,891 & 5,052 & 2,846 & 2,206 \\
\hline $\begin{array}{l}\text { chromosomal abnormalities .........(Q00-Q99) } \\
\text { Symptoms, signs and abnormal clinical and } \\
\text { laboratory findings, not elsewhere }\end{array}$ & 10,421 & 5,455 & 4,966 & 8,155 & 4,297 & 3,858 & 1,807 & 924 & 883 \\
\hline classified . . . . . . . . . . . . . (R00-R99) & 33,500 & 14,282 & 19,218 & 28,126 & 11,642 & 16,484 & 4,651 & 2,264 & 2,387 \\
\hline $\begin{array}{l}\text { All other diseases . . . . . . . . . . . . . (residual) } \\
\text { Accidents (unintentional injuries) . . . . (V01-X59, }\end{array}$ & 238,192 & 96,447 & 141,745 & 206,947 & 82,943 & 124,004 & 26,371 & 11,280 & 15,091 \\
\hline Y85-Y86) & 123,706 & 79,827 & 43,879 & 106,252 & 68,059 & 38,193 & 13,559 & 9,268 & 4,291 \\
\hline $\begin{array}{r}\text { Transport accidents ......... (V01-V99,Y85) } \\
\text { Motor-vehicle accidents. . . . . . (V02-V04, } \\
\text { V09.0,V09.2,V12-V14,V19.0-V19.2, } \\
\text { V19.4-V19.6,V20-V79,V80.3-V80.5, } \\
\text { V81.0-V81.1,V82.0-V82.1,V83-V86, }\end{array}$ & 46,844 & 33,434 & 13,410 & 39,086 & 27,864 & 11,222 & 5,859 & 4,336 & 1,523 \\
\hline $\begin{array}{r}\text { V87.0-V87.8,V88.0-V88.8,V89.0,V89.2) } \\
\text { Other land transport accidents. . . . . (V01, } \\
\text { V05-V06,V09.1,V09.3-V09.9,V10-V11, } \\
\text { V15-V18,V19.3,V19.8-V19.9,V80.0-V80.2, } \\
\text { V80.6-V80.9,V81.2-V81.9,V82.2-V82.9, }\end{array}$ & 43,945 & 31,102 & 12,843 & 36,653 & 25,903 & 10,750 & 5,519 & 4,058 & 1,461 \\
\hline $\begin{array}{l}\text { V87.9,V88.9,V89.1,V89.3,V89.9) } \\
\text { Water, air and space, and other and } \\
\text { unspecified transport accidents }\end{array}$ & 1,083 & 856 & 227 & 852 & 676 & 176 & 165 & 129 & 36 \\
\hline and their sequelae $\ldots \ldots($ V90-V99,Y85) & 1,816 & 1,476 & 340 & 1,581 & 1,285 & 296 & 175 & 149 & 26 \\
\hline
\end{tabular}

See footnotes at end of table. 
Table 12. Number of deaths from 113 selected causes and Enterocolitis due to Clostridium difficile, by race and sex: United States, 2007-Con.

[Data for specified races other than white and black should be interpreted with caution because of inconsistencies between reporting race on death certificates and on censuses and surveys; see "Technical Notes." The asterisks $\left({ }^{*}\right)$ preceding the cause-of-death codes indicate that they are not part of the International Classification of Diseases, Tenth Revision (ICD-10), Second Edition; see "Technical Notes"]

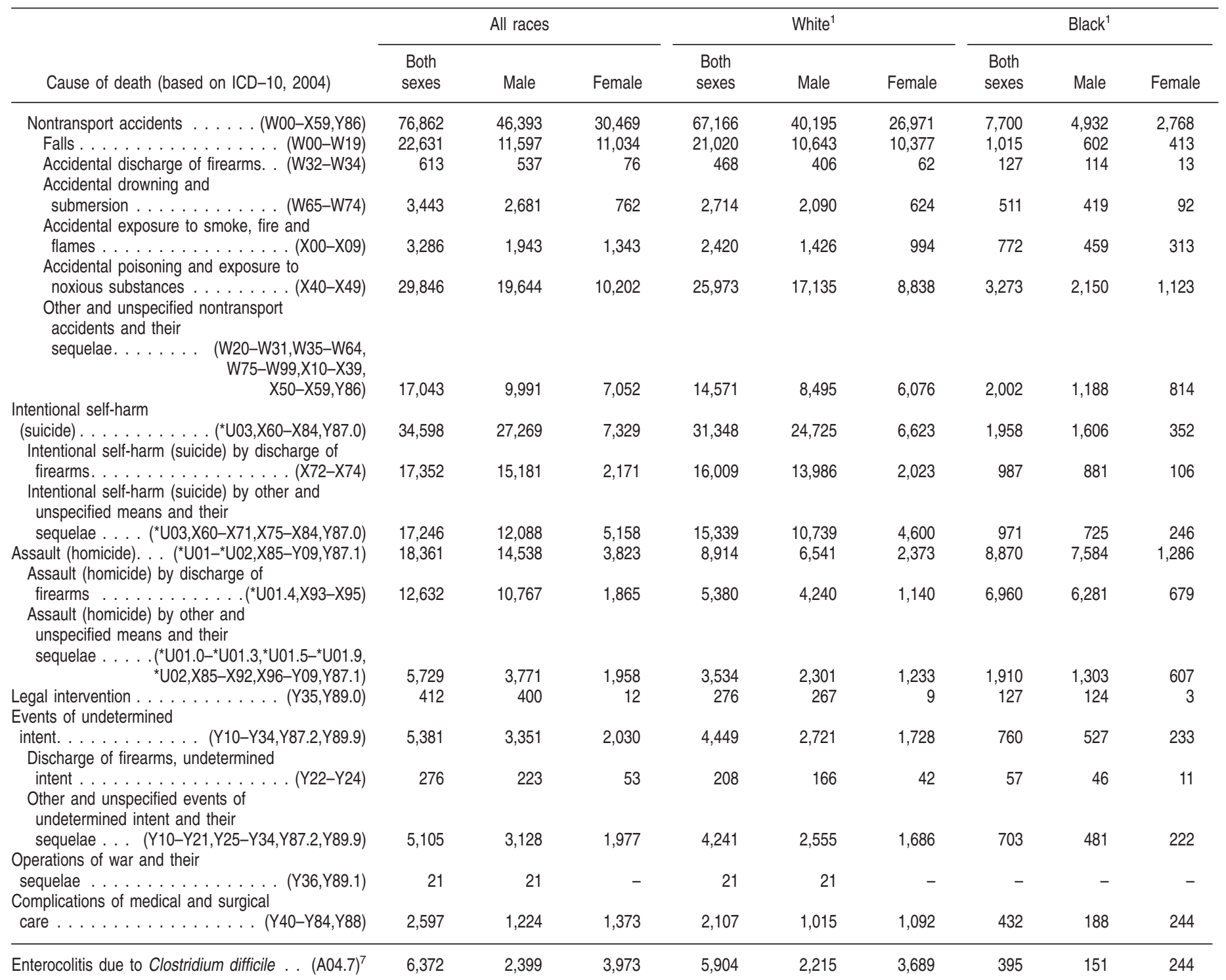

See footnotes at end of table. 
Table 12. Number of deaths from 113 selected causes and Enterocolitis due to Clostridium difficile, by race and sex: United States, 2007-Con.

[Data for specified races other than white and black should be interpreted with caution because of inconsistencies between reporting race on death certificates and on censuses and surveys; see "Technical Notes." The asterisks $\left({ }^{*}\right)$ preceding the cause-of-death codes indicate that they are not part of the International Classification of Diseases, Tenth Revision (ICD-10), Second Edition; see "Technical Notes"]

\begin{tabular}{|c|c|c|c|c|c|c|}
\hline \multirow[b]{2}{*}{ Cause of death (based on ICD-10, 2004) } & \multicolumn{3}{|c|}{ American Indian or Alaska Native ${ }^{1,2}$} & \multicolumn{3}{|c|}{ Asian or Pacific Islander ${ }^{1,3}$} \\
\hline & $\begin{array}{l}\text { Both } \\
\text { sexes }\end{array}$ & Male & Female & $\begin{array}{l}\text { Both } \\
\text { sexes }\end{array}$ & Male & Female \\
\hline All causes $\ldots \ldots \ldots$. & 14,367 & 7,885 & 6,482 & 45,609 & 23,823 & 21,786 \\
\hline Salmonella infections & 1 & 1 & - & 2 & 1 & 1 \\
\hline Shigellosis and amebiasis . . . . . . . (A03,A06) & - & - & - & - & - & - \\
\hline Certain other intestinal infections . (A04,A07-A09) & 31 & 13 & 18 & 50 & 22 & 28 \\
\hline Tuberculosis $\ldots \ldots \ldots \ldots \ldots \ldots$ (A16-A19) & 12 & 5 & 7 & 77 & 45 & 32 \\
\hline Respiratory tuberculosis . . . . . . . . . . . (A16) & 8 & 3 & 5 & 63 & 37 & 26 \\
\hline Other tuberculosis $\ldots \ldots \ldots \ldots$ (A17-A19) & 4 & 2 & 2 & 14 & 8 & 6 \\
\hline Whooping cough . & - & - & - & - & - & - \\
\hline Scarlet fever and erysipelas ...... & 1 & - & 1 & - & - & - \\
\hline Meningococcal infection . . . . . . . . . . (A39) & 2 & 1 & 1 & 1 & 1 & - \\
\hline Septicemia $\ldots \ldots \ldots \ldots \ldots$ (A40-A41) & 230 & 102 & 128 & 551 & 302 & 249 \\
\hline Syphilis $\ldots \ldots \ldots \ldots \ldots$ (A50-A53) & 1 & 1 & - & - & - & - \\
\hline Acute poliomyelitis $\ldots \ldots \ldots \ldots \ldots$ (A80) & - & - & - & - & - & - \\
\hline \multicolumn{7}{|l|}{ Arthropod-borne viral } \\
\hline encephalitis $\ldots \ldots \ldots \ldots$. . . . A83-A84,A85.2) & - & - & - & - & - & - \\
\hline Measles $\ldots \ldots \ldots \ldots \ldots \ldots \ldots$ (B05) & - & - & - & - & - & - \\
\hline$\ldots \ldots \ldots(\mathrm{B} 15-\mathrm{B} 19)$ & 105 & 66 & 39 & 294 & 149 & 145 \\
\hline \multicolumn{7}{|l|}{ Human immunodeficiency virus (HIV) } \\
\hline disease $\ldots \ldots \ldots \ldots \ldots \ldots$ (B20-B24) & 78 & 53 & 25 & 75 & 61 & 14 \\
\hline Malaria . . . . . . . . . . . . . . . . . . . (B50-B54) & - & - & - & 1 & 1 & - \\
\hline \multicolumn{7}{|l|}{$\begin{array}{l}\text { Other and unspecified infectious and parasitic } \\
\text { diseases and their sequelae } \\
\text { A20-A36,A42-A44,A48-A49,A54-A79,A00,A05, } \\
\text { A85.0-A85.1,A85.8,A86-B04,B06-B09, }\end{array}$} \\
\hline B25-B49,B55-B99) & 51 & 29 & 22 & 157 & 83 & 74 \\
\hline Malignant neoplasms .. & 2,561 & 1,345 & 1,216 & 12,326 & 6,394 & 5,932 \\
\hline \multicolumn{7}{|l|}{ Malignant neoplasms of lip, oral cavity and } \\
\hline pharynx $\ldots \ldots \ldots \ldots \ldots \ldots(C 00-C 14)$ & 43 & 26 & 17 & 242 & 160 & 82 \\
\hline Malignant neoplasm of esophagus . . . . . (C15) & 52 & 43 & 9 & 209 & 159 & 50 \\
\hline Malignant neoplasm of stomach . . . . . . (C16) & 90 & 53 & 37 & 749 & 412 & 337 \\
\hline \multicolumn{7}{|l|}{ Malignant neoplasms of colon, rectum } \\
\hline and anus $\ldots \ldots \ldots \ldots \ldots(\mathrm{C} 18-\mathrm{C} 21)$ & 252 & 132 & 120 & 1,238 & 624 & 614 \\
\hline \multicolumn{7}{|l|}{ Malignant neoplasms of liver and } \\
\hline intrahepatic bile ducts . . . . . . & 151 & 100 & 51 & 1,118 & 767 & 351 \\
\hline Malignant neoplasm of pancreas & 143 & 75 & 68 & 809 & 390 & 419 \\
\hline Malignant neoplasm of larynx . . . . . . . (C32) & 21 & 18 & 3 & 49 & 42 & 7 \\
\hline \multicolumn{7}{|l|}{ Malignant neoplasms of trachea, } \\
\hline bronchus and lung . . . . . . . . (C33-C34) & 695 & 384 & 311 & 2,841 & 1,647 & 1,194 \\
\hline Malignant melanoma of skin $\ldots \ldots \ldots \ldots$ (C43) & 22 & 14 & 8 & 45 & 25 & 20 \\
\hline Malignant neoplasm of breast . & 170 & 2 & 168 & 788 & 1 & 787 \\
\hline Malignant neoplasm of cervix uteri $\ldots \ldots$ (C53) & 32 & $\ldots$ & 32 & 147 & $\ldots$ & 147 \\
\hline \multicolumn{7}{|l|}{ Malignant neoplasms of corpus uteri } \\
\hline and uterus, part unspecified $\ldots \ldots$ (C54-C55) & 32 & $\ldots$ & 32 & 167 & $\ldots$ & 167 \\
\hline Malignant neoplasm of ovary $\ldots \ldots \ldots$ (C56) & 56 & $\ldots$ & 56 & 312 & $\ldots$ & 312 \\
\hline Malignant neoplasm of prostate . . . . . . (C61) & 105 & 105 & $\ldots$ & 414 & 414 & $\ldots$ \\
\hline \multicolumn{7}{|l|}{ Malignant neoplasms of kidney and } \\
\hline renal pelvis $\ldots \ldots \ldots \ldots \ldots \ldots$ (C64-C65) & 102 & 71 & 31 & 237 & 153 & 84 \\
\hline Malignant neoplasm of bladder . . . . . . (C67) & 31 & 22 & 9 & 158 & 107 & 51 \\
\hline \multicolumn{7}{|l|}{$\begin{array}{l}\text { Malignant neoplasms of meninges, } \\
\text { brain and other parts of central }\end{array}$} \\
\hline nervous system $\ldots \ldots \ldots \ldots(\mathrm{C} 70-\mathrm{C} 72)$ & 44 & 24 & 20 & 222 & 128 & 94 \\
\hline \multicolumn{7}{|l|}{ Malignant neoplasms of lymphoid, } \\
\hline hematopoietic and related tissue . . (C81-C96) & 209 & 118 & 91 & 1,150 & 652 & 498 \\
\hline Hodgkin's disease $\ldots . . . \ldots \ldots \ldots$. . . (C81) & 3 & 3 & - & 17 & 8 & 9 \\
\hline Non-Hodgkin's lymphoma . . . . . . (C82-C85) & 77 & 42 & 35 & 480 & 272 & 208 \\
\hline Leukemia . . . . . . . . . . . (C91-C95) & 78 & 42 & 36 & 435 & 248 & 187 \\
\hline \multicolumn{7}{|l|}{ Multiple myeloma and immunoproliferative } \\
\hline neoplasms $\ldots \ldots \ldots \ldots(\mathrm{C} 88, \mathrm{C} 90)$ & 49 & 29 & 20 & 217 & 123 & 94 \\
\hline
\end{tabular}

See footnotes at end of table. 
Table 12. Number of deaths from 113 selected causes and Enterocolitis due to Clostridium difficile, by race and sex: United States, 2007-Con.

[Data for specified races other than white and black should be interpreted with caution because of inconsistencies between reporting race on death certificates and on censuses and surveys; see "Technical Notes." The asterisks ( $\left(^{*}\right)$ preceding the cause-of-death codes indicate that they are not part of the International Classification of Diseases, Tenth Revision (ICD-10), Second Edition; see "Technical Notes"]

\begin{tabular}{|c|c|c|c|c|c|c|}
\hline \multirow[b]{2}{*}{ Cause of death (based on ICD-10, 2004) } & \multicolumn{3}{|c|}{ American Indian or Alaska Native ${ }^{1,2}$} & \multicolumn{3}{|c|}{ Asian or Pacific Islander 1,3} \\
\hline & $\begin{array}{l}\text { Both } \\
\text { sexes }\end{array}$ & Male & Female & $\begin{array}{l}\text { Both } \\
\text { sexes }\end{array}$ & Male & Female \\
\hline \multicolumn{7}{|l|}{$\begin{array}{l}\text { Other and unspecified malignant neoplasms of } \\
\text { lymphoid, hematopoietic and }\end{array}$} \\
\hline related tissue $\ldots \ldots \ldots \ldots \ldots$. . . . . (C96) & 2 & 2 & - & 1 & 1 & - \\
\hline \multicolumn{7}{|l|}{ All other and unspecified malignant } \\
\hline \multicolumn{6}{|l|}{ C37-C 41,C44-C49,C51-C52,C57-C60, } & 718 \\
\hline \multicolumn{7}{|l|}{$\begin{array}{l}\text { In situ neoplasms, benign neoplasms and } \\
\text { neoplasms of uncertain or unknown }\end{array}$} \\
\hline behavior $\ldots \ldots \ldots \ldots \ldots \ldots$ (D00-D48) & 51 & 26 & 25 & 308 & 166 & 142 \\
\hline$\ldots \ldots \ldots \ldots \ldots \ldots(D 50-D 64)$ & 21 & 9 & 12 & 104 & 47 & 57 \\
\hline Diabetes mellitus $\ldots \ldots \ldots \ldots \ldots$ (E10-E14) & 790 & 381 & 409 & 1,743 & 860 & 883 \\
\hline Nutritional deficiencies . . . . . . . . . (E40-E64) & 26 & 10 & 16 & 58 & 22 & 36 \\
\hline Malnutrition . . . . . . . . . . . . (E40-E46) & 24 & 9 & 15 & 52 & 20 & 32 \\
\hline Other nutritional deficiencies $\ldots \ldots$ (E50-E64) & 2 & 1 & 1 & 6 & 2 & 4 \\
\hline Meningitis $\ldots \ldots \ldots \ldots \ldots \ldots(\mathrm{G} 00, \mathrm{G} 03)$ & 6 & 4 & 2 & 18 & 8 & 10 \\
\hline Parkinson's disease $\ldots \ldots \ldots \ldots \ldots$ (G20-G21) & 55 & 32 & 23 & 337 & 190 & 147 \\
\hline Alzheimer's disease . . . . . . . . . (G30) & 191 & 69 & 122 & 748 & 245 & 503 \\
\hline Major cardiovascular diseases . . . . . . (100-178) & 3,482 & 1,913 & 1,569 & 15,340 & 7,878 & 7,462 \\
\hline Diseases of heart . . . (100-109,|111,|13,|120-151) & 2,648 & 1,520 & 1,128 & 10,574 & 5,724 & 4,850 \\
\hline \multicolumn{7}{|l|}{ Acute rheumatic fever and chronic } \\
\hline rheumatic heart diseases $\ldots \ldots .(100-109)$ & 17 & 7 & 10 & 86 & 28 & 58 \\
\hline Hypertensive heart disease . . . . . . . . (I11) & 135 & 79 & 56 & 599 & 292 & 307 \\
\hline Hypertensive heart and renal disease ....(113) & 8 & 4 & 4 & 90 & 38 & 52 \\
\hline Ischemic heart diseases . . . . . . . (120-I25) & 1,777 & 1,048 & 729 & 7,414 & 4,178 & 3,236 \\
\hline Acute myocardial infarction . . . . (121-122) & 591 & 359 & 232 & 2,380 & 1,345 & 1,035 \\
\hline Other acute ischemic heart diseases . . (I24) & 61 & 34 & 27 & 51 & 30 & 21 \\
\hline \multicolumn{7}{|l|}{ Other forms of chronic ischemic } \\
\hline heart disease $\ldots \ldots \ldots \ldots(120,125)$ & 1,125 & 655 & 470 & 4,983 & 2,803 & 2,180 \\
\hline \multicolumn{7}{|l|}{ Atherosclerotic cardiovascular disease, } \\
\hline so described $\ldots \ldots \ldots \ldots \ldots$ (125.0) & 325 & 200 & 125 & 1,145 & 681 & 464 \\
\hline \multicolumn{7}{|l|}{ All other forms of chronic ischemic } \\
\hline heart disease $\ldots \ldots \ldots(120,125.1-125.9)$ & 800 & 455 & 345 & 3,838 & 2,122 & 1,716 \\
\hline Other heart diseases . . . . . . . . . (I26-I51) & 711 & 382 & 329 & 2,385 & 1,188 & 1,197 \\
\hline Acute and subacute endocarditis .....(I33) & 14 & 9 & 5 & 21 & 11 & 10 \\
\hline \multicolumn{7}{|l|}{ Diseases of pericardium and acute } \\
\hline myocarditis $\ldots \ldots \ldots \ldots(|30-| 31, \mid 40)$ & 4 & 3 & 1 & 20 & 11 & 9 \\
\hline Heart failure . . . . . . . . . . .(150) & 188 & 85 & 103 & 513 & 226 & 287 \\
\hline \multicolumn{7}{|l|}{ All other forms of heart disease (126-I28, } \\
\hline |34-|38,|42-|49,|51) & 505 & 285 & 220 & 1,831 & 940 & 891 \\
\hline \multicolumn{7}{|l|}{ Essential hypertension and hypertensive } \\
\hline renal disease $\ldots \ldots \ldots \ldots \ldots(110,|12| 15)$, & 108 & 46 & 62 & 634 & 286 & 348 \\
\hline Cerebrovascular diseases . . . . . . (160-169) & 586 & 267 & 319 & 3,586 & 1,581 & 2,005 \\
\hline Atherosclerosis . . . . . . . . . . . . . (170) & 30 & 15 & 15 & 111 & 48 & 63 \\
\hline Other diseases of circulatory system . . (171-178) & 110 & 65 & 45 & 435 & 239 & 196 \\
\hline Aortic aneurysm and dissection ......(171) & 60 & 41 & 19 & 321 & 187 & 134 \\
\hline \multicolumn{7}{|l|}{ Other diseases of arteries, arterioles and } \\
\hline capillaries . . . . . . . . . . . . (172-178) & 50 & 24 & 26 & 114 & 52 & 62 \\
\hline Other disorders of circulatory system . . . (180-199) & 17 & 6 & 11 & 47 & 25 & 22 \\
\hline Influenza and pneumonia $\ldots \ldots \ldots\left(\right.$ J09-J18) ${ }^{4}$ & 280 & 150 & 130 & 1,335 & 703 & 632 \\
\hline Influenza. . . . . . . . . . . . (J09-J11) & 5 & 2 & 3 & 6 & 3 & 3 \\
\hline Pneumonia . . . . . . . & 275 & 148 & 127 & 1,329 & 700 & 629 \\
\hline \multicolumn{7}{|l|}{ Other acute lower respiratory } \\
\hline infections ........... & 2 & 1 & 1 & 3 & 2 & 1 \\
\hline Acute bronchitis and bronchiolitis . . . .(J20-J21) & 2 & 1 & 1 & 2 & 2 & - \\
\hline Other and unspecified acute lower respiratory & & & & & & \\
\hline infections $\ldots \ldots \ldots \ldots(\mathrm{J} 22, \mathrm{U} 04)^{5,6}$ & - & - & - & 1 & - & 1 \\
\hline Chronic lower respiratory diseases . . . .(J40-J47) & 611 & 299 & 312 & 1,331 & 795 & 536 \\
\hline Bronchitis, chronic and unspecified . . .(J40-J42) & 2 & 1 & 1 & 17 & 10 & 7 \\
\hline Emphysema $\ldots \ldots \ldots \ldots \ldots \ldots$ (J43) & 49 & 25 & 24 & 122 & 84 & 38 \\
\hline ..(J45-J46) & 34 & 12 & 22 & 135 & 67 & 68 \\
\hline
\end{tabular}

See footnotes at end of table. 
Table 12. Number of deaths from 113 selected causes and Enterocolitis due to Clostridium difficile, by race and sex: United States, 2007-Con.

[Data for specified races other than white and black should be interpreted with caution because of inconsistencies between reporting race on death certificates and on censuses and surveys; see "Technical Notes." The asterisks $\left({ }^{*}\right)$ preceding the cause-of-death codes indicate that they are not part of the International Classification of Diseases, Tenth Revision (ICD-10), Second Edition; see "Technical Notes"]

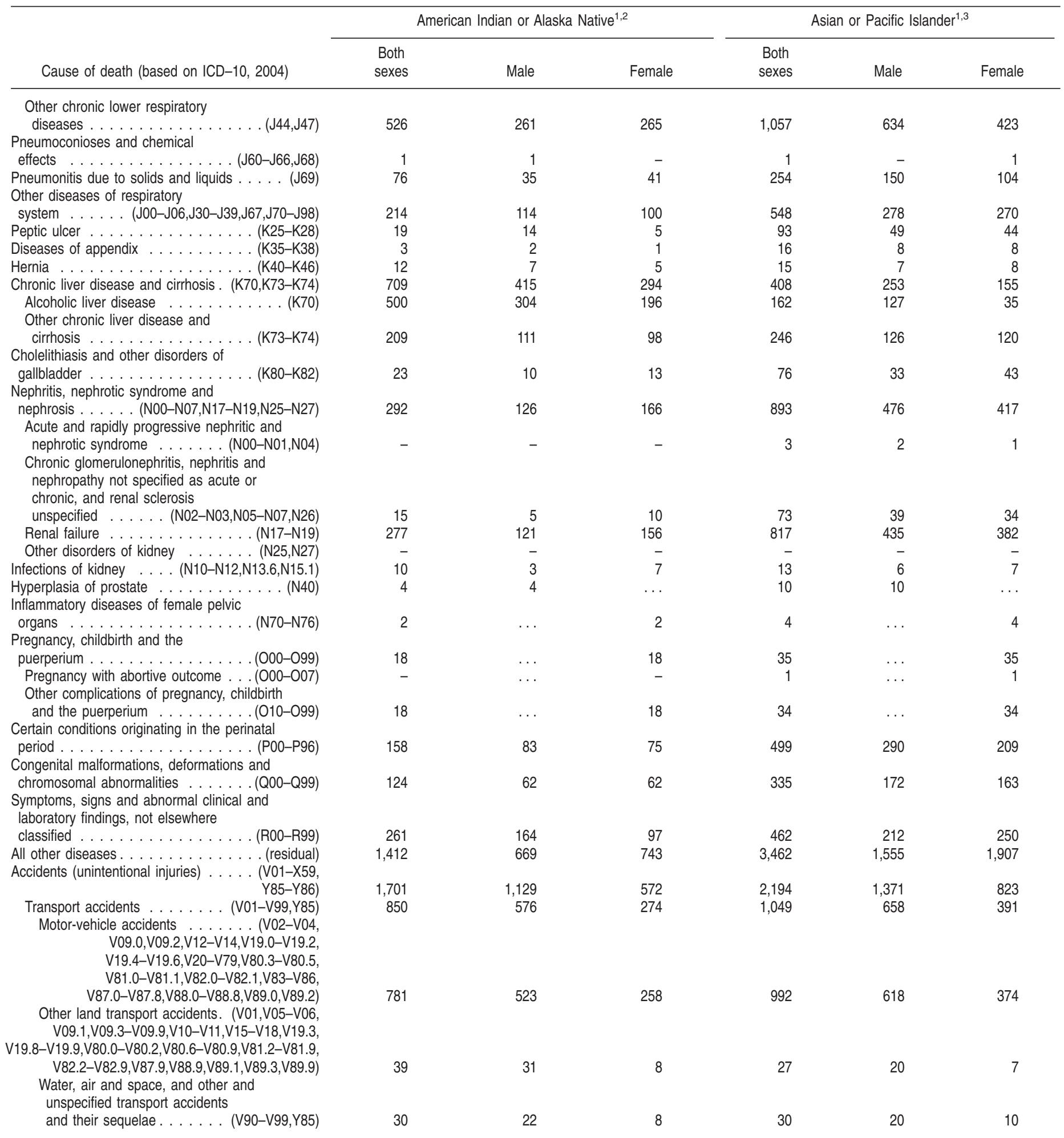

See footnotes at end of table. 


\section{Table 12. Number of deaths from 113 selected causes and Enterocolitis due to Clostridium difficile, by race and sex:} United States, 2007-Con.

[Data for specified races other than white and black should be interpreted with caution because of inconsistencies between reporting race on death certificates and on censuses and surveys; see "Technical Notes." The asterisks $\left(^{*}\right)$ preceding the cause-of-death codes indicate that they are not part of the International Classification of Diseases, Tenth Revision (ICD-10), Second Edition; see "Technical Notes"]

\begin{tabular}{|c|c|c|c|c|c|c|}
\hline \multirow[b]{2}{*}{ Cause of death (based on ICD-10, 2004) } & \multicolumn{3}{|c|}{ American Indian or Alaska Native $e^{1,2}$} & \multicolumn{3}{|c|}{ Asian or Pacific Islander ${ }^{1,3}$} \\
\hline & $\begin{array}{l}\text { Both } \\
\text { sexes }\end{array}$ & Male & Female & $\begin{array}{l}\text { Both } \\
\text { sexes }\end{array}$ & Male & Female \\
\hline Nontransport accidents . . . . . (W00-X59,Y86) & 851 & 553 & 298 & 1,145 & 713 & 432 \\
\hline $\begin{array}{l}\text { Falls . . . . . . . . . . . . . . (W00-W19) } \\
\text { Accidental discharge of }\end{array}$ & 132 & 89 & 43 & 464 & 263 & 201 \\
\hline $\begin{array}{c}\text { firearms } \ldots \ldots \ldots \ldots \text { (W32-W34) } \\
\text { Accidental drowning and }\end{array}$ & 11 & 10 & 1 & 7 & 7 & - \\
\hline $\begin{array}{l}\text { submersion .............. (W65-W74) } \\
\text { Accidental exposure to smoke, fire and }\end{array}$ & 67 & 57 & 10 & 151 & 115 & 36 \\
\hline $\begin{array}{l}\text { flames } \ldots \ldots \ldots \ldots \ldots \ldots \ldots(X 00-X 09) \\
\text { Accidental poisoning and exposure to }\end{array}$ & 42 & 24 & 18 & 52 & 34 & 18 \\
\hline $\begin{array}{l}\text { noxious substances . . . . . . . (X40-X49) } \\
\text { Other and unspecified nontransport } \\
\text { accidents and their sequelae... (W20-W31, }\end{array}$ & 375 & 213 & 162 & 225 & 146 & 79 \\
\hline $\begin{array}{l}\text { W35-W64,W75-W99,X10-X39,X50-X59,Y86) } \\
\text { Intentional self-harm }\end{array}$ & 224 & 160 & 64 & 246 & 148 & 98 \\
\hline $\begin{array}{l}\left.\text { (suicide) . . . . . . . . . . ( }{ }^{*} \mathrm{U} 03, \mathrm{X} 60-\mathrm{X} 84, \mathrm{Y} 87.0\right) \\
\text { Intentional self-harm (suicide) by discharge of }\end{array}$ & 392 & 310 & 82 & 900 & 628 & 272 \\
\hline $\begin{array}{l}\text { firearms. . . . . . . . . . . . . X72-X74) } \\
\text { Intentional self-harm (suicide) by other and } \\
\text { unspecified means and their }\end{array}$ & 135 & 116 & 19 & 221 & 198 & 23 \\
\hline sequelae . . . . (*U03,X60-X71,X75-X84,Y87.0) & 257 & 194 & 63 & 679 & 430 & 249 \\
\hline $\begin{array}{l}\left.\text { Assault (homicide). . ( ( }{ }^{*} \mathrm{U} 01-{ }^{*} \mathrm{U} 02, \mathrm{X} 85-\mathrm{Y} 09, \mathrm{Y} 87.1\right) \\
\text { Assault (homicide) by discharge of }\end{array}$ & 220 & 163 & 57 & 357 & 250 & 107 \\
\hline $\begin{array}{l}\left.\text { firearms. . . . . . . . . . ( }{ }^{*} \cup 01.4, \mathrm{X} 93-\mathrm{X} 95\right) \\
\text { Assault (homicide) by other and } \\
\text { unspecified means and their } \\
\text { sequelae . . . . . ( }{ }^{*} \cup 01.0-^{*} \cup 01.3,{ }^{*} \cup 01.5-{ }^{*} \cup 01.9,\end{array}$ & 91 & 78 & 13 & 201 & 168 & 33 \\
\hline *U02,X85-X92,X96-Y09,Y87.1) & 129 & 85 & 44 & 156 & 82 & 74 \\
\hline $\begin{array}{l}\text { Legal intervention . . . . . . . . . . . (Y35,Y89.0) } \\
\text { Events of undetermined }\end{array}$ & 3 & 3 & - & 6 & 6 & - \\
\hline $\begin{array}{l}\text { intent } \ldots \ldots \ldots \ldots \ldots \text { (Y . . . . . . } 10-Y 4, Y 87.2, Y 89.9) \\
\text { Discharge of firearms, undetermined }\end{array}$ & 92 & 51 & 41 & 80 & 52 & 28 \\
\hline $\begin{array}{l}\text { intent . . . . . . . . . . . . . . (Y22-Y24) } \\
\text { Other and unspecified events of } \\
\text { undetermined intent and their }\end{array}$ & 6 & 6 & - & 5 & 5 & - \\
\hline $\begin{array}{l}\text { sequelae .... (Y10-Y21,Y25-Y34,Y87.2,Y89.9) } \\
\text { Operations of war and their }\end{array}$ & 86 & 45 & 41 & 75 & 47 & 28 \\
\hline $\begin{array}{c}\text { sequelae ................... }(Y 36, Y 89.1) \\
\text { Complications of medical and surgical }\end{array}$ & - & - & - & - & - & - \\
\hline care $\ldots \ldots \ldots \ldots \ldots \ldots(\mathrm{Y} 40-\mathrm{Y} 84, \mathrm{Y} 88)$ & 16 & 4 & 12 & 42 & 17 & 25 \\
\hline Enterocolitis due to Clostridium difficile . (A04.7) $)^{7}$ & 26 & 11 & 15 & 47 & 22 & 25 \\
\hline
\end{tabular}

- Quantity zero.

... Category not applicable.

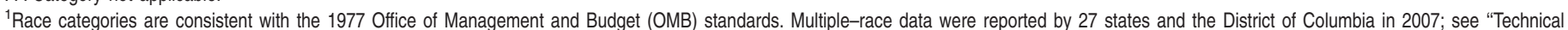
Notes." The multiple-race data for these reporting areas were bridged to the single-race categories of the 1977 OMB standards for comparability with other reporting areas; see "Technical Notes."

${ }^{2}$ Includes Aleuts and Eskimos.

${ }^{3}$ Includes Chinese, Filipino, Hawaiian, Japanese, and Other Asian or Pacific Islander.

${ }^{4} \mathrm{New}$ ICD-10 code J09 (Influenza due to identified avian influenza virus) was added to the category in 2007. No deaths occurred from this cause in 2007.

${ }^{5} \mathrm{New}$ ICD-10 code U04 (Severe acute respiratory syndrome [SARS]) was added to the category in 2007. No deaths occurred from this cause in 2007.

${ }^{6}$ Cause-of-death title was changed in 2007 to reflect the addition of SARS (ICD-10 code U04).

${ }^{7}$ Included in "Certain other intestinal infections (A04,A07-A09)" shown above. Beginning with data year 2006, "Enterocolitis due to Clostridium difficile (A04.7)" is shown separately at the bottom of tables showing 113 selected causes and is included in the list of rankable causes, see "Technical Notes."

NOTE: Complete confirmation of deaths from selected causes of death, considered to be of public health concern, was not provided by the following states: Connecticut, Florida, Indiana, Kentucky, Maryland, North Carolina, Oklahoma, Pennsylvania, Rhode Island, South Carolina, Washington, and West Virginia; see "Technical Notes." 
Table 13. Number of deaths from 113 selected causes and Enterocolitis due to Clostridium difficile, by Hispanic origin, race for non-Hispanic population, and sex: United States, 2007

[Race and Hispanic origin are reported separately on the death certificate. Persons of Hispanic origin may be of any race. Data for Hispanic persons are not tabulated separately by race; data for non-Hispanic persons are tabulated by race. Data for Hispanic origin should be interpreted with caution because of inconsistencies between reporting Hispanic origin on death certificates and on censuses and surveys; see "Technical Notes." The asterisks (*) preceding the cause-of-death codes indicate that they are not part of the International Classification of Diseases, Tenth Revision (ICD-10), Second Edition; see "Technical Notes"]

\begin{tabular}{|c|c|c|c|c|c|c|c|c|c|}
\hline \multirow[b]{2}{*}{ Cause of death (based on ICD-10, 2004) } & \multicolumn{3}{|c|}{ All origins } & \multicolumn{3}{|c|}{ Hispanic } & \multicolumn{3}{|c|}{ Non-Hispanic ${ }^{1}$} \\
\hline & $\begin{array}{l}\text { Both } \\
\text { sexes }\end{array}$ & Male & Female & $\begin{array}{l}\text { Both } \\
\text { sexes }\end{array}$ & Male & Female & $\begin{array}{l}\text { Both } \\
\text { sexes }\end{array}$ & Male & Female \\
\hline All causes & $2,423,712$ & $1,203,968$ & $1,219,744$ & 135,519 & 75,708 & 59,811 & $2,284,446$ & $1,125,974$ & $1,158,472$ \\
\hline Salmonella infections & 30 & 14 & 16 & 1 & - & 1 & 28 & 14 & 14 \\
\hline Shigellosis and amebiasis . . . . . . . & 4 & 2 & 2 & 2 & 2 & - & 2 & - & 2 \\
\hline Certain other intestinal infections $\ldots .(A 04, A 07-A 09)$ & 6,758 & 2,541 & 4,217 & 277 & 118 & 159 & 6,477 & 2,421 & 4,056 \\
\hline Tuberculosis $\ldots \ldots \ldots \ldots \ldots \ldots$ (A16-A19) & 554 & 342 & 212 & 92 & 63 & 29 & 459 & 278 & 181 \\
\hline Respiratory tuberculosis . . . . . . . . . (A16) & 424 & 266 & 158 & 75 & 52 & 23 & 347 & 213 & 134 \\
\hline Other tuberculosis. & 130 & 76 & 54 & 17 & 11 & 6 & 112 & 65 & 47 \\
\hline Whooping cough. & 9 & 1 & 8 & 6 & 1 & 5 & 3 & - & 3 \\
\hline Scarlet fever and erysipelas & 3 & 1 & 2 & 1 & - & 1 & 2 & 1 & 1 \\
\hline Meningococcal infection & 87 & 48 & 39 & 9 & 5 & 4 & 78 & 43 & 35 \\
\hline Septicemia $\ldots \ldots \ldots \ldots$ & 34,828 & 15,839 & 18,989 & 1,894 & 944 & 950 & 32,865 & 14,857 & 18,008 \\
\hline (A50-A53) & 42 & 25 & 17 & 4 & 3 & 1 & 38 & 22 & 16 \\
\hline Acute poliomyelitis $\ldots \ldots \ldots \ldots \ldots \ldots \ldots$ (A80) & - & - & - & - & - & - & - & - & - \\
\hline \multicolumn{10}{|l|}{ Arthropod-borne viral } \\
\hline$\ldots \ldots($ A83-A84,A85.2) & 3 & 2 & 1 & - & - & - & 3 & 2 & 1 \\
\hline$\ldots \ldots \ldots \ldots(\mathrm{B} 05)$ & - & - & - & - & - & - & - & - & - \\
\hline$\ldots \ldots(\mathrm{B} 15-\mathrm{B} 19)$ & 7,407 & 4,910 & 2,497 & 1,121 & 758 & 363 & 6,264 & 4,137 & 2,127 \\
\hline \multicolumn{10}{|l|}{ Human immunodeficiency virus (HIV) } \\
\hline disease $\ldots \ldots \ldots \ldots \ldots \ldots$ & 11,295 & 8,097 & 3,198 & 1,516 & 1,180 & 336 & 9,726 & 6,874 & 2,852 \\
\hline 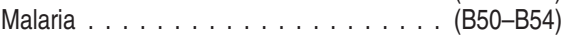 & 5 & 3 & 2 & - & - & - & 5 & 3 & 2 \\
\hline \multicolumn{10}{|l|}{$\begin{array}{r}\text { Other and unspecified infectious and parasitic } \\
\text { diseases and their sequelae... (A00,A05, } \\
\text { A20-A36,A42-A44,A48-A49, A54-A79,A81-A82, } \\
\text { A85.0-A85.1,A85.8, A86-B04,B06-B09, }\end{array}$} \\
\hline B25-B49,B55-B99) & 5,825 & 2,982 & 2,843 & 505 & 272 & 233 & 5,313 & 2,707 & 2,606 \\
\hline Malignant neoplasms & 562,875 & 292,857 & 270,018 & 27,660 & 14,493 & 13,167 & 534,614 & 278,027 & 256,587 \\
\hline \multicolumn{10}{|l|}{ Malignant neoplasms of lip, oral cavity } \\
\hline and pharynx .... & 8,067 & 5,510 & 2,557 & 364 & 260 & 104 & 7,693 & 5,242 & 2,451 \\
\hline Malignant neoplasm of esophagus $\ldots$ & 13,592 & 10,750 & 2,842 & 483 & 403 & 80 & 13,096 & 10,337 & 2,759 \\
\hline Malignant neoplasm of stomach . . . . . . . (C16) & 11,388 & 6,757 & 4,631 & 1,305 & 759 & 546 & 10,075 & 5,991 & 4,084 \\
\hline \multicolumn{10}{|l|}{ Malignant neoplasms of colon, rectum } \\
\hline \multicolumn{10}{|l|}{ Malignant neoplasms of liver and } \\
\hline intrahepatic bile ducts & 17,146 & 11,343 & 5,803 & 1,903 & 1,256 & 647 & 15,217 & 10,067 & 5,150 \\
\hline Malignant neoplasm of pancreas . . . . . & 34,117 & 17,132 & 16,985 & 1,779 & 869 & 910 & 32,300 & 16,240 & 16,060 \\
\hline Malignant neoplasm of larynx $\ldots \ldots \ldots \ldots$ (C32) & 3,634 & 2,890 & 744 & 164 & 147 & 17 & 3,461 & 2,737 & 724 \\
\hline \multicolumn{10}{|l|}{ Malignant neoplasms of trachea, } \\
\hline Malignant melanoma of skin $\ldots \ldots$ & 8,461 & 5,506 & 2,955 & $\begin{array}{r}4,0<2 \\
194\end{array}$ & $\begin{array}{r}2,0<2 \\
112\end{array}$ & 82 & 8,262 & 5,390 & 2,872 \\
\hline Malignant neoplasm of breast & 40,970 & 371 & 40,599 & 2,092 & 16 & 2,076 & 38,842 & 355 & 38,487 \\
\hline Malignant neoplasm of cervix uteri . & 4,021 & $\ldots$ & 4,021 & 473 & $\ldots$ & 473 & 3,546 & $\ldots$ & 3,546 \\
\hline \multicolumn{10}{|l|}{ Malignant neoplasms of corpus uteri } \\
\hline and uterus, part unspecified. & 7,456 & . & 7,456 & 397 & $\ldots$ & 397 & 7,049 & $\cdots$ & 7,049 \\
\hline Malignant neoplasm of ovary . . . . . & 14,621 & & 14,621 & 773 & & 773 & 13,837 & & 13,837 \\
\hline Malignant neoplasm of prostate $\ldots . . . .$. (C61) & 29,093 & 29,093 & $\ldots$ & 1,432 & 1,432 & $\ldots$ & 27,633 & 27,633 & \\
\hline \multicolumn{10}{|l|}{ Malignant neoplasms of kidney and } \\
\hline renal pelvis $\ldots$. & 12,703 & 7,964 & 4,739 & 867 & 579 & 288 & 11,824 & 7,378 & 4,446 \\
\hline Malignant neoplasm of bladder . . . & 13,843 & 9,644 & 4,199 & 477 & 348 & 129 & 13,355 & 9,288 & 4,067 \\
\hline \multicolumn{10}{|l|}{$\begin{array}{l}\text { Malignant neoplasms of meninges, } \\
\text { brain and other parts of central }\end{array}$} \\
\hline nervous system . . . & 13,234 & 7,315 & 5,919 & 815 & 437 & 378 & 12,407 & 6,873 & 5,534 \\
\hline \multicolumn{10}{|l|}{ Malignant neoplasms of lymphoid, } \\
\hline hematopoietic and related tissue . . . . (C81-C96) & 54,991 & 30,198 & 24,793 & 3,289 & 1,760 & 1,529 & 51,639 & 28,412 & 23,227 \\
\hline Hodgkin's disease $\ldots \ldots \ldots \ldots$ & 1,271 & 706 & 565 & 126 & 66 & 60 & 1,144 & 640 & 504 \\
\hline Non-Hodgkin's lymphoma . . . . . . . (C82-C85) & 20,528 & 11,004 & 9,524 & 1,224 & 655 & 569 & 19,283 & 10,339 & 8,944 \\
\hline Leukemia . . . . . . . . . . . (C91-C95) & 21,825 & 12,388 & 9,437 & 1,279 & 696 & 583 & 20,521 & 11,679 & 8,842 \\
\hline \multicolumn{10}{|l|}{ Multiple myeloma and immunoproliferative } \\
\hline$\ldots \ldots \ldots(\mathrm{C} 88, \mathrm{C} 90)$ & 11,307 & 6,066 & 5,241 & 660 & 343 & 317 & 10,631 & 5,720 & 4,911 \\
\hline
\end{tabular}

See footnotes at end of table. 
Table 13. Number of deaths from 113 selected causes and Enterocolitis due to Clostridium difficile, by Hispanic origin, race for non-Hispanic population, and sex: United States, 2007-Con.

[Race and Hispanic origin are reported separately on the death certificate. Persons of Hispanic origin may be of any race. Data for Hispanic persons are not tabulated separately by race; data for non-Hispanic persons are tabulated by race. Data for Hispanic origin should be interpreted with caution because of inconsistencies between reporting Hispanic origin on death certificates and on censuses and surveys; see "Technical Notes." The asterisks (*) preceding the cause-of-death codes indicate that they are not part of the International Classification of Diseases, Tenth Revision (ICD-10), Second Edition; see "Technical Notes"]

\begin{tabular}{|c|c|c|c|c|c|c|c|c|c|}
\hline \multirow[b]{2}{*}{ Cause of death (based on ICD-10, 2004) } & \multicolumn{3}{|c|}{ All origins } & \multicolumn{3}{|c|}{ Hispanic } & \multicolumn{3}{|c|}{ Non-Hispanic ${ }^{1}$} \\
\hline & $\begin{array}{l}\text { Both } \\
\text { sexes }\end{array}$ & Male & Female & $\begin{array}{l}\text { Both } \\
\text { sexes }\end{array}$ & Male & Female & $\begin{array}{l}\text { Both } \\
\text { sexes }\end{array}$ & Male & Female \\
\hline \multicolumn{10}{|l|}{$\begin{array}{l}\text { Other and unspecified malignant neoplasms } \\
\text { of lymphoid, hematopoietic and }\end{array}$} \\
\hline 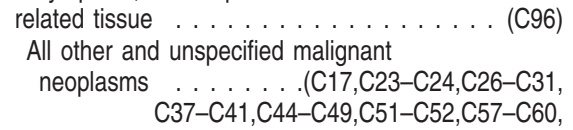 & 60 & 34 & 26 & - & - & - & 60 & 34 & 26 \\
\hline $\mathrm{C} 62-\mathrm{C} 63, \mathrm{C} 66, \mathrm{C} 68-\mathrm{C} 69, \mathrm{C} 73-\mathrm{C} 80, \mathrm{C} 97)$ & 63,192 & 32,887 & 30,305 & 3,427 & 1,784 & 1,643 & 59,687 & 31,052 & 28,635 \\
\hline \multicolumn{10}{|l|}{$\begin{array}{l}\text { In situ neoplasms, benign neoplasms and } \\
\text { neoplasms of uncertain or unknown }\end{array}$} \\
\hline behavior . . . . . . . . . . . . (D00-D48) & 14,204 & 7,256 & 6,948 & 696 & 329 & 367 & 13,495 & 6,918 & 6,577 \\
\hline$\ldots \ldots \ldots \ldots(\mathrm{D} 50-\mathrm{D} 64)$ & 4,829 & 1,940 & 2,889 & 217 & 106 & 111 & 4,604 & 1,828 & 2,776 \\
\hline$\ldots \ldots \ldots(\mathrm{E} 10-\mathrm{E} 14)$ & 71,382 & 35,478 & 35,904 & 6,417 & 3,199 & 3,218 & 64,863 & 32,226 & 32,637 \\
\hline Nutritional deficiencies $\ldots \ldots \ldots \ldots \ldots$ (E40-E64) & 2,852 & 1,071 & 1,781 & 115 & 40 & 75 & 2,734 & 1,029 & 1,705 \\
\hline$\ldots \ldots \ldots \ldots(E 40-E 46)$ & 2,644 & 991 & 1,653 & 108 & 38 & 70 & 2,533 & 951 & 1,582 \\
\hline Other nutritional deficiencies . . . . . . . (E50-E64) & 208 & 80 & 128 & 7 & 2 & 5 & 201 & 78 & 123 \\
\hline Meningitis $\ldots \ldots \ldots \ldots \ldots \ldots \ldots$ (G00,G03) & 655 & 346 & 309 & 71 & 36 & 35 & 583 & 310 & 273 \\
\hline$\ldots \ldots \ldots(\mathrm{G} 20-\mathrm{G} 21)$ & 20,058 & 11,554 & 8,504 & 771 & 442 & 329 & 19,276 & 11,109 & 8,167 \\
\hline Alzheimer's disease $\ldots \ldots \ldots \ldots \ldots \ldots \ldots$ (G30) & 74,632 & 21,800 & 52,832 & 2,471 & 801 & 1,670 & 72,101 & 20,976 & 51,125 \\
\hline Major cardiovascular diseases . . . . . . . (100-178) & 806,156 & 388,049 & 418,107 & 38,694 & 20,236 & 18,458 & 766,265 & 367,072 & 399,193 \\
\hline Diseases of heart $\ldots \ldots(100-109,|11| 113,, \mid 20-151)$ & 616,067 & 309,821 & 306,246 & 29,021 & 15,657 & 13,364 & 586,077 & 293,547 & 292,530 \\
\hline \multicolumn{10}{|l|}{ Acute rheumatic fever and chronic } \\
\hline rheumatic heart diseases $\ldots \ldots \ldots(100-109)$ & 3,201 & 1,026 & 2,175 & 155 & 52 & 103 & 3,042 & 971 & 2,071 \\
\hline Hypertensive heart disease $\ldots \ldots \ldots \ldots$ (111) & 30,780 & 14,249 & 16,531 & 1,710 & 919 & 791 & 28,991 & 13,276 & 15,715 \\
\hline Hypertensive heart and renal disease . . & 2,987 & 1,318 & 1,669 & 193 & 97 & 96 & 2,792 & 1,220 & 1,572 \\
\hline Ischemic heart diseases $\ldots \ldots \ldots \ldots$ (I20-I25) & 406,351 & 216,050 & 190,301 & 20,452 & 11,303 & 9,149 & 385,210 & 204,295 & 180,915 \\
\hline Acute myocardial infarction . . . . . . (121-I22) & 132,968 & 71,712 & 61,256 & 6,792 & 3,767 & 3,025 & 126,005 & 67,829 & 58,176 \\
\hline Other acute ischemic heart diseases . . . (I24) & 4,092 & 2,159 & 1,933 & 113 & 65 & 48 & 3,970 & 2,086 & 1,884 \\
\hline \multicolumn{10}{|l|}{ Other forms of chronic ischemic } \\
\hline heart disease $\ldots \ldots \ldots \ldots \ldots(120,125)$ & 269,291 & 142,179 & 127,112 & 13,547 & 7,471 & 6,076 & 255,235 & 134,380 & 120,855 \\
\hline \multicolumn{7}{|l|}{ Atherosclerotic cardiovascular } & 55,559 & 31,155 & 24,404 \\
\hline \multicolumn{10}{|l|}{ All other forms of chronic ischemic } \\
\hline heart disease $\ldots \ldots \ldots(120,|25.1-| 25.9)$ & 210,240 & 108,778 & 101,462 & 10,314 & 5,412 & 4,902 & 199,676 & 103,225 & 96,451 \\
\hline Other heart diseases . . . . . . . . . (I26-I51) & 172,748 & 77,178 & 95,570 & 6,511 & 3,286 & 3,225 & 166,042 & 73,785 & 92,257 \\
\hline Acute and subacute endocarditis $\ldots \ldots$ (I33) & 1,225 & 718 & 507 & 79 & 54 & 25 & 1,145 & 663 & 482 \\
\hline \multicolumn{10}{|l|}{ Diseases of pericardium and acute } \\
\hline myocarditis $\ldots \ldots \ldots \ldots(|30-| 31, \mid 40)$ & 867 & 454 & 413 & 66 & 42 & 24 & 800 & 412 & 388 \\
\hline 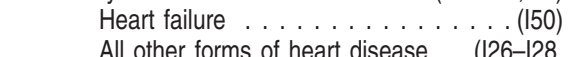 & 56,565 & 22,914 & 33,651 & 1,890 & 844 & 1,046 & 54,628 & 22,047 & 32,581 \\
\hline |34-|38,|42-|49,|51) & 114,091 & 53,092 & 60,999 & 4,476 & 2,346 & 2,130 & 109,469 & 50,663 & 58,806 \\
\hline \multicolumn{10}{|l|}{ Essential hypertension and hypertensive renal } \\
\hline disease $\ldots \ldots \ldots \ldots(110,112,115)$ & 23,965 & 9,417 & 14,548 & 1,395 & 639 & 756 & 22,542 & 8,768 & 13,774 \\
\hline Cerebrovascular diseases $\ldots \ldots \ldots \ldots(160-169)$ & 135,952 & 54,111 & 81,841 & 7,078 & 3,319 & 3,759 & 128,705 & 50,697 & 78,008 \\
\hline Atherosclerosis . & 8,232 & 3,220 & 5,012 & 326 & 138 & 188 & 7,898 & 3,078 & 4,820 \\
\hline Other diseases of circulatory system . . . (171-178) & 21,940 & 11,480 & 10,460 & 874 & 483 & 391 & 21,043 & 10,982 & 10,061 \\
\hline Aortic aneurysm and dissection & 12,986 & 7,543 & 5,443 & 472 & 295 & 177 & 12,505 & 7,241 & 5,264 \\
\hline \multicolumn{10}{|l|}{ Other diseases of arteries, arterioles and } \\
\hline$\ldots \ldots \ldots(172-178)$ & 8,954 & 3,937 & 5,017 & 402 & 188 & 214 & 8,538 & 3,741 & 4,797 \\
\hline Other disorders of circulatory system . . . . (180-199) & 4,101 & 1,902 & 2,199 & 241 & 123 & 118 & 3,847 & 1,772 & 2,075 \\
\hline Influenza and pneumonia & 52,717 & 24,071 & 28,646 & 2,735 & 1,361 & 1,374 & 49,896 & 22,662 & 27,234 \\
\hline Influenza. . . . . . . . . . & 411 & 187 & 224 & 32 & 18 & 14 & 378 & 168 & 210 \\
\hline Pneumonia $\ldots \ldots \ldots \ldots \ldots \ldots .($ (J12-J18) & 52,306 & 23,884 & 28,422 & 2,703 & 1,343 & 1,360 & 49,518 & 22,494 & 27,024 \\
\hline \multicolumn{10}{|l|}{ Other acute lower respiratory } \\
\hline$(\mathrm{J} 20-\mathrm{J} 22, \mathrm{U} 04)^{5}$ & 255 & 121 & 134 & 23 & 12 & 11 & 232 & 109 & 123 \\
\hline Acute bronchitis and bronchiolitis . . . . (J20-J21) & 213 & 104 & 109 & 23 & 12 & 11 & 190 & 92 & 98 \\
\hline \multicolumn{10}{|l|}{ Other and unspecified acute lower respiratory } \\
\hline 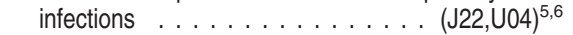 & 42 & 17 & 25 & - & - & - & 42 & 17 & 25 \\
\hline Chronic lower respiratory diseases & 127,924 & 61,235 & 66,689 & 3,531 & 1,894 & 1,637 & 124,217 & 59,234 & 64,983 \\
\hline Bronchitis, chronic and unspecified & 667 & 273 & 394 & 37 & 21 & 16 & 629 & 251 & 378 \\
\hline Emphysema . . . . . . . . . . . . . . (J43) & 12,790 & 6,598 & 6,192 & 294 & 181 & 113 & 12,473 & 6,403 & 6,070 \\
\hline Asthma $\ldots \ldots \ldots \ldots \ldots$ & 3,447 & 1,274 & 2,173 & 239 & 109 & 130 & 3,207 & 1,164 & 2,043 \\
\hline
\end{tabular}


Table 13. Number of deaths from 113 selected causes and Enterocolitis due to Clostridium difficile, by Hispanic origin, race for non-Hispanic population, and sex: United States, 2007-Con.

[Race and Hispanic origin are reported separately on the death certificate. Persons of Hispanic origin may be of any race. Data for Hispanic persons are not tabulated separately by race; data for non-Hispanic persons are tabulated by race. Data for Hispanic origin should be interpreted with caution because of inconsistencies between reporting Hispanic origin on death certificates and on censuses and surveys; see "Technical Notes." The asterisks (*) preceding the cause-of-death codes indicate that they are not part of the International Classification of Diseases, Tenth Revision (ICD-10), Second Edition; see "Technical Notes"]

\begin{tabular}{|c|c|c|c|c|c|c|c|c|c|}
\hline \multirow[b]{2}{*}{ Cause of death (based on ICD-10, 2004) } & \multicolumn{3}{|c|}{ All origins } & \multicolumn{3}{|c|}{ Hispanic } & \multicolumn{3}{|c|}{ Non-Hispanic ${ }^{1}$} \\
\hline & $\begin{array}{l}\text { Both } \\
\text { sexes }\end{array}$ & Male & Female & $\begin{array}{l}\text { Both } \\
\text { sexes }\end{array}$ & Male & Female & $\begin{array}{l}\text { Both } \\
\text { sexes }\end{array}$ & Male & Female \\
\hline Other chronic lower respiratory diseases $(\mathrm{J} 44, \mathrm{~J} 47)$ & 111,020 & 53,090 & 57,930 & 2,961 & 1,583 & 1,378 & 107,908 & 51,416 & 56,492 \\
\hline Pneumoconioses and chemical & & & & & & & & & \\
\hline effects $\ldots \ldots \ldots \ldots \ldots$ & 915 & 875 & 40 & 21 & 20 & 1 & 893 & 855 & 38 \\
\hline $\begin{array}{l}\text { Pneumonitis due to solids and liquids . . . . . . . (J69) } \\
\text { Other diseases of respiratory }\end{array}$ & 16,988 & 8,934 & 8,054 & 545 & 291 & 254 & 16,420 & 8,628 & 7,792 \\
\hline system . . . . . . (J00-J06,J30-J39,J67,J70-J98) & 28,508 & 14,291 & 14,217 & 1,721 & 892 & 829 & 26,757 & 13,380 & 13,377 \\
\hline Peptic ulcer $\ldots . . . \ldots$. & 3,045 & 1,469 & 1,576 & 152 & 90 & 62 & 2,890 & 1,377 & 1,513 \\
\hline Diseases of appendix $\ldots \ldots \ldots \ldots \ldots($ K35-K38) & 426 & 259 & 167 & 28 & 17 & 11 & 397 & 241 & 156 \\
\hline Hernia . . . . . . . & 1,698 & 708 & 990 & 97 & 37 & 60 & 1,597 & 669 & 928 \\
\hline Chronic liver disease and cirrhosis . .(K70,K73-K74) & 29,165 & 19,151 & 10,014 & 3,913 & 2,799 & 1,114 & 25,190 & 16,299 & 8,891 \\
\hline Alcoholic liver disease $\ldots \ldots \ldots \ldots \ldots$ (K70) & 14,406 & 10,549 & 3,857 & 2,129 & 1,776 & 353 & 12,240 & 8,740 & 3,500 \\
\hline $\begin{array}{l}\text { Other chronic liver disease and } \\
\text { cirrhosis } \ldots \ldots \ldots \ldots \ldots \ldots \ldots \ldots \text { K73-K74) }\end{array}$ & 14,759 & 8,602 & 6,157 & 1,784 & 1,023 & 761 & 12,950 & 7,559 & 5,391 \\
\hline $\begin{array}{l}\text { Cholelithiasis and other disorders of } \\
\text { gallbladder . . . . . . . . . (K80-K82) }\end{array}$ & 3,237 & 1,441 & 1,796 & 237 & 121 & 116 & 2,997 & 1,319 & 1,678 \\
\hline Nephritis, nephrotic syndrome and & & & & & & & & & \\
\hline $\begin{array}{c}\text { nephrosis . . . . . . . (N00-N07,N17-N19,N25-N27) } \\
\text { Acute and rapidly progressive nephritic and }\end{array}$ & 46,448 & 22,616 & 23,832 & 2,691 & 1,363 & 1,328 & 43,694 & 21,216 & 22,478 \\
\hline $\begin{array}{l}\text { nephrotic syndrome } \ldots . . . . . \text { (NO0-N01,N04) } \\
\text { Chronic glomerulonephritis, nephritis and } \\
\text { nephropathy not specified as acute or } \\
\text { chronic, and renal sclerosis }\end{array}$ & 206 & 111 & 95 & 25 & 12 & 13 & 181 & 99 & 82 \\
\hline unspecified $\ldots \ldots . . .(\mathrm{N} 02-\mathrm{N} 03, \mathrm{~N} 05-\mathrm{N} 07, \mathrm{~N} 26)$ & 2,958 & 1,452 & 1,506 & 170 & 94 & 76 & 2,787 & 1,357 & 1,430 \\
\hline Renal failure $\ldots \ldots \ldots \ldots \ldots \ldots(\mathrm{N} 17-\mathrm{N} 19)$ & 43,263 & 21,038 & 22,225 & 2,495 & 1,257 & 1,238 & 40,706 & 19,745 & 20,961 \\
\hline Other disorders of kidney & 21 & 15 & 6 & 1 & - & 1 & 20 & 15 & 5 \\
\hline Infections of kidney $\ldots \ldots(\mathrm{N} 10-\mathrm{N} 12, \mathrm{~N} 13.6, \mathrm{~N} 15.1)$ & 628 & 214 & 414 & 48 & 11 & 37 & 579 & 203 & 376 \\
\hline $\begin{array}{l}\text { Hyperplasia of prostate } \ldots \ldots \ldots \ldots \text {. . . . . . . } \\
\text { Inflammatory diseases of female pelvic }\end{array}$ & 491 & 491 & $\ldots$ & 30 & 30 & .. & 458 & 458 & $\ldots$ \\
\hline $\begin{array}{l}\text { organs } \ldots \ldots \ldots \ldots \ldots \text { (N70-N76) } \\
\text { Pregnancy, childbirth and the }\end{array}$ & 116 & $\ldots$ & 116 & 6 & $\ldots$ & 6 & 110 & $\ldots$ & 110 \\
\hline puerperium . . . . . . . . . . . . . . . (000-099) & 769 & $\ldots$ & 769 & 134 & $\ldots$ & 134 & 634 & $\ldots$ & 634 \\
\hline $\begin{array}{l}\text { Pregnancy with abortive outcome } \ldots(\mathrm{O}) 00-007) \\
\text { Other complications of pregnancy, childbirth }\end{array}$ & 31 & $\cdots$ & 31 & 5 & $\cdots$ & 5 & 26 & $\cdots$ & 26 \\
\hline $\begin{array}{l}\text { and the puerperium } \ldots \ldots \ldots \ldots(010-099) \\
\text { Certain conditions originating in the perinatal }\end{array}$ & 738 & $\ldots$ & 738 & 129 & $\ldots$ & 129 & 608 & $\ldots$ & 608 \\
\hline 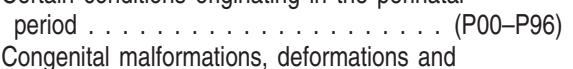 & 14,599 & 8,218 & 6,381 & 2,946 & 1,643 & 1,303 & 11,525 & 6,502 & 5,023 \\
\hline $\begin{array}{l}\text { chromosomal abnormalities .......... (Q00-Q99) } \\
\text { Symptoms, signs and abnormal clinical and } \\
\text { laboratory findings, not elsewhere }\end{array}$ & 10,421 & 5,455 & 4,966 & 1,991 & 1,063 & 928 & 8,392 & 4,371 & 4,021 \\
\hline classified . . . . . . . . . . . . . (R00-R99) & 33,500 & 14,282 & 19,218 & 1,952 & 1,134 & 818 & 31,464 & 13,099 & 18,365 \\
\hline $\begin{array}{l}\text { All other diseases . . . . . . . . . . . . (residual) } \\
\text { Accidents (unintentional injuries) (V01-X59, }\end{array}$ & 238,192 & 96,447 & 141,745 & 11,711 & 5,534 & 6,177 & 226,142 & 90,742 & 135,400 \\
\hline Y85-Y86) & 123,706 & 79,827 & 43,879 & 11,723 & 8,844 & 2,879 & 111,641 & 70,739 & 40,902 \\
\hline $\begin{array}{r}\text { Transport accidents } \ldots \ldots \ldots . . .(\mathrm{V} 01-\mathrm{V} 99, \mathrm{Y} 85) \\
\text { Motor-vehicle accidents } \ldots . . . .(\mathrm{V} 02-\mathrm{V} 04, \\
\text { V09.0,V09.2,V12-V14,V19.0-V19.2, } \\
\text { V19.4-V19.6,V20-V79,V80.3-V80.5, } \\
\text { V81.0-V81.1,V82.0-V82.1,V83-V86, }\end{array}$ & 46,844 & 33,434 & 13,410 & 6,117 & 4,641 & 1,476 & 40,604 & 28,695 & 11,909 \\
\hline V87.0-V87.8,V88.0-V88.8,V89.0,V89.2) & 43,945 & 31,102 & 12,843 & 5,824 & 4,390 & 1,434 & 38,013 & 26,626 & 11,387 \\
\hline $\begin{array}{r}\text { Other land transport accidents . . . . . (V01, } \\
\text { V05-V06,V09.1,V09.3-V09.9,V10-V11, } \\
\text { V15-V18,V19.3,V19.8-V19.9,V80.0-V80.2, } \\
\text { V80.6-V80.9,V81.2-V81.9,V82.2-V82.9, } \\
\text { V87.9,V88.9,V89.1,V89.3,V89.9) }\end{array}$ & 1,083 & 856 & 227 & 167 & 144 & 23 & 907 & 705 & 202 \\
\hline $\begin{array}{l}\text { Water, air and space, and other and } \\
\text { unspecified transport accidents } \\
\text { and their sequelae .........(V90-V99,Y85) }\end{array}$ & 1,816 & 1,476 & 340 & 126 & 107 & 19 & 1,684 & 1,364 & 320 \\
\hline
\end{tabular}

See footnotes at end of table. 
Table 13. Number of deaths from 113 selected causes and Enterocolitis due to Clostridium difficile, by Hispanic origin, race for non-Hispanic population, and sex: United States, 2007-Con.

[Race and Hispanic origin are reported separately on the death certificate. Persons of Hispanic origin may be of any race. Data for Hispanic persons are not tabulated separately by race; data for non-Hispanic persons are tabulated by race. Data for Hispanic origin should be interpreted with caution because of inconsistencies between reporting Hispanic origin on death certificates and on censuses and surveys; see "Technical Notes." The asterisks $\left({ }^{*}\right)$ preceding the cause-of-death codes indicate that they are not part of the International Classification of Diseases, Tenth Revision (ICD-10), Second Edition; see "Technical Notes"]

\begin{tabular}{|c|c|c|c|c|c|c|c|c|c|}
\hline \multirow[b]{2}{*}{ Cause of death (based on ICD-10, 2004) } & \multicolumn{3}{|c|}{ All origins } & \multicolumn{3}{|c|}{ Hispanic } & \multicolumn{3}{|c|}{ Non-Hispanic ${ }^{1}$} \\
\hline & $\begin{array}{l}\text { Both } \\
\text { sexes }\end{array}$ & Male & Female & $\begin{array}{l}\text { Both } \\
\text { sexes }\end{array}$ & Male & Female & $\begin{array}{l}\text { Both } \\
\text { sexes }\end{array}$ & Male & Female \\
\hline Nontransport accidents . . . . . . . (W00-X59,Y86) & 76,862 & 46,393 & 30,469 & 5,606 & 4,203 & 1,403 & 71,037 & 42,044 & 28,993 \\
\hline Falls $\ldots \ldots \ldots \ldots \ldots$ (W00-W19) & 22,631 & 11,597 & 11,034 & 1,212 & 762 & 450 & 21,374 & 10,803 & 10,571 \\
\hline $\begin{array}{l}\text { Accidental discharge of firearms. . . . (W32-W34) } \\
\text { Accidental drowning and }\end{array}$ & 613 & 537 & 76 & 65 & 58 & 7 & 548 & 479 & 69 \\
\hline $\begin{array}{l}\text { submersion . . . . . . . . . . . . . . .(W65-W74) } \\
\text { Accidental exposure to smoke, fire and }\end{array}$ & 3,443 & 2,681 & 762 & 480 & 410 & 70 & 2,946 & 2,256 & 690 \\
\hline $\begin{array}{l}\text { flames .................... } \\
\text { Accidental poisoning and exposure to }\end{array}$ & 3,286 & 1,943 & 1,343 & 230 & 150 & 80 & 3,041 & 1,786 & 1,255 \\
\hline $\begin{array}{l}\text { noxious substances } \ldots . . . \ldots . . .(\mathrm{X} 40-\mathrm{X} 49) \\
\text { Other and unspecified nontransport }\end{array}$ & 29,846 & 19,644 & 10,202 & 2,436 & 1,943 & 493 & 27,307 & 17,640 & 9,667 \\
\hline $\begin{array}{l}\text { accidents and their sequelae.... (W20-W31, } \\
\text { W35-W64,W75-W99,X10-X39,X50-X59,Y86) } \\
\text { Intentional self-harm }\end{array}$ & 17,043 & 9,991 & 7,052 & 1,183 & 880 & 303 & 15,821 & 9,080 & 6,741 \\
\hline $\begin{array}{l}\text { (suicide) . . . . . . . . . . . ( }{ }^{*} \mathrm{U} 03, \mathrm{X} 60-\mathrm{X} 84, \mathrm{Y} 87.0 \text { ) } \\
\text { Intentional self-harm (suicide) by }\end{array}$ & 34,598 & 27,269 & 7,329 & 2,465 & 2,078 & 387 & 32,061 & 25,133 & 6,928 \\
\hline $\begin{array}{l}\text { discharge of firearms . . . . . . . . . (X72-X74) } \\
\text { Intentional self-harm (suicide) by other and } \\
\text { unspecified means and their }\end{array}$ & 17,352 & 15,181 & 2,171 & 931 & 855 & 76 & 16,386 & 14,295 & 2,091 \\
\hline sequelae . . . . . ( $\left.{ }^{*} \mathrm{U} 03, \mathrm{X} 60-\mathrm{X} 71, \mathrm{X} 75-\mathrm{X} 84, \mathrm{Y} 87.0\right)$ & 17,246 & 12,088 & 5,158 & 1,534 & 1,223 & 311 & 15,675 & 10,838 & 4,837 \\
\hline $\begin{array}{l}\text { Assault (homicide). . . . . (*U01-*U02,X85-Y09,Y87.1) } \\
\text { Assault (homicide) by discharge of }\end{array}$ & 18,361 & 14,538 & 3,823 & 3,466 & 2,926 & 540 & 14,798 & 11,529 & 3,269 \\
\hline $\begin{array}{l}\text { firearms. . . . . . . . . (*U01.4,X93-X95) } \\
\text { Assault (homicide) by other and } \\
\text { unspecified means and their } \\
\text { sequelae . . . . ( }{ }^{*} \cup 01.0-^{*} \cup 01.3,{ }^{*} \cup 01.5-{ }^{*} \cup 01.9,\end{array}$ & 12,632 & 10,767 & 1,865 & 2,385 & 2,133 & 252 & 10,193 & 8,584 & 1,609 \\
\hline *U02,X85-X92,X96-Y09,Y87.1) & 5,729 & 3,771 & 1,958 & 1,081 & 793 & 288 & 4,605 & 2,945 & 1,660 \\
\hline $\begin{array}{l}\text { Legal intervention . . . . . . . . . . . . . (Y35,YY89.0) } \\
\text { Events of undetermined }\end{array}$ & 412 & 400 & 12 & 95 & 95 & - & 316 & 304 & 12 \\
\hline $\begin{array}{l}\text { intent. . . . . . . . . . . . . (Y10-Y34,Y87.2,Y89.9) } \\
\text { Discharge of firearms, undetermined }\end{array}$ & 5,381 & 3,351 & 2,030 & 331 & 232 & 99 & 5,030 & 3,105 & 1,925 \\
\hline $\begin{array}{l}\text { intent . . . . . . . . . . . . . . . . (Y22-Y24) } \\
\text { Other and unspecified events of } \\
\text { undetermined intent and their }\end{array}$ & 276 & 223 & 53 & 31 & 29 & 2 & 244 & 193 & 51 \\
\hline $\begin{array}{l}\text { sequelae ..... (Y10-Y21,Y25-Y34,Y87.2,Y89.9) } \\
\text { Operations of war and their }\end{array}$ & 5,105 & 3,128 & 1,977 & 300 & 203 & 97 & 4,786 & 2,912 & 1,874 \\
\hline $\begin{array}{l}\text { sequelae . . . . . . . . . . . . . . . (Y36,Y89.1) } \\
\text { Complications of medical and surgical }\end{array}$ & 21 & 21 & - & 3 & 3 & - & 18 & 18 & - \\
\hline care $\ldots \ldots \ldots \ldots \ldots \ldots .(\mathrm{Y} 40-\mathrm{Y} 84, \mathrm{Y} 88)$ & 2,597 & 1,224 & 1,373 & 143 & 67 & 76 & 2,453 & 1,156 & 1,297 \\
\hline Enterocolitis due to Clostridium difficile . & 6,372 & 2,399 & 3,973 & 250 & 107 & 143 & 6,119 & 2,291 & 3,828 \\
\hline
\end{tabular}

See footnotes at end of table. 
Table 13. Number of deaths from 113 selected causes and Enterocolitis due to Clostridium difficile, by Hispanic origin, race for non-Hispanic population, and sex: United States, 2007-Con.

[Race and Hispanic origin are reported separately on the death certificate. Persons of Hispanic origin may be of any race. Data for Hispanic persons are not tabulated separately by race; data for non-Hispanic persons are tabulated by race. Data for Hispanic origin should be interpreted with caution because of inconsistencies between reporting Hispanic origin on death certificates and on censuses and surveys; see "Technical Notes." The asterisks (*) preceding the cause-of-death codes indicate that they are not part of the International Classification of Diseases, Tenth Revision (ICD-10), Second Edition; see "Technical Notes"]

\begin{tabular}{|c|c|c|c|c|c|c|c|c|c|}
\hline \multirow[b]{2}{*}{ Cause of death (based on ICD-10, 2004) } & \multicolumn{3}{|c|}{ Non-Hispanic white ${ }^{2}$} & \multicolumn{3}{|c|}{ Non-Hispanic black ${ }^{2}$} & \multicolumn{3}{|c|}{ Origin not stated ${ }^{3}$} \\
\hline & $\begin{array}{l}\text { Both } \\
\text { sexes }\end{array}$ & Male & Female & $\begin{array}{l}\text { Both } \\
\text { sexes }\end{array}$ & Male & Female & $\begin{array}{l}\text { Both } \\
\text { sexes }\end{array}$ & Male & Female \\
\hline All causes $\ldots \ldots \ldots \ldots \ldots \ldots \ldots$ & $1,939,606$ & 948,662 & 990,944 & 286,366 & 146,474 & 139,892 & 3,747 & 2,286 & 1,461 \\
\hline Salmonella infections & 22 & 11 & 11 & 3 & 1 & 2 & 1 & - & 1 \\
\hline Shigellosis and amebiasis $\ldots \ldots \ldots \ldots(\mathrm{A} 03, \mathrm{A06})$ & 1 & - & 1 & 1 & - & 1 & - & - & - \\
\hline Certain other intestinal infections $\ldots .(\mathrm{A} 04, \mathrm{~A} 07-\mathrm{A} 09)$ & 5,978 & 2,228 & 3,750 & 420 & 158 & 262 & 4 & 2 & 2 \\
\hline Tuberculosis $\ldots \ldots \ldots \ldots \ldots \ldots$ (A16-A19) & 250 & 146 & 104 & 121 & 82 & 39 & 3 & 1 & 2 \\
\hline Respiratory tuberculosis . . . . . . . . . . . (A16) & 187 & 112 & 75 & 89 & 61 & 28 & 2 & 1 & 1 \\
\hline Other tuberculosis $\ldots \ldots \ldots \ldots \ldots$ (A17-A19) & 63 & 34 & 29 & 32 & 21 & 11 & 1 & - & 1 \\
\hline Whooping cough . & 3 & - & 3 & - & - & - & - & - & - \\
\hline Scarlet fever and erysipelas & 1 & 1 & - & - & - & - & - & - & - \\
\hline Meningococcal infection ... . & 49 & 27 & 22 & 26 & 14 & 12 & - & - & - \\
\hline Septicemia $\ldots . . . \ldots$. . & 25,856 & 11,653 & 14,203 & 6,241 & 2,807 & 3,434 & 69 & 38 & 31 \\
\hline Syphilis $\ldots \ldots \ldots \ldots \ldots \ldots$ (A50-A53) & 16 & 9 & 7 & 21 & 12 & 9 & - & - & - \\
\hline Acute poliomyelitis $\ldots \ldots \ldots \ldots \ldots \ldots \ldots$ (A80) & - & - & - & - & - & - & - & - & - \\
\hline Arthropod-borne viral encephalitis . (A83-A84,A85.2) & 2 & 1 & 1 & 1 & 1 & - & - & - & - \\
\hline Measles $\ldots \ldots \ldots \ldots \ldots \ldots \ldots \ldots$ (B05) & - & - & - & - & - & - & - & - & - \\
\hline$\ldots \ldots \ldots \ldots \ldots$ (B15-B19) & 4,855 & 3,256 & 1,599 & 1,025 & 673 & 352 & 22 & 15 & 7 \\
\hline \multicolumn{10}{|l|}{ Human immunodeficiency virus (HIV) } \\
\hline disease $\ldots \ldots \ldots \ldots \ldots \ldots$. . . . . . . . . . . & 3,182 & 2,638 & 544 & 6,398 & 4,127 & 2,271 & 53 & 43 & 10 \\
\hline Malaria $\ldots \ldots \ldots \ldots \ldots \ldots \ldots$ (B50-B54) & 3 & 2 & 1 & 1 & - & 1 & - & - & - \\
\hline \multicolumn{10}{|l|}{$\begin{array}{r}\text { Other and unspecified infectious and parasitic } \\
\text { diseases and their sequelae .......A00,A05, } \\
\text { A20-A36,A42-A44,A48-A49, A54-A79,A81-A82, } \\
\text { A85.0-A85.1,A85.8, A86-B04,B06-B09, }\end{array}$} \\
\hline B25-B49,B55-B99) & 4,357 & 2,208 & 2,149 & 758 & 392 & 366 & 7 & 3 & 4 \\
\hline Malignant neoplasms & 456,576 & 237,672 & 218,904 & 63,441 & 32,766 & 30,675 & 601 & 337 & 264 \\
\hline \multicolumn{10}{|l|}{ Malignant neoplasms of lip, oral cavity } \\
\hline Malignant neoplasm of esophagus . . . . . (C15) & 11,393 & 9,083 & 2,310 & 1,445 & 1,054 & 391 & 13 & 10 & 3 \\
\hline Malignant neoplasm of stomach . . . . . . . (C16) & 7,272 & 4,355 & 2,917 & 1,973 & 1,175 & 798 & 8 & 7 & 1 \\
\hline \multicolumn{10}{|l|}{ Malignant neoplasms of colon, rectum } \\
\hline and anus $\ldots \ldots \ldots \ldots \ldots \ldots$ (C18-C21) & 42,410 & 21,431 & 20,979 & 6,853 & 3,412 & 3,441 & 63 & 35 & 28 \\
\hline \multicolumn{10}{|l|}{ Malignant neoplasms of liver and } \\
\hline intrahepatic bile ducts $\ldots \ldots \ldots \ldots \ldots \ldots$ (C22) & 11,722 & 7,639 & 4,083 & 2,243 & 1,575 & 668 & 26 & 20 & 6 \\
\hline Malignant neoplasm of pancreas & 27,336 & 13,905 & 13,431 & 4,033 & 1,878 & 2,155 & 38 & 23 & 15 \\
\hline Malignant neoplasm of larynx $\ldots \ldots \ldots \ldots$ (C32) & 2,741 & 2,137 & 604 & 651 & 541 & 110 & 9 & 6 & 3 \\
\hline \multicolumn{10}{|l|}{ Malignant neoplasms of trachea, } \\
\hline bronchus and lung $\ldots \ldots \ldots$ & 134,134 & 73,717 & 60,417 & 16,360 & 9,744 & 6,616 & 166 & 99 & 67 \\
\hline Malignant melanoma of skin . . . . . . . . . (C43) & 8,063 & 5,294 & 2,769 & 133 & 57 & 76 & 5 & 4 & 1 \\
\hline Malignant neoplasm of breast . . . . . . . . . (C50) & 32,106 & 300 & 31,806 & 5,800 & 52 & 5,748 & 36 & - & 36 \\
\hline Malignant neoplasm of cervix uteri $\ldots . .$. (C53) & 2,576 & $\ldots$ & 2,576 & 796 & $\ldots$ & 796 & 2 & $\ldots$ & 2 \\
\hline \multicolumn{10}{|l|}{ Malignant neoplasms of corpus uteri and } \\
\hline uterus, part unspecified $\ldots \ldots \ldots \ldots$ (C54-C55) & 5,570 & $\ldots$ & 5,570 & 1,283 & $\ldots$ & 1,283 & 10 & $\ldots$ & 10 \\
\hline Malignant neoplasm of ovary $\ldots \ldots \ldots$ (C56) & 12,330 & & 12,330 & 1,150 & & 1,150 & 11 & & 11 \\
\hline Malignant neoplasm of prostate $\ldots \ldots \ldots$ (C61) & 22,259 & 22,259 & $\ldots$ & 4,865 & 4,865 & $\ldots$ & 28 & 28 & $\ldots$ \\
\hline \multicolumn{10}{|l|}{ Malignant neoplasms of kidney and } \\
\hline renal pelvis $\ldots \ldots \ldots \ldots \ldots$ (C64-C65) & 10,286 & 6,422 & 3,864 & 1,211 & 739 & 472 & 12 & 7 & 5 \\
\hline Malignant neoplasm of bladder $\ldots \ldots \ldots$ (C67) & 12,139 & 8,600 & 3,539 & 1,030 & 562 & 468 & 11 & 8 & 3 \\
\hline \multicolumn{10}{|l|}{$\begin{array}{l}\text { Malignant neoplasms of meninges, } \\
\text { brain and other parts of central }\end{array}$} \\
\hline nervous system $\ldots \ldots \ldots \ldots$ & 11,375 & 6,328 & 5,047 & 773 & 397 & 376 & 12 & 5 & 7 \\
\hline \multicolumn{10}{|l|}{ Malignant neoplasms of lymphoid, } \\
\hline hematopoietic and related tissue $\ldots$ (C81-C96) & 45,120 & 25,020 & 20,100 & 5,189 & 2,638 & 2,551 & 63 & 26 & 37 \\
\hline Hodgkin's disease $\ldots \ldots \ldots \ldots \ldots$ (C81) & 997 & 555 & 442 & 127 & 74 & 53 & 1 & - & 1 \\
\hline Non-Hodgkin's lymphoma . . . . . . . (C82-C85) & 17,356 & 9,284 & 8,072 & 1,379 & 745 & 634 & 21 & 10 & 11 \\
\hline Leukemia . . . . . . . . . . . (C91-C95) & 18,213 & 10,454 & 7,759 & 1,805 & 940 & 865 & 25 & 13 & 12 \\
\hline \multicolumn{10}{|l|}{ Multiple myeloma and immunoproliferative } \\
\hline neoplasms $\ldots \ldots \ldots \ldots \ldots(\mathrm{C} 88, \mathrm{C} 90)$ & 8,508 & 4,703 & 3,805 & 1,867 & 872 & 995 & 16 & 3 & 13 \\
\hline \multicolumn{10}{|l|}{$\begin{array}{l}\text { Other and unspecified malignant } \\
\text { neoplasms of lymphoid, hematopoietic }\end{array}$} \\
\hline and related tissue $\ldots \ldots \ldots \ldots \ldots$ (C96) & 46 & 24 & 22 & 11 & 7 & 4 & - & - & - \\
\hline
\end{tabular}

See footnotes at end of table. 
Table 13. Number of deaths from 113 selected causes and Enterocolitis due to Clostridium difficile, by Hispanic origin, race for non-Hispanic population, and sex: United States, 2007-Con.

[Race and Hispanic origin are reported separately on the death certificate. Persons of Hispanic origin may be of any race. Data for Hispanic persons are not tabulated separately by race; data for non-Hispanic persons are tabulated by race. Data for Hispanic origin should be interpreted with caution because of inconsistencies between reporting Hispanic origin on death certificates and on censuses and surveys; see "Technical Notes." The asterisks (*) preceding the cause-of-death codes indicate that they are not part of the International Classification of Diseases, Tenth Revision (ICD-10), Second Edition; see "Technical Notes"]

\begin{tabular}{|c|c|c|c|c|c|c|c|c|c|}
\hline \multirow[b]{2}{*}{ Cause of death (based on ICD-10, 2004) } & \multicolumn{3}{|c|}{ Non-Hispanic white ${ }^{2}$} & \multicolumn{3}{|c|}{ Non-Hispanic black ${ }^{2}$} & \multicolumn{3}{|c|}{ Origin not stated ${ }^{3}$} \\
\hline & $\begin{array}{l}\text { Both } \\
\text { sexes }\end{array}$ & Male & Female & $\begin{array}{l}\text { Both } \\
\text { sexes }\end{array}$ & Male & Female & $\begin{array}{l}\text { Both } \\
\text { sexes }\end{array}$ & Male & Female \\
\hline \\
\hline $\begin{array}{r}\text { neoplasms } \ldots \ldots .(C 17, C 23-C 24, C 26-C 31 \\
\text { C37-C41,C44-C49,C51-C52,C57-C60, }\end{array}$ & & & & & & & & & \\
\hline $\mathrm{C} 62-\mathrm{C} 63, \mathrm{C} 66, \mathrm{C} 68-\mathrm{C} 69, \mathrm{C} 73-\mathrm{C} 80, \mathrm{C} 97)$ & 51,383 & 26,886 & 24,497 & 6,599 & 3,314 & 3,285 & 78 & 51 & 27 \\
\hline \multicolumn{10}{|l|}{$\begin{array}{l}\text { In situ neoplasms, benign neoplasms and } \\
\text { neoplasms of uncertain or unknown }\end{array}$} \\
\hline behavior $\ldots \ldots \ldots \ldots \ldots \ldots$ (D00-D48) & 11,965 & 6,141 & 5,824 & 1,178 & 586 & 592 & 13 & 9 & 4 \\
\hline Anemias . . . . . . . . . . . . . (D50-D64) & 3,523 & 1,343 & 2,180 & 958 & 430 & 528 & 8 & 6 & 2 \\
\hline Diabetes mellitus $\ldots \ldots \ldots \ldots \ldots \ldots$ (E10-E14) & 50,046 & 25,584 & 24,462 & 12,343 & 5,434 & 6,909 & 102 & 53 & 49 \\
\hline Nutritional deficiencies $\ldots \ldots \ldots \ldots \ldots$. E40-E64) & 2,260 & 834 & 1,426 & 391 & 163 & 228 & 3 & 2 & 1 \\
\hline Malnutrition $\ldots \ldots \ldots \ldots \ldots \ldots$ (E40-E46) & 2,084 & 763 & 1,321 & 373 & 159 & 214 & 3 & 2 & 1 \\
\hline Other nutritional deficiencies . . . . . . (E50-E64) & 176 & 71 & 105 & 18 & 4 & 14 & - & - & - \\
\hline Meningitis .... & 428 & 222 & 206 & 132 & 77 & 55 & 1 & - & 1 \\
\hline Parkinson's disease ... . & 18,098 & 10,457 & 7,641 & 789 & 431 & 358 & 11 & 3 & 8 \\
\hline Alzheimer's disease & 66,453 & 19,378 & 47,075 & 4,729 & 1,293 & 3,436 & 60 & 23 & 37 \\
\hline Major cardiovascular diseases $\ldots$ & 652,763 & 311,345 & 341,418 & 95,059 & 46,159 & 48,900 & 1,197 & 741 & 456 \\
\hline Diseases of heart . . . . (100-109,|111,|13,|20-I51) & 502,683 & 251,229 & 251,454 & 70,443 & 35,243 & 35,200 & 969 & 617 & 352 \\
\hline \multicolumn{10}{|l|}{ Acute rheumatic fever and chronic } \\
\hline rheumatic heart diseases $\ldots . . . .(100-109)$ & 2,698 & 853 & 1,845 & 242 & 83 & 159 & 4 & 3 & 1 \\
\hline Hypertensive heart disease $\ldots \ldots \ldots \ldots$. (111) & 21,244 & 9,292 & 11,952 & 7,023 & 3,618 & 3,405 & 79 & 54 & 25 \\
\hline Hypertensive heart and renal disease . . . . (113) & 1,874 & 769 & 1,105 & 820 & 409 & 411 & 2 & 1 & 1 \\
\hline Ischemic heart diseases . . . . . . . . (I20-125) & 334,047 & 177,716 & 156,331 & 42,152 & 21,473 & 20,679 & 689 & 452 & 237 \\
\hline Acute myocardial infarction . . . . . (121-I22) & 109,134 & 59,246 & 49,888 & 13,967 & 6,923 & 7,044 & 171 & 116 & 55 \\
\hline Other acute ischemic heart diseases . . . (I24) & 3,331 & 1,728 & 1,603 & 530 & 296 & 234 & 9 & 8 & 1 \\
\hline \multicolumn{10}{|l|}{ Other forms of chronic ischemic heart } \\
\hline disease . . . . . . . . . . . . . . (120,125) & 221,582 & 116,742 & 104,840 & 27,655 & 14,254 & 13,401 & 509 & 328 & 181 \\
\hline \multicolumn{10}{|l|}{ Atherosclerotic cardiovascular } \\
\hline disease, so described . . . . . . . . (125.0) & 45,326 & 25,224 & 20,102 & 8,796 & 5,070 & 3,726 & 259 & 187 & 72 \\
\hline \multicolumn{10}{|l|}{ All other forms of chronic ischemic } \\
\hline heart disease $\ldots \ldots \ldots(120,125.1-\mid 25.9)$ & 176,256 & 91,518 & 84,738 & 18,859 & 9,184 & 9,675 & 250 & 141 & 109 \\
\hline Other heart diseases. & 142,820 & 62,599 & 80,221 & 20,206 & 9,660 & 10,546 & 195 & 107 & 88 \\
\hline Acute and subacute endocarditis & 889 & 500 & 389 & 222 & 143 & 79 & 1 & 1 & - \\
\hline \multicolumn{10}{|l|}{ Diseases of pericardium and acute } \\
\hline myocarditis . . . . . . . . . . (I30-I31,|40) & 633 & 324 & 309 & 143 & 74 & 69 & 1 & - & 1 \\
\hline Heart failure . . . . . . . . . . (150) & 48,480 & 19,413 & 29,067 & 5,464 & 2,328 & 3,136 & 47 & 23 & 24 \\
\hline All other forms of heart disease . (I26-I28, & & & & & & & & & \\
\hline |34-|38,|142-|49,|151) & 92,818 & 42,362 & 50,456 & 14,377 & 7,115 & 7,262 & 146 & 83 & 63 \\
\hline \multicolumn{10}{|l|}{ Essential hypertension and hypertensive } \\
\hline renal disease $\ldots \ldots \ldots \ldots \ldots(110,|12| 115)$, & 17,216 & 6,480 & 10,736 & 4,597 & 1,963 & 2,634 & 28 & 10 & 18 \\
\hline Cerebrovascular diseases . . . . . . . (160-169) & 107,678 & 41,416 & 66,262 & 16,934 & 7,470 & 9,464 & 169 & 95 & 74 \\
\hline Atherosclerosis . . . . . . . . . . . . . (170) & 7,119 & 2,758 & 4,361 & 643 & 259 & 384 & 8 & 4 & 4 \\
\hline Other diseases of circulatory system . . . (171-178) & 18,067 & 9,462 & 8,605 & 2,442 & 1,224 & 1,218 & 23 & 15 & 8 \\
\hline Aortic aneurysm and dissection $\ldots \ldots \ldots$ (171) & 10,890 & 6,325 & 4,565 & 1,241 & 694 & 547 & 9 & 7 & 2 \\
\hline \multicolumn{10}{|l|}{ Other diseases of arteries, arterioles and } \\
\hline$\ldots \ldots \ldots \ldots \ldots .(172-178)$ & 7,177 & 3,137 & 4,040 & 1,201 & 530 & 671 & 14 & 8 & 6 \\
\hline Other disorders of circulatory system & 3,092 & 1,398 & 1,694 & 692 & 344 & 348 & 13 & 7 & 6 \\
\hline Influenza and pneumonia . . . . . . & 43,219 & 19,361 & 23,858 & 5,091 & 2,464 & 2,627 & 86 & 48 & 38 \\
\hline Influenza. . . . . . . . . & 342 & 151 & 191 & 26 & 13 & 13 & 1 & 1 & - \\
\hline . .(J12-J18) & 42,877 & 19,210 & 23,667 & 5,065 & 2,451 & 2,614 & 85 & 47 & 38 \\
\hline \multicolumn{10}{|l|}{ Other acute lower respiratory } \\
\hline infections ........... & 195 & 90 & 105 & 32 & 16 & 16 & - & - & - \\
\hline Acute bronchitis and bronchiolitis . . . . (J20-J21) & 158 & 74 & 84 & 28 & 15 & 13 & - & - & - \\
\hline \multicolumn{10}{|l|}{ Other and unspecified acute lower respiratory } \\
\hline infections $\ldots \ldots \ldots \ldots \ldots(\mathrm{J} 22, \mathrm{U} 04)^{5,6}$ & 37 & 16 & 21 & 4 & 1 & 3 & - & - & - \\
\hline Chronic lower respiratory diseases. & 114,480 & 53,989 & 60,491 & 7,830 & 4,167 & 3,663 & 176 & 107 & 69 \\
\hline Bronchitis, chronic and unspecified . . . .(J40-J42) & 554 & 216 & 338 & 56 & 24 & 32 & 1 & 1 & - \\
\hline Emphysema $\ldots \ldots \ldots \ldots \ldots \ldots \ldots$ (J43) & 11,575 & 5,840 & 5,735 & 728 & 455 & 273 & 23 & 14 & 9 \\
\hline Asthma $\ldots \ldots \ldots \ldots \ldots \ldots \ldots \ldots$ (J45-J46) & 2,151 & 686 & 1,465 & 893 & 402 & 491 & 1 & 1 & - \\
\hline
\end{tabular}

See footnotes at end of table. 
Table 13. Number of deaths from 113 selected causes and Enterocolitis due to Clostridium difficile, by Hispanic origin, race for non-Hispanic population, and sex: United States, 2007-Con.

[Race and Hispanic origin are reported separately on the death certificate. Persons of Hispanic origin may be of any race. Data for Hispanic persons are not tabulated separately by race; data for non-Hispanic persons are tabulated by race. Data for Hispanic origin should be interpreted with caution because of inconsistencies between reporting Hispanic origin on death certificates and on censuses and surveys; see "Technical Notes." The asterisks (*) preceding the cause-of-death codes indicate that they are not part of the International Classification of Diseases, Tenth Revision (ICD-10), Second Edition; see "Technical Notes"]

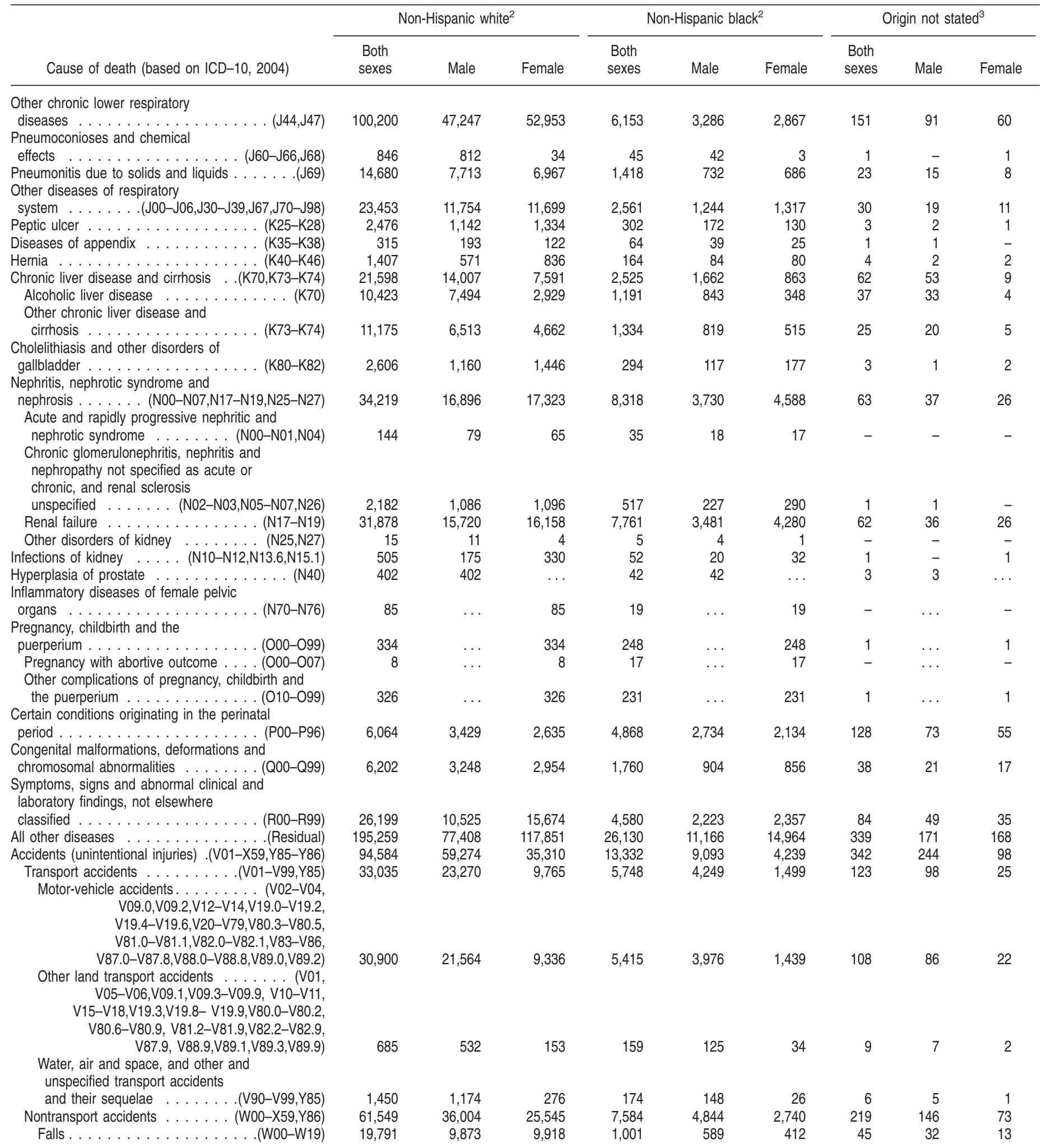

See footnotes at end of table. 
Table 13. Number of deaths from 113 selected causes and Enterocolitis due to Clostridium difficile, by Hispanic origin, race for non-Hispanic population, and sex: United States, 2007-Con.

[Race and Hispanic origin are reported separately on the death certificate. Persons of Hispanic origin may be of any race. Data for Hispanic persons are not tabulated separately by race; data for non-Hispanic persons are tabulated by race. Data for Hispanic origin should be interpreted with caution because of inconsistencies between reporting Hispanic origin on death certificates and on censuses and surveys; see "Technical Notes." The asterisks $\left({ }^{*}\right)$ preceding the cause-of-death codes indicate that they are not part of the International Classification of Diseases, Tenth Revision (ICD-10), Second Edition; see "Technical Notes"]

\begin{tabular}{|c|c|c|c|c|c|c|c|c|c|}
\hline \multirow[b]{2}{*}{ Cause of death (based on ICD-10, 2004) } & \multicolumn{3}{|c|}{ Non-Hispanic white ${ }^{2}$} & \multicolumn{3}{|c|}{ Non-Hispanic black ${ }^{2}$} & \multicolumn{3}{|c|}{ Origin not stated ${ }^{3}$} \\
\hline & $\begin{array}{l}\text { Both } \\
\text { sexes }\end{array}$ & Male & Female & $\begin{array}{l}\text { Both } \\
\text { sexes }\end{array}$ & Male & Female & $\begin{array}{l}\text { Both } \\
\text { sexes }\end{array}$ & Male & Female \\
\hline Accidental discharge of firearms. . . .(W32-W34) & 404 & 349 & 55 & 126 & 113 & 13 & - & - & - \\
\hline $\begin{array}{l}\text { Accidental drowning and } \\
\text { submersion .................(W65-W74) } \\
\text { Accidental exposure to smoke, fire and }\end{array}$ & 2,243 & 1,688 & 555 & 502 & 410 & 92 & 17 & 15 & 2 \\
\hline $\begin{array}{l}\text { flames .................... (X00-X09) } \\
\text { Accidental poisoning and exposure to }\end{array}$ & 2,188 & 1,276 & 912 & 764 & 454 & 310 & 15 & 7 & 8 \\
\hline $\begin{array}{l}\text { noxious substances ........... (X40-X49) } \\
\text { Other and unspecified nontransport } \\
\text { accidents and their sequelae .....W20-W31, }\end{array}$ & 23,531 & 15,204 & 8,327 & 3,215 & 2,107 & 1,108 & 103 & 61 & 42 \\
\hline $\begin{array}{l}\text { W35-W64,W75-W99,X10-X39,X50-X59,Y86) } \\
\text { Intentional self-harm }\end{array}$ & 13,392 & 7,614 & 5,778 & 1,976 & 1,171 & 805 & 39 & 31 & 8 \\
\hline $\begin{array}{l}\left.\text { (suicide) . . . . . . . . . . . ( }{ }^{*} \cup 03, \mathrm{X} 60-\mathrm{X} 84, \mathrm{Y} 87.0\right) \\
\text { Intentional self-harm (suicide) by discharge of }\end{array}$ & 28,897 & 22,660 & 6,237 & 1,916 & 1,571 & 345 & 72 & 58 & 14 \\
\hline $\begin{array}{l}\text { firearms. } \ldots \text { ntional self-harm (suicide) by other and } \\
\text { unspecified means and their }\end{array}$ & 15,073 & 13,129 & 1,944 & 975 & 870 & 105 & 35 & 31 & 4 \\
\hline sequelae ..... (*U03,X60-X71,X75-X84,Y87.0) & 13,824 & 9,531 & 4,293 & 941 & 701 & 240 & 37 & 27 & 10 \\
\hline $\begin{array}{l}\text { Assault (homicide). . . . . (*U01-*U02,X85-Y09,Y87.1) } \\
\text { Assault (homicide) by discharge of }\end{array}$ & 5,512 & 3,669 & 1,843 & 8,746 & 7,477 & 1,269 & 97 & 83 & 14 \\
\hline 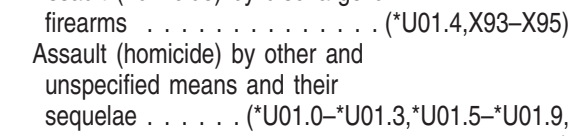 & 3,053 & 2,165 & 888 & 6,867 & 6,192 & 675 & 54 & 50 & 4 \\
\hline$\left.{ }^{*} \cup 02, X 85-X 92, X 96-Y 09, Y 87.1\right)$ & 2,459 & 1,504 & 955 & 1,879 & 1,285 & 594 & 43 & 33 & 10 \\
\hline $\begin{array}{l}\text { Legal intervention . . . . . . . . . . . (Y35,Y89.0) } \\
\text { Events of undetermined }\end{array}$ & 183 & 174 & 9 & 124 & 121 & 3 & 1 & 1 & - \\
\hline $\begin{array}{l}\text { intent. . . . . . . . . . . . . (Y10-Y34,Y87.2,Y89.9) } \\
\text { Discharge of firearms, undetermined }\end{array}$ & 4,122 & 2,490 & 1,632 & 747 & 517 & 230 & 20 & 14 & 6 \\
\hline $\begin{array}{l}\text { intent . . . . . . . . . . . . . . . (Y22-Y24) } \\
\text { Other and unspecified events of } \\
\text { undetermined intent and their }\end{array}$ & 178 & 138 & 40 & 57 & 46 & 11 & 1 & 1 & - \\
\hline sequelae . . . . . (Y10-Y21,Y25-Y34,Y87.2,Y89.9) & 3,944 & 2,352 & 1,592 & 690 & 471 & 219 & 19 & 13 & 6 \\
\hline $\begin{array}{l}\text { Operations of war and their sequelae . . . (Y36,Y89.1) } \\
\text { Complications of medical and surgical }\end{array}$ & 18 & 18 & - & - & - & - & - & - & - \\
\hline care . . . . . . . . . . . . . . (Y40-Y84,Y88) & 1,967 & 948 & 1,019 & 430 & 187 & 243 & 1 & 1 & - \\
\hline Enterocolitis due to Clostridium difficile . . . (A04.7) & 5,657 & 2,109 & 3,548 & 391 & 149 & 242 & 3 & 1 & 2 \\
\hline
\end{tabular}

\footnotetext{
- Quantity zero.
}

.. Category not applicable.

${ }^{1}$ Includes races other than white and black.

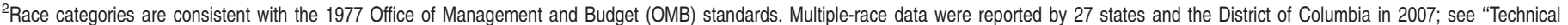
Notes." The multiple-race data for these reporting areas were bridged to the single-race categories of the 1977 OMB standards for comparability with other reporting areas; see "Technical Notes."

${ }^{3}$ Includes deaths for which Hispanic origin was not reported on the death certificate.

${ }^{4} \mathrm{New}$ ICD-10 code J09 (Influenza due to identified avian influenza virus) was added to the category in 2007. No deaths occurred from this cause in 2007.

${ }^{5} \mathrm{New}$ ICD-10 code U04 (Severe acute respiratory syndrome [SARS]) was added to the category in 2007. No deaths occurred from this cause in 2007.

${ }^{6}$ Cause-of-death title was changed in 2007 to reflect the addition of SARS (ICD-10 code U04).

${ }^{7}$ Included in "Certain other intestinal infections (A04,A07-A09)" shown above. Beginning with data year 2006, "Enterocolitis due to Clostridium difficile (A04.7)" is shown separately at the bottom of tables showing 113 selected causes and is included in the list of rankable causes; see "Technical Notes."

NOTE: Complete confirmation of deaths from selected causes of death, considered to be of public health concern, was not provided by the following states: Connecticut, Florida, Indiana, Kentucky, Maryland, North Carolina, Oklahoma, Pennsylvania, Rhode Island, South Carolina, Washington, and West Virginia; see "Technical Notes." 
Table 14. Death rates for 113 selected causes and Enterocolitis due to Clostridium difficile, by race and sex: United States, 2007

[Rates per 100,000 population in specified group. Populations used for computing death rates are postcensal estimates based on the 2000 census, estimated as of July 1, 2007; see "Technical Notes." Data for specified races other than white and black should be interpreted with caution because of inconsistencies between reporting race on death certificates and on censuses and surveys; see "Technical Notes." The asterisks $\left(^{*}\right)$ preceding the cause-of-death codes indicate that they are not part of the International Classification of Diseases, Tenth Revision (ICD-10), Second Edition; see "Technical Notes"]

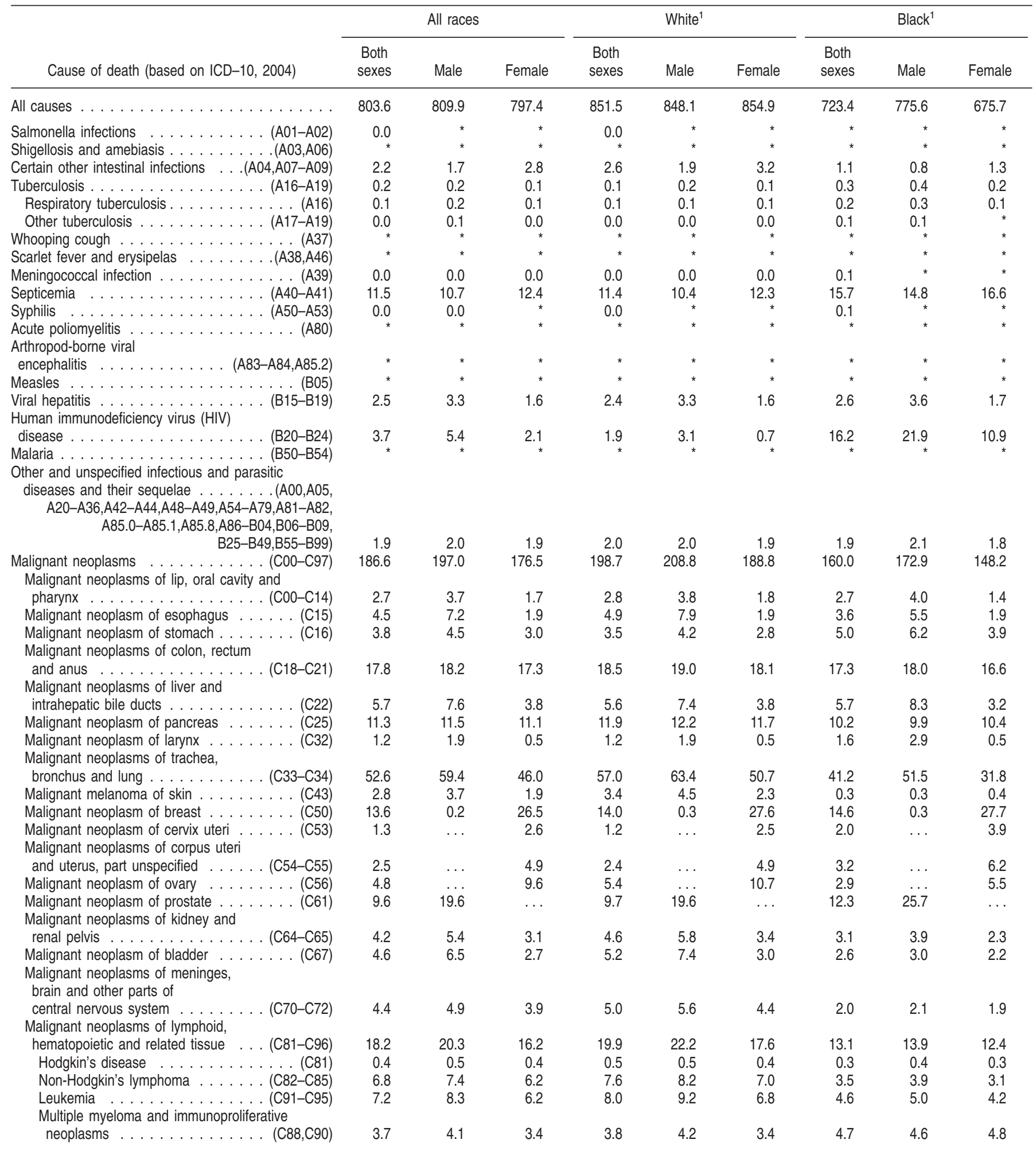

See footnotes at end of table. 

Table 14. Death rates for 113 selected causes and Enterocolitis due to Clostridium difficile, by race and sex:
United States, 2007-Con.

[Rates per 100,000 population in specified group. Populations used for computing death rates are postcensal estimates based on the 2000 census, estimated as of July 1, 2007; see "Technical Notes." Data for specified races other than white and black should be interpreted with caution because of inconsistencies between reporting race on death certificates and on censuses and surveys; see "Technical Notes." The asterisks (*) preceding the cause-of-death codes indicate that they are not part of the International Classification of Diseases, Tenth Revision (ICD-10), Second Edition; see "Technical Notes"]

\begin{tabular}{|c|c|c|c|c|c|c|c|c|c|}
\hline \multirow[b]{2}{*}{ Cause of death (based on ICD-10, 2004) } & \multicolumn{3}{|c|}{ All races } & \multicolumn{3}{|c|}{ White ${ }^{1}$} & \multicolumn{3}{|c|}{ Black $^{1}$} \\
\hline & $\begin{array}{l}\text { Both } \\
\text { sexes }\end{array}$ & Male & Female & $\begin{array}{l}\text { Both } \\
\text { sexes }\end{array}$ & Male & Female & $\begin{array}{l}\text { Both } \\
\text { sexes }\end{array}$ & Male & Female \\
\hline \multicolumn{10}{|l|}{$\begin{array}{l}\text { Other and unspecified malignant neoplasms } \\
\text { of lymphoid, hematopoietic and }\end{array}$} \\
\hline related tissue $\ldots \ldots \ldots \ldots \ldots \ldots$ (C96) & 0.0 & 0.0 & 0.0 & 0.0 & 0.0 & 0.0 & * & * & * \\
\hline \multicolumn{10}{|l|}{$\begin{array}{l}\text { All other and unspecified malignant } \\
\text { neoplasms }\end{array}$} \\
\hline $\begin{array}{l}\text { C37-C41,C44-C49,C51- C52,C57-C60, } \\
\text { C62-C63,C66,C68-C69,C73-C80, C97) }\end{array}$ & 21.0 & 22.1 & 19.8 & 22.5 & 23.7 & 21.3 & 16.7 & 17.5 & 15.9 \\
\hline \multicolumn{10}{|l|}{$\begin{array}{l}\text { In situ neoplasms, benign neoplasms and } \\
\text { neoplasms of uncertain or unknown }\end{array}$} \\
\hline behavior $\ldots \ldots \ldots \ldots \ldots \ldots \ldots$. (D00-D48) & 4.7 & 4.9 & 4.5 & 5.2 & 5.4 & 5.0 & 3.0 & 3.1 & 2.9 \\
\hline Anemias . . . . . . . . . . . . . . . . . . (D50-D64) & 1.6 & 1.3 & 1.9 & 1.5 & 1.2 & 1.9 & 2.4 & 2.3 & 2.5 \\
\hline$\ldots \ldots \ldots \ldots(\mathrm{E} 10-\mathrm{E} 14)$ & 23.7 & 23.9 & 23.5 & 23.2 & 23.8 & 22.5 & 31.1 & 28.7 & 33.3 \\
\hline Nutritional deficiencies & 0.9 & 0.7 & 1.2 & 1.0 & 0.7 & 1.2 & 1.0 & 0.9 & 1.1 \\
\hline Malnutrition . . . . . & 0.9 & 0.7 & 1.1 & 0.9 & 0.7 & 1.1 & 0.9 & 0.8 & 1.0 \\
\hline Other nutritional deficiencies . . . . . . . (E50-E64) & 0.1 & 0.1 & 0.1 & 0.1 & 0.1 & 0.1 & * & * & * \\
\hline Meningitis $\ldots \ldots \ldots \ldots \ldots \ldots(\mathrm{G}, \ldots \ldots, \mathrm{G} 03)$ & 0.2 & 0.2 & 0.2 & 0.2 & 0.2 & 0.2 & 0.3 & 0.4 & 0.3 \\
\hline Parkinson's disease $\ldots \ldots \ldots \ldots \ldots \ldots$ (G20-G21) & 6.7 & 7.8 & 5.6 & 7.7 & 9.0 & 6.5 & 2.0 & 2.3 & 1.7 \\
\hline Alzheimer's disease . . . . . . . . . . . . . . (G30) & 24.7 & 14.7 & 34.5 & 28.3 & 16.7 & 39.7 & 11.9 & 6.8 & 16.5 \\
\hline Major cardiovascular diseases . . . . . . . (100-178) & 267.3 & 261.0 & 273.3 & 283.8 & 274.6 & 292.8 & 240.0 & 244.2 & 236.1 \\
\hline Diseases of heart $\ldots . .(100-109,|11| 13,,|20-| 51)$ & 204.3 & 208.4 & 200.2 & 218.3 & 221.1 & 215.5 & 177.9 & 186.5 & 170.0 \\
\hline \multicolumn{10}{|l|}{ Acute rheumatic fever and chronic } \\
\hline rheumatic heart diseases $\ldots .$. & 1.1 & 0.7 & 1.4 & 1.2 & 0.8 & 1.6 & 0.6 & 0.4 & 0.8 \\
\hline Hypertensive heart disease $\ldots \ldots \ldots$. . . (111) & 10.2 & 9.6 & 10.8 & 9.4 & 8.5 & 10.4 & 17.8 & 19.2 & 16.4 \\
\hline Hypertensive heart and renal disease . . . . (113) & 1.0 & 0.9 & 1.1 & 0.8 & 0.7 & 1.0 & 2.1 & 2.2 & 2.0 \\
\hline Ischemic heart diseases . . . . . . . (I20-I25) & 134.7 & 145.3 & 124.4 & 145.5 & 156.6 & 134.7 & 106.6 & 113.8 & 100.0 \\
\hline Acute myocardial infarction . . . . . . (121-122) & 44.1 & 48.2 & 40.0 & 47.6 & 52.2 & 43.1 & 35.2 & 36.6 & 34.0 \\
\hline Other acute ischemic heart diseases . . . (I24) & 1.4 & 1.5 & 1.3 & 1.4 & 1.5 & 1.3 & 1.3 & 1.6 & 1.1 \\
\hline \multicolumn{10}{|l|}{ Other forms of chronic ischemic heart } \\
\hline disease . . . . . . . . . . . . . . (120,|25) & 89.3 & 95.6 & 83.1 & 96.5 & 102.9 & 90.3 & 70.1 & 75.7 & 64.9 \\
\hline \multicolumn{10}{|l|}{ Atherosclerotic cardiovascular disease, } \\
\hline so described $\ldots \ldots \ldots \ldots \ldots$ (125.0) & 19.6 & 22.5 & 16.8 & 20.0 & 22.7 & 17.3 & 22.3 & 27.0 & 18.0 \\
\hline \multicolumn{10}{|l|}{ All other forms of chronic ischemic heart } \\
\hline disease $\ldots \ldots \ldots \ldots(120,|25.1-| 25.9)$ & 69.7 & 73.2 & 66.3 & 76.6 & 80.2 & 72.9 & 47.8 & 48.7 & 46.9 \\
\hline Other heart diseases . . . . . . . . . . (I26-151) & 57.3 & 51.9 & 62.5 & 61.3 & 54.6 & 67.9 & 50.8 & 50.9 & 50.8 \\
\hline Acute and subacute endocarditis . . . . (I33) & 0.4 & 0.5 & 0.3 & 0.4 & 0.5 & 0.3 & 0.6 & 0.8 & 0.4 \\
\hline \multicolumn{10}{|l|}{ Diseases of pericardium and acute } \\
\hline myocarditis $\ldots \ldots \ldots \ldots(|30-| 31, \mid 40)$ & 0.3 & 0.3 & 0.3 & 0.3 & 0.3 & 0.3 & 0.4 & 0.4 & 0.3 \\
\hline Heart failure $\ldots \ldots \ldots \ldots \ldots$. $\ldots \ldots 0)$ & 18.8 & 15.4 & 22.0 & 20.7 & 16.8 & 24.5 & 13.7 & 12.2 & 15.1 \\
\hline All other forms of heart disease . (I26-I28, & & & & & & & & & \\
\hline |34-|38,|42-|49,|51) & 37.8 & 35.7 & 39.9 & 39.9 & 37.0 & 42.8 & 36.2 & 37.5 & 35.0 \\
\hline \multicolumn{10}{|l|}{ Essential hypertension and hypertensive renal } \\
\hline disease $\ldots \ldots \ldots \ldots \ldots \ldots(110,|12| 115)$, & 7.9 & 6.3 & 9.5 & 7.6 & 5.9 & 9.3 & 11.6 & 10.3 & 12.7 \\
\hline Cerebrovascular diseases . . . . . . . . (160-169) & 45.1 & 36.4 & 53.5 & 47.1 & 37.0 & 57.0 & 42.7 & 39.5 & 45.6 \\
\hline Atherosclerosis . . . . . . . . . . . . . . . (170) & 2.7 & 2.2 & 3.3 & 3.1 & 2.4 & 3.7 & 1.6 & 1.4 & 1.9 \\
\hline Other diseases of circulatory system . . . (171-178) & 7.3 & 7.7 & 6.8 & 7.8 & 8.2 & 7.3 & 6.2 & 6.5 & 5.9 \\
\hline Aortic aneurysm and dissection $\ldots \ldots \ldots$ (171) & 4.3 & 5.1 & 3.6 & 4.7 & 5.5 & 3.9 & 3.1 & 3.7 & 2.6 \\
\hline \multicolumn{10}{|l|}{ Other diseases of arteries, arterioles and } \\
\hline capillaries . . . . . . . . . . . . . (172-178) & 3.0 & 2.6 & 3.3 & 3.1 & 2.8 & 3.5 & 3.0 & 2.8 & 3.2 \\
\hline Other disorders of circulatory system . & 1.4 & 1.3 & 1.4 & 1.4 & 1.3 & 1.5 & 1.8 & 1.8 & 1.7 \\
\hline Influenza and pneumonia . . . . . . . . (J09-J18) & 17.5 & 16.2 & 18.7 & 18.9 & 17.2 & 20.5 & 12.9 & 13.1 & 12.7 \\
\hline Influenza. . . . . . . . . & 0.1 & 0.1 & 0.1 & 0.2 & 0.1 & 0.2 & 0.1 & * & * \\
\hline Pneumonia ..... & 17.3 & 16.1 & 18.6 & 18.7 & 17.0 & 20.4 & 12.8 & 13.0 & 12.6 \\
\hline \multicolumn{10}{|l|}{ Other acute lower respiratory } \\
\hline infections ............ & 0.1 & 0.1 & 0.1 & 0.1 & 0.1 & 0.1 & 0.1 & * & * \\
\hline Acute bronchitis and bronchiolitis . . . . (J20-J21) & 0.1 & 0.1 & 0.1 & 0.1 & 0.1 & 0.1 & 0.1 & * & * \\
\hline $\begin{array}{l}\text { Other and unspecified acute lower } \\
\text { respiratory infections }\end{array}$ & & & & & * & CO & * & * & * \\
\hline $\begin{array}{l}\text { respiratory intectlons ........... } \\
\text { Chronic lower respiratory diseases . }\end{array}$ & $\begin{array}{r}0.0 \\
42.4\end{array}$ & 41.2 & $\begin{array}{r}0.0 \\
43.6\end{array}$ & 48.5 & 46.3 & $\begin{array}{r}0.0 \\
50.6\end{array}$ & 19.7 & 22.0 & 17.7 \\
\hline Bronchitis, chronic and unspecified . . . (J40-J42) & 0.2 & 0.2 & 0.3 & 0.2 & 0.2 & 0.3 & 0.1 & 0.1 & 0.2 \\
\hline Emphysema . . . . . . . . . . . . . . . . (J43) & 4.2 & 4.4 & 4.0 & 4.9 & 5.0 & 4.8 & 1.8 & 2.4 & 1.3 \\
\hline
\end{tabular}

See footnotes at end of table. 
Table 14. Death rates for 113 selected causes and Enterocolitis due to Clostridium difficile, by race and sex: United States, 2007-Con.

[Rates per 100,000 population in specified group. Populations used for computing death rates are postcensal estimates based on the 2000 census, estimated as of July 1, 2007; see "Technical Notes." Data for specified races other than white and black should be interpreted with caution because of inconsistencies between reporting race on death certificates and on censuses and surveys; see "Technical Notes." The asterisks (*) preceding the cause-of-death codes indicate that they are not part of the International Classification of Diseases, Tenth Revision (ICD-10), Second Edition; see "Technical Notes"]

\begin{tabular}{|c|c|c|c|c|c|c|c|c|c|}
\hline \multirow[b]{2}{*}{ Cause of death (based on ICD-10, 2004) } & \multicolumn{3}{|c|}{ All races } & \multicolumn{3}{|c|}{ White $^{1}$} & \multicolumn{3}{|c|}{ Black $^{1}$} \\
\hline & $\begin{array}{l}\text { Both } \\
\text { sexes }\end{array}$ & Male & Female & $\begin{array}{l}\text { Both } \\
\text { sexes }\end{array}$ & Male & Female & $\begin{array}{l}\text { Both } \\
\text { sexes }\end{array}$ & Male & Female \\
\hline $\begin{array}{l}\text { Asthma . . . . . . . . . . . . . . . . . (J45-J46) } \\
\text { Other chronic lower respiratory }\end{array}$ & 1.1 & 0.9 & 1.4 & 1.0 & 0.7 & 1.3 & 2.3 & 2.1 & 2.4 \\
\hline diseases . . . . . . . . . . . . . (J44,J47) & 36.8 & 35.7 & 37.9 & 42.4 & 40.5 & 44.2 & 15.5 & 17.4 & 13.8 \\
\hline Pneumoconioses and chemical effects $(\mathrm{J} 60-\mathrm{J} 66, \mathrm{~J} 68)$ & 0.3 & 0.6 & 0.0 & 0.4 & 0.7 & 0.0 & 0.1 & 0.2 & * \\
\hline $\begin{array}{l}\text { Pneumonitis due to solids and liquids . . . . . . . (J69) } \\
\text { Other diseases of respiratory }\end{array}$ & 5.6 & 6.0 & 5.3 & 6.3 & 6.6 & 5.9 & 3.6 & 3.9 & 3.3 \\
\hline system . . . . . (J00-J06,J30- J39,J67,J70-J98) & 9.5 & 9.6 & 9.3 & 10.3 & 10.5 & 10.2 & 6.5 & 6.6 & 6.4 \\
\hline Peptic ulcer . . . . . . . . . . . . . . . . . (K25-K28) & 1.0 & 1.0 & 1.0 & 1.1 & 1.0 & 1.1 & 0.8 & 0.9 & 0.6 \\
\hline Diseases of appendix $\ldots \ldots \ldots \ldots \ldots($ K35-K38) & 0.1 & 0.2 & 0.1 & 0.1 & 0.2 & 0.1 & 0.2 & 0.2 & 0.1 \\
\hline Hernia . . . . . . . . . . . . . . . . . . . . (K40-K46) & 0.6 & 0.5 & 0.6 & 0.6 & 0.5 & 0.7 & 0.4 & 0.4 & 0.4 \\
\hline Chronic liver disease and cirrhosis ..(K70,K73-K74) & 9.7 & 12.9 & 6.5 & 10.5 & 13.9 & 7.1 & 6.4 & 8.8 & 4.2 \\
\hline $\begin{array}{l}\text { Alcoholic liver disease ... . . . . . . . . . (K70) } \\
\text { Other chronic liver disease and }\end{array}$ & 4.8 & 7.1 & 2.5 & 5.1 & 7.7 & 2.7 & 3.0 & 4.5 & 1.7 \\
\hline cirrhosis .................. (K73-K74) & 4.9 & 5.8 & 4.0 & 5.3 & 6.2 & 4.4 & 3.4 & 4.3 & 2.5 \\
\hline $\begin{array}{l}\text { Cholelithiasis and other disorders of } \\
\text { gallbladder .................... (K80-K82) } \\
\text { Nephritis, nephrotic syndrome and }\end{array}$ & 1.1 & 1.0 & 1.2 & 1.2 & 1.1 & 1.3 & 0.7 & 0.6 & 0.9 \\
\hline $\begin{array}{l}\text { nephrotic syndrome ........... (N00-N01,N04) } \\
\text { Chronic glomerulonephritis, nephritis and } \\
\text { nephropathy not specified as acute or } \\
\text { chronic, and renal sclerosis }\end{array}$ & 0.1 & 0.1 & 0.1 & 0.1 & 0.1 & 0.1 & 0.1 & * & * \\
\hline unspecified $\ldots \ldots \ldots$ (N02-N03,N05-N07,N26) & 1.0 & 1.0 & 1.0 & 1.0 & 1.0 & 1.0 & 1.3 & 1.2 & 1.4 \\
\hline Renal failure . . . . . . . . . . (N17-N19) & 14.3 & 14.2 & 14.5 & 14.1 & 14.0 & 14.1 & 19.6 & 18.4 & 20.6 \\
\hline Other disorders of kidney $\ldots \ldots \ldots(\mathrm{N} 25, \mathrm{~N} 27)$ & 0.0 & * & * & * & * & * & * & * & * \\
\hline Infections of kidney $\ldots \ldots(\mathrm{N} 10-\mathrm{N} 12, \mathrm{~N} 13.6, \mathrm{~N} 15.1)$ & 0.2 & 0.1 & 0.3 & 0.2 & 0.2 & 0.3 & 0.1 & 0.1 & 0.2 \\
\hline $\begin{array}{l}\text { Hyperplasia of prostate } \ldots \ldots \ldots \ldots \ldots \text { (N40) } \\
\text { Inflammatory diseases of female pelvic }\end{array}$ & 0.2 & 0.3 & $\cdots$ & 0.2 & 0.4 & $\cdots$ & 0.1 & 0.2 & $\ldots$ \\
\hline $\begin{array}{l}\text { organs } \ldots \ldots \ldots \ldots \text { (N70-N76) } \\
\text { Pregnancy, childbirth and the }\end{array}$ & 0.0 & $\ldots$ & 0.1 & 0.0 & $\ldots$ & 0.1 & 0.0 & $\ldots$ & 0.1 \\
\hline puerperium . . . . . . . . . . . . (000-099) & 0.3 & $\ldots$ & 0.5 & 0.2 & $\ldots$ & 0.4 & 0.6 & $\ldots$ & 1.2 \\
\hline $\begin{array}{l}\text { Pregnancy with abortive outcome .... (O00-O07) } \\
\text { Other complications of pregnancy, childbirth and }\end{array}$ & 0.0 & $\ldots$ & 0.0 & 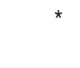 & $\ldots$ & * & * & $\ldots$ & * \\
\hline $\begin{array}{l}\text { the puerperium } \ldots \ldots \ldots \ldots \ldots(010-099) \\
\text { Certain conditions originating in the perinatal }\end{array}$ & 0.2 & $\ldots$ & 0.5 & 0.2 & $\ldots$ & 0.4 & 0.6 & $\ldots$ & 1.1 \\
\hline $\begin{array}{l}\text { period ........................... P00-P96) } \\
\text { Congenital malformations, deformations and }\end{array}$ & 4.8 & 5.5 & 4.2 & 3.6 & 4.1 & 3.2 & 12.6 & 14.9 & 10.6 \\
\hline chromosomal abnormalities ......... (Q00-Q99) & 3.5 & 3.7 & 3.2 & 3.3 & 3.6 & 3.1 & 4.5 & 4.8 & 4.2 \\
\hline $\begin{array}{l}\text { Symptoms, signs and abnormal clinical and } \\
\text { laboratory findings, not elsewhere }\end{array}$ & & & & & & & & & \\
\hline classified . . . . . . . . . . . . . (R00-R99) & 11.1 & 9.6 & 12.6 & 11.5 & 9.6 & 13.4 & 11.6 & 11.8 & 11.4 \\
\hline $\begin{array}{l}\text { All other diseases } \ldots \ldots \ldots \text {. } \ldots \ldots \ldots \text { (residual) } \\
\text { Accidents (unintentional injuries) } \ldots \ldots \ldots \text { (V01-X59, }\end{array}$ & 79.0 & 64.9 & 92.7 & 85.0 & 68.7 & 100.9 & 65.9 & 59.0 & 72.2 \\
\hline Y85-Y86) & 41.0 & 53.7 & 28.7 & 43.6 & 56.4 & 31.1 & 33.9 & 48.5 & 20.5 \\
\hline $\begin{array}{r}\text { Transport accidents } \ldots \ldots \ldots \text {. } \ldots 01-V 99, Y 85) \\
\text { Motor-vehicle accidents . . . . . (V02-V04, } \\
\text { V09.0,V09.2,V12-V14,V19.0-V19.2, } \\
\text { V19.4-V19.6,V20-V79,V80.3-V80.5, } \\
\text { V81.0-V81.1,V82.0-V82.1,V83-V86, }\end{array}$ & 15.5 & 22.5 & 8.8 & 16.0 & 23.1 & 9.1 & 14.6 & 22.7 & 7.3 \\
\hline V87.0-V87.8,V88.0-V88.8,V89.0,V89.2) & 14.6 & 20.9 & 8.4 & 15.0 & 21.5 & 8.8 & 13.8 & 21.2 & 7.0 \\
\hline $\begin{array}{r}\text { Other land transport accidents. . . . . (V01, } \\
\text { V05-V06,V09.1,V09.3-V09.9,V10-V11, } \\
\text { V15-V18,V19.3,V19.8-V19.9,V80.0-V80.2, } \\
\text { V80.6-V80.9,V81.2-V81.9,V82.2-V82.9, } \\
\text { V87.9,V88.9,V89.1,V89.3,V89.9) }\end{array}$ & 0.4 & 0.6 & 0.1 & 0.3 & 0.6 & 0.1 & 0.4 & 0.7 & 0.2 \\
\hline
\end{tabular}

See footnotes at end of table. 
Table 14. Death rates for 113 selected causes and Enterocolitis due to Clostridium difficile, by race and sex: United States, 2007-Con.

[Rates per 100,000 population in specified group. Populations used for computing death rates are postcensal estimates based on the 2000 census, estimated as of July 1, 2007; see "Technical Notes." Data for specified races other than white and black should be interpreted with caution because of inconsistencies between reporting race on death certificates and on censuses and surveys; see "Technical Notes." The asterisks (*) preceding the cause-of-death codes indicate that they are not part of the International Classification of Diseases, Tenth Revision (ICD-10), Second Edition; see "Technical Notes"]

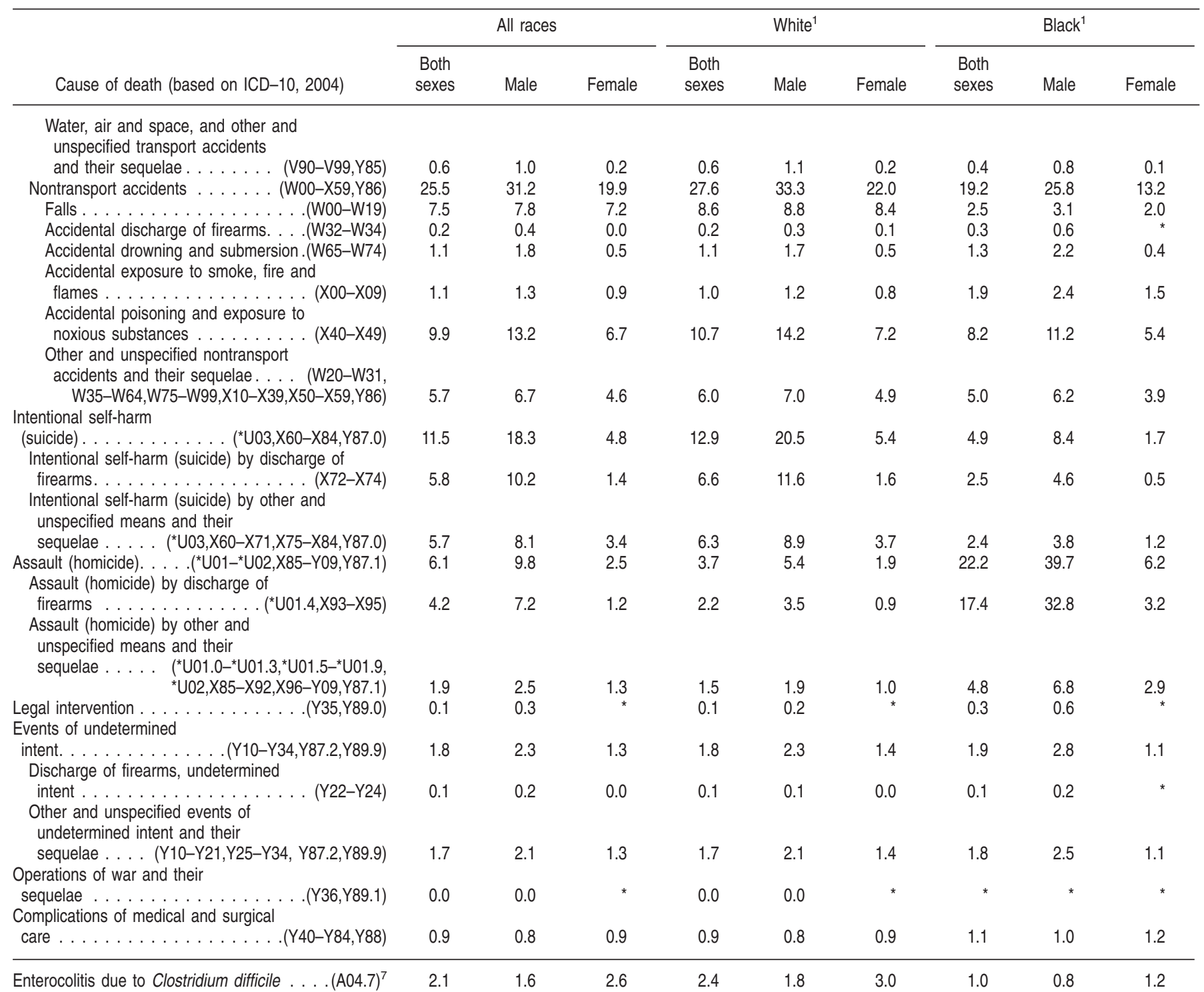

See footnotes at end of table. 
Table 14. Death rates for 113 selected causes and Enterocolitis due to Clostridium difficile, by race and sex: United States, 2007-Con.

[Rates per 100,000 population in specified group. Populations used for computing death rates are postcensal estimates based on the 2000 census, estimated as of July 1, 2007; see "Technical Notes." Data for specified races other than white and black should be interpreted with caution because of inconsistencies between reporting race on death certificates and on censuses and surveys; see "Technical Notes." The asterisks (*) preceding the cause-of-death codes indicate that they are not part of the International Classification of Diseases, Tenth Revision (ICD-10), Second Edition; see "Technical Notes"]

\begin{tabular}{|c|c|c|c|c|c|c|}
\hline \multirow[b]{2}{*}{ Cause of death (based on ICD-10, 2004) } & \multicolumn{3}{|c|}{ American Indian or Alaska Native $e^{1,2}$} & \multicolumn{3}{|c|}{ Asian or Pacific Islander ${ }^{1,3}$} \\
\hline & $\begin{array}{l}\text { Both } \\
\text { sexes }\end{array}$ & Male & Female & $\begin{array}{l}\text { Both } \\
\text { sexes }\end{array}$ & Male & Female \\
\hline$\ldots \ldots \ldots \ldots \ldots$ & 444.0 & 488.2 & 400.0 & 308.7 & 331.4 & 287.2 \\
\hline Salmonella infections & * & * & * & * & * & * \\
\hline Shigellosis and amebiasis . . . . . . . . . (A03,A06) & * & * & * & * & * & * \\
\hline Certain other intestinal infections $\ldots$ (A04,A07-A09) & 1.0 & * & * & 0.3 & 0.3 & 0.4 \\
\hline Tuberculosis . . . . . . . . . . . . . . . (A16-A19) & * & * & * & 0.5 & 0.6 & 0.4 \\
\hline Respiratory tuberculosis. & * & * & * & 0.4 & 0.5 & 0.3 \\
\hline Other tuberculosis $\ldots \ldots \ldots \ldots$ (A17-A19) & * & * & * & * & * & * \\
\hline Whooping cough $\ldots \ldots \ldots \ldots \ldots$ (A37) & * & * & * & * & * & * \\
\hline Scarlet fever and erysipelas $\ldots \ldots \ldots \ldots(\mathrm{A} 38, \mathrm{~A} 46)$ & * & * & * & * & * & * \\
\hline Meningococcal infection . . . . . . . . . . . . . (A39) & * & * & * & * & * & * \\
\hline Septicemia $\ldots \ldots \ldots \ldots \ldots$ (A40-A41) & 7.1 & 6.3 & 7.9 & 3.7 & 4.2 & 3.3 \\
\hline Syphilis . . . . . . . . . . . . . (A50-A53) & * & * & * & * & * & * \\
\hline Acute poliomyelitis . . . . . . . . . . . (A80) & * & * & * & * & * & * \\
\hline Arthropod-borne viral encephalitis . (A83-A84,A85.2) & * & * & * & * & * & * \\
\hline Measles . . . . . . . . . . . . . . . (B05) & * & * & * & * & * & * \\
\hline Viral hepatitis . . . . . . . . . (B15-B19) & 3.2 & 4.1 & 2.4 & 2.0 & 2.1 & 1.9 \\
\hline \multicolumn{7}{|l|}{ Human immunodeficiency virus (HIV) } \\
\hline disease $\ldots \ldots \ldots \ldots \ldots \ldots \ldots$ (B20-B24) & 2.4 & 3.3 & 1.5 & 0.5 & 0.8 & * \\
\hline 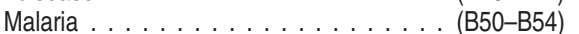 & * & * & * & * & * & * \\
\hline \multicolumn{7}{|l|}{$\begin{array}{r}\text { diseases and their sequelae } . . . . . A 00, A 05 \\
\text { A20-A36,A42-A44,A48-A49,A54-A79,A81-A82, } \\
\text { A85.0-A85.1,A85.8,A86-B04,B06-B09, }\end{array}$} \\
\hline B25-B49,B55-B99) & 1.6 & 1.8 & 1.4 & 1.1 & 1.2 & 1.0 \\
\hline Malignant neoplasms $\ldots \ldots \ldots \ldots \ldots$ (COO-C97) & 79.1 & 83.3 & 75.0 & 83.4 & 89.0 & 78.2 \\
\hline \multicolumn{7}{|l|}{ Malignant neoplasms of lip, oral cavity } \\
\hline and pharynx $\ldots \ldots \ldots \ldots \ldots(\mathrm{C} 00-\mathrm{C} 14)$ & 1.3 & 1.6 & * & 1.6 & 2.2 & 1.1 \\
\hline Malignant neoplasm of esophagus . . . . . (C15) & 1.6 & 2.7 & * & 1.4 & 2.2 & 0.7 \\
\hline Malignant neoplasm of stomach . . . . . . . (C16) & 2.8 & 3.3 & 2.3 & 5.1 & 5.7 & 4.4 \\
\hline \multicolumn{7}{|l|}{ Malignant neoplasms of colon, rectum } \\
\hline and anus $\ldots \ldots \ldots \ldots \ldots(\mathrm{C} 18-\mathrm{C} 21)$ & 7.8 & 8.2 & 7.4 & 8.4 & 8.7 & 8.1 \\
\hline \multicolumn{7}{|l|}{ Malignant neoplasms of liver and } \\
\hline intrahepatic bile ducts $\ldots \ldots \ldots \ldots \ldots \ldots$ (C22) & 4.7 & 6.2 & 3.1 & 7.6 & 10.7 & 4.6 \\
\hline Malignant neoplasm of pancreas $\ldots \ldots \ldots$ (C25) & 4.4 & 4.6 & 4.2 & 5.5 & 5.4 & 5.5 \\
\hline Malignant neoplasm of larynx . . . . . . . . (C32) & 0.6 & * & * & 0.3 & 0.6 & * \\
\hline \multicolumn{7}{|l|}{ Malignant neoplasms of trachea, } \\
\hline bronchus and lung ......... & 21.5 & 23.8 & 19.2 & 19.2 & 22.9 & 15.7 \\
\hline Malignant melanoma of skin . & 0.7 & * & * & 0.3 & 0.3 & 0.3 \\
\hline Malignant neoplasm of breast . . & 5.3 & * & 10.4 & 5.3 & * & 10.4 \\
\hline Malignant neoplasm of cervix uteri & 1.0 & $\ldots$ & 2.0 & 1.0 & $\ldots$ & 1.9 \\
\hline \multicolumn{7}{|l|}{ Malignant neoplasms of corpus uteri and } \\
\hline uterus, part unspecified . . . . . . (C54-C55) & 1.0 & $\ldots$ & 2.0 & 1.1 & $\ldots$ & 2.2 \\
\hline Malignant neoplasm of ovary $\ldots \ldots \ldots \ldots$ (C56) & 1.7 & & 3.5 & 2.1 & $\ldots$ & 4.1 \\
\hline Malignant neoplasm of prostate . . . . . . (C61) & 3.2 & 6.5 & $\ldots$ & 2.8 & 5.8 & $\ldots$ \\
\hline \multicolumn{7}{|l|}{ Malignant neoplasms of kidney and } \\
\hline renal pelvis $\ldots \ldots \ldots \ldots \ldots$ (C64-C65) & 3.2 & 4.4 & 1.9 & 1.6 & 2.1 & 1.1 \\
\hline Malignant neoplasm of bladder . . . . . . (C67) & 1.0 & 1.4 & * & 1.1 & 1.5 & 0.7 \\
\hline \multicolumn{7}{|l|}{$\begin{array}{l}\text { Malignant neoplasms of meninges, } \\
\text { brain and other parts of central }\end{array}$} \\
\hline nervous system $\ldots \ldots \ldots \ldots$ (C70-C72) & 1.4 & 1.5 & 1.2 & 1.5 & 1.8 & 1.2 \\
\hline \multicolumn{7}{|l|}{ Malignant neoplasms of lymphoid, } \\
\hline hematopoietic and related tissue $\ldots($ (C81-C96) & 6.5 & 7.3 & 5.6 & 7.8 & 9.1 & 6.6 \\
\hline Hodgkin's disease ............ (C81) & & & * & * & * & \\
\hline Non-Hodgkin's lymphoma . . . . . . (C82-C85) & 2.4 & 2.6 & 2.2 & 3.2 & 3.8 & 2.7 \\
\hline Leukemia . . . . . . . . . . . & 2.4 & 2.6 & 2.2 & 2.9 & 3.5 & 2.5 \\
\hline \multicolumn{7}{|l|}{ Multiple myeloma and immunoproliferative } \\
\hline neoplasms $\ldots \ldots \ldots \ldots(\mathrm{C} 8 \mathrm{\ldots}, \mathrm{C90})$ & 1.5 & 1.8 & 1.2 & 1.5 & 1.7 & 1.2 \\
\hline Other and unspecified malignant & & & & & & \\
\hline $\begin{array}{l}\text { neoplasms of lymphoid, hematopoietic and } \\
\text { related tissue } \ldots \ldots \ldots \ldots \ldots \ldots \text { (C96) }\end{array}$ & * & * & * & * & * & * \\
\hline
\end{tabular}

See footnotes at end of table. 
Table 14. Death rates for 113 selected causes and Enterocolitis due to Clostridium difficile, by race and sex: United States, 2007-Con.

[Rates per 100,000 population in specified group. Populations used for computing death rates are postcensal estimates based on the 2000 census, estimated as of July 1, 2007; see "Technical Notes." Data for specified races other than white and black should be interpreted with caution because of inconsistencies between reporting race on death certificates and on censuses and surveys; see "Technical Notes." The asterisks (*) preceding the cause-of-death codes indicate that they are not part of the International Classification of Diseases, Tenth Revision (ICD-10), Second Edition; see "Technical Notes"]

\begin{tabular}{|c|c|c|c|c|c|c|}
\hline \multirow[b]{2}{*}{ Cause of death (based on ICD-10, 2004) } & \multicolumn{3}{|c|}{ American Indian or Alaska Native ${ }^{1,2}$} & \multicolumn{3}{|c|}{ Asian or Pacific Islander ${ }^{1,3}$} \\
\hline & $\begin{array}{l}\text { Both } \\
\text { sexes }\end{array}$ & Male & Female & $\begin{array}{l}\text { Both } \\
\text { sexes }\end{array}$ & Male & Female \\
\hline $\begin{array}{l}\text { All other and unspecified malignant } \\
\text { neoplasms . . . . (C17,C23-C24,C26-C31, } \\
\text { C37-C41,C44-C49,C51-C52,C57-C60, } \\
\text { C62-C63,C66,C68-C69,C73-C80,C97) }\end{array}$ & 9.6 & 9.8 & 9.4 & 9.7 & 9.9 & 9.5 \\
\hline \multicolumn{7}{|l|}{$\begin{array}{l}\text { In situ neoplasms, benign neoplasms and } \\
\text { neoplasms of uncertain or unknown }\end{array}$} \\
\hline behavior . . . . . . . . . . . . (D00-D48) & 1.6 & 1.6 & 1.5 & 2.1 & 2.3 & 1.9 \\
\hline (D50-D64) & 0.6 & * & * & 0.7 & 0.7 & 0.8 \\
\hline Diabetes mellitus. & 24.4 & 23.6 & 25.2 & 11.8 & 12.0 & 11.6 \\
\hline Nutritional deficiencies & 0.8 & * & * & 0.4 & 0.3 & 0.5 \\
\hline$\ldots \ldots($ E40-E46) & 0.7 & * & * & 0.4 & 0.3 & 0.4 \\
\hline Other nutritional deficiencies . . . . . . . (E50-E64) & * & * & * & * & * & * \\
\hline 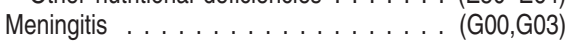 & * & * & * & * & * & * \\
\hline Parkinson's disease . . . . . . . . . (G20-G21) & 1.7 & 2.0 & 1.4 & 2.3 & 2.6 & 1.9 \\
\hline Alzheimer's disease $\ldots \ldots \ldots \ldots \ldots \ldots \ldots$ (G30) & 5.9 & 4.3 & 7.5 & 5.1 & 3.4 & 6.6 \\
\hline Major cardiovascular diseases . . . . . . . (100-178) & 107.6 & 118.4 & 96.8 & 103.8 & 109.6 & 98.4 \\
\hline Diseases of heart $\ldots . .(100-109,|11| 113,, \mid 20-151)$ & 81.8 & 94.1 & 69.6 & 71.6 & 79.6 & 63.9 \\
\hline \multicolumn{7}{|l|}{ Acute rheumatic fever and chronic } \\
\hline rheumatic heart diseases $\ldots \ldots \ldots$ (100-109) & * & * & * & 0.6 & 0.4 & 0.8 \\
\hline Hypertensive heart disease $\ldots \ldots \ldots \ldots$ (l11) & 4.2 & 4.9 & 3.5 & 4.1 & 4.1 & 4.0 \\
\hline Hypertensive heart and renal disease . . . . (l13) & * & * & * & 0.6 & 0.5 & 0.7 \\
\hline Ischemic heart diseases . . . . . . . (I20-I25) & 54.9 & 64.9 & 45.0 & 50.2 & 58.1 & 42.7 \\
\hline Acute myocardial infarction ....... (121-I22) & 18.3 & 22.2 & 14.3 & 16.1 & 18.7 & 13.6 \\
\hline Other acute ischemic heart diseases .... (124) & 1.9 & 2.1 & 1.7 & 0.3 & 0.4 & 0.3 \\
\hline \multicolumn{7}{|l|}{ Other forms of chronic ischemic heart } \\
\hline disease . . . . . . . . . . . . . . (120,|25) & 34.8 & 40.6 & 29.0 & 33.7 & 39.0 & 28.7 \\
\hline \multicolumn{7}{|l|}{ Atherosclerotic cardiovascular } \\
\hline disease, so described . . . . & 10.0 & 12.4 & 7.7 & 7.8 & 9.5 & 6.1 \\
\hline \multicolumn{7}{|l|}{ All other forms of chronic ischemic } \\
\hline heart disease $\ldots \ldots \ldots(|20| 25.1-,\mid 25.9)$ & 24.7 & 28.2 & 21.3 & 26.0 & 29.5 & 22.6 \\
\hline Other heart diseases . . . . . . . . . (I26-151) & 22.0 & 23.6 & 20.3 & 16.1 & 16.5 & 15.8 \\
\hline Acute and subacute endocarditis ..... (I33) & * & * & * & 0.1 & * & * \\
\hline \multicolumn{7}{|l|}{ Diseases of pericardium and acute } \\
\hline myocarditis $\ldots \ldots \ldots \ldots(|30-131| 40)$, & * & * & * & 0.1 & * & * \\
\hline Heart failure . . . . . . . . (150) & 5.8 & 5.3 & 6.4 & 3.5 & 3.1 & 3.8 \\
\hline \multicolumn{7}{|l|}{ All other forms of heart disease (126-I28, } \\
\hline$|34-| 38,|42-| 49, \mid 51)$ & 15.6 & 17.6 & 13.6 & 12.4 & 13.1 & 11.7 \\
\hline \multicolumn{7}{|l|}{ Essential hypertension and hypertensive } \\
\hline renal disease $\ldots \ldots \ldots \ldots \ldots(110,112,115)$ & 3.3 & 2.8 & 3.8 & 4.3 & 4.0 & 4.6 \\
\hline Cerebrovascular diseases $\ldots \ldots \ldots \ldots(160-169)$ & 18.1 & 16.5 & 19.7 & 24.3 & 22.0 & 26.4 \\
\hline Atherosclerosis . . . . . . . . . . . . . . . (170) & 0.9 & * & * & 0.8 & 0.7 & 0.8 \\
\hline Other diseases of circulatory system $\ldots .(171-178)$ & 3.4 & 4.0 & 2.8 & 2.9 & 3.3 & 2.6 \\
\hline Aortic aneurysm and dissection ....... (171) & 1.9 & 2.5 & * & 2.2 & 2.6 & 1.8 \\
\hline \multicolumn{7}{|l|}{ Other diseases of arteries, arterioles and } \\
\hline capillaries . . . . . . . . . . . . . (172-178) & 1.5 & 1.5 & 1.6 & 0.8 & 0.7 & 0.8 \\
\hline Other disorders of circulatory system . . . . (180-199) & * & * & * & 0.3 & 0.3 & 0.3 \\
\hline Influenza and pneumonia . . . . . . . (J09-J18 $)^{4}$ & 8.7 & 9.3 & 8.0 & 9.0 & 9.8 & 8.3 \\
\hline 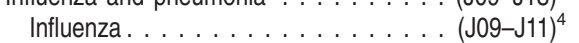 & * & * & * & * & * & *. \\
\hline Pneumonia . . . . . . . . . . . . . . (J12-J18) & 8.5 & 9.2 & 7.8 & 9.0 & 9.7 & 8.3 \\
\hline \multicolumn{7}{|l|}{ Other acute lower respiratory } \\
\hline infections. & * & * & * & * & * & * \\
\hline Acute bronchitis and bronchiolitis . . . . (J20-J21) & * & * & * & * & * & * \\
\hline 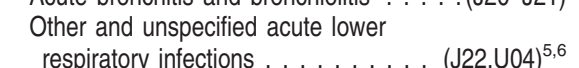 & * & * & * & * & * & * \\
\hline $\begin{array}{l}\text { Chronic lower respiratory diseases } \\
\text { (J40-J47) }\end{array}$ & 18.9 & 18.5 & 19.3 & 90 & 111 & 71 \\
\hline Bronchitis, chronic and unspecified . . . . (J40-J42) & * & * & * & * & * & * \\
\hline Emphysema $\ldots \ldots \ldots \ldots \ldots \ldots$ (J43) & 1.5 & 1.5 & 1.5 & 0.8 & 1.2 & 0.5 \\
\hline Asthma . . . . . . . . . . . . . . . . . (J45-J46) & 1.1 & * & 1.4 & 0.9 & 0.9 & 0.9 \\
\hline Other chronic lower respiratory & & & & & & \\
\hline 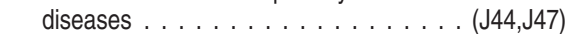 & 16.3 & 16.2 & 16.4 & 7.2 & 8.8 & 5.6 \\
\hline
\end{tabular}

See footnotes at end of table. 
Table 14. Death rates for 113 selected causes and Enterocolitis due to Clostridium difficile, by race and sex: United States, 2007-Con.

[Rates per 100,000 population in specified group. Populations used for computing death rates are postcensal estimates based on the 2000 census, estimated as of July 1, 2007; see "Technical Notes." Data for specified races other than white and black should be interpreted with caution because of inconsistencies between reporting race on death certificates and on censuses and surveys; see "Technical Notes." The asterisks (*) preceding the cause-of-death codes indicate that they are not part of the International Classification of Diseases, Tenth Revision (ICD-10), Second Edition; see "Technical Notes"]

\begin{tabular}{|c|c|c|c|c|c|c|}
\hline \multirow[b]{2}{*}{ Cause of death (based on ICD-10, 2004) } & \multicolumn{3}{|c|}{ American Indian or Alaska Native ${ }^{1,2}$} & \multicolumn{3}{|c|}{ Asian or Pacific Islander ${ }^{1,3}$} \\
\hline & $\begin{array}{l}\text { Both } \\
\text { sexes }\end{array}$ & Male & Female & $\begin{array}{l}\text { Both } \\
\text { sexes }\end{array}$ & Male & Female \\
\hline $\begin{array}{l}\text { Pneumoconioses and chemical } \\
\text { effects } \ldots \ldots \ldots \ldots \ldots \ldots \ldots \ldots(\mathrm{J} 60-\mathrm{J} 66, \mathrm{~J} 68)\end{array}$ & * & * & * & * & * & * \\
\hline $\begin{array}{l}\text { Pneumonitis due to solids and liquids . . . . . (J69) } \\
\text { Other diseases of respiratory } \\
\text { system } \ldots \ldots \ldots \ldots \ldots \text { (J00-J06,J30- J39, }\end{array}$ & 2.3 & 2.2 & 2.5 & 1.7 & 2.1 & 1.4 \\
\hline $\begin{array}{r}\text { J67,J70-J98) }\end{array}$ & 6.6 & 7.1 & 6.2 & 3.7 & 3.9 & 3.6 \\
\hline Peptic ulcer . . . . . . . . . . . . . . (K25-K28) & * & * & * & 0.6 & 0.7 & 0.6 \\
\hline Diseases of appendix . . . . . . . . (K35-K38) & * & * & * & * & * & * \\
\hline Hernia . . . . . . . . & * & * & * & * & * & * \\
\hline $\begin{array}{c}\text { Chronic liver disease and cirrhosis } \ldots(\mathrm{K} 70, \mathrm{~K} 73-\mathrm{K} 74) \\
\text { Alcoholic liver disease } \ldots \ldots \ldots \ldots \ldots \text { (K70) }\end{array}$ & $\begin{array}{l}21.9 \\
15.5\end{array}$ & $\begin{array}{l}25.7 \\
18.8\end{array}$ & $\begin{array}{l}18.1 \\
12.1\end{array}$ & $\begin{array}{l}2.8 \\
1.1\end{array}$ & $\begin{array}{l}3.5 \\
1.8\end{array}$ & $\begin{array}{l}2.0 \\
0.5\end{array}$ \\
\hline $\begin{array}{l}\text { Other chronic liver disease and } \\
\text { cirrhosis } \ldots \ldots \ldots \ldots \text { (K73-K74) }\end{array}$ & 6.5 & 6.9 & 6.0 & 1.7 & 1.8 & 1.6 \\
\hline $\begin{array}{l}\text { Cholelithiasis and other disorders of } \\
\text { gallbladder .................. (K80-K82) } \\
\text { Nephritis, nephrotic syndrome and }\end{array}$ & 0.7 & * & * & 0.5 & 0.5 & 0.6 \\
\hline $\begin{array}{c}\text { nephrosis . . . . . . . (N00-N07,N17-N19,N25-N27) } \\
\text { Acute and rapidly progressive nephritic and }\end{array}$ & 9.0 & 7.8 & 10.2 & 6.0 & 6.6 & 5.5 \\
\hline $\begin{array}{l}\text { nephrotic syndrome . . . . . . (N00-N01,N04) } \\
\text { nephroplomerulonephritis, nephritis and } \\
\text { chronic, and renal speciferosis }\end{array}$ & * & * & * & * & * & * \\
\hline unspecified $\ldots \ldots \ldots$ (N02-N03,N05-N07,N26) & * & * & * & 0.5 & 0.5 & 0.4 \\
\hline Renal failure $\ldots \ldots \ldots \ldots \ldots$ (N17-N19) & 8.6 & 7.5 & 9.6 & 5.5 & 6.1 & 5.0 \\
\hline Other disorders of kidney $\ldots \ldots \ldots(\mathrm{N} 25, \mathrm{~N} 27)$ & * & * & * & * & * & * \\
\hline Infections of kidney $\ldots \ldots$ (N10-N12,N13.6,N15.1) & * & * & * & * & * & * \\
\hline $\begin{array}{l}\text { Hyperplasia of prostate .............. (N40) } \\
\text { Inflammatory diseases of female pelvic }\end{array}$ & * & * & $\ldots$ & * & * & $\ldots$ \\
\hline $\begin{array}{l}\text { organs } \ldots \ldots \ldots \ldots \ldots \ldots \ldots \text { (N70-N76) } \\
\text { Pregnancy, childbirth and the }\end{array}$ & * & $\ldots$ & * & * & $\ldots$ & * \\
\hline puerperium . . . . . . . . . . . . . (000-099) & * & $\ldots$ & * & 0.2 & $\ldots$ & 0.5 \\
\hline $\begin{array}{l}\text { Pregnancy with abortive outcome } \ldots(\mathrm{O}) 00-007) \\
\text { Other complications of pregnancy, childbirth and }\end{array}$ & * & $\cdots$ & * & * & $\cdots$ & * \\
\hline the puerperium $\ldots \ldots \ldots \ldots$ (010-099) & * & $\ldots$ & * & 0.2 & $\ldots$ & 0.4 \\
\hline $\begin{array}{l}\text { Certain conditions originating in the perinatal } \\
\text { period } \ldots \ldots \ldots \ldots \ldots \ldots \ldots \ldots \ldots \ldots \text { (P00-P96) }\end{array}$ & 4.9 & 5.1 & 4.6 & 3.4 & 4.0 & 2.8 \\
\hline $\begin{array}{l}\text { Congenital malformations, deformations and } \\
\text { chromosomal abnormalities ........(Q00-Q99) }\end{array}$ & 3.8 & 3.8 & 3.8 & 2.3 & 2.4 & 2.1 \\
\hline $\begin{array}{l}\text { Symptoms, signs and abnormal clinical and } \\
\text { laboratory findings, not elsewhere }\end{array}$ & & & & & & \\
\hline classified $\ldots \ldots \ldots \ldots \ldots \ldots \ldots$ (R00-R99) & 8.1 & 10.2 & 6.0 & 3.1 & 2.9 & 3.3 \\
\hline $\begin{array}{l}\text { All other diseases . . . . . . . . . . . . . (residual) } \\
\text { Accidents (unintentional injuries) } \ldots \ldots \text { (V01-X59, }\end{array}$ & 43.6 & 41.4 & 45.9 & 23.4 & 21.6 & 25.1 \\
\hline Y85-Y86) & 52.6 & 69.9 & 35.3 & 14.9 & 19.1 & 10.8 \\
\hline $\begin{array}{r}\text { Transport accidents . . . . . . . . V01-V99,Y85) } \\
\text { Motor-vehicle accidents. . . . . (V02-V04, } \\
\text { V09.0,V09.2,V12-V14,V19.0-V19.2, } \\
\text { V19.4-V19.6,V20-V79,V80.3-V80.5, } \\
\text { V81.0-V81.1,V82.0-V82.1,V83-V86, }\end{array}$ & 26.3 & 35.7 & 16.9 & 7.1 & 9.2 & 5.2 \\
\hline V87.0-V87.8,V88.0-V88.8,V89.0,V89.2) & 24.1 & 32.4 & 15.9 & 6.7 & 8.6 & 4.9 \\
\hline $\begin{array}{r}\text { Other land transport accidents. . . . . . (V01, } \\
\text { V05-V06,V09.1,V09.3-V09.9,V10-V11, } \\
\text { V15-V18,V19.3,V19.8-V19.9,V80.0-V80.2, } \\
\text { V80.6-V80.9,V81.2-V81.9,V82.2-V82.9, } \\
\text { V87.9,V88.9,V89.1,V89.3,V89.9) }\end{array}$ & 1.2 & 1.9 & * & 0.2 & 0.3 & * \\
\hline $\begin{array}{l}\text { Water, air and space, and other and } \\
\text { unspecified transport accidents and } \\
\text { their sequelae }\end{array}$ & & & & & & \\
\hline Nontransport accidents $\ldots \ldots \ldots$ (W00-X59,Y86) & $\begin{array}{r}0.9 \\
26.3\end{array}$ & $\begin{array}{r}1.4 \\
34.2\end{array}$ & 18.4 & $\begin{array}{l}0.2 \\
7.8\end{array}$ & $\begin{array}{l}0.3 \\
9.9\end{array}$ & 5.7 \\
\hline
\end{tabular}

See footnotes at end of table. 
Table 14. Death rates for 113 selected causes and Enterocolitis due to Clostridium difficile, by race and sex: United States, 2007-Con.

[Rates per 100,000 population in specified group. Populations used for computing death rates are postcensal estimates based on the 2000 census, estimated as of July 1, 2007; see "Technical Notes." Data for specified races other than white and black should be interpreted with caution because of inconsistencies between reporting race on death certificates and on censuses and surveys; see "Technical Notes." The asterisks (*) preceding the cause-of-death codes indicate that they are not part of the International Classification of Diseases, Tenth Revision (ICD-10), Second Edition; see "Technical Notes"]

\begin{tabular}{|c|c|c|c|c|c|c|}
\hline \multirow[b]{2}{*}{ Cause of death (based on ICD-10, 2004) } & \multicolumn{3}{|c|}{ American Indian or Alaska Native $e^{1,2}$} & \multicolumn{3}{|c|}{ Asian or Pacific Islander ${ }^{1,3}$} \\
\hline & $\begin{array}{l}\text { Both } \\
\text { sexes }\end{array}$ & Male & Female & $\begin{array}{l}\text { Both } \\
\text { sexes }\end{array}$ & Male & Female \\
\hline Falls . . . . . . . . . . . . . . . . (W00-W19) & 4.1 & 5.5 & 2.7 & 3.1 & 3.7 & 2.6 \\
\hline Accidental discharge of firearms. . . .(W32-W34) & * & * & * & * & * & * \\
\hline Accidental drowning and submersion.(W65-W74) & 2.1 & 3.5 & * & 1.0 & 1.6 & 0.5 \\
\hline $\begin{array}{l}\text { Accidental exposure to smoke, fire and } \\
\text { flames . . . . . . . . . . . . . (X00-X09) } \\
\text { Accidental poisoning and exposure to }\end{array}$ & 1.3 & 1.5 & * & 0.4 & 0.5 & * \\
\hline noxious substances $\ldots \ldots \ldots(\mathrm{X} 40-\mathrm{X} 49)$ & 11.6 & 13.2 & 10.0 & 1.5 & 2.0 & 1.0 \\
\hline $\begin{array}{l}\text { Other and unspecified nontransport accidents } \\
\text { and their sequelae.... (W20-W31,W35-W64, } \\
\text { W75-W99,X10-X39,X50-X59,Y86) }\end{array}$ & 6.9 & 9.9 & 3.9 & 1.7 & 2.1 & 1.3 \\
\hline Intentional self-harm & & & & & & \\
\hline $\begin{array}{l}\left.\text { (suicide) . . . . . . . . . . . ( }{ }^{*} \cup 03, \mathrm{X} 60-\mathrm{X} 84, \mathrm{Y} 87.0\right) \\
\text { Intentional self-harm (suicide) by discharge of }\end{array}$ & 12.1 & 19.2 & 5.1 & 6.1 & 8.7 & 3.6 \\
\hline $\begin{array}{l}\text { firearms. . . . . . . . . . . . . . X (X72-X74) } \\
\text { Intentional self-harm (suicide) by other and } \\
\text { unspecified means and their }\end{array}$ & 4.2 & 7.2 & * & 1.5 & 2.8 & 0.3 \\
\hline sequelae ...... ( $\left.{ }^{*} \mathrm{U} 03, \mathrm{X} 60-\mathrm{X} 71, \mathrm{X} 75-\mathrm{X} 84, \mathrm{Y} 87.0\right)$ & 7.9 & 12.0 & 3.9 & 4.6 & 6.0 & 3.3 \\
\hline $\begin{array}{l}\text { Assault (homicide). . . . . (*U01-*U02,X85-Y09,Y87.1) } \\
\text { Assault (homicide) by discharge of }\end{array}$ & 6.8 & 10.1 & 3.5 & 2.4 & 3.5 & 1.4 \\
\hline firearms. . . . . . . . . . ( $\left.{ }^{*} \cup 01.4, \mathrm{X93}-\mathrm{X95}\right)$ & 2.8 & 4.8 & * & 1.4 & 2.3 & 0.4 \\
\hline $\begin{array}{l}\text { Assault (homicide) by other and } \\
\text { unspecified means and their } \\
\text { sequelae..... (* }{ }^{*} 01.0-{ }^{*} \cup 01.3,{ }^{*} \cup 01.5-{ }^{*} \cup 01.9\end{array}$ & & & & & & \\
\hline *U02,X85-X92,X96-Y09,Y87.1) & 4.0 & 5.3 & 2.7 & 1.1 & 1.1 & 1.0 \\
\hline $\begin{array}{l}\text { Legal intervention . . . . . . . . . . . (Y35,Y89.0) } \\
\text { Events of undetermined }\end{array}$ & * & * & * & * & * & * \\
\hline $\begin{array}{l}\text { intent. . . . . . . . . . . (Y10-Y34,Y87.2,Y89.9) } \\
\text { Discharge of firearms, undetermined }\end{array}$ & 2.8 & 3.2 & 2.5 & 0.5 & 0.7 & 0.4 \\
\hline 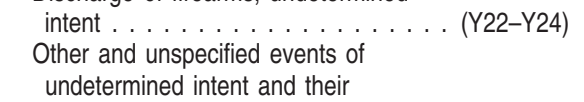 & * & * & * & * & * & * \\
\hline sequelae .... (Y10-Y21,Y25-Y34, Y87.2,Y89.9) & 2.7 & 2.8 & 2.5 & 0.5 & 0.7 & 0.4 \\
\hline Operations of war and their sequelae . . (Y36,Y89.1) & 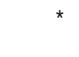 & * & * & * & * & * \\
\hline $\begin{array}{l}\text { Complications of medical and surgical } \\
\text { care } \ldots \ldots \ldots \ldots \ldots \ldots \ldots \ldots(Y 40-Y 84, Y 88)\end{array}$ & * & * & * & 0.3 & * & 0.3 \\
\hline Enterocolitis due to Clostridium difficile $\ldots . .(\text { A04.7 })^{7}$ & 0.8 & * & * & 0.3 & 0.3 & 0.3 \\
\hline
\end{tabular}

0.0 Quantity more than zero but less than 0.05 .

* Figure does not meet standards of reliability or precision; see "Technical Notes."

... Category not applicable.

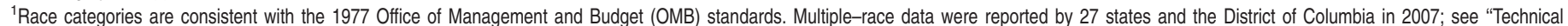
Notes." The multiple-race data for these reporting areas were bridged to the single-race categories of the 1977 OMB standards for comparability with other reporting areas; see "Technical Notes."

${ }^{2}$ Includes Aleuts and Eskimos.

${ }^{3}$ Includes Chinese, Filipino, Hawaiian, Japanese, and Other Asian or Pacific Islander.

${ }^{4} \mathrm{New}$ ICD-10 code J09 (Influenza due to identified avian influenza virus) was added to the category in 2007. No deaths occurred from this cause in 2007.

${ }^{5} \mathrm{New}$ ICD-10 code U04 (Severe acute respiratory syndrome [SARS]) was added to the category in 2007. No deaths occurred from this cause in 2007.

${ }^{6}$ Cause-of-death title was changed in 2007 to reflect the addition of SARS (ICD-10 code U04).

${ }^{7}$ Included in "Certain other intestinal infections (A04,A07-A09)" shown above. Beginning with data year 2006, "Enterocolitis due to Clostridium difficile (A04.7)" is shown separately at the bottom of tables showing 113 selected causes and is included in the list of rankable causes, see "Technical Notes."

NOTE: Complete confirmation of deaths from selected causes of death, considered to be of public health concern, was not provided by the following states: Connecticut, Florida, Indiana, Kentucky, Maryland, North Carolina, Oklahoma, Pennsylvania, Rhode Island, South Carolina, Washington, and West Virginia; see "Technical Notes." 
Table 15. Death rates for 113 selected causes and Enterocolitis due to Clostridium difficile, by Hispanic origin, race for non-Hispanic population, and sex: United States, 2007

[Rates per 100,000 population in specified group. Populations used for computing death rates are postcensal estimates based on the 2000 census, estimated as of July 1 , 2007; see "Technical Notes." Race and Hispanic origin are reported separately on the death certificate. Persons of Hispanic origin may be of any race. Data for Hispanic persons are not tabulated separately by race; data for non-Hispanic persons are tabulated by race. Data for Hispanic origin should be interpreted with caution because of inconsistencies between reporting Hispanic origin on death certificates and on censuses and surveys; see "Technical Notes." The asterisks $\left({ }^{*}\right)$ preceding the cause-of-death codes indicate that they are not part of the International Classification of Diseases, Tenth Revision (ICD-10), Second Edition; see "Technical Notes"]

\begin{tabular}{|c|c|c|c|c|c|c|c|c|c|}
\hline \multirow[b]{2}{*}{ Cause of death (based on ICD-10, 2004) } & \multicolumn{3}{|c|}{ All origins ${ }^{1}$} & \multicolumn{3}{|c|}{ Hispanic } & \multicolumn{3}{|c|}{ Non-Hispanic ${ }^{2}$} \\
\hline & $\begin{array}{l}\text { Both } \\
\text { sexes }\end{array}$ & Male & Female & $\begin{array}{l}\text { Both } \\
\text { sexes }\end{array}$ & Male & Female & $\begin{array}{l}\text { Both } \\
\text { sexes }\end{array}$ & Male & Female \\
\hline All causes $\ldots \ldots \ldots \ldots \ldots \ldots \ldots$ & 803.6 & 809.9 & 797.4 & 297.8 & 321.8 & 272.1 & 892.0 & 899.8 & 884.5 \\
\hline Salmonella infections & 0.0 & * & * & * & * & * & 0.0 & * & * \\
\hline Shigellosis and amebiasis . . . . . . . . . (A03,A06) & * & * & * & * & * & * & * & * & * \\
\hline Certain other intestinal infections $\ldots(\mathrm{A} 04, \mathrm{~A} 07-\mathrm{A} 09)$ & 2.2 & 1.7 & 2.8 & 0.6 & 0.5 & 0.7 & 2.5 & 1.9 & 3.1 \\
\hline Tuberculosis . . . . . . . . . . . . . (A16-A19) & 0.2 & 0.2 & 0.1 & 0.2 & 0.3 & 0.1 & 0.2 & 0.2 & 0.1 \\
\hline Respiratory tuberculosis . . . . . . . . . (A16) & 0.1 & 0.2 & 0.1 & 0.2 & 0.2 & 0.1 & 0.1 & 0.2 & 0.1 \\
\hline Other tuberculosis . . . . . . . . . (A17-A19) & 0.0 & 0.1 & 0.0 & * & * & * & 0.0 & 0.1 & 0.0 \\
\hline Whooping cough $\ldots \ldots \ldots \ldots \ldots \ldots$ (A37) & * & * & * & * & * & * & * & * & * \\
\hline Scarlet fever and erysipelas $\ldots \ldots \ldots(\mathrm{A} 38, \mathrm{~A} 46)$ & * & * & * & * & * & * & * & * & * \\
\hline Meningococcal infection $\ldots \ldots \ldots \ldots \ldots$ (A39) & 0.0 & 0.0 & 0.0 & * & * & * & 0.0 & 0.0 & 0.0 \\
\hline Septicemia $\ldots . \ldots \ldots \ldots \ldots$. . . A40-A41) & 11.5 & 10.7 & 12.4 & 4.2 & 4.0 & 4.3 & 12.8 & 11.9 & 13.7 \\
\hline Syphilis $\ldots \ldots \ldots \ldots \ldots \ldots \ldots$ (A50-A53) & 0.0 & 0.0 & * & * & * & * & 0.0 & 0.0 & * \\
\hline Acute poliomyelitis & * & * & * & * & * & * & * & * & * \\
\hline Arthropod-borne viral encephalitis . (A83-A84,A85.2) & * & * & * & * & * & * & * & * & * \\
\hline Measles .................... (B05) & * & * & * & * & * & * & * & * & * \\
\hline Viral hepatitis . . . . . . . . . . . (B15-B19) & 2.5 & 3.3 & 1.6 & 2.5 & 3.2 & 1.7 & 2.4 & 3.3 & 1.6 \\
\hline \multicolumn{10}{|l|}{ Human immunodeficiency virus (HIV) } \\
\hline disease $\ldots \ldots \ldots \ldots \ldots \ldots$ (B20-B24) & 3.7 & 5.4 & 2.1 & 3.3 & 5.0 & 1.5 & 3.8 & 5.5 & 2.2 \\
\hline Malaria . . . . . . . . . . . . . . (B50-B54) & * & * & * & * & * & * & * & * & * \\
\hline \multirow{2}{*}{\multicolumn{10}{|c|}{$\begin{array}{r}\text { Other and unspecified infectious and parasitic } \\
\text { diseases and their sequelae } \ldots . . . . . . .(A 00, A 05, \\
\text { A20-A36,A42-A44,A48-A49, A54-A79,A81-A82, } \\
\text { A85.0-A85.1,A85.8, A86-B04,B06-B09, }\end{array}$}} \\
\hline & 1.9 & 2.0 & 1.9 & 1.1 & 1.2 & 1.1 & 2.1 & 2.2 & 2.0 \\
\hline \multirow{2}{*}{\multicolumn{10}{|c|}{ Malignant neoplasms of lip, oral cavity and }} \\
\hline & & & & & & & & & \\
\hline Malignant neoplasm of esophagus . . . . . (C15) & 4.5 & 7.2 & 1.9 & 1.1 & 1.7 & 0.4 & 5.1 & $\begin{array}{l}4.2 \\
8.3\end{array}$ & 2.1 \\
\hline Malignant neoplasm of stomach . . . . . . . (C16) & 3.8 & 4.5 & 3.0 & 2.9 & 3.2 & 2.5 & 3.9 & 4.8 & 3.1 \\
\hline \multicolumn{10}{|l|}{ Malignant neoplasms of colon, rectum } \\
\hline and anus $\ldots \ldots \ldots \ldots \ldots$ (C18-C21) & 17.8 & 18.2 & 17.3 & 6.2 & 6.4 & 5.9 & 19.8 & 20.4 & 19.2 \\
\hline \multicolumn{10}{|l|}{ Malignant neoplasms of liver and } \\
\hline intrahepatic bile ducts $\ldots \ldots \ldots \ldots \ldots \ldots$ (C22) & 5.7 & 7.6 & 3.8 & 4.2 & 5.3 & 2.9 & 5.9 & 8.0 & 3.9 \\
\hline Malignant neoplasm of pancreas $\ldots \ldots \ldots$ (C25) & 11.3 & 11.5 & 11.1 & 3.9 & 3.7 & 4.1 & 12.6 & 13.0 & 12.3 \\
\hline Malignant neoplasm of larynx $\ldots \ldots \ldots \ldots$ (C32) & 1.2 & 1.9 & 0.5 & 0.4 & 0.6 & * & 1.4 & 2.2 & 0.6 \\
\hline \multicolumn{10}{|l|}{ Malignant neoplasms of trachea, } \\
\hline bronchus and lung $\ldots \ldots \ldots$ & 52.6 & 59.4 & 46.0 & 10.2 & 12.0 & 8.2 & 60.1 & 68.3 & 52.3 \\
\hline Malignant melanoma of skin . & 2.8 & 3.7 & 1.9 & 0.4 & 0.5 & 0.4 & 3.2 & 4.3 & 2.2 \\
\hline Malignant neoplasm of breast & 13.6 & 0.2 & 26.5 & 4.6 & * & 9.4 & 15.2 & 0.3 & 29.4 \\
\hline Malignant neoplasm of cervix uteri & 1.3 & $\ldots$ & 2.6 & 1.0 & $\ldots$ & 2.2 & 1.4 & $\ldots$ & 2.7 \\
\hline \multicolumn{10}{|l|}{ Malignant neoplasms of corpus uteri } \\
\hline and uterus, part unspecified $\ldots \ldots$ (C54-C55) & 2.5 & $\ldots$ & 4.9 & 0.9 & $\ldots$ & 1.8 & 2.8 & $\ldots$ & 5.4 \\
\hline Malignant neoplasm of ovary $\ldots \ldots \ldots$ (C56) & 4.8 & . & 9.6 & 1.7 & $\ldots$ & 3.5 & 5.4 & $\ldots$ & 10.6 \\
\hline Malignant neoplasm of prostate $\ldots \ldots \ldots$ (C61) & 9.6 & 19.6 & $\ldots$ & 3.1 & 6.1 & $\ldots$ & 10.8 & 22.1 & $\ldots$ \\
\hline \multicolumn{10}{|l|}{ Malignant neoplasms of kidney and } \\
\hline renal pelvis $\ldots \ldots \ldots \ldots \ldots$ & 4.2 & 5.4 & 3.1 & 1.9 & 2.5 & 1.3 & 4.6 & 5.9 & 3.4 \\
\hline Malignant neoplasm of bladder $\ldots \ldots \ldots$ (C67) & 4.6 & 6.5 & 2.7 & 1.0 & 1.5 & 0.6 & 5.2 & 7.4 & 3.1 \\
\hline \multicolumn{10}{|l|}{$\begin{array}{l}\text { Malignant neoplasms of meninges, } \\
\text { brain and other parts of central }\end{array}$} \\
\hline nervous system $\ldots \ldots \ldots \ldots \ldots$. . C70-C72) & 4.4 & 4.9 & 3.9 & 1.8 & 1.9 & 1.7 & 4.8 & 5.5 & 4.2 \\
\hline \multicolumn{10}{|l|}{ Malignant neoplasms of lymphoid, } \\
\hline hematopoietic and related tissue $\ldots($ C81-C96) & 18.2 & 20.3 & 16.2 & 7.2 & 7.5 & 7.0 & 20.2 & 22.7 & 17.7 \\
\hline Hodgkin's disease $\ldots \ldots \ldots \ldots \ldots$ (C81) & 0.4 & 0.5 & 0.4 & 0.3 & 0.3 & 0.3 & 0.4 & 0.5 & 0.4 \\
\hline Non-Hodgkin's lymphoma & 6.8 & 7.4 & 6.2 & 2.7 & 2.8 & 2.6 & 7.5 & 8.3 & 6.8 \\
\hline Leukemia . . . . . . . & 7.2 & 8.3 & 6.2 & 2.8 & 3.0 & 2.7 & 8.0 & 9.3 & 6.8 \\
\hline \multicolumn{10}{|l|}{ Multiple myeloma and immunoproliferative } \\
\hline 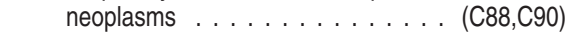 & 3.7 & 4.1 & 3.4 & 1.5 & 1.5 & 1.4 & 4.2 & 4.6 & 3.7 \\
\hline
\end{tabular}

See footnotes at end of table. 
Table 15. Death rates for 113 selected causes and Enterocolitis due to Clostridium difficile, by Hispanic origin, race for non-Hispanic population, and sex: United States, 2007-Con.

[Rates per 100,000 population in specified group. Populations used for computing death rates are postcensal estimates based on the 2000 census, estimated as of July 1 , 2007; see "Technical Notes." Race and Hispanic origin are reported separately on the death certificate. Persons of Hispanic origin may be of any race. Data for Hispanic persons are not tabulated separately by race; data for non-Hispanic persons are tabulated by race. Data for Hispanic origin should be interpreted with caution because of inconsistencies between reporting Hispanic origin on death certificates and on censuses and surveys; see "Technical Notes." The asterisks $\left({ }^{*}\right)$ preceding the cause-of-death codes indicate that they are not part of the International Classification of Diseases, Tenth Revision (ICD-10), Second Edition; see "Technical Notes"]

\begin{tabular}{|c|c|c|c|c|c|c|c|c|c|}
\hline \multirow[b]{2}{*}{ Cause of death (based on ICD-10, 2004) } & \multicolumn{3}{|c|}{ All origins ${ }^{1}$} & \multicolumn{3}{|c|}{ Hispanic } & \multicolumn{3}{|c|}{ Non-Hispanic ${ }^{2}$} \\
\hline & $\begin{array}{l}\text { Both } \\
\text { sexes }\end{array}$ & Male & Female & $\begin{array}{l}\text { Both } \\
\text { sexes }\end{array}$ & Male & Female & $\begin{array}{l}\text { Both } \\
\text { sexes }\end{array}$ & Male & Female \\
\hline \multicolumn{10}{|l|}{$\begin{array}{l}\text { Other and unspecified malignant neoplasms } \\
\text { of lymphoid, hematopoietic and }\end{array}$} \\
\hline related tissue $\ldots \ldots \ldots \ldots \ldots$ (C96) & 0.0 & 0.0 & 0.0 & * & * & * & 0.0 & 0.0 & 0.0 \\
\hline \multicolumn{4}{|l|}{$\begin{array}{l}\text { All other and unspecified malignant } \\
\text { neoplasms . (C17,C23-C24,C26-C31, C37-C41, }\end{array}$} & 75 & 76 & 75 & 233 & 248 & 210 \\
\hline \multicolumn{10}{|l|}{$\begin{array}{l}\text { In situ neoplasms, benign neoplasms } \\
\text { and neoplasms of uncertain or unknown }\end{array}$} \\
\hline behavior $\ldots \ldots \ldots \ldots \ldots \ldots$ (D00-D48) & 4.7 & 4.9 & 4.5 & 1.5 & 1.4 & 1.7 & 5.3 & 5.5 & 5.0 \\
\hline Anemias $\ldots \ldots \ldots \ldots \ldots \ldots \ldots$ (D50-D64) & 1.6 & 1.3 & 1.9 & 0.5 & 0.5 & 0.5 & 1.8 & 1.5 & 2.1 \\
\hline Diabetes mellitus $\ldots \ldots \ldots \ldots \ldots \ldots$ (E10-E14) & 23.7 & 23.9 & 23.5 & 14.1 & 13.6 & 14.6 & 25.3 & 25.8 & 24.9 \\
\hline Nutritional deficiencies . . . . . . . . . . . . (E40-E64) & 0.9 & 0.7 & 1.2 & 0.3 & 0.2 & 0.3 & 1.1 & 0.8 & 1.3 \\
\hline Malnutrition $\ldots \ldots \ldots \ldots \ldots \ldots$ (E40-E46) & 0.9 & 0.7 & 1.1 & 0.2 & 0.2 & 0.3 & 1.0 & 0.8 & 1.2 \\
\hline Other nutritional deficiencies . . . . . . . (E50-E64) & 0.1 & 0.1 & 0.1 & * & * & * & 0.1 & 0.1 & 0.1 \\
\hline 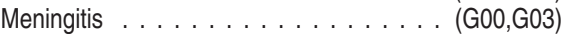 & 0.2 & 0.2 & 0.2 & 0.2 & 0.2 & 0.2 & 0.2 & 0.2 & 0.2 \\
\hline 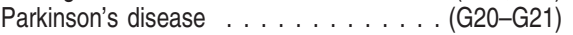 & 6.7 & 7.8 & 5.6 & 1.7 & 1.9 & 1.5 & 7.5 & 8.9 & 6.2 \\
\hline Alzheimer's disease $\ldots \ldots \ldots \ldots \ldots \ldots \ldots$ (G30) & 24.7 & 14.7 & 34.5 & 5.4 & 3.4 & 7.6 & 28.2 & 16.8 & 39.0 \\
\hline Major cardiovascular diseases . . . . . . . (100-178) & 267.3 & 261.0 & 273.3 & 85.0 & 86.0 & 84.0 & 299.2 & 293.3 & 304.8 \\
\hline \multirow{2}{*}{\multicolumn{10}{|c|}{ Acute rheumatic fever and chronic }} \\
\hline & & & & & & & & & \\
\hline rheumatic heart diseases $\ldots \ldots \ldots$ (100-109) & 1.1 & 0.7 & 1.4 & 0.3 & 0.2 & 0.5 & 1.2 & 0.8 & 1.6 \\
\hline Hypertensive heart disease $\ldots \ldots \ldots \ldots$ (l11) & 10.2 & 9.6 & 10.8 & 3.8 & 3.9 & 3.6 & 11.3 & 10.6 & 12.0 \\
\hline Hypertensive heart and renal disease $\ldots .$. (113) & 1.0 & 0.9 & 1.1 & 0.4 & 0.4 & 0.4 & 1.1 & 1.0 & 1.2 \\
\hline Ischemic heart diseases $\ldots \ldots \ldots \ldots$ (I20-125) & 134.7 & 145.3 & 124.4 & 44.9 & 48.0 & 41.6 & 150.4 & 163.3 & 138.1 \\
\hline Acute myocardial infarction . & 44.1 & 48.2 & 40.0 & 14.9 & 16.0 & 13.8 & 49.2 & 54.2 & 44.4 \\
\hline Other acute ischemic heart diseases . . . (I24) & 1.4 & 1.5 & 1.3 & 0.2 & 0.3 & 0.2 & 1.6 & 1.7 & 1.4 \\
\hline \multicolumn{10}{|l|}{ Other forms of chronic ischemic heart } \\
\hline disease . . . . . . . . . . . . . $(120,125)$ & 89.3 & 95.6 & 83.1 & 29.8 & 31.8 & 27.6 & 99.7 & 107.4 & 92.3 \\
\hline $\begin{array}{l}\text { Atherosclerotic cardiovascular } \\
\text { disease, so described . . . . . . . (125.0) }\end{array}$ & 19.6 & 22.5 & 16.8 & 7.1 & 8.8 & 5.3 & 21.7 & 24.9 & 18.6 \\
\hline \multicolumn{10}{|l|}{ All other forms of chronic ischemic heart } \\
\hline disease $\ldots \ldots \ldots \ldots(|20| 25.1-,\mid 25.9)$ & 69.7 & 73.2 & 66.3 & 22.7 & 23.0 & 22.3 & 78.0 & 82.5 & 73.6 \\
\hline Other heart diseases . . . . . . . . (I26-I51) & 57.3 & 51.9 & 62.5 & 14.3 & 14.0 & 14.7 & 64.8 & 59.0 & 70.4 \\
\hline Acute and subacute endocarditis ...... (I33) & 0.4 & 0.5 & 0.3 & 0.2 & 0.2 & 0.1 & 0.4 & 0.5 & 0.4 \\
\hline \multicolumn{10}{|l|}{ Diseases of pericardium and acute } \\
\hline myocarditis $\ldots \ldots \ldots \ldots(130-131,140)$ & 0.3 & 0.3 & 0.3 & 0.1 & 0.2 & 0.1 & 0.3 & 0.3 & 0.3 \\
\hline Heart failure $\ldots \ldots \ldots \ldots \ldots$ (150) & 18.8 & 15.4 & 22.0 & 4.2 & 3.6 & 4.8 & 21.3 & 17.6 & 24.9 \\
\hline \multicolumn{10}{|l|}{ All other forms of heart disease . (I26-I28, } \\
\hline$|34-| 38,|42-| 49, \mid 51)$ & 37.8 & 35.7 & 39.9 & 9.8 & 10.0 & 9.7 & 42.7 & 40.5 & 44.9 \\
\hline \multicolumn{10}{|l|}{ Essential hypertension and } \\
\hline hypertensive renal disease $\ldots \ldots \ldots(|110| 12,, \mid 15)$ & 7.9 & 6.3 & 9.5 & 3.1 & 2.7 & 3.4 & 8.8 & 7.0 & 10.5 \\
\hline Cerebrovascular diseases .... & 45.1 & 36.4 & 53.5 & 15.6 & 14.1 & 17.1 & 50.3 & 40.5 & 59.6 \\
\hline Atherosclerosis . . . . . . . . . . . . . . . . (170) & 2.7 & 2.2 & 3.3 & 0.7 & 0.6 & 0.9 & 3.1 & 2.5 & 3.7 \\
\hline Other diseases of circulatory system . . . (171-178) & 7.3 & 7.7 & 6.8 & 1.9 & 2.1 & 1.8 & 8.2 & 8.8 & 7.7 \\
\hline Aortic aneurysm and dissection $\ldots \ldots \ldots$ (171) & 4.3 & 5.1 & 3.6 & 1.0 & 1.3 & 0.8 & 4.9 & 5.8 & 4.0 \\
\hline \multicolumn{10}{|l|}{ Other diseases of arteries, arterioles and } \\
\hline capillaries . . . . . . . . . . . . . (172-178) & 3.0 & 2.6 & 3.3 & 0.9 & 0.8 & 1.0 & 3.3 & 3.0 & 3.7 \\
\hline Other disorders of circulatory system . . . (180-199) & 1.4 & 1.3 & 1.4 & 0.5 & 0.5 & 0.5 & 1.5 & 1.4 & 1.6 \\
\hline Influenza and pneumonia $\ldots \ldots \ldots$ & 17.5 & 16.2 & 18.7 & 6.0 & 5.8 & 6.3 & 19.5 & 18.1 & 20.8 \\
\hline Influenza. . . . . . . . . . & 0.1 & 0.1 & 0.1 & 0.1 & * & * & 0.1 & 0.1 & 0.2 \\
\hline Pneumonia ... & 17.3 & 16.1 & 18.6 & 5.9 & 5.7 & 6.2 & 19.3 & 18.0 & 20.6 \\
\hline \multicolumn{10}{|l|}{ Other acute lower respiratory } \\
\hline infections . . . . . . . . & 0.1 & 0.1 & 0.1 & 0.1 & * & * & 0.1 & 0.1 & 0.1 \\
\hline Acute bronchitis and bronchiolitis . . . . (J20-J21) & 0.1 & 0.1 & 0.1 & 0.1 & * & * & 0.1 & 0.1 & 0.1 \\
\hline Other and unspecified acute lower & & & & & & & & & \\
\hline respiratory infections . . . . . . . & 0.0 & * & 0.0 & * & * & * & 0.0 & * & 0.0 \\
\hline Chronic lower respiratory diseases & 42.4 & 41.2 & 43.6 & 7.8 & 8.1 & 7.4 & 48.5 & 47.3 & 49.6 \\
\hline Bronchitis, chronic and unspecified . . . . (J40-J42) & 0.2 & 0.2 & 0.3 & 0.1 & 0.1 & * & 0.2 & 0.2 & 0.3 \\
\hline Emphysema . . . . . . . . . . & 4.2 & 4.4 & 4.0 & 0.6 & 0.8 & 0.5 & 4.9 & 5.1 & 4.6 \\
\hline
\end{tabular}

See footnotes at end of table. 
Table 15. Death rates for 113 selected causes and Enterocolitis due to Clostridium difficile, by Hispanic origin, race for non-Hispanic population, and sex: United States, 2007-Con.

[Rates per 100,000 population in specified group. Populations used for computing death rates are postcensal estimates based on the 2000 census, estimated as of July 1 , 2007; see "Technical Notes." Race and Hispanic origin are reported separately on the death certificate. Persons of Hispanic origin may be of any race. Data for Hispanic persons are not tabulated separately by race; data for non-Hispanic persons are tabulated by race. Data for Hispanic origin should be interpreted with caution because of inconsistencies between reporting Hispanic origin on death certificates and on censuses and surveys; see "Technical Notes." The asterisks (*) preceding the cause-of-death codes indicate that they are not part of the International Classification of Diseases, Tenth Revision (ICD-10), Second Edition; see "Technical Notes"]

\begin{tabular}{|c|c|c|c|c|c|c|c|c|c|}
\hline \multirow[b]{2}{*}{ Cause of death (based on ICD-10, 2004) } & \multicolumn{3}{|c|}{ All origins ${ }^{1}$} & \multicolumn{3}{|c|}{ Hispanic } & \multicolumn{3}{|c|}{ Non-Hispanic ${ }^{2}$} \\
\hline & $\begin{array}{l}\text { Both } \\
\text { sexes }\end{array}$ & Male & Female & $\begin{array}{l}\text { Both } \\
\text { sexes }\end{array}$ & Male & Female & $\begin{array}{l}\text { Both } \\
\text { sexes }\end{array}$ & Male & Female \\
\hline $\begin{array}{l}\text { Asthma . . . . . . . . . . . . . . . . . . (J45-J46) } \\
\text { Other chronic lower respiratory }\end{array}$ & 1.1 & 0.9 & 1.4 & 0.5 & 0.5 & 0.6 & 1.3 & 0.9 & 1.6 \\
\hline $\begin{array}{c}\text { diseases . . . . . . . . . . . . . . (J44,J47) } \\
\text { Pneumoconioses and chemical }\end{array}$ & 36.8 & 35.7 & 37.9 & 6.5 & 6.7 & 6.3 & 42.1 & 41.1 & 43.1 \\
\hline effects $\ldots \ldots \ldots \ldots \ldots \ldots(\ldots \ldots$ (J60-J66,J68) & 0.3 & 0.6 & 0.0 & 0.0 & 0.1 & * & 0.3 & 0.7 & 0.0 \\
\hline $\begin{array}{l}\text { Pneumonitis due to solids and liquids . . . . . . . (J69) } \\
\text { Other diseases of respiratory }\end{array}$ & 5.6 & 6.0 & 5.3 & 1.2 & 1.2 & 1.2 & 6.4 & 6.9 & 5.9 \\
\hline system . . . . . . .(J00-J06,J30-J39,J67,J70-J98) & 9.5 & 9.6 & 9.3 & 3.8 & 3.8 & 3.8 & 10.4 & 10.7 & 10.2 \\
\hline Peptic ulcer . . . . . . . . . . . . . . . . (K25-K28) & 1.0 & 1.0 & 1.0 & 0.3 & 0.4 & 0.3 & 1.1 & 1.1 & 1.2 \\
\hline Diseases of appendix $\ldots \ldots \ldots \ldots \ldots \ldots$ (K35-K38) & 0.1 & 0.2 & 0.1 & 0.1 & * & * & 0.2 & 0.2 & 0.1 \\
\hline Hernia $\ldots \ldots \ldots \ldots \ldots \ldots \ldots$ (K40-K46) & 0.6 & 0.5 & 0.6 & 0.2 & 0.2 & 0.3 & 0.6 & 0.5 & 0.7 \\
\hline Chronic liver disease and cirrhosis $\ldots(\mathrm{K} 70, \mathrm{~K} 73-\mathrm{K} 74)$ & 9.7 & 12.9 & 6.5 & 8.6 & 11.9 & 5.1 & 9.8 & 13.0 & 6.8 \\
\hline $\begin{array}{l}\text { Alcoholic liver disease } \ldots \ldots \ldots \ldots \ldots \text { (K70) } \\
\text { Other chronic liver disease and }\end{array}$ & 4.8 & 7.1 & 2.5 & 4.7 & 7.5 & 1.6 & 4.8 & 7.0 & 2.7 \\
\hline $\begin{array}{l}\text { cirrhosis . . . . . . . . . . . . (K73-K74) } \\
\text { Cholelithiasis and other disorders of }\end{array}$ & 4.9 & 5.8 & 4.0 & 3.9 & 4.3 & 3.5 & 5.1 & 6.0 & 4.1 \\
\hline $\begin{array}{l}\text { gallbladder ................... (K80-K82) } \\
\text { Nephritis, nephrotic syndrome and }\end{array}$ & 1.1 & 1.0 & 1.2 & 0.5 & 0.5 & 0.5 & 1.2 & 1.1 & 1.3 \\
\hline $\begin{array}{l}\text { nephrosis ........... (N00-N07,N17-N19,N25-N27) } \\
\text { Acute and rapidly progressive nephritic and }\end{array}$ & 15.4 & 15.2 & 15.6 & 5.9 & 5.8 & 6.0 & 17.1 & 17.0 & 17.2 \\
\hline $\begin{array}{l}\text { nephrotic syndrome ........ (N00-N01,N04) } \\
\text { Chronic glomerulonephritis, nephritis and } \\
\text { nephropathy not specified as acute or } \\
\text { chronic, and renal sclerosis }\end{array}$ & 0.1 & 0.1 & 0.1 & 0.1 & * & * & 0.1 & 0.1 & 0.1 \\
\hline unspecified $\ldots \ldots . .$. (N02-N03,N05-N07,N26) & 1.0 & 1.0 & 1.0 & 0.4 & 0.4 & 0.3 & 1.1 & 1.1 & 1.1 \\
\hline Renal failure $\ldots \ldots \ldots \ldots \ldots \ldots$ (N17-N19) & 14.3 & 14.2 & 14.5 & 5.5 & 5.3 & 5.6 & 15.9 & 15.8 & 16.0 \\
\hline Other disorders of kidney $\ldots \ldots \ldots$ (N25,N27) & 0.0 & * & * & * & * & * & 0.0 & * & * \\
\hline Infections of kidney $\ldots \ldots$ (N10-N12,N13.6,N15.1) & 0.2 & 0.1 & 0.3 & 0.1 & * & 0.2 & 0.2 & 0.2 & 0.3 \\
\hline $\begin{array}{l}\text { Hyperplasia of prostate } \ldots \ldots \ldots \ldots \ldots \text {. . . . . . . } \\
\text { Inflammatory diseases of female pelvic }\end{array}$ & 0.2 & 0.3 & $\ldots$ & 0.1 & 0.1 & $\ldots$ & 0.2 & 0.4 & $\ldots$ \\
\hline $\begin{array}{l}\text { organs } \ldots \ldots \ldots \ldots \ldots \text { (N70-N76) } \\
\text { Pregnancy, childbirth and }\end{array}$ & 0.0 & $\ldots$ & 0.1 & * & $\ldots$ & * & 0.0 & $\ldots$ & 0.1 \\
\hline the puerperium $\ldots \ldots \ldots \ldots$ (000-099) & 0.3 & $\ldots$ & 0.5 & 0.3 & $\ldots$ & 0.6 & 0.2 & $\ldots$ & 0.5 \\
\hline $\begin{array}{l}\text { Pregnancy with abortive outcome } \ldots . . .(000-007) \\
\text { Other complications of pregnancy, childbirth and }\end{array}$ & 0.0 & $\ldots$ & 0.0 & * & $\ldots$ & * & 0.0 & $\ldots$ & 0.0 \\
\hline $\begin{array}{l}\text { the puerperium } \ldots \ldots \ldots \ldots \ldots(010-099) \\
\text { Certain conditions originating in the perinatal }\end{array}$ & 0.2 & $\ldots$ & 0.5 & 0.3 & $\ldots$ & 0.6 & 0.2 & $\ldots$ & 0.5 \\
\hline $\begin{array}{l}\text { period ....................... (P00-P96) } \\
\text { Congenital malformations, deformations and }\end{array}$ & 4.8 & 5.5 & 4.2 & 6.5 & 7.0 & 5.9 & 4.5 & 5.2 & 3.8 \\
\hline $\begin{array}{l}\text { chromosomal abnormalities . . . . . . . (Q00-Q99) } \\
\text { Symptoms, signs and abnormal clinical and } \\
\text { laboratory findings, not elsewhere }\end{array}$ & 3.5 & 3.7 & 3.2 & 4.4 & 4.5 & 4.2 & 3.3 & 3.5 & 3.1 \\
\hline classified . . . . . . . . . . . . . . . (R00-R99) & 11.1 & 9.6 & 12.6 & 4.3 & 4.8 & 3.7 & 12.3 & 10.5 & 14.0 \\
\hline All other diseases $\ldots \ldots \ldots \ldots \ldots$ (Residual) & 79.0 & 64.9 & 92.7 & 25.7 & 23.5 & 28.1 & 88.3 & 72.5 & 103.4 \\
\hline 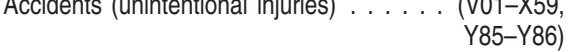 & 41.0 & 53.7 & 28.7 & 25.8 & 37.6 & 13.1 & 43.6 & 56.5 & 31.2 \\
\hline $\begin{array}{r}\text { Transport accidents } \ldots \ldots \ldots .(\mathrm{V} 01-\mathrm{V} 99, \mathrm{Y} 85) \\
\text { Motor-vehicle accidents. . . . . . (V02-V04, } \\
\text { V09.0,V09.2,V12-V14,V19.0-V19.2, } \\
\text { V19.4-V19.6,V20-V79,V80.3-V80.5, } \\
\text { V81.0-V81.1,V82.0-V82.1,V83-V86, }\end{array}$ & 15.5 & 22.5 & 8.8 & 13.4 & 19.7 & 6.7 & 15.9 & 22.9 & 9.1 \\
\hline $\begin{array}{r}\text { V87.0-V87.8,V88.0-V88.8,V89.0, V89.2) } \\
\text { Other land transport accidents ....... (V01, } \\
\text { V05-V06,V09.1,V09.3-V09.9, V10-V11, } \\
\text { V15-V18,V19.3,V19.8- V19.9,V80.0-V80.2, } \\
\text { V80.6-V80.9, V81.2-V81.9,V82.2-V82.9, }\end{array}$ & 14.6 & 20.9 & 8.4 & 12.8 & 18.7 & 6.5 & 14.8 & 21.3 & 8.7 \\
\hline V87.9, V88.9,V89.1,V89.3,V89.9) & 0.4 & 0.6 & 0.1 & 0.4 & 0.6 & 0.1 & 0.4 & 0.6 & 0.2 \\
\hline
\end{tabular}

See footnotes at end of table. 
Table 15. Death rates for 113 selected causes and Enterocolitis due to Clostridium difficile, by Hispanic origin, race for non-Hispanic population, and sex: United States, 2007-Con.

[Rates per 100,000 population in specified group. Populations used for computing death rates are postcensal estimates based on the 2000 census, estimated as of July 1 , 2007; see "Technical Notes." Race and Hispanic origin are reported separately on the death certificate. Persons of Hispanic origin may be of any race. Data for Hispanic persons are not tabulated separately by race; data for non-Hispanic persons are tabulated by race. Data for Hispanic origin should be interpreted with caution because of inconsistencies between reporting Hispanic origin on death certificates and on censuses and surveys; see "Technical Notes." The asterisks (*) preceding the cause-of-death codes indicate that they are not part of the International Classification of Diseases, Tenth Revision (ICD-10), Second Edition; see "Technical Notes"]

\begin{tabular}{|c|c|c|c|c|c|c|c|c|c|}
\hline \multirow[b]{2}{*}{ Cause of death (based on ICD-10, 2004) } & \multicolumn{3}{|c|}{ All origins ${ }^{1}$} & \multicolumn{3}{|c|}{ Hispanic } & \multicolumn{3}{|c|}{ Non-Hispanic ${ }^{2}$} \\
\hline & $\begin{array}{l}\text { Both } \\
\text { sexes }\end{array}$ & Male & Female & $\begin{array}{l}\text { Both } \\
\text { sexes }\end{array}$ & Male & Female & $\begin{array}{l}\text { Both } \\
\text { sexes }\end{array}$ & Male & Female \\
\hline \multicolumn{10}{|l|}{$\begin{array}{l}\text { Water, air and space, and other and } \\
\text { unspecified transport accidents }\end{array}$} \\
\hline and their sequelae $\ldots \ldots \ldots($ V90-V99,Y85) & 0.6 & 1.0 & 0.2 & 0.3 & 0.5 & * & 0.7 & 1.1 & 0.2 \\
\hline Nontransport accidents . . . . . . (WO0-X59,Y86) & 25.5 & 31.2 & 19.9 & 12.3 & 17.9 & 6.4 & 27.7 & 33.6 & 22.1 \\
\hline Falls . . . . . . . . . . . . . . (W00-W19) & 7.5 & 7.8 & 7.2 & 2.7 & 3.2 & 2.0 & 8.3 & 8.6 & 8.1 \\
\hline $\begin{array}{l}\text { Accidental discharge of firearms. . . (W32-W34) } \\
\text { Accidental drowning and }\end{array}$ & 0.2 & 0.4 & 0.0 & 0.1 & 0.2 & * & 0.2 & 0.4 & 0.1 \\
\hline $\begin{array}{l}\text { submersion . . . . . . . . . . . . . (W65-W74) } \\
\text { Accidental exposure to smoke, fire and }\end{array}$ & 1.1 & 1.8 & 0.5 & 1.1 & 1.7 & 0.3 & 1.2 & 1.8 & 0.5 \\
\hline 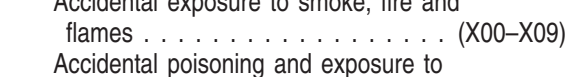 & 1.1 & 1.3 & 0.9 & 0.5 & 0.6 & 0.4 & 1.2 & 1.4 & 1.0 \\
\hline $\begin{array}{l}\text { noxious substances } \ldots . . . . . .(X 40-X 49) \\
\text { Other and unspecified nontransport }\end{array}$ & 9.9 & 13.2 & 6.7 & 5.4 & 8.3 & 2.2 & 10.7 & 14.1 & 7.4 \\
\hline $\begin{array}{l}\text { accidents and their sequelae.... (W20- W31, } \\
\text { W35-W64,W75-W99,X10-X39, X50-X59,Y86) } \\
\text { Intentional self-harm }\end{array}$ & 5.7 & 6.7 & 4.6 & 2.6 & 3.7 & 1.4 & 6.2 & 7.3 & 5.1 \\
\hline $\begin{array}{l}\text { Intentional self-harm } \\
\text { (suicide) . . . . . . . . . ( (*U03,X60-X84,Y87.0) } \\
\text { Intentional self-harm (suicide) by discharge of }\end{array}$ & 11.5 & 18.3 & 4.8 & 5.4 & 8.8 & 1.8 & 12.5 & 20.1 & 5.3 \\
\hline $\begin{array}{l}\text { firearms. } \ldots \ldots \ldots \ldots \ldots \ldots \text { (Xntional self-harm (suicide) by other and } \\
\text { Intenspecified means and their }\end{array}$ & 5.8 & 10.2 & 1.4 & 2.0 & 3.6 & 0.3 & 6.4 & 11.4 & 1.6 \\
\hline sequelae ...... ( $\left.{ }^{*} \mathrm{U} 03, \mathrm{X} 60-\mathrm{X} 71, \mathrm{X} 75-\mathrm{X} 84, \mathrm{Y} 87.0\right)$ & 5.7 & 8.1 & 3.4 & 3.4 & 5.2 & 1.4 & 6.1 & 8.7 & 3.7 \\
\hline $\begin{array}{l}\text { Assault (homicide). . . . . (*U01-*U02,X85-Y09,Y87.1) } \\
\text { Assault (homicide) by discharge of }\end{array}$ & 6.1 & 9.8 & 2.5 & 7.6 & 12.4 & 2.5 & 5.8 & 9.2 & 2.5 \\
\hline $\begin{array}{l}\left.\text { firearms. . . . . . . . . . . ( }{ }^{*} \cup 01.4, \mathrm{X} 93-\mathrm{X} 95\right) \\
\text { Assault (homicide) by other and } \\
\text { unspecified means and their } \\
\text { sequelae. }\end{array}$ & 4.2 & 7.2 & 1.2 & 5.2 & 9.1 & 1.1 & 4.0 & 6.9 & 1.2 \\
\hline$\left.{ }^{*} \mathrm{U} 02, \mathrm{X} 85-\mathrm{X} 92, \mathrm{X} 96-Y 09, Y 87.1\right)$ & 1.9 & 2.5 & 1.3 & 2.4 & 3.4 & 1.3 & 1.8 & 2.4 & 1.3 \\
\hline $\begin{array}{l}\text { Legal intervention . . . . . . . . . . . (Y35,Y89.0) } \\
\text { Events of undetermined }\end{array}$ & 0.1 & 0.3 & * & 0.2 & 0.4 & * & 0.1 & 0.2 & * \\
\hline $\begin{array}{l}\text { intent. . . . . . . . . . . . (Y10-Y34,Y87.2,Y89.9) } \\
\text { Discharge of firearms, undetermined }\end{array}$ & 1.8 & 2.3 & 1.3 & 0.7 & 1.0 & 0.5 & 2.0 & 2.5 & 1.5 \\
\hline $\begin{array}{l}\text { intent ..................... (Y22-Y24) } \\
\text { Other and unspecified events of } \\
\text { undetermined intent and their }\end{array}$ & 0.1 & 0.2 & 0.0 & 0.1 & 0.1 & * & 0.1 & 0.2 & 0.0 \\
\hline sequelae . . . . . (Y10-Y21,Y25-Y34,Y87.2,Y89.9) & 1.7 & 2.1 & 1.3 & 0.7 & 0.9 & 0.4 & 1.9 & 2.3 & 1.4 \\
\hline $\begin{array}{l}\text { Operations of war and their sequelae ....(Y36,Y89.1) } \\
\text { Complications of medical and surgical }\end{array}$ & 0.0 & 0.0 & * & * & * & * & * & * & * \\
\hline care $\ldots \ldots \ldots \ldots \ldots \ldots \ldots(Y 40-Y 84, Y 88)$ & 0.9 & 0.8 & 0.9 & 0.3 & 0.3 & 0.3 & 1.0 & 0.9 & 1.0 \\
\hline Enterocolitis due to Clostridium difficile . . . (A04.7) ${ }^{7}$ & 2.1 & 1.6 & 2.6 & 0.5 & 0.5 & 0.7 & 2.4 & 1.8 & 2.9 \\
\hline
\end{tabular}

See footnotes at end of table. 
Table 15. Death rates for 113 selected causes and Enterocolitis due to Clostridium difficile, by Hispanic origin, race for non-Hispanic population, and sex: United States, 2007-Con.

[Rates per 100,000 population in specified group. Populations used for computing death rates are postcensal estimates based on the 2000 census, estimated as of July 1 , 2007; see "Technical Notes." Race and Hispanic origin are reported separately on the death certificate. Persons of Hispanic origin may be of any race. Data for Hispanic persons are not tabulated separately by race; data for non-Hispanic persons are tabulated by race. Data for Hispanic origin should be interpreted with caution because of inconsistencies between reporting Hispanic origin on death certificates and on censuses and surveys; see "Technical Notes." The asterisks (*) preceding the cause-of-death codes indicate that they are not part of the International Classification of Diseases, Tenth Revision (ICD-10), Second Edition; see "Technical Notes"]

\begin{tabular}{|c|c|c|c|c|c|c|}
\hline \multirow[b]{2}{*}{ Cause of death (based on ICD-10, 2004) } & \multicolumn{3}{|c|}{ Non-Hispanic white ${ }^{3}$} & \multicolumn{3}{|c|}{ Non-Hispanic black ${ }^{3}$} \\
\hline & $\begin{array}{l}\text { Both } \\
\text { sexes }\end{array}$ & Male & Female & $\begin{array}{l}\text { Both } \\
\text { sexes }\end{array}$ & Male & Female \\
\hline All causes $\ldots \ldots \ldots \ldots \ldots \ldots \ldots$ & 964.1 & 960.4 & 967.6 & 750.7 & 805.1 & 701.0 \\
\hline Salmonella infections . . . . . . . . . (A01-A02) & 0.0 & * & * & * & * & * \\
\hline Shigellosis and amebiasis . . . . . . . . . (A03,A06) & * & * & * & * & * & * \\
\hline Certain other intestinal infections $\ldots(\mathrm{A} 04, \mathrm{~A} 07-\mathrm{A} 09)$ & 3.0 & 2.3 & 3.7 & 1.1 & 0.9 & 1.3 \\
\hline Tuberculosis. . . . . . . . (A16-A19) & 0.1 & 0.1 & 0.1 & 0.3 & 0.5 & 0.2 \\
\hline Respiratory tuberculosis. . . . . . (A16) & 0.1 & 0.1 & 0.1 & 0.2 & 0.3 & 0.1 \\
\hline Other tuberculosis . . . . . (A17-A19) & 0.0 & 0.0 & 0.0 & 0.1 & 0.1 & * \\
\hline Whooping cough $\ldots \ldots \ldots \ldots \ldots \ldots$ (A37) & * & * & * & * & * & * \\
\hline Scarlet fever and erysipelas $\ldots \ldots \ldots(\mathrm{A} 38, \mathrm{~A} 46)$ & * & * & * & * & * & * \\
\hline Meningococcal infection . . . . . . . . . . . (A39) & 0.0 & 0.0 & 0.0 & 0.1 & * & * \\
\hline Septicemia $\ldots \ldots \ldots \ldots$. . . . . . . (A40-A41) & 12.9 & 11.8 & 13.9 & 16.4 & 15.4 & 17.2 \\
\hline Syphilis $\ldots \ldots \ldots \ldots \ldots \ldots \ldots$ (A50-A53) & * & * & * & 0.1 & * & * \\
\hline Acute poliomyelitis . . . . . . . . . . . (A80) & * & * & * & * & * & * \\
\hline Arthropod-borne viral encephalitis . (A83-A84,A85.2) & * & * & * & * & * & * \\
\hline Measles . . . . . . . . . . . . . . . (B05) & * & * & * & * & * & * \\
\hline Viral hepatitis . . . . . . . . . . . (B15-B19) & 2.4 & 3.3 & 1.6 & 2.7 & 3.7 & 1.8 \\
\hline \multicolumn{7}{|l|}{ Human immunodeficiency virus (HIV) } \\
\hline disease $\ldots \ldots \ldots \ldots \ldots$ (B20-B24) & 1.6 & 2.7 & 0.5 & 16.8 & 22.7 & 11.4 \\
\hline Malaria . . . . . . . . . . . . . (B50-B54) & * & * & * & * & * & * \\
\hline \multicolumn{7}{|l|}{$\begin{array}{r}\text { Other and unspecified infectious and parasitic } \\
\text { diseases and their sequelae . . . . . (A00,A05, } \\
\text { A20-A36,A42-A44,A48-A49, A54-A79,A81-A82, } \\
\text { A85.0-A85.1,A85.8, A86-B04,B06-B09, }\end{array}$} \\
\hline Malignant neoplasms . . . . . . . . . (C00-C97) & 226.9 & 240.6 & 213.7 & 166.3 & 180.1 & 153.7 \\
\hline \multicolumn{7}{|l|}{ Malignant neoplasms of lip, oral cavity and } \\
\hline pharynx ... . . . . . . . . . . . . (CO0-C14) & 3.2 & 4.3 & 2.0 & 2.8 & 4.2 & 1.5 \\
\hline Malignant neoplasm of esophagus . . . . . . (C15) & 5.7 & 9.2 & 2.3 & 3.8 & 5.8 & 2.0 \\
\hline Malignant neoplasm of stomach . . & 3.6 & 4.4 & 2.8 & 5.2 & 6.5 & 4.0 \\
\hline \multicolumn{7}{|l|}{ Malignant neoplasms of colon, rectum } \\
\hline and anus $\ldots \ldots \ldots \ldots(C 18-C 21)$ & 21.1 & 21.7 & 20.5 & 18.0 & 18.8 & 17.2 \\
\hline \multicolumn{7}{|l|}{ Malignant neoplasms of liver and } \\
\hline intrahepatic bile ducts $\ldots \ldots \ldots$ & 5.8 & 7.7 & 4.0 & 5.9 & 8.7 & 3.3 \\
\hline Malignant neoplasm of pancreas . & 13.6 & 14.1 & 13.1 & 10.6 & 10.3 & 10.8 \\
\hline Malignant neoplasm of larynx $\ldots \ldots \ldots \ldots$ (C32) & 1.4 & 2.2 & 0.6 & 1.7 & 3.0 & 0.6 \\
\hline \multicolumn{7}{|l|}{ Malignant neoplasms of trachea, } \\
\hline bronchus and lung $\ldots \ldots \ldots$ & 66.7 & 74.6 & 59.0 & 42.9 & 53.6 & 33.2 \\
\hline Malignant melanoma of skin ... & 4.0 & 5.4 & 2.7 & 0.3 & 0.3 & 0.4 \\
\hline Malignant neoplasm of breast . & 16.0 & 0.3 & 31.1 & 15.2 & 0.3 & 28.8 \\
\hline Malignant neoplasm of cervix uteri & 1.3 & $\ldots$ & 2.5 & 2.1 & $\ldots$ & 4.0 \\
\hline \multicolumn{7}{|l|}{ Malignant neoplasms of corpus uteri } \\
\hline and uterus, part unspecified $\ldots \ldots$ (C54-C55) & 2.8 & $\ldots$ & 5.4 & 3.4 & $\ldots$ & 6.4 \\
\hline Malignant neoplasm of ovary . . . . . . . . (C56) & 6.1 & & 12.0 & 3.0 & & 5.8 \\
\hline Malignant neoplasm of prostate $\ldots \ldots \ldots$ (C61) & 11.1 & 22.5 & $\ldots$ & 12.8 & 26.7 & $\ldots$ \\
\hline \multicolumn{7}{|l|}{ Malignant neoplasms of kidney and } \\
\hline renal pelvis $\ldots \ldots \ldots \ldots \ldots$ (C64-C65) & 5.1 & 6.5 & 3.8 & 3.2 & 4.1 & 2.4 \\
\hline Malignant neoplasm of bladder $\ldots \ldots \ldots$ (C67) & 6.0 & 8.7 & 3.5 & 2.7 & 3.1 & 2.3 \\
\hline \multicolumn{7}{|l|}{$\begin{array}{l}\text { Malignant neoplasms of meninges, } \\
\text { brain and other parts of central }\end{array}$} \\
\hline nervous system $\ldots \ldots \ldots \ldots$ (C70-C72) & 5.7 & 6.4 & 4.9 & 2.0 & 2.2 & 1.9 \\
\hline \multicolumn{7}{|l|}{ Malignant neoplasms of lymphoid, } \\
\hline hematopoietic and related tissue $\ldots($ C81-C96) & 22.4 & 25.3 & 19.6 & 13.6 & 14.5 & 12.8 \\
\hline Hodgkin's disease $\ldots \ldots \ldots \ldots \ldots$ (C81) & 0.5 & 0.6 & 0.4 & 0.3 & 0.4 & 0.3 \\
\hline Non-Hodgkin's lymphoma . . . . . . (C82-C85) & 8.6 & 9.4 & 7.9 & 3.6 & 4.1 & 3.2 \\
\hline Leukemia . . . . . . . . . . . . (C91-C95) & 9.1 & 10.6 & 7.6 & 4.7 & 5.2 & 4.3 \\
\hline \multicolumn{7}{|l|}{ Multiple myeloma and immunoproliferative } \\
\hline neoplasms $\ldots \ldots \ldots \ldots(\mathrm{C} 88, \mathrm{C90})$ & 4.2 & 4.8 & 3.7 & 4.9 & 4.8 & 5.0 \\
\hline
\end{tabular}

See footnotes at end of table. 
Table 15. Death rates for 113 selected causes and Enterocolitis due to Clostridium difficile, by Hispanic origin, race for non-Hispanic population, and sex: United States, 2007-Con.

[Rates per 100,000 population in specified group. Populations used for computing death rates are postcensal estimates based on the 2000 census, estimated as of July 1 , 2007; see "Technical Notes." Race and Hispanic origin are reported separately on the death certificate. Persons of Hispanic origin may be of any race. Data for Hispanic persons are not tabulated separately by race; data for non-Hispanic persons are tabulated by race. Data for Hispanic origin should be interpreted with caution because of inconsistencies between reporting Hispanic origin on death certificates and on censuses and surveys; see "Technical Notes." The asterisks ( $\left.{ }^{*}\right)$ preceding the cause-of-death codes indicate that they are not part of the International Classification of Diseases, Tenth Revision (ICD-10), Second Edition; see "Technical Notes"]

\begin{tabular}{|c|c|c|c|c|c|c|}
\hline Cause of death (based on ICD-10, 2004) & \multicolumn{3}{|c|}{ Non-Hispanic white ${ }^{3}$} & \multicolumn{3}{|c|}{ Non-Hispanic black ${ }^{3}$} \\
\hline $\begin{array}{l}\text { All other and unspecified malignant } \\
\text { neoplasms . (C17,C23-C24,C26-C31, C37-C41, } \\
\text { C44-C49,C51-C52,C57-C60, C62-C63, }\end{array}$ & & & & & & \\
\hline \multicolumn{7}{|l|}{$\begin{array}{l}\text { In situ neoplasms, benign neoplasms and } \\
\text { neoplasms of uncertain or unknown }\end{array}$} \\
\hline behavior . . . . . . . . . . . . (D00-D48) & 5.9 & 6.2 & 5.7 & 3.1 & 3.2 & 3.0 \\
\hline Anemias $\ldots \ldots \ldots \ldots \ldots \ldots \ldots$ (D50-D64) & 1.8 & 1.4 & 2.1 & 2.5 & 2.4 & 2.6 \\
\hline Diabetes mellitus $\ldots \ldots \ldots \ldots \ldots$ (E10-E14) & 24.9 & 25.9 & 23.9 & 32.4 & 29.9 & 34.6 \\
\hline Nutritional deficiencies . . . . . . . . . . (E40-E64) & 1.1 & 0.8 & 1.4 & 1.0 & 0.9 & 1.1 \\
\hline Major cardiovascular diseases $\ldots \ldots \ldots$. $\ldots$ (100-178) & 324.4 & 315.2 & 333.4 & 249.2 & 253.7 & 245.1 \\
\hline \multirow{2}{*}{\multicolumn{7}{|c|}{ Acute rheumatic fever and chronic }} \\
\hline & & & & & & \\
\hline rheumatic heart diseases $\ldots \ldots \ldots(100-109)$ & 1.3 & 0.9 & 1.8 & 0.6 & 0.5 & 0.8 \\
\hline Hypertensive heart disease $\ldots \ldots$. & 10.6 & 9.4 & 11.7 & 18.4 & 19.9 & 17.1 \\
\hline Hypertensive heart and renal disease . . . . (113) & 0.9 & 0.8 & 1.1 & 2.1 & 2.2 & 2.1 \\
\hline Ischemic heart diseases $\ldots \ldots \ldots \ldots$ (I20-125) & 166.0 & 179.9 & 152.6 & 110.5 & 118.0 & 103.6 \\
\hline Acute myocardial infarction . . . . . (121-I22) & 54.2 & 60.0 & 48.7 & 36.6 & 38.1 & 35.3 \\
\hline Other acute ischemic heart diseases . . . (124) & 1.7 & 1.7 & 1.6 & 1.4 & 1.6 & 1.2 \\
\hline \multicolumn{7}{|l|}{ Other forms of chronic ischemic heart } \\
\hline disease . . . . . . . . . . . . . . (120,125) & 110.1 & 118.2 & 102.4 & 72.5 & 78.4 & 67.2 \\
\hline Atherosclerotic cardiovascular disease, so & & & & & & \\
\hline $\begin{array}{l}\text { described .................. (I25.0) } \\
\text { All other forms of chronic ischemic heart }\end{array}$ & 22.5 & 25.5 & 19.6 & 23.1 & 27.9 & 18.7 \\
\hline renal disease $\ldots \ldots \ldots \ldots \ldots(110,|112| 15)$, & 8.6 & 6.6 & 10.5 & 12.1 & 10.8 & 13.2 \\
\hline Cerebrovascular diseases $\ldots \ldots \ldots \ldots(160-169)$ & 53.5 & 41.9 & 64.7 & 44.4 & 41.1 & 47.4 \\
\hline Atherosclerosis . . . . . . . . . . . . . . . . . (170) & 3.5 & 2.8 & 4.3 & 1.7 & 1.4 & 1.9 \\
\hline Other diseases of circulatory system . . (171-178) & 9.0 & 9.6 & 8.4 & 6.4 & 6.7 & 6.1 \\
\hline Aortic aneurysm and dissection ....... (171) & 5.4 & 6.4 & 4.5 & 3.3 & 3.8 & 2.7 \\
\hline \multicolumn{7}{|l|}{ Other diseases of arteries, arterioles and } \\
\hline capillaries . . . . . . . . . . . . . . (172-178) & 3.6 & 3.2 & 3.9 & 3.1 & 2.9 & 3.4 \\
\hline Other disorders of circulatory system . . . (180-199) & 1.5 & 1.4 & 1.7 & 1.8 & 1.9 & 1.7 \\
\hline Influenza and pneumonia $\ldots \ldots \ldots\left(\right.$ J09-J18) ${ }^{4}$ & 21.5 & 19.6 & 23.3 & 13.3 & 13.5 & 13.2 \\
\hline Influenza . . . . . . . . . . . . . (J09-J111 & 0.2 & 0.2 & 0.2 & 0.1 & * & * \\
\hline Pneumonia $\ldots \ldots \ldots \ldots \ldots \ldots$ (J12-J18) & 21.3 & 19.4 & 23.1 & 13.3 & 13.5 & 13.1 \\
\hline \multicolumn{7}{|l|}{ Other acute lower respiratory } \\
\hline infections $\ldots \ldots \ldots \ldots$ & 0.1 & 0.1 & 0.1 & 0.1 & * & * \\
\hline Acute bronchitis and bronchiolitis . . . . (J20-J21) & 0.1 & 0.1 & 0.1 & 0.1 & * & * \\
\hline $\begin{array}{l}\text { Other and unspecified acute lower } \\
\text { respiratory infections }\end{array}$ & 0.0 & * & 00 & * & * & * \\
\hline Chronic lower respiratory diseases $\ldots . . .(J 40-J 47)$ & 56.9 & 54.7 & 59.1 & 20.5 & 22.9 & 18.4 \\
\hline Bronchitis, chronic and unspecified . . . . (J40-J42) & 0.3 & 0.2 & 0.3 & 0.1 & 0.1 & 0.2 \\
\hline
\end{tabular}

See footnotes at end of table. 
Table 15. Death rates for 113 selected causes and Enterocolitis due to Clostridium difficile, by Hispanic origin, race for non-Hispanic population, and sex: United States, 2007-Con.

[Rates per 100,000 population in specified group. Populations used for computing death rates are postcensal estimates based on the 2000 census, estimated as of July 1 , 2007; see "Technical Notes." Race and Hispanic origin are reported separately on the death certificate. Persons of Hispanic origin may be of any race. Data for Hispanic persons are not tabulated separately by race; data for non-Hispanic persons are tabulated by race. Data for Hispanic origin should be interpreted with caution because of inconsistencies between reporting Hispanic origin on death certificates and on censuses and surveys; see "Technical Notes." The asterisks (*) preceding the cause-of-death codes indicate that they are not part of the International Classification of Diseases, Tenth Revision (ICD-10), Second Edition; see "Technical Notes"]

\begin{tabular}{|c|c|c|c|c|c|c|}
\hline \multirow[b]{2}{*}{ Cause of death (based on ICD-10, 2004) } & \multicolumn{3}{|c|}{ Non-Hispanic white ${ }^{3}$} & \multicolumn{3}{|c|}{ Non-Hispanic black ${ }^{3}$} \\
\hline & $\begin{array}{l}\text { Both } \\
\text { sexes }\end{array}$ & Male & Female & $\begin{array}{l}\text { Both } \\
\text { sexes }\end{array}$ & Male & Female \\
\hline Emphysema . . . . . . . . . . . . . . . . . . .(J43) & 5.8 & 5.9 & 5.6 & 1.9 & 2.5 & 1.4 \\
\hline Asthma $\ldots \ldots \ldots \ldots \ldots \ldots \ldots \ldots \ldots$ (J45-J46) & 1.1 & 0.7 & 1.4 & 2.3 & 2.2 & 2.5 \\
\hline Other chronic lower respiratory & & & & & & \\
\hline $\begin{array}{l}\text { diseases . . . . . . . . . . . . . . (J44,J47) } \\
\text { Pneumoconioses and chemical }\end{array}$ & 49.8 & 47.8 & 51.7 & 16.1 & 18.1 & 14.4 \\
\hline 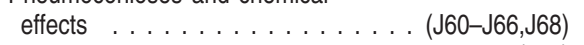 & 0.4 & 0.8 & 0.0 & 0.1 & 0.2 & * \\
\hline $\begin{array}{l}\text { Pneumonitis due to solids and liquids . . . . . . . (J69) } \\
\text { Other diseases of respiratory }\end{array}$ & 7.3 & 7.8 & 6.8 & 3.7 & 4.0 & 3.4 \\
\hline system . . . . . . (J00-J06,J30-J39,J67,J70-J98) & 11.7 & 11.9 & 11.4 & 6.7 & 6.8 & 6.6 \\
\hline Peptic ulcer $\ldots \ldots \ldots \ldots \ldots \ldots$ (K25-K28) & 1.2 & 1.2 & 1.3 & 0.8 & 0.9 & 0.7 \\
\hline Diseases of appendix $\ldots \ldots \ldots \ldots \ldots($ K35-K38) & 0.2 & 0.2 & 0.1 & 0.2 & 0.2 & 0.1 \\
\hline 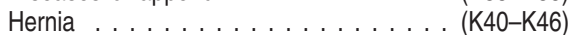 & 0.7 & 0.6 & 0.8 & 0.4 & 0.5 & 0.4 \\
\hline Chronic liver disease and cirrhosis ..(K70,K73-K74) & 10.7 & 14.2 & 7.4 & 6.6 & 9.1 & 4.3 \\
\hline $\begin{array}{l}\text { Alcoholic liver disease .... . . . . . . . (K70) } \\
\text { Other chronic liver disease and }\end{array}$ & 5.2 & 7.6 & 2.9 & 3.1 & 4.6 & 1.7 \\
\hline cirrhosis . . . . . . . . . & 5.6 & 6.6 & 4.6 & 3.5 & 4.5 & 2.6 \\
\hline $\begin{array}{l}\text { Cholelithiasis and other disorders of } \\
\text { gallbladder .................. (K80-K82) } \\
\text { Nephritis, nephrotic syndrome and }\end{array}$ & 1.3 & 1.2 & 1.4 & 0.8 & 0.6 & 0.9 \\
\hline nephrosis . . . . . . (N00-N07,N17-N19,N25-N27) & 17.0 & 17.1 & 16.9 & 21.8 & 20.5 & 23.0 \\
\hline $\begin{array}{l}\text { Acute and rapidly progressive nephritic and } \\
\text { nephrotic syndrome ......... (N00-N01,N04) } \\
\text { Chronic glomerulonephritis, nephritis and } \\
\text { nephropathy not specified as acute or } \\
\text { chronic, and renal sclerosis }\end{array}$ & 0.1 & 0.1 & 0.1 & 0.1 & * & * \\
\hline unspecified ....... (N02-N03,N05-N07,N26) & 1.1 & 1.1 & 1.1 & 1.4 & 1.2 & 1.5 \\
\hline Renal failure $\ldots \ldots \ldots \ldots \ldots \ldots$ (N17-N19) & 15.8 & 15.9 & 15.8 & 20.3 & 19.1 & 21.4 \\
\hline Other disorders of kidney $\ldots \ldots \ldots(\mathrm{N} 25, \mathrm{~N} 27)$ & * & * & * & * & * & * \\
\hline Infections of kidney $\ldots \ldots(\mathrm{N} 10-\mathrm{N} 12, \mathrm{~N} 13.6, \mathrm{~N} 15.1)$ & 0.3 & 0.2 & 0.3 & 0.1 & 0.1 & 0.2 \\
\hline $\begin{array}{l}\text { Hyperplasia of prostate } \ldots \ldots \ldots \ldots \ldots \text { (N40) } \\
\text { Inflammatory diseases of female pelvic }\end{array}$ & 0.2 & 0.4 & $\ldots$ & 0.1 & 0.2 & $\ldots$ \\
\hline $\begin{array}{l}\text { organs } \ldots \ldots \ldots \ldots \ldots \ldots \ldots \text { (N70-N76) } \\
\text { Pregnancy, childbirth and the }\end{array}$ & 0.0 & $\ldots$ & 0.1 & * & $\ldots$ & * \\
\hline puerperium . . . . . . . . . . . . . (000-099) & 0.2 & $\ldots$ & 0.3 & 0.7 & $\ldots$ & 1.2 \\
\hline $\begin{array}{l}\text { Pregnancy with abortive outcome .... (O00-007) } \\
\text { Other complications of pregnancy, childbirth and }\end{array}$ & * & $\ldots$ & * & * & $\ldots$ & * \\
\hline $\begin{array}{l}\text { the puerperium } \ldots \ldots \ldots \ldots \ldots \text { (010-099) } \\
\text { Certain conditions originating in the perinatal }\end{array}$ & 0.2 & $\ldots$ & 0.3 & 0.6 & $\ldots$ & 1.2 \\
\hline $\begin{array}{l}\text { period } \ldots \ldots \ldots \ldots \ldots \ldots \ldots \ldots \text { (P00-P96) } \\
\text { Congenital malformations, deformations and }\end{array}$ & 3.0 & 3.5 & 2.6 & 12.8 & 15.0 & 10.7 \\
\hline $\begin{array}{l}\text { chromosomal abnormalities ......... (Q00-Q99) } \\
\text { Symptoms, signs and abnormal clinical and } \\
\text { laboratory findings, not elsewhere }\end{array}$ & 3.1 & 3.3 & 2.9 & 4.6 & 5.0 & 4.3 \\
\hline classified . . . . . . . . . . . . . . . (R00-R99) & 13.0 & 10.7 & 15.3 & 12.0 & 12.2 & 11.8 \\
\hline All other diseases & 97.1 & 78.4 & 115.1 & 68.5 & 61.4 & 75.0 \\
\hline Accidents (unintentional injuries) .(V01-X59,Y85-Y86) & 47.0 & 60.0 & 34.5 & 34.9 & 50.0 & 21.2 \\
\hline $\begin{array}{r}\text { Transport accidents } \ldots . . . . .(\mathrm{V} 01-\mathrm{V} 99, \mathrm{Y} 85) \\
\text { Motor-vehicle accidents. . . . . (V02-V04, } \\
\text { V09.0,V09.2,V12-V14,V19.0-V19.2, } \\
\text { V19.4-V19.6,V20-V79,V80.3-V80.5, } \\
\text { V81.0-V81.1,V82.0-V82.1,V83-V86, }\end{array}$ & 16.4 & 23.6 & 9.5 & 15.1 & 23.4 & 7.5 \\
\hline $\begin{array}{r}\text { V87.0-V87.8,V88.0-V88.8,V89.0, V89.2) } \\
\text { Other land transport accidents ...... (V01, } \\
\text { V05-V06,V09.1,V09.3-V09.9, V10-V11, } \\
\text { V15-V18,V19.3,V19.8- V19.9,V80.0-V80.2, } \\
\text { V80.6-V80.9, V81.2-V81.9,V82.2-V82.9, } \\
\text { V87.9, V88.9,V89.1,V89.3,V89.9) }\end{array}$ & 15.4 & 21.8 & 9.1 & 14.2 & 21.9 & 7.2 \\
\hline
\end{tabular}

See footnotes at end of table. 
Table 15. Death rates for 113 selected causes and Enterocolitis due to Clostridium difficile, by Hispanic origin, race for non-Hispanic population, and sex: United States, 2007-Con.

[Rates per 100,000 population in specified group. Populations used for computing death rates are postcensal estimates based on the 2000 census, estimated as of July 1 , 2007; see "Technical Notes." Race and Hispanic origin are reported separately on the death certificate. Persons of Hispanic origin may be of any race. Data for Hispanic persons are not tabulated separately by race; data for non-Hispanic persons are tabulated by race. Data for Hispanic origin should be interpreted with caution because of inconsistencies between reporting Hispanic origin on death certificates and on censuses and surveys; see "Technical Notes." The asterisks (*) preceding the cause-of-death codes indicate that they are not part of the International Classification of Diseases, Tenth Revision (ICD-10), Second Edition; see "Technical Notes"]

\begin{tabular}{|c|c|c|c|c|c|c|}
\hline \multirow[b]{2}{*}{ Cause of death (based on ICD-10, 2004) } & \multicolumn{3}{|c|}{ Non-Hispanic white ${ }^{3}$} & \multicolumn{3}{|c|}{ Non-Hispanic black ${ }^{3}$} \\
\hline & $\begin{array}{l}\text { Both } \\
\text { sexes }\end{array}$ & Male & Female & $\begin{array}{l}\text { Both } \\
\text { sexes }\end{array}$ & Male & Female \\
\hline \multicolumn{7}{|l|}{$\begin{array}{l}\text { Water, air and space, and other and } \\
\text { unspecified transport accidents }\end{array}$} \\
\hline and their sequelae . . . . . . . . (V90-V99,Y85) & 0.7 & 1.2 & 0.3 & 0.5 & 0.8 & 0.1 \\
\hline Nontransport accidents . . . . . . (W00-X59,Y86) & 30.6 & 36.5 & 24.9 & 19.9 & 26.6 & 13.7 \\
\hline $\begin{array}{l}\text { Falls ...................... (W00-W19) } \\
\text { Accidental discharge of }\end{array}$ & 9.8 & 10.0 & 9.7 & 2.6 & 3.2 & 2.1 \\
\hline firearms . . . . . . . . . . . . . . . (W32-W34) & 0.2 & 0.4 & 0.1 & 0.3 & 0.6 & * \\
\hline $\begin{array}{l}\text { Accidental drowning and } \\
\text { submersion ................... (W65-W74) } \\
\text { Accidental exposure to smoke, fire and }\end{array}$ & 1.1 & 1.7 & 0.5 & 1.3 & 2.3 & 0.5 \\
\hline $\begin{array}{l}\text { flames } \ldots \ldots \ldots \ldots \ldots \ldots \ldots \text { (X00-X09) } \\
\text { Accidental poisoning and exposure to }\end{array}$ & 1.1 & 1.3 & 0.9 & 2.0 & 2.5 & 1.6 \\
\hline $\begin{array}{l}\text { noxious substances } \ldots \ldots \ldots \ldots(\mathrm{X} 40-\mathrm{X} 49) \\
\text { Other and unspecified nontransport }\end{array}$ & 11.7 & 15.4 & 8.1 & 8.4 & 11.6 & 5.6 \\
\hline $\begin{array}{l}\text { accidents and their sequelae.... (W20- W31, } \\
\text { W35-W64,W75-W99,X10-X39, X50-X59,Y86) }\end{array}$ & 6.7 & 7.7 & 5.6 & 5.2 & 6.4 & 4.0 \\
\hline $\begin{array}{l}\left.\text { Intentional self-harm (suicide) . ( }{ }^{*} \cup 03, \mathrm{X} 60-\mathrm{X} 84, \mathrm{Y} 87.0\right) \\
\text { Intentional self-harm (suicide) by discharge of }\end{array}$ & 14.4 & 22.9 & 6.1 & 5.0 & 8.6 & 1.7 \\
\hline $\begin{array}{l}\text { firearms. . . . . . . . . . . . . (X72-X74) } \\
\text { Intentional self-harm (suicide) by other and } \\
\text { unspecified means and their }\end{array}$ & 7.5 & 13.3 & 1.9 & 2.6 & 4.8 & 0.5 \\
\hline sequelae . . . . . ( $\left.{ }^{*} \mathrm{U} 03, \mathrm{X} 60-\mathrm{X} 71, \mathrm{X} 75-\mathrm{X} 84, \mathrm{Y} 87.0\right)$ & 6.9 & 9.6 & 4.2 & 2.5 & 3.9 & 1.2 \\
\hline $\begin{array}{l}\text { Assault (homicide). . . . . (*U01-*U02,X85-Y09,Y87.1) } \\
\text { Assault (homicide) by discharge of }\end{array}$ & 2.7 & 3.7 & 1.8 & 22.9 & 41.1 & 6.4 \\
\hline $\begin{array}{l}\left.\text { firearms. . . . . . . . . . ( }{ }^{*} \cup 01.4, \mathrm{X} 93-\mathrm{X} 95\right) \\
\text { unspecified means and their }\end{array}$ & 1.5 & 2.2 & 0.9 & 18.0 & 34.0 & 3.4 \\
\hline $\begin{array}{r}\text { sequelae . . . . . . ( }{ }^{*} \cup 01.0{ }^{*} \cup 01.3,{ }^{*} \cup 01.5-{ }^{*} \cup 01.9, \\
\end{array}$ & 1.2 & 1.5 & 0.9 & 4.9 & 7.1 & 3.0 \\
\hline $\begin{array}{l}\text { Legal intervention . . . . . . . . . . . . (Y35,Y89.0) } \\
\text { Events of undetermined }\end{array}$ & 0.1 & 0.2 & * & 0.3 & 0.7 & * \\
\hline $\begin{array}{l}\text { intent. . . . . . . . . . . . . (Y10-Y34,Y87.2,Y89.9) } \\
\text { Discharge of firearms, undetermined }\end{array}$ & 2.0 & 2.5 & 1.6 & 2.0 & 2.8 & 1.2 \\
\hline $\begin{array}{l}\text { intent ..................... (Y22-Y24) } \\
\text { Other and unspecified events of } \\
\text { undetermined intent and their }\end{array}$ & 0.1 & 0.1 & 0.0 & 0.1 & 0.3 & * \\
\hline sequelae . . . . . (Y10-Y21,Y25-Y34,Y87.2,Y89.9) & 2.0 & 2.4 & 1.6 & 1.8 & 2.6 & 1.1 \\
\hline Operations of war and their sequelae . . (Y36,Y89.1) & * & * & * & * & * & * \\
\hline $\begin{array}{l}\text { Complications of medical and surgical } \\
\text { care } \ldots \ldots \ldots \ldots \ldots \ldots \ldots(Y 40-Y 84, Y 88)\end{array}$ & 1.0 & 1.0 & 1.0 & 1.1 & 1.0 & 1.2 \\
\hline Enterocolitis due to Clostridium difficile . . . (A04.7) & 2.8 & 2.1 & 3.5 & 1.0 & 0.8 & 1.2 \\
\hline
\end{tabular}

0.0 Quantity more than zero but less than 0.05 .

*Figure does not meet standards of reliability or precision; see "Technical Notes."

... Category not applicable.

"Figures for origin not stated are included in "all origins" but not distributed among specified origins.

${ }^{2}$ Includes races other than white and black.

${ }^{3}$ Race categories are consistent with the 1977 Office of Management and Budget (OMB) standards. Multiple-race data were reported by 27 states and the District of Columbia in 2007 ; see "Technical Notes." The multiple-race data for these reporting areas were bridged to the single-race categories of the 1977 OMB standards for comparability with other reporting areas; see "Technical Notes."

${ }^{4} \mathrm{New}$ ICD-10 code J09 (Influenza due to identified avian influenza virus) was added to the category in 2007. No deaths occurred from this cause in 2007.

${ }^{5} \mathrm{New}$ ICD-10 code U04 (Severe acute respiratory syndrome [SARS]) was added to the category in 2007. No deaths occurred from this cause in 2007.

${ }^{6}$ Cause-of-death title was changed in 2007 to reflect the addition of SARS (ICD-10 code U04).

"Included in "Certain other intestinal infections (A04,A07-A09)" shown above. Beginning with data year 2006, "Enterocolitis due to Clostridium difficile (A04.7)" is shown separately at the bottom of tables showing 113 selected causes and is included in the list of rankable causes; see "Technical Notes."

NOTE: Complete confirmation of deaths from selected causes of death, considered to be of public health concern, was not provided by the following states: Connecticut, Florida, Indiana, Kentucky, Maryland, North Carolina, Oklahoma, Pennsylvania, Rhode Island, South Carolina, Washington, and West Virginia; see "Technical Notes." 
Table 16. Age-adjusted death rates for 113 selected causes and Enterocolitis due to Clostridium difficile, by race and sex: United States, 2007

[Age-adjusted rates per 100,000 U.S. standard population; see "Technical Notes." Populations used for computing death rates are postcensal estimates based on the 2000 census, estimated as of July 1, 2007; see "Technical Notes." Data for specified races other than white and black should be interpreted with caution because of inconsistencies between reporting race on death certificates and on censuses and surveys; see "Technical Notes." The asterisks $\left({ }^{*}\right)$ preceding the cause-of-death codes indicate that they are not part of the International Classification of Diseases, Tenth Revision (ICD-10), Second Edition; see "Technical Notes"]

\begin{tabular}{|c|c|c|c|c|c|c|c|c|c|}
\hline \multirow[b]{2}{*}{ Cause of death (based on ICD-10, 2004) } & \multicolumn{3}{|c|}{ All races } & \multicolumn{3}{|c|}{ White $^{1}$} & \multicolumn{3}{|c|}{ Black $^{1}$} \\
\hline & $\begin{array}{l}\text { Both } \\
\text { sexes }\end{array}$ & Male & Female & $\begin{array}{l}\text { Both } \\
\text { sexes }\end{array}$ & Male & Female & $\begin{array}{l}\text { Both } \\
\text { sexes }\end{array}$ & Male & Female \\
\hline$\ldots \ldots \ldots \ldots \ldots \ldots \ldots \ldots$ & 760.2 & 905.6 & 643.4 & 749.4 & 890.5 & 634.8 & 958.0 & $1,184.4$ & 793.8 \\
\hline$\ldots \ldots(\mathrm{A} 01-\mathrm{A} 02)$ & 0.0 & * & * & 0.0 & * & * & * & * & * \\
\hline Shigellosis and amebiasis. . & * & * & * & * & * & * & * & * & * \\
\hline Certain other intestinal infections $\ldots(\mathrm{A} 04, \mathrm{~A} 07-\mathrm{A} 09)$ & 2.1 & 2.1 & 2.2 & 2.2 & 2.2 & 2.3 & 1.6 & 1.6 & 1.6 \\
\hline Tuberculosis . . . . . . . . . . . . . . . . . (A16-A19) & 0.2 & 0.2 & 0.1 & 0.1 & 0.2 & 0.1 & 0.4 & 0.6 & 0.2 \\
\hline Respiratory tuberculosis . . . . . . . . . . . (A16) & 0.1 & 0.2 & 0.1 & 0.1 & 0.1 & 0.0 & 0.3 & 0.5 & 0.2 \\
\hline Other tuberculosis $\ldots \ldots \ldots \ldots \ldots \ldots$. A17-A19) & 0.0 & 0.0 & 0.0 & 0.0 & 0.0 & 0.0 & 0.1 & 0.2 & * \\
\hline Whooping cough $\ldots \ldots \ldots \ldots \ldots \ldots$ (A37) & * & * & * & * & * & * & * & * & * \\
\hline Scarlet fever and erysipelas $\ldots \ldots \ldots(\mathrm{A} 38, \mathrm{~A} 46)$ & * & * & * & * & * & * & * & * & * \\
\hline Meningococcal infection . . . . . . . . . (A39) & 0.0 & 0.0 & 0.0 & 0.0 & 0.0 & 0.0 & 0.0 & * & * \\
\hline Septicemia $\ldots \ldots \ldots \ldots \ldots$. . . . . . . A40-A41) & 11.0 & 12.1 & 10.2 & 10.0 & 11.1 & 9.3 & 21.7 & 24.6 & 19.9 \\
\hline$\ldots \ldots \ldots \ldots \ldots$ (A50-A53) & 0.0 & 0.0 & * & 0.0 & * & * & 0.1 & * & * \\
\hline Acute poliomyelitis . & * & * & * & * & * & * & * & * & * \\
\hline Arthropod-borne viral encephalitis . (A83-A84,A85.2) & * & * & * & * & * & * & * & * & * \\
\hline Measles . . . . . . . . . . . . . . . (B05) & * & * & * & * & * & * & * & * & * \\
\hline 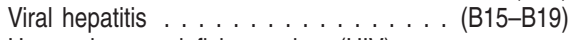 & 2.3 & 3.1 & 1.5 & 2.2 & 3.0 & 1.4 & 2.9 & 4.2 & 1.9 \\
\hline \multicolumn{10}{|l|}{ Human immunodeficiency virus (HIV) } \\
\hline disease . . . . . . . . . . . . . . . . . . (B20-B24) & 3.7 & 5.4 & 2.1 & 1.9 & 3.1 & 0.7 & 17.3 & 24.5 & 11.3 \\
\hline Malaria . . . . . . . . . . . . . . (B50-B54) & * & * & * & * & * & * & * & * & * \\
\hline \multicolumn{10}{|l|}{$\begin{array}{r}\text { Other and unspecified infectious and parasitic } \\
\text { diseases and their sequelae ........A00,A05, } \\
\text { A20-A36,A42-A44,A48-A49,A54-A79,A81-A82, } \\
\text { A85.0-A85.1,A85.8,A86-B04,B06-B09, }\end{array}$} \\
\hline B25-B49,B55-B99) & 1.8 & 2.2 & 1.6 & 1.8 & 2.1 & 1.5 & 2.4 & 2.9 & 2.0 \\
\hline Malignant neoplasms & 178.4 & 217.5 & 151.3 & 177.5 & 215.1 & 151.2 & 215.5 & 282.3 & 174.9 \\
\hline \multicolumn{10}{|l|}{ Malignant neoplasms of lip, oral cavity and } \\
\hline pharynx . . . . . . . . . . . . . . . (CO0-C14) & 2.5 & 3.9 & 1.4 & 2.5 & 3.7 & 1.4 & 3.3 & 5.7 & 1.6 \\
\hline Malignant neoplasm of esophagus . . . . . (C15) & 4.3 & 7.7 & 1.6 & 4.3 & 7.8 & 1.5 & 4.7 & 8.3 & 2.2 \\
\hline Malignant neoplasm of stomach . . . . . . . . (C16) & 3.6 & 5.0 & 2.6 & 3.1 & 4.4 & 2.2 & 6.9 & 10.4 & 4.6 \\
\hline \multicolumn{10}{|l|}{ Malignant neoplasms of colon, rectum } \\
\hline and anus $\ldots \ldots \ldots \ldots \ldots \ldots$ (C18-C21) & 16.9 & 20.1 & 14.4 & 16.4 & 19.6 & 13.9 & 23.5 & 29.0 & 19.8 \\
\hline \multicolumn{10}{|l|}{ Malignant neoplasms of liver and } \\
\hline intrahepatic bile ducts . . . . . . . . . . . . (C22) & 5.4 & 7.9 & 3.2 & 5.0 & 7.2 & 3.1 & 7.1 & 11.5 & 3.8 \\
\hline Malignant neoplasm of pancreas . . . . . . (C25) & 10.8 & 12.5 & 9.4 & 10.6 & 12.4 & 9.2 & 14.0 & 15.5 & 12.7 \\
\hline Malignant neoplasm of larynx $\ldots \ldots \ldots \ldots$ (C32) & 1.1 & 2.1 & 0.4 & 1.1 & 1.9 & 0.4 & 2.1 & 4.3 & 0.6 \\
\hline \multicolumn{10}{|l|}{ Malignant neoplasms of trachea, } \\
\hline bronchus and lung .... & 50.6 & 65.1 & 40.0 & 51.2 & 64.8 & 41.2 & 55.6 & 82.2 & 38.1 \\
\hline Malignant melanoma of skin . . . & 2.7 & 4.0 & 1.7 & 3.0 & 4.5 & 1.9 & 0.5 & 0.5 & 0.4 \\
\hline Malignant neoplasm of breast & 12.9 & 0.3 & 22.9 & 12.5 & 0.3 & 22.3 & 18.6 & 0.4 & 31.4 \\
\hline Malignant neoplasm of cervix uteri & 1.3 & $\ldots$ & 2.4 & 1.2 & $\ldots$ & 2.2 & 2.5 & $\ldots$ & 4.3 \\
\hline \multicolumn{10}{|l|}{ Malignant neoplasms of corpus uteri } \\
\hline and uterus, part unspecified $\ldots \ldots \ldots$ (C54-C55) & 2.3 & $\ldots$ & 4.2 & 2.2 & $\ldots$ & 3.9 & 4.5 & $\ldots$ & 7.5 \\
\hline Malignant neoplasm of ovary $\ldots \ldots$. . . . . (C56) & 4.6 & & 8.2 & 4.8 & $\ldots$ & 8.6 & 3.9 & $\ldots$ & 6.5 \\
\hline Malignant neoplasm of prostate . . . . . . . . (C61) & 9.2 & 23.5 & $\ldots$ & 8.5 & 21.6 & $\ldots$ & 18.6 & 51.5 & $\ldots$ \\
\hline \multicolumn{10}{|l|}{ Malignant neoplasms of kidney and } \\
\hline renal pelvis . . . . . . . . . . . . . (C64-C65) & 4.0 & 5.8 & 2.6 & 4.1 & 5.9 & 2.7 & 4.1 & 5.9 & 2.7 \\
\hline Malignant neoplasm of bladder . . . . . . . (C67) & 4.4 & 7.6 & 2.2 & 4.6 & 8.0 & 2.2 & 3.8 & 5.4 & 2.8 \\
\hline \multicolumn{10}{|l|}{$\begin{array}{l}\text { Malignant neoplasms of meninges, } \\
\text { brain and other parts of central }\end{array}$} \\
\hline nervous system $\ldots \ldots \ldots \ldots$ & 4.2 & 5.1 & 3.5 & 4.6 & 5.5 & 3.8 & 2.4 & 2.8 & 2.1 \\
\hline \multicolumn{10}{|l|}{ Malignant neoplasms of lymphoid, } \\
\hline hematopoietic and related tissue $\ldots$ (C81-C96) & 17.6 & 22.8 & 13.7 & 17.8 & 23.2 & 13.8 & 17.6 & 21.9 & 14.8 \\
\hline Hodgkin's disease $\ldots \ldots \ldots$ & 0.4 & 0.5 & 0.3 & 0.4 & 0.5 & 0.3 & 0.4 & 0.5 & 0.3 \\
\hline Non-Hodgkin's lymphoma & 6.5 & 8.3 & 5.2 & 6.8 & 8.6 & 5.5 & 4.5 & 5.7 & 3.6 \\
\hline Leukemia . . . . . . . . & 7.0 & 9.4 & 5.3 & 7.2 & 9.7 & 5.4 & 6.1 & 7.9 & 5.0 \\
\hline \multicolumn{10}{|l|}{ Multiple myeloma and immunoproliferative } \\
\hline neoplasms . . . . . . . . . . (C88,C90) & 3.6 & 4.6 & 2.9 & 3.3 & 4.4 & 2.6 & 6.6 & 7.8 & 5.9 \\
\hline \multicolumn{10}{|l|}{$\begin{array}{l}\text { Other and unspecified malignant neoplasms } \\
\text { of lymphoid, hematopoietic and }\end{array}$} \\
\hline related tissue $\ldots \ldots \ldots \ldots \ldots \ldots$ (C96) & 0.0 & 0.0 & 0.0 & 0.0 & 0.0 & 0.0 & * & * & * \\
\hline
\end{tabular}

See footnotes at end of table. 
Table 16. Age-adjusted death rates for 113 selected causes and Enterocolitis due to Clostridium difficile, by race and sex: United States, 2007-Con.

[Age-adjusted rates per 100,000 U.S. standard population; see "Technical Notes." Populations used for computing death rates are postcensal estimates based on the 2000 census, estimated as of July 1, 2007; see "Technical Notes." Data for specified races other than white and black should be interpreted with caution because of inconsistencies between reporting race on death certificates and on censuses and surveys; see "Technical Notes." The asterisks $\left({ }^{*}\right)$ preceding the cause-of-death codes indicate that they are not part of the International Classification of Diseases, Tenth Revision (ICD-10), Second Edition; see "Technical Notes"]

\begin{tabular}{|c|c|c|c|c|c|c|c|c|c|}
\hline \multirow[b]{2}{*}{ Cause of death (based on ICD-10, 2004) } & \multicolumn{3}{|c|}{ All races } & \multicolumn{3}{|c|}{ White $^{1}$} & \multicolumn{3}{|c|}{ Black $^{1}$} \\
\hline & $\begin{array}{l}\text { Both } \\
\text { sexes }\end{array}$ & Male & Female & $\begin{array}{l}\text { Both } \\
\text { sexes }\end{array}$ & Male & Female & $\begin{array}{l}\text { Both } \\
\text { sexes }\end{array}$ & Male & Female \\
\hline $\begin{array}{l}\text { All other and unspecified malignant } \\
\text { neoplasms . . . . (C17,C23-C24,C26-C31, } \\
\text { C37-C41,C44-C49,C51-C52,C57-C60, } \\
\text { C62-C63,C66,C68-C69,C73-C80,C97) }\end{array}$ & 20.0 & 24.3 & 16.8 & 20.0 & 24.5 & 16.7 & 22.0 & 26.8 & 18.8 \\
\hline \multicolumn{10}{|l|}{$\begin{array}{l}\text { In situ neoplasms, benign neoplasms and } \\
\text { neoplasms of uncertain or unknown }\end{array}$} \\
\hline behavior . . . . . . . . . . . (D00-D48) & 4.5 & 5.6 & 3.7 & 4.6 & 5.8 & 3.8 & 4.1 & 5.2 & 3.4 \\
\hline Anemias $\ldots \ldots \ldots \ldots \ldots \ldots$ (D50-D64) & 1.5 & 1.5 & 1.5 & 1.3 & 1.3 & 1.3 & 3.0 & 3.1 & 2.9 \\
\hline Diabetes mellitus & 22.5 & 26.4 & 19.5 & 20.5 & 24.6 & 17.2 & 42.8 & 45.9 & 40.2 \\
\hline Nutritional deficiencies. & 0.9 & 0.9 & 0.9 & 0.8 & 0.8 & 0.8 & 1.5 & 1.6 & 1.3 \\
\hline Malnutrition . . . . . . & 0.8 & 0.8 & 0.8 & 0.8 & 0.7 & 0.8 & 1.4 & 1.6 & 1.3 \\
\hline Other nutritional deficiencies . . . . . . (E50-E64) & 0.1 & 0.1 & 0.1 & 0.1 & 0.1 & 0.0 & * & * & * \\
\hline Meningitis $\ldots \ldots \ldots \ldots \ldots \ldots \ldots\left(G 00 \ldots \ldots, \ldots^{G} 03\right)$ & 0.2 & 0.2 & 0.2 & 0.2 & 0.2 & 0.2 & 0.3 & 0.4 & 0.3 \\
\hline Parkinson's disease $\ldots \ldots \ldots \ldots \ldots \ldots$ (G20-G21) & 6.4 & 9.6 & 4.3 & 6.8 & 10.2 & 4.6 & 3.2 & 4.9 & 2.2 \\
\hline Alzheimer's disease $\ldots \ldots \ldots \ldots \ldots \ldots$. . . . (G30) & 22.7 & 18.5 & 24.9 & 23.5 & 19.1 & 25.9 & 19.0 & 15.6 & 20.6 \\
\hline Major cardiovascular diseases . . . . . . . (100-178) & 249.9 & 298.9 & 210.4 & 244.1 & 292.7 & 204.6 & 335.0 & 403.5 & 284.2 \\
\hline Diseases of heart $\ldots . .(100-109,|11| 13,, \mid 20-151)$ & 190.9 & 237.7 & 154.0 & 187.8 & 234.8 & 150.5 & 247.3 & 305.9 & 204.5 \\
\hline Acute rheumatic fever and chronic rheumatic & & & & & & & & & \\
\hline heart diseases . . . . . . . . . . (100-109) & 1.0 & 0.8 & 1.2 & 1.0 & 0.8 & 1.2 & 0.8 & 0.7 & 0.9 \\
\hline Hypertensive heart disease $\ldots \ldots \ldots \ldots$. . (111) & 9.5 & 10.3 & 8.4 & 8.1 & 8.6 & 7.2 & 23.3 & 28.4 & 19.3 \\
\hline Hypertensive heart and renal disease . . . . (I13) & 0.9 & 1.0 & 0.9 & 0.7 & 0.8 & 0.7 & 2.8 & 3.4 & 2.4 \\
\hline Ischemic heart diseases $\ldots \ldots \ldots \ldots$ (I20-125) & 126.0 & 165.4 & 95.7 & 125.5 & 165.6 & 94.2 & 150.6 & 191.6 & 121.5 \\
\hline Acute myocardial infarction . . . . . (121-122) & 41.4 & 54.0 & 31.5 & 41.3 & 54.3 & 30.9 & 49.5 & 60.9 & 41.2 \\
\hline Other acute ischemic heart diseases ... (I24) & 1.3 & 1.6 & 1.0 & 1.2 & 1.5 & 1.0 & 1.8 & 2.4 & 1.4 \\
\hline \multicolumn{10}{|l|}{ Other forms of chronic ischemic heart } \\
\hline disease . . . . . . . . . . . $(120,125)$ & 83.3 & 109.8 & 63.2 & 82.9 & 109.8 & 62.3 & 99.3 & 128.2 & 78.9 \\
\hline $\begin{array}{l}\text { Atherosclerotic cardiovascular } \\
\text { disease so described }\end{array}$ & & & & & & & & & \\
\hline $\begin{array}{l}\text { disease, so described ............ (125.0) } \\
\text { All other forms of chronic ischemic heart }\end{array}$ & 18.2 & 24.3 & 13.1 & 17.3 & 23.0 & 12.3 & 30.3 & 42.3 & 21.6 \\
\hline disease . . . . . . . . . (120,|25.1-|25.9) & 65.1 & 85.5 & 50.1 & 65.6 & 86.7 & 49.9 & 69.1 & 85.8 & 57.4 \\
\hline Other heart diseases . . . . . . . . . (I26-151) & 53.4 & 60.2 & 48.0 & 52.6 & 59.0 & 47.3 & 69.7 & 81.9 & 60.4 \\
\hline Acute and subacute endocarditis . . . . (I33) & 0.4 & 0.5 & 0.3 & 0.4 & 0.5 & 0.3 & 0.7 & 1.0 & 0.4 \\
\hline \multicolumn{10}{|l|}{ Diseases of pericardium and acute } \\
\hline myocarditis $\ldots \ldots \ldots(130-131,140)$ & 0.3 & 0.3 & 0.2 & 0.3 & 0.3 & 0.2 & 0.4 & 0.4 & 0.4 \\
\hline Heart failure . . . . . . . . . (150) & 17.3 & 18.6 & 16.2 & 17.3 & 18.7 & 16.3 & 20.1 & 22.3 & 18.4 \\
\hline \multicolumn{10}{|l|}{ All other forms of heart disease . (126-128, } \\
\hline \multirow{2}{*}{\multicolumn{10}{|c|}{ Essential hypertension and hypertensive renal }} \\
\hline & & & & & & & & & \\
\hline 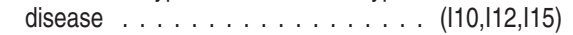 & 7.4 & 7.3 & 7.2 & 6.5 & 6.3 & 6.4 & 16.3 & 17.4 & 15.3 \\
\hline Cerebrovascular diseases . . . . . . . (160-169) & 42.2 & 42.5 & 41.3 & 40.5 & 40.2 & 39.9 & 60.3 & 67.1 & 55.0 \\
\hline Atherosclerosis . . . . . . . . . . . . . . . . (170) & 2.5 & 2.6 & 2.4 & 2.6 & 2.7 & 2.4 & 2.4 & 2.6 & 2.3 \\
\hline Other diseases of circulatory system . . . (171-178) & 6.9 & 8.8 & 5.5 & 6.8 & 8.8 & 5.3 & 8.6 & 10.6 & 7.2 \\
\hline Aortic aneurysm and dissection $\ldots . . . .(171)$ & 4.1 & 5.7 & 2.9 & 4.1 & 5.8 & 2.9 & 4.2 & 5.5 & 3.2 \\
\hline \multicolumn{10}{|l|}{ Other diseases of arteries, arterioles and } \\
\hline capillaries . . . . . . . . . . . . . . (172-178) & 2.8 & 3.1 & 2.5 & 2.7 & 3.0 & 2.5 & 4.4 & 5.1 & 4.0 \\
\hline Other disorders of circulatory system . . . . (180-199) & 1.3 & 1.4 & 1.2 & 1.2 & 1.3 & 1.1 & 2.2 & 2.4 & 1.9 \\
\hline Influenza and pneumonia $\ldots \ldots \ldots \ldots(\text { J09-J18 })^{4}$ & 16.2 & 19.3 & 14.2 & 16.0 & 18.9 & 14.1 & 18.4 & 23.6 & 15.2 \\
\hline Influenza. . . . . . . . . . . . . (J09-J11) & 0.1 & 0.1 & 0.1 & 0.1 & 0.1 & 0.1 & 0.0 & * & * \\
\hline Pneumonia . . . . . . . . . . . . . (J12-J18) & 16.1 & 19.2 & 14.1 & 15.9 & 18.8 & 14.0 & 18.4 & 23.6 & 15.2 \\
\hline \multicolumn{10}{|l|}{ Other acute lower respiratory } \\
\hline infections ............ & 0.1 & 0.1 & 0.1 & 0.1 & 0.1 & 0.1 & 0.1 & * & * \\
\hline Acute bronchitis and bronchiolitis . . . . (J20-J21) & 0.1 & 0.1 & 0.0 & 0.1 & 0.1 & 0.0 & 0.1 & * & * \\
\hline Other and unspecified acute lower & & & & & & & & & \\
\hline respiratory infections $\ldots \ldots \ldots \ldots($ (J22,U04) $\ldots \ldots$ & 0.0 & * & 0.0 & 0.0 & * & 0.0 & * & * & * \\
\hline Chronic lower respiratory diseases ......(J40-J47) & 40.8 & 48.0 & 36.0 & 43.0 & 49.8 & 38.5 & 28.1 & 39.6 & 21.4 \\
\hline Bronchitis, chronic and unspecified . . . . (J40-J42) & 0.2 & 0.2 & 0.2 & 0.2 & 0.2 & 0.2 & 0.2 & 0.2 & 0.2 \\
\hline Emphysema $\ldots \ldots \ldots \ldots$ & 4.1 & 5.1 & 3.4 & 4.3 & 5.3 & 3.7 & 2.6 & 4.2 & 1.6 \\
\hline Asthma . . . . . . . . . . & 1.1 & 0.9 & 1.2 & 0.9 & 0.7 & 1.0 & 2.5 & 2.5 & 2.6 \\
\hline $\begin{array}{l}\text { Other chronic lower respiratory } \\
\text { diseases } \ldots \ldots \ldots \ldots \ldots\end{array}$ & 35.3 & 41.9 & 31.2 & 37.5 & 43.7 & 33.6 & 22.8 & 32.7 & 17.1 \\
\hline
\end{tabular}

See footnotes at end of table. 
Table 16. Age-adjusted death rates for 113 selected causes and Enterocolitis due to Clostridium difficile, by race and sex: United States, 2007-Con.

[Age-adjusted rates per 100,000 U.S. standard population; see "Technical Notes." Populations used for computing death rates are postcensal estimates based on the 2000 census, estimated as of July 1, 2007; see "Technical Notes." Data for specified races other than white and black should be interpreted with caution because of inconsistencies between reporting race on death certificates and on censuses and surveys; see "Technical Notes." The asterisks $\left({ }^{*}\right)$ preceding the cause-of-death codes indicate that they are not part of the International Classification of Diseases, Tenth Revision (ICD-10), Second Edition; see "Technical Notes"]

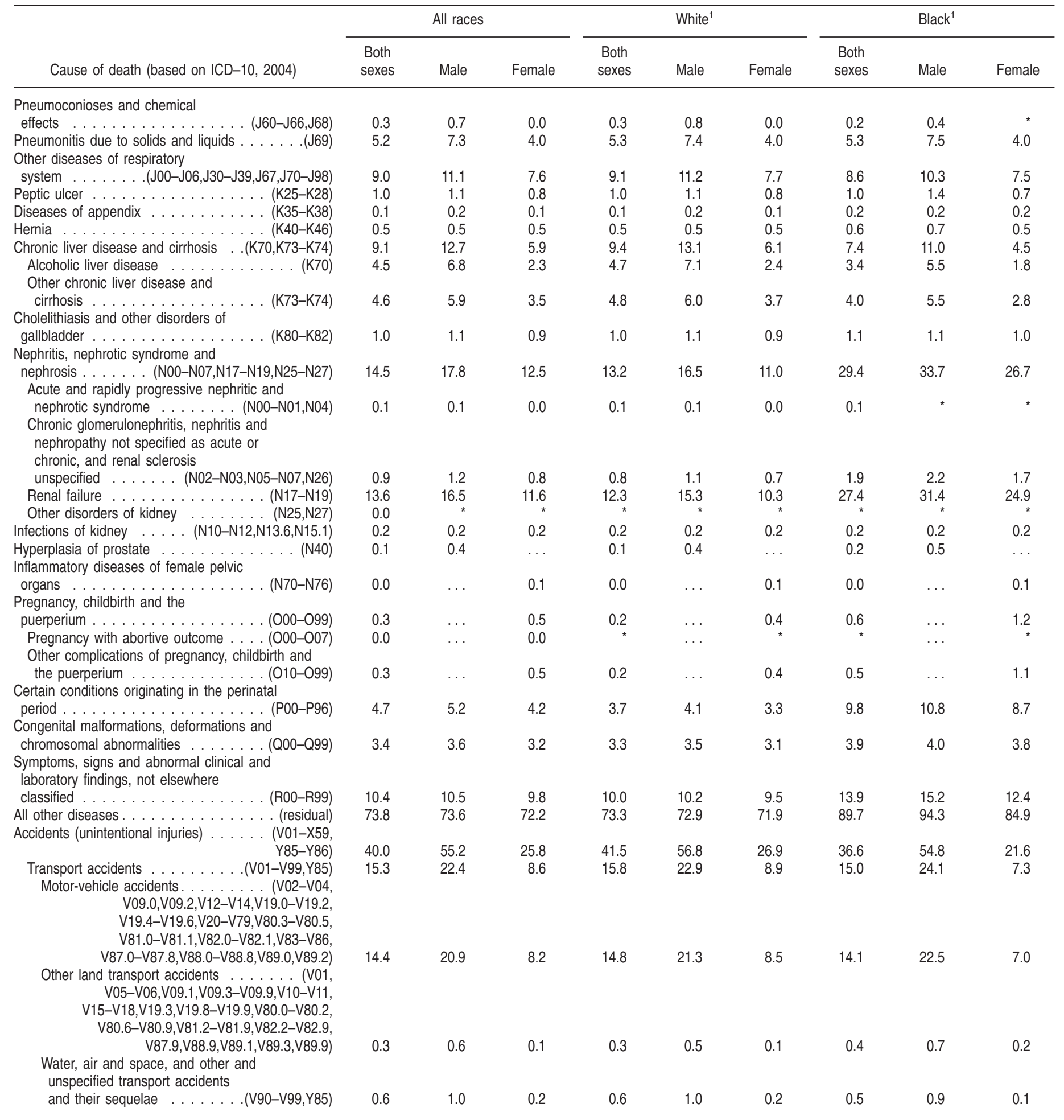

See footnotes at end of table. 
Table 16. Age-adjusted death rates for 113 selected causes and Enterocolitis due to Clostridium difficile, by race and sex: United States, 2007-Con.

[Age-adjusted rates per 100,000 U.S. standard population; see "Technical Notes." Populations used for computing death rates are postcensal estimates based on the 2000 census, estimated as of July 1, 2007; see "Technical Notes." Data for specified races other than white and black should be interpreted with caution because of inconsistencies between reporting race on death certificates and on censuses and surveys; see "Technical Notes." The asterisks $\left({ }^{*}\right)$ preceding the cause-of-death codes indicate that they are not part of the International Classification of Diseases, Tenth Revision (ICD-10), Second Edition; see "Technical Notes"]

\begin{tabular}{|c|c|c|c|c|c|c|c|c|c|}
\hline \multirow[b]{2}{*}{ Cause of death (based on ICD-10, 2004) } & \multicolumn{3}{|c|}{ All races } & \multicolumn{3}{|c|}{ White $^{1}$} & \multicolumn{3}{|c|}{ Black $^{1}$} \\
\hline & $\begin{array}{l}\text { Both } \\
\text { sexes }\end{array}$ & Male & Female & $\begin{array}{l}\text { Both } \\
\text { sexes }\end{array}$ & Male & Female & $\begin{array}{l}\text { Both } \\
\text { sexes }\end{array}$ & Male & Female \\
\hline Nontransport accidents $\ldots \ldots \ldots($ W00-X59,Y86) & 24.6 & 32.7 & 17.2 & 25.7 & 34.0 & 18.0 & 21.6 & 30.8 & 14.2 \\
\hline 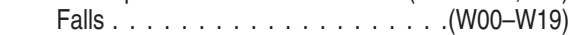 & 7.0 & 9.0 & 5.5 & 7.5 & 9.5 & 5.9 & 3.4 & 4.9 & 2.4 \\
\hline Accidental discharge of firearms. . . .(W32-W34) & 0.2 & 0.4 & 0.0 & 0.2 & 0.3 & 0.1 & 0.3 & 0.5 & * \\
\hline Accidental drowning and submersion.(W65-W74) & 1.1 & 1.8 & 0.5 & 1.1 & 1.7 & 0.5 & 1.2 & 2.1 & 0.4 \\
\hline $\begin{array}{l}\text { Accidental exposure to smoke, fire and } \\
\text { flames .................. X00-X09) }\end{array}$ & & & & & & & & & \\
\hline $\begin{array}{l}\text { tlames } \ldots \ldots \ldots \ldots \ldots \ldots \ldots \\
\text { Accidental poisoning and exposure to }\end{array}$ & 1.1 & 1.3 & 0.8 & 0.9 & 1.2 & 0.7 & 2.2 & 3.0 & 1.6 \\
\hline $\begin{array}{l}\text { noxious substances .......... (X40-X49) } \\
\text { Other and unspecified nontransport } \\
\text { accidents and their sequelae..... (W20-W31, }\end{array}$ & 9.8 & 13.0 & 6.6 & 10.6 & 14.0 & 7.2 & 8.6 & 12.2 & 5.5 \\
\hline W35-W64, W75-W99,X10-X39,X50-X59,Y86) & 5.4 & 7.2 & 3.7 & 5.4 & 7.2 & 3.7 & 5.9 & 8.0 & 4.2 \\
\hline $\begin{array}{l}\left.\text { Intentional self-harm (suicide) . ( }{ }^{*} \cup 03, \mathrm{X} 60-\mathrm{X} 84, \mathrm{Y} 87.0\right) \\
\text { Intentional self-harm (suicide) by discharge of }\end{array}$ & 11.3 & 18.4 & 4.7 & 12.5 & 20.2 & 5.2 & 5.0 & 8.8 & 1.7 \\
\hline $\begin{array}{l}\text { Iirearms. } \ldots \ldots \ldots \ldots \text { (X72-X74) } \\
\text { unspecified means and their }\end{array}$ & 5.6 & 10.3 & 1.4 & 6.3 & 11.4 & 1.6 & 2.5 & 4.9 & 0.5 \\
\hline sequelae . . . . . ( $\left.{ }^{*} \mathrm{U} 03, \mathrm{X} 60-\mathrm{X} 71, \mathrm{X} 75-\mathrm{X} 84, \mathrm{Y} 87.0\right)$ & 5.6 & 8.1 & 3.3 & 6.2 & 8.7 & 3.6 & 2.5 & 3.9 & 1.2 \\
\hline 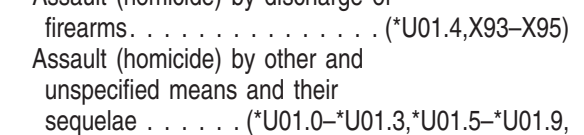 & 4.2 & 7.1 & 1.2 & 2.2 & 3.5 & 1.0 & 16.3 & 30.1 & 3.2 \\
\hline *U02,X85-X92,X96-Y09,Y87.1) & 1.9 & 2.5 & 1.3 & 1.4 & 1.9 & 1.0 & 4.8 & 7.0 & 2.9 \\
\hline $\begin{array}{l}\text { Legal intervention . . . . . . . . . . . (Y35,Y89.0) } \\
\text { Events of undetermined }\end{array}$ & 0.1 & 0.3 & * & 0.1 & 0.2 & * & 0.3 & 0.6 & * \\
\hline $\begin{array}{l}\text { intent. . . . . . . . . . . . . (Y10-Y34,Y87.2,Y89.9) } \\
\text { Discharge of firearms, undetermined }\end{array}$ & 1.8 & 2.2 & 1.3 & 1.8 & 2.2 & 1.4 & 2.0 & 3.0 & 1.1 \\
\hline 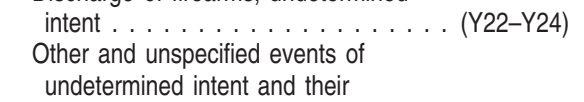 & 0.1 & 0.1 & 0.0 & 0.1 & 0.1 & 0.0 & 0.1 & 0.2 & * \\
\hline sequelae . . . . . (Y10-Y21,Y25-Y34,Y87.2,Y89.9) & 1.7 & 2.1 & 1.3 & 1.7 & 2.1 & 1.4 & 1.8 & 2.7 & 1.1 \\
\hline Operations of war and their sequelae ....(Y36,Y89.1) & 0.0 & 0.0 & * & 0.0 & 0.0 & * & * & * & * \\
\hline care $\ldots \ldots \ldots \ldots \ldots \ldots \ldots(\mathrm{Y} 40-\mathrm{Y} 84, \mathrm{Y} 88)$ & 0.8 & 0.9 & 0.8 & 0.8 & 0.9 & 0.7 & 1.4 & 1.6 & 1.4 \\
\hline Enterocolitis due to Clostridium difficile $\ldots . .(\text { A04.7 })^{7}$ & 2.0 & 2.0 & 2.0 & 2.1 & 2.0 & 2.1 & 1.5 & 1.5 & 1.4 \\
\hline
\end{tabular}

See footnotes at end of table. 
Table 16. Age-adjusted death rates for 113 selected causes and Enterocolitis due to Clostridium difficile, by race and sex: United States, 2007-Con.

[Age-adjusted rates per 100,000 U.S. standard population; see "Technical Notes." Populations used for computing death rates are postcensal estimates based on the 2000 census, estimated as of July 1, 2007; see "Technical Notes." Data for specified races other than white and black should be interpreted with caution because of inconsistencies between reporting race on death certificates and on censuses and surveys; see "Technical Notes." The asterisks (*) preceding the cause-of-death codes indicate that they are not part of the International Classification of Diseases, Tenth Revision (ICD-10), Second Edition; see "Technical Notes"]

\begin{tabular}{|c|c|c|c|c|c|c|}
\hline \multirow[b]{2}{*}{ Cause of death (based on ICD-10, 2004) } & \multicolumn{3}{|c|}{ American Indian or Alaska Native ${ }^{1,2}$} & \multicolumn{3}{|c|}{ Asian or Pacific Islander ${ }^{1,3}$} \\
\hline & $\begin{array}{l}\text { Both } \\
\text { sexes }\end{array}$ & Male & Female & $\begin{array}{l}\text { Both } \\
\text { sexes }\end{array}$ & Male & Female \\
\hline All causes $\ldots \ldots \ldots \ldots \ldots \ldots \ldots \ldots$ & 627.2 & 736.7 & 533.2 & 415.0 & 499.2 & 350.6 \\
\hline$\ldots \ldots \ldots($ A01-A02) & * & * & * & * & * & * \\
\hline Shigellosis and amebiasis . & * & * & * & * & * & * \\
\hline Certain other intestinal infections $\ldots$. (A04,A07-A09) & 1.5 & * & * & 0.5 & 0.5 & 0.5 \\
\hline Tuberculosis . . . . . . . . . . . . . . . . (A16-A19) & * & * & * & 0.7 & 0.9 & 0.5 \\
\hline Respiratory tuberculosis. . . . . . . & * & * & * & 0.6 & 0.8 & 0.4 \\
\hline Other tuberculosis $\ldots \ldots \ldots \ldots$. A17-A19) & * & * & * & * & * & * \\
\hline Whooping cough $\ldots \ldots \ldots \ldots \ldots$ (A37) & * & * & * & * & * & * \\
\hline Scarlet fever and erysipelas $\ldots \ldots \ldots($ A38,A46) & * & * & * & * & * & * \\
\hline Meningococcal infection . . . . . . . . . . . . (A39) & * & * & * & * & * & * \\
\hline Septicemia . . . . . . . . . . . . (A40-A41) & 10.1 & 9.5 & 10.5 & 5.1 & 6.5 & 4.1 \\
\hline Syphilis . . . . . . . . . . . . . . (A50-A53) & * & * & * & * & * & * \\
\hline Acute poliomyelitis ..... & * & * & * & * & * & * \\
\hline Arthropod-borne viral encephalitis . (A83-A84,A85.2) & * & * & * & * & * & * \\
\hline Measles . . . . . . . . . . . . . . . . (B05) & * & * & * & * & * & * \\
\hline Viral hepatitis . . . . . . . . . . . (B15-B19) & 3.6 & 4.9 & 2.5 & 2.4 & 2.5 & 2.2 \\
\hline \multicolumn{7}{|l|}{ Human immunodeficiency virus (HIV) } \\
\hline disease $\ldots \ldots \ldots \ldots \ldots \ldots \ldots$ (B20-B24) & 2.6 & 3.6 & 1.7 & 0.5 & 0.8 & * \\
\hline Malaria $\ldots \ldots \ldots \ldots \ldots \ldots \ldots$ (B50-B54) & * & * & * & * & * & * \\
\hline \multicolumn{7}{|l|}{$\begin{array}{r}\text { Other and unspecified infectious and parasitic } \\
\text { diseases and their sequelae .........A00,A05, } \\
\text { A20-A36,A42-A44,A48-A49,A54-A79,A81-A82, } \\
\text { A85.0-A85.1,A85.8,A86-B04,B06-B09, }\end{array}$} \\
\hline B25-B49,B55-B99) & 2.2 & 2.6 & 1.7 & 1.3 & 1.6 & 1.1 \\
\hline$\ldots \ldots \ldots(\mathrm{COO}-\mathrm{C} 97)$ & 117.8 & 139.4 & 102.1 & 106.7 & 130.2 & 90.0 \\
\hline \multicolumn{7}{|l|}{ Malignant neoplasms of lip, oral cavity and } \\
\hline pharynx $\ldots \ldots \ldots \ldots \ldots \ldots(\mathrm{C} 00-\mathrm{C} 14)$ & 2.0 & 2.6 & * & 1.9 & 2.8 & 1.2 \\
\hline Malignant neoplasm of esophagus . . . . . . (C15) & 2.3 & 4.1 & * & 1.8 & 3.2 & 0.8 \\
\hline Malignant neoplasm of stomach . . & 4.0 & 5.0 & 3.1 & 6.6 & 8.6 & 5.1 \\
\hline \multicolumn{7}{|l|}{ Malignant neoplasms of colon, rectum } \\
\hline and anus $\ldots \ldots \ldots \ldots \ldots(\mathrm{C} 18-\mathrm{C} 21)$ & 11.5 & 13.2 & 10.2 & 10.9 & 12.5 & 9.6 \\
\hline \multicolumn{7}{|l|}{ Malignant neoplasms of liver and } \\
\hline intrahepatic bile ducts . . . . . . . . . . (C22) & 6.5 & 8.8 & 4.4 & 9.2 & 13.9 & 5.5 \\
\hline Malignant neoplasm of pancreas $\ldots . .$. (C25) & 6.6 & 7.8 & 5.8 & 7.2 & 8.1 & 6.6 \\
\hline Malignant neoplasm of larynx . . . . . . . . (C32) & 0.9 & * & * & 0.4 & 0.9 & * \\
\hline \multicolumn{7}{|l|}{ Malignant neoplasms of trachea, } \\
\hline bronchus and lung $\ldots \ldots \ldots$ & 32.7 & 40.7 & 26.8 & 25.3 & 34.7 & 18.5 \\
\hline Malignant melanoma of skin . . . & 1.0 & * & * & 0.4 & 0.5 & 0.3 \\
\hline Malignant neoplasm of breast . & 7.1 & * & 12.7 & 6.1 & * & 11.1 \\
\hline Malignant neoplasm of cervix uteri & 1.3 & $\ldots$ & 2.3 & 1.1 & $\ldots$ & 2.0 \\
\hline \multicolumn{7}{|l|}{ Malignant neoplasms of corpus uteri } \\
\hline and uterus, part unspecified $\ldots \ldots$ (C54-C55) & 1.5 & $\ldots$ & 2.7 & 1.4 & $\ldots$ & 2.5 \\
\hline Malignant neoplasm of ovary $\ldots \ldots \ldots \ldots$ (C56) & 2.7 & $\ldots$ & 4.8 & 2.6 & $\ldots$ & 4.6 \\
\hline Malignant neoplasm of prostate . . . . . . . (C61) & 5.8 & 14.1 & $\ldots$ & 4.2 & 10.4 & $\ldots$ \\
\hline \multicolumn{7}{|l|}{ Malignant neoplasms of kidney and } \\
\hline renal pelvis $\ldots \ldots$ & 4.5 & 7.1 & 2.5 & 2.1 & 3.0 & 1.3 \\
\hline Malignant neoplasm of bladder $\ldots \ldots \ldots$ (C67) & 1.6 & 3.0 & * & 1.5 & 2.4 & 0.9 \\
\hline \multicolumn{7}{|l|}{$\begin{array}{l}\text { Malignant neoplasms of meninges, } \\
\text { brain and other parts of central }\end{array}$} \\
\hline nervous system $\ldots \ldots \ldots \ldots$ (C70-C72) & 1.8 & 1.8 & 1.7 & 1.7 & 2.1 & 1.3 \\
\hline \multicolumn{7}{|l|}{ Malignant neoplasms of lymphoid, } \\
\hline hematopoietic and related tissue $\ldots$ (C81-C96) & 9.5 & 11.6 & 7.7 & 10.0 & 13.2 & 7.7 \\
\hline$\ldots \ldots \ldots \ldots(\mathrm{C} 81)$ & * & * & * & * & * & * \\
\hline Non-Hodgkin's lymphoma . . . . . . (C82-C85) & 3.8 & 4.5 & 3.2 & 4.3 & 5.6 & 3.3 \\
\hline Leukemia . . . . . . . . . . . . . . (C91-C95) & 3.3 & 3.6 & 3.0 & 3.7 & 4.9 & 2.8 \\
\hline \multicolumn{7}{|l|}{ Multiple myeloma and immunoproliferative } \\
\hline neoplasms $\ldots \ldots \ldots \ldots \ldots(\mathrm{C} 88, \mathrm{C} 90)$ & 2.2 & 3.2 & 1.6 & 2.0 & 2.5 & 1.5 \\
\hline
\end{tabular}

See footnotes at end of table. 
Table 16. Age-adjusted death rates for 113 selected causes and Enterocolitis due to Clostridium difficile, by race and sex: United States, 2007-Con.

[Age-adjusted rates per 100,000 U.S. standard population; see "Technical Notes." Populations used for computing death rates are postcensal estimates based on the 2000 census, estimated as of July 1, 2007; see "Technical Notes." Data for specified races other than white and black should be interpreted with caution because of inconsistencies between reporting race on death certificates and on censuses and surveys; see "Technical Notes." The asterisks $\left({ }^{*}\right)$ preceding the cause-of-death codes indicate that they are not part of the International Classification of Diseases, Tenth Revision (ICD-10), Second Edition; see "Technical Notes"]

\begin{tabular}{|c|c|c|c|c|c|c|}
\hline \multirow[b]{2}{*}{ Cause of death (based on ICD-10, 2004) } & \multicolumn{3}{|c|}{ American Indian or Alaska Native ${ }^{1,2}$} & \multicolumn{3}{|c|}{ Asian or Pacific Islander ${ }^{1,3}$} \\
\hline & $\begin{array}{l}\text { Both } \\
\text { sexes }\end{array}$ & Male & Female & $\begin{array}{l}\text { Both } \\
\text { sexes }\end{array}$ & Male & Female \\
\hline $\begin{array}{l}\text { Other and unspecified malignant } \\
\text { neoplasms of lymphoid, hematopoietic and } \\
\text { related tissue } \ldots \ldots \ldots \ldots \ldots \ldots \text { (C96) }\end{array}$ & * & * & * & * & * & * \\
\hline $\begin{array}{l}\text { All other and unspecified malignant } \\
\text { neoplasms } \ldots \ldots \ldots(\mathrm{C} 17, \mathrm{C} 23-\mathrm{C} 24, \mathrm{C} 26-\mathrm{C} 31\end{array}$ & & & & & & \\
\hline $\mathrm{C} 62-\mathrm{C} 63, \mathrm{C} 66, \mathrm{C} 68-\mathrm{C} 69, \mathrm{C} 73-\mathrm{C} 80, \mathrm{C} 97)$ & 14.5 & 16.1 & 13.4 & 12.4 & 14.1 & 11.0 \\
\hline \multicolumn{7}{|l|}{$\begin{array}{l}\text { In situ neoplasms, benign neoplasms and } \\
\text { neoplasms of uncertain or unknown }\end{array}$} \\
\hline behavior . . . . . . . . . . . . (D00-D48) & 2.6 & 2.9 & 2.3 & 2.8 & 3.7 & 2.2 \\
\hline Anemias $\ldots \ldots \ldots \ldots \ldots$ (D50-D64) & 1.0 & * & * & 1.0 & 1.1 & 0.9 \\
\hline Diabetes mellitus $\ldots \ldots \ldots \ldots \ldots$ (E10-E14) & 37.2 & 38.1 & 36.1 & 16.2 & 18.3 & 14.5 \\
\hline Nutritional deficiencies . . . . . . . . . . . (E40-E64) & 1.3 & * & * & 0.6 & 0.6 & 0.6 \\
\hline Malnutrition $\ldots \ldots \ldots \ldots \ldots \ldots$ (E40-E46) & 1.3 & * & * & 0.5 & 0.5 & 0.6 \\
\hline Other nutritional deficiencies . . . . . . (E50-E64) & * & * & * & * & * & * \\
\hline Meningitis . . . . . . . . . . . . (G00,G03) & * & * & * & * & * & * \\
\hline Parkinson's disease . . . . . . . . . (G20-G21) & 3.2 & 4.7 & 2.2 & 3.5 & 5.0 & 2.6 \\
\hline Alzheimer's disease $\ldots \ldots \ldots \ldots \ldots \ldots \ldots$. . . . . . . . . & 11.3 & 10.8 & 11.9 & 8.1 & 6.7 & 8.9 \\
\hline Major cardiovascular diseases $\ldots \ldots \ldots \ldots(100-178)$ & 169.7 & 205.0 & 139.5 & 147.1 & 174.8 & 125.5 \\
\hline Diseases of heart ..... (100-109,|111,|13,|20-I51) & 127.3 & 159.8 & 99.8 & 101.2 & 126.0 & 82.0 \\
\hline \multicolumn{7}{|l|}{ Acute rheumatic fever and chronic } \\
\hline rheumatic heart diseases $\ldots \ldots \ldots(100-109)$ & * & * & * & 0.7 & 0.5 & 0.9 \\
\hline Hypertensive heart disease . . . . . . . . (I11) & 5.8 & 7.0 & 4.7 & 5.6 & 6.2 & 5.1 \\
\hline Hypertensive heart and renal disease . . . . (113) & * & * & * & 0.9 & 0.9 & 0.9 \\
\hline Ischemic heart diseases $\ldots \ldots \ldots \ldots$ (I20-125) & 86.7 & 112.2 & 65.6 & 71.0 & 91.7 & 55.0 \\
\hline Acute myocardial infarction $\ldots \ldots$. . (121-122) & 27.9 & 37.6 & 20.3 & 22.6 & 29.3 & 17.5 \\
\hline Other acute ischemic heart diseases . . . (124) & 2.7 & 3.3 & 2.2 & 0.4 & 0.6 & 0.3 \\
\hline \multicolumn{7}{|l|}{ Other forms of chronic ischemic heart } \\
\hline disease . . . . . . . . . . . . (120,125) & 56.1 & 71.2 & 43.1 & 47.9 & 61.9 & 37.2 \\
\hline \multicolumn{7}{|l|}{ Atherosclerotic cardiovascular disease } \\
\hline so described $\ldots \ldots \ldots \ldots$ (125.0) & 15.4 & 19.9 & 11.3 & 10.3 & 13.4 & 7.8 \\
\hline \multicolumn{7}{|l|}{ All other forms of chronic ischemic heart } \\
\hline disease $\ldots \ldots \ldots \ldots(120,|25.1-| 25.9)$ & 40.7 & 51.3 & 31.8 & 37.6 & 48.5 & 29.4 \\
\hline Other heart diseases $\ldots \ldots \ldots \ldots$ (I26-I51) & 33.6 & 39.6 & 28.4 & 22.9 & 26.7 & 20.1 \\
\hline Acute and subacute endocarditis ...... (I33) & * & * & * & 0.2 & * & * \\
\hline \multicolumn{7}{|l|}{ Diseases of pericardium and acute } \\
\hline myocarditis $\ldots \ldots \ldots \ldots(|30-| 31, \mid 40)$ & * & * & * & 0.1 & * & * \\
\hline Heart failure . . . . . . . . . . . (150) & 10.0 & 11.1 & 9.2 & 5.3 & 5.7 & 5.0 \\
\hline All other forms of heart disease . (I26-I28, & & & & & & \\
\hline$|34-| 38,|42-| 49,|51|$ & 23.0 & 27.7 & 18.8 & 17.3 & 20.6 & 14.8 \\
\hline \multicolumn{7}{|l|}{ Essential hypertension and hypertensive renal } \\
\hline disease $\ldots \ldots \ldots \ldots \ldots \ldots(110,|12| 15)$, & 5.7 & 5.4 & 5.9 & 6.4 & 6.9 & 6.0 \\
\hline Cerebrovascular diseases $\ldots$. & 29.8 & 31.1 & 28.4 & 34.3 & 35.5 & 33.2 \\
\hline Atherosclerosis . . . . . . . & 1.6 & * & * & 1.1 & 1.2 & 1.1 \\
\hline Other diseases of circulatory system $\ldots .(171-178)$ & 5.3 & 7.0 & 3.9 & 4.1 & 5.2 & 3.2 \\
\hline Aortic aneurysm and dissection ........ (171) & 2.9 & 4.4 & * & 2.9 & 4.0 & 2.2 \\
\hline \multicolumn{7}{|l|}{ Other diseases of arteries, arterioles and } \\
\hline capillaries . . . . . . . . . . . (172-178) & 2.4 & 2.6 & 2.3 & 1.1 & 1.2 & 1.1 \\
\hline Other disorders of circulatory system . . . (180-199) & * & * & * & 0.4 & 0.5 & 0.3 \\
\hline Influenza and pneumonia . . . . . . . . (J09-J18) & 13.8 & 16.0 & 11.7 & 13.6 & 17.8 & 10.9 \\
\hline 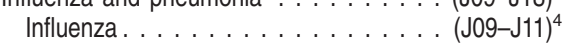 & * & * & * & * & * & * \\
\hline Pneumonia . . . . . . . . . . . . . . . (J12-J18) & 13.6 & 15.8 & 11.5 & 13.6 & 17.7 & 10.8 \\
\hline \multicolumn{7}{|l|}{ Other acute lower respiratory } \\
\hline infections ............ & * & * & * & * & * & * \\
\hline Acute bronchitis and bronchiolitis . . . . (J20-J21) & * & * & * & * & * & * \\
\hline \multicolumn{7}{|l|}{ Other and unspecified acute lower respiratory } \\
\hline infections $\ldots \ldots \ldots \ldots \ldots(\mathrm{J} 22, \mathrm{U} 04)^{5,6}$ & * & * & * & * & * & * \\
\hline Chronic lower respiratory diseases & 30.9 & 34.9 & 28.1 & 13.4 & 19.6 & 9.1 \\
\hline Bronchitis, chronic and unspecified ..... (J40-J42) & * & * & * & * & $*$ & * \\
\hline Emphysema . . . . . . . . & 2.4 & 2.8 & 2.2 & 1.2 & 2.0 & 0.6 \\
\hline$\ldots(\mathrm{J} 45-\mathrm{J} 46)$ & 1.3 & * & 1.6 & 1.2 & 1.3 & 1.1 \\
\hline
\end{tabular}

See footnotes at end of table. 
Table 16. Age-adjusted death rates for 113 selected causes and Enterocolitis due to Clostridium difficile, by race and sex: United States, 2007-Con.

[Age-adjusted rates per 100,000 U.S. standard population; see "Technical Notes." Populations used for computing death rates are postcensal estimates based on the 2000 census, estimated as of July 1, 2007; see "Technical Notes." Data for specified races other than white and black should be interpreted with caution because of inconsistencies between reporting race on death certificates and on censuses and surveys; see "Technical Notes." The asterisks (*) preceding the cause-of-death codes indicate that they are not part of the International Classification of Diseases, Tenth Revision (ICD-10), Second Edition; see "Technical Notes"]

\begin{tabular}{|c|c|c|c|c|c|c|}
\hline \multirow[b]{2}{*}{ Cause of death (based on ICD-10, 2004) } & \multicolumn{3}{|c|}{ American Indian or Alaska Native ${ }^{1,2}$} & \multicolumn{3}{|c|}{ Asian or Pacific Islander ${ }^{1,3}$} \\
\hline & $\begin{array}{l}\text { Both } \\
\text { sexes }\end{array}$ & Male & Female & $\begin{array}{l}\text { Both } \\
\text { sexes }\end{array}$ & Male & Female \\
\hline Other chronic lower respiratory & & & & & & \\
\hline $\begin{array}{r}\text { diseases } \ldots \ldots \ldots \ldots \ldots(\ldots \ldots \ldots \\
\text { Pneumoconioses and chemical effects }(\mathrm{J} 60-\mathrm{J} 66, \mathrm{~J} 647)\end{array}$ & 27.1 & $\begin{array}{r}31.2 \\
*\end{array}$ & 24.3 & $\begin{aligned} 10.8 \\
*\end{aligned}$ & 16.0 & 7.3 \\
\hline $\begin{array}{l}\text { Pneumonitis due to solids and liquids . . . . . . . (J69) } \\
\text { Other diseases of respiratory }\end{array}$ & 4.0 & 5.0 & 3.6 & 2.7 & 3.9 & 1.8 \\
\hline system $\ldots \ldots \ldots$. . .J00-J06,J30-J39,J67,J70-J98) & 10.4 & 12.0 & 8.9 & 5.2 & 6.2 & 4.4 \\
\hline Peptic ulcer $\ldots \ldots \ldots \ldots$. . . . . . . . . (K25-K28) & * & * & * & 0.9 & 1.2 & 0.7 \\
\hline Diseases of appendix $\ldots \ldots \ldots \ldots \ldots($ K35-K38) & * & * & * & * & * & * \\
\hline Hernia $\ldots \ldots \ldots \ldots \ldots \ldots \ldots($ (K40-K46) & * & * & * & * & * & * \\
\hline Chronic liver disease and cirrhosis . . (K70,K73-K74) & 24.8 & 30.2 & 19.9 & 3.3 & 4.2 & 2.4 \\
\hline Alcoholic liver disease $\ldots \ldots \ldots \ldots \ldots$ (K70) & 17.0 & 21.5 & 12.8 & 1.1 & 1.9 & 0.5 \\
\hline $\begin{array}{l}\text { Other chronic liver disease and } \\
\text { cirrhosis ................. K73-K74) }\end{array}$ & 7.8 & 8.7 & 7.0 & 2.1 & 2.3 & 1.9 \\
\hline $\begin{array}{l}\text { Cholelithiasis and other disorders of } \\
\text { gallbladder . . . . . . . . . . . . . (K80-K82) } \\
\text { Nephritis, nephrotic syndrome and }\end{array}$ & 1.1 & * & * & 0.7 & 0.7 & 0.7 \\
\hline $\begin{array}{c}\text { nephrosis ......... (N00-N07,N17-N19,N25-N27) } \\
\text { Acute and rapidly progressive nephritic and }\end{array}$ & 14.3 & 14.1 & 14.6 & 8.6 & 10.8 & 7.0 \\
\hline $\begin{array}{l}\text { Acute and rapidly progressive nephritic and } \\
\text { nephrotic syndrome .......... (N00-N01,N04) } \\
\text { Chronic glomerulonephritis, nephritis and } \\
\text { nephropathy not specified as acute or } \\
\text { chronic, and renal sclerosis }\end{array}$ & * & * & * & * & * & * \\
\hline unspecified $\ldots \ldots \ldots$ (N02-N03,N05-N07,N26) & * & * & * & 0.7 & 1.0 & 0.6 \\
\hline Renal failure $\ldots \ldots \ldots \ldots \ldots \ldots(\mathrm{N} 17-\mathrm{N} 19)$ & 13.5 & 13.4 & 13.7 & 7.9 & 9.8 & 6.4 \\
\hline Other disorders of kidney $\ldots \ldots \ldots$ (N25,N27) & * & * & * & * & * & * \\
\hline Infections of kidney . . . . (N10-N12,N13.6,N15.1) & * & * & * & * & * & * \\
\hline $\begin{array}{l}\text { Hyperplasia of prostate } \ldots \ldots \ldots \ldots \ldots \text { (N40) } \\
\text { Inflammatory diseases of female pelvic }\end{array}$ & * & * & $\ldots$ & * & * & $\cdots$ \\
\hline $\begin{array}{l}\text { Organs } \ldots \ldots \ldots \ldots \ldots \ldots \text { (N70-N76) } \\
\text { Pregnancy, childbirth and the }\end{array}$ & * & $\ldots$ & * & * & $\ldots$ & * \\
\hline puerperium . . . . . . . . . . . . . (000-099) & * & $\ldots$ & * & 0.2 & $\ldots$ & 0.4 \\
\hline $\begin{array}{l}\text { Pregnancy with abortive outcome } \ldots(\mathrm{O} 00-007) \\
\text { Other complications of pregnancy, childbirth and }\end{array}$ & * & $\cdots$ & * & * & $\cdots$ & * \\
\hline $\begin{array}{l}\text { the puerperium } \ldots \ldots \ldots \ldots \ldots(010-099) \\
\text { Certain conditions oriainating in the perinatal }\end{array}$ & * & $\ldots$ & * & 0.2 & $\ldots$ & 0.4 \\
\hline period ...................... (P00-P96) & 4.7 & 4.8 & 4.5 & 3.2 & 3.6 & 2.7 \\
\hline $\begin{array}{l}\text { Congenital malformations, deformations and } \\
\text { chromosomal abnormalities . . . . . . (Q00-Q99) }\end{array}$ & 3.8 & 3.8 & 3.8 & 2.2 & 2.3 & 2.2 \\
\hline $\begin{array}{l}\text { Symptoms, signs and abnormal clinical and } \\
\text { laboratory findings, not elsewhere }\end{array}$ & & & & & & \\
\hline 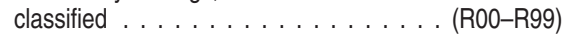 & 9.6 & 12.7 & 7.1 & 4.1 & 4.3 & 4.0 \\
\hline All other diseases $\ldots \ldots \ldots \ldots$. . . . . . . . . . . & 63.2 & 65.1 & 61.2 & 32.8 & 34.0 & 31.6 \\
\hline 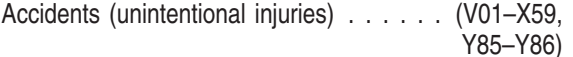 & 55.7 & 75.4 & 37.1 & 17.0 & 22.6 & 12.2 \\
\hline $\begin{array}{r}\text { Transport accidents ........... (V01-V99,Y85) } \\
\text { Motor-vehicle accidents ..... (V02-V04, } \\
\text { V09.0,V09.2,V12-V14,V19.0-V19.2, } \\
\text { V19.4-V19.6,V20-V79,V80.3-V80.5, } \\
\text { V81.0-V81.1,V82.0-V82.1,V83-V86, }\end{array}$ & 25.8 & 35.3 & 16.6 & 7.6 & 10.1 & 5.4 \\
\hline V87.0-V87.8,V88.0-V88.8,V89.0,V89.2) & 23.7 & 32.0 & 15.6 & 7.2 & 9.4 & 5.2 \\
\hline $\begin{array}{r}\text { Other land transport accidents. . . . . . (V01, } \\
\text { V05-V06,V09.1,V09.3-V09.9,V10-V11, } \\
\text { V15-V18,V19.3,V19.8-V19.9,V80.0-V80.2, } \\
\text { V80.6-V80.9,V81.2-V81.9,V82.2-V82.9, } \\
\text { V87.9.V88.9.V89.1.V89.3.V89.9) }\end{array}$ & 1.2 & 2.0 & * & 0.2 & 0.3 & * \\
\hline $\begin{array}{l}\text { Water, air and space, and other and } \\
\text { unspecified transport accidents } \\
\text { and their sequelae......... (V90-V99,Y85) }\end{array}$ & 0.9 & 1.4 & * & 0.2 & 0.3 & * \\
\hline
\end{tabular}

See footnotes at end of table. 


\section{Table 16. Age-adjusted death rates for 113 selected causes and Enterocolitis due to Clostridium difficile, by race} and sex: United States, 2007-Con.

[Age-adjusted rates per 100,000 U.S. standard population; see "Technical Notes." Populations used for computing death rates are postcensal estimates based on the 2000 census, estimated as of July 1, 2007; see "Technical Notes." Data for specified races other than white and black should be interpreted with caution because of inconsistencies between reporting race on death certificates and on censuses and surveys; see "Technical Notes." The asterisks (*) preceding the cause-of-death codes indicate that they are not part of the International Classification of Diseases, Tenth Revision (ICD-10), Second Edition; see "Technical Notes"]

\begin{tabular}{|c|c|c|c|c|c|c|}
\hline \multirow[b]{2}{*}{ Cause of death (based on ICD-10, 2004) } & \multicolumn{3}{|c|}{ American Indian or Alaska Native ${ }^{1,2}$} & \multicolumn{3}{|c|}{ Asian or Pacific Islander ${ }^{1,3}$} \\
\hline & $\begin{array}{l}\text { Both } \\
\text { sexes }\end{array}$ & Male & Female & $\begin{array}{l}\text { Both } \\
\text { sexes }\end{array}$ & Male & Female \\
\hline Nontransport accidents . . . . . . (W00-X59,Y86) & 30.0 & 40.0 & 20.5 & 9.4 & 12.6 & 6.7 \\
\hline Falls . . . . . . . . . . . . . . . . (W00-W19) & 6.2 & 9.2 & 3.8 & 4.4 & 5.7 & 3.4 \\
\hline $\begin{array}{l}\text { Accidental discharge of firearms. . . . (W32-W34) } \\
\text { Accidental drowning and }\end{array}$ & * & * & * & * & * & * \\
\hline $\begin{array}{l}\text { submersion . . . . . . . . . . . . . . (W65-W74) } \\
\text { Accidental exposure to smoke, fire and }\end{array}$ & 2.0 & 3.4 & * & 1.1 & 1.6 & 0.5 \\
\hline $\begin{array}{l}\text { flames } \ldots \ldots \ldots \ldots \ldots \ldots \\
\text { Accidental poisoning and exposure to }\end{array}$ & 1.6 & 1.9 & * & 0.4 & 0.5 & * \\
\hline $\begin{array}{l}\text { noxious substances .......... (X40-X49) } \\
\text { Other and unspecified nontransport }\end{array}$ & 11.6 & 13.2 & 10.0 & 1.5 & 1.9 & 1.0 \\
\hline $\begin{array}{l}\text { accidents and their sequelae .... (W20-W31, } \\
\text { W35-W64,W75-W99,X10-X39,X50-X59,Y86) }\end{array}$ & 8.2 & 11.8 & 4.8 & 2.1 & 2.7 & 1.6 \\
\hline Intentional self-harm & & & & & & \\
\hline $\begin{array}{l}\left.\text { (suicide) . . . . . . . . . . . ( }{ }^{*} \mathrm{U} 03, \mathrm{X} 60-\mathrm{X} 84, \mathrm{Y} 87.0\right) \\
\text { Intentional self-harm (suicide) by discharge of }\end{array}$ & 11.5 & 18.1 & 4.9 & 6.1 & 9.0 & 3.5 \\
\hline $\begin{array}{l}\text { firearms. . . . . . . . . . . . . (X72-X74) } \\
\text { Intentional self-harm (suicide) by other and } \\
\text { unspecified means and their }\end{array}$ & 4.1 & 7.2 & * & 1.5 & 2.8 & 0.3 \\
\hline sequelae ...... ( $\left.{ }^{*} \mathrm{U} 03, \mathrm{X} 60-\mathrm{X} 71, \mathrm{X} 75-\mathrm{X} 84, \mathrm{Y} 87.0\right)$ & 7.3 & 10.9 & 3.7 & 4.6 & 6.2 & 3.2 \\
\hline $\begin{array}{l}\text { Assault (homicide). . . . . (*U01-*U02,X85-Y09,Y87.1) } \\
\text { Assault (homicide) by discharge of }\end{array}$ & 6.5 & 9.2 & 3.6 & 2.3 & 3.3 & 1.3 \\
\hline 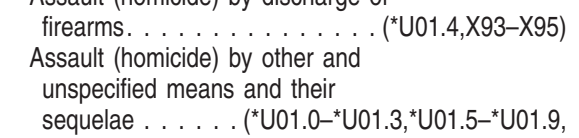 & 2.6 & 4.3 & * & 1.3 & 2.2 & 0.4 \\
\hline *U02,X85-X92,X96-Y09,Y87.1) & 3.9 & 4.9 & 2.8 & 1.0 & 1.1 & 0.9 \\
\hline $\begin{array}{l}\text { Legal intervention . . . . . . . . . . . . (Y35,Y89.0) } \\
\text { Events of undetermined }\end{array}$ & * & * & * & * & * & * \\
\hline $\begin{array}{l}\text { intent. . . . . . . . . . . . . (Y10-Y34,Y87.2,Y89.9) } \\
\text { Discharge of firearms, undetermined }\end{array}$ & 2.9 & 3.4 & 2.5 & 0.5 & 0.7 & 0.4 \\
\hline $\begin{array}{l}\text { intent . . . . . . . . . . . . . . . (Y22-Y24) } \\
\text { Other and unspecified events of } \\
\text { undetermined intent and their }\end{array}$ & * & * & * & * & * & * \\
\hline sequelae . . . . . (Y10-Y21,Y25-Y34,Y87.2,Y89.9) & 2.8 & 3.1 & 2.5 & 0.5 & 0.6 & 0.4 \\
\hline $\begin{array}{l}\text { Operations of war and their sequelae } \ldots .(\mathrm{Y} 36, \mathrm{Y} 89.1) \\
\text { Complications of medical and surgical }\end{array}$ & 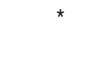 & * & * & * & * & * \\
\hline care $(Y 40-Y 84, Y 88)$ & * & * & * & 0.4 & * & 0.4 \\
\hline Enterocolitis due to Clostridium difficile $\ldots . .(\text { A04.7 })^{7}$ & 1.4 & * & * & 0.5 & 0.5 & 0.4 \\
\hline
\end{tabular}

0.0 Quantity more than zero but less than 0.05 .

* Figure does not meet standards of reliability or precision; see "Technical Notes."

... Category not applicable.

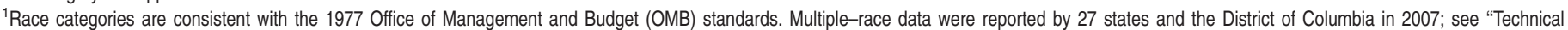
Notes." The multiple-race data for these reporting areas were bridged to the single-race categories of the 1977 OMB standards for comparability with other reporting areas; see "Technical Notes."

${ }^{2}$ Includes Aleuts and Eskimos.

${ }^{3}$ Includes Chinese, Filipino, Hawaiian, Japanese, and Other Asian or Pacific Islander.

${ }^{4}$ New ICD-10 code J09 (Influenza due to identified avian influenza virus) was added to the category in 2007. No deaths occurred from this cause in 2007.

${ }^{5} \mathrm{New}$ ICD-10 code U04 (Severe acute respiratory syndrome [SARS]) was added to the category in 2007. No deaths occurred from this cause in 2007.

${ }^{6}$ Cause-of-death title was changed in 2007 to reflect the addition of SARS (ICD-10 code U04).

${ }^{7}$ Included in "Certain other intestinal infections (A04,A07-A09)" shown above. Beginning with data year 2006, "Enterocolitis due to Clostridium difficile (A04.7)" is shown separately at the bottom of tables showing 113 selected causes and is included in the list of rankable causes, see "Technical Notes."

NOTE: Complete confirmation of deaths from selected causes of death, considered to be of public health concern, was not provided by the following states: Connecticut, Florida, Indiana, Kentucky, Maryland, North Carolina, Oklahoma, Pennsylvania, Rhode Island, South Carolina, Washington, and West Virginia; see "Technical Notes." 
Table 17. Age-adjusted death rates for 113 selected causes and Enterocolitis due to Clostridium difficile, by Hispanic origin, race for non-Hispanic population, and sex: United States, 2007

[Age-adjusted rates per 100,000 U.S. standard population; see "Technical Notes." Populations used for computing death rates are postcensal estimates based on the 2000 census, estimated as of July 1, 2007; see "Technical Notes." Race and Hispanic origin are reported separately on the death certificate. Persons of Hispanic origin may be of any race. Data for Hispanic persons are not tabulated separately by race; data for non-Hispanic persons are tabulated by race. Data for Hispanic origin should be interpreted with caution because of inconsistencies between reporting Hispanic origin on death certificates and on censuses and surveys; see "Technical Notes." The asterisks (*) preceding the cause-of-death codes indicate that they are not part of the International Classification of Diseases, Tenth Revision (ICD-10), Second Edition; see "Technical Notes"]

\begin{tabular}{|c|c|c|c|c|c|c|c|c|c|}
\hline \multirow[b]{2}{*}{ Cause of death (based on ICD-10, 2004) } & \multicolumn{3}{|c|}{ All origins ${ }^{1}$} & \multicolumn{3}{|c|}{ Hispanic } & \multicolumn{3}{|c|}{ Non-Hispanic ${ }^{2}$} \\
\hline & Both sexes & Male & Female & Both sexes & Male & Female & Both sexes & Male & Female \\
\hline$\ldots \ldots \ldots$ & 760.2 & 905.6 & 643.4 & 546.1 & 654.5 & 452.7 & 776.3 & 924.9 & 657.7 \\
\hline Salmonella infections & 0.0 & * & * & * & * & * & 0.0 & * & * \\
\hline Shigellosis and amebiasis . . . . . . . . (A03,A06) & * & * & * & * & * & * & * & * & * \\
\hline Certain other intestinal infections $\ldots(\mathrm{A} 04, \mathrm{~A} 07-\mathrm{A} 09)$ & 2.1 & 2.1 & 2.2 & 1.4 & 1.4 & 1.3 & 2.2 & 2.1 & 2.2 \\
\hline Tuberculosis . . . . . . . . . . . . . . (A16-A19) & 0.2 & 0.2 & 0.1 & 0.4 & 0.6 & 0.2 & 0.1 & 0.2 & 0.1 \\
\hline Respiratory tuberculosis . . . . . . . . . (A16) & 0.1 & 0.2 & 0.1 & 0.3 & 0.5 & 0.2 & 0.1 & 0.2 & 0.1 \\
\hline Other tuberculosis $\ldots \ldots \ldots \ldots$ & 0.0 & 0.0 & 0.0 & * & * & * & 0.0 & 0.0 & 0.0 \\
\hline Whooping cough $\ldots \ldots \ldots \ldots \ldots \ldots$ (A37) & * & * & * & * & * & * & * & * & * \\
\hline Scarlet fever and erysipelas $\ldots \ldots \ldots(\mathrm{A} 38, \mathrm{~A} 46)$ & * & * & * & * & * & * & * & * & * \\
\hline Meningococcal infection $\ldots \ldots \ldots \ldots \ldots \ldots$ (A39) & 0.0 & 0.0 & 0.0 & * & * & * & 0.0 & 0.0 & 0.0 \\
\hline Septicemia . . . . . . . . . . . (A40-A41) & 11.0 & 12.1 & 10.2 & 8.2 & 9.2 & 7.4 & 11.2 & 12.3 & 10.4 \\
\hline (A50-A53) & 0.0 & 0.0 & * & * & * & * & 0.0 & 0.0 & * \\
\hline Acute poliomyelitis & * & * & * & * & * & * & * & * & * \\
\hline Arthropod-borne viral encephalitis . (A83-A84,A85.2) & * & * & * & * & * & * & * & * & * \\
\hline Measles . . . . . . . . . . . . . . . (B05) & * & * & * & * & * & * & * & * & * \\
\hline Viral hepatitis . . . . . . . . . . . (B15-B19) & 2.3 & 3.1 & 1.5 & 3.8 & 5.1 & 2.5 & 2.1 & 2.9 & 1.4 \\
\hline \multicolumn{10}{|l|}{ Human immunodeficiency virus (HIV) } \\
\hline disease $\ldots \ldots \ldots \ldots \ldots \ldots$ & 3.7 & 5.4 & 2.1 & 4.1 & 6.3 & 1.8 & 3.7 & 5.3 & 2.2 \\
\hline 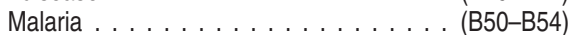 & * & * & * & * & * & * & * & * & * \\
\hline \multirow{2}{*}{\multicolumn{10}{|c|}{$\begin{array}{r}\text { Other and unspecified infectious and parasitic } \\
\text { diseases and their sequelae ..........A00,A05, } \\
\text { A20-A36,A42-A44,A48-A49,A54-A79,A81-A82, } \\
\text { A85.0-A85.1,A85.8,A86-B04,B06-B09, }\end{array}$}} \\
\hline & & & & & 2.0 & 1.6 & 1.8 & 2.2 & 1.6 \\
\hline Malignant neoplasms . . . . . . . . . (C00-C97) & 178.4 & 217.5 & 151.3 & 116.2 & 141.4 & 98.6 & 183.1 & 223.2 & 155.3 \\
\hline \multicolumn{10}{|l|}{ Malignant neoplasms of lip, oral cavity } \\
\hline and pharynx $\ldots \ldots \ldots \ldots \ldots \ldots(\mathrm{C} 00-C 14)$ & 2.5 & 3.9 & 1.4 & 1.5 & 2.3 & 0.8 & 2.6 & 4.0 & 1.5 \\
\hline Malignant neoplasm of esophagus $\ldots \ldots \ldots$ (C15) & 4.3 & 7.7 & 1.6 & 2.0 & 3.7 & 0.7 & 4.5 & 8.0 & 1.6 \\
\hline Malignant neoplasm of stomach . . . . . . . (C16) & 3.6 & 5.0 & 2.6 & 5.5 & 7.4 & 4.1 & 3.4 & 4.8 & 2.4 \\
\hline \multicolumn{10}{|l|}{ Malignant neoplasms of colon, rectum } \\
\hline and anus $\ldots \ldots \ldots \ldots \ldots$ (C18-C21) & 16.9 & 20.1 & 14.4 & 12.0 & 14.5 & 10.1 & 17.2 & 20.5 & 14.7 \\
\hline \multicolumn{10}{|l|}{ Malignant neoplasms of liver and } \\
\hline intrahepatic bile ducts $\ldots \ldots \ldots \ldots \ldots \ldots$ (C22) & 5.4 & 7.9 & 3.2 & 7.8 & 11.1 & 5.1 & 5.2 & 7.7 & 3.1 \\
\hline Malignant neoplasm of pancreas . . . . . . . . (C25) & 10.8 & 12.5 & 9.4 & 7.9 & 8.4 & 7.4 & 11.0 & 12.8 & 9.6 \\
\hline Malignant neoplasm of larynx $\ldots \ldots \ldots$. . . (C32) & 1.1 & 2.1 & 0.4 & 0.7 & 1.5 & * & 1.2 & 2.1 & 0.4 \\
\hline \multicolumn{10}{|l|}{ Malignant neoplasms of trachea, } \\
\hline bronchus and lung ...... & 50.6 & 65.1 & 40.0 & 20.9 & 29.6 & 14.4 & 53.0 & 67.8 & 42.0 \\
\hline Malignant melanoma of skin & 2.7 & 4.0 & 1.7 & 0.8 & 1.0 & 0.6 & 2.8 & 4.2 & 1.8 \\
\hline Malignant neoplasm of breast & 12.9 & 0.3 & 22.9 & 7.9 & * & 14.5 & 13.2 & 0.3 & 23.6 \\
\hline Malignant neoplasm of cervix uteri $\ldots \ldots$. . (C53) & 1.3 & $\ldots$ & 2.4 & 1.5 & $\ldots$ & 3.0 & 1.2 & $\ldots$ & 2.4 \\
\hline \multicolumn{10}{|l|}{ Malignant neoplasms of corpus uteri } \\
\hline and uterus, part unspecified . . . . . . . (C54-C55) & 2.3 & $\ldots$ & 4.2 & 1.6 & $\ldots$ & 2.9 & 2.4 & $\ldots$ & 4.3 \\
\hline Malignant neoplasm of ovary $\ldots \ldots \ldots \ldots \ldots$ (C56) & 4.6 & & 8.2 & 3.2 & & 5.7 & 4.7 & & 8.4 \\
\hline Malignant neoplasm of prostate . . . . . . . (C61) & 9.2 & 23.5 & $\ldots$ & 7.2 & 17.7 & $\ldots$ & 9.3 & 23.9 & $\ldots$ \\
\hline \multicolumn{10}{|l|}{ Malignant neoplasms of kidney and } \\
\hline$\ldots \ldots \ldots(\mathrm{C} 64-\mathrm{C} 65)$ & 4.0 & 5.8 & 2.6 & 3.7 & 5.5 & 2.2 & 4.0 & 5.8 & 2.7 \\
\hline Malignant neoplasm of bladder . . . . . . . . (C67) & 4.4 & 7.6 & 2.2 & 2.3 & 4.0 & 1.1 & 4.5 & 7.8 & 2.3 \\
\hline \multicolumn{10}{|l|}{$\begin{array}{l}\text { Malignant neoplasms of meninges, } \\
\text { brain and other parts of central }\end{array}$} \\
\hline nervous system $\ldots \ldots \ldots \ldots \ldots \ldots$ (C70-C72) & 4.2 & 5.1 & 3.5 & 2.8 & 3.1 & 2.5 & 4.4 & 5.3 & 3.6 \\
\hline \multicolumn{10}{|l|}{ Malignant neoplasms of lymphoid, hematopoietic } \\
\hline$\ldots \ldots \ldots(\mathrm{C} 81-\mathrm{C} 96)$ & 17.6 & 22.8 & 13.7 & 13.0 & 15.5 & 11.1 & 17.8 & 23.3 & 13.8 \\
\hline Hodgkin's disease . . . . . . . . . . (C81) & 0.4 & 0.5 & 0.3 & 0.4 & 0.5 & 0.4 & 0.4 & 0.5 & 0.3 \\
\hline Non-Hodgkin's lymphoma $\ldots \ldots \ldots$ (C82-C85) & 6.5 & 8.3 & 5.2 & 5.1 & 6.2 & 4.4 & 6.6 & 8.4 & 5.2 \\
\hline Leukemia . . . . . . . . . . . . . (C91-C95) & 7.0 & 9.4 & 5.3 & 4.5 & 5.5 & 3.8 & 7.1 & 9.6 & 5.3 \\
\hline \multicolumn{10}{|l|}{ Multiple myeloma and immunoproliferative } \\
\hline neoplasms $\ldots \ldots \ldots \ldots \ldots(\mathrm{C} 88, \mathrm{C} 90)$ & 3.6 & 4.6 & 2.9 & 2.9 & 3.4 & 2.5 & 3.7 & 4.7 & 2.9 \\
\hline $\begin{array}{l}\text { Other and unspecified malignant neoplasms } \\
\text { of lymphoid, hematopoietic and }\end{array}$ & & & & & & & & & \\
\hline related tissue $\ldots \ldots \ldots \ldots \ldots \ldots$ (C96) & 0.0 & 0.0 & 0.0 & * & * & * & 0.0 & 0.0 & 0.0 \\
\hline
\end{tabular}

See footnotes at end of table. 
Table 17. Age-adjusted death rates for 113 selected causes and Enterocolitis due to Clostridium difficile, by Hispanic origin, race for non-Hispanic population, and sex: United States, 2007-Con.

[Age-adjusted rates per 100,000 U.S. standard population; see "Technical Notes." Populations used for computing death rates are postcensal estimates based on the 2000 census, estimated as of July 1, 2007; see "Technical Notes." Race and Hispanic origin are reported separately on the death certificate. Persons of Hispanic origin may be of any race. Data for Hispanic persons are not tabulated separately by race; data for non-Hispanic persons are tabulated by race. Data for Hispanic origin should be interpreted with caution because of inconsistencies between reporting Hispanic origin on death certificates and on censuses and surveys; see "Technical Notes." The asterisks $\left({ }^{*}\right)$ preceding the cause-of-death codes indicate that they are not part of the International Classification of Diseases, Tenth Revision (ICD-10), Second Edition; see "Technical Notes"]

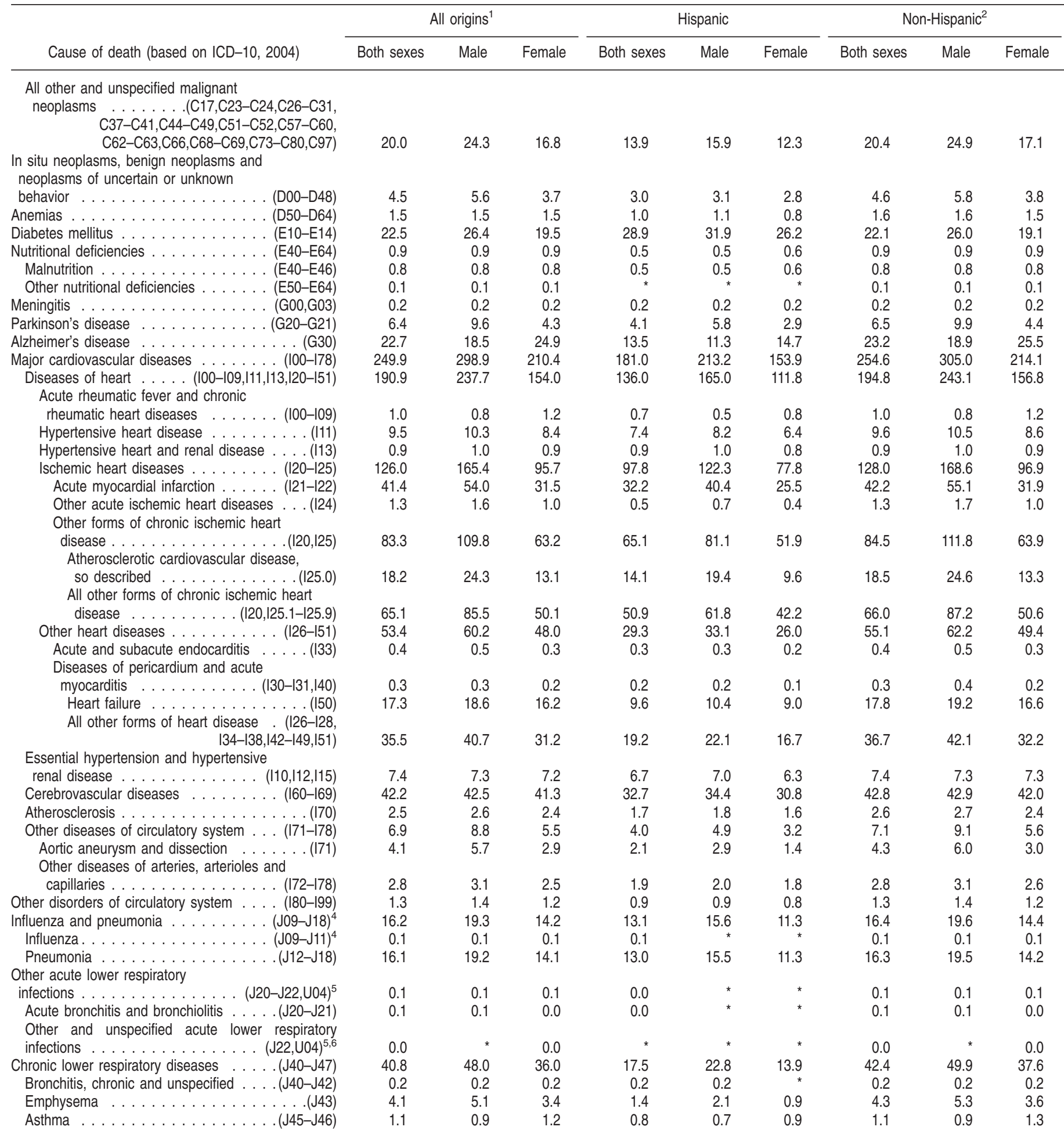

See footnotes at end of table. 
Table 17. Age-adjusted death rates for 113 selected causes and Enterocolitis due to Clostridium difficile, by Hispanic origin, race for non-Hispanic population, and sex: United States, 2007-Con.

[Age-adjusted rates per 100,000 U.S. standard population; see "Technical Notes." Populations used for computing death rates are postcensal estimates based on the 2000 census, estimated as of July 1, 2007; see "Technical Notes." Race and Hispanic origin are reported separately on the death certificate. Persons of Hispanic origin may be of any race. Data for Hispanic persons are not tabulated separately by race; data for non-Hispanic persons are tabulated by race. Data for Hispanic origin should be interpreted with caution because of inconsistencies between reporting Hispanic origin on death certificates and on censuses and surveys; see "Technical Notes." The asterisks $\left(^{*}\right)$ preceding the cause-of-death codes indicate that they are not part of the International Classification of Diseases, Tenth Revision (ICD-10), Second Edition; see "Technical Notes"]

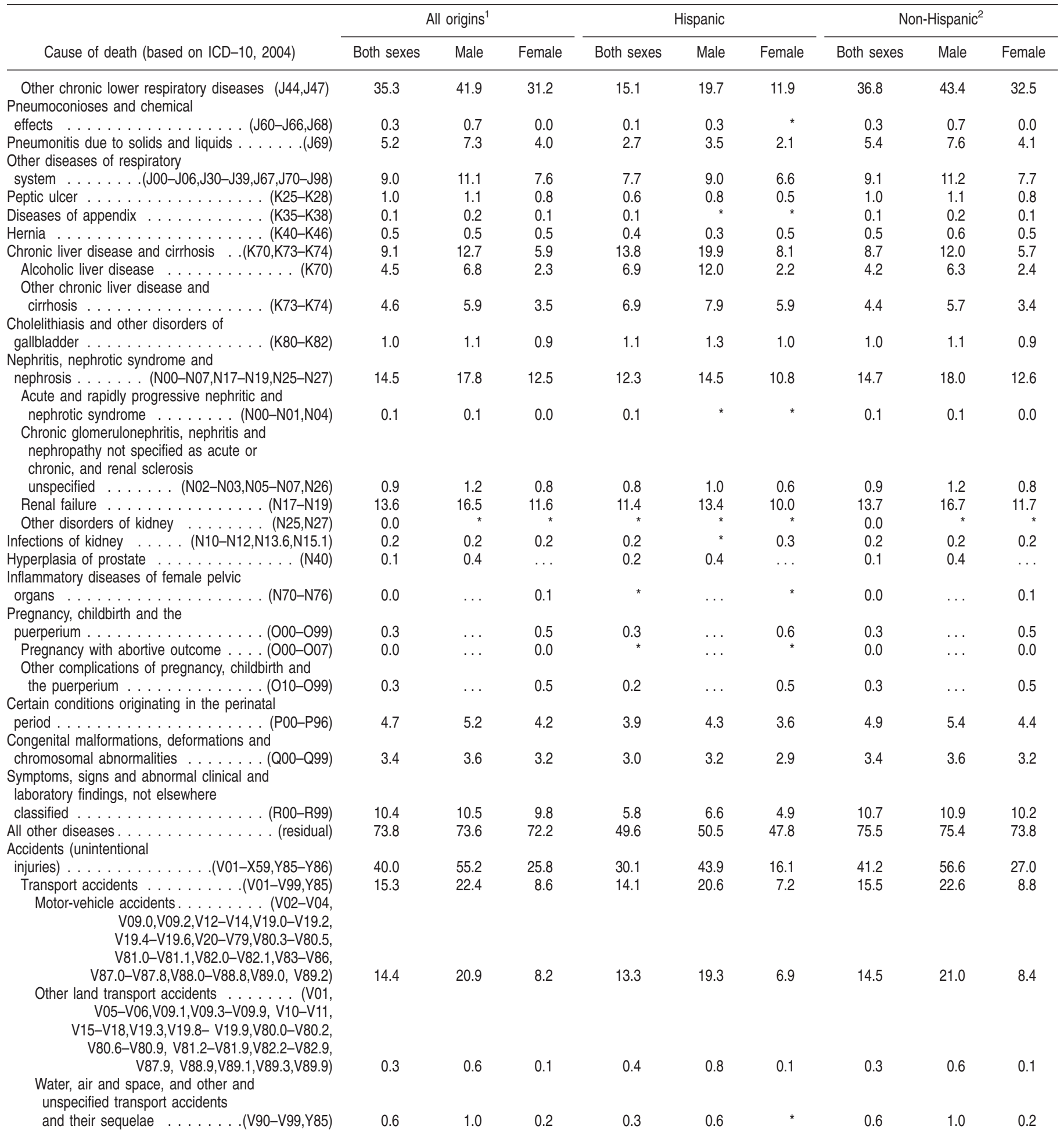

See footnotes at end of table. 
Table 17. Age-adjusted death rates for 113 selected causes and Enterocolitis due to Clostridium difficile, by Hispanic origin, race for non-Hispanic population, and sex: United States, 2007-Con.

[Age-adjusted rates per 100,000 U.S. standard population; see "Technical Notes." Populations used for computing death rates are postcensal estimates based on the 2000 census, estimated as of July 1, 2007; see "Technical Notes." Race and Hispanic origin are reported separately on the death certificate. Persons of Hispanic origin may be of any race. Data for Hispanic persons are not tabulated separately by race; data for non-Hispanic persons are tabulated by race. Data for Hispanic origin should be interpreted with caution because of inconsistencies between reporting Hispanic origin on death certificates and on censuses and surveys; see "Technical Notes." The asterisks $\left({ }^{*}\right)$ preceding the cause-of-death codes indicate that they are not part of the International Classification of Diseases, Tenth Revision (ICD-10), Second Edition; see "Technical Notes"]

\begin{tabular}{|c|c|c|c|c|c|c|c|c|c|}
\hline \multirow[b]{2}{*}{ Cause of death (based on ICD-10, 2004) } & \multicolumn{3}{|c|}{ All origins ${ }^{1}$} & \multicolumn{3}{|c|}{ Hispanic } & \multicolumn{3}{|c|}{ Non-Hispanic ${ }^{2}$} \\
\hline & Both sexes & Male & Female & Both sexes & Male & Female & Both sexes & Male & Female \\
\hline Nontransport accidents . . . . . . (W00-X59,Y86) & 24.6 & 32.7 & 17.2 & 16.1 & 23.2 & 8.9 & 25.7 & 34.0 & 18.2 \\
\hline Falls $\ldots \ldots \ldots \ldots \ldots$ (W00-W19) & 7.0 & 9.0 & 5.5 & 5.0 & 6.4 & 3.7 & 7.1 & 9.1 & 5.6 \\
\hline $\begin{array}{l}\text { Accidental discharge of firearms ....(W32-W34) } \\
\text { Accidental drowning and }\end{array}$ & 0.2 & 0.4 & 0.0 & 0.1 & 0.2 & * & 0.2 & 0.4 & 0.1 \\
\hline $\begin{array}{l}\text { submersion . . . . . . . . . . . . . (W65-W74) } \\
\text { Accidental exposure to smoke, fire and }\end{array}$ & 1.1 & 1.8 & 0.5 & 1.0 & 1.7 & 0.3 & 1.1 & 1.8 & 0.5 \\
\hline $\begin{array}{l}\text { flames } \ldots \ldots \ldots \ldots \ldots \ldots \ldots \text { (X00-X09) } \\
\text { Accidental poisoning and exposure to noxious }\end{array}$ & 1.1 & 1.3 & 0.8 & 0.6 & 0.9 & 0.4 & 1.1 & 1.4 & 0.9 \\
\hline $\begin{array}{l}\text { substances ............... (X40-X49) } \\
\text { Other and unspecified nontransport } \\
\text { accidents and their sequelae..... (W20- W31, }\end{array}$ & 9.8 & 13.0 & 6.6 & 5.8 & 8.9 & 2.5 & 10.5 & 13.8 & 7.2 \\
\hline $\begin{array}{l}\text { W35-W64,W75-W99,X10-X39, X50-X59,Y86) } \\
\text { Intentional self-harm }\end{array}$ & 5.4 & 7.2 & 3.7 & 3.5 & 5.2 & 1.9 & 5.5 & 7.4 & 3.9 \\
\hline $\begin{array}{l}\text { (suicide) . . . . . . . . . . . ( }{ }^{*} \cup 03, \mathrm{X} 60-\mathrm{X} 84, \mathrm{Y} 87.0 \text { ) } \\
\text { Intentional self-harm (suicide) by discharge of }\end{array}$ & 11.3 & 18.4 & 4.7 & 6.0 & 10.1 & 1.9 & 12.0 & 19.7 & 5.0 \\
\hline $\begin{array}{l}\text { firearms } \ldots \ldots \ldots \ldots \ldots \ldots \ldots \ldots(X 72-X 74) \\
\text { Intentional self-harm (suicide) by other and } \\
\text { unspecified means and their }\end{array}$ & 5.6 & 10.3 & 1.4 & 2.4 & 4.4 & 0.4 & 6.1 & 11.2 & 1.5 \\
\hline sequelae . . . . . ( $\left.{ }^{*} \cup 03, \mathrm{X} 60-\mathrm{X} 71, \mathrm{X} 75-\mathrm{X} 84, \mathrm{Y} 87.0\right)$ & 5.6 & 8.1 & 3.3 & 3.6 & 5.7 & 1.5 & 6.0 & 8.5 & 3.5 \\
\hline $\begin{array}{l}\text { Assault (homicide) . .(*U01-*U02,X85-Y09,Y87.1) } \\
\text { Assault (homicide) by discharge of }\end{array}$ & 6.1 & 9.6 & 2.5 & 6.9 & 11.2 & 2.3 & 5.9 & 9.2 & 2.5 \\
\hline $\begin{array}{l}\left.\text { firearms } \ldots \ldots \ldots \text {. . . . . . ( }{ }^{*} \cup 01.4, \mathrm{X} 93-\mathrm{X} 95\right) \\
\text { Assault (homicide) by other and } \\
\text { unspecified means and their } \\
\text { sequelae . . . . . ( }{ }^{*} \cup 01.0-{ }^{*} \cup 01.3,{ }^{*} \cup 01.5-{ }^{*} \cup 01.9\end{array}$ & 4.2 & 7.1 & 1.2 & 4.7 & 7.8 & 1.1 & 4.1 & 6.9 & 1.3 \\
\hline *U02,X85-X92,X96-Y09,Y87.1) & 1.9 & 2.5 & 1.3 & 2.3 & 3.3 & 1.2 & 1.8 & 2.3 & 1.3 \\
\hline $\begin{array}{l}\text { Legal intervention } \ldots \ldots \ldots \ldots \ldots \ldots \ldots \text {. } \ldots 335, Y 89.0) \\
\text { Events of undetermined }\end{array}$ & 0.1 & 0.3 & * & 0.2 & 0.4 & * & 0.1 & 0.3 & * \\
\hline $\begin{array}{l}\text { intent } \ldots \ldots \ldots \ldots \ldots . . .(Y 10-Y 34, Y 87.2, Y 89.9) \\
\text { Discharge of firearms, undetermined }\end{array}$ & 1.8 & 2.2 & 1.3 & 0.8 & 1.1 & 0.5 & 1.9 & 2.4 & 1.4 \\
\hline $\begin{array}{l}\text { intent . . . . . . . . . . . . . . . . (Y22-Y24) } \\
\text { Other and unspecified events of } \\
\text { undetermined intent and their }\end{array}$ & 0.1 & 0.1 & 0.0 & 0.0 & 0.1 & * & 0.1 & 0.1 & 0.0 \\
\hline sequelae . . . . . (Y10-Y21,Y25-Y34,Y87.2,Y89.9) & 1.7 & 2.1 & 1.3 & 0.7 & 0.9 & 0.5 & 1.8 & 2.3 & 1.4 \\
\hline $\begin{array}{l}\text { Operations of war and their sequelae } \ldots(Y 36, Y 89.1) \\
\text { Complications of medical and surgical }\end{array}$ & 0.0 & 0.0 & $*$ & * & * & * & * & * & * \\
\hline care $\ldots \ldots \ldots \ldots \ldots \ldots \ldots .(\mathrm{Y} 40-\mathrm{Y} 84, \mathrm{Y}$.Y88) & 0.8 & 0.9 & 0.8 & 0.5 & 0.5 & 0.5 & 0.9 & 0.9 & 0.8 \\
\hline Enterocolitis due to Clostridium difficile $\ldots . .(A 04.7)^{7}$ & 2.0 & 2.0 & 2.0 & 1.3 & 1.3 & 1.2 & 2.0 & 2.0 & 2.1 \\
\hline
\end{tabular}

See footnotes at end of table. 
Table 17. Age-adjusted death rates for 113 selected causes and Enterocolitis due to Clostridium difficile, by Hispanic origin, race for non-Hispanic population, and sex: United States, 2007-Con.

[Age-adjusted rates per 100,000 U.S. standard population; see "Technical Notes." Populations used for computing death rates are postcensal estimates based on the 2000 census, estimated as of July 1, 2007; see "Technical Notes." Race and Hispanic origin are reported separately on the death certificate. Persons of Hispanic origin may be of any race. Data for Hispanic persons are not tabulated separately by race; data for non-Hispanic persons are tabulated by race. Data for Hispanic origin should be interpreted with caution because of inconsistencies between reporting Hispanic origin on death certificates and on censuses and surveys; see "Technical Notes." The asterisks (*) preceding the cause-of-death codes indicate that they are not part of the International Classification of Diseases, Tenth Revision (ICD-10), Second Edition; see "Technical Notes"]

\begin{tabular}{|c|c|c|c|c|c|c|}
\hline \multirow[b]{2}{*}{ Cause of death (based on ICD-10, 2004) } & \multicolumn{3}{|c|}{ Non-Hispanic white ${ }^{3}$} & \multicolumn{3}{|c|}{ Non-Hispanic black ${ }^{3}$} \\
\hline & Both sexes & Male & Female & Both sexes & Male & Female \\
\hline$\ldots \ldots \ldots \ldots \ldots$ & 763.3 & 906.8 & 647.7 & 978.6 & $1,210.9$ & 810.4 \\
\hline$\ldots \ldots \ldots(\mathrm{A} 01-\mathrm{A} 02)$ & 0.0 & * & * & * & * & * \\
\hline Shigellosis and amebiasis . . . . . . . . (A03,A06) & * & * & * & * & * & * \\
\hline Certain other intestinal infections $\ldots .(A 04, A 07-A 09)$ & 2.3 & 2.2 & 2.3 & 1.6 & 1.6 & 1.6 \\
\hline Tuberculosis . . . . . . . . . . . . . . . . (A16-A19) & 0.1 & 0.1 & 0.1 & 0.4 & 0.6 & 0.2 \\
\hline Respiratory tuberculosis . . . . . . . . . (A16) & 0.1 & 0.1 & 0.0 & 0.3 & 0.5 & 0.2 \\
\hline Other tuberculosis $\ldots \ldots \ldots \ldots \ldots$ (A17-A19) & 0.0 & 0.0 & 0.0 & 0.1 & 0.2 & * \\
\hline Whooping cough $\ldots \ldots \ldots \ldots \ldots \ldots$ (A37) & * & * & * & * & * & * \\
\hline Scarlet fever and erysipelas $\ldots \ldots \ldots(\mathrm{A} 38, \mathrm{~A} 46)$ & * & * & * & * & * & * \\
\hline Meningococcal infection . . . . . . . . . . . (A39) & 0.0 & 0.0 & 0.0 & 0.0 & * & * \\
\hline Septicemia . . . . . . . . . . . (A40-A41) & 10.1 & 11.2 & 9.4 & 22.2 & 25.2 & 20.3 \\
\hline Syphilis $\ldots \ldots \ldots \ldots$ (A50-A53) & * & * & * & 0.1 & * & * \\
\hline Acute poliomyelitis $\ldots \ldots \ldots \ldots \ldots \ldots$ (A80) & * & * & * & * & * & * \\
\hline Arthropod-borne viral encephalitis. . (A83-A84,A85.2) & * & * & * & * & * & * \\
\hline Measles ..................... (B05) & * & * & * & * & * & * \\
\hline Viral hepatitis . . . . . . . . . . . (B15-B19) & 2.0 & 2.7 & 1.3 & 3.0 & 4.3 & 1.9 \\
\hline \multicolumn{7}{|l|}{ Human immunodeficiency virus (HIV) } \\
\hline disease $\ldots \ldots \ldots \ldots \ldots \ldots \ldots$ (B20-B24) & 1.5 & 2.5 & 0.5 & 17.8 & 25.2 & 11.8 \\
\hline 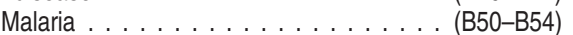 & * & * & * & * & * & * \\
\hline \multicolumn{7}{|l|}{$\begin{array}{r}\text { diseases and their sequelae } \\
\text { A20-A36,A42-A44,A48-A49, A54-A79,A81-A82, } \\
\text { A85.0-A85.1,A85.8, A86-B04,B06-B09, }\end{array}$} \\
\hline B25-B49,B55-B99) & 1.8 & 2.1 & 1.5 & 2.4 & 3.0 & 2.1 \\
\hline Malignant neoplasms $\ldots \ldots \ldots \ldots \ldots$ (CO0-C97) & 182.3 & 220.8 & 155.3 & 220.0 & 288.6 & 178.4 \\
\hline \multicolumn{7}{|l|}{ Malignant neoplasms of lip, oral cavity } \\
\hline and pharynx $\ldots \ldots \ldots \ldots \ldots(\mathrm{CO0}-\mathrm{C} 14)$ & 2.5 & 3.8 & 1.4 & 3.4 & 5.9 & 1.6 \\
\hline Malignant neoplasm of esophagus . . . . . (C15) & 4.5 & 8.2 & 1.6 & 4.8 & 8.6 & 2.3 \\
\hline Malignant neoplasm of stomach . . . . . . . (C16) & 2.9 & 4.1 & 2.0 & 7.0 & 10.5 & 4.7 \\
\hline \multicolumn{7}{|l|}{ Malignant neoplasms of colon, rectum } \\
\hline and anus $\ldots \ldots \ldots \ldots \ldots \ldots($ C18-C21) & 16.7 & 19.9 & 14.2 & 24.0 & 29.6 & 20.2 \\
\hline \multicolumn{7}{|l|}{ Malignant neoplasms of liver and } \\
\hline intrahepatic bile ducts $\ldots \ldots \ldots \ldots \ldots$ (C22) & 4.7 & 6.9 & 2.9 & 7.2 & 11.8 & 3.9 \\
\hline Malignant neoplasm of pancreas . . . . . . (C25) & 10.8 & 12.7 & 9.3 & 14.3 & 15.9 & 12.9 \\
\hline Malignant neoplasm of larynx . . . . . . . . (C32) & 1.1 & 1.9 & 0.4 & 2.2 & 4.4 & 0.6 \\
\hline \multicolumn{7}{|l|}{ Malignant neoplasms of trachea, } \\
\hline bronchus and lung . . . . . . . & 53.9 & 67.7 & 43.5 & 56.8 & 84.0 & 39.0 \\
\hline Malignant melanoma of skin ... & 3.3 & 4.9 & 2.1 & 0.5 & 0.5 & 0.4 \\
\hline Malignant neoplasm of breast . . & 12.8 & 0.3 & 23.0 & 19.0 & 0.4 & 32.2 \\
\hline Malignant neoplasm of cervix uteri $\ldots . .$. (C53) & 1.1 & $\ldots$ & 2.1 & 2.5 & $\ldots$ & 4.4 \\
\hline \multicolumn{7}{|l|}{ Malignant neoplasms of corpus uteri and } \\
\hline uterus, part unspecified $\ldots \ldots \ldots$ (C54-C55) & 2.2 & $\ldots$ & 3.9 & 4.6 & $\ldots$ & 7.7 \\
\hline Malignant neoplasm of ovary. & 4.9 & & 8.9 & 4.0 & & 6.7 \\
\hline Malignant neoplasm of prostate . . . . . . (C61) & 8.6 & 21.9 & $\ldots$ & 18.9 & 52.6 & $\ldots$ \\
\hline \multicolumn{7}{|l|}{ Malignant neoplasms of kidney and } \\
\hline$\ldots \ldots \ldots(\mathrm{C} 64-\mathrm{C} 65)$ & 4.1 & 5.9 & 2.7 & 4.1 & 6.1 & 2.8 \\
\hline Malignant neoplasm of bladder $\ldots \ldots \ldots$ (C67) & 4.7 & 8.2 & 2.3 & 3.8 & 5.5 & 2.8 \\
\hline \multicolumn{7}{|l|}{$\begin{array}{l}\text { Malignant neoplasms of meninges, } \\
\text { brain and other parts of central }\end{array}$} \\
\hline nervous system $\ldots \ldots \ldots \ldots$ (C70-C72) & 4.8 & 5.8 & 3.9 & 2.4 & 2.9 & 2.1 \\
\hline \multicolumn{7}{|l|}{ Malignant neoplasms of lymphoid, } \\
\hline hematopoietic and related tissue ... (C81-C96) & 18.1 & 23.7 & 13.9 & 17.9 & 22.5 & 14.9 \\
\hline Hodgkin's disease & 0.4 & 0.5 & 0.3 & 0.4 & 0.5 & 0.3 \\
\hline Non-Hodgkin's lymphoma $\ldots \ldots \ldots$ (C82-C85) & 6.9 & 8.7 & 5.5 & 4.6 & 5.9 & 3.7 \\
\hline Leukemia $\ldots \ldots \ldots \ldots \ldots$. . . . . . & 7.3 & 10.0 & 5.4 & 6.2 & 8.0 & 5.0 \\
\hline \multicolumn{7}{|l|}{ Multiple myeloma and immunoproliferative } \\
\hline neoplasms $\ldots \ldots \ldots \ldots \ldots(\mathrm{C} 88, \mathrm{C} 90)$ & 3.4 & 4.4 & 2.6 & 6.7 & 8.0 & 5.9 \\
\hline $\begin{array}{l}\text { Other and unspecified malignant } \\
\text { neoplasms of lymphoid, hematopoietic and }\end{array}$ & & & & & & \\
\hline related tissue $\ldots \ldots \ldots \ldots \ldots$ (C96) & 0.0 & 0.0 & 0.0 & * & * & * \\
\hline
\end{tabular}

See footnotes at end of table. 
Table 17. Age-adjusted death rates for 113 selected causes and Enterocolitis due to Clostridium difficile, by Hispanic origin, race for non-Hispanic population, and sex: United States, 2007-Con.

[Age-adjusted rates per 100,000 U.S. standard population; see "Technical Notes." Populations used for computing death rates are postcensal estimates based on the 2000 census, estimated as of July 1, 2007; see "Technical Notes." Race and Hispanic origin are reported separately on the death certificate. Persons of Hispanic origin may be of any race. Data for Hispanic persons are not tabulated separately by race; data for non-Hispanic persons are tabulated by race. Data for Hispanic origin should be interpreted with caution because of inconsistencies between reporting Hispanic origin on death certificates and on censuses and surveys; see "Technical Notes." The asterisks (*) preceding the cause-of-death codes indicate that they are not part of the International Classification of Diseases, Tenth Revision (ICD-10), Second Edition; see "Technical Notes"]

\begin{tabular}{|c|c|c|c|c|c|c|}
\hline \multirow[b]{2}{*}{ Cause of death (based on ICD-10, 2004) } & \multicolumn{3}{|c|}{ Non-Hispanic white ${ }^{3}$} & \multicolumn{3}{|c|}{ Non-Hispanic black ${ }^{3}$} \\
\hline & Both sexes & Male & Female & Both sexes & Male & Female \\
\hline \multicolumn{7}{|l|}{ All other and unspecified malignant } \\
\hline neoplasms $\ldots \ldots \ldots(\mathrm{C} 17, \mathrm{C} 23-\mathrm{C} 24, \mathrm{C} 26-\mathrm{C} 31$ & & & & & & \\
\hline C37-C41,C44-C49,C51-C52,C57-C60, & & & & & & \\
\hline $\mathrm{C} 62-\mathrm{C} 63, \mathrm{C} 66, \mathrm{C} 68-\mathrm{C} 69, \mathrm{C} 73-\mathrm{C} 80, \mathrm{C} 97)$ & 20.5 & 25.1 & 17.1 & 22.5 & 27.5 & 19.1 \\
\hline behavior . . . . . . . . . . . . (D00-D48) & 4.7 & 5.9 & 3.8 & 4.2 & 5.3 & 3.5 \\
\hline Anemias $\ldots \ldots \ldots \ldots \ldots$ (D50-D64) & 1.3 & 1.3 & 1.3 & 3.1 & 3.2 & 2.9 \\
\hline Diabetes mellitus $\ldots \ldots \ldots \ldots \ldots \ldots$ (E10-E14) & 19.8 & 23.9 & 16.4 & 43.7 & 46.9 & 41.0 \\
\hline Nutritional deficiencies $\ldots \ldots \ldots \ldots \ldots$ (E40-E64) & 0.8 & 0.8 & 0.8 & 1.5 & 1.7 & 1.4 \\
\hline Malnutrition . . . . . . . . . . . . (E40-E46) & 0.8 & 0.8 & 0.8 & 1.4 & 1.6 & 1.3 \\
\hline Other nutritional deficiencies $\ldots \ldots \ldots$ (E50-E64) & 0.1 & 0.1 & 0.1 & * & * & * \\
\hline Meningitis $\ldots \ldots \ldots \ldots \ldots(\mathrm{G} 00, \mathrm{G} 03)$ & 0.2 & 0.2 & 0.2 & 0.4 & 0.4 & 0.3 \\
\hline Parkinson's disease . . . . . . . . . (G20-G21) & 6.9 & 10.5 & 4.7 & 3.2 & 5.0 & 2.2 \\
\hline Alzheimer's disease $\ldots \ldots \ldots \ldots \ldots \ldots$ (G30) & 24.1 & 19.6 & 26.6 & 19.4 & 16.0 & 20.9 \\
\hline Major cardiovascular diseases . . . . . . . (100-178) & 248.2 & 298.2 & 207.6 & 341.4 & 411.5 & 289.5 \\
\hline Diseases of heart $\ldots \ldots(100-109,|11| 13,,|20-| 51)$ & 191.4 & 239.8 & 153.0 & 251.9 & 311.8 & 208.2 \\
\hline \multicolumn{7}{|l|}{ Acute rheumatic fever and chronic } \\
\hline rheumatic heart diseases $\ldots \ldots \ldots(100-109)$ & 1.0 & 0.8 & 1.2 & 0.8 & 0.7 & 0.9 \\
\hline Hypertensive heart disease $\ldots \ldots \ldots \ldots$ (l11) & 8.1 & 8.6 & 7.3 & 23.8 & 28.9 & 19.7 \\
\hline Hypertensive heart and renal disease . . . . (I13) & 0.7 & 0.8 & 0.7 & 2.9 & 3.5 & 2.4 \\
\hline Ischemic heart diseases . . . . . . . (I20-I25) & 127.4 & 168.8 & 95.2 & 153.1 & 194.9 & 123.5 \\
\hline Acute myocardial infarction $\ldots \ldots$. (121-122) & 42.1 & 55.5 & 31.3 & 50.5 & 62.2 & 42.0 \\
\hline Other acute ischemic heart diseases . . . (124) & 1.3 & 1.6 & 1.0 & 1.8 & 2.5 & 1.4 \\
\hline \multicolumn{7}{|l|}{ Other forms of chronic ischemic heart } \\
\hline disease . . . . . . . . . . . . . . (120,125) & 84.0 & 111.7 & 62.8 & 100.8 & 130.2 & 80.0 \\
\hline \multicolumn{7}{|l|}{$\begin{array}{l}\text { disease } \ldots \ldots \ldots \ldots \ldots \ldots \ldots \ldots \ldots \\
\text { Atherosclerotic cardiovascular }\end{array}$} \\
\hline disease, so described . . . . . . . . (I25.0) & 17.4 & 23.2 & 12.5 & 30.8 & 43.0 & 21.9 \\
\hline \multicolumn{7}{|l|}{ All other forms of chronic ischemic } \\
\hline heart disease $\ldots \ldots \ldots$ (I20,125.1-|25.9) & 66.5 & 88.4 & 50.4 & 70.0 & 87.2 & 58.1 \\
\hline Other heart diseases . . . . . . . . (I26-I51) & 54.1 & 60.8 & 48.7 & 71.3 & 83.9 & 61.8 \\
\hline Acute and subacute endocarditis ...... (I33) & 0.4 & 0.5 & 0.3 & 0.7 & 1.1 & 0.4 \\
\hline \multicolumn{7}{|l|}{ Diseases of pericardium and acute } \\
\hline myocarditis $\ldots \ldots \ldots \ldots(130-131, \mid 40)$ & 0.3 & 0.3 & 0.2 & 0.4 & 0.5 & 0.4 \\
\hline Heart failure . . . . . . . . . . . . . . (150) & 17.8 & 19.2 & 16.7 & 20.5 & 22.9 & 18.8 \\
\hline \multicolumn{7}{|l|}{ All other forms of heart disease $\quad$ (I26-I28, } \\
\hline$|34-| 38,|42-| 49, \mid 151)$ & 35.7 & 40.8 & 31.4 & 49.6 & 59.5 & 42.2 \\
\hline \multicolumn{7}{|l|}{ Essential hypertension and hypertensive } \\
\hline renal disease $\ldots \ldots \ldots \ldots(110,112,115)$ & 6.4 & 6.2 & 6.3 & 16.6 & 17.8 & 15.5 \\
\hline Cerebrovascular diseases $\ldots \ldots \ldots \ldots(160-169)$ & 40.7 & 40.3 & 40.3 & 61.6 & 68.5 & 56.1 \\
\hline Atherosclerosis . . . . . . . . . . . . (170) & 2.6 & 2.7 & 2.5 & 2.5 & 2.7 & 2.3 \\
\hline Other diseases of circulatory system $\ldots .(171-178)$ & 7.0 & 9.0 & 5.5 & 8.7 & 10.8 & 7.3 \\
\hline Aortic aneurysm and dissection ....... (171) & 4.3 & 6.0 & 3.0 & 4.3 & 5.6 & 3.3 \\
\hline \multicolumn{7}{|l|}{ Other diseases of arteries, arterioles and } \\
\hline capillaries . . . . . . . . . . . . (172-178) & 2.7 & 3.0 & 2.5 & 4.5 & 5.2 & 4.0 \\
\hline Other disorders of circulatory system . . . (180-199) & 1.3 & 1.3 & 1.1 & 2.2 & 2.5 & 2.0 \\
\hline Influenza and pneumonia . . . . . . . (J09-J18 $)^{4}$ & 16.2 & 19.1 & 14.3 & 18.8 & 24.0 & 15.5 \\
\hline Influenza. . . . . . . . . . . . . . & 0.1 & 0.1 & 0.1 & 0.0 & * & * \\
\hline Pneumonia . . . . . . . . . . . . . (J12-J18) & 16.0 & 19.0 & 14.2 & 18.7 & 24.0 & 15.4 \\
\hline \multicolumn{7}{|l|}{ Other acute lower respiratory } \\
\hline 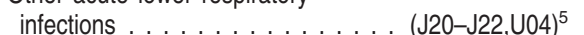 & 0.1 & 0.1 & 0.1 & 0.1 & * & * \\
\hline Acute bronchitis and bronchiolitis . . . . (J20-J21) & 0.1 & 0.1 & 0.0 & 0.1 & * & * \\
\hline \multicolumn{7}{|l|}{ Other and unspecified acute lower respiratory } \\
\hline infections $\ldots \ldots \ldots \ldots \ldots(\text { J22,U04 })^{5,6}$ & 0.0 & * & 0.0 & * & * & * \\
\hline Chronic lower respiratory diseases $\ldots . .$. (J40-J47) & 44.9 & 51.8 & 40.4 & 28.7 & 40.4 & 21.9 \\
\hline Bronchitis, chronic and unspecified ..... (J40-J42) & 0.2 & 0.2 & 0.2 & 0.2 & 0.2 & 0.2 \\
\hline 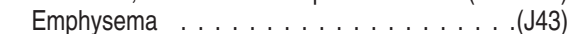 & 4.6 & 5.5 & 3.9 & 2.7 & 4.4 & 1.7 \\
\hline 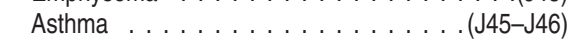 & 0.9 & 0.7 & 1.1 & 2.6 & 2.5 & 2.6 \\
\hline
\end{tabular}

See footnotes at end of table. 
Table 17. Age-adjusted death rates for 113 selected causes and Enterocolitis due to Clostridium difficile, by Hispanic origin, race for non-Hispanic population, and sex: United States, 2007-Con.

[Age-adjusted rates per 100,000 U.S. standard population; see "Technical Notes." Populations used for computing death rates are postcensal estimates based on the 2000 census, estimated as of July 1, 2007; see "Technical Notes." Race and Hispanic origin are reported separately on the death certificate. Persons of Hispanic origin may be of any race. Data for Hispanic persons are not tabulated separately by race; data for non-Hispanic persons are tabulated by race. Data for Hispanic origin should be interpreted with caution because of inconsistencies between reporting Hispanic origin on death certificates and on censuses and surveys; see "Technical Notes." The asterisks (*) preceding the cause-of-death codes indicate that they are not part of the International Classification of Diseases, Tenth Revision (ICD-10), Second Edition; see "Technical Notes"]

\begin{tabular}{|c|c|c|c|c|c|c|}
\hline \multirow[b]{2}{*}{ Cause of death (based on ICD-10, 2004) } & \multicolumn{3}{|c|}{ Non-Hispanic white ${ }^{3}$} & \multicolumn{3}{|c|}{ Non-Hispanic black ${ }^{3}$} \\
\hline & Both sexes & Male & Female & Both sexes & Male & Female \\
\hline $\begin{array}{l}\text { Other chronic lower respiratory } \\
\text { diseases } \ldots \ldots \ldots \ldots \ldots \ldots \ldots \ldots \ldots \ldots \\
(\mathrm{J} 44, \mathrm{~J} 47)\end{array}$ & 39.2 & 45.4 & 35.2 & 23.2 & 33.3 & 17.4 \\
\hline Pneumoconioses and chemical & & & & & & \\
\hline effects $\ldots \ldots \ldots \ldots \ldots \ldots($ (J60-J66,J68) & 0.3 & 0.8 & 0.0 & 0.2 & 0.4 & * \\
\hline $\begin{array}{l}\text { Pneumonitis due to solids and liquids . . . . . . . (J69) } \\
\text { Other diseases of respiratory }\end{array}$ & 5.5 & 7.7 & 4.2 & 5.4 & 7.7 & 4.1 \\
\hline system . . . . . . (J00-J06,J30-J39,J67,J70-J98) & 9.2 & 11.3 & 7.8 & 8.8 & 10.5 & 76 \\
\hline Peptic ulcer .................. (K25-K28) & 1.0 & 1.1 & 0.8 & 1.0 & 1.4 & 0.8 \\
\hline Diseases of appendix . . . . . . . . (K35-K38) & 0.1 & 0.2 & 0.1 & 0.2 & 0.3 & 0.2 \\
\hline 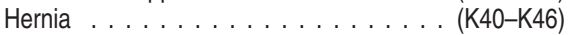 & 0.5 & 0.6 & 0.5 & 0.6 & 0.8 & 0.5 \\
\hline Chronic liver disease and cirrhosis $\quad .(\mathrm{K} 70, \mathrm{~K} 73-\mathrm{K} 74)$ & 8.9 & 12.2 & 5.9 & 7.5 & 11.3 & 4.6 \\
\hline Alcoholic liver disease $\ldots \ldots \ldots \ldots \ldots$. . . . . . . & 4.4 & 6.5 & 2.4 & 3.5 & 5.6 & 1.8 \\
\hline $\begin{array}{l}\text { Other chronic liver disease and } \\
\text { cirrhosis } \ldots \ldots \ldots \ldots \text { (K73-K74) }\end{array}$ & 4.5 & 5.8 & 3.4 & 4.1 & 5.7 & 2.8 \\
\hline $\begin{array}{l}\text { Cholelithiasis and other disorders of } \\
\text { gallbladder ................ K80-K82) }\end{array}$ & 1.0 & 1.1 & 0.9 & 1.1 & 1.2 & 1.0 \\
\hline $\begin{array}{l}\text { Nephritis, nephrotic syndrome and } \\
\text { nephrosis ....... (N00-N07,N17-N19,N25-N27) }\end{array}$ & 13.1 & 16.5 & 11.0 & 30.0 & 34.4 & 27.2 \\
\hline $\begin{array}{l}\text { Acute and rapidly progressive nephritic and } \\
\text { nephrotic syndrome ........... (N00-N01,N04) } \\
\text { Chronic glomerulonephritis, nephritis and } \\
\text { nephropathy not specified as acute or } \\
\text { chronic, and renal sclerosis }\end{array}$ & 0.1 & 0.1 & 0.0 & 0.1 & * & * \\
\hline unspecified $\ldots \ldots \ldots$ (N02-N03,N05-N07,N26) & 0.8 & 1.1 & 0.7 & 1.9 & 2.3 & 1.7 \\
\hline Renal failure $\ldots \ldots \ldots \ldots \ldots$ (N17-N19) & 12.2 & 15.3 & 10.3 & 28.0 & 32.0 & 25.4 \\
\hline Other disorders of kidney $\ldots \ldots \ldots(\mathrm{N} 25, \mathrm{~N} 27)$ & * & * & * & * & * & * \\
\hline Infections of kidney $\ldots \ldots$ (N10-N12,N13.6,N15.1) & 0.2 & 0.2 & 0.2 & 0.2 & 0.2 & 0.2 \\
\hline $\begin{array}{l}\text { Hyperplasia of prostate } \ldots \ldots \ldots \ldots \ldots \text {. } \ldots \ldots \text { (N40) } \\
\text { Inflammatory diseases of female pelvic }\end{array}$ & 0.1 & 0.4 & $\ldots$ & 0.2 & 0.5 & $\ldots$ \\
\hline $\begin{array}{l}\text { organs } \ldots \ldots \ldots \ldots \ldots \ldots \ldots \text { (N70-N76) } \\
\text { Pregnancy, childbirth and }\end{array}$ & 0.0 & $\ldots$ & 0.1 & * & $\ldots$ & * \\
\hline the puerperium $\ldots \ldots \ldots \ldots$ (O00-099) & 0.2 & $\ldots$ & 0.4 & 0.6 & $\ldots$ & 1.2 \\
\hline $\begin{array}{l}\text { Pregnancy with abortive outcome . . . . (O00-007) } \\
\text { Other complications of pregnancy, childbirth and }\end{array}$ & * & $\ldots$ & * & * & $\ldots$ & * \\
\hline $\begin{array}{l}\text { the puerperium } \ldots \ldots \ldots \ldots \ldots(010-099) \\
\text { Certain conditions originating in the perinatal }\end{array}$ & 0.2 & $\ldots$ & 0.4 & 0.6 & $\ldots$ & 1.1 \\
\hline $\begin{array}{l}\text { chromosomal abnormalities ......... (Q00-Q99) } \\
\text { Symptoms, signs and abnormal clinical and } \\
\text { laboratory findings, not elsewhere }\end{array}$ & 3.3 & 3.4 & 3.1 & 4.1 & 4.2 & 3.9 \\
\hline classified . . . . . . . . . . . . . . (R00-R99) & 10.4 & 10.5 & 9.9 & 14.3 & 15.6 & 12.8 \\
\hline $\begin{array}{l}\text { All other diseases . . . . . . . . . . . . (residual) } \\
\text { Accidents (unintentional }\end{array}$ & 74.9 & 74.5 & 73.4 & 91.7 & 96.6 & 86.7 \\
\hline injuries) . . . . . . . . . . . . . .(V01-X59,Y85-Y86) & 43.0 & 58.6 & 28.4 & 37.6 & 56.2 & 22.2 \\
\hline $\begin{array}{r}\text { Transport accidents } \ldots \ldots \ldots . . . . .(V 01-V 99, Y 85) \\
\text { Motor-vehicle accidents. . . . . . (V02-V04, } \\
\text { V09.0,V09.2,V12-V14,V19.0-V19.2, } \\
\text { V19.4-V19.6,V20-V79,V80.3-V80.5, } \\
\text { V81.0-V81.1,V82.0-V82.1,V83-V86, }\end{array}$ & 15.9 & 23.0 & 9.1 & 15.4 & 24.7 & 7.5 \\
\hline $\begin{array}{r}\text { V87.0-V87.8,V88.0-V88.8,V89.0, V89.2) } \\
\text { Other land transport accidents ...... (V01, } \\
\text { V05-V06,V09.1,V09.3-V09.9, V10-V11, } \\
\text { V15-V18,V19.3,V19.8- V19.9,V80.0-V80.2, } \\
\text { V80.6-V80.9, V81.2-V81.9,V82.2-V82.9, }\end{array}$ & 14.9 & 21.4 & 8.7 & 14.5 & 23.1 & 7.2 \\
\hline $\begin{array}{l}\text { V87.9, V88.9,V89.1,V89.3,V89.9) } \\
\text { Water, air and space, and other and } \\
\text { unspecified transport accidents }\end{array}$ & 0.3 & 0.5 & 0.1 & 0.4 & 0.7 & 0.2 \\
\hline and their sequelae . . . . . . . . (V90-V99,Y85) & 0.7 & 1.1 & 0.3 & 0.5 & 0.9 & 0.1 \\
\hline
\end{tabular}

See footnotes at end of table. 
Table 17. Age-adjusted death rates for 113 selected causes and Enterocolitis due to Clostridium difficile, by Hispanic origin, race for non-Hispanic population, and sex: United States, 2007-Con.

[Age-adjusted rates per 100,000 U.S. standard population; see "Technical Notes." Populations used for computing death rates are postcensal estimates based on the 2000 census, estimated as of July 1, 2007; see "Technical Notes." Race and Hispanic origin are reported separately on the death certificate. Persons of Hispanic origin may be of any race. Data for Hispanic persons are not tabulated separately by race; data for non-Hispanic persons are tabulated by race. Data for Hispanic origin should be interpreted with caution because of inconsistencies between reporting Hispanic origin on death certificates and on censuses and surveys; see "Technical Notes." The asterisks ( $\left.{ }^{*}\right)$ preceding the cause-of-death codes indicate that they are not part of the International Classification of Diseases, Tenth Revision (ICD-10), Second Edition; see "Technical Notes"]

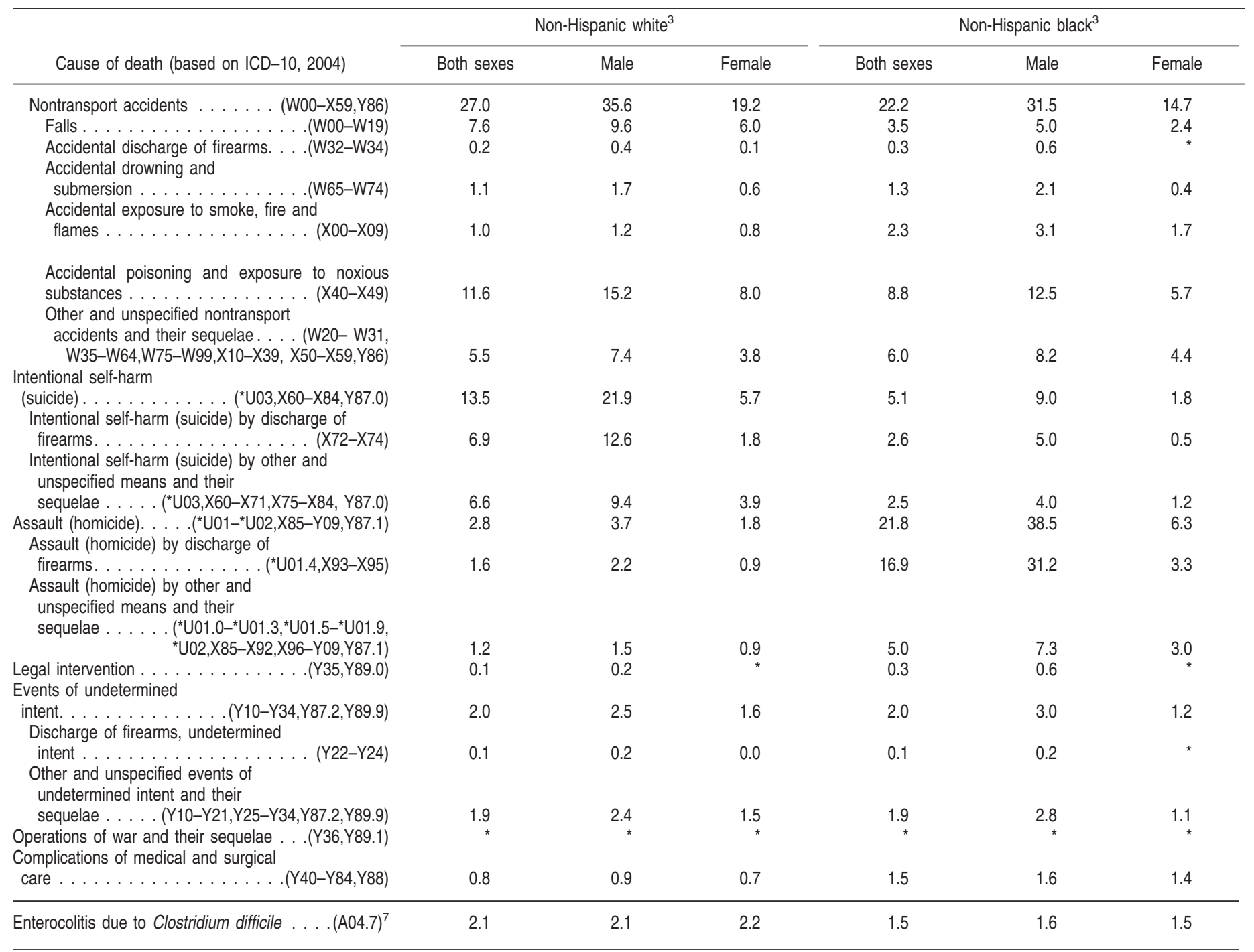

0.0 Quantity more than zero but less than 0.05 .

* Figure does not meet standards of reliability or precision; see "Technical Notes."

... Category not applicable.

${ }^{1}$ Figures for origin not stated are included in "all origins" but not distributed among specified origins.

${ }^{2}$ Includes races other than white and black.

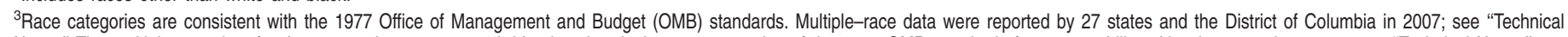
Notes." The multiple-race data for these reporting areas were bridged to the single-race categories of the 1977 OMB standards for comparability with other reporting areas; see "Technical Notes."

${ }^{4} \mathrm{New}$ ICD-10 code $\mathrm{J09}$ (Influenza due to identified avian influenza virus) was added to the category in 2007. No deaths occurred from this cause in 2007.

${ }^{5} \mathrm{New}$ ICD-10 code U04 (Severe acute respiratory syndrome [SARS]) was added to the category in 2007. No deaths occurred from this cause in 2007.

${ }^{6}$ Cause-of-death title was changed in 2007 to reflect the addition of SARS (ICD-10 code U04).

${ }^{7}$ Included in "Certain other intestinal infections (A04,A07-A09)" shown above. Beginning with data year 2006, "Enterocolitis due to Clostridium difficile (A04.7)" is shown separately at the bottom of tables showing 113 selected causes and is included in the list of rankable causes, see "Technical Notes."

NOTE: Complete confirmation of deaths from selected causes of death, considered to be of public health concern, was not provided by the following states: Connecticut, Florida, Indiana, Kentucky, Maryland, North Carolina, Oklahoma, Pennsylvania, Rhode Island, South Carolina, Washington, and West Virginia; see "Technical Notes." 
Table 18. Number of deaths, death rates, and age-adjusted death rates for injury deaths, by mechanism and intent of death: United States, 2007

[Totals for selected causes of death differ from those shown in other tables that utilize standard mortality tabulation lists; see "Technical Notes." Populations used for computing death rates are postcensal estimates based on the 2000 census, estimated as of July 1, 2007. Rates are per 100,000 population; age-adjusted rates are per 100,000 U.S. standard population; see "Technical Notes." Figure(s) in brackets [] applies to the code or range of codes preceding it. For explanation of asterisks preceding cause-of-death codes, see "Technical Notes"]

\begin{tabular}{|c|c|c|c|}
\hline $\begin{array}{c}\text { Mechanism and intent of death (based on the International Classification of } \\
\text { Diseases, Tenth Revision, Second Edition, 2004) }\end{array}$ & Number & Rate & $\begin{array}{l}\text { Age-adjusted } \\
\text { rate }^{1}\end{array}$ \\
\hline$\ldots \ldots \ldots \ldots \ldots\left({ }^{*} \mathrm{U} 01-{ }^{*} \mathrm{U} 03, \mathrm{~V} 01-\mathrm{Y} 36, \mathrm{Y} 85-\mathrm{Y} 87, \mathrm{Y} 89\right)$ & 182,479 & 60.5 & 59.2 \\
\hline 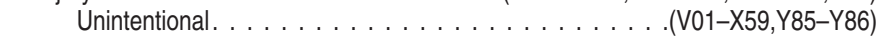 & 123,706 & 41.0 & 40.0 \\
\hline 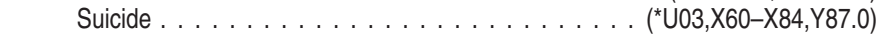 & 34,598 & 11.5 & 11.3 \\
\hline Homicide . . . . . . . . . . . . . . . . . . . . . . . .(*U01-*U02,X85-Y09,Y87.1) & 18,361 & 6.1 & 6.1 \\
\hline Undetermined . . . . . . . . . . . . . . . . . . . . .(Y10-Y34,Y87.2,Y89.9) & 5,381 & 1.8 & 1.8 \\
\hline Legal intervention/war . . . . . . . . . . . . . . (Y35-Y36,Y89[.0,.1]) & 433 & 0.1 & 0.1 \\
\hline Cut $/$ pierce . . . . . . . . . . . . . . . (W25-W29,W45,X78,X99,Y28,Y35.4) & 2,734 & 0.9 & 0.9 \\
\hline Unintentional. . . . . . . . . . . . . . . . . . . . . (W25-W29,W45) & 111 & 0.0 & 0.0 \\
\hline 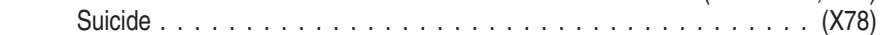 & 619 & 0.2 & 0.2 \\
\hline Homicide . . . . . . . . . . . . . . . . . . . . . . . . . . (X99) & 1,981 & 0.7 & 0.6 \\
\hline 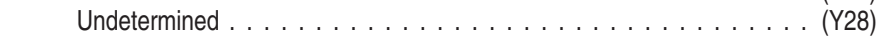 & 23 & 0.0 & 0.0 \\
\hline Legal intervention/war . . . . . . . . . . . . . . . . . (Y35.4) & - & * & * \\
\hline Drowning. . . . . . . . . . . . . . . . . (W65-W74,X71,X92,Y21) & 4,086 & 1.4 & 1.4 \\
\hline 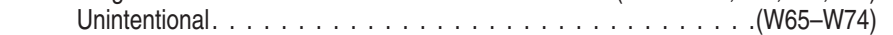 & 3,443 & 1.1 & 1.1 \\
\hline Suicide . . . . . . . . . . . . . . . . . . . (X71) & 358 & 0.1 & 0.1 \\
\hline Homicide . . . . . . . . . . . . . . . . . & 49 & 0.0 & 0.0 \\
\hline Undetermined $\ldots \ldots \ldots \ldots \ldots \ldots \ldots$ & 236 & 0.1 & 0.1 \\
\hline 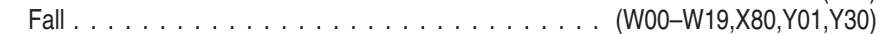 & 23,443 & 7.8 & 7.3 \\
\hline Unintentional. . . . . . . . . . . . . . . . . . . . . . . (W00-W19) & 22,631 & 7.5 & 7.0 \\
\hline Suicide . . . . . . . . . . . . . . . . . . . . . . . . . . (X80) & 731 & 0.2 & 0.2 \\
\hline Homicide . . . . . . . . . . . . . . . . . . . . . . (Y01) & 15 & * & * \\
\hline Undetermined $\ldots \ldots \ldots \ldots \ldots \ldots \ldots$ & 66 & 0.0 & 0.0 \\
\hline \multicolumn{4}{|l|}{ Fire/hot object or substance . . . . . . (*U01.3,X00-X19,X76-X77,X97-X98, } \\
\hline$Y 26-Y 27, Y 36.3)^{2}$ & 3,774 & 1.3 & 1.2 \\
\hline Unintentional. . . . . . . . . . . . . . . . . . . . . . . . . (X00-X19) & 3,375 & 1.1 & 1.1 \\
\hline 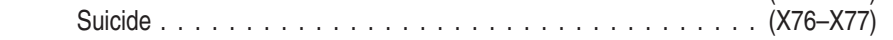 & 157 & 0.1 & 0.1 \\
\hline Homicide . . . . . . . . . . . . . . . . . . . . . . . . . ( ${ }^{*} \mathrm{U} 01.3, \mathrm{X97-X98)}$ & 141 & 0.0 & 0.0 \\
\hline 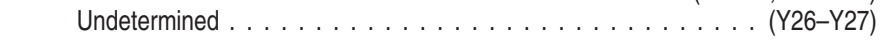 & 101 & 0.0 & 0.0 \\
\hline Legal intervention/war . . . . . . . . . . . . . . . . . . . (Y36.3) & - & * & * \\
\hline 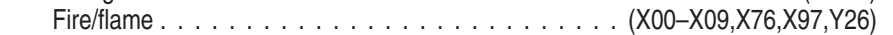 & 3,680 & 1.2 & 1.2 \\
\hline Unintentional. . . . . . . . . . . . . . . . . . . . . . . . . . . . . (X00-X09) & 3,286 & 1.1 & 1.1 \\
\hline 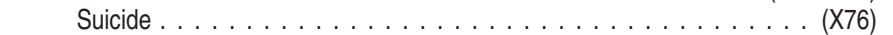 & 157 & 0.1 & 0.1 \\
\hline$\ldots \ldots \ldots \ldots$ & 139 & 0.0 & 0.0 \\
\hline Undetermined $\ldots \ldots \ldots \ldots \ldots \ldots \ldots \ldots$ & 98 & 0.0 & 0.0 \\
\hline Hot object/substance . . . . . . . . . . . . . . . . (X10-X19,X77,X98,Y27) & 94 & 0.0 & 0.0 \\
\hline Unintentional. . . . . . . . . . . . . . . . . . . . . . (X10-X19) & 89 & 0.0 & 0.0 \\
\hline Suicide . . . . . . . . . . . . . . . . . . . . . . . . . . . . . . (X77) & - & * & * \\
\hline Homicide . . . . . . . . . . . . . . . . . . . . . . . . . . . (X98) & 2 & * & * \\
\hline Undetermined . . . . . . . . . . . . & 3 & * & * \\
\hline Firearm. . . . . . . . . . (*U01.4,W32-W34,X72-X74,X93-X95,Y22-Y24,Y35.0) & 31,224 & 10.4 & 10.2 \\
\hline Unintentional. . . . . . . . . . . . . . . . . . . . . . . (W32-W34) & 613 & 0.2 & 0.2 \\
\hline 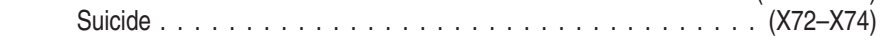 & 17,352 & 5.8 & 5.6 \\
\hline Homicide . . . . . . . . . . . . . . . . . . . . . . . . ( $\left.{ }^{*} \mathrm{U} 01.4, \mathrm{X93}-\mathrm{X95}\right)$ & 12,632 & 4.2 & 4.2 \\
\hline Undetermined . . . . . . . . . . . . . . . . . . . . . . . (Y22-Y24) & 276 & 0.1 & 0.1 \\
\hline Legal intervention/war . . . . . . . . . . . . . . . . . . . . . . . . . (Y35.0) & 351 & 0.1 & 0.1 \\
\hline 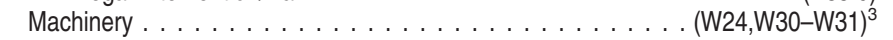 & 659 & 0.2 & 0.2 \\
\hline$\ldots \ldots \ldots \ldots \ldots$. . . .U01.1,V01-V99,X82,Y03,Y32,Y36.1) & 46,250 & 15.3 & 15.2 \\
\hline Unintentional. $\ldots \ldots \ldots \ldots \ldots \ldots \ldots \ldots \ldots \ldots \ldots$ (V01-V99) & 46,067 & 15.3 & 15.1 \\
\hline Suicide . . . . . . . . . . . . . . . . . . . . . . . . (X82) & 131 & 0.0 & 0.1 \\
\hline$\left.\ldots \ldots \ldots \ldots \ldots \ldots \ldots \ldots{ }^{*} \mathrm{U} 01.1, \mathrm{Y} 03\right)$ & 30 & 0.0 & 0.0 \\
\hline Undetermined $\ldots \ldots \ldots \ldots \ldots$. . . . . . . . . . . . . . . . . . . . . . (Y32) & 22 & 0.0 & 0.0 \\
\hline Legal intervention/war . . . . . . . . . . . . . . . . . (Y36.1) & - & * & * \\
\hline \multirow{2}{*}{\multicolumn{4}{|c|}{ Motor vehicle traffic . . . . . . . . . V02-V04[.1,.9],V09.2,V12-V14[.3-.9], }} \\
\hline & & & \\
\hline V83-V86[.0-.3],V87[.0-.8],V89.2) & 42,031 & 13.9 & 13.8 \\
\hline 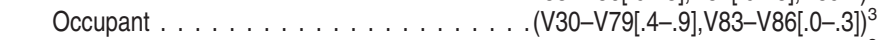 & 16,560 & 5.5 & 5.4 \\
\hline 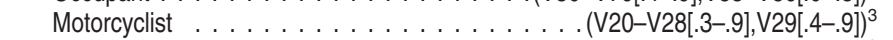 & 4,889 & 1.6 & 1.6 \\
\hline Pedal cyclist . . . . . . . . . . . . . . . (V12-V14[.3-.9],V19[.4-.6]) & 578 & 0.2 & 0.2 \\
\hline 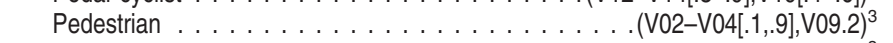 & 4,820 & 1.6 & 1.6 \\
\hline 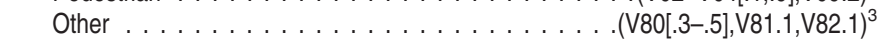 & 6 & * & * \\
\hline 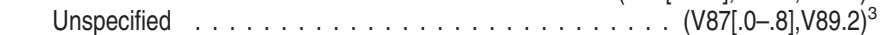 & 15,178 & 5.0 & 5.0 \\
\hline Pedal cyclist, other . . . . . (V10-V11,V12-V14[.0-.2],V15-V18,V19[.0-.3,.8,.9]) & 242 & 0.1 & 0.1 \\
\hline Pedestrian, other . . . . . . . . . (V01,V02-V04[.0],V05,V06,V09[.0,1,.3,.9]) & 1,138 & 0.4 & 0.4 \\
\hline
\end{tabular}

See footnotes at end of table. 
Table 18. Number of deaths, death rates, and age-adjusted death rates for injury deaths, by mechanism and intent of death: United States, 2007-Con.

[Totals for selected causes of death differ from those shown in other tables that utilize standard mortality tabulation lists; see "Technical Notes." Populations used for computing death rates are postcensal estimates based on the 2000 census, estimated as of July 1, 2007. Rates are per 100,000 population; age-adjusted rates are per 100,000 U.S. standard population; see "Technical Notes." Figure(s) in brackets [] applies to the code or range of codes preceding it. For explanation of asterisks preceding cause-of-death codes, see "Technical Notes"]

\begin{tabular}{|c|c|c|c|}
\hline $\begin{array}{c}\text { Mechanism and intent of death (based on the International Classification of } \\
\text { Diseases, Tenth Revision, Second Edition, 2004) }\end{array}$ & Number & Rate & $\begin{array}{l}\text { Age-adjusted } \\
\text { rate }^{1}\end{array}$ \\
\hline \multicolumn{4}{|l|}{ Other land transport . . . . . . (V20-V28[.0-.2],V29-V79[.0-.3],V80[.0-.2,.6-.9], } \\
\hline V81-V82[.0,.2-.9],V83-V86[.4-.9],V87.9,V88[.0-.9],V89[.0,.1,.3,.9],X82,Y03,Y32) & 1,800 & 0.6 & 0.6 \\
\hline Unintentional $\ldots \ldots \ldots$ (V20-V28[.0-.2],V29-V79[.0-.3],V80[.0-.2,.6-.9], & & & \\
\hline V81-V82[.0,.2-.9],V83-V86[.4-.9],V87.9,V88[.0-.9],V89[.0,.1,.3,.9]) & 1,617 & 0.5 & 0.5 \\
\hline Suicide . . . . . . . . . . . . . . . . . . . . . . . . (X82) & 131 & 0.0 & 0.1 \\
\hline Homicide . . . . . . . . . . . . . . . . . . . . . . . . . (YO3) & 30 & 0.0 & 0.0 \\
\hline$\ldots \ldots \ldots \ldots \ldots \ldots$ (Y32) & 22 & 0.0 & 0.0 \\
\hline Other transport (*U01.1,V90-V99,Y36.1) & 1,039 & 0.3 & 0.3 \\
\hline Unintentional. . . . . . . . . . . . & 1,039 & 0.3 & 0.3 \\
\hline Homicide . . . . . . . . . . . . . . . & - & * & * \\
\hline Legal intervention/war . . & - & * & * \\
\hline Natural/environmental ... (W42-W43,W53-W64,W92-W99,X20-X39,X51-X57) & 1,449 & 0.5 & 0.5 \\
\hline 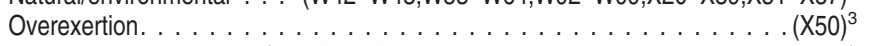 & 9 & * & * \\
\hline Poisoning . . . . . ( ${ }^{*}$ U01[.6-.7],X40-X49,X60-X69,X85-X90,Y10-Y19,Y35.2) & 40,059 & 13.3 & 13.1 \\
\hline 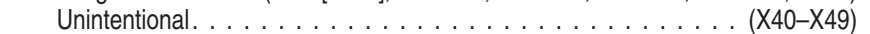 & 29,846 & 9.9 & 9.8 \\
\hline Suicide . . . . . . . . . . . . . . . . . . . . . (X60-X69) & 6,358 & 2.1 & 2.1 \\
\hline$\left.\ldots \ldots \ldots \ldots \ldots \ldots \ldots{ }^{*} \cup 01[.6-.7], \mathrm{X} 85-\mathrm{X} 90\right)$ & 85 & 0.0 & 0.0 \\
\hline$\ldots(Y 10-Y 19)$ & 3,770 & 1.2 & 1.3 \\
\hline Legal intervention/war . . . . . . & - & * & * \\
\hline Struck by or against . . . . . . . (W20-W22,W50-W52,X79,Y00,Y04,Y29,Y35.3) & 1,009 & 0.3 & 0.3 \\
\hline Unintentional. . . . . . . . . . . . . . . . . (W20-W22,W50-W52) & 832 & 0.3 & 0.3 \\
\hline Suicide . . . . . . . . . . . . . . . . . . . . . . . (X79) & 1 & * & * \\
\hline Homicide . . . . . . . . . . . . . . . . . . . . . . . . (YOO,YO4) & 173 & 0.1 & 0.1 \\
\hline Undetermined . . . . . . . . . . . . . . . . . . . . . . . . . (Y29) & 3 & * & * \\
\hline Legal intervention/war . . . . . . . . . . . . & - & * & * \\
\hline Suffocation. . . . . . . . . . . . . . . . . (W75-W84,X70,X91,Y20) & 14,930 & 4.9 & 4.9 \\
\hline Unintentional. . . . . . . . . . . . . . . . . . . . . . . . (W75-W84) & 5,997 & 2.0 & 1.9 \\
\hline Suicide . . . . . . . . . . . . . . . . . . . . . . . . (X70) & 8,161 & 2.7 & 2.7 \\
\hline Homicide $\ldots \ldots \ldots \ldots \ldots \ldots \ldots \ldots \ldots$ (X91) & 637 & 0.2 & 0.2 \\
\hline Undetermined. & 135 & 0.0 & 0.0 \\
\hline \multicolumn{4}{|l|}{ Other specified, classifiable . . . (*U01[.0,.2,.5], ,U03.0,W23,W35-W41,W44,W49, } \\
\hline W85-W91,X75,X81,X96,Y02, Y05-Y07,Y25,Y31, Y35[.1,.5],Y36[.0,.2,.4-.8],Y85) & 2,121 & 0.7 & 0.7 \\
\hline Unintentional. . . . . . . . . . . (W23,W35-W41,W44,W49,W85-W91,Y85) & 1,542 & 0.5 & 0.5 \\
\hline 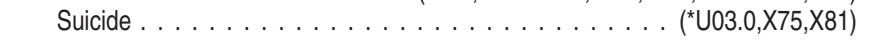 & 331 & 0.1 & 0.1 \\
\hline 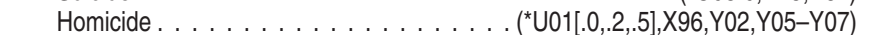 & 186 & 0.1 & 0.1 \\
\hline 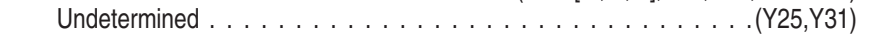 & 17 & * & * \\
\hline Legal intervention/war . . . . . . . . . . . . (Y35[.1,.5],Y36[.0,.2,.4-.8]) & 45 & 0.0 & 0.0 \\
\hline \multicolumn{4}{|l|}{ Other specified, not elsewhere classified . . . . . .(*U01.8, ${ }^{*} \cup 02, X 58, X 83, Y 08, Y 33$, } \\
\hline Y35.6,Y86-Y87,Y89[.0-.1]) & 2,165 & 0.7 & 0.7 \\
\hline$\ldots \ldots \ldots \ldots \ldots(\mathrm{X} 58, \mathrm{Y} 86)$ & 1,113 & 0.4 & 0.4 \\
\hline .(X83,Y87.0) & 236 & 0.1 & 0.1 \\
\hline$\ldots \ldots \ldots\left({ }^{*} \mathrm{U} 01.8,{ }^{*} \mathrm{U} 02, \mathrm{Y} 08, \mathrm{Y} 87.1\right)$ & 586 & 0.2 & 0.2 \\
\hline 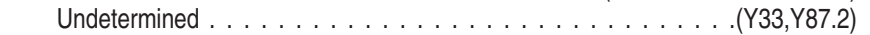 & 197 & 0.1 & 0.1 \\
\hline$\ldots \ldots \ldots(Y 35.6, Y 89[\cdot 0, .1])$ & 33 & 0.0 & 0.0 \\
\hline Unspecified . . . . . . . . . (*'U01.9, $\left.{ }^{*} \mathrm{U} 03.9, \mathrm{X} 59, \mathrm{X} 84, \mathrm{Y} 09, \mathrm{Y} 34, \mathrm{Y} 35.7, \mathrm{Y} 36.9, \mathrm{Y} 89.9\right)$ & 8,567 & 2.8 & 2.7 \\
\hline Unintentional. . . . . . . . . . . . . . . . . . . . (X59) & 6,019 & 2.0 & 1.9 \\
\hline 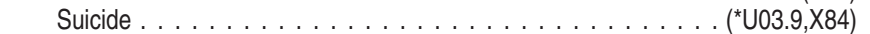 & 163 & 0.1 & 0.1 \\
\hline$\left({ }^{*} \cup 01.9, Y 09\right)$ & 1,846 & 0.6 & 0.6 \\
\hline Undetermined. & 535 & 0.2 & 0.2 \\
\hline Legal intervention/war. & 4 & * & * \\
\hline
\end{tabular}

0.0 Quantity more than zero but less than 0.05 .

- Quantity zero.

"Figure does not meet standard of reliability or precision; see "Technical Notes."

${ }^{1}$ For method of computation, see "Technical Notes."

${ }^{2}$ Codes ${ }^{*} \mathrm{U} 01.3$ and Y 36.3 cannot be divided separately into the subcategories shown below; therefore, subcategories may not add to total.

${ }^{3}$ Intent of death is unintentional. 
Table 19. Number of deaths, death rates, and age-adjusted death rates for injury by firearms, by race and sex: United States, 1999-2007

[Rates on an annual basis per 100,000 population in specified group; age-adjusted rates are per 100,000 U.S. standard population; see "Technical Notes." Rates are based on populations enumerated as of April 1 for 2000 and estimated as of July 1 for all other years; see "Technical Notes." Race categories are consistent with the 1977 Office of Management and Budget (OMB) standards. Data for specified races other than white and black should be interpreted with caution because of inconsistencies between reporting race on death certificates and on censuses and surveys; see "Technical Notes." A listing of the International Classification of Diseases, Tenth Revision (ICD-10), Second Edition codes included in this table can be found in the note at the bottom of the table]

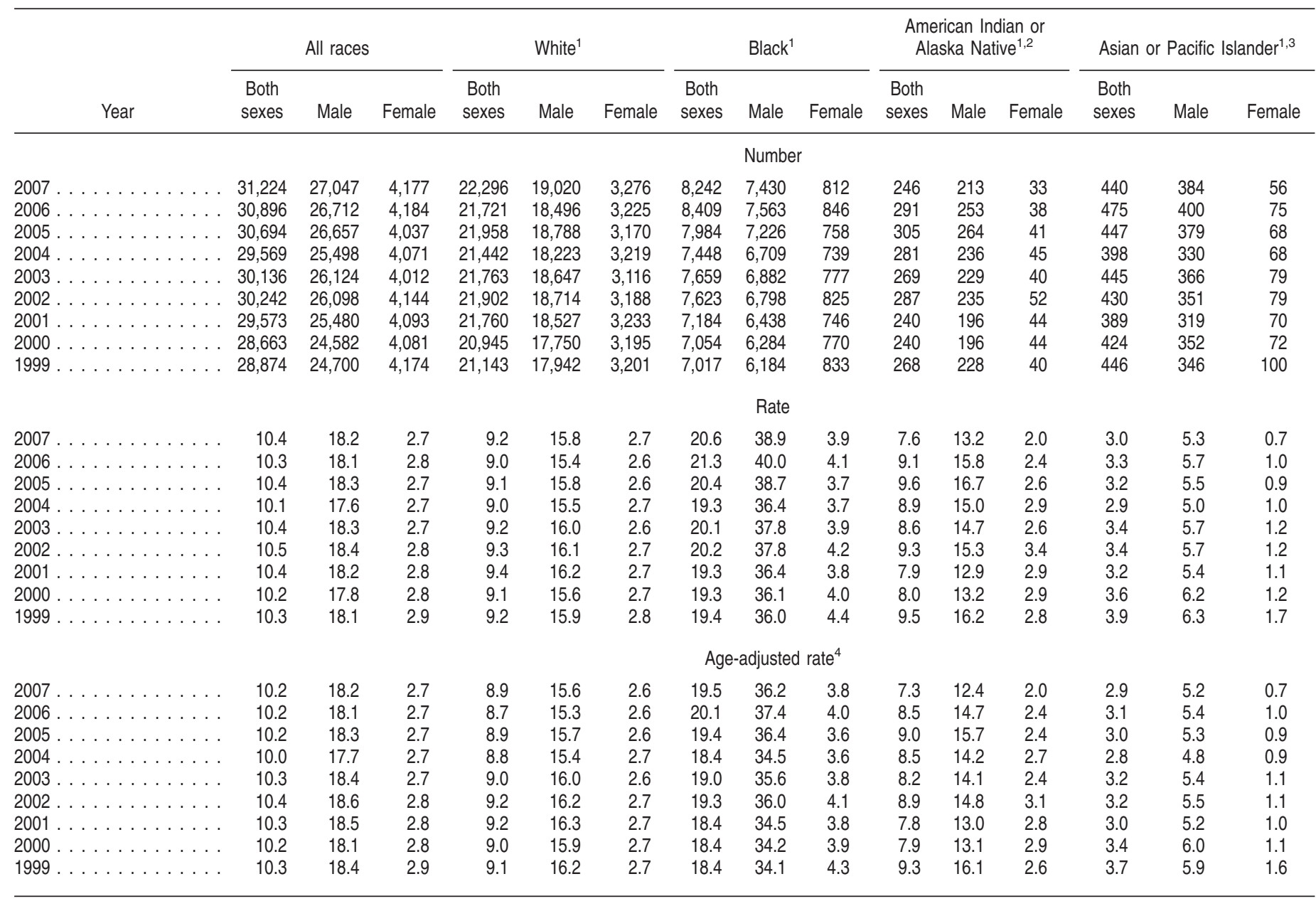

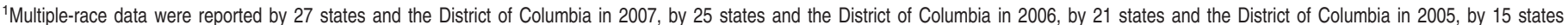

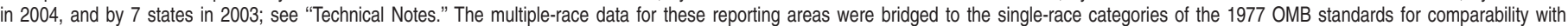
other reporting areas; see "Technical Notes."

${ }^{2}$ Includes Aleuts and Eskimos.

${ }^{3}$ Includes Chinese, Filipino, Hawaiian, Japanese, and Other Asian or Pacific Islander.

${ }^{4}$ For method of computation, see "Technical Notes."

NOTE: Causes of death attributable to injury by firearms include ICD-10 codes *U01.4, W32-W34, X72-X74, X93-X95, Y22-Y24, and Y35.0. 
Table 20. Number of deaths, death rates, and age-adjusted death rates for injury by firearms, by Hispanic origin, race for non-Hispanic population, and sex: United States, 1999-2007

[ Rates on an annual basis per 100,000 population in specified group; age-adjusted rates are per 100,000 U.S. standard population; see "Technical Notes." Rates are based on populations enumerated as of April 1 for 2000 and estimated as of July 1 for all other years; see "Technical Notes." Race and Hispanic origin are reported separately on the death certificate. Persons of Hispanic origin may be of any race. Data for Hispanic persons are not tabulated separately by race; data for non-Hispanic persons are tabulated by race. Data for Hispanic origin should be interpreted with caution because of inconsistencies between reporting Hispanic origin on death certificates and on censuses and surveys; see "Technical Notes." Race categories are consistent with the 1977 Office of Management and Budget (OMB) standards. A listing of the International Classification of Diseases, Tenth Revision (ICD-10), Second Edition codes included in this table can be found in the note at the bottom of the table]

\begin{tabular}{|c|c|c|c|c|c|c|c|c|c|c|c|c|c|c|c|c|}
\hline & \multirow[b]{2}{*}{ Year } & \multicolumn{3}{|c|}{ All origins ${ }^{1}$} & \multicolumn{3}{|c|}{ Hispanic } & \multicolumn{3}{|c|}{ Non-Hispanic ${ }^{2}$} & \multicolumn{3}{|c|}{ Non-Hispanic white ${ }^{3}$} & \multicolumn{3}{|c|}{ Non-Hispanic black ${ }^{3}$} \\
\hline & & $\begin{array}{l}\text { Both } \\
\text { sexes }\end{array}$ & Male & Female & $\begin{array}{l}\text { Both } \\
\text { sexes }\end{array}$ & Male & Female & $\begin{array}{l}\text { Both } \\
\text { sexes }\end{array}$ & Male & Female & $\begin{array}{l}\text { Both } \\
\text { sexes }\end{array}$ & Male & Female & $\begin{array}{l}\text { Both } \\
\text { sexes }\end{array}$ & Male & Female \\
\hline & & \multicolumn{15}{|c|}{ Number } \\
\hline 2007 & $\ldots$ & 31,224 & 27,047 & 4,177 & 3,492 & 3,155 & 337 & 27,641 & 23,809 & 3,832 & 18,861 & 15,925 & 2,936 & 8,133 & 7,326 & 807 \\
\hline 2006 & & 30,896 & 26,712 & 4,184 & 3,464 & 3,142 & 322 & 27,329 & 23,482 & 3,847 & 18,312 & 15,411 & 2,901 & 8,294 & 7,460 & 834 \\
\hline 2005 & & 30,694 & 26,657 & 4,037 & 3,469 & 3,144 & 325 & 27,103 & 23,406 & 3,697 & 18,521 & 15,672 & 2,849 & 7,865 & 7,122 & 743 \\
\hline 2004 & . & 29,569 & 25,498 & 4,071 & 3,278 & 2,973 & 305 & 26,189 & 22,436 & 3,753 & 18,200 & 15,283 & 2,917 & 7,347 & 6,620 & 727 \\
\hline 2003 & $\ldots \ldots \ldots \ldots \ldots$ & 30,136 & 26,124 & 4,012 & 3,319 & 2,998 & 321 & 26,710 & 23,036 & 3,674 & 18,457 & 15,670 & 2,787 & 7,566 & 6,794 & 772 \\
\hline 2002 & $\ldots \ldots \ldots \ldots \ldots$ & 30,242 & 26,098 & 4,144 & 3,143 & 2,834 & 309 & 26,944 & 23,127 & 3,817 & 18,762 & 15,881 & 2,881 & 7,494 & 6,681 & 813 \\
\hline 2001 & & 29,573 & 25,480 & 4,093 & 3,087 & 2,774 & 313 & 26,341 & 22,573 & 3,768 & 18,676 & 15,760 & 2,916 & 7,063 & 6,323 & 740 \\
\hline 2000 & & 28,663 & 24,582 & 4,081 & 2,891 & 2,582 & 309 & 25,637 & 21,881 & 3,756 & 18,042 & 15,160 & 2,882 & 6,958 & 6,193 & 765 \\
\hline \multirow[t]{2}{*}{1999} & . & 28,874 & 24,700 & 4,174 & 2,878 & 2,549 & 329 & 25,877 & 22,050 & 3,827 & 18,260 & 15,384 & 2,876 & 6,933 & 6,114 & 819 \\
\hline & & \multicolumn{15}{|c|}{ Rate } \\
\hline 2007 & & 10.4 & 18.2 & 2.7 & 7.7 & 13.4 & 1.5 & 10.8 & 19.0 & 2.9 & 9.4 & 16.1 & 2.9 & 21.3 & 40.3 & 4.0 \\
\hline 2006 & & 10.3 & 18.1 & 2.8 & 7.8 & 13.7 & 1.5 & 10.7 & 18.8 & 2.9 & 9.1 & 15.6 & 2.8 & 22.0 & 41.4 & 4.2 \\
\hline 2005 & & 10.4 & 18.3 & 2.7 & 8.1 & 14.2 & 1.6 & 10.7 & 18.9 & 2.8 & 9.2 & 15.9 & 2.8 & 21.1 & 40.0 & 3.8 \\
\hline 2004 & & 10.1 & 17.6 & 2.7 & 7.9 & 13.9 & 1.5 & 10.4 & 18.2 & 2.9 & 9.1 & 15.6 & 2.9 & 19.9 & 37.6 & 3.8 \\
\hline 2003 & & 10.4 & 18.3 & 2.7 & 8.3 & 14.6 & 1.7 & 10.6 & 18.8 & 2.9 & 9.3 & 16.0 & 2.7 & 20.7 & 39.1 & 4.0 \\
\hline 2002 & $\ldots$ & 10.5 & 18.4 & 2.8 & 8.1 & 14.2 & 1.6 & 10.8 & 19.0 & 3.0 & 9.4 & 16.3 & 2.8 & 20.7 & 38.9 & 4.3 \\
\hline 2001 & $\ldots \ldots \ldots \ldots$ & 10.4 & 18.2 & 2.8 & 8.3 & 14.6 & 1.7 & 10.6 & 18.7 & 3.0 & 9.4 & 16.3 & 2.9 & 19.8 & 37.3 & 4.0 \\
\hline 2000 & $\ldots \ldots \ldots \ldots$ & 10.2 & 17.8 & 2.8 & 8.2 & 14.2 & 1.8 & 10.4 & 18.3 & 3.0 & 9.1 & 15.7 & 2.9 & 19.8 & 37.1 & 4.2 \\
\hline \multirow[t]{2}{*}{1999} & & 10.3 & 18.1 & 2.9 & 8.5 & 14.6 & 2.0 & 10.6 & 18.5 & 3.0 & 9.3 & 15.9 & 2.9 & 20.0 & 37.1 & 4.5 \\
\hline & & \multicolumn{15}{|c|}{ Age-adjusted rate ${ }^{4}$} \\
\hline 2007 & $\cdots$ & 10.2 & 18.2 & 2.7 & 7.4 & 12.9 & 1.5 & 10.6 & 18.8 & 2.9 & 8.8 & 15.4 & 2.8 & 20.2 & 37.6 & 4.0 \\
\hline 2006 & & 10.2 & 18.1 & 2.7 & 7.3 & 12.7 & 1.5 & 10.5 & 18.7 & 2.9 & 8.6 & 15.0 & 2.7 & 20.8 & 38.8 & 4.1 \\
\hline 2005 & & 10.2 & 18.3 & 2.7 & 7.6 & 13.3 & 1.6 & 10.5 & 18.8 & 2.8 & 8.8 & 15.3 & 2.7 & 20.0 & 37.7 & 3.7 \\
\hline 2004 & & 10.0 & 17.7 & 2.7 & 7.5 & 13.1 & 1.5 & 10.2 & 18.2 & 2.9 & 8.7 & 15.1 & 2.8 & 19.0 & 35.7 & 3.7 \\
\hline 2003 & & 10.3 & 18.4 & 2.7 & 7.8 & 13.6 & 1.6 & 10.5 & 18.8 & 2.8 & 8.8 & 15.6 & 2.7 & 19.7 & 36.8 & 3.9 \\
\hline 2002 & & 10.4 & 18.6 & 2.8 & 7.6 & 13.4 & 1.6 & 10.7 & 19.1 & 3.0 & 9.0 & 16.0 & 2.8 & 19.8 & 37.0 & 4.2 \\
\hline 2001 & & 10.3 & 18.5 & 2.8 & 7.8 & 13.7 & 1.7 & 10.5 & 18.8 & 3.0 & 9.1 & 16.0 & 2.8 & 18.9 & 35.4 & 3.9 \\
\hline 2000 & & 10.2 & 18.1 & 2.8 & 7.8 & 13.6 & 1.8 & 10.3 & 18.4 & 3.0 & 8.8 & 15.5 & 2.8 & 18.9 & 35.2 & 4.1 \\
\hline 1999 & & 10.3 & 18.4 & 2.9 & 8.2 & 14.2 & 2.0 & 10.5 & 18.7 & 3.0 & 8.9 & 15.8 & 2.8 & 19.0 & 35.2 & 4.4 \\
\hline
\end{tabular}

${ }^{1}$ Figures for origin not stated are included in "all origins" but not distributed among specified origins.

${ }^{2}$ Includes races other than white and black.

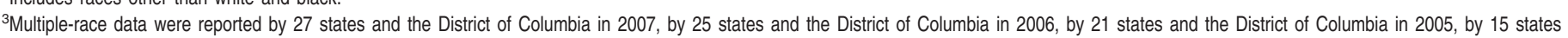

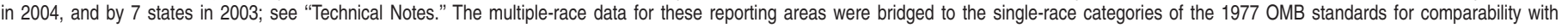
other reporting areas; see "Technical Notes."

${ }^{4}$ For method of computation, see "Technical Notes."

NOTE: Causes of death attributable to injury by firearms include ICD-10 codes *U01.4, W32-W34, X72-X74, X93-X95, Y22-Y24, and Y35.0. 
Table 21. Number of deaths, death rates, and age-adjusted death rates for drug-induced causes, by race and sex: United States, 1999-2007

[Rates on an annual basis per 100,000 population in specified group; age-adjusted rates are per 100,000 U.S. standard population; see "Technical Notes." Rates are based on populations enumerated as of April 1 for 2000 and estimated as of July 1 for all other years; see "Technical Notes." Race categories are consistent with the 1977 Office of Management and Budget (OMB) standards. Data for specified races other than white and black should be interpreted with caution because of inconsistencies between reporting race on death certificates and on censuses and surveys; see "Technical Notes." A listing of the International Classification of Diseases, Tenth Revision (ICD-10), Second Edition codes included in this table can be found in the note at the bottom of the table]

\begin{tabular}{|c|c|c|c|c|c|c|c|c|c|c|c|c|c|c|c|c|}
\hline & \multirow[b]{2}{*}{ Year } & \multicolumn{3}{|c|}{ All races } & \multicolumn{3}{|c|}{ White $^{1}$} & \multicolumn{3}{|c|}{ Black $^{1}$} & \multicolumn{3}{|c|}{$\begin{array}{l}\text { American Indian or } \\
\text { Alaska Native }\end{array}$} & \multicolumn{3}{|c|}{ Asian or Pacific Islander ${ }^{1,3}$} \\
\hline & & $\begin{array}{l}\text { Both } \\
\text { sexes }\end{array}$ & Male & Female & $\begin{array}{l}\text { Both } \\
\text { sexes }\end{array}$ & Male & Female & $\begin{array}{l}\text { Both } \\
\text { sexes }\end{array}$ & Male & Female & $\begin{array}{l}\text { Both } \\
\text { sexes }\end{array}$ & Male & Female & $\begin{array}{l}\text { Both } \\
\text { sexes }\end{array}$ & Male & Female \\
\hline & & \multicolumn{15}{|c|}{ Number } \\
\hline 2007 & $\ldots \ldots$ & 38,371 & 23,883 & 14,488 & 33,480 & 20,795 & 12,685 & 4,194 & 2,708 & 1,486 & 388 & 205 & 183 & 309 & 175 & 134 \\
\hline 2006 & & 38,396 & 24,507 & 13,889 & 32,866 & 20,786 & 12,080 & 4,790 & 3,261 & 1,529 & 407 & 253 & 154 & 333 & 207 & 126 \\
\hline 2005 & & 33,541 & 21,208 & 12,333 & 28,804 & 18,152 & 10,652 & 4,098 & 2,677 & 1,421 & 362 & 205 & 157 & 277 & 174 & 103 \\
\hline 2004 & . & 30,711 & 19,362 & 11,349 & 26,474 & 16,634 & 9,840 & 3,633 & 2,352 & 1,281 & 354 & 212 & 142 & 250 & 164 & 86 \\
\hline 2003 & $\cdots$ & 28,723 & 18,426 & 10,297 & 24,683 & 15,824 & 8,859 & 3,527 & 2,303 & 1,224 & 295 & 168 & 127 & 218 & 131 & 87 \\
\hline 2002 & & 26,040 & 16,734 & 9,306 & 22,146 & 14,170 & 7,976 & 3,463 & 2,307 & 1,156 & 230 & 136 & 94 & 201 & 121 & 80 \\
\hline 2001 & 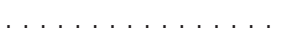 & 21,705 & 14,253 & 7,452 & 18,195 & 11,882 & 6,313 & 3,165 & 2,163 & 1,002 & 184 & 100 & 84 & 161 & 108 & 53 \\
\hline 2000 & $\ldots \ldots \ldots \ldots$ & 19,720 & 13,137 & 6,583 & 16,388 & 10,857 & 5,531 & 3,034 & 2,094 & 940 & 160 & 99 & 61 & 138 & 87 & 51 \\
\hline \multirow[t]{2}{*}{1999} & $\ldots \ldots \ldots \ldots$ & 19,128 & 12,885 & 6,243 & 15,714 & 10,506 & 5,208 & 3,100 & 2,191 & 909 & 164 & 96 & 68 & 150 & 92 & 58 \\
\hline & & \multicolumn{15}{|c|}{ Rate } \\
\hline 2007 & $\ldots$ & 12.7 & 16.1 & 9.5 & 13.7 & 17.2 & 10.3 & 10.5 & 14.2 & 7.1 & 12.0 & 12.7 & 11.3 & 2.1 & 2.4 & 1.8 \\
\hline 2006 & $\ldots \ldots$ & 12.8 & 16.6 & 9.1 & 13.6 & 17.3 & 9.9 & 12.1 & 17.3 & 7.4 & 12.7 & 15.8 & 9.6 & 2.3 & 2.9 & 1.7 \\
\hline 2005 & . & 11.3 & 14.5 & 8.2 & 12.0 & 15.3 & 8.8 & 10.5 & 14.3 & 7.0 & 11.5 & 13.0 & 9.9 & 2.0 & 2.5 & 1.4 \\
\hline 2004 & & 10.5 & 13.4 & 7.6 & 11.1 & 14.1 & 8.2 & 9.4 & 12.8 & 6.3 & 11.2 & 13.5 & 9.0 & 1.8 & 2.5 & 1.2 \\
\hline 2003 & $\ldots$ & 9.9 & 12.9 & 7.0 & 10.4 & 13.5 & 7.4 & 9.2 & 12.7 & 6.1 & 9.5 & 10.8 & 8.2 & 1.7 & 2.0 & 1.3 \\
\hline 2002 & $\ldots \ldots \ldots$ & 9.0 & 11.8 & 6.3 & 9.4 & 12.2 & 6.7 & 9.2 & 12.8 & 5.8 & 7.5 & 8.9 & 6.1 & 1.6 & 2.0 & 1.2 \\
\hline 2001 & $\ldots \ldots \ldots \ldots$ & 7.6 & 10.2 & 5.1 & 7.8 & 10.4 & 5.4 & 8.5 & 12.2 & 5.1 & 6.0 & 6.6 & 5.5 & 1.3 & 1.8 & 0.8 \\
\hline 2000 & $\ldots \ldots \ldots \ldots$ & 7.0 & 9.5 & 4.6 & 7.1 & 9.6 & 4.7 & 8.3 & 12.0 & 4.9 & 5.4 & 6.7 & 4.1 & 1.2 & 1.5 & 0.8 \\
\hline \multirow[t]{2}{*}{1999} & $\ldots \ldots \ldots$ & 6.9 & 9.4 & 4.4 & 6.9 & 9.3 & 4.5 & 8.6 & 12.7 & 4.8 & 5.8 & 6.8 & 4.8 & 1.3 & 1.7 & 1.0 \\
\hline & & \multicolumn{15}{|c|}{ Age-adjusted rate ${ }^{4}$} \\
\hline 2007 & $\cdots \cdots \cdots \cdots$ & 12.6 & 15.8 & 9.3 & 13.6 & 16.9 & 10.2 & 11.0 & 15.4 & 7.3 & 12.1 & 12.6 & 11.5 & 2.0 & 2.3 & 1.7 \\
\hline 2006 & & 12.7 & 16.4 & 9.0 & 13.5 & 17.1 & 9.8 & 12.9 & 18.9 & 7.7 & 13.4 & 16.3 & 10.5 & 2.3 & 3.0 & 1.7 \\
\hline 2005 & & 11.3 & 14.4 & 8.1 & 11.9 & 15.1 & 8.7 & 11.2 & 15.8 & 7.2 & 11.9 & 13.4 & 10.4 & 1.9 & 2.6 & 1.4 \\
\hline 2004 & & 10.4 & 13.3 & 7.6 & 11.1 & 13.9 & 8.1 & 10.1 & 14.3 & 6.6 & 11.6 & 13.8 & 9.4 & 1.8 & 2.5 & 1.2 \\
\hline 2003 & & 9.9 & 12.8 & 7.0 & 10.4 & 13.4 & 7.4 & 9.9 & 14.1 & 6.4 & 9.9 & 11.2 & 8.6 & 1.6 & 1.9 & 1.3 \\
\hline 2002 & . & 9.0 & 11.7 & 6.3 & 9.4 & 12.1 & 6.7 & 9.9 & 14.2 & 6.1 & 7.8 & 9.0 & 6.4 & 1.6 & 2.1 & 1.2 \\
\hline 2001 & & 7.6 & 10.1 & 5.1 & 7.8 & 10.2 & 5.3 & 9.2 & 13.6 & 5.4 & 6.6 & 7.3 & 5.8 & 1.3 & 1.8 & 0.8 \\
\hline 2000 & & 7.0 & 9.5 & 4.6 & 7.1 & 9.4 & 4.7 & 9.0 & 13.5 & 5.2 & 5.6 & 6.9 & 4.3 & 1.1 & 1.5 & 0.8 \\
\hline 1999 & & 6.8 & 9.4 & 4.4 & 6.8 & 9.2 & 4.4 & 9.3 & 14.3 & 5.1 & 6.1 & 7.2 & 5.0 & 1.4 & 1.7 & 1.0 \\
\hline
\end{tabular}

${ }^{1}$ Multiple-race data were reported by 27 states and the District of Columbia in 2007, by 25 states and the District of Columbia in 2006, by 21 states and the District of Columbia in 2005 , by 15 states in 2004, and by 7 states in 2003; see "Technical Notes." The multiple-race data for these reporting areas were bridged to the single-race categories of the 1977 OMB standards for comparability with other reporting areas; see "Technical Notes."

${ }^{2}$ Includes Aleuts and Eskimos.

Includes Chinese, Filipino, Hawaiian, Japanese, and Other Asian or Pacific Islander.

${ }^{4}$ For method of computation, see "Technical Notes."

NOTES: Causes of death attributable to drug-induced mortality include ICD-10 codes D52.1, D59.0, D59.2, D61.1, D64.2, E06.4, E16.0, E23.1, E24.2, E27.3, E66.1, F11.0-F11.5, F11.7-F11.9, F12.0-F12.5, F12.7-F12.9, F13.0-F13.5, F13.7-F13.9, F14.0-F14.5, F14.7-F14.9, F15.0-F15.5, F15.7-F15.9, F16.0-F16.5, F16.7-F16.9, F17.0, F17.3-F17.5, F17.7-F17.9, F18.0-F18.5, F18.7-F18.9, F19.0-F19.5, F19.7-F19.9, G21.1, G24.0, G25.1, G25.4, G25.6, G44.4, G62.0, G72.0, 195.2, J70.2-J70.4, K85.3, L10.5, L27.0-L27.1, M10.2, M32.0, M80.4, M81.4, M83.5, M87.1, R50.2, R78.1-R78.5, X40-X44, X60-X64, X85, and Y10-Y14. In 2006, the list of drug-induced codes was modified to include two new ICD-10 codes, Drug-induced acute pancreatitis (K85.3) and Drug-induced fever (R50.2); see "Technical Notes." 
Table 22. Number of deaths, death rates, and age-adjusted death rates for drug-induced causes, by Hispanic origin, race for non-Hispanic population, and sex: United States, 1999-2007

[Rates on an annual basis per 100,000 population in specified group; age-adjusted rates are per 100,000 U.S. standard population; see "Technical Notes." Rates are based on populations enumerated as of April 1 for 2000 and estimated as of July 1 for all other years; see "Technical Notes." Race and Hispanic origin are reported separately on the death certificate. Persons of Hispanic origin may be of any race. Data for Hispanic persons are not tabulated separately by race; data for non-Hispanic persons are tabulated by race. Data for Hispanic origin should be interpreted with caution because of inconsistencies between reporting Hispanic origin on death certificates and on censuses and surveys; see "Technical Notes." Race categories are consistent with the 1977 Office of Management and Budget (OMB) standards. A listing of the International Classification of Diseases, Tenth Revision (ICD-10), Second Edition codes included in this table can be found in the note at the bottom of the table]

\begin{tabular}{|c|c|c|c|c|c|c|c|c|c|c|c|c|c|c|c|c|}
\hline & \multirow[b]{2}{*}{ Year } & \multicolumn{3}{|c|}{ All origins ${ }^{1}$} & \multicolumn{3}{|c|}{ Hispanic } & \multicolumn{3}{|c|}{ Non-Hispanic ${ }^{2}$} & \multicolumn{3}{|c|}{ Non-Hispanic white ${ }^{3}$} & \multicolumn{3}{|c|}{ Non-Hispanic black ${ }^{3}$} \\
\hline & & $\begin{array}{l}\text { Both } \\
\text { sexes }\end{array}$ & Male & Female & $\begin{array}{l}\text { Both } \\
\text { sexes }\end{array}$ & Male & Female & $\begin{array}{l}\text { Both } \\
\text { sexes }\end{array}$ & Male & Female & $\begin{array}{l}\text { Both } \\
\text { sexes }\end{array}$ & Male & Female & $\begin{array}{l}\text { Both } \\
\text { sexes }\end{array}$ & Male & Female \\
\hline & & \multicolumn{15}{|c|}{ Number } \\
\hline 2007 & . & 38,371 & 23,883 & 14,488 & 2,723 & 2,045 & 678 & 35,532 & 21,771 & 13,761 & 30,755 & 18,760 & 11,995 & 4,126 & 2,662 & 1,464 \\
\hline 2006 & & 38,396 & 24,507 & 13,889 & 2,871 & 2,135 & 736 & 35,379 & 22,262 & 13,117 & 29,970 & 18,634 & 11,336 & 4,722 & 3,212 & 1,510 \\
\hline 2005 & & 33,541 & 21,208 & 12,333 & 2,596 & 1,969 & 627 & 30,809 & 19,140 & 11,669 & 26,186 & 16,170 & 10,016 & 4,019 & 2,612 & 1,407 \\
\hline 2004 & $\cdots$ & 30,711 & 19,362 & 11,349 & 2,257 & 1,671 & 586 & 28,339 & 17,605 & 10,734 & 24,201 & 14,952 & 9,249 & 3,577 & 2,309 & 1,268 \\
\hline 2003 & $\ldots$ & 28,723 & 18,426 & 10,297 & 2,358 & 1,800 & 558 & 26,199 & 16,497 & 9,702 & 22,245 & 13,959 & 8,286 & 3,466 & 2,256 & 1,210 \\
\hline 2002 & 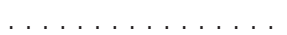 & 26,040 & 16,734 & 9,306 & 2,137 & 1,647 & 490 & 23,756 & 14,978 & 8,778 & 19,949 & 12,478 & 7,471 & 3,404 & 2,264 & 1,140 \\
\hline 2001 & & 21,705 & 14,253 & 7,452 & 1,731 & 1,335 & 396 & 19,799 & 12,778 & 7,021 & 16,367 & 10,465 & 5,902 & 3,099 & 2,113 & 986 \\
\hline 2000 & $\ldots$ & 19,720 & 13,137 & 6,583 & 1,700 & 1,348 & 352 & 17,835 & 11,656 & 6,179 & 14,585 & 9,439 & 5,146 & 2,977 & 2,050 & 927 \\
\hline \multirow[t]{2}{*}{1999} & $\ldots \ldots \ldots$ & 19,128 & 12,885 & 6,243 & 1,965 & 1,605 & 360 & 16,966 & 11,136 & 5,830 & 13,644 & 8,831 & 4,813 & 3,030 & 2,134 & 896 \\
\hline & & \multicolumn{15}{|c|}{ Rate } \\
\hline 2007 & & 12.7 & 16.1 & 9.5 & 6.0 & 8.7 & 3.1 & 13.9 & 17.4 & 10.5 & 15.3 & 19.0 & 11.7 & 10.8 & 14.6 & 7.3 \\
\hline 2006 & $\ldots$ & 12.8 & 16.6 & 9.1 & 6.5 & 9.3 & 3.4 & 13.9 & 17.9 & 10.1 & 14.9 & 18.9 & 11.1 & 12.5 & 17.8 & 7.6 \\
\hline 2005 & $\ldots$ & 11.3 & 14.5 & 8.2 & 6.1 & 8.9 & 3.0 & 12.1 & 15.4 & 9.0 & 13.1 & 16.4 & 9.8 & 10.8 & 14.7 & 7.2 \\
\hline 2004 & & 10.5 & 13.4 & 7.6 & 5.5 & 7.8 & 2.9 & 11.2 & 14.3 & 8.3 & 12.1 & 15.3 & 9.1 & 9.7 & 13.1 & 6.6 \\
\hline 2003 & & 9.9 & 12.9 & 7.0 & 5.9 & 8.7 & 2.9 & 10.4 & 13.5 & 7.6 & 11.2 & 14.3 & 8.2 & 9.5 & 13.0 & 6.3 \\
\hline 2002 & & 9.0 & 11.8 & 6.3 & 5.5 & 8.2 & 2.6 & 9.5 & 12.3 & 6.9 & 10.0 & 12.8 & 7.4 & 9.4 & 13.2 & 6.0 \\
\hline 2001 & 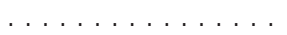 & 7.6 & 10.2 & 5.1 & 4.7 & 7.0 & 2.2 & 8.0 & 10.6 & 5.5 & 8.3 & 10.8 & 5.8 & 8.7 & 12.5 & 5.3 \\
\hline 2000 & $\ldots$ & 7.0 & 9.5 & 4.6 & 4.8 & 7.4 & 2.1 & 7.2 & 9.7 & 4.9 & 7.4 & 9.8 & 5.1 & 8.5 & 12.3 & 5.0 \\
\hline \multirow[t]{2}{*}{1999} & $\ldots$ & 6.9 & 9.4 & 4.4 & 5.8 & 9.2 & 2.2 & 6.9 & 9.3 & 4.6 & 6.9 & 9.2 & 4.8 & 8.7 & 12.9 & 4.9 \\
\hline & & \multicolumn{15}{|c|}{ Age-adjusted rate ${ }^{4}$} \\
\hline 2007 & $\cdots$ & 12.6 & 15.8 & 9.3 & 6.5 & 9.5 & 3.4 & 13.6 & 17.0 & 10.2 & 15.1 & 18.7 & 11.4 & 11.4 & 15.8 & 7.5 \\
\hline 2006 & & 12.7 & 16.4 & 9.0 & 7.2 & 10.3 & 3.9 & 13.6 & 17.5 & 9.8 & 14.7 & 18.6 & 10.8 & 13.2 & 19.4 & 7.9 \\
\hline 2005 & & 11.3 & 14.4 & 8.1 & 6.8 & 10.0 & 3.5 & 11.9 & 15.2 & 8.8 & 12.8 & 16.2 & 9.6 & 11.4 & 16.1 & 7.4 \\
\hline 2004 & & 10.4 & 13.3 & 7.6 & 6.2 & 8.9 & 3.4 & 11.1 & 14.1 & 8.2 & 12.0 & 15.0 & 8.9 & 10.4 & 14.6 & 6.8 \\
\hline 2003 & & 9.9 & 12.8 & 7.0 & 6.7 & 9.9 & 3.3 & 10.3 & 13.3 & 7.4 & 11.0 & 14.1 & 8.0 & 10.1 & 14.4 & 6.6 \\
\hline 2002 & & 9.0 & 11.7 & 6.3 & 6.2 & 9.3 & 3.0 & 9.4 & 12.1 & 6.8 & 9.9 & 12.6 & 7.2 & 10.1 & 14.5 & 6.3 \\
\hline 2001 & & 7.6 & 10.1 & 5.1 & 5.3 & 8.0 & 2.5 & 7.9 & 10.4 & 5.4 & 8.1 & 10.6 & 5.7 & 9.3 & 13.8 & 5.5 \\
\hline 2000 & & 7.0 & 9.5 & 4.6 & 5.4 & 8.3 & 2.4 & 7.1 & 9.5 & 4.8 & 7.2 & 9.6 & 4.9 & 9.1 & 13.6 & 5.3 \\
\hline 1999 & 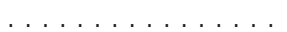 & 6.8 & 9.4 & 4.4 & 6.4 & 10.3 & 2.5 & 6.8 & 9.2 & 4.6 & 6.8 & 8.9 & 4.6 & 9.4 & 14.4 & 5.2 \\
\hline
\end{tabular}

1Figures for origin not stated are included in "all origins" but not distributed among specified origins.

${ }^{2}$ Includes races other than white and black.

${ }^{3}$ Multiple-race data were reported by 27 states and the District of Columbia in 2007, by 25 states and the District of Columbia in 2006, by 21 states and the District of Columbia in 2005 , by 15 states in 2004, and by 7 states in 2003; see "Technical Notes." The multiple-race data for these reporting areas were bridged to the single-race categories of the 1977 OMB standards for comparability with other reporting areas; see "Technical Notes."

${ }^{4}$ For method of computation, see "Technical Notes."

NOTES: Causes of death attributable to drug-induced mortality include ICD-10 codes D52.1, D59.0, D59.2, D61.1, D64.2, E06.4, E16.0, E23.1, E24.2, E27.3, E66.1, F11.0-F11.5, F11.7-F11.9, F12.0-F12.5, F12.7-F12.9, F13.0-F13.5, F13.7-F13.9, F14.0-F14.5, F14.7-F14.9, F15.0-F15.5, F15.7-F15.9, F16.0-F16.5, F16.7-F16.9, F17.0, F17.3-F17.5, F17.7-F17.9, F18.0-F18.5, F18.7-F18.9, F19.0-F19.5, F19.7-F19.9, G21.1, G24.0, G25.1, G25.4, G25.6, G44.4, G62.0, G72.0, 195.2, J70.2-J70.4, K85.3, L10.5, L27.0-L27.1, M10.2, M32.0, M80.4, M81.4, M83.5, M87.1, R50.2, R78.1-R78.5, X40-X44, X60-X64, X85, and Y10-Y14. In 2006, the list of drug-induced codes was modified to include two new ICD-10 codes, Drug-induced acute pancreatitis (K85.3) and Drug-induced fever (R50.2). 
Table 23. Number of deaths, death rates, and age-adjusted death rates for alcohol-induced causes, by race and sex: United States, 1999-2007

[Rates on an annual basis per 100,000 population in specified group; age-adjusted rates are per 100,000 U.S. standard population; see "Technical Notes." Rates are based on populations enumerated as of April 1 for 2000 and estimated as of July 1 for all other years; see "Technical Notes." Race categories are consistent with the 1977 Office of Management and Budget (OMB) standards. Data for specified races other than white and black should be interpreted with caution because of inconsistencies between reporting race on death certificates and on censuses and surveys; see "Technical Notes." A listing of the International Classification of Diseases, Tenth Revision (ICD-10), Second Edition codes included in this table can be found in the note at the bottom of the table]

\begin{tabular}{|c|c|c|c|c|c|c|c|c|c|c|c|c|c|c|c|c|}
\hline & \multirow[b]{2}{*}{ Year } & \multicolumn{3}{|c|}{ All races } & \multicolumn{3}{|c|}{ White $^{1}$} & \multicolumn{3}{|c|}{ Black $^{1}$} & \multicolumn{3}{|c|}{$\begin{array}{l}\text { American Indian or } \\
\text { Alaska Native }\end{array}$} & \multicolumn{3}{|c|}{ Asian or Pacific Islander ${ }^{1,3}$} \\
\hline & & $\begin{array}{l}\text { Both } \\
\text { sexes }\end{array}$ & Male & Female & $\begin{array}{l}\text { Both } \\
\text { sexes }\end{array}$ & Male & Female & $\begin{array}{l}\text { Both } \\
\text { sexes }\end{array}$ & Male & Female & $\begin{array}{l}\text { Both } \\
\text { sexes }\end{array}$ & Male & Female & $\begin{array}{l}\text { Both } \\
\text { sexes }\end{array}$ & Male & Female \\
\hline & & \multicolumn{15}{|c|}{ Number } \\
\hline 2007 & $\ldots$ & 23,199 & 17,428 & 5,771 & 19,921 & 15,027 & 4,894 & 2,248 & 1,703 & 545 & 774 & 495 & 279 & 256 & 203 & 53 \\
\hline 2006 & & 22,073 & 16,472 & 5,601 & 18,917 & 14,186 & 4,731 & 2,172 & 1,599 & 573 & 717 & 458 & 259 & 267 & 229 & 38 \\
\hline 2005 & & 21,634 & 16,238 & 5,396 & 18,432 & 13,917 & 4,515 & 2,316 & 1,698 & 618 & 655 & 432 & 223 & 231 & 191 & 40 \\
\hline 2004 & . & 21,081 & 15,906 & 5,175 & 17,875 & 13,525 & 4,350 & 2,351 & 1,784 & 567 & 645 & 417 & 228 & 210 & 180 & 30 \\
\hline 2003 & & 20,687 & 15,630 & 5,057 & 17,437 & 13,218 & 4,219 & 2,406 & 1,824 & 582 & 664 & 431 & 233 & 180 & 157 & 23 \\
\hline 2002 & & 20,218 & 15,272 & 4,946 & 16,988 & 12,926 & 4,062 & 2,434 & 1,798 & 636 & 597 & 387 & 210 & 199 & 161 & 38 \\
\hline 2001 & 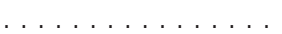 & 20,114 & 15,149 & 4,965 & 16,640 & 12,588 & 4,052 & 2,723 & 2,048 & 675 & 591 & 387 & 204 & 160 & 126 & 34 \\
\hline 2000 & $\ldots \ldots \ldots \ldots$ & 19,643 & 14,993 & 4,650 & 16,223 & 12,509 & 3,714 & 2,712 & 1,993 & 719 & 537 & 353 & 184 & 171 & 138 & 33 \\
\hline \multirow[t]{2}{*}{1999} & $\ldots \ldots \ldots \ldots$ & 19,469 & 14,894 & 4,575 & 15,903 & 12,277 & 3,626 & 2,832 & 2,100 & 732 & 589 & 397 & 192 & 145 & 120 & 25 \\
\hline & & \multicolumn{15}{|c|}{ Rate } \\
\hline 2007 & $\ldots$ & 7.7 & 11.7 & 3.8 & 8.2 & 12.4 & 4.0 & 5.6 & 8.9 & 2.6 & 23.9 & 30.6 & 17.2 & 1.7 & 2.8 & 0.7 \\
\hline 2006 & $\ldots \ldots \ldots \ldots$ & 7.4 & 11.2 & 3.7 & 7.8 & 11.8 & 3.9 & 5.5 & 8.5 & 2.8 & 22.4 & 28.6 & 16.2 & 1.8 & 3.2 & 0.5 \\
\hline 2005 & $\ldots \ldots \ldots \ldots$ & 7.3 & 11.1 & 3.6 & 7.7 & 11.7 & 3.7 & 5.9 & 9.1 & 3.0 & 20.7 & 27.4 & 14.1 & 1.6 & 2.8 & 0.6 \\
\hline 2004 & & 7.2 & 11.0 & 3.5 & 7.5 & 11.5 & 3.6 & 6.1 & 9.7 & 2.8 & 20.5 & 26.5 & 14.5 & 1.5 & 2.7 & 0.4 \\
\hline 2003 & . & 7.1 & 10.9 & 3.4 & 7.4 & 11.3 & 3.5 & 6.3 & 10.0 & 2.9 & 21.3 & 27.8 & 15.0 & 1.4 & 2.4 & 0.3 \\
\hline 2002 & $\ldots \ldots \ldots \ldots$ & 7.0 & 10.8 & 3.4 & 7.2 & 11.1 & 3.4 & 6.4 & 10.0 & 3.2 & 19.4 & 25.2 & 13.6 & 1.6 & 2.6 & 0.6 \\
\hline 2001 & $\ldots \ldots \ldots$ & 7.1 & 10.8 & 3.4 & 7.2 & 11.0 & 3.4 & 7.3 & 11.6 & 3.5 & 19.3 & 25.4 & 13.3 & 1.3 & 2.1 & 0.5 \\
\hline 2000 & $\ldots \ldots \ldots \ldots$ & 7.0 & 10.9 & 3.2 & 7.1 & 11.0 & 3.2 & 7.4 & 11.4 & 3.7 & 18.0 & 23.7 & 12.3 & 1.5 & 2.4 & 0.5 \\
\hline \multirow[t]{2}{*}{1999} & $\ldots \ldots \ldots$ & 7.0 & 10.9 & 3.2 & 7.0 & 10.9 & 3.1 & 7.8 & 12.2 & 3.9 & 20.8 & 28.1 & 13.5 & 1.3 & 2.2 & 0.4 \\
\hline & & \multicolumn{15}{|c|}{ Age-adjusted rate ${ }^{4}$} \\
\hline 2007 & …...... & 7.3 & 11.3 & 3.5 & 7.5 & 11.6 & 3.6 & 6.3 & 11.0 & 2.7 & 26.3 & 35.1 & 18.2 & 1.8 & 3.0 & 0.7 \\
\hline 2006 & & 7.0 & 10.9 & 3.4 & 7.2 & 11.2 & 3.5 & 6.2 & 10.4 & 3.0 & 25.2 & 33.3 & 17.8 & 1.9 & 3.5 & 0.5 \\
\hline 2005 & . & 7.0 & 11.0 & 3.4 & 7.2 & 11.1 & 3.4 & 6.8 & 11.4 & 3.3 & 23.7 & 32.5 & 15.6 & 1.7 & 3.1 & 0.6 \\
\hline 2004 & & 7.0 & 11.0 & 3.3 & 7.1 & 11.0 & 3.3 & 7.2 & 12.3 & 3.1 & 23.8 & 32.4 & 16.1 & 1.7 & 3.2 & 0.5 \\
\hline 2003 & & 7.0 & 11.0 & 3.3 & 7.0 & 11.0 & 3.3 & 7.4 & 12.8 & 3.3 & 25.1 & 34.0 & 16.9 & 1.5 & 2.8 & 0.4 \\
\hline 2002 & & 6.9 & 11.0 & 3.3 & 6.9 & 10.9 & 3.2 & 7.8 & 13.1 & 3.6 & 23.2 & 31.5 & 15.6 & 1.8 & 3.2 & 0.6 \\
\hline 2001 & & 7.0 & 11.2 & 3.3 & 6.9 & 10.9 & 3.3 & 8.9 & 15.1 & 3.9 & 23.6 & 33.0 & 15.4 & 1.5 & 2.6 & 0.6 \\
\hline 2000 & & 7.0 & 11.4 & 3.2 & 6.9 & 11.1 & 3.0 & 9.1 & 15.3 & 4.3 & 22.7 & 31.4 & 14.9 & 1.7 & 2.9 & 0.7 \\
\hline 1999 & & 7.1 & 11.5 & 3.2 & 6.8 & 11.0 & 3.0 & 9.8 & 16.7 & 4.5 & 26.7 & 38.8 & 16.2 & 1.6 & 2.8 & 0.5 \\
\hline
\end{tabular}

${ }^{1}$ Multiple-race data were reported by 27 states and the District of Columbia in 2007, by 25 states and the District of Columbia in 2006, by 21 states and the District of Columbia in 2005 , by 15 states in 2004, and by 7 states in 2003; see "Technical Notes." The multiple-race data for these reporting areas were bridged to the single-race categories of the 1977 OMB standards for comparability with other reporting areas; see "Technical Notes."

${ }^{2}$ Includes Aleuts and Eskimos.

${ }^{3}$ Includes Chinese, Filipino, Hawaiian, Japanese, and Other Asian or Pacific Islander.

${ }^{4}$ For method of computation, see "Technical Notes."

NOTES: Causes of death attributable to alcohol-induced mortality include ICD-10 codes E24.4, F10, G31.2, G62.1, G72.1, I42.6, K29.2, K70, K85.2, K86.0, R78.0, X45, X65, and Y15. In 2006, the list of alcohol-induced codes was modified to include a new ICD-10 code, Alcohol-induced acute pancreatitis (K85.2); see "Technical Notes." 
Table 24. Number of deaths, death rates, and age-adjusted death rates for alcohol-induced causes, by Hispanic origin, race for non-Hispanic population, and sex: United States, 1999-2007

[Rates on an annual basis per 100,000 population in specified group; age-adjusted rates are per 100,000 U.S. standard population; see "Technical Notes." Rates are based on populations enumerated as of April 1 for 2000 and estimated as of July 1 for all other years; see "Technical Notes." Race and Hispanic origin are reported separately on the death certificate. Persons of Hispanic origin may be of any race. Data for Hispanic persons are not tabulated separately by race; data for non-Hispanic persons are tabulated by race. Data for Hispanic origin should be interpreted with caution because of inconsistencies between reporting Hispanic origin on death certificates and on censuses and surveys; see "Technical Notes." Race categories are consistent with the 1977 Office of Management and Budget (OMB) standards. A listing of the International Classification of Diseases, Tenth Revision (ICD-10), Second Edition codes included in this table can be found in the note at the bottom of the table]

\begin{tabular}{|c|c|c|c|c|c|c|c|c|c|c|c|c|c|c|c|c|}
\hline & \multirow[b]{2}{*}{ Year } & \multicolumn{3}{|c|}{ All origins ${ }^{1}$} & \multicolumn{3}{|c|}{ Hispanic } & \multicolumn{3}{|c|}{ Non-Hispanic ${ }^{2}$} & \multicolumn{3}{|c|}{ Non-Hispanic white ${ }^{3}$} & \multicolumn{3}{|c|}{ Non-Hispanic black ${ }^{3}$} \\
\hline & & $\begin{array}{l}\text { Both } \\
\text { sexes }\end{array}$ & Male & Female & $\begin{array}{l}\text { Both } \\
\text { sexes }\end{array}$ & Male & Female & $\begin{array}{l}\text { Both } \\
\text { sexes }\end{array}$ & Male & Female & $\begin{array}{l}\text { Both } \\
\text { sexes }\end{array}$ & Male & Female & $\begin{array}{l}\text { Both } \\
\text { sexes }\end{array}$ & Male & Female \\
\hline & & \multicolumn{15}{|c|}{ Number } \\
\hline 2007 & . . & 23,199 & 17,428 & 5,771 & 2,977 & 2,539 & 438 & 20,130 & 14,811 & 5,319 & 16,935 & 12,474 & 4,461 & 2,210 & 1,674 & 536 \\
\hline 2006 & & 22,073 & 16,472 & 5,601 & 2,804 & 2,341 & 463 & 19,187 & 14,055 & 5,132 & 16,114 & 11,837 & 4,277 & 2,128 & 1,561 & 567 \\
\hline 2005 & & 21,634 & 16,238 & 5,396 & 2,658 & 2,265 & 393 & 18,877 & 13,890 & 4,987 & 15,729 & 11,610 & 4,119 & 2,282 & 1,670 & 612 \\
\hline 2004 & $\ldots \ldots$ & 21,081 & 15,906 & 5,175 & 2,406 & 2,056 & 350 & 18,567 & 13,761 & 4,806 & 15,418 & 11,428 & 3,990 & 2,318 & 1,754 & 564 \\
\hline 2003 & $\ldots$ & 20,687 & 15,630 & 5,057 & 2,422 & 2,048 & 374 & 18,160 & 13,490 & 4,670 & 14,977 & 11,133 & 3,844 & 2,367 & 1,787 & 580 \\
\hline 2002 & & 20,218 & 15,272 & 4,946 & 2,408 & 2,065 & 343 & 17,661 & 13,078 & 4,583 & 14,494 & 10,783 & 3,711 & 2,396 & 1,768 & 628 \\
\hline 2001 & & 20,114 & 15,149 & 4,965 & 2,381 & 2,026 & 355 & 17,593 & 13,009 & 4,584 & 14,186 & 10,497 & 3,689 & 2,677 & 2,016 & 661 \\
\hline 2000 & $\ldots$ & 19,643 & 14,993 & 4,650 & 2,323 & 2,024 & 299 & 17,177 & 12,843 & 4,334 & 13,815 & 10,408 & 3,407 & 2,672 & 1,959 & 713 \\
\hline \multirow[t]{2}{*}{1999} & $\ldots \ldots \ldots$ & 19,469 & 14,894 & 4,575 & 2,184 & 1,864 & 320 & 17,143 & 12,905 & 4,238 & 13,633 & 10,337 & 3,296 & 2,794 & 2,066 & 728 \\
\hline & & \multicolumn{15}{|c|}{ Rate } \\
\hline 2007 & & 7.7 & 11.7 & 3.8 & 6.5 & 10.8 & 2.0 & 7.9 & 11.8 & 4.1 & 8.4 & 12.6 & 4.4 & 5.8 & 9.2 & 2.7 \\
\hline 2006 & $\ldots$ & 7.4 & 11.2 & 3.7 & 6.3 & 10.2 & 2.2 & 7.5 & 11.3 & 3.9 & 8.0 & 12.0 & 4.2 & 5.6 & 8.7 & 2.9 \\
\hline 2005 & 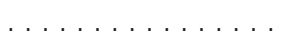 & 7.3 & 11.1 & 3.6 & 6.2 & 10.3 & 1.9 & 7.4 & 11.2 & 3.8 & 7.9 & 11.8 & 4.0 & 6.1 & 9.4 & 3.1 \\
\hline 2004 & & 7.2 & 11.0 & 3.5 & 5.8 & 9.6 & 1.8 & 7.4 & 11.2 & 3.7 & 7.7 & 11.7 & 3.9 & 6.3 & 10.0 & 2.9 \\
\hline 2003 & & 7.1 & 10.9 & 3.4 & 6.1 & 9.9 & 1.9 & 7.2 & 11.0 & 3.6 & 7.5 & 11.4 & 3.8 & 6.5 & 10.3 & 3.0 \\
\hline 2002 & & 7.0 & 10.8 & 3.4 & 6.2 & 10.3 & 1.8 & 7.1 & 10.7 & 3.6 & 7.3 & 11.1 & 3.7 & 6.6 & 10.3 & 3.3 \\
\hline 2001 & & 7.1 & 10.8 & 3.4 & 6.4 & 10.7 & 2.0 & 7.1 & 10.8 & 3.6 & 7.2 & 10.8 & 3.6 & 7.5 & 11.9 & 3.5 \\
\hline 2000 & . . & 7.0 & 10.9 & 3.2 & 6.6 & 11.1 & 1.7 & 7.0 & 10.7 & 3.4 & 7.0 & 10.8 & 3.4 & 7.6 & 11.7 & 3.9 \\
\hline \multirow[t]{2}{*}{1999} & $\ldots \ldots \ldots$ & 7.0 & 10.9 & 3.2 & 6.4 & 10.7 & 1.9 & 7.0 & 10.8 & 3.4 & 6.9 & 10.7 & 3.3 & 8.0 & 12.5 & 4.0 \\
\hline & & \multicolumn{15}{|c|}{ Age-adjusted rate ${ }^{4}$} \\
\hline 2007 & & 7.3 & 11.3 & 3.5 & 9.3 & 16.4 & 2.7 & 7.0 & 10.8 & 3.6 & 7.2 & 10.9 & 3.8 & 6.5 & 11.2 & 2.8 \\
\hline 2006 & & 7.0 & 10.9 & 3.4 & 9.1 & 15.7 & 3.0 & 6.8 & 10.4 & 3.5 & 6.9 & 10.5 & 3.6 & 6.3 & 10.5 & 3.0 \\
\hline 2005 & & 7.0 & 11.0 & 3.4 & 9.1 & 16.2 & 2.6 & 6.8 & 10.4 & 3.5 & 6.8 & 10.4 & 3.5 & 7.0 & 11.6 & 3.4 \\
\hline 2004 & & 7.0 & 11.0 & 3.3 & 8.6 & 15.1 & 2.5 & 6.8 & 10.6 & 3.4 & 6.8 & 10.5 & 3.4 & 7.3 & 12.5 & 3.2 \\
\hline 2003 & & 7.0 & 11.0 & 3.3 & 9.2 & 16.2 & 2.8 & 6.8 & 10.5 & 3.3 & 6.7 & 10.4 & 3.4 & 7.6 & 12.9 & 3.3 \\
\hline 2002 & & 6.9 & 11.0 & 3.3 & 9.5 & 17.0 & 2.7 & 6.7 & 10.4 & 3.3 & 6.6 & 10.2 & 3.3 & 7.9 & 13.3 & 3.7 \\
\hline 2001 & & 7.0 & 11.2 & 3.3 & 10.1 & 18.1 & 2.9 & 6.7 & 10.5 & 3.4 & 6.5 & 10.1 & 3.3 & 9.0 & 15.4 & 4.0 \\
\hline 2000 & & 7.0 & 11.4 & 3.2 & 10.5 & 19.4 & 2.6 & 6.7 & 10.6 & 3.2 & 6.4 & 10.1 & 3.1 & 9.3 & 15.5 & 4.4 \\
\hline 1999 & $\ldots \ldots \ldots \ldots \ldots$ & 7.1 & 11.5 & 3.2 & 10.3 & 18.6 & 3.0 & 6.8 & 10.8 & 3.2 & 6.4 & 10.2 & 3.0 & 10.0 & 16.9 & 4.6 \\
\hline
\end{tabular}

1Figures for origin not stated are included in "all origins" but not distributed among specified origins.

${ }^{2}$ Includes races other than white and black.

${ }^{3}$ Multiple-race data were reported by 27 states and the District of Columbia in 2007, by 25 states and the District of Columbia in 2006, by 21 states and the District of Columbia in 2005, by 15 states in 2004, and by 7 states in 2003; see "Technical Notes." The multiple-race data for these reporting areas were bridged to the single-race categories of the 1977 OMB standards for comparability with other reporting areas; see "Technical Notes."

${ }^{4}$ For method of computation, see "Technical Notes."

NOTES: Causes of death attributable to alcohol-induced mortality include ICD-10 codes E24.4, F10, G31.2, G62.1, G72.1, 142.6, K29.2, K70, K85.2, K86.0, R78.0, X45, X65, and Y15. In 2006, the list of alcohol-induced codes was modified to include a new ICD-10 code, Alcohol-induced acute pancreatitis (K85.2). 
Table 25. Number of deaths, death rates, and age-adjusted death rates for ages 15 years and over, by marital status and sex: United States, 2007

[Rates per 100,000 population in specified group; age-adjusted rates per 100,000 U.S. standard population; see "Technical Notes." Population estimates used for computing death rates are based on the Current Population Survey adjusted to July 1, 2007, resident population control totals for the United States; see "Technical Notes"]

\begin{tabular}{|c|c|c|c|c|c|c|c|c|c|}
\hline Marital status and sex & $\begin{array}{l}15 \text { years } \\
\text { and over }\end{array}$ & $\begin{array}{l}15-24 \\
\text { years }\end{array}$ & $\begin{array}{l}25-34 \\
\text { years }\end{array}$ & $\begin{array}{l}35-44 \\
\text { years }\end{array}$ & $\begin{array}{l}45-54 \\
\text { years }\end{array}$ & $\begin{array}{l}55-64 \\
\text { years }\end{array}$ & $\begin{array}{l}65-74 \\
\text { years }\end{array}$ & $\begin{array}{l}75 \text { years } \\
\text { and over }\end{array}$ & $\begin{array}{c}\text { Age-adjusted } \\
\text { rate }^{2}\end{array}$ \\
\hline & \multicolumn{9}{|c|}{ Number } \\
\hline$\ldots \ldots \ldots$ & $2,383,523$ & 33,982 & 42,572 & 79,606 & 184,686 & 287,110 & 389,238 & $1,366,329$ & $\ldots$ \\
\hline Never married. . . . . . . . . . . . . . & 260,281 & 31,424 & 25,552 & 28,859 & 44,192 & 36,602 & 27,231 & 66,421 & $\ldots$ \\
\hline Ever married $\ldots \ldots \ldots \ldots \ldots \ldots$ & $2,110,875$ & 2,473 & 16,771 & 49,955 & 138,263 & 247,629 & 359,522 & $1,296,262$ & $\ldots$ \\
\hline Married . . . . . . . . . . . . & 917,839 & 2,131 & 12,244 & 31,797 & 81,017 & 146,861 & 206,907 & 436,882 & $\ldots$ \\
\hline Widowed . . . . . . . . . . . . & 879,173 & 54 & 259 & 1,291 & 6,879 & 24,821 & 81,699 & 764,170 & $\ldots$ \\
\hline Divorced. . . . . . . . . . . . . . & 313,863 & 288 & 4,268 & 16,867 & 50,367 & 75,947 & 70,916 & 95,210 & $\ldots$ \\
\hline Not stated . . . . . . . . . . . & 12,367 & 85 & 249 & 792 & 2,231 & 2,879 & 2,485 & 3,646 & $\cdots$ \\
\hline$\ldots \ldots \ldots \ldots$ & $1,181,300$ & 25,316 & 29,792 & 50,105 & 114,456 & 173,618 & 218,344 & 569,669 & $\ldots$ \\
\hline Never married. . . . . . . . . . . . . & 161,119 & 23,715 & 18,972 & 20,213 & 30,402 & 23,948 & 16,670 & 27,199 & $\ldots$ \\
\hline Ever married . . . . . . . . . . . & $1,011,370$ & 1,533 & 10,638 & 29,313 & 82,304 & 147,368 & 199,795 & 540,419 & $\ldots$ \\
\hline Married . . . . . . . . . . . & 618,209 & 1,348 & 7,795 & 18,629 & 48,067 & 92,682 & 136,653 & 313,035 & $\ldots$ \\
\hline Widowed . . . . . . . . . . . . & 223,656 & 22 & 115 & 506 & 2,465 & 7,907 & 23,823 & 188,818 & $\ldots$ \\
\hline Divorced. . . . . . . . . . . . . . & 169,505 & 163 & 2,728 & 10,178 & 31,772 & 46,779 & 39,319 & 38,566 & $\ldots$ \\
\hline Not stated . . . . . . . . . . . . & 8,811 & 68 & 182 & 579 & 1,750 & 2,302 & 1,879 & 2,051 & $\cdots$ \\
\hline$\ldots \ldots \ldots$ & $1,202,223$ & 8,666 & 12,780 & 29,501 & 70,230 & 113,492 & 170,894 & 796,660 & $\ldots$ \\
\hline Never married. . . . . . . . . . . . . . . & 99,162 & 7,709 & 6,580 & 8,646 & 13,790 & 12,654 & 10,561 & 39,222 & $\ldots$ \\
\hline Ever married $\ldots \ldots \ldots \ldots \ldots$ & $1,099,505$ & 940 & 6,133 & 20,642 & 55,959 & 100,261 & 159,727 & 755,843 & $\ldots$ \\
\hline Married . . . . . . . . . . . . & 299,630 & 783 & 4,449 & 13,168 & 32,950 & 54,179 & 70,254 & 123,847 & $\ldots$ \\
\hline Widowed . . . . . . . . . . . . . & 655,517 & 32 & 144 & 785 & 4,414 & 16,914 & 57,876 & 575,352 & $\ldots$ \\
\hline Divorced. . . . . . . . . . . . . . . . & 144,358 & 125 & 1,540 & 6,689 & 18,595 & 29,168 & 31,597 & 56,644 & $\ldots$ \\
\hline \multirow[t]{2}{*}{ Not stated . . . . . . . . . . . . } & 3,556 & 17 & 67 & 213 & 481 & 577 & 606 & 1,595 & $\cdots$ \\
\hline & \multicolumn{9}{|c|}{ Rate $^{3}$} \\
\hline$\ldots \ldots \ldots \ldots$ & 990.1 & 79.9 & 104.9 & 184.4 & 420.9 & 877.7 & $2,011.3$ & $7,371.3$ & $1,167.9$ \\
\hline Never married. . . . . . . . . . . . . . & 365.4 & 82.1 & 155.3 & 385.0 & 835.1 & $1,627.3$ & $3,363.1$ & $9,792.5$ & $1,780.4$ \\
\hline Ever married $\ldots \ldots \ldots \ldots \ldots$ & $1,245.4$ & 58.4 & 69.5 & 140.1 & 358.4 & 812.9 & $1,938.9$ & $7,258.9$ & $1,109.6$ \\
\hline Married . . . . . . . . . . . . & 699.8 & 54.6 & 56.2 & 105.3 & 263.9 & 630.9 & $1,599.4$ & $5,213.4$ & 828.3 \\
\hline Widowed . . . . . . . . . . . . & $5,831.3$ & * & 216.5 & 379.8 & 753.0 & $1,333.3$ & $2,425.6$ & $9,056.1$ & $1,570.7$ \\
\hline Divorced. . . . . . . . . . . . . & $1,349.4$ & 96.7 & 190.2 & 328.0 & 722.3 & $1,426.4$ & $3,168.6$ & $9,159.7$ & $1,643.8$ \\
\hline$\ldots \ldots \ldots$ & $1,005.3$ & 115.8 & 144.0 & 231.8 & 530.0 & $1,100.6$ & $2,456.9$ & $8,035.4$ & $1,348.3$ \\
\hline Never married. . . . . . . . . . . . . . & 413.2 & 117.0 & 198.9 & 457.4 & $1,013.5$ & $2,142.8$ & $4,329.8$ & $10,268.3$ & $2,057.6$ \\
\hline Ever married $\ldots \ldots \ldots \ldots \ldots \ldots$ & $1,288.1$ & 96.2 & 95.5 & 170.4 & 442.6 & $1,005.4$ & $2,350.0$ & $7,918.7$ & $1,269.8$ \\
\hline Married . . . . . . . . . . . . & 940.3 & 91.8 & 76.6 & 125.6 & 315.4 & 765.6 & $1,955.5$ & $6,349.7$ & $1,009.0$ \\
\hline Widowed . . . . . . . . . . . . & $7,618.2$ & * & * & 553.5 & $1,098.3$ & $2,201.5$ & $3,577.7$ & $12,099.4$ & $2,273.0$ \\
\hline Divorced. . . . . . . . . . . . & $1,723.7$ & 146.0 & 288.8 & 447.9 & $1,015.3$ & $2,133.0$ & $4,636.1$ & $11,543.2$ & $2,223.4$ \\
\hline Female. & 975.6 & 42.0 & 64.2 & 136.9 & 315.2 & 670.1 & $1,633.0$ & $6,960.0$ & $1,020.3$ \\
\hline Never married. . . . . . . . . . . . . . & 307.5 & 42.8 & 95.2 & 281.1 & 601.7 & $1,118.2$ & $2,486.8$ & $9,487.7$ & $1,506.4$ \\
\hline Ever married . . . . . . . . . . . & $1,208.6$ & 35.6 & 47.2 & 111.8 & 280.0 & 634.4 & $1,590.8$ & $6,850.8$ & 983.8 \\
\hline Married . . . . . . . . . . . . . & 458.1 & 32.2 & 38.4 & 85.8 & 213.2 & 485.0 & $1,181.1$ & $3,589.8$ & 595.1 \\
\hline Widowed ............... & $5,399.2$ & * & 146.5 & 315.9 & 640.5 & $1,125.8$ & $2,141.8$ & $8,365.6$ & $1,395.0$ \\
\hline Divorced. . . . . . . . . . . . . . . & $1,075.2$ & 67.1 & 118.5 & 233.0 & 483.7 & 931.5 & $2,273.2$ & $8,030.7$ & $1,291.6$ \\
\hline
\end{tabular}

Category not applicable

* Figure does not meet standards of reliability or precision; see "Technical Notes."

${ }^{1}$ Excludes figures for age not stated.

${ }^{2}$ Calculated based on ages 25 years and over. For method of computation, see "Technical Notes."

${ }^{3}$ Figures for marital status not stated are included in totals for "both sexes," "male," and "female" but are not distributed among specified marital status groups. 
Table 26. Number of deaths, death rates, and age-adjusted death rates for ages 25-64 years, by educational attainment and sex: Total of 22 reporting states and the District of Columbia using the 2003 version of the U.S. Standard Certificate of Death and total of 26 reporting states using the 1989 version of the U.S. Standard Certificate of Death, 2007

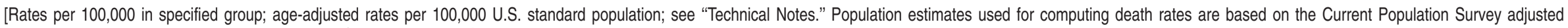
to July 1,2007 , resident population control totals for reporting areas]

\begin{tabular}{|c|c|c|c|c|c|c|c|c|c|c|c|c|c|}
\hline \multicolumn{7}{|c|}{$\begin{array}{l}22 \text { reporting states and the District of Columbia }{ }^{1} \text { using } \\
2003 \text { version of U.S. Standard Certificate of Death }\end{array}$} & \multicolumn{7}{|c|}{$\begin{array}{c}26 \text { reporting states }{ }^{2} \text { using } 1989 \\
\text { version of U.S. Standard Certificate of Death }\end{array}$} \\
\hline Education level and sex & $\begin{array}{l}25-64 \\
\text { years }^{3}\end{array}$ & $\begin{array}{l}25-34 \\
\text { years }\end{array}$ & $\begin{array}{l}35-44 \\
\text { years }\end{array}$ & $\begin{array}{l}45-54 \\
\text { years }\end{array}$ & $\begin{array}{l}55-64 \\
\text { years }\end{array}$ & $\begin{array}{l}\text { Age-adjusted } \\
\text { rate }^{4}\end{array}$ & Years of school completed ${ }^{5}$ and sex & $\begin{array}{l}25-64 \\
\text { years }^{3}\end{array}$ & $\begin{array}{l}25-34 \\
\text { years }\end{array}$ & $\begin{array}{l}35-44 \\
\text { years }\end{array}$ & $\begin{array}{l}45-54 \\
\text { years }\end{array}$ & $\begin{array}{l}55-64 \\
\text { years }\end{array}$ & $\begin{array}{l}\text { Age-adjusted } \\
\text { rate }^{4}\end{array}$ \\
\hline & \multicolumn{6}{|c|}{ Number } & & \multicolumn{6}{|c|}{ Number } \\
\hline $\begin{array}{l}\text { Both sexes . . . . . . . . . . . . . . } \\
\text { Less than high school diploma or GED . } \\
\text { High school diploma or GED ........ } \\
\text { Some college or collegiate degree. . . } \\
\text { Not stated }{ }^{6} \ldots \ldots\end{array}$ & $\begin{array}{r}310,903 \\
60,991 \\
126,184 \\
109,248 \\
14,480\end{array}$ & $\begin{array}{r}22,011 \\
5,265 \\
8,901 \\
6,707 \\
1,138\end{array}$ & $\begin{array}{r}41,335 \\
9,025 \\
17,251 \\
13,021 \\
2,038\end{array}$ & $\begin{array}{r}97,727 \\
18,932 \\
41,320 \\
32,858 \\
4,617\end{array}$ & $\begin{array}{r}149,830 \\
27,769 \\
58,712 \\
56,662 \\
6,687\end{array}$ & $\begin{array}{l}\cdots \\
\cdots \\
\cdots \\
\cdots \\
\cdots\end{array}$ & $\begin{array}{l}\text { Both sexes. } \ldots \ldots \ldots \ldots \ldots \ldots \\
\text { Under } 12 \text { years. } \ldots \ldots \ldots \ldots \ldots \\
12 \text { years. } \ldots \ldots \ldots \ldots \ldots \\
13 \text { years or more. } \ldots \ldots \ldots \ldots \\
\text { Not stated }^{7} \ldots \ldots \ldots \ldots\end{array}$ & $\begin{array}{r}260,661 \\
53,458 \\
115,327 \\
81,214 \\
10,662\end{array}$ & $\begin{array}{r}18,840 \\
4,234 \\
8,564 \\
5,202 \\
840\end{array}$ & $\begin{array}{r}35,041 \\
7,339 \\
16,037 \\
10,163 \\
1,502\end{array}$ & $\begin{array}{r}80,060 \\
15,742 \\
36,652 \\
24,398 \\
3,268\end{array}$ & $\begin{array}{r}126,720 \\
26,143 \\
54,074 \\
41,451 \\
5,052\end{array}$ & $\begin{array}{l}\cdots \\
\cdots \\
\cdots \\
\cdots\end{array}$ \\
\hline $\begin{array}{l}\text { Male. . . . . . . . . . . . . . . . . } \\
\text { Less than high school diploma or GED . } \\
\text { High school diploma or GED . . . . . } \\
\text { Some college or collegiate degree. . } \\
\text { Not stated }{ }^{6} \ldots \ldots \ldots \ldots \ldots \ldots \ldots\end{array}$ & $\begin{array}{r}192,543 \\
39,161 \\
78,550 \\
64,707 \\
10,125\end{array}$ & $\begin{array}{r}15,385 \\
3,846 \\
6,414 \\
4,271 \\
854\end{array}$ & $\begin{array}{r}26,132 \\
5,926 \\
11,323 \\
7,452 \\
1,431\end{array}$ & $\begin{array}{r}60,609 \\
12,444 \\
26,244 \\
18,669 \\
3,252\end{array}$ & $\begin{array}{r}90,417 \\
16,945 \\
34,569 \\
34,315 \\
4,588\end{array}$ & $\begin{array}{l}\cdots \\
\cdots \\
\cdots \\
\cdots \\
\cdots\end{array}$ & $\begin{array}{l}\text { Male } \ldots \ldots \ldots \ldots \\
\text { Under } 12 \text { years. } \ldots \ldots \ldots \ldots \ldots \\
12 \text { years. } \ldots \ldots \ldots \ldots \ldots \\
13 \text { years or more. } \ldots \ldots \ldots \ldots \\
\quad \text { Not stated } \ldots \ldots \ldots \ldots \ldots \ldots\end{array}$ & $\begin{array}{r}161,632 \\
34,775 \\
71,833 \\
47,697 \\
7,327\end{array}$ & $\begin{array}{r}13,278 \\
3,094 \\
6,267 \\
3,291 \\
626\end{array}$ & $\begin{array}{r}21,947 \\
4,828 \\
10,491 \\
5,635 \\
993\end{array}$ & $\begin{array}{r}49,660 \\
10,509 \\
23,294 \\
13,588 \\
2,269\end{array}$ & $\begin{array}{r}76,747 \\
16,344 \\
31,781 \\
25,183 \\
3,439\end{array}$ & $\begin{array}{l}\cdots \\
\cdots \\
\cdots \\
\cdots \\
\cdots\end{array}$ \\
\hline $\begin{array}{l}\text { Female } \ldots \ldots \ldots \\
\text { Less than high school diploma or GED } \ldots \\
\text { High school diploma or GED . . . . . } \\
\text { Some college or collegiate degree. . } \\
\text { Not stated }\end{array}$ & $\begin{array}{r}118,360 \\
21,830 \\
47,634 \\
44,541 \\
4,355\end{array}$ & $\begin{array}{r}6,626 \\
1,419 \\
2,487 \\
2,436 \\
284\end{array}$ & $\begin{array}{r}15,203 \\
3,099 \\
5,928 \\
5,569 \\
607\end{array}$ & $\begin{array}{r}37,118 \\
6,488 \\
15,076 \\
14,189 \\
1,365\end{array}$ & $\begin{array}{r}59,413 \\
10,824 \\
24,143 \\
22,347 \\
2,099\end{array}$ & $\begin{array}{l}\cdots \\
\cdots \\
\cdots \\
\cdots \\
\cdots\end{array}$ & $\begin{array}{l}\text { Female } \ldots \ldots \ldots \\
\text { Under } 12 \text { years. } \ldots \ldots \ldots \ldots \ldots \\
12 \text { years. } \ldots \ldots \ldots \ldots \ldots \\
13 \text { years or more. } \ldots \ldots \ldots \ldots \\
\text { Not stated }{ }^{7} \ldots \ldots \ldots \ldots\end{array}$ & $\begin{array}{r}99,029 \\
18,683 \\
43,494 \\
33,517 \\
3,335\end{array}$ & $\begin{array}{r}5,562 \\
1,140 \\
2,297 \\
1,911 \\
214\end{array}$ & $\begin{array}{r}13,094 \\
2,511 \\
5,546 \\
4,528 \\
509\end{array}$ & $\begin{array}{r}30,400 \\
5,233 \\
13,358 \\
10,810 \\
999\end{array}$ & $\begin{array}{r}49,973 \\
9,799 \\
22,293 \\
16,268 \\
1,613\end{array}$ & $\begin{array}{l}\cdots \\
\cdots \\
\cdots \\
\cdots \\
\cdots\end{array}$ \\
\hline Not stated ${ }^{6} \ldots \ldots \ldots \ldots \ldots$ & \multicolumn{6}{|c|}{ Rate $^{8}$} & & \multicolumn{6}{|c|}{ Rate $^{8}$} \\
\hline $\begin{array}{l}\text { Both sexes . . . . . . . . . . . . . . . } \\
\text { Less than high school diploma or GED . } \\
\text { High school diploma or GED . . . . . . } \\
\text { Some college or collegiate degree. . . . }\end{array}$ & $\begin{array}{l}355.8 \\
547.5 \\
503.3 \\
213.5\end{array}$ & $\begin{array}{r}98.3 \\
169.3 \\
145.9 \\
50.9\end{array}$ & $\begin{array}{r}173.2 \\
292.6 \\
254.8 \\
92.9\end{array}$ & $\begin{array}{l}411.7 \\
666.3 \\
571.2 \\
240.5\end{array}$ & $\begin{array}{r}861.5 \\
1,319.6 \\
1,182.1 \\
549.1\end{array}$ & $\begin{array}{l}330.9 \\
529.5 \\
463.9 \\
196.7\end{array}$ & $\begin{array}{l}\text { Both sexes. } \ldots \ldots \ldots \ldots \ldots \\
\quad \text { Under } 12 \text { years. } \ldots \ldots \ldots \ldots \ldots \\
12 \text { years. } \ldots \ldots \ldots \ldots \ldots \\
13 \text { years or more. } \ldots \ldots \ldots \ldots \ldots\end{array}$ & $\begin{array}{l}388.6 \\
747.3 \\
535.9 \\
211.5\end{array}$ & $\begin{array}{r}113.0 \\
235.2 \\
172.2 \\
52.5\end{array}$ & $\begin{array}{r}198.9 \\
415.5 \\
295.6 \\
97.4\end{array}$ & $\begin{array}{l}430.2 \\
844.9 \\
576.4 \\
234.9\end{array}$ & $\begin{array}{r}894.1 \\
1,516.5 \\
1,135.4 \\
539.3\end{array}$ & $\begin{array}{l}353.0 \\
664.4 \\
477.0 \\
195.4\end{array}$ \\
\hline $\begin{array}{l}\text { Male. . . . . . . . . . . . . . . . . . } \\
\text { Less than high school diploma or GED . } \\
\text { High school diploma or GED . . . . . . . } \\
\text { Some college or collegiate degree. . . }\end{array}$ & $\begin{array}{l}443.7 \\
653.5 \\
609.6 \\
263.9\end{array}$ & $\begin{array}{r}135.0 \\
215.3 \\
186.1 \\
69.3\end{array}$ & $\begin{array}{l}218.0 \\
347.3 \\
315.0 \\
111.5\end{array}$ & $\begin{array}{l}516.3 \\
824.9 \\
709.0 \\
286.0\end{array}$ & $\begin{array}{r}1,092.2 \\
1,710.1 \\
1,611.5 \\
667.3\end{array}$ & $\begin{array}{l}420.3 \\
665.2 \\
600.9 \\
238.9\end{array}$ & $\begin{array}{l}\text { Male } \ldots \ldots \ldots \ldots \\
\text { Under } 12 \text { years. } \ldots \ldots \ldots \ldots \ldots \\
12 \text { years. } \ldots \ldots \ldots \ldots \ldots \\
13 \text { years or more. } \ldots \ldots \ldots \ldots\end{array}$ & $\begin{array}{l}484.4 \\
873.3 \\
647.1 \\
260.9\end{array}$ & $\begin{array}{r}156.4 \\
294.4 \\
222.6 \\
71.2\end{array}$ & $\begin{array}{l}249.7 \\
470.7 \\
362.3 \\
115.8\end{array}$ & $\begin{array}{r}543.5 \\
1,044.4 \\
713.4 \\
279.3\end{array}$ & $\begin{array}{r}1,103.9 \\
1,817.8 \\
1,495.2 \\
641.1\end{array}$ & $\begin{array}{l}444.8 \\
799.3 \\
606.9 \\
234.6\end{array}$ \\
\hline $\begin{array}{l}\text { Female . . . . . . . . . . . . . . . . . } \\
\text { Less than high school diploma or GED . } \\
\text { High school diploma or GED . . . . . . } \\
\text { Some college or collegiate degree. . . . }\end{array}$ & $\begin{array}{l}269.1 \\
424.0 \\
390.9 \\
167.1\end{array}$ & $\begin{array}{r}60.3 \\
107.2 \\
93.7 \\
34.7\end{array}$ & $\begin{array}{r}127.9 \\
224.9 \\
186.6 \\
76.0\end{array}$ & $\begin{array}{l}309.4 \\
486.8 \\
426.7 \\
199.0\end{array}$ & $\begin{array}{l}652.0 \\
972.0 \\
855.6 \\
431.7\end{array}$ & $\begin{array}{l}245.2 \\
387.4 \\
336.8 \\
156.8\end{array}$ & 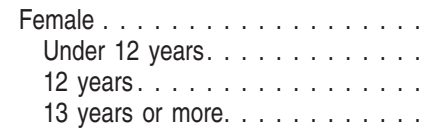 & $\begin{array}{l}293.8 \\
589.1 \\
417.5 \\
166.6\end{array}$ & $\begin{array}{r}68.0 \\
152.2 \\
106.4 \\
36.2\end{array}$ & $\begin{array}{r}148.2 \\
339.0 \\
219.2 \\
81.4\end{array}$ & $\begin{array}{l}320.9 \\
610.5 \\
431.8 \\
195.7\end{array}$ & $\begin{array}{r}692.2 \\
1,188.2 \\
845.4 \\
432.9\end{array}$ & $\begin{array}{l}263.3 \\
503.1 \\
349.9 \\
158.2\end{array}$ \\
\hline
\end{tabular}

Category not applicable.

'Includes data for California, Connecticut, Delaware, District of Columbia, Florida, Idaho, Kansas, Michigan, Montana, Nebraska, New Hampshire, New Jersey, New Mexico, New York, Ohio, Oklahoma, Oregon, South Carolina, South Dakota, Texas, Utah, Washington, and Wyoming; see "Technical Notes."

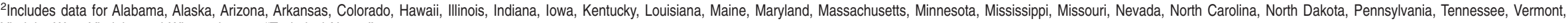
Virginia, West Virginia, and Wisconsin; see "Technical Notes."

${ }^{3}$ Excludes figures for age not stated. $\quad \quad{ }^{4}$ Calculated based on ages 25-64 years. For method of computation, see "Technical Notes."

${ }^{5}$ For rates, the definition of educational attainment differs for the numerator and denominator; see "Technical Notes."

${ }^{6}$ Includes deaths that occurred in states that reported the unrevised education attainment item on the death certificate and in states that did not have an education item on the death certificate.

Includes deaths that occurred in states that reported the revised education attainment item on the death certificate and in states that did not have an education item on the death certificate.

${ }^{8}$ Figures for education not stated are included in totals for "both sexes," "male," and "female" but are not distributed among specified years of education. 
Table 27. Number of deaths, death rates, and age-adjusted death rates for injury at work and ages 15 years and over, by race and sex: United States, 2007

[Rates per 100,000 population in specified group; age-adjusted rates per 100,000 U.S. standard population; see "Technical Notes." Rates are based on populations estimated as of July 1, 2007; see "Technical Notes." For a discussion of injury at work, see "Technical Notes"]

\begin{tabular}{|c|c|c|c|c|c|c|c|c|}
\hline Race and sex & $\begin{array}{l}15 \text { years } \\
\text { and } \text { over }^{1}\end{array}$ & $\begin{array}{l}15-24 \\
\text { years }\end{array}$ & $\begin{array}{l}25-34 \\
\text { years }\end{array}$ & $\begin{array}{l}35-44 \\
\text { years }\end{array}$ & $\begin{array}{l}45-54 \\
\text { years }\end{array}$ & $\begin{array}{l}55-64 \\
\text { years }\end{array}$ & $\begin{array}{l}65 \text { years } \\
\text { and over }\end{array}$ & $\begin{array}{c}\text { Age-adjusted } \\
\text { rate }^{2}\end{array}$ \\
\hline & \multicolumn{8}{|c|}{ Number } \\
\hline All races $^{3}$, both sexes . . & 5,025 & 489 & 824 & 1,021 & 1,266 & 837 & 588 & $\ldots$ \\
\hline$\ldots \ldots \ldots \ldots \ldots$ & 4,606 & 446 & 776 & 947 & 1,158 & 764 & 515 & ... \\
\hline Female $\ldots \ldots \ldots \ldots \ldots \ldots$ & 419 & 43 & 48 & 74 & 108 & 73 & 73 & $\cdots$ \\
\hline White $^{4}$, both sexes . . . . . . . . . . & 4,257 & 412 & 701 & 822 & 1,061 & 728 & 533 & $\ldots$ \\
\hline Male $\ldots \ldots \ldots \ldots \ldots \ldots$ & 3,914 & 378 & 663 & 764 & 969 & 673 & 467 & $\ldots$ \\
\hline Female . . . . . . . . . . . & 343 & 34 & 38 & 58 & 92 & 55 & 66 & $\ldots$ \\
\hline Black $^{4}$, both sexes . . . . . . . . . . & 579 & 58 & 87 & 150 & 156 & 83 & 45 & $\ldots$ \\
\hline$\ldots \ldots \ldots$ & 521 & 53 & 78 & 139 & 141 & 71 & 39 & $\ldots$ \\
\hline \multirow[t]{2}{*}{ Female } & 58 & 5 & 9 & 11 & 15 & 12 & 6 & $\ldots$ \\
\hline & \multicolumn{8}{|c|}{ Rate } \\
\hline All races $^{3}$, both sexes $\ldots \ldots \ldots$ & 2.1 & 1.2 & 2.0 & 2.4 & 2.9 & 2.6 & 1.6 & 2.1 \\
\hline$\ldots \ldots \ldots \ldots$ & 3.9 & 2.0 & 3.8 & 4.4 & 5.4 & 4.8 & 3.2 & 3.9 \\
\hline Female $\ldots \ldots \ldots \ldots \ldots \ldots$ & 0.3 & 0.2 & 0.2 & 0.3 & 0.5 & 0.4 & 0.3 & 0.3 \\
\hline White ${ }^{4}$, both sexes . . . . . . . . . . . . & 2.2 & 1.2 & 2.2 & 2.4 & 2.9 & 2.6 & 1.6 & 2.1 \\
\hline Male $\ldots \ldots \ldots \ldots \ldots \ldots$ & 4.1 & 2.2 & 4.0 & 4.4 & 5.4 & 5.0 & 3.3 & 4.0 \\
\hline Female $\ldots \ldots \ldots \ldots \ldots$ & 0.3 & 0.2 & 0.2 & 0.3 & 0.5 & 0.4 & 0.3 & 0.3 \\
\hline Black ${ }^{4}$, both sexes . . . . . . . . & 1.9 & 0.9 & 1.5 & 2.6 & 2.9 & 2.5 & 1.4 & 2.0 \\
\hline Male. & 3.7 & 1.5 & 2.8 & 5.2 & 5.8 & 4.7 & 3.1 & 3.8 \\
\hline Female $\ldots \ldots \ldots \ldots$ & 0.4 & * & * & * & * & * & * & 0.4 \\
\hline
\end{tabular}

... Category not applicable.

" Figure does not meet standards of reliability or precision; see "Technical Notes."

${ }^{1}$ Excludes figures for age not stated.

${ }^{2}$ Calculated based on ages 15 years and over. For method of computation, see "Technical Notes."

${ }^{3}$ Includes races other than white and black.

${ }^{4}$ Race categories are consistent with the 1977 Office of Management and Budget (OMB) standards. Multiple-race data were reported by 27 states and the District of Columbia in 2007 ; see "Technical Notes." The multiple-race data for these reporting areas were bridged to the single-race categories of the 1977 OMB standards for comparability with other reporting areas; see "Technical Notes." 
100 National Vital Statistics Reports, Vol. 58, No. 19, May 20, 2010

Table 28. Number of deaths, death rates, and age-adjusted death rates for injury at work, by race and sex: United States, 1993-2007

[Includes ages 15 years and over; excludes figures for age not stated. Rates on annual basis per 100,000 population in specified group; age-adjusted rates are per 100,000 U.S. standard population; see "Technical Notes." Rates are based on populations enumerated as of April 1 for 2000 and estimated as of July 1 for all other years; see "Technical Notes." Race categories are consistent with the 1977 Office of Management and Budget (OMB) standards. For a discussion of injury at work, see "Technical Notes"]

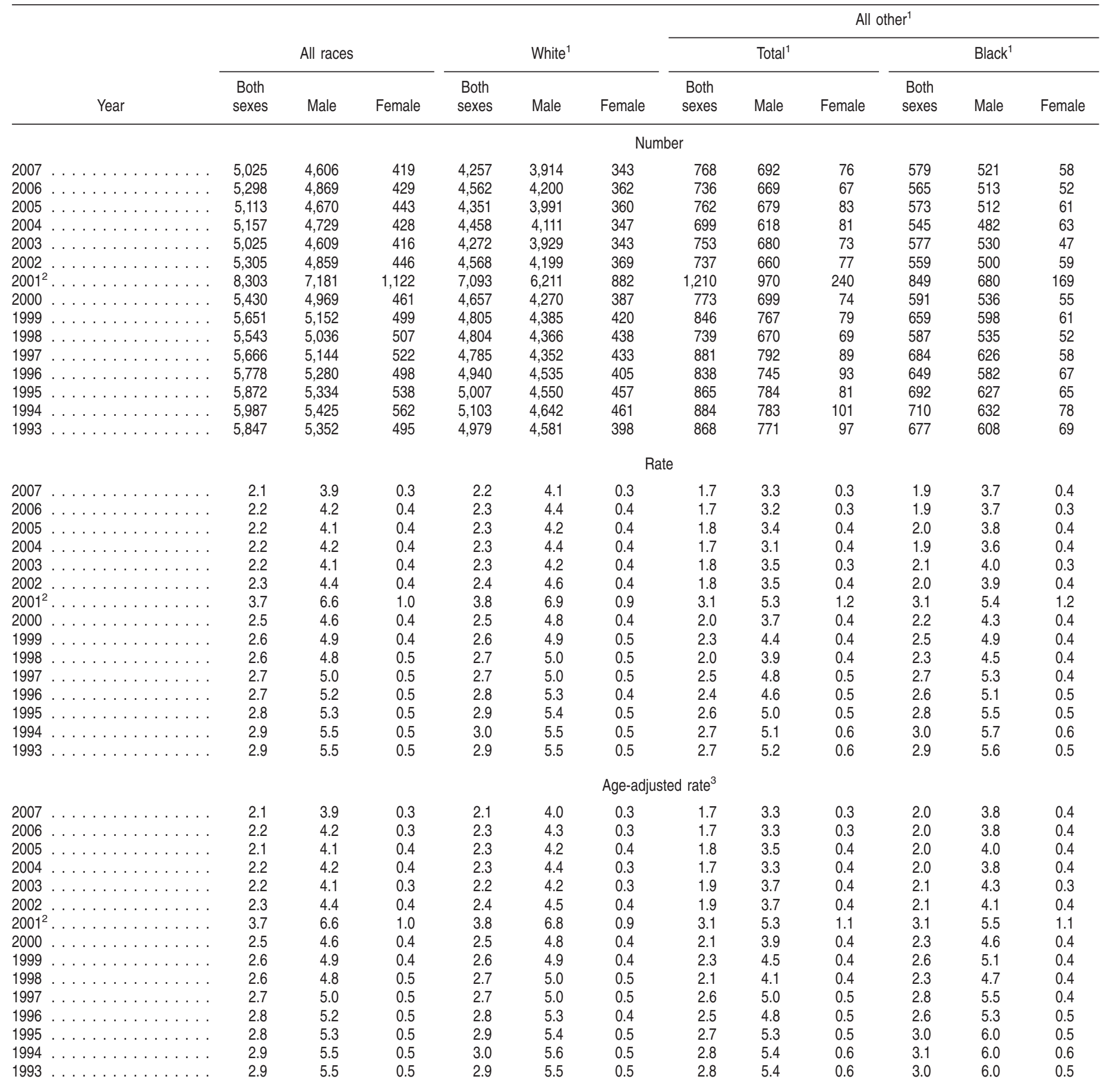

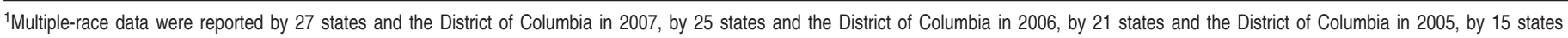

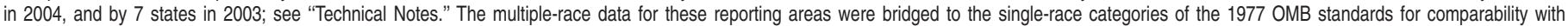
other reporting areas; see "Technical Notes."

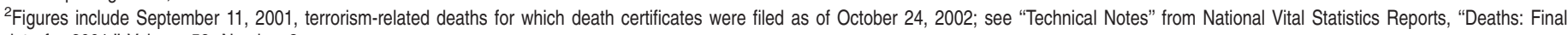
data for 2001," Volume 52, Number 3.

${ }^{3}$ For method of computation, see "Technical Notes." 
Table 29. Number of deaths, death rates, and age-adjusted death rates for major causes of death: United States, each state, Puerto Rico, Virgin Islands, Guam, American Samoa, and Northern Marianas, 2007

[Rates per 100,000 population; age-adjusted rates are per 100,000 U.S. standard population; see "Technical Notes." Populations used for computing death rates are postcensal estimates based on the 2000 census estimated as of July 1, 2007; see "Technical Notes." Numbers after causes of death are categories of the International Classification of Diseases, Tenth Revision (ICD-10). The asterisks $\left(^{*}\right)$ preceding the cause-of-death codes indicate that they are not part of ICD-10; see "Technical Notes." For explanation of asterisks preceding cause-of-death codes, see "Technical Notes"]

\begin{tabular}{|c|c|c|c|c|c|c|c|c|c|c|c|c|}
\hline \multirow[b]{2}{*}{ Area } & \multicolumn{3}{|c|}{ All causes } & \multicolumn{3}{|c|}{$\begin{array}{l}\text { Human immunodeficiency virus } \\
\text { (HIV) disease (B20-B24) }\end{array}$} & \multicolumn{3}{|c|}{$\begin{array}{l}\text { Malignant neoplasms } \\
\quad(\mathrm{C} 00-\mathrm{C} 97)\end{array}$} & \multicolumn{3}{|c|}{$\begin{array}{l}\text { Diabetes mellitus } \\
\quad(\text { E10-E14) }\end{array}$} \\
\hline & Number & Rate & $\begin{array}{l}\text { Age- } \\
\text { adjusted } \\
\text { rate }^{1}\end{array}$ & Number & Rate & $\begin{array}{l}\text { Age- } \\
\text { adjusted } \\
\text { rate }^{1}\end{array}$ & Number & Rate & $\begin{array}{l}\text { Age- } \\
\text { adjusted } \\
\text { rate }^{1}\end{array}$ & Number & Rate & $\begin{array}{l}\text { Age- } \\
\text { adjusted } \\
\text { rate }^{1}\end{array}$ \\
\hline United States ${ }^{2}$. & $2,423,712$ & 803.6 & 760.2 & 11,295 & 3.7 & 3.7 & 562,875 & 186.6 & 178.4 & 71,382 & 23.7 & 22.5 \\
\hline Alabama. ... . & 46,696 & $1,009.0$ & 930.7 & 183 & 4.0 & 4.0 & 10,025 & 216.6 & 197.3 & 1,313 & 28.4 & 26.0 \\
\hline$\ldots \ldots \ldots$ & 3,463 & 506.7 & 755.1 & 7 & * & * & 839 & 122.8 & 179.9 & 105 & 15.4 & 23.4 \\
\hline$\ldots \ldots \ldots \ldots$ & 45,554 & 718.7 & 682.1 & 109 & 1.7 & 1.8 & 10,134 & 159.9 & 152.8 & 1,159 & 18.3 & 17.4 \\
\hline Arkansas & 28,191 & 994.5 & 882.8 & 91 & 3.2 & 3.2 & 6,388 & 225.3 & 200.4 & 838 & 29.6 & 26.5 \\
\hline California & 233,720 & 639.4 & 674.2 & 1,101 & 3.0 & 3.0 & 55,011 & 150.5 & 161.7 & 7,413 & 20.3 & 21.8 \\
\hline Colorado. & 29,993 & 616.9 & 700.8 & 82 & 1.7 & 1.6 & 6,617 & 136.1 & 153.7 & 710 & 14.6 & 16.7 \\
\hline Connecticut. & 28,651 & 818.1 & 694.1 & 140 & 4.0 & 3.6 & 6,827 & 194.9 & 170.7 & 646 & 18.4 & 15.8 \\
\hline Delaware & 7,327 & 847.3 & 773.6 & 55 & 6.4 & 6.2 & 1,853 & 214.3 & 193.9 & 223 & 25.8 & 23.4 \\
\hline District of Columbia . . . . . . & 5,188 & 881.9 & 866.9 & 194 & 33.0 & 32.8 & 1,169 & 198.7 & 199.1 & 152 & 25.8 & 25.2 \\
\hline Florida $\ldots \ldots \ldots \ldots \ldots$ & 168,096 & 921.0 & 685.9 & 1,530 & 8.4 & 8.3 & 40,088 & 219.6 & 166.6 & 5,110 & 28.0 & 21.1 \\
\hline$\ldots \ldots \ldots \ldots$ & 68,331 & 715.9 & 839.8 & 689 & 7.2 & 7.1 & 14,983 & 157.0 & 181.8 & 1,604 & 16.8 & 19.5 \\
\hline$\ldots \ldots \ldots \ldots$ & 9,495 & 739.8 & 607.4 & 21 & 1.6 & 1.6 & 2,214 & 172.5 & 146.2 & 291 & 22.7 & 18.5 \\
\hline$\ldots \ldots \ldots$ & 10,822 & 721.8 & 734.6 & 6 & * & * & 2,405 & 160.4 & 165.6 & 331 & 22.1 & 22.7 \\
\hline$\ldots \ldots \ldots$ & 100,503 & 782.0 & 759.8 & 303 & 2.4 & 2.3 & 24,115 & 187.6 & 185.9 & 2,851 & 22.2 & 21.8 \\
\hline 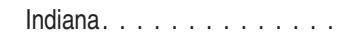 & 54,000 & 851.0 & 809.9 & 97 & 1.5 & 1.5 & 12,778 & 201.4 & 193.2 & 1,564 & 24.6 & 23.4 \\
\hline$\ldots \ldots \ldots \ldots$ & 27,221 & 911.0 & 718.6 & 18 & * & * & 6,376 & 213.4 & 177.7 & 767 & 25.7 & 20.5 \\
\hline Kansas. . . . . . . . . & 24,491 & 882.2 & 783.0 & 22 & 0.8 & 0.8 & 5,406 & 194.7 & 180.0 & 702 & 25.3 & 22.8 \\
\hline Kentucky. . . . . . . . . . . & 40,090 & 945.2 & 896.9 & 55 & 1.3 & 1.3 & 9,692 & 228.5 & 213.5 & 1,091 & 25.7 & 24.1 \\
\hline Louisiana . . . . . . . . . & 39,966 & 930.9 & 926.4 & 344 & 8.0 & 8.3 & 8,736 & 203.5 & 200.3 & 1,437 & 33.5 & 33.3 \\
\hline$\ldots \ldots \ldots$ & 12,493 & 948.4 & 773.6 & 13 & * & * & 3,112 & 236.3 & 191.9 & 355 & 27.0 & 21.9 \\
\hline Maryland. & 43,757 & 778.8 & 782.7 & 436 & 7.8 & 7.4 & 10,179 & 181.2 & 180.7 & 1,301 & 23.2 & 23.4 \\
\hline Massachusetts . & 52,917 & 820.4 & 707.5 & 143 & 2.2 & 2.0 & 13,003 & 201.6 & 179.8 & 1,222 & 18.9 & 16.6 \\
\hline Michigan. . . . . & 86,721 & 861.0 & 806.1 & 187 & 1.9 & 1.8 & 20,087 & 199.4 & 187.3 & 2,826 & 28.1 & 26.3 \\
\hline Minnesota . & 37,138 & 714.5 & 661.5 & 49 & 0.9 & 0.9 & 9,176 & 176.5 & 169.7 & 1,084 & 20.9 & 19.6 \\
\hline Mississippi. . & 28,255 & 968.0 & 943.0 & 163 & 5.6 & 5.9 & 6,002 & 205.6 & 200.4 & 654 & 22.4 & 21.8 \\
\hline Missouri . . . . . & 54,166 & 921.4 & 826.7 & 128 & 2.2 & 2.2 & 12,380 & 210.6 & 191.6 & 1,444 & 24.6 & 22.3 \\
\hline Montana . . . . . . . . . . & 8,624 & 900.3 & 772.7 & 5 & * & * & 1,921 & 200.6 & 172.1 & 258 & 26.9 & 23.1 \\
\hline Nebraska . . . . . . . . . & 15,263 & 860.1 & 743.7 & 28 & 1.6 & 1.7 & 3,479 & 196.0 & 177.3 & 472 & 26.6 & 23.3 \\
\hline$\ldots \ldots \ldots$ & 18,687 & 728.4 & 803.5 & 80 & 3.1 & 3.1 & 4,331 & 168.8 & 180.2 & 312 & 12.2 & 12.9 \\
\hline New Hampshire . . . . . . . & 10,303 & 783.0 & 727.0 & 10 & * & * & 2,609 & 198.3 & 184.5 & 280 & 21.3 & 20.1 \\
\hline New Jersey. & 69,662 & 802.0 & 724.2 & 495 & 5.7 & 5.3 & 17,096 & 196.8 & 180.4 & 2,329 & 26.8 & 24.4 \\
\hline New Mexico. . . . . . . . . . & 15,482 & 785.9 & 755.9 & 35 & 1.8 & 1.9 & 3,238 & 164.4 & 157.3 & 673 & 34.2 & 32.7 \\
\hline New York . . . . . . . . . & 147,680 & 765.3 & 686.4 & 1,342 & 7.0 & 6.6 & 35,485 & 183.9 & 168.0 & 3,715 & 19.3 & 17.5 \\
\hline North Carolina . . . . . . . . & 76,046 & 839.3 & 834.4 & 384 & 4.2 & 4.1 & 17,478 & 192.9 & 189.0 & 2,156 & 23.8 & 23.4 \\
\hline North Dakota . . . . . . . . . & 5,561 & 869.3 & 679.5 & 3 & * & * & 1,264 & 197.6 & 165.3 & 226 & 35.3 & 28.3 \\
\hline$\ldots \ldots \ldots \ldots$ & 106,534 & 929.1 & 830.8 & 207 & 1.8 & 1.8 & 25,230 & 220.0 & 197.9 & 3,722 & 32.5 & 29.1 \\
\hline Oklahoma . . . . . . . . . & 36,032 & 996.1 & 920.4 & 91 & 2.5 & 2.7 & 7,727 & 213.6 & 198.2 & 1,148 & 31.7 & 29.3 \\
\hline$\ldots \ldots$ & 31,403 & 838.0 & 753.9 & 54 & 1.4 & 1.4 & 7,393 & 197.3 & 179.3 & 1,113 & 29.7 & 27.0 \\
\hline Pennsylvania & 125,104 & $1,006.2$ & 790.1 & 378 & 3.0 & 2.9 & 29,014 & 233.4 & 188.2 & 3,442 & 27.7 & 22.0 \\
\hline Rhode Island. & 9,723 & 919.1 & 750.0 & 24 & 2.3 & 2.2 & 2,213 & 209.2 & 179.6 & 248 & 23.4 & 19.2 \\
\hline South Carolina & 39,439 & 894.8 & 849.7 & 315 & 7.1 & 7.1 & 8,867 & 201.2 & 186.7 & 1,231 & 27.9 & 26.2 \\
\hline South Dakota. . & 6,826 & 857.3 & 693.5 & 5 & * & * & 1,612 & 202.5 & 171.3 & 247 & 31.0 & 25.6 \\
\hline Tennessee. . . . . . . . . . & 57,087 & 927.2 & 885.2 & 252 & 4.1 & 4.1 & 13,161 & 213.8 & 200.3 & 1,700 & 27.6 & 26.2 \\
\hline$\ldots \ldots \ldots \ldots$ & 160,548 & 671.6 & 777.7 & 988 & 4.1 & 4.3 & 35,074 & 146.7 & 170.3 & 5,109 & 21.4 & 24.9 \\
\hline $\operatorname{Utah} \ldots \ldots \ldots \ldots$ & 14,143 & 534.6 & 694.2 & 23 & 0.9 & 1.0 & 2,572 & 97.2 & 128.8 & 548 & 20.7 & 27.6 \\
\hline Vermont . . . . . & 5,179 & 833.6 & 729.3 & 2 & * & * & 1,346 & 216.7 & 188.5 & 170 & 27.4 & 24.4 \\
\hline 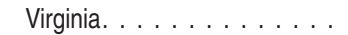 & 58,225 & 755.0 & 770.6 & 230 & 3.0 & 2.8 & 14,009 & 181.6 & 182.7 & 1,507 & 19.5 & 19.7 \\
\hline Washington . . . . . . . . & 47,323 & 731.6 & 722.2 & 107 & 1.7 & 1.6 & 11,568 & 178.8 & 177.7 & 1,508 & 23.3 & 23.2 \\
\hline West Virginia . . & 21,086 & $1,163.7$ & 951.7 & 22 & 1.2 & 1.2 & 4,690 & 258.8 & 207.6 & 800 & 44.1 & 35.5 \\
\hline Wisconsin . . . . . . . . . . & 46,241 & 825.5 & 732.3 & 49 & 0.9 & 0.9 & 10,963 & 195.7 & 177.9 & 1,136 & 20.3 & 18.3 \\
\hline Wyoming $\ldots \ldots \ldots \ldots$ & 4,266 & 815.9 & 802.0 & - & * & * & 940 & 179.8 & 174.4 & 139 & 26.6 & 26.2 \\
\hline Puerto Rico ${ }^{3}$. & 29,169 & 739.9 & 726.7 & 385 & 9.8 & 10.3 & 4,975 & 126.2 & 121.3 & 2,724 & 69.1 & 66.5 \\
\hline Virgin Islands ${ }^{3}$. & 703 & 640.1 & 700.2 & 8 & * & * & 116 & 105.6 & 109.2 & 45 & 41.0 & 45.4 \\
\hline Guam $^{3} \ldots \ldots$ & 778 & 448.5 & 694.9 & 4 & * & * & 129 & 74.4 & 122.3 & 43 & 24.8 & 44.0 \\
\hline American Samoa ${ }^{3}$. & 250 & 390.5 & $1,054.4$ & - & * & * & 29 & 45.3 & 150.1 & 33 & 51.5 & 126.1 \\
\hline Northern Marianas ${ }^{3}$. & 137 & 162.0 & 913.0 & - & * & * & 30 & 35.5 & 239.5 & 9 & & \\
\hline
\end{tabular}

See footnotes at end of table. 
Table 29. Number of deaths, death rates, and age-adjusted death rates for major causes of death: United States, each state, Puerto Rico, Virgin Islands, Guam, American Samoa, and Northern Marianas, 2007-Con.

[Rates per 100,000 population; age-adjusted rates are per 100,000 U.S. standard population; see "Technical Notes." Populations used for computing death rates are postcensal estimates based on the 2000 census estimated as of July 1, 2007; see "Technical Notes." Numbers after causes of death are categories of the International Classification of Diseases, Tenth Revision (ICD-10). The asterisks $\left(^{*}\right)$ preceding the cause-of-death codes indicate that they are not part of ICD-10; see "Technical Notes." For explanation of asterisks preceding cause-of-death codes, see "Technical Notes"]

\begin{tabular}{|c|c|c|c|c|c|c|c|c|c|c|c|c|}
\hline \multirow[b]{2}{*}{ Area } & \multicolumn{3}{|c|}{ Parkinson's disease (G20-G21) } & \multicolumn{3}{|c|}{ Alzheimer's disease (G30) } & \multicolumn{3}{|c|}{$\begin{array}{c}\text { Diseases of heart } \\
(|100-109,| 11,|13,| 20-\mid 51)\end{array}$} & \multicolumn{3}{|c|}{$\begin{array}{l}\text { Essential hypertension } \\
\text { and hypertensive renal } \\
\text { disease }(|10,| 12, \mid 15)\end{array}$} \\
\hline & Number & Rate & $\begin{array}{l}\text { Age- } \\
\text { adjusted } \\
\text { rate }^{1}\end{array}$ & Number & Rate & $\begin{array}{l}\text { Age- } \\
\text { adjusted } \\
\text { rate }^{1}\end{array}$ & Number & Rate & $\begin{array}{l}\text { Age- } \\
\text { adjusted } \\
\text { rate }^{1}\end{array}$ & Number & Rate & $\begin{array}{l}\text { Age- } \\
\text { adjusted } \\
\text { rate }^{1}\end{array}$ \\
\hline United States ${ }^{2}$. & 20,058 & 6.7 & 6.4 & 74,632 & 24.7 & 22.7 & 616,067 & 204.3 & 190.9 & 23,965 & 7.9 & 7.4 \\
\hline Alabama $\ldots \ldots \ldots \ldots$ & 321 & 6.9 & 6.4 & 1,517 & 32.8 & 30.1 & 11,926 & 257.7 & 235.5 & 475 & 10.3 & 9.5 \\
\hline$\ldots \ldots \ldots$ & 16 & * & * & 65 & 9.5 & 20.8 & 613 & 89.7 & 147.9 & 15 & * & * \\
\hline$\ldots \ldots \ldots$ & 458 & 7.2 & 6.8 & 2,051 & 32.4 & 29.6 & 10,302 & 162.5 & 152.5 & 397 & 6.3 & 5.9 \\
\hline Arkansas & 171 & 6.0 & 5.3 & 824 & 29.1 & 24.6 & 7,214 & 254.5 & 221.8 & 257 & 9.1 & 7.9 \\
\hline California & 1,992 & 5.4 & 6.0 & 8,497 & 23.2 & 24.3 & 61,690 & 168.8 & 177.9 & 3,193 & 8.7 & 9.2 \\
\hline Colorado. & 287 & 5.9 & 7.3 & 1,109 & 22.8 & 27.8 & 6,106 & 125.6 & 145.3 & 217 & 4.5 & 5.3 \\
\hline Connecticut . & 267 & 7.6 & 6.4 & 764 & 21.8 & 16.9 & 7,289 & 208.1 & 171.0 & 300 & 8.6 & 7.0 \\
\hline Delaware & 55 & 6.4 & 5.8 & 201 & 23.2 & 20.9 & 1,914 & 221.3 & 200.2 & 46 & 5.3 & 4.8 \\
\hline District of Columbia . . . . . . & 23 & 3.9 & 3.8 & 140 & 23.8 & 21.8 & 1,434 & 243.8 & 239.4 & 63 & 10.7 & 10.6 \\
\hline 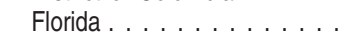 & 1,573 & 8.6 & 5.8 & 4,644 & 25.4 & 16.2 & 42,254 & 231.5 & 162.4 & 1,716 & 9.4 & 6.6 \\
\hline Georgia $\ldots \ldots \ldots \ldots$ & 412 & 4.3 & 5.7 & 1,849 & 19.4 & 25.3 & 16,184 & 169.6 & 203.0 & 934 & 9.8 & 11.5 \\
\hline$\ldots \ldots \ldots \ldots$ & 99 & 7.7 & 6.0 & 247 & 19.2 & 14.1 & 2,227 & 173.5 & 140.2 & 102 & 7.9 & 6.1 \\
\hline$\ldots \ldots \ldots \ldots$ & 110 & 7.3 & 7.6 & 416 & 27.7 & 28.2 & 2,433 & 162.3 & 164.1 & 71 & 4.7 & 4.8 \\
\hline$\ldots \ldots \ldots \ldots$ & 817 & 6.4 & 6.2 & 2,734 & 21.3 & 19.9 & 25,813 & 200.8 & 192.8 & 856 & 6.7 & 6.3 \\
\hline Indiana. . . . . . . . . & 460 & 7.2 & 7.0 & 1,663 & 26.2 & 24.2 & 13,682 & 215.6 & 203.0 & 517 & 8.1 & 7.6 \\
\hline$\ldots \ldots \ldots \ldots$ & 291 & 9.7 & 7.5 & 1,202 & 40.2 & 27.9 & 6,880 & 230.3 & 174.8 & 253 & 8.5 & 6.2 \\
\hline Kansas. . . . . . . . & 239 & 8.6 & 7.5 & 860 & 31.0 & 25.2 & 5,749 & 207.1 & 178.7 & 173 & 6.2 & 5.2 \\
\hline Kentucky. . . . . . . . . . & 255 & 6.0 & 5.8 & 1,198 & 28.2 & 27.1 & 9,916 & 233.8 & 220.9 & 294 & 6.9 & 6.6 \\
\hline Louisiana . . . . . . . . . . & 288 & 6.7 & 7.0 & 1,324 & 30.8 & 31.3 & 9,947 & 231.7 & 230.0 & 395 & 9.2 & 9.1 \\
\hline Maine & 120 & 9.1 & 7.5 & 470 & 35.7 & 27.9 & 2,852 & 216.5 & 172.9 & 74 & 5.6 & 4.4 \\
\hline Maryland. & 327 & 5.8 & 6.1 & 881 & 15.7 & 16.0 & 11,314 & 201.4 & 202.4 & 411 & 7.3 & 7.3 \\
\hline Massachusetts & 464 & 7.2 & 6.1 & 1,695 & 26.3 & 20.9 & 12,710 & 197.1 & 165.5 & 458 & 7.1 & 5.9 \\
\hline Michigan. . . . & 754 & 7.5 & 7.1 & 2,432 & 24.1 & 22.1 & 24,149 & 239.8 & 221.5 & 773 & 7.7 & 7.1 \\
\hline Minnesota . . . . . . . . . & 433 & 8.3 & 7.8 & 1,179 & 22.7 & 19.6 & 7,477 & 143.9 & 129.8 & 407 & 7.8 & 7.0 \\
\hline Mississippi. . . . . . . . . . . & 144 & 4.9 & 5.0 & 797 & 27.3 & 26.5 & 8,037 & 275.4 & 266.5 & 438 & 15.0 & 14.5 \\
\hline Missouri . . . . . . . . . & 418 & 7.1 & 6.4 & 1,681 & 28.6 & 24.3 & 14,338 & 243.9 & 214.4 & 404 & 6.9 & 6.0 \\
\hline Montana . . . . . . . . . . . & 81 & 8.5 & 7.3 & 260 & 27.1 & 22.1 & 1,870 & 195.2 & 163.1 & 74 & 7.7 & 6.3 \\
\hline Nebraska . . . . . . . . . . & 163 & 9.2 & 7.8 & 512 & 28.9 & 22.9 & 3,520 & 198.4 & 165.3 & 186 & 10.5 & 8.4 \\
\hline Nevada & 98 & 3.8 & 4.6 & 248 & 9.7 & 12.3 & 4,591 & 179.0 & 200.0 & 129 & 5.0 & 5.9 \\
\hline New Hampshire & 86 & 6.5 & 6.3 & 418 & 31.8 & 28.9 & 2,511 & 190.8 & 174.9 & 69 & 5.2 & 4.8 \\
\hline New Jersey . . . . . . . . . & 601 & 6.9 & 6.2 & 1,823 & 21.0 & 18.0 & 18,831 & 216.8 & 191.9 & 595 & 6.9 & 6.0 \\
\hline New Mexico. . . . . . . . . . & 163 & 8.3 & 8.1 & 322 & 16.3 & 15.5 & 3,305 & 167.8 & 159.2 & 99 & 5.0 & 4.8 \\
\hline New York . . . . . . . . . & 878 & 4.5 & 4.1 & 1,999 & 10.4 & 8.8 & 49,528 & 256.7 & 225.1 & 1,645 & 8.5 & 7.5 \\
\hline North Carolina . . . . . . . . & 542 & 6.0 & 6.2 & 2,460 & 27.1 & 27.7 & 17,395 & 192.0 & 191.0 & 680 & 7.5 & 7.5 \\
\hline North Dakota . . . . . . . . . & 58 & 9.1 & 6.9 & 395 & 61.7 & 40.4 & 1,414 & 221.0 & 164.1 & 72 & 11.3 & 8.0 \\
\hline$\ldots \ldots \ldots \ldots$ & 870 & 7.6 & 6.8 & 3,671 & 32.0 & 27.4 & 26,757 & 233.3 & 204.8 & 1,211 & 10.6 & 9.2 \\
\hline Oklahoma ... . & 294 & 8.1 & 7.6 & 927 & 25.6 & 23.0 & 9,602 & 265.4 & 241.6 & 245 & 6.8 & 6.1 \\
\hline Oregon. & 328 & 8.8 & 8.0 & 1,200 & 32.0 & 27.6 & 6,655 & 177.6 & 156.9 & 356 & 9.5 & 8.3 \\
\hline Pennsylvania & 1,162 & 9.3 & 7.0 & 3,505 & 28.2 & 19.8 & 32,862 & 264.3 & 199.4 & 996 & 8.0 & 6.0 \\
\hline Rhode Island $\ldots \ldots \ldots \ldots$ & 96 & 9.1 & 7.5 & 328 & 31.0 & 22.2 & 2,751 & 260.1 & 203.6 & 59 & 5.6 & 4.4 \\
\hline South Carolina . . . . . . . . & 276 & 6.3 & 6.1 & 1,396 & 31.7 & 30.9 & 8,992 & 204.0 & 192.9 & 406 & 9.2 & 8.7 \\
\hline South Dakota. . . . . . . . . . & 61 & 7.7 & 6.0 & 346 & 43.5 & 30.2 & 1,633 & 205.1 & 159.1 & 69 & 8.7 & 6.4 \\
\hline Tennessee. . . . . . . . . . . . & 387 & 6.3 & 6.2 & 2,276 & 37.0 & 35.9 & 14,280 & 231.9 & 220.6 & 528 & 8.6 & 8.2 \\
\hline 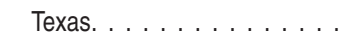 & 1,293 & 5.4 & 6.8 & 4,814 & 20.1 & 24.7 & 38,912 & 162.8 & 191.9 & 1,515 & 6.3 & 7.5 \\
\hline$\ldots \ldots$ & 158 & 6.0 & 8.4 & 393 & 14.9 & 20.5 & 2,980 & 112.7 & 152.1 & 138 & 5.2 & 7.1 \\
\hline$\ldots \ldots \ldots$ & 55 & 8.9 & 7.9 & 205 & 33.0 & 28.4 & 1,166 & 187.7 & 161.2 & 40 & 6.4 & 5.7 \\
\hline$\ldots \ldots \ldots \ldots$ & 473 & 6.1 & 6.6 & 1,703 & 22.1 & 23.4 & 13,750 & 178.3 & 182.7 & 484 & 6.3 & 6.4 \\
\hline Washington ........... & 503 & 7.8 & 8.0 & 2,689 & 41.6 & 40.7 & 11,037 & 170.6 & 167.3 & 473 & 7.3 & 7.2 \\
\hline West Virginia . . . . . . . & 133 & 7.3 & 5.9 & 534 & 29.5 & 23.1 & 5,208 & 287.4 & 229.4 & 226 & 12.5 & 9.7 \\
\hline Wisconsin . . . . . . . . . & 470 & 8.4 & 7.4 & 1,658 & 29.6 & 24.3 & 11,110 & 198.3 & 171.9 & 400 & 7.1 & 6.0 \\
\hline Wyoming $\ldots \ldots \ldots \ldots$ & 35 & 6.7 & 6.8 & 110 & 21.0 & 21.0 & 957 & 183.0 & 178.3 & 31 & 5.9 & 5.7 \\
\hline Puerto Rico ${ }^{3}$. & 133 & 3.4 & 3.4 & 1,574 & 39.9 & 40.7 & 5,585 & 141.7 & 138.4 & 455 & 11.5 & 11.4 \\
\hline Virgin Islands ${ }^{3}$. & 2 & * & $\stackrel{0.7}{*}$ & 14 & * & * & 222 & 202.1 & 234.7 & 9 & * & * \\
\hline Guam $^{3} \ldots .$. & 8 & * & * & 3 & * & * & 234 & 134.9 & 228.4 & 4 & * & * \\
\hline American Samoa ${ }^{3}$. & - & * & * & - & * & * & 52 & 81.2 & 278.0 & 11 & * & * \\
\hline Northern Marianas ${ }^{3}$ & 1 & * & * & - & * & * & 26 & 30.8 & 167.0 & 1 & * & * \\
\hline
\end{tabular}

See footnotes at end of table. 
Table 29. Number of deaths, death rates, and age-adjusted death rates for major causes of death: United States, each state, Puerto Rico, Virgin Islands, Guam, American Samoa, and Northern Marianas, 2007-Con.

[Rates per 100,000 population; age-adjusted rates are per 100,000 U.S. standard population; see "Technical Notes." Populations used for computing death rates are postcensal estimates based on the 2000 census estimated as of July 1, 2007; see "Technical Notes." Numbers after causes of death are categories of the International Classification of Diseases, Tenth Revision (ICD-10). The asterisks $\left(^{*}\right)$ preceding the cause-of-death codes indicate that they are not part of ICD-10; see "Technical Notes." For explanation of asterisks preceding cause-of-death codes, see "Technical Notes"]

\begin{tabular}{|c|c|c|c|c|c|c|c|c|c|c|c|c|}
\hline \multirow[b]{2}{*}{ Area } & \multicolumn{3}{|c|}{$\begin{array}{l}\text { Cerebrovascular } \\
\text { diseases (160-169) }\end{array}$} & \multicolumn{3}{|c|}{$\begin{array}{l}\text { Influenza and pneumonia } \\
(\mathrm{J} 09-\mathrm{J} 18)^{4}\end{array}$} & \multicolumn{3}{|c|}{$\begin{array}{l}\text { Chronic lower respiratory } \\
\text { diseases (J40-J47) }\end{array}$} & \multicolumn{3}{|c|}{$\begin{array}{c}\text { Chronic liver disease } \\
\text { and cirrhosis (K70,K73-K74) }\end{array}$} \\
\hline & Number & Rate & $\begin{array}{l}\text { Age- } \\
\text { adjusted } \\
\text { rate }^{1}\end{array}$ & Number & Rate & $\begin{array}{l}\text { Age- } \\
\text { adjusted } \\
\text { rate }^{1}\end{array}$ & Number & Rate & $\begin{array}{c}\text { Age- } \\
\text { adjusted } \\
\text { rate }^{1}\end{array}$ & Number & Rate & $\begin{array}{l}\text { Age- } \\
\text { adjusted } \\
\text { rate }^{1}\end{array}$ \\
\hline United States ${ }^{2}$. & 135,952 & 45.1 & 42.2 & 52,717 & 17.5 & 16.2 & 127,924 & 42.4 & 40.8 & 29,165 & 9.7 & 9.1 \\
\hline Alabama. . & 2,747 & 59.4 & 54.5 & 898 & 19.4 & 17.8 & 2,530 & 54.7 & 50.2 & 505 & 10.9 & 9.9 \\
\hline$\ldots \ldots \ldots$ & 157 & 23.0 & 44.3 & 48 & 7.0 & 12.9 & 175 & 25.6 & 44.4 & 70 & 10.2 & 11.4 \\
\hline$\ldots \ldots \ldots$ & 2,207 & 34.8 & 32.7 & 905 & 14.3 & 13.5 & 2,686 & 42.4 & 40.2 & 752 & 11.9 & 11.7 \\
\hline$\ldots \ldots \ldots$ & 1,873 & 66.1 & 57.4 & 734 & 25.9 & 22.3 & 1,656 & 58.4 & 51.7 & 271 & 9.6 & 8.6 \\
\hline$\ldots \ldots \ldots$ & 14,557 & 39.8 & 42.2 & 6,546 & 17.9 & 18.9 & 12,532 & 34.3 & 37.4 & 4,065 & 11.1 & 11.3 \\
\hline Colorado . . . . . . . . . & 1,600 & 32.9 & 39.0 & 592 & 12.2 & 14.3 & 2,002 & 41.2 & 49.1 & 542 & 11.1 & 11.0 \\
\hline Connecticut . . . . . . . . . . & 1,463 & 41.8 & 34.2 & 776 & 22.2 & 17.6 & 1,353 & 38.6 & 33.1 & 288 & 8.2 & 7.3 \\
\hline Delaware $\ldots \ldots \ldots \ldots$ & 374 & 43.2 & 39.4 & 117 & 13.5 & 12.2 & 379 & 43.8 & 40.1 & 70 & 8.1 & 7.5 \\
\hline District of Columbia . . . . . . & 220 & 37.4 & 36.9 & 82 & 13.9 & 13.2 & 129 & 21.9 & 22.4 & 45 & 7.6 & 7.5 \\
\hline Florida . . . . . . . & 8,781 & 48.1 & 33.6 & 2,246 & 12.3 & 8.6 & 9,357 & 51.3 & 36.7 & 2,260 & 12.4 & 10.4 \\
\hline Georgia & 3,894 & 40.8 & 49.7 & 1,407 & 14.7 & 18.3 & 3,384 & 35.5 & 43.8 & 686 & 7.2 & 7.6 \\
\hline Hawaii . & 643 & 50.1 & 39.6 & 199 & 15.5 & 11.6 & 299 & 23.3 & 19.3 & 112 & 8.7 & 7.7 \\
\hline Idaho. & 640 & 42.7 & 43.2 & 228 & 15.2 & 15.1 & 666 & 44.4 & 46.6 & 156 & 10.4 & 10.2 \\
\hline$\ldots \ldots \ldots$ & 5,864 & 45.6 & 43.9 & 2,550 & 19.8 & 18.8 & 4,742 & 36.9 & 36.7 & 1,035 & 8.1 & 7.9 \\
\hline$\ldots \ldots \ldots$ & 3,083 & 48.6 & 45.7 & 1,098 & 17.3 & 16.1 & 3,227 & 50.9 & 49.2 & 489 & 7.7 & 7.3 \\
\hline lowa . . . . . . . . . . . & 1,686 & 56.4 & 42.1 & 749 & 25.1 & 18.0 & 1,660 & 55.6 & 44.7 & 239 & 8.0 & 7.2 \\
\hline Kansas. . . . . . . . . & 1,498 & 54.0 & 46.0 & 665 & 24.0 & 19.9 & 1,476 & 53.2 & 48.8 & 230 & 8.3 & 7.8 \\
\hline$\ldots \ldots \ldots$ & 2,144 & 50.5 & 48.1 & 897 & 21.1 & 20.1 & 2,629 & 62.0 & 59.0 & 390 & 9.2 & 8.5 \\
\hline$\ldots \ldots \ldots$ & 2,147 & 50.0 & 50.1 & 870 & 20.3 & 20.3 & 1,685 & 39.2 & 39.7 & 357 & 8.3 & 8.0 \\
\hline$\ldots \ldots \ldots$ & 664 & 50.4 & 40.3 & 236 & 17.9 & 14.1 & 728 & 55.3 & 44.8 & 156 & 11.8 & 9.6 \\
\hline$\ldots \ldots \ldots$ & 2,364 & 42.1 & 42.7 & 994 & 17.7 & 17.9 & 1,901 & 33.8 & 35.1 & 447 & 8.0 & 7.4 \\
\hline Massachusetts . . . . . . . & 2,832 & 43.9 & 36.5 & 1,538 & 23.8 & 19.5 & 2,332 & 36.2 & 31.6 & 609 & 9.4 & 8.4 \\
\hline Michigan. . . . . . . . . . & 4,798 & 47.6 & 44.3 & 1,637 & 16.3 & 15.0 & 4,624 & 45.9 & 43.6 & 1,008 & 10.0 & 9.1 \\
\hline Minnesota . . . . . . . . . . & 2,193 & 42.2 & 38.1 & 603 & 11.6 & 10.2 & 1,758 & 33.8 & 32.8 & 379 & 7.3 & 6.9 \\
\hline Mississippi. . . . . . . . . . . . & 1,589 & 54.4 & 53.0 & 554 & 19.0 & 18.3 & 1,408 & 48.2 & 47.5 & 263 & 9.0 & 8.8 \\
\hline Missouri . . . . . . . . . & 3,229 & 54.9 & 48.2 & 1,289 & 21.9 & 18.9 & 3,081 & 52.4 & 47.4 & 433 & 7.4 & 6.7 \\
\hline Montana . & 443 & 46.2 & 38.5 & 183 & 19.1 & 15.8 & 604 & 63.1 & 55.0 & 138 & 14.4 & 12.6 \\
\hline Nebraska & 921 & 51.9 & 43.1 & 331 & 18.7 & 15.1 & 919 & 51.8 & 45.8 & 116 & 6.5 & 6.3 \\
\hline Nevada & 850 & 33.1 & 38.3 & 408 & 15.9 & 18.4 & 1,050 & 40.9 & 47.5 & 268 & 10.4 & 10.0 \\
\hline New Hampshire .. & 489 & 37.2 & 34.3 & 207 & 15.7 & 14.5 & 611 & 46.4 & 44.0 & 116 & 8.8 & 7.8 \\
\hline New Jersey . . . . . . . . . & 3,492 & 40.2 & 35.8 & 1,343 & 15.5 & 13.7 & 2,991 & 34.4 & 31.3 & 646 & 7.4 & 6.7 \\
\hline New Mexico. . . . . . . . . . . & 804 & 40.8 & 39.2 & 298 & 15.1 & 14.6 & 884 & 44.9 & 43.6 & 372 & 18.9 & 18.4 \\
\hline New York . . . . . . . . . & 6,160 & 31.9 & 28.2 & 4,431 & 23.0 & 20.0 & 6,561 & 34.0 & 30.8 & 1,308 & 6.8 & 6.2 \\
\hline North Carolina . . . . . . . . . & 4,530 & 50.0 & 50.3 & 1,645 & 18.2 & 18.4 & 4,231 & 46.7 & 47.1 & 888 & 9.8 & 9.2 \\
\hline North Dakota . . . . . . . . . & 330 & 51.6 & 37.3 & 133 & 20.8 & 14.6 & 265 & 41.4 & 32.9 & 43 & 6.7 & 6.3 \\
\hline$\ldots \ldots \ldots$ & 5,905 & 51.5 & 45.3 & 1,743 & 15.2 & 13.3 & 6,454 & 56.3 & 50.7 & 1,152 & 10.0 & 9.1 \\
\hline Oklahoma . . . . . . . . . . & 2,126 & 58.8 & 53.8 & 801 & 22.1 & 20.1 & 2,386 & 66.0 & 61.2 & 505 & 14.0 & 13.2 \\
\hline Oregon. $\ldots \ldots \ldots \ldots$ & 1,835 & 49.0 & 43.6 & 477 & 12.7 & 11.1 & 1,892 & 50.5 & 46.3 & 466 & 12.4 & 11.1 \\
\hline Pennsylvania . . . . . . . . . . & 7,152 & 57.5 & 42.9 & 2,555 & 20.6 & 15.2 & 6,077 & 48.9 & 38.1 & 1,084 & 8.7 & 7.4 \\
\hline Rhode Island $\ldots \ldots \ldots \ldots$ & 457 & 43.2 & 33.5 & 224 & 21.2 & 16.1 & 421 & 39.8 & 32.7 & 119 & 11.2 & 9.9 \\
\hline South Carolina . . . . . . . . & 2,466 & 55.9 & 53.4 & 723 & 16.4 & 15.8 & 2,036 & 46.2 & 44.0 & 512 & 11.6 & 10.5 \\
\hline South Dakota. . . . . . . . . & 410 & 51.5 & 38.7 & 189 & 23.7 & 17.9 & 457 & 57.4 & 47.0 & 95 & 11.9 & 11.3 \\
\hline Tennessee. . . . . . . . . . . . . & 3,450 & 56.0 & 53.9 & 1,438 & 23.4 & 22.5 & 3,167 & 51.4 & 49.2 & 629 & 10.2 & 9.2 \\
\hline Texas. ... & 9,796 & 41.0 & 49.0 & 3,230 & 13.5 & 16.1 & 8,107 & 33.9 & 41.2 & 2,535 & 10.6 & 11.4 \\
\hline Utah & 755 & 28.5 & 38.9 & 313 & 11.8 & 16.0 & 617 & 23.3 & 31.9 & 142 & 5.4 & 6.5 \\
\hline Vermont & 269 & 43.3 & 37.6 & 70 & 11.3 & 9.8 & 316 & 50.9 & 44.9 & 54 & 8.7 & 7.0 \\
\hline Virginia. . & 3,313 & 43.0 & 44.5 & 1,231 & 16.0 & 16.6 & 2,770 & 35.9 & 37.7 & 613 & 7.9 & 7.5 \\
\hline Washington . & 2,692 & 41.6 & 41.4 & 743 & 11.5 & 11.1 & 2,684 & 41.5 & 42.4 & 661 & 10.2 & 9.5 \\
\hline West Virginia $\ldots \ldots \ldots \ldots$ & 1,113 & 61.4 & 48.9 & 408 & 22.5 & 17.8 & 1,331 & 73.5 & 58.6 & 273 & 15.1 & 12.5 \\
\hline Wisconsin . & 2,738 & 48.9 & 42.3 & 1,022 & 18.2 & 15.5 & 2,399 & 42.8 & 38.7 & 487 & 8.7 & 7.9 \\
\hline Wyoming $\ldots \ldots \ldots \ldots$ & 209 & 40.0 & 39.5 & 113 & 21.6 & 20.9 & 295 & 56.4 & 56.5 & 86 & 16.4 & 15.5 \\
\hline Puerto Rico ${ }^{3}$. & 1,650 & 41.9 & 41.3 & 1,015 & 25.7 & 25.6 & 1,108 & 28.1 & 27.9 & 232 & 5.9 & 5.5 \\
\hline Virgin Islands ${ }^{3}$. & 40 & 36.4 & 36.8 & 14 & * & * & 17 & * & * & 13 & * & * \\
\hline Guam $^{3} \ldots \ldots$ & 55 & 31.7 & 50.1 & 14 & * & * & 21 & 12.1 & 23.2 & 27 & 15.6 & 18.9 \\
\hline American Samoa ${ }^{3}$. & 22 & 34.4 & 94.2 & 6 & * & * & 12 & * & * & 1 & * & * \\
\hline Northern Marianas ${ }^{3} \ldots \ldots \ldots$ & 8 & * & * & 2 & * & * & 5 & * & * & 3 & * & * \\
\hline
\end{tabular}

See footnotes at end of table. 
Table 29. Number of deaths, death rates, and age-adjusted death rates for major causes of death: United States, each state, Puerto Rico, Virgin Islands, Guam, American Samoa, and Northern Marianas, 2007-Con.

[Rates per 100,000 population; age-adjusted rates are per 100,000 U.S. standard population; see "Technical Notes." Populations used for computing death rates are postcensal estimates based on the 2000 census estimated as of July 1, 2007; see "Technical Notes." Numbers after causes of death are categories of the International Classification of Diseases, Tenth Revision (ICD-10). The asterisks (*) preceding the cause-of-death codes indicate that they are not part of ICD-10; see "Technical Notes." For explanation of asterisks preceding cause-of-death codes, see "Technical Notes"]

\begin{tabular}{|c|c|c|c|c|c|c|c|c|c|c|c|c|}
\hline \multirow[b]{2}{*}{ Area } & \multicolumn{3}{|c|}{$\begin{array}{l}\text { Nephritis, nephrotic syndrome } \\
\text { and nephrosis } \\
\text { (N00-N07,N17-N19,N25-N27) }\end{array}$} & \multicolumn{3}{|c|}{$\begin{array}{c}\text { Accidents } \\
\text { (V01-X59,Y85-Y86) }\end{array}$} & \multicolumn{3}{|c|}{$\begin{array}{l}\text { Motor-vehicle } \\
\text { accidents }^{5}\end{array}$} & \multicolumn{3}{|c|}{$\begin{array}{c}\text { Intentional self-harm } \\
\text { (suicide) (*U03,X60-X84,Y87.0) }\end{array}$} \\
\hline & Number & Rate & $\begin{array}{l}\text { Age- } \\
\text { adjusted } \\
\text { rate }^{1}\end{array}$ & Number & Rate & $\begin{array}{l}\text { Age- } \\
\text { adjusted }^{1} \\
\text { rate }^{1}\end{array}$ & Number & Rate & $\begin{array}{l}\text { Age- } \\
\text { adjusted } \\
\text { rate }^{1}\end{array}$ & Number & Rate & $\begin{array}{l}\text { Age- } \\
\text { adjusted } \\
\text { rate }^{1}\end{array}$ \\
\hline United States ${ }^{2}$. & 46,448 & 15.4 & 14.5 & 123,706 & 41.0 & 40.0 & 43,945 & 14.6 & 14.4 & 34,598 & 11.5 & 11.3 \\
\hline Alabama. & 1,051 & 22.7 & 20.7 & 2,542 & 54.9 & 53.9 & 1,212 & 26.2 & 25.9 & 592 & 12.8 & 12.5 \\
\hline$\ldots \ldots$ & 40 & 5.9 & 10.5 & 354 & 51.8 & 55.3 & 107 & 15.7 & 15.2 & 149 & 21.8 & 22.1 \\
\hline$\ldots \ldots$ & 528 & 8.3 & 7.9 & 3,161 & 49.9 & 49.4 & 1,104 & 17.4 & 17.6 & 1,016 & 16.0 & 16.1 \\
\hline Arkansas $\ldots \ldots \ldots \ldots$ & 666 & 23.5 & 20.6 & 1,391 & 49.1 & 47.6 & 675 & 23.8 & 23.7 & 402 & 14.2 & 14.3 \\
\hline California . . . . . . . . & 2,835 & 7.8 & 8.3 & 11,614 & 31.8 & 31.9 & 4,306 & 11.8 & 11.7 & 3,602 & 9.9 & 9.8 \\
\hline Colorado . . . . . . . . & 444 & 9.1 & 10.8 & 2,056 & 42.3 & 44.2 & 593 & 12.2 & 12.3 & 811 & 16.7 & 16.4 \\
\hline Connecticut . . & 566 & 16.2 & 13.4 & 1,343 & 38.3 & 35.8 & 309 & 8.8 & 8.7 & 271 & 7.7 & 7.4 \\
\hline Delaware ... & 163 & 18.8 & 17.0 & 309 & 35.7 & 34.8 & 118 & 13.6 & 13.6 & 95 & 11.0 & 10.7 \\
\hline District of Columbia . . . . . . & 67 & 11.4 & 11.2 & 193 & 32.8 & 32.4 & 54 & 9.2 & 8.9 & 36 & 6.1 & 5.8 \\
\hline Florida $\ldots \ldots \ldots \ldots \ldots$ & 2,923 & 16.0 & 11.4 & 9,113 & 49.9 & 46.5 & 3,329 & 18.2 & 18.1 & 2,587 & 14.2 & 13.3 \\
\hline Georgia $\ldots \ldots \ldots \ldots$ & 1,689 & 17.7 & 21.5 & 4,012 & 42.0 & 44.2 & 1,745 & 18.3 & 18.5 & 997 & 10.4 & 10.7 \\
\hline Hawaii . . . . . . . . . . . . & 176 & 13.7 & 11.1 & 470 & 36.6 & 33.3 & 136 & 10.6 & 10.3 & 133 & 10.4 & 9.7 \\
\hline Idaho $\ldots \ldots \ldots \ldots \ldots$ & 134 & 8.9 & 9.1 & 641 & 42.8 & 43.1 & 273 & 18.2 & 18.5 & 223 & 14.9 & 15.1 \\
\hline$\ldots \ldots \ldots \ldots$ & 2,536 & 19.7 & 19.2 & 4,367 & 34.0 & 33.4 & 1,375 & 10.7 & 10.6 & 1,108 & 8.6 & 8.5 \\
\hline$\ldots \ldots \ldots \ldots$ & 1,293 & 20.4 & 19.3 & 2,499 & 39.4 & 38.7 & 942 & 14.8 & 14.8 & 790 & 12.5 & 12.4 \\
\hline lowa. & 272 & 9.1 & 6.8 & 1,252 & 41.9 & 37.3 & 459 & 15.4 & 15.0 & 322 & 10.8 & 10.6 \\
\hline$\ldots \ldots$ & 554 & 20.0 & 17.5 & 1,205 & 43.4 & 41.2 & 447 & 16.1 & 15.9 & 382 & 13.8 & 13.7 \\
\hline$\ldots \ldots$ & 994 & 23.4 & 22.4 & 2,372 & 55.9 & 55.1 & 853 & 20.1 & 20.0 & 649 & 15.3 & 15.1 \\
\hline Louisiana . . . . . . . . . . . . & 1,152 & 26.8 & 26.9 & 2,466 & 57.4 & 57.6 & 1,036 & 24.1 & 24.0 & 522 & 12.2 & 12.2 \\
\hline$\ldots \ldots \ldots \ldots$ & 269 & 20.4 & 16.5 & 584 & 44.3 & 41.5 & 198 & 15.0 & 14.7 & 191 & 14.5 & 13.7 \\
\hline Maryland. . . . . . . . . . . . . & 731 & 13.0 & 13.1 & 1,480 & 26.3 & 26.2 & 675 & 12.0 & 12.0 & 518 & 9.2 & 9.0 \\
\hline Massachusetts . . . . . . . & 1,361 & 21.1 & 17.9 & 2,139 & 33.2 & 30.8 & 450 & 7.0 & 6.7 & 516 & 8.0 & 7.6 \\
\hline Michigan. . . . . . . . . . . . . & 1,610 & 16.0 & 14.9 & 3,764 & 37.4 & 36.1 & 1,229 & 12.2 & 12.0 & 1,131 & 11.2 & 11.0 \\
\hline Minnesota . . . . . . . . . . & 780 & 15.0 & 13.7 & 2,066 & 39.7 & 37.4 & 618 & 11.9 & 11.7 & 572 & 11.0 & 10.8 \\
\hline Mississippi. . . . . . . . . . . & 697 & 23.9 & 23.4 & 1,808 & 61.9 & 61.9 & 914 & 31.3 & 31.6 & 396 & 13.6 & 13.8 \\
\hline Missouri $\ldots \ldots \ldots \ldots$ & 1,184 & 20.1 & 17.8 & 2,975 & 50.6 & 48.4 & 1,054 & 17.9 & 17.6 & 808 & 13.7 & 13.5 \\
\hline Montana . . . . . . . . . . . & 110 & 11.5 & 9.7 & 614 & 64.1 & 60.2 & 268 & 28.0 & 27.6 & 196 & 20.5 & 19.4 \\
\hline Nebraska . . . . . . . . . . . . & 269 & 15.2 & 12.6 & 674 & 38.0 & 35.7 & 284 & 16.0 & 15.7 & 181 & 10.2 & 10.2 \\
\hline Nevada $\ldots \ldots \ldots \ldots \ldots$ & 461 & 18.0 & 20.6 & 1,212 & 47.2 & 48.4 & 407 & 15.9 & 16.0 & 471 & 18.4 & 18.3 \\
\hline New Hampshire . . . . . . . . & 149 & 11.3 & 10.7 & 527 & 40.1 & 38.5 & 138 & 10.5 & 10.3 & 158 & 12.0 & 11.1 \\
\hline New Jersey. . . . . . . . . . & 1,690 & 19.5 & 17.4 & 2,425 & 27.9 & 26.8 & 719 & 8.3 & 8.2 & 596 & 6.9 & 6.7 \\
\hline New Mexico. . . . . . . . . . . & 252 & 12.8 & 12.3 & 1,329 & 67.5 & 66.7 & 379 & 19.2 & 19.2 & 401 & 20.4 & 20.4 \\
\hline New York ... & 2,387 & 12.4 & 11.0 & 5,160 & 26.7 & 25.3 & 1,478 & 7.7 & 7.4 & 1,396 & 7.2 & 7.0 \\
\hline North Carolina & 1,723 & 19.0 & 19.0 & 4,389 & 48.4 & 48.3 & 1,818 & 20.1 & 20.0 & 1,077 & 11.9 & 11.7 \\
\hline North Dakota. & 56 & 8.8 & 6.2 & 279 & 43.6 & 39.3 & 115 & 18.0 & 17.5 & 95 & 14.9 & 14.4 \\
\hline Ohio $\ldots \ldots \ldots \ldots \ldots$ & 1,747 & 15.2 & 13.5 & 4,922 & 42.9 & 41.1 & 1,399 & 12.2 & 12.1 & 1,295 & 11.3 & 11.0 \\
\hline Oklahoma . . . . . . . . . . . . & 623 & 17.2 & 15.7 & 2,149 & 59.4 & 58.4 & 743 & 20.5 & 20.4 & 531 & 14.7 & 14.7 \\
\hline Oregon. . . . . . . . . . & 426 & 11.4 & 10.2 & 1,646 & 43.9 & 41.5 & 490 & 13.1 & 13.0 & 594 & 15.9 & 15.2 \\
\hline Pennsylvania . & 2,965 & 23.8 & 18.2 & 5,568 & 44.8 & 40.9 & 1,604 & 12.9 & 12.5 & 1,441 & 11.6 & 11.2 \\
\hline Rhode Island $\ldots \ldots \ldots$. . & 167 & 15.8 & 12.7 & 416 & 39.3 & 34.6 & 85 & 8.0 & 7.6 & 96 & 9.1 & 8.7 \\
\hline South Carolina . . . . . . . . . & 806 & 18.3 & 17.3 & 2,364 & 53.6 & 53.0 & 1,062 & 24.1 & 24.2 & 530 & 12.0 & 11.7 \\
\hline South Dakota. . . . . . . . . & 77 & 9.7 & 7.4 & 366 & 46.0 & 41.8 & 149 & 18.7 & 18.3 & 102 & 12.8 & 12.5 \\
\hline Tennessee. . . . . . . . . . . . . & 831 & 13.5 & 13.0 & 3,257 & 52.9 & 52.1 & 1,303 & 21.2 & 21.0 & 844 & 13.7 & 13.3 \\
\hline Texas. . . . . . . . . . . & 3,291 & 13.8 & 16.3 & 9,392 & 39.3 & 41.4 & 3,800 & 15.9 & 16.2 & 2,433 & 10.2 & 10.4 \\
\hline$\ldots \ldots \ldots \ldots$ & 220 & 8.3 & 11.4 & 811 & 30.7 & 34.4 & 320 & 12.1 & 12.4 & 378 & 14.3 & 15.4 \\
\hline Vermont ... & 54 & 8.7 & 7.5 & 303 & 48.8 & 44.7 & 71 & 11.4 & 10.9 & 89 & 14.3 & 13.8 \\
\hline$\ldots \ldots$ & 1,439 & 18.7 & 19.3 & 2,931 & 38.0 & 38.1 & 1,081 & 14.0 & 13.9 & 880 & 11.4 & 11.2 \\
\hline Washington . & 440 & 6.8 & 6.7 & 2,637 & 40.8 & 39.8 & 649 & 10.0 & 9.9 & 865 & 13.4 & 13.0 \\
\hline West Virginia . & 480 & 26.5 & 21.1 & 1,241 & 68.5 & 65.9 & 429 & 23.7 & 23.6 & 300 & 16.6 & 15.9 \\
\hline Wisconsin . . . & 1,002 & 17.9 & 15.6 & 2,619 & 46.8 & 43.8 & 809 & 14.4 & 14.2 & 729 & 13.0 & 12.7 \\
\hline Wyoming . & 68 & 13.0 & 13.1 & 299 & 57.2 & 57.0 & 134 & 25.6 & 25.3 & 101 & 19.3 & 19.7 \\
\hline Puerto Rico ${ }^{3}$. & 1,026 & 26.0 & 25.2 & 1,190 & 30.2 & 29.9 & 443 & 11.2 & 11.0 & 271 & 6.9 & 6.9 \\
\hline Virgin Islands ${ }^{3}$. & 9 & * & * & 32 & 29.1 & 30.7 & 11 & * & * & 11 & * & * \\
\hline Guam $^{3}$. & 25 & 14.4 & 21.7 & 41 & 23.6 & 26.2 & 26 & 15.0 & 16.0 & 28 & 16.1 & 16.0 \\
\hline American Samoa ${ }^{3}$. & 8 & * & * & 12 & * & * & 1 & * & * & 1 & * & * \\
\hline Northern Marianas ${ }^{3} . \ldots \ldots$. & 8 & * & * & 11 & * & * & 3 & * & * & 3 & * & * \\
\hline
\end{tabular}

See footnotes at end of table. 
Table 29. Number of deaths, death rates, and age-adjusted death rates for major causes of death: United States, each state, Puerto Rico, Virgin Islands, Guam, American Samoa, and Northern Marianas, 2007-Con.

[Rates per 100,000 population; age-adjusted rates are per 100,000 U.S. standard population; see "Technical Notes." Populations used for computing death rates are postcensal estimates based on the 2000 census estimated as of July 1, 2007; see "Technical Notes." Numbers after causes of death are categories of the International Classification of Diseases, Tenth Revision (ICD-10). The asterisks $\left(^{*}\right)$ preceding the cause-of-death codes indicate that they are not part of ICD-10; see "Technical Notes." For explanation of asterisks preceding cause-of-death codes, see "Technical Notes"]

\begin{tabular}{|c|c|c|c|c|c|c|c|c|c|c|c|c|}
\hline \multirow[b]{2}{*}{ Area } & \multicolumn{3}{|c|}{$\begin{array}{c}\text { Assault (homicide) } \\
\left({ }^{*} \text { 01- }{ }^{*} \text { 02,X85-Y09,Y87.1) }\right.\end{array}$} & \multicolumn{3}{|c|}{ Alcohol-induced causes ${ }^{6}$} & \multicolumn{3}{|c|}{ Drug-induced causes ${ }^{7}$} & \multicolumn{3}{|c|}{ Injury by firearms ${ }^{8}$} \\
\hline & Number & Rate & $\begin{array}{l}\text { Age- } \\
\text { adjusted } \\
\text { rate }^{1}\end{array}$ & Number & Rate & $\begin{array}{l}\text { Age- } \\
\text { adjusted } \\
\text { rate }^{1}\end{array}$ & Number & Rate & $\begin{array}{l}\text { Age- } \\
\text { adjusted } \\
\text { rate }^{1}\end{array}$ & Number & Rate & $\begin{array}{l}\text { Age- } \\
\text { adjusted } \\
\text { rate }^{1}\end{array}$ \\
\hline United States ${ }^{2}$. & 18,361 & 6.1 & 6.1 & 23,199 & 7.7 & 7.3 & 38,371 & 12.7 & 12.6 & 31,224 & 10.4 & 10.2 \\
\hline Alabama $\ldots \ldots \ldots \ldots$ & 480 & 10.4 & 10.5 & 254 & 5.5 & 5.0 & 554 & 12.0 & 12.1 & 812 & 17.5 & 17.5 \\
\hline Alaska . . . . . . . . . . . . & 50 & 7.3 & 7.1 & 144 & 21.1 & 20.6 & 75 & 11.0 & 10.3 & 120 & 17.6 & 17.8 \\
\hline$\ldots \ldots \ldots \ldots$ & 528 & 8.3 & 8.3 & 747 & 11.8 & 11.9 & 981 & 15.5 & 15.9 & 951 & 15.0 & 15.1 \\
\hline Arkansas & 243 & 8.6 & 8.8 & 173 & 6.1 & 5.6 & 326 & 11.5 & 11.8 & 426 & 15.0 & 15.2 \\
\hline California & 2,376 & 6.5 & 6.3 & 4,027 & 11.0 & 11.1 & 4,178 & 11.4 & 11.3 & 3,268 & 8.9 & 8.8 \\
\hline Colorado. & 173 & 3.6 & 3.4 & 610 & 12.5 & 11.9 & 747 & 15.4 & 14.7 & 505 & 10.4 & 10.3 \\
\hline Connecticut . . . . . . . . & 106 & 3.0 & 3.1 & 214 & 6.1 & 5.5 & 444 & 12.7 & 12.6 & 149 & 4.3 & 4.2 \\
\hline$\ldots \ldots \ldots$ & 48 & 5.6 & 5.7 & 57 & 6.6 & 6.1 & 102 & 11.8 & 12.1 & 79 & 9.1 & 9.0 \\
\hline District of Columbia . . . . . . & 159 & 27.0 & 23.8 & 60 & 10.2 & 9.6 & 90 & 15.3 & 15.1 & 144 & 24.5 & 21.7 \\
\hline 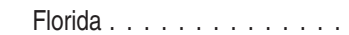 & 1,324 & 7.3 & 7.6 & 1,770 & 9.7 & 8.6 & 2,936 & 16.1 & 16.3 & 2,272 & 12.4 & 12.2 \\
\hline$\ldots \ldots \ldots$ & 771 & 8.1 & 8.0 & 505 & 5.3 & 5.3 & 973 & 10.2 & 10.0 & 1,244 & 13.0 & 13.2 \\
\hline$\ldots \ldots \ldots \ldots$ & 24 & 1.9 & 1.8 & 82 & 6.4 & 5.8 & 142 & 11.1 & 10.8 & 36 & 2.8 & 2.6 \\
\hline$\ldots \ldots \ldots$ & 48 & 3.2 & 3.2 & 152 & 10.1 & 10.0 & 133 & 8.9 & 9.1 & 187 & 12.5 & 12.7 \\
\hline Illinois & 863 & 6.7 & 6.6 & 630 & 4.9 & 4.7 & 1,239 & 9.6 & 9.6 & 1,032 & 8.0 & 7.9 \\
\hline$\ldots \ldots \ldots \ldots$ & 374 & 5.9 & 5.9 & 315 & 5.0 & 4.7 & 827 & 13.0 & 13.1 & 670 & 10.6 & 10.6 \\
\hline lowa . . . . . . . . . . . & 51 & 1.7 & 1.8 & 212 & 7.1 & 6.5 & 211 & 7.1 & 7.1 & 157 & 5.3 & 5.0 \\
\hline Kansas. . . . . . . . . & 115 & 4.1 & 4.2 & 211 & 7.6 & 7.2 & 294 & 10.6 & 10.8 & 292 & 10.5 & 10.3 \\
\hline$\ldots \ldots \ldots$ & 213 & 5.0 & 5.0 & 283 & 6.7 & 6.2 & 722 & 17.0 & 16.9 & 612 & 14.4 & 14.2 \\
\hline$\ldots \ldots \ldots$ & 627 & 14.6 & 14.6 & 202 & 4.7 & 4.5 & 862 & 20.1 & 20.5 & 869 & 20.2 & 20.2 \\
\hline$\ldots \ldots \ldots$ & 26 & 2.0 & 2.0 & 133 & 10.1 & 8.1 & 161 & 12.2 & 12.4 & 107 & 8.1 & 7.6 \\
\hline Maryland. & 573 & 10.2 & 10.4 & 338 & 6.0 & 5.5 & 807 & 14.4 & 14.0 & 678 & 12.1 & 12.1 \\
\hline Massachusetts . . . . . . . & 188 & 2.9 & 3.0 & 440 & 6.8 & 6.1 & 1,003 & 15.6 & 15.1 & 235 & 3.6 & 3.6 \\
\hline Michigan. . . . . . . . . . & 704 & 7.0 & 7.1 & 713 & 7.1 & 6.4 & 1,542 & 15.3 & 15.0 & 1,095 & 10.9 & 10.8 \\
\hline Minnesota . . . . . . . . . . . & 120 & 2.3 & 2.3 & 333 & 6.4 & 6.0 & 359 & 6.9 & 6.7 & 344 & 6.6 & 6.5 \\
\hline Mississippi. . . . . . . . . . . . & 286 & 9.8 & 10.0 & 160 & 5.5 & 5.4 & 334 & 11.4 & 11.7 & 535 & 18.3 & 18.5 \\
\hline Missouri . . . . . . . . & 384 & 6.5 & 6.6 & 324 & 5.5 & 5.2 & 730 & 12.4 & 12.4 & 759 & 12.9 & 12.8 \\
\hline Montana. & 24 & 2.5 & 2.5 & 143 & 14.9 & 13.1 & 132 & 13.8 & 13.4 & 139 & 14.5 & 13.7 \\
\hline Nebraska & 71 & 4.0 & 4.1 & 103 & 5.8 & 5.7 & 92 & 5.2 & 5.4 & 142 & 8.0 & 7.9 \\
\hline Nevada & 188 & 7.3 & 7.4 & 253 & 9.9 & 9.4 & 515 & 20.1 & 19.8 & 414 & 16.1 & 16.3 \\
\hline New Hampshire & 14 & * & * & 120 & 9.1 & 8.0 & 187 & 14.2 & 13.8 & 78 & 5.9 & 5.5 \\
\hline New Jersey . . . . . . . . . & 403 & 4.6 & 4.8 & 414 & 4.8 & 4.3 & 797 & 9.2 & 9.1 & 446 & 5.1 & 5.2 \\
\hline New Mexico. . . . . . . . . . & 160 & 8.1 & 8.2 & 404 & 20.5 & 20.1 & 471 & 23.9 & 24.1 & 295 & 15.0 & 15.0 \\
\hline New York . . . . . . . . . & 840 & 4.4 & 4.4 & 1,167 & 6.0 & 5.5 & 1,909 & 9.9 & 9.6 & 985 & 5.1 & 5.0 \\
\hline North Carolina . . . . . . . . . & 674 & 7.4 & 7.5 & 676 & 7.5 & 7.0 & 1,125 & 12.4 & 12.3 & 1,116 & 12.3 & 12.3 \\
\hline North Dakota . . . . . . . . . & 14 & * & * & 57 & 8.9 & 8.6 & 37 & 5.8 & 5.5 & 57 & 8.9 & 8.6 \\
\hline$\ldots \ldots \ldots$ & 639 & 5.6 & 5.7 & 780 & 6.8 & 6.2 & 1,691 & 14.7 & 14.7 & 1,105 & 9.6 & 9.6 \\
\hline Oklahoma . . . . . . . . . . & 251 & 6.9 & 6.9 & 412 & 11.4 & 11.0 & 687 & 19.0 & 19.5 & 482 & 13.3 & 13.2 \\
\hline Oregon. $\ldots \ldots \ldots \ldots$ & 81 & 2.2 & 2.1 & 543 & 14.5 & 13.0 & 564 & 15.1 & 14.5 & 387 & 10.3 & 9.8 \\
\hline Pennsylvania . . . . . . . . . . & 750 & 6.0 & 6.3 & 548 & 4.4 & 3.9 & 1,812 & 14.6 & 14.5 & 1,325 & 10.7 & 10.6 \\
\hline Rhode Island $\ldots \ldots \ldots \ldots$ & 24 & 2.3 & 2.2 & 93 & 8.8 & 7.9 & 142 & 13.4 & 13.0 & 37 & 3.5 & 3.4 \\
\hline South Carolina . . . . . . . . & 382 & 8.7 & 8.7 & 363 & 8.2 & 7.6 & 584 & 13.2 & 13.1 & 592 & 13.4 & 13.3 \\
\hline South Dakota. . . . . . . . . & 15 & * & * & 81 & 10.2 & 9.8 & 34 & 4.3 & 4.3 & 52 & 6.5 & 6.1 \\
\hline Tennessee. & 475 & 7.7 & 7.8 & 481 & 7.8 & 7.1 & 1,035 & 16.8 & 16.6 & 924 & 15.0 & 14.8 \\
\hline Texas. . . . & 1,495 & 6.3 & 6.2 & 1,360 & 5.7 & 6.0 & 2,343 & 9.8 & 9.9 & 2,561 & 10.7 & 10.9 \\
\hline Utah & 68 & 2.6 & 2.4 & 144 & 5.4 & 6.4 & 546 & 20.6 & 22.4 & 253 & 9.6 & 10.4 \\
\hline Vermont . & 13 & * & * & 63 & 10.1 & 8.1 & 68 & 10.9 & 11.1 & 52 & 8.4 & 8.1 \\
\hline$\ldots \ldots \ldots$ & 420 & 5.4 & 5.4 & 445 & 5.8 & 5.3 & 713 & 9.2 & 9.1 & 825 & 10.7 & 10.5 \\
\hline Washington . . . . & 201 & 3.1 & 3.1 & 718 & 11.1 & 10.3 & 1,003 & 15.5 & 14.8 & 548 & 8.5 & 8.3 \\
\hline West Virginia . . . . & 86 & 4.7 & 4.9 & 165 & 9.1 & 7.9 & 405 & 22.4 & 22.9 & 267 & 14.7 & 14.2 \\
\hline Wisconsin . & 202 & 3.6 & 3.6 & 489 & 8.7 & 7.9 & 639 & 11.4 & 11.3 & 488 & 8.7 & 8.6 \\
\hline Wyoming & 17 & * & * & 88 & 16.8 & 15.6 & 68 & 13.0 & 13.3 & 76 & 14.5 & 15.0 \\
\hline
\end{tabular}

See footnotes at end of table. 
106 National Vital Statistics Reports, Vol. 58, No. 19, May 20, 2010

Table 29. Number of deaths, death rates, and age-adjusted death rates for major causes of death: United States, each state, Puerto Rico, Virgin Islands, Guam, American Samoa, and Northern Marianas, 2007-Con.

[Rates per 100,000 population; age-adjusted rates are per 100,000 U.S. standard population; see "Technical Notes." Populations used for computing death rates are postcensal estimates based on the 2000 census estimated as of July 1, 2007; see "Technical Notes." Numbers after causes of death are categories of the International Classification of Diseases, Tenth Revision (ICD-10). The asterisks $\left(^{*}\right)$ preceding the cause-of-death codes indicate that they are not part of ICD-10; see "Technical Notes." For explanation of asterisks preceding cause-of-death codes, see "Technical Notes"]

\begin{tabular}{|c|c|c|c|c|c|c|c|c|c|c|c|c|}
\hline \multirow[b]{2}{*}{ Area } & \multicolumn{3}{|c|}{$\begin{array}{c}\text { Assault (homicide) } \\
\text { (*U01-*U02,X85-Y09,Y87.1) }\end{array}$} & \multicolumn{3}{|c|}{ Alcohol-induced causes ${ }^{6}$} & \multicolumn{3}{|c|}{ Drug-induced causes ${ }^{7}$} & \multicolumn{3}{|c|}{ Injury by firearms ${ }^{8}$} \\
\hline & Number & Rate & $\begin{array}{l}\text { Age- } \\
\text { adjusted } \\
\text { rate }^{1}\end{array}$ & Number & Rate & $\begin{array}{l}\text { Age- } \\
\text { adjusted } \\
\text { rate }^{1}\end{array}$ & Number & Rate & $\begin{array}{l}\text { Age- } \\
\text { adjusted } \\
\text { rate }^{1}\end{array}$ & Number & Rate & $\begin{array}{c}\text { Age- } \\
\text { adjusted } \\
\text { rate }^{1}\end{array}$ \\
\hline Puerto Rico ${ }^{3}$. & 720 & 18.3 & 18.0 & 204 & 5.2 & 4.8 & 265 & 6.7 & 7.0 & 696 & 17.7 & 17.4 \\
\hline Virgin Islands ${ }^{3}$. & 43 & 39.2 & 42.1 & 22 & 20.0 & 16.3 & 6 & * & * & 38 & 34.6 & 37.6 \\
\hline Guam $^{3} \ldots \ldots \ldots$ & 5 & * & * & 5 & * & * & - & * & * & 5 & * & * \\
\hline American Samoa ${ }^{3} . . . . . .$. & - & * & * & 1 & * & * & - & * & * & - & * & * \\
\hline Northern Marianas ${ }^{3} \ldots \ldots \ldots$ & - & * & * & 3 & * & * & - & * & * & 1 & * & * \\
\hline
\end{tabular}

* Figure does not meet standards of reliability or precision; see "Technical Notes."

- Quantity zero.

${ }^{1}$ Death rates are affected by the population composition of the area. Age-adjusted death rates should be used for comparisons between areas; for method of computation, see "Technical Notes."

2Excludes data for Puerto Rico, Virgin Islands, Guam, American Samoa, and Northern Marianas.

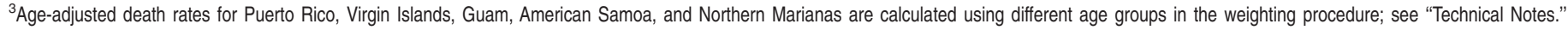

${ }^{4} \mathrm{New}$ ICD-10 code J09 (Influenza due to identified avian influenza virus) was added to the category in 2007. No deaths occurred from this cause in 2007.

${ }^{5} \mathrm{ICD}-10$ codes for Motor vehicle accidents are V02-V04, V09.0, V09.2, V12-V14, V19.0-V19.2, V19.4-V19.6, V20-V79, V80.3-V80.5, V81.0-V81.1, V82.0-V82.1, V83-V86, V87.0-V87.8,

V88.0-V88.8, V89.0, and V89.2; see "Technical Notes."

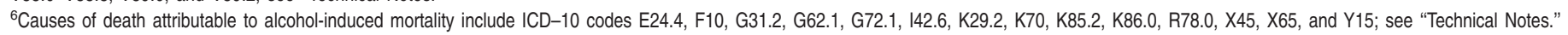

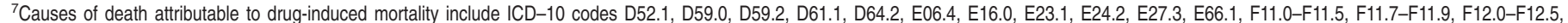

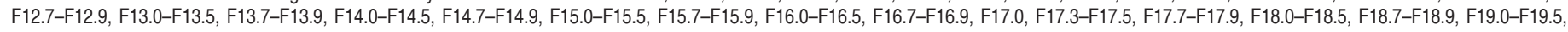

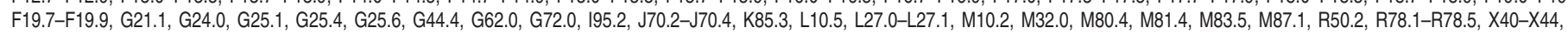

X60-X64, X85, and Y10-Y14; see "Technical Notes."

${ }^{8}$ ICD-10 codes for Injury by firearms are *U01.4, W32-W34, X72-X74, X93-X95, Y22-Y24, and Y35.0; see "Technical Notes." 
Table 30. Infant, neonatal, and postneonatal mortality rates, by race and sex: United States, 1940, 1950, 1960, 1970, and 1975-2007

[Rates are infant (under 1 year), neonatal (under 28 days), and postneonatal (28 days-11 months) deaths per 1,000 live births in specified group. Beginning in 1980, race for live births is tabulated according to race of mother; see "Technical Notes." Race categories are consistent with the 1977 Office of Management and Budget (OMB) standards]

\begin{tabular}{|c|c|c|c|c|c|c|c|c|c|c|c|c|}
\hline \multirow[b]{3}{*}{ Year } & \multirow{2}{*}{\multicolumn{3}{|c|}{ All races }} & \multirow{2}{*}{\multicolumn{3}{|c|}{ White $^{1}$}} & \multicolumn{6}{|c|}{ All other ${ }^{1}$} \\
\hline & & & & & & & \multicolumn{3}{|c|}{ Total $^{1}$} & \multicolumn{3}{|c|}{ Black $^{1}$} \\
\hline & $\begin{array}{l}\text { Both } \\
\text { sexes }\end{array}$ & Male & Female & $\begin{array}{l}\text { Both } \\
\text { sexes }\end{array}$ & Male & Female & $\begin{array}{l}\text { Both } \\
\text { sexes }\end{array}$ & Male & Female & $\begin{array}{l}\text { Both } \\
\text { sexes }\end{array}$ & Male & Female \\
\hline Race of mother ${ }^{2}$ & \multicolumn{12}{|c|}{ Infant mortality rate } \\
\hline 2007 & 6.75 & 7.38 & 6.09 & 5.64 & 6.17 & 5.08 & 10.55 & 11.51 & 9.54 & 13.24 & 14.49 & 11.94 \\
\hline 2006 & 6.69 & 7.32 & 6.03 & 5.56 & 6.10 & 4.99 & 10.60 & 11.54 & 9.61 & 13.29 & 14.38 & 12.16 \\
\hline 2005 & 6.87 & 7.56 & 6.15 & 5.73 & 6.32 & 5.11 & 10.92 & 11.98 & 9.82 & 13.73 & 15.15 & 12.27 \\
\hline 2004 & 6.79 & 7.47 & 6.09 & 5.66 & 6.22 & 5.07 & 10.92 & 12.01 & 9.77 & 13.79 & 15.19 & 12.33 \\
\hline$\ldots \ldots \ldots \ldots \ldots \ldots$ & 6.85 & 7.60 & 6.07 & 5.72 & 6.36 & 5.05 & 11.09 & 12.24 & 9.90 & 14.01 & 15.53 & 12.43 \\
\hline$\ldots \ldots \ldots \ldots \ldots \ldots$ & 6.97 & 7.64 & 6.27 & 5.79 & 6.42 & 5.13 & 11.41 & 12.24 & 10.55 & 14.36 & 15.43 & 13.25 \\
\hline 2001. & 6.85 & 7.52 & 6.14 & 5.65 & 6.21 & 5.06 & 11.33 & 12.44 & 10.18 & 14.02 & 15.48 & 12.52 \\
\hline 2000 & 6.91 & 7.57 & 6.21 & 5.68 & 6.22 & 5.11 & 11.44 & 12.57 & 10.26 & 14.09 & 15.50 & 12.63 \\
\hline 1999 & 7.06 & 7.72 & 6.36 & 5.77 & 6.35 & 5.15 & 11.94 & 12.94 & 10.90 & 14.56 & 15.92 & 13.16 \\
\hline 1998 & 7.20 & 7.83 & 6.54 & 5.95 & 6.47 & 5.41 & 11.92 & 13.01 & 10.79 & 14.31 & 15.75 & 12.82 \\
\hline 1997 & 7.23 & 7.95 & 6.47 & 6.03 & 6.67 & 5.36 & 11.76 & 12.83 & 10.65 & 14.16 & 15.47 & 12.82 \\
\hline 1996 & 7.32 & 8.02 & 6.59 & 6.07 & 6.67 & 5.44 & 12.18 & 13.31 & 11.01 & 14.68 & 16.04 & 13.27 \\
\hline$\ldots \ldots \ldots$ & 7.59 & 8.33 & 6.81 & 6.29 & 6.99 & 5.55 & 12.61 & 13.53 & 11.65 & 15.12 & 16.34 & 13.86 \\
\hline$\ldots \ldots \ldots \ldots \ldots \ldots$ & 8.02 & 8.81 & 7.20 & 6.57 & 7.22 & 5.89 & 13.47 & 14.82 & 12.08 & 15.83 & 17.49 & 14.12 \\
\hline $1993 \ldots \ldots \ldots \ldots \ldots \ldots$ & 8.37 & 9.25 & 7.43 & 6.82 & 7.56 & 6.05 & 14.07 & 15.58 & 12.52 & 16.52 & 18.33 & 14.67 \\
\hline (5) & 8.52 & 9.39 & 7.61 & 6.92 & 7.69 & 6.12 & 14.44 & 15.72 & 13.10 & 16.85 & 18.38 & 15.26 \\
\hline$\ldots \ldots \ldots \ldots \ldots \ldots$ & 8.94 & 10.00 & 7.84 & 7.30 & 8.26 & 6.30 & 15.07 & 16.53 & 13.57 & 17.57 & 19.38 & 15.71 \\
\hline$\ldots \ldots \ldots \ldots \ldots$ & 9.22 & 10.26 & 8.13 & 7.56 & 8.51 & 6.56 & 15.52 & 16.96 & 14.03 & 17.96 & 19.62 & 16.25 \\
\hline$\ldots \ldots \ldots \ldots \ldots \ldots \ldots$ & 9.81 & 10.81 & 8.77 & 8.08 & 9.01 & 7.10 & 16.33 & 17.60 & 15.02 & 18.61 & 20.02 & 17.15 \\
\hline 1988 & 9.95 & 10.99 & 8.86 & 8.36 & 9.35 & 7.31 & 16.08 & 17.33 & 14.79 & 18.54 & 20.04 & 16.99 \\
\hline 1987 & 10.08 & 11.17 & 8.94 & 8.48 & 9.45 & 7.45 & 16.46 & 18.06 & 14.80 & 18.75 & 20.63 & 16.83 \\
\hline$\ldots \ldots \ldots \ldots$ & 10.35 & 11.55 & 9.10 & 8.80 & 9.87 & 7.67 & 16.72 & 18.45 & 14.91 & 18.90 & 20.91 & 16.81 \\
\hline 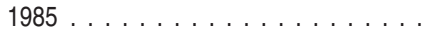 & 10.64 & 11.91 & 9.32 & 9.17 & 10.39 & 7.88 & 16.84 & 18.33 & 15.28 & 19.01 & 20.76 & 17.22 \\
\hline 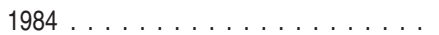 & 10.79 & 11.90 & 9.62 & 9.30 & 10.38 & 8.17 & 17.05 & 18.37 & 15.69 & 19.15 & 20.67 & 17.58 \\
\hline 1983 & 11.16 & 12.31 & 9.96 & 9.61 & 10.66 & 8.49 & 17.80 & 19.44 & 16.11 & 19.98 & 21.95 & 17.96 \\
\hline 1982 & 11.52 & 12.77 & 10.21 & 9.94 & 11.08 & 8.73 & 18.31 & 20.07 & 16.49 & 20.48 & 22.45 & 18.44 \\
\hline 1981 & 11.93 & 13.14 & 10.66 & 10.34 & 11.50 & 9.12 & 18.82 & 20.36 & 17.24 & 20.81 & 22.54 & 19.03 \\
\hline 1980 & 12.60 & 13.93 & 11.21 & 10.86 & 12.12 & 9.52 & 20.19 & 21.89 & 18.43 & 22.19 & 24.16 & 20.15 \\
\hline \multicolumn{13}{|l|}{ Race of child ${ }^{3}$} \\
\hline 1980 & 12.60 & 13.93 & 11.21 & 11.00 & 12.27 & 9.65 & 19.12 & 20.73 & 17.47 & 21.37 & 23.27 & 19.43 \\
\hline$\ldots \ldots \ldots$ & 13.07 & 14.50 & 11.56 & 11.42 & 12.82 & 9.94 & 19.81 & 21.47 & 18.09 & 21.78 & 23.66 & 19.85 \\
\hline$\ldots \ldots \ldots \ldots \ldots \ldots$ & 13.78 & 15.26 & 12.23 & 12.01 & 13.37 & 10.58 & 21.06 & 23.15 & 18.90 & 23.11 & 25.39 & 20.77 \\
\hline 1977 & 14.12 & 15.75 & 12.40 & 12.34 & 13.90 & 10.68 & 21.68 & 23.71 & 19.58 & 23.64 & 25.91 & 21.30 \\
\hline 1976 & 15.24 & 16.82 & 13.57 & 13.31 & 14.81 & 11.71 & 23.50 & 25.51 & 21.42 & 25.54 & 27.83 & 23.19 \\
\hline 1975. & 16.07 & 17.86 & 14.18 & 14.17 & 15.94 & 12.30 & 24.23 & 26.24 & 22.17 & 26.21 & 28.32 & 24.03 \\
\hline 1970 & 20.01 & 22.37 & 17.52 & 17.75 & 19.95 & 15.42 & 30.92 & 34.20 & 27.53 & 32.65 & 36.18 & 29.01 \\
\hline 1960 & 26.04 & 29.33 & 22.59 & 22.91 & 26.01 & 19.64 & 43.21 & 47.88 & 38.46 & 44.32 & 49.12 & 39.43 \\
\hline 1950 & 29.21 & 32.75 & 25.48 & 26.77 & 30.21 & 23.13 & 44.46 & 48.87 & 39.93 & 43.91 & 48.27 & 39.44 \\
\hline 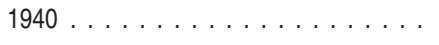 & 47.02 & 52.45 & 41.29 & 43.23 & 48.32 & 37.84 & 73.78 & 82.21 & 65.19 & 72.94 & 81.07 & 64.61 \\
\hline Race of mother ${ }^{2}$ & \multicolumn{12}{|c|}{ Neonatal mortality rate } \\
\hline 2007 & 4.42 & 4.79 & 4.02 & 3.70 & 4.01 & 3.37 & 6.86 & 7.49 & 6.22 & 8.65 & 9.48 & 7.78 \\
\hline 2006 & 4.45 & 4.84 & 4.05 & 3.72 & 4.05 & 3.37 & 7.00 & 7.58 & 6.40 & 8.82 & 9.49 & 8.12 \\
\hline 2005 & 4.54 & 4.93 & 4.12 & 3.79 & 4.10 & 3.46 & 7.18 & 7.88 & 6.47 & 9.07 & 9.96 & 8.14 \\
\hline 2004 & 4.52 & 4.94 & 4.09 & 3.78 & 4.14 & 3.41 & 7.19 & 7.82 & 6.54 & 9.13 & 9.95 & 8.27 \\
\hline 2003 & 4.62 & 5.08 & 4.14 & 3.87 & 4.26 & 3.46 & 7.40 & 8.14 & 6.64 & 9.40 & 10.40 & 8.37 \\
\hline 2002 & 4.66 & 5.06 & 4.25 & 3.89 & 4.27 & 3.50 & 7.55 & 8.03 & 7.05 & 9.51 & 10.13 & 8.87 \\
\hline 2001. & 4.54 & 4.97 & 4.08 & 3.78 & 4.15 & 3.39 & 7.37 & 8.06 & 6.65 & 9.21 & 10.15 & 8.25 \\
\hline $2000 \ldots$ & 4.63 & 5.06 & 4.17 & 3.82 & 4.16 & 3.46 & 7.60 & 8.39 & 6.79 & 9.38 & 10.39 & 8.35 \\
\hline $1999 \ldots \ldots$ & 4.73 & 5.11 & 4.33 & 3.88 & 4.19 & 3.56 & 7.94 & 8.60 & 7.25 & 9.77 & 10.72 & 8.79 \\
\hline 1998 & 4.80 & 5.21 & 4.37 & 3.98 & 4.31 & 3.63 & 7.91 & 8.63 & 7.17 & 9.55 & 10.51 & 8.56 \\
\hline 1997 & 4.77 & 5.20 & 4.32 & 3.99 & 4.37 & 3.59 & 7.74 & 8.36 & 7.09 & 9.40 & 10.12 & 8.65 \\
\hline 1996 & 4.77 & 5.18 & 4.34 & 3.97 & 4.31 & 3.62 & 7.86 & 8.59 & 7.12 & 9.56 & 10.45 & 8.65 \\
\hline 1995 & 4.91 & 5.36 & 4.44 & 4.08 & 4.50 & 3.64 & 8.13 & 8.71 & 7.53 & 9.85 & 10.63 & 9.05 \\
\hline 1994 & 5.12 & 5.58 & 4.64 & 4.20 & 4.55 & 3.83 & 8.60 & 9.51 & 7.65 & 10.21 & 11.32 & 9.07 \\
\hline 1993 & 5.29 & 5.75 & 4.81 & 4.29 & 4.64 & 3.92 & 9.02 & 9.90 & 8.11 & 10.69 & 11.76 & 9.59 \\
\hline 1992 & 5.37 & 5.84 & 4.89 & 4.35 & 4.72 & 3.96 & 9.19 & 10.02 & 8.32 & 10.83 & 11.83 & 9.79 \\
\hline
\end{tabular}

See footnotes at end of table. 
108 National Vital Statistics Reports, Vol. 58, No. 19, May 20, 2010

Table 30. Infant, neonatal, and postneonatal mortality rates, by race and sex: United States, 1940, 1950, 1960, 1970, and 1975-2007-Con.

[Rates are infant (under 1 year), neonatal (under 28 days), and postneonatal (28 days-11 months) deaths per 1,000 live births in specified group. Beginning in 1980, race for live births is tabulated according to race of mother; see "Technical Notes." Race categories are consistent with the 1977 Office of Management and Budget (OMB) standards]

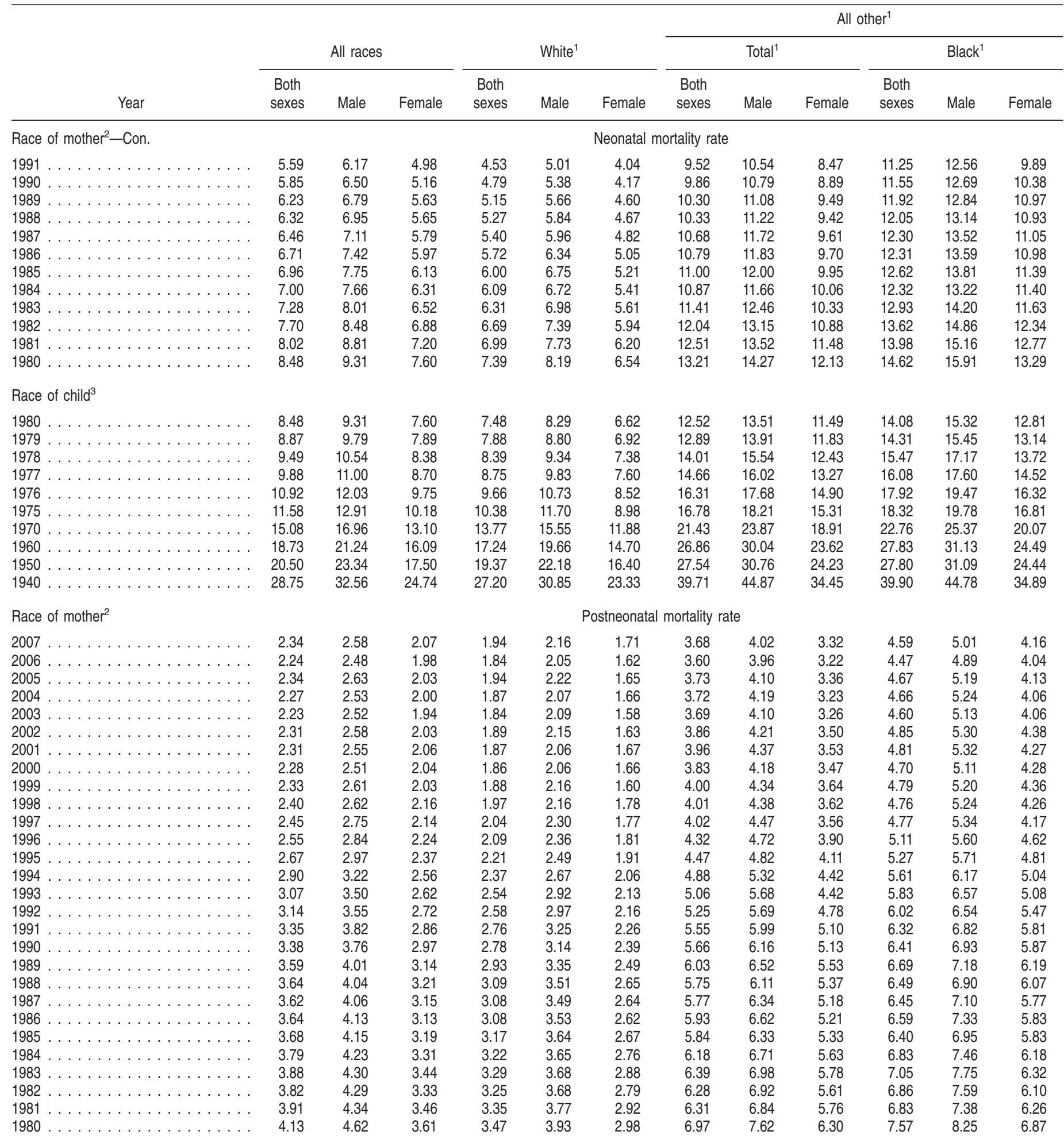

See footnotes at end of table. 
Table 30. Infant, neonatal, and postneonatal mortality rates, by race and sex: United States, 1940, 1950, 1960, 1970, and 1975-2007-Con.

[Rates are infant (under 1 year), neonatal (under 28 days), and postneonatal (28 days-11 months) deaths per 1,000 live births in specified group. Beginning in 1980, race for live births is tabulated according to race of mother; see "Technical Notes." Race categories are consistent with the 1977 Office of Management and Budget (OMB) standards]

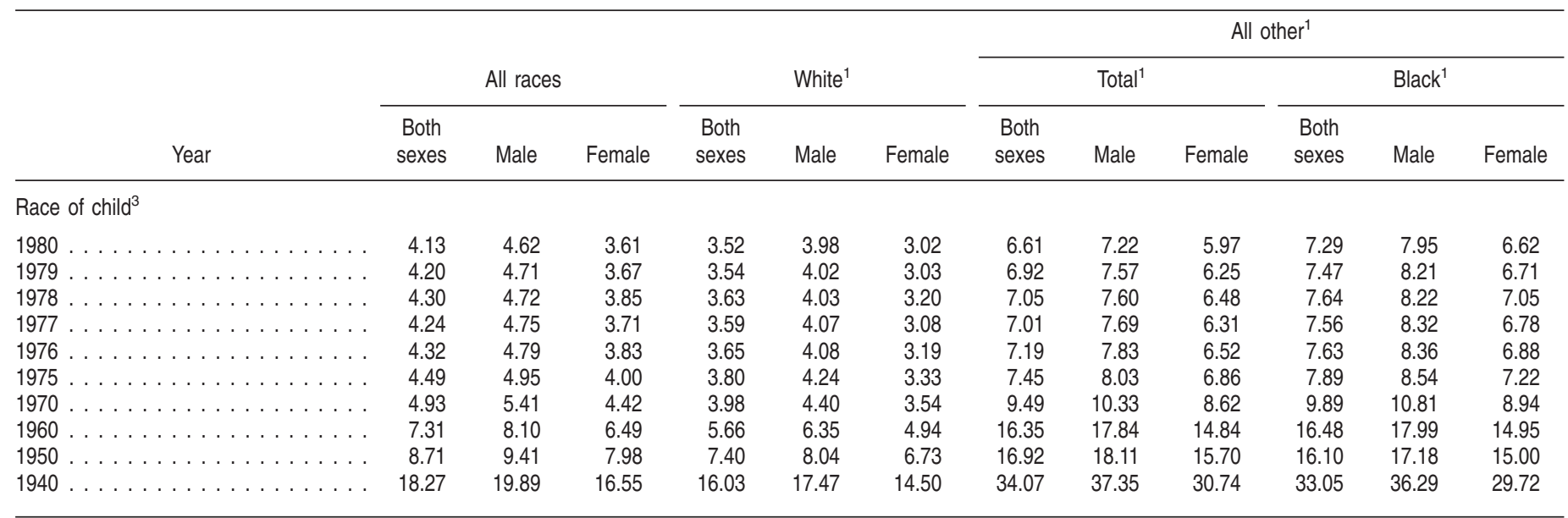

${ }^{1}$ Multiple-race data were reported for deaths by 27 states and the District of Columbia in 2007, by 25 states and the District of Columbia in 2006, by 21 states and the District of Columbia in 2005, by 15 states in 2004, and by 7 states in 2003; see "Technical Notes." Multiple-race data were reported for births by 27 areas in 2007, by 23 areas in 2006, by 19 areas in 2005, by 15 areas in 2004, and by 6 areas in 2003; see "Technical Notes." The multiple-race data for these reporting areas were bridged to the single-race categories of the 1977 OMB standards for comparability with other reporting areas; see "Technical Notes."

2Infant deaths are based on race of child as stated on the death certificate; live births are based on race of mother as stated on the birth certificate; see "Technical Notes."

${ }^{3}$ Infant deaths are based on race of child as stated on the death certificate; live births are based on race of parents as stated on the birth certificate; see "Technical Notes." 
Table 31. Number of infant deaths and infant mortality rates for 130 selected causes, by race: United States, 2007

[Rates are infant deaths (under 1 year) per 100,000 live births in specified group. Infant deaths are based on race of decedent; live births are based on race of mother. The asterisks $\left(^{*}\right)$ preceding the cause-of-death codes indicate that they are not part of the International Classification of Diseases, Tenth Revision (ICD-10), Second Edition; see "Technical Notes"]

\begin{tabular}{|c|c|c|c|c|c|c|}
\hline \multirow[b]{2}{*}{ Cause of death (based on ICD-10, 2004) } & \multicolumn{3}{|c|}{ Number } & \multicolumn{3}{|c|}{ Rate } \\
\hline & $\begin{array}{c}\text { All } \\
\text { races }^{1}\end{array}$ & White $^{2}$ & Black $^{2}$ & $\begin{array}{c}\text { All } \\
\text { races }^{1}\end{array}$ & White $^{2}$ & Black $^{2}$ \\
\hline All causes & 29,138 & 18,807 & 8,944 & 675.1 & 563.7 & $1,323.7$ \\
\hline Certain infectious and parasitic diseases & 482 & 293 & 170 & 11.2 & 8.8 & 25.2 \\
\hline Certain intestinal infectious diseases. & 13 & 7 & 4 & * & * & * \\
\hline Diarrhea and gastroenteritis of infectious origin $\ldots \ldots \ldots \ldots \ldots$ & - & - & - & * & * & * \\
\hline Tuberculosis . . . . . . . . . . . . . . . . . . . . . . (A16-A19) & 2 & 1 & 1 & * & * & * \\
\hline 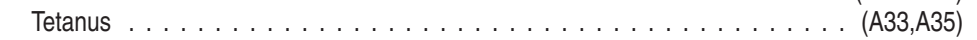 & - & - & - & * & * & * \\
\hline 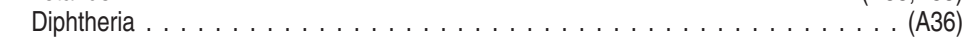 & - & - & - & * & * & * \\
\hline 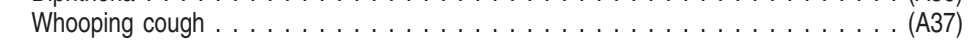 & 8 & 8 & - & * & * & * \\
\hline Meningococcal infection & 11 & 7 & 4 & * & * & * \\
\hline Septicemia . . . . . . . & 283 & 158 & 116 & 6.6 & 4.7 & 17.2 \\
\hline Congenital syphilis & 5 & 2 & 3 & * & * & * \\
\hline$\ldots \ldots \ldots \ldots \ldots$ (A54) & - & - & - & * & * & * \\
\hline$\ldots \ldots \ldots \ldots \ldots($ A80-B34) & 115 & 81 & 29 & 2.7 & 2.4 & 4.3 \\
\hline 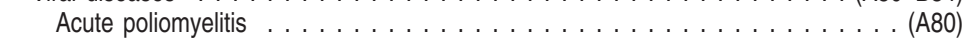 & - & - & - & * & * & * \\
\hline 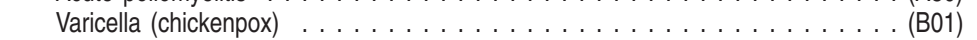 & - & - & - & * & * & * \\
\hline Measles . . . . . . . . . . . . . . . & - & - & - & * & * & * \\
\hline Human immunodeficiency virus (HIV) disease & 5 & 2 & 1 & * & * & * \\
\hline Mumps $\ldots \ldots \ldots \ldots \ldots \ldots \ldots$. & - & - & - & * & * & * \\
\hline Other and unspecified viral diseases $\ldots$. (A81-B00,B02-B04,B06-B19,B25,B27-B34) & 110 & 79 & 28 & 2.5 & 2.4 & 4.1 \\
\hline 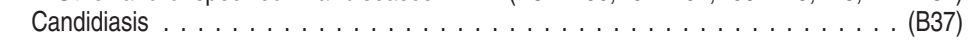 & 13 & 8 & 4 & * & * & * \\
\hline 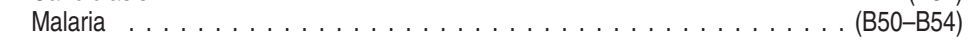 & - & - & - & * & * & * \\
\hline 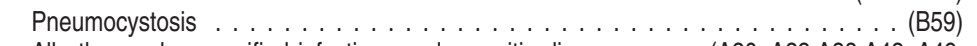 & 1 & - & 1 & * & * & * \\
\hline All other and unspecified infectious and parasitic diseases . . . (A20-A32,A38,A42-A49, & & & & & & \\
\hline A51-A53,A55-A79,B35-B36,B38-B49,B55-B58,B60-B99) & 31 & 21 & 8 & 0.7 & 0.6 & * \\
\hline$\ldots \ldots \ldots \ldots \ldots \ldots \ldots \ldots \ldots \ldots \ldots \ldots \ldots \ldots \ldots \ldots \ldots$ & 131 & 107 & 17 & 3.0 & 3.2 & * \\
\hline Malignant neoplasms & 72 & 60 & 9 & 1.7 & 1.8 & * \\
\hline Hodgkin's disease and non-Hodgkin's lymphomas. & 2 & 2 & - & * & * & * \\
\hline Leukemia . . . . . . . . . . . . . . . . . . . . . . . . (C91-C95) & 21 & 19 & 1 & 0.5 & * & * \\
\hline Other and unspecified malignant neoplasms . . . . . (C00-C80,C88,C90,C96-C97) & 49 & 39 & 8 & 1.1 & 1.2 & * \\
\hline In situ neoplasms, benign neoplasms and neoplasms of uncertain or & & & & & & \\
\hline unknown behavior $\ldots \ldots \ldots \ldots \ldots \ldots \ldots \ldots$ & 59 & 47 & 8 & 1.4 & 1.4 & * \\
\hline Diseases of the blood and blood-forming organs and certain disorders & & & & & & \\
\hline involving the immune mechanism $\ldots \ldots \ldots \ldots \ldots \ldots \ldots \ldots \ldots \ldots \ldots \ldots$ & 116 & 78 & 24 & 2.7 & 2.3 & 3.6 \\
\hline 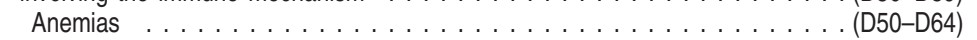 & 17 & 4 & 7 & * & * & * \\
\hline Hemorrhagic conditions and other diseases of blood and & & & & & & \\
\hline blood-forming organs $\ldots \ldots \ldots \ldots \ldots \ldots$ & 77 & 56 & 14 & 1.8 & 1.7 & * \\
\hline Certain disorders involving the immune mechanism $\ldots \ldots \ldots \ldots \ldots \ldots$ (D80-D89) & 22 & 18 & 3 & 0.5 & * & * \\
\hline 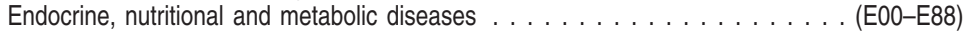 & 252 & 171 & 63 & 5.8 & 5.1 & 9.3 \\
\hline Short stature, not elsewhere classified $\ldots \ldots \ldots \ldots \ldots$ & 5 & 2 & 3 & * & * & * \\
\hline Nutritional deficiencies $\ldots \ldots \ldots \ldots \ldots \ldots$ (E40-E64) & 7 & 4 & 3 & * & * & * \\
\hline Cystic fibrosis ....... & 11 & 9 & 1 & * & * & * \\
\hline Volume depletion, disorders of fluid, electrolyte and acid-base balance. . . & 60 & 30 & 27 & 1.4 & 0.9 & 4.0 \\
\hline All other endocrine, nutritional and metabolic diseases ...... (E00-E32,E34.0-E34.2, & & & & & & \\
\hline Diseases of the nervous system $\ldots \ldots \ldots \ldots \ldots \ldots \ldots \ldots \ldots \ldots$ E34.4-E34.9,E65-E83,E85,E88) & $\begin{array}{l}169 \\
413\end{array}$ & $\begin{array}{l}126 \\
282\end{array}$ & $\begin{array}{r}29 \\
103\end{array}$ & $\begin{array}{l}3.9 \\
9.6\end{array}$ & $\begin{array}{l}3.8 \\
8.5\end{array}$ & $\begin{array}{r}4.3 \\
15.2\end{array}$ \\
\hline 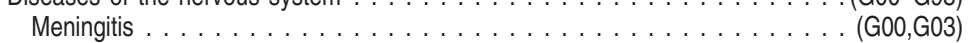 & 82 & 51 & 23 & 1.9 & 1.5 & 3.4 \\
\hline Infantile spinal muscular atrophy, type I (Werdnig-Hoffman) . . . . . . . . . . (G12.0) & 13 & 12 & 1 & * & * & * \\
\hline Infantile cerebral palsy .. & 11 & 8 & 2 & * & * & * \\
\hline Anoxic brain damage, not elsewhere classified & 64 & 30 & 31 & 1.5 & 0.9 & 4.6 \\
\hline Other diseases of nervous system $\ldots \ldots \ldots$ & & & & & & \\
\hline G81-G92,G93.0,G93.2-G93.9,G95-G98) & 243 & 181 & 46 & 5.6 & 5.4 & 6.8 \\
\hline Diseases of the ear and mastoid process & 3 & 3 & - & * & * & \\
\hline Diseases of the circulatory system $\ldots \ldots \ldots \ldots \ldots$. . . . . . . . . . . . . . . . & 624 & 399 & 187 & 14.5 & 12.0 & 27.7 \\
\hline Pulmonary heart disease and diseases of pulmonary circulation . . . . . . . . (I26-I28) & 100 & 61 & 30 & 2.3 & 1.8 & 4.4 \\
\hline Pericarditis, endocarditis and myocarditis $\ldots \ldots \ldots \ldots \ldots \ldots \ldots(130,|33| 40)$, & 21 & 10 & 10 & 0.5 & * & * \\
\hline Cardiomyopathy $\ldots \ldots \ldots \ldots \ldots \ldots$ & 120 & 74 & 38 & 2.8 & 2.2 & 5.6 \\
\hline Cardiac arrest. & 29 & 17 & 12 & 0.7 & * & * \\
\hline Cerebrovascular diseases & 132 & 94 & 34 & 3.1 & 2.8 & 5.0 \\
\hline All other diseases of circulatory system . . . (100-125,|31,|34-|38,|144-|45,|47-|51,|170-199) & 222 & 143 & 63 & 5.1 & 4.3 & 9.3 \\
\hline Diseases of the respiratory system $\ldots \ldots \ldots \ldots \ldots \ldots \ldots \ldots \ldots$ & 640 & 382 & 221 & 14.8 & 11.4 & 32.7 \\
\hline
\end{tabular}

See footnotes at end of table. 
Table 31. Number of infant deaths and infant mortality rates for 130 selected causes, by race: United States, 2007-Con.

[Rates are infant deaths (under 1 year) per 100,000 live births in specified group. Infant deaths are based on race of decedent; live births are based on race of mother. The asterisks $\left({ }^{*}\right)$ preceding the cause-of-death codes indicate that they are not part of the International Classification of Diseases, Tenth Revision (ICD-10), Second Edition; see "Technical Notes"]

\begin{tabular}{|c|c|c|c|c|c|c|}
\hline \multirow[b]{2}{*}{ Cause of death (based on ICD-10, 2004) } & \multicolumn{3}{|c|}{ Number } & \multicolumn{3}{|c|}{ Rate } \\
\hline & $\begin{array}{c}\text { All } \\
\text { races }^{1}\end{array}$ & White $^{2}$ & Black $^{2}$ & $\begin{array}{c}\text { All } \\
\text { races }^{1}\end{array}$ & White $^{2}$ & Black $^{2}$ \\
\hline Acute upper respiratory infections. & 14 & 8 & 5 & * & * & * \\
\hline$\ldots \ldots \ldots \ldots \ldots .(\mathrm{J} 09-\mathrm{J} 18)^{3}$ & 222 & 132 & 75 & 5.1 & 4.0 & 11.1 \\
\hline$\ldots \ldots \ldots \ldots \ldots \ldots \ldots \ldots$ & 13 & 9 & 4 & * & * & * \\
\hline$\ldots \ldots \ldots \ldots \ldots \ldots \ldots \ldots \ldots \ldots \ldots \ldots \ldots$ & 209 & 123 & 71 & 4.8 & 3.7 & 10.5 \\
\hline 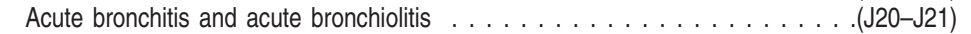 & 45 & 28 & 15 & 1.0 & 0.8 & * \\
\hline Bronchitis, chronic and unspecified $\ldots \ldots \ldots \ldots \ldots \ldots \ldots \ldots \ldots \ldots \ldots \ldots \ldots \ldots \ldots \ldots \ldots \ldots$ (J40-J42) & 24 & 13 & 9 & 0.6 & * & * \\
\hline Asthma . . . . . . . . . . . . . . & 4 & 2 & 2 & * & * & * \\
\hline Pneumonitis due to solids and liquids $\ldots \ldots \ldots$. & 10 & 7 & 3 & * & * & * \\
\hline \multicolumn{7}{|l|}{ Other and unspecified diseases of respiratory system . . . . . . . . . (J22,J30-J39, } \\
\hline J43-J44,J47-J68,J70-J98,U04) ${ }^{4}$ & 321 & 192 & 112 & 7.4 & 5.8 & 16.6 \\
\hline Diseases of the digestive system & 677 & 383 & 260 & 15.7 & 11.5 & 38.5 \\
\hline Gastritis, duodenitis, and noninfective enteritis and colitis . . . . . . (K29,K50-K55) & 413 & 220 & 173 & 9.6 & 6.6 & 25.6 \\
\hline Hernia of abdominal cavity and intestinal obstruction without hernia $\ldots(\mathrm{K} 40-\mathrm{K} 46, \mathrm{~K} 56)$ & 68 & 48 & 17 & 1.6 & 1.4 & * \\
\hline All other and unspecified diseases of digestive system ....(K00-K28,K30-K38,K57-K92) & 196 & 115 & 70 & 4.5 & 3.4 & 10.4 \\
\hline Diseases of the genitourinary system & 169 & 105 & 61 & 3.9 & 3.1 & 9.0 \\
\hline Renal failure and other disorders of kidney & 138 & 89 & 46 & 3.2 & 2.7 & 6.8 \\
\hline \multicolumn{7}{|l|}{ Other and unspecified diseases of genitourinary system } \\
\hline N26,N28-N95) & 31 & 16 & 15 & 0.7 & * & * \\
\hline Certain conditions originating in the perinatal period & 14,466 & 8,801 & 5,013 & 335.2 & 263.8 & 741.9 \\
\hline \multicolumn{7}{|l|}{ Newborn affected by maternal factors and by complications of pregnancy, } \\
\hline 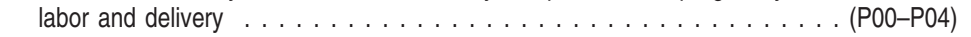 & 3,274 & 2,036 & 1,099 & 75.9 & 61.0 & 162.7 \\
\hline Newborn affected by maternal hypertensive disorders $\ldots \ldots \ldots \ldots \ldots$ (P00.0) & 89 & 53 & 33 & 2.1 & 1.6 & 4.9 \\
\hline \multicolumn{7}{|l|}{ Newborn affected by other maternal conditions which may be unrelated } \\
\hline$\ldots \ldots \ldots \ldots \ldots \ldots \ldots \ldots \ldots .1-P 00.9)$ & 91 & 59 & 26 & 2.1 & 1.8 & 3.8 \\
\hline Newborn affected by maternal complications of pregnancy & 1,769 & 1,067 & 624 & 41.0 & 32.0 & 92.4 \\
\hline Newborn affected by incompetent cervix . . . . . . . . . . . . . & 488 & 266 & 192 & 11.3 & 8.0 & 28.4 \\
\hline Newborn affected by premature rupture of membranes $\ldots \ldots \ldots \ldots \ldots$ (P01.1) & 851 & 507 & 311 & 19.7 & 15.2 & 46.0 \\
\hline Newborn affected by multiple pregnancy $\ldots \ldots \ldots \ldots \ldots$. . . . . . . . . . . . & 238 & 160 & 72 & 5.5 & 4.8 & 10.7 \\
\hline \multicolumn{7}{|l|}{ Newborn affected by other maternal complications of } \\
\hline pregnancy ................... & 192 & 134 & 49 & 4.4 & 4.0 & 7.3 \\
\hline Newborn affected by complications of placenta, cord and membranes . . . & 1,135 & 735 & 358 & 26.3 & 22.0 & 53.0 \\
\hline Newborn affected by complications involving placenta . . . . . . . . (P02.0-P02.3) & 579 & 411 & 149 & 13.4 & 12.3 & 22.1 \\
\hline Newborn affected by complications involving cord & 43 & 32 & 10 & 1.0 & 1.0 & * \\
\hline Newborn affected by chorioamnionitis $\ldots . . .$. & 511 & 290 & 199 & 11.8 & 8.7 & 29.5 \\
\hline \multicolumn{7}{|l|}{ Newborn affected by other and unspecified abnormalities of } \\
\hline membranes $\ldots \ldots \ldots \ldots \ldots \ldots \ldots \ldots$ & 2 & 2 & - & * & * & * \\
\hline Newborn affected by other complications of labor and delivery $\ldots \ldots \ldots \ldots$ (P03) & 127 & 86 & 34 & 2.9 & 2.6 & 5.0 \\
\hline \multicolumn{7}{|l|}{ Newborn affected by noxious influences transmitted via placenta or } \\
\hline breast milk $\ldots \ldots \ldots \ldots \ldots \ldots \ldots \ldots \ldots \ldots \ldots$ & 63 & 36 & 24 & 1.5 & 1.1 & 3.6 \\
\hline Disorders related to length of gestation and fetal malnutrition . . . . . & 4,961 & 2,783 & 1,960 & 114.9 & 83.4 & 290.1 \\
\hline Slow fetal growth and fetal malnutrition $\ldots \ldots$ & 104 & 65 & 35 & 2.4 & 1.9 & 5.2 \\
\hline \multicolumn{7}{|l|}{ Disorders related to short gestation and low birth weight, not elsewhere } \\
\hline 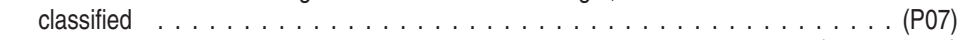 & 4,857 & 2,718 & 1,925 & 112.5 & 81.5 & 284.9 \\
\hline Extremely low birth weight or extreme immaturity $\ldots \ldots \ldots \ldots$ (P07.0,P07.2) & 3,706 & 2,061 & 1,484 & 85.9 & 61.8 & 219.6 \\
\hline Other low birth weight or preterm $\ldots \ldots \ldots \ldots \ldots$ (P07.1,P07.3) & 1,151 & 657 & 441 & 26.7 & 19.7 & 65.3 \\
\hline Disorders related to long gestation and high birth weight $\ldots \ldots \ldots \ldots$ (P08) & - & - & - & * & * & * \\
\hline Birth trauma $\ldots \ldots \ldots \ldots \ldots \ldots \ldots \ldots \ldots \ldots$ & 12 & 8 & 2 & * & * & * \\
\hline Intrauterine hypoxia and birth asphyxia & 356 & 240 & 100 & 8.2 & 7.2 & 14.8 \\
\hline Intrauterine hypoxia . . . . . . . . . & 106 & 63 & 34 & 2.5 & 1.9 & 5.0 \\
\hline Birth asphyxia .... & 250 & 177 & 66 & 5.8 & 5.3 & 9.8 \\
\hline Respiratory distress of newborn. & 789 & 479 & 280 & 18.3 & 14.4 & 41.4 \\
\hline Other respiratory conditions originating in the perinatal period & 1,117 & 701 & 372 & 25.9 & 21.0 & 55.1 \\
\hline 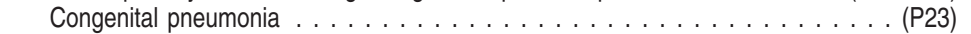 & 103 & 67 & 30 & 2.4 & 2.0 & 4.4 \\
\hline Neonatal aspiration syndromes. & 51 & 36 & 10 & 1.2 & 1.1 & \\
\hline \multicolumn{7}{|l|}{ Interstitial emphysema and related conditions originating in the perinatal } \\
\hline$\ldots(\mathrm{P} 25)$ & 124 & 83 & 37 & 2.9 & 2.5 & 5.5 \\
\hline Pulmonary hemorrhage originating in the perinatal period & 161 & 88 & 68 & 3.7 & 2.6 & 10.1 \\
\hline Chronic respiratory disease originating in the perinatal period $\ldots \ldots \ldots$ & 243 & 133 & 101 & 5.6 & 4.0 & 14.9 \\
\hline Atelectasis $\ldots \ldots \ldots \ldots \ldots \ldots \ldots \ldots \ldots \ldots$ & 366 & 256 & 100 & 8.5 & 7.7 & 14.8 \\
\hline All other respiratory conditions originating in the perinatal period $\ldots \ldots$ (P28.2-P28.9) & 69 & 38 & 26 & 1.6 & 1.1 & 3.8 \\
\hline Infections specific to the perinatal period $\ldots \ldots \ldots \ldots \ldots \ldots \ldots$ (P35-P39) & 1,057 & 661 & 344 & 24.5 & 19.8 & 50.9 \\
\hline
\end{tabular}

See footnotes at end of table. 
Table 31. Number of infant deaths and infant mortality rates for 130 selected causes, by race: United States, 2007-Con.

[Rates are infant deaths (under 1 year) per 100,000 live births in specified group. Infant deaths are based on race of decedent; live births are based on race of mother. The asterisks $\left({ }^{*}\right)$ preceding the cause-of-death codes indicate that they are not part of the International Classification of Diseases, Tenth Revision (ICD-10), Second Edition; see "Technical Notes"]

\begin{tabular}{|c|c|c|c|c|c|c|}
\hline \multirow[b]{2}{*}{ Cause of death (based on ICD-10, 2004) } & \multicolumn{3}{|c|}{ Number } & \multicolumn{3}{|c|}{ Rate } \\
\hline & $\begin{array}{c}\text { All } \\
\text { races }^{1}\end{array}$ & White ${ }^{2}$ & Black $^{2}$ & $\begin{array}{c}\text { All } \\
\text { races }^{1}\end{array}$ & White $^{2}$ & Black $^{2}$ \\
\hline Bacterial sepsis of newborn & 820 & 516 & 264 & 19.0 & 15.5 & 39.1 \\
\hline Omphalitis of newborn with or without mild hemorrhage $\ldots \ldots \ldots \ldots$ & 4 & 2 & 1 & * & * & * \\
\hline All other infections specific to the perinatal period $\ldots \ldots \ldots \ldots \ldots$ (P35,P37,P39) & 233 & 143 & 79 & 5.4 & 4.3 & 11.7 \\
\hline Hemorrhagic and hematological disorders of newborn . . . . . . . . . . (P50-P61) & 711 & 501 & 171 & 16.5 & 15.0 & 25.3 \\
\hline Neonatal hemorrhage $\ldots \ldots \ldots \ldots \ldots \ldots$ & 597 & 422 & 148 & 13.8 & 12.6 & 21.9 \\
\hline Hemorrhagic disease of newborn & - & - & - & * & * & * \\
\hline \multicolumn{7}{|l|}{ Hemolytic disease of newborn due to isoimmunization and other } \\
\hline perinatal jaundice & 15 & 8 & 5 & * & * & * \\
\hline Hematological disorders & 99 & 71 & 18 & 2.3 & 2.1 & * \\
\hline Syndrome of infant of a diabetic mother and neonatal diabetes mellitus . . (P70.0-P70.2) & 14 & 9 & 4 & * & * & * \\
\hline Necrotizing enterocolitis of newborn $\ldots \ldots \ldots \ldots \ldots \ldots \ldots \ldots$ (P77) & 554 & 310 & 218 & 12.8 & 9.3 & 32.3 \\
\hline Hydrops fetalis not due to hemolytic disease . . . . & 177 & 141 & 23 & 4.1 & 4.2 & 3.4 \\
\hline \multirow{2}{*}{\multicolumn{7}{|c|}{$\begin{array}{r}\text { Other perinatal conditions } \ldots \ldots \ldots(\text { P29,P70.3-P70.9,P71-P76,P78-P81,P83.0-P83.1, } \\
\text { P83.3-P83.9,P90-P96) }\end{array}$}} \\
\hline & 1,444 & 932 & 440 & 33.5 & 27.9 & 65.1 \\
\hline Congenital malformations, deformations and chromosomal abnormalities . . . . (Q00-Q99) & 5,785 & 4,351 & 1,137 & 134.0 & 130.4 & 168.3 \\
\hline Anencephaly and similar malformations $\ldots \ldots \ldots \ldots \ldots \ldots$ & 321 & 261 & 46 & 7.4 & 7.8 & 6.8 \\
\hline Congenital hydrocephalus $\ldots \ldots \ldots \ldots \ldots$. . . . & 93 & 64 & 21 & 2.2 & 1.9 & 3.1 \\
\hline Spina bifida . . . . . . . & 19 & 12 & 5 & * & * & * \\
\hline Other congenital malformations of nervous system & 393 & 310 & 63 & 9.1 & 9.3 & 9.3 \\
\hline Congenital malformations of heart $\ldots \ldots \ldots \ldots$ & 1,363 & 1,010 & 282 & 31.6 & 30.3 & 41.7 \\
\hline Other congenital malformations of circulatory system $\ldots \ldots \ldots \ldots \ldots \ldots$ (Q25-Q28) & 254 & 174 & 67 & 5.9 & 5.2 & 9.9 \\
\hline Congenital malformations of respiratory system $\ldots \ldots \ldots \ldots \ldots$ & 410 & 304 & 85 & 9.5 & 9.1 & 12.6 \\
\hline Congenital malformations of digestive system . . . . & 132 & 93 & 31 & 3.1 & 2.8 & 4.6 \\
\hline Congenital malformations of genitourinary system . & 514 & 395 & 105 & 11.9 & 11.8 & 15.5 \\
\hline \multicolumn{7}{|l|}{ Congenital malformations and deformations of musculoskeletal system, limbs } \\
\hline and integument $\ldots \ldots \ldots \ldots \ldots \ldots \ldots \ldots \ldots \ldots \ldots \ldots \ldots \ldots \ldots \ldots$ & 623 & 465 & 128 & 14.4 & 13.9 & 18.9 \\
\hline 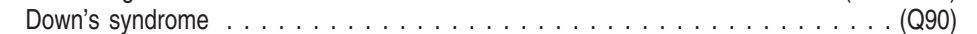 & 82 & 56 & 20 & 1.9 & 1.7 & 3.0 \\
\hline Edward's syndrome . . . . . . . . . . . . . . . . . . . . . . . . . (Q91.0-Q91.3) & 525 & 408 & 90 & 12.2 & 12.2 & 13.3 \\
\hline 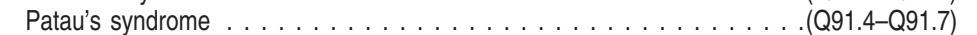 & 295 & 219 & 58 & 6.8 & 6.6 & 8.6 \\
\hline Other congenital malformations and deformations & 552 & 422 & 101 & 12.8 & 12.6 & 14.9 \\
\hline Other chromosomal abnormalities, not elsewhere classified . . . . . . . . . (Q92-Q99) & 209 & 158 & 35 & 4.8 & 4.7 & 5.2 \\
\hline \multicolumn{7}{|l|}{ Symptoms, signs and abnormal clinical and laboratory } \\
\hline findings, not elsewhere classified. & 3,617 & 2,347 & 1,106 & 83.8 & 70.3 & 163.7 \\
\hline Sudden infant death syndrome & 2,453 & 1,612 & 738 & 56.8 & 48.3 & 109.2 \\
\hline \multicolumn{7}{|l|}{ Other symptoms, signs and abnormal clinical and laboratory } \\
\hline findings, not elsewhere classified $\ldots \ldots \ldots \ldots \ldots$ (R00-R53,R55-R94,R96-R99) & 1,164 & 735 & 368 & 27.0 & 22.0 & 54.5 \\
\hline$\ldots \ldots \ldots \ldots$ (residual) & 16 & 11 & 4 & * & * & * \\
\hline External causes of mortality & 1,747 & 1,094 & 578 & 40.5 & 32.8 & 85.5 \\
\hline Accidents (unintentional injuries) & 1,285 & 803 & 428 & 29.8 & 24.1 & 63.3 \\
\hline Transport accidents . . . . . . . & 127 & 93 & 28 & 2.9 & 2.8 & 4.1 \\
\hline \multicolumn{7}{|l|}{$\begin{array}{r}\text { Motor-vehicle accidents . . . . . . . . (V02-V04,V09.0,V09.2,V12-V14,V19.0-V19.2, } \\
\text { V19.4-V19.6,V20-V79,V80.3-V80.5,V81.0-V81.1,V82.0-V82.1,V83-V86, }\end{array}$} \\
\hline V87.0-V87.8,V88.0-V88.8,V89.0,V89.2) & 124 & 91 & 27 & 2.9 & 2.7 & 4.0 \\
\hline \multicolumn{7}{|l|}{ Other and unspecified transport accidents . . . . (V01,V05-V06,V09.1,V09.3-V09.9, } \\
\hline \multicolumn{7}{|l|}{ 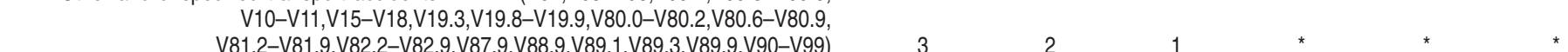 } \\
\hline V81.2-V81.9,V82.2-V82.9,V87.9,V88.9,V89.1,V89.3,V89.9,V90-V99) & 3 & 2 & 1 & * & * & * \\
\hline$\ldots \ldots \ldots \ldots \ldots$ (W00-W19) & 24 & 20 & 4 & 0.6 & 0.6 & * \\
\hline Accidental discharge of firearms & 1 & - & 1 & * & * & * \\
\hline Accidental drowning and submersion & 57 & 39 & 16 & 1.3 & 1.2 & * \\
\hline Accidental suffocation and strangulation in bed & 669 & 389 & 252 & 15.5 & 11.7 & 37.3 \\
\hline Other accidental suffocation and strangulation. & 220 & 147 & 66 & 5.1 & 4.4 & 9.8 \\
\hline \multicolumn{7}{|l|}{ Accidental inhalation and ingestion of food or other objects causing } \\
\hline obstruction of respiratory tract $\ldots \ldots \ldots \ldots \ldots \ldots \ldots$ & 70 & 46 & 20 & 1.6 & 1.4 & 3.0 \\
\hline Accidents caused by exposure to smoke, fire and flames & 38 & 23 & 15 & 0.9 & 0.7 & \\
\hline Accidental poisoning and exposure to noxious substances & 19 & 8 & 8 & * & * & * \\
\hline Other and unspecified accidents . (W20-W31,W35-W64,W85-W99,X10-X39,X50-X59) & 60 & 38 & 18 & 1.4 & 1.1 & * \\
\hline
\end{tabular}

See footnotes at end of table. 
Table 31. Number of infant deaths and infant mortality rates for 130 selected causes, by race: United States, 2007-Con.

[Rates are infant deaths (under 1 year) per 100,000 live births in specified group. Infant deaths are based on race of decedent; live births are based on race of mother. The asterisks $\left({ }^{*}\right)$ preceding the cause-of-death codes indicate that they are not part of the International Classification of Diseases, Tenth Revision (ICD-10), Second Edition; see "Technical Notes"]

\begin{tabular}{|c|c|c|c|c|c|c|}
\hline \multirow[b]{2}{*}{ Cause of death (based on ICD-10, 2004) } & \multicolumn{3}{|c|}{ Number } & \multicolumn{3}{|c|}{ Rate } \\
\hline & $\begin{array}{c}\text { All } \\
\text { races }^{1}\end{array}$ & White ${ }^{2}$ & Black $^{2}$ & $\begin{array}{c}\text { All } \\
\text { races }^{1}\end{array}$ & White ${ }^{2}$ & Black $^{2}$ \\
\hline$\ldots \ldots\left({ }^{*} \cup 01, X 85-Y 09\right)$ & 352 & 228 & 110 & 8.2 & 6.8 & 16.3 \\
\hline Assault (homicide) by hanging, strangulation and suffocation & 30 & 24 & 4 & 0.7 & 0.7 & * \\
\hline Assault (homicide) by discharge of firearms & 15 & 10 & 5 & * & * & * \\
\hline Neglect, abandonment and other maltreatment syndromes & 86 & 55 & 28 & 2.0 & 1.6 & 4.1 \\
\hline Assault (homicide) by other and unspecified means $\ldots$. & & & & & & \\
\hline *U01.5-*U01.9,X85-X90,X92,X96-X99,Y00-Y05,Y08-Y09) & 221 & 139 & 73 & 5.1 & 4.2 & 10.8 \\
\hline Complications of medical and surgical care . . . . . . . . . . . . . . (Y40-Y84) & 22 & 9 & 11 & 0.5 & * & * \\
\hline Other external causes. . . . . . . . . . . . . . (Y10-Y36) & 88 & 54 & 29 & 2.0 & 1.6 & 4.3 \\
\hline
\end{tabular}

* Figure does not meet standards of reliability or precision; see "Technical Notes."

- Quantity zero.

${ }^{1}$ Includes races other than white and black.

${ }^{2}$ Race categories are consistent with the 1977 Office of Management and Budget (OMB) standards. Multiple-race data were reported for deaths by 27 states and the District of Columbia and, for births, by 27 areas; see "Technical Notes." The multiple-race data for these reporting areas were bridged to the single-race categories of the 1977 OMB standards for comparability with other reporting areas; see "Technical Notes."

${ }^{3} \mathrm{New}$ ICD-10 code J09 (Influenza due to identified avian influenza virus) was added to the category in 2007.

${ }^{4} \mathrm{New}$ ICD-10 code U04 (Severe acute respiratory syndrome [SARS]) was added to the category in 2007.

NOTE: Complete confirmation of deaths from selected causes of death, considered to be of public health concern, was not provided by the following states: Connecticut, Florida, Indiana, Kentucky, Maryland, North Carolina, Oklahoma, Pennsylvania, Rhode Island, South Carolina, Washington, and West Virginia; see "Technical Notes." 
Table 32. Number of infant and neonatal deaths and mortality rates, by race for the United States, each state, Puerto Rico, Virgin Islands, Guam, American Samoa, and Northern Marianas, and by sex for the United States, 2007

[Rates are infant deaths (under 1 year) per 1,000 live births in specified group. Infant deaths are based on race of decedent; live births are based on race of mother. See "Technical Notes"]

\begin{tabular}{|c|c|c|c|c|c|c|c|c|c|c|c|c|}
\hline \multirow[b]{3}{*}{ Sex and area } & \multicolumn{6}{|c|}{ Infant deaths } & \multicolumn{6}{|c|}{ Neonatal deaths } \\
\hline & \multicolumn{2}{|c|}{ All races ${ }^{1}$} & \multicolumn{2}{|c|}{ White $^{2}$} & \multicolumn{2}{|c|}{ Black $^{2}$} & \multicolumn{2}{|c|}{ All races ${ }^{1}$} & \multicolumn{2}{|c|}{ White $^{2}$} & \multicolumn{2}{|c|}{ Black $^{2}$} \\
\hline & Number & Rate & Number & Rate & Number & Rate & Number & Rate & Number & Rate & Number & Rate \\
\hline $\begin{array}{l}\text { United } \text { States }^{3} \ldots \ldots \ldots \ldots \ldots \ldots \\
\text { Male } \ldots \ldots \ldots \ldots \ldots \ldots \\
\text { Female } \ldots \ldots \ldots \ldots \ldots \ldots\end{array}$ & $\begin{array}{l}29,138 \\
16,293 \\
12,845\end{array}$ & $\begin{array}{l}6.75 \\
7.38 \\
6.09\end{array}$ & $\begin{array}{r}18,807 \\
10,540 \\
8,267\end{array}$ & $\begin{array}{l}5.64 \\
6.17 \\
5.08\end{array}$ & $\begin{array}{l}8,944 \\
4,975 \\
3,969\end{array}$ & $\begin{array}{l}13.24 \\
14.49 \\
11.94\end{array}$ & $\begin{array}{r}19,058 \\
10,587 \\
8,471\end{array}$ & $\begin{array}{l}4.42 \\
4.79 \\
4.02\end{array}$ & $\begin{array}{r}12,333 \\
6,845 \\
5,488\end{array}$ & $\begin{array}{l}3.70 \\
4.01 \\
3.37\end{array}$ & $\begin{array}{l}5,842 \\
3,256 \\
2,586\end{array}$ & $\begin{array}{l}8.65 \\
9.48 \\
7.78\end{array}$ \\
\hline 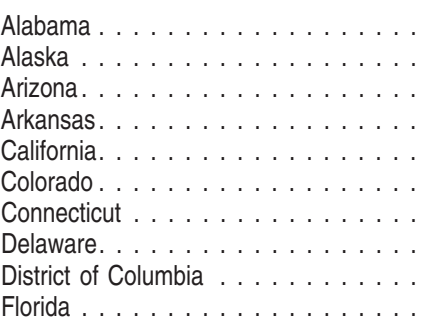 & $\begin{array}{r}641 \\
72 \\
711 \\
317 \\
2,944 \\
433 \\
276 \\
91 \\
116 \\
1,685\end{array}$ & $\begin{array}{r}9.89 \\
6.51 \\
6.90 \\
7.66 \\
5.20 \\
6.12 \\
6.63 \\
7.48 \\
13.09 \\
7.05\end{array}$ & $\begin{array}{r}350 \\
36 \\
581 \\
209 \\
2,223 \\
375 \\
197 \\
51 \\
29 \\
950\end{array}$ & $\begin{array}{l}7.98 \\
5.20 \\
6.57 \\
6.47 \\
4.89 \\
5.85 \\
5.90 \\
6.12 \\
8.52 \\
5.52\end{array}$ & $\begin{array}{r}284 \\
3 \\
65 \\
107 \\
421 \\
45 \\
68 \\
39 \\
87 \\
709\end{array}$ & $\begin{array}{r}14.35 \\
* \\
14.96 \\
13.17 \\
12.35 \\
13.16 \\
12.07 \\
11.84 \\
16.61 \\
12.21\end{array}$ & $\begin{array}{r}406 \\
35 \\
488 \\
179 \\
2,009 \\
295 \\
211 \\
65 \\
86 \\
1,058\end{array}$ & $\begin{array}{l}6.27 \\
3.17 \\
4.74 \\
4.33 \\
3.55 \\
4.17 \\
5.06 \\
5.34 \\
9.70 \\
4.42\end{array}$ & $\begin{array}{r}215 \\
21 \\
406 \\
109 \\
1,518 \\
257 \\
149 \\
36 \\
20 \\
607\end{array}$ & $\begin{array}{l}4.90 \\
3.03 \\
4.59 \\
3.38 \\
3.34 \\
4.01 \\
4.46 \\
4.32 \\
5.87 \\
3.53\end{array}$ & $\begin{array}{r}188 \\
2 \\
44 \\
70 \\
272 \\
28 \\
53 \\
29 \\
66 \\
434\end{array}$ & $\begin{array}{r}9.50 \\
* \\
10.13 \\
8.61 \\
7.98 \\
8.19 \\
9.41 \\
8.80 \\
12.60 \\
7.47\end{array}$ \\
\hline 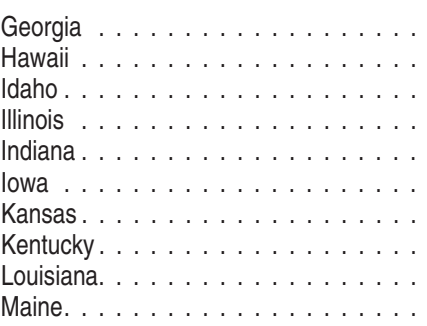 & $\begin{array}{r}1,206 \\
124 \\
169 \\
1,217 \\
681 \\
225 \\
333 \\
397 \\
608 \\
89\end{array}$ & $\begin{array}{l}7.98 \\
6.48 \\
6.75 \\
6.73 \\
7.58 \\
5.50 \\
7.93 \\
6.69 \\
9.17 \\
6.30\end{array}$ & $\begin{array}{r}516 \\
34 \\
158 \\
725 \\
513 \\
201 \\
260 \\
316 \\
238 \\
85\end{array}$ & $\begin{array}{l}5.56 \\
6.11 \\
6.59 \\
5.22 \\
6.61 \\
5.34 \\
7.03 \\
6.01 \\
6.14 \\
6.33\end{array}$ & $\begin{array}{r}664 \\
8 \\
3 \\
452 \\
166 \\
22 \\
62 \\
72 \\
365 \\
4\end{array}$ & $\begin{array}{r}12.77 \\
* \\
* \\
14.16 \\
15.99 \\
11.58 \\
18.98 \\
12.69 \\
14.08 \\
*\end{array}$ & $\begin{array}{r}766 \\
81 \\
113 \\
865 \\
430 \\
135 \\
210 \\
241 \\
355 \\
63\end{array}$ & $\begin{array}{l}5.07 \\
4.23 \\
4.52 \\
4.78 \\
4.79 \\
3.30 \\
5.00 \\
4.06 \\
5.35 \\
4.46\end{array}$ & $\begin{array}{r}332 \\
20 \\
107 \\
534 \\
321 \\
123 \\
166 \\
196 \\
135 \\
61\end{array}$ & $\begin{array}{l}3.58 \\
3.59 \\
4.46 \\
3.84 \\
4.13 \\
3.27 \\
4.49 \\
3.73 \\
3.49 \\
4.54\end{array}$ & $\begin{array}{r}411 \\
8 \\
2 \\
302 \\
108 \\
11 \\
38 \\
43 \\
217 \\
2\end{array}$ & $\begin{array}{r}7.90 \\
* \\
* \\
9.46 \\
10.40 \\
* \\
11.63 \\
7.58 \\
8.37 \\
*\end{array}$ \\
\hline 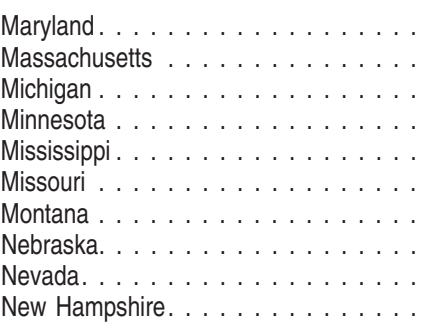 & $\begin{array}{r}625 \\
384 \\
995 \\
409 \\
467 \\
613 \\
79 \\
182 \\
262 \\
76\end{array}$ & $\begin{array}{r}8.00 \\
4.93 \\
7.94 \\
5.55 \\
10.04 \\
7.48 \\
6.35 \\
6.76 \\
6.36 \\
5.36\end{array}$ & $\begin{array}{r}219 \\
283 \\
595 \\
280 \\
165 \\
393 \\
63 \\
144 \\
201 \\
71\end{array}$ & $\begin{array}{l}4.79 \\
4.54 \\
6.11 \\
4.69 \\
6.65 \\
5.89 \\
5.94 \\
6.12 \\
6.03 \\
5.34\end{array}$ & $\begin{array}{r}371 \\
83 \\
370 \\
82 \\
290 \\
210 \\
3 \\
28 \\
47 \\
3\end{array}$ & $\begin{array}{r}13.63 \\
8.76 \\
16.39 \\
11.70 \\
13.87 \\
16.48 \\
* \\
14.04 \\
12.35 \\
\text { * }\end{array}$ & $\begin{array}{r}453 \\
267 \\
696 \\
280 \\
274 \\
409 \\
45 \\
131 \\
166 \\
46\end{array}$ & $\begin{array}{l}5.80 \\
3.42 \\
5.56 \\
3.80 \\
5.89 \\
4.99 \\
3.62 \\
4.86 \\
4.03 \\
3.25\end{array}$ & $\begin{array}{r}162 \\
194 \\
426 \\
196 \\
95 \\
252 \\
37 \\
103 \\
127 \\
44\end{array}$ & $\begin{array}{l}3.54 \\
3.11 \\
4.37 \\
3.28 \\
3.83 \\
3.77 \\
3.49 \\
4.38 \\
3.81 \\
3.31\end{array}$ & $\begin{array}{r}263 \\
58 \\
251 \\
50 \\
175 \\
149 \\
1 \\
21 \\
31 \\
2\end{array}$ & $\begin{array}{r}9.67 \\
6.12 \\
11.12 \\
7.13 \\
8.37 \\
11.69 \\
* \\
10.53 \\
8.15 \\
*\end{array}$ \\
\hline 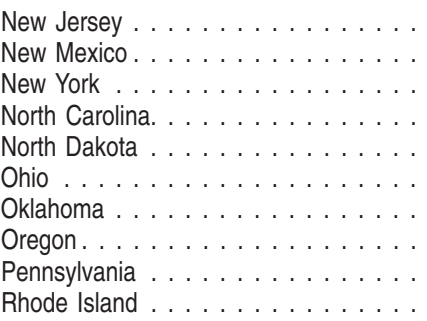 & $\begin{array}{r}601 \\
192 \\
1,412 \\
1,112 \\
66 \\
1,160 \\
469 \\
284 \\
1,139 \\
91\end{array}$ & $\begin{array}{l}5.18 \\
6.27 \\
5.57 \\
8.49 \\
7.47 \\
7.69 \\
8.52 \\
5.75 \\
7.56 \\
7.35\end{array}$ & $\begin{array}{r}342 \\
153 \\
861 \\
600 \\
51 \\
768 \\
307 \\
254 \\
721 \\
68\end{array}$ & $\begin{array}{l}4.13 \\
5.99 \\
4.95 \\
6.35 \\
6.80 \\
6.34 \\
7.25 \\
5.70 \\
6.12 \\
6.52\end{array}$ & $\begin{array}{r}234 \\
8 \\
482 \\
467 \\
1 \\
382 \\
90 \\
12 \\
394 \\
20\end{array}$ & $\begin{array}{r}11.02 \\
* \\
8.82 \\
15.14 \\
* \\
14.81 \\
18.03 \\
* \\
15.07 \\
16.00\end{array}$ & $\begin{array}{r}399 \\
119 \\
937 \\
747 \\
43 \\
781 \\
263 \\
195 \\
751 \\
67\end{array}$ & $\begin{array}{l}3.44 \\
3.89 \\
3.70 \\
5.70 \\
4.86 \\
5.18 \\
4.78 \\
3.95 \\
4.98 \\
5.41\end{array}$ & $\begin{array}{r}237 \\
99 \\
569 \\
400 \\
38 \\
510 \\
176 \\
175 \\
485 \\
50\end{array}$ & $\begin{array}{l}2.86 \\
3.88 \\
3.27 \\
4.24 \\
5.07 \\
4.21 \\
4.16 \\
3.93 \\
4.12 \\
4.79\end{array}$ & $\begin{array}{r}146 \\
6 \\
325 \\
317 \\
1 \\
263 \\
54 \\
8 \\
247 \\
15\end{array}$ & $\begin{array}{r}6.87 \\
* \\
5.95 \\
10.28 \\
* \\
10.20 \\
10.82 \\
\text { * } \\
9.45 \\
\text { * }\end{array}$ \\
\hline $\begin{array}{l}\text { South Carolina } \ldots \ldots \ldots \ldots \ldots \\
\text { South Dakota . . . . . . . . . . . }\end{array}$ & $\begin{array}{r}539 \\
79\end{array}$ & $\begin{array}{l}8.57 \\
6.44\end{array}$ & $\begin{array}{r}242 \\
54\end{array}$ & $\begin{array}{l}6.03 \\
5.55\end{array}$ & $\begin{array}{r}291 \\
3\end{array}$ & $\begin{array}{r}13.69 \\
*\end{array}$ & $\begin{array}{r}356 \\
51\end{array}$ & $\begin{array}{l}5.66 \\
4.16\end{array}$ & $\begin{array}{r}158 \\
39\end{array}$ & $\begin{array}{l}3.93 \\
4.01\end{array}$ & $\begin{array}{r}194 \\
2\end{array}$ & $\begin{array}{r}9.13 \\
*\end{array}$ \\
\hline $\begin{array}{l}\text { Tennessee . . . . . . . . . . . . . . . . . . . . . } \\
\text { Texas. . . . }\end{array}$ & $\begin{array}{r}721 \\
2,564\end{array}$ & $\begin{array}{l}8.31 \\
6.29\end{array}$ & $\begin{array}{r}421 \\
1,934\end{array}$ & $\begin{array}{l}6.44 \\
5.68\end{array}$ & $\begin{array}{l}295 \\
573\end{array}$ & $\begin{array}{l}15.74 \\
11.51\end{array}$ & $\begin{array}{r}450 \\
1,572\end{array}$ & $\begin{array}{l}5.19 \\
3.86\end{array}$ & $\begin{array}{r}256 \\
1,167\end{array}$ & $\begin{array}{l}3.91 \\
3.43\end{array}$ & $\begin{array}{l}191 \\
367\end{array}$ & $\begin{array}{r}10.19 \\
7.37\end{array}$ \\
\hline 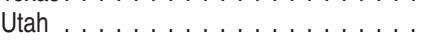 & 280 & 5.08 & 259 & 4.98 & 4 & * & 187 & 3.39 & 174 & 3.35 & 3 & * \\
\hline Vermont & 33 & 5.07 & 30 & 4.76 & 1 & * & 20 & 3.07 & 17 & * & 1 & * \\
\hline Virginia. . & 848 & 7.79 & 444 & 5.80 & 375 & 15.41 & 583 & 5.35 & 288 & 3.76 & 274 & 11.26 \\
\hline Washington . & 429 & 4.82 & 313 & 4.33 & 50 & 10.28 & 254 & 2.85 & 190 & 2.63 & 27 & 5.55 \\
\hline West Virginia & 164 & 7.46 & 146 & 6.95 & 17 & * & 103 & 4.68 & 91 & 4.33 & 11 & \\
\hline Wisconsin . . . . . & 470 & 6.46 & 329 & 5.37 & 111 & 15.18 & 293 & 4.03 & 218 & 3.56 & 61 & 8.34 \\
\hline Wyoming. . . & 58 & 7.35 & 49 & 6.65 & 1 & & 29 & 3.67 & 27 & 3.66 & - & \\
\hline
\end{tabular}

See footnotes at end of table. 
Table 32. Number of infant and neonatal deaths and mortality rates, by race for the United States, each state, Puerto Rico, Virgin Islands, Guam, American Samoa, and Northern Marianas, and by sex for the United States, 2007-Con.

[Rates are infant deaths (under 1 year) per 1,000 live births in specified group. Infant deaths are based on race of decedent; live births are based on race of mother. See "Technical Notes"]

\begin{tabular}{|c|c|c|c|c|c|c|c|c|c|c|c|c|}
\hline \multirow[b]{3}{*}{ Sex and area } & \multicolumn{6}{|c|}{ Infant deaths } & \multicolumn{6}{|c|}{ Neonatal deaths } \\
\hline & \multicolumn{2}{|c|}{ All races ${ }^{1}$} & \multicolumn{2}{|c|}{ White ${ }^{2}$} & \multicolumn{2}{|c|}{ Black $^{2}$} & \multicolumn{2}{|c|}{ All races ${ }^{1}$} & \multicolumn{2}{|c|}{ White $^{2}$} & \multicolumn{2}{|c|}{ Black $^{2}$} \\
\hline & Number & Rate & Number & Rate & Number & Rate & Number & Rate & Number & Rate & Number & Rate \\
\hline Puerto Rico . . . . . . . . . . . . . . . . & 394 & 8.45 & 378 & 9.06 & 16 & * & 278 & 5.96 & 271 & 6.49 & 7 & * \\
\hline Virgin Islands & 12 & * & 2 & * & 10 & * & 9 & * & 2 & * & 7 & * \\
\hline Guam. . . . . . . . . . . . . . . . & 36 & 10.34 & 2 & * & - & * & 23 & 6.60 & - & * & - & * \\
\hline American Samoa . . . . . . . . . . . . . & 11 & * & - & * & - & * & 7 & * & - & * & - & * \\
\hline Northern Marianas . . . . . . . . . . . . & 5 & * & - & * & - & * & 4 & * & - & * & - & * \\
\hline
\end{tabular}

*Figure does not meet standards of reliability or precision; see "Technical Notes."

- Quantity zero.

${ }^{1}$ Includes races other than white and black.

${ }^{2}$ Race categories are consistent with the 1977 Office of Management and Budget (OMB) standards. Multiple-race data were reported for deaths by 27 states and the District of Columbia and, for

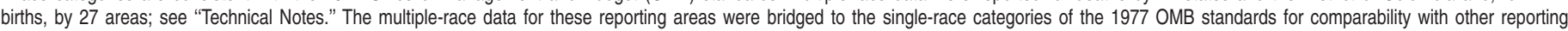
areas; see "Technical Notes."

${ }^{3}$ Excludes data for Puerto Rico, Virgin Islands, Guam, American Samoa, and Northern Marianas.

Table 33. Number of maternal deaths and maternal mortality rates for selected causes, by race: United States, 2007

[Maternal causes are those assigned to categories A34, 000-095, and 098-099 of the International Classification of Diseases, Tenth Revision (ICD-10), Second Edition. An increasing number of states use a separate item regarding pregnancy status on the death certificate to help identify these deaths; see "Technical Notes." Rates are per 100,000 live births in specified group; see "Technical Notes"]

\begin{tabular}{|c|c|c|c|c|c|c|c|c|}
\hline \multirow[b]{3}{*}{ Cause of death (based on ICD-10, 2004) } & \multicolumn{4}{|c|}{ Number } & \multicolumn{4}{|c|}{ Rate } \\
\hline & \multirow{2}{*}{$\begin{array}{l}\text { All } \\
\text { races }\end{array}$} & \multirow[b]{2}{*}{ White $^{1}$} & \multicolumn{2}{|c|}{ All other ${ }^{1}$} & \multirow{2}{*}{$\begin{array}{l}\text { All } \\
\text { races }\end{array}$} & \multirow[b]{2}{*}{ White $^{1}$} & \multicolumn{2}{|c|}{ All other ${ }^{1}$} \\
\hline & & & Total $^{1}$ & Black $^{1}$ & & & Total $^{1}$ & Black $^{1}$ \\
\hline$\ldots \ldots \ldots \ldots \ldots \ldots . \ldots . .434,000-095,098-099)$ & 548 & 335 & 213 & 179 & 12.7 & 10.0 & 21.7 & 26.5 \\
\hline$\ldots \ldots \ldots \ldots \ldots(000-007)$ & 31 & 12 & 19 & 18 & 0.7 & * & * & * \\
\hline Ectopic pregnancy $\ldots . . \ldots \ldots$ & 14 & 3 & 11 & 11 & * & * & * & * \\
\hline$\ldots \ldots \ldots \ldots \ldots \ldots \ldots \ldots(003)$ & 9 & 5 & 4 & 3 & * & * & * & * \\
\hline 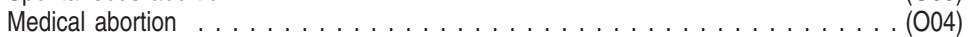 & - & - & - & - & * & * & * & * \\
\hline Other abortion . & 1 & - & 1 & 1 & * & * & * & * \\
\hline Other and unspecified pregnancy with abortive outcome $\ldots \ldots \ldots(001-002,006-007)$ & 7 & 4 & 3 & 3 & * & * & * & * \\
\hline Other direct obstetric causes . . . . . . . . . . . . . . . . . . . . (A34,010-092) & 362 & 219 & 143 & 117 & 8.4 & 6.6 & 14.6 & 17.3 \\
\hline$\ldots \ldots \ldots \ldots \ldots \ldots(011,013-016)$ & 64 & 42 & 22 & 19 & 1.5 & 1.3 & 2.2 & * \\
\hline Hemorrhage of pregnancy and childbirth and placenta previa . . . (020,044-046,067,072) & 41 & 30 & 11 & 9 & 0.9 & 0.9 & * & * \\
\hline Complications predominately related to the puerperium $\ldots \ldots \ldots \ldots(\mathrm{A} 34,085-092)$ & 93 & 49 & 44 & 31 & 2.2 & 1.5 & 4.5 & 4.6 \\
\hline 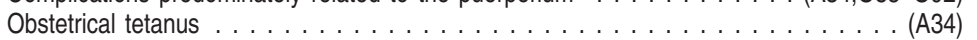 & - & - & - & - & * & * & * & * \\
\hline Obstetric embolism $\ldots \ldots \ldots \ldots \ldots \ldots \ldots \ldots \ldots \ldots \ldots \ldots \ldots \ldots \ldots$ (088) & 33 & 18 & 15 & 8 & 0.8 & * & * & * \\
\hline Other complications predominately related to the puerperium $\ldots \ldots(085-087,089-092)$ & 60 & 31 & 29 & 23 & 1.4 & 0.9 & 3.0 & 3.4 \\
\hline All other direct obstetric causes . . . . (010,012,021-043,047-066,068-071,073-075) & 164 & 98 & 66 & 58 & 3.8 & 2.9 & 6.7 & 8.6 \\
\hline Obstetric death of unspecified cause $\ldots \ldots \ldots \ldots \ldots \ldots \ldots \ldots \ldots$ (095) & 20 & 11 & 9 & 7 & 0.5 & * & * & * \\
\hline Indirect obstetric causes $\ldots \ldots \ldots \ldots \ldots \ldots \ldots \ldots \ldots$ (098-099) & 135 & 93 & 42 & 37 & 3.1 & 2.8 & 4.3 & 5.5 \\
\hline $\begin{array}{l}\text { Maternal causes more than } 42 \text { days after delivery or termination of pregnancy } \\
\text { Death from any obstetric cause occurring more than } 42 \text { days but }\end{array}$ & 221 & 130 & 91 & 72 & 5.1 & 3.9 & 9.3 & 10.7 \\
\hline less than 1 year after delivery $\ldots \ldots \ldots \ldots \ldots \ldots \ldots$ & 215 & 129 & 86 & 68 & 5.0 & 3.9 & 8.8 & 10.1 \\
\hline Death from sequelae of direct obstetric causes . . . . . . . . . . . & 6 & 1 & 5 & 4 & * & * & * & * \\
\hline
\end{tabular}

" Figure does not meet standards of reliability or precision; see "Technical Notes."

- Quantity zero.

${ }^{1}$ Race categories are consistent with the 1977 Office of Management and Budget (OMB) standards. Multiple-race data were reported for deaths by 27 states and the District of Columbia and, for births, by 27 areas; see "Technical Notes." The multiple-race data for these reporting areas were bridged to the single-race categories of the 1977 OMB standards for comparability with other reporting areas; see "Technical Notes." 
Table 34. Number of maternal deaths and maternal mortality rates for selected causes, by Hispanic origin and race for non-Hispanic population:

\section{United States, 2007}

[Maternal causes are those assigned to categories A34, 000-095, and 098-099 of the International Classification of Diseases, Tenth Revision (ICD-10), Second Edition. An increasing number of states use a separate item regarding pregnancy status on the death certificate to help identify these deaths; see "Technical Notes." Rates are per 100,000 live births in specified group; see "Technical Notes." Race and Hispanic origin are reported

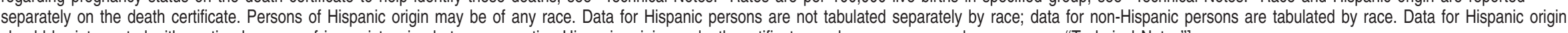
should be interpreted with caution because of inconsistencies between reporting Hispanic origin on death certificates and on censuses and surveys; see "Technical Notes"]

\begin{tabular}{|c|c|c|c|c|c|c|c|c|c|c|}
\hline \multirow[b]{2}{*}{ Cause of death (based on ICD-10, 2004) } & \multicolumn{5}{|c|}{ Number } & \multicolumn{5}{|c|}{ Rate } \\
\hline & $\begin{array}{c}\text { All } \\
\text { origins }^{1}\end{array}$ & Hispanic & Non-Hispanic ${ }^{2}$ & $\begin{array}{l}\text { Non-Hispanic } \\
\text { white }^{3}\end{array}$ & $\begin{array}{c}\text { Non-Hispanic } \\
\text { black }^{3}\end{array}$ & $\begin{array}{c}\text { All } \\
\text { origins }^{1}\end{array}$ & Hispanic & Non-Hispanic ${ }^{2}$ & $\begin{array}{l}\text { Non-Hispanic } \\
\text { white }^{3}\end{array}$ & $\begin{array}{l}\text { Non-Hispanic } \\
\text { black }^{3}\end{array}$ \\
\hline.$(A 34,000-095,098-099)$ & 548 & 95 & 453 & 242 & 178 & 12.7 & 8.9 & 14.1 & 10.5 & 28.4 \\
\hline Pregnancy with abortive outcome & 31 & 5 & 26 & 8 & 17 & 0.7 & * & 0.8 & * & * \\
\hline Ectopic pregnancy . . . . . . & 14 & 1 & 13 & 2 & 11 & * & * & * & * & * \\
\hline Spontaneous abortion . . . . . . . . . . . . . . . . . . . . . . . . . . . . . . . (003) & 9 & 2 & 7 & 3 & 3 & * & * & * & * & * \\
\hline Medical abortion ......... & - & - & - & - & - & * & * & * & * & * \\
\hline Other abortion . & 1 & - & 1 & - & 1 & * & * & * & * & * \\
\hline Other and unspecified pregnancy with abortive outcome $\ldots . .(001-002,006-007)$ & 7 & 2 & 5 & 3 & 2 & * & * & * & * & * \\
\hline Other direct obstetric causes $\ldots \ldots \ldots \ldots \ldots \ldots$. . . . & 362 & 67 & 295 & 153 & 117 & 8.4 & 6.3 & 9.2 & 6.6 & 18.7 \\
\hline Eclampsia and pre-eclampsia & 64 & 13 & 51 & 29 & 19 & 1.5 & * & 1.6 & 1.3 & * \\
\hline $\begin{array}{l}\text { Hemorrhage of pregnancy and childbirth and placenta } \\
\text { previa } \ldots \ldots \ldots \ldots \ldots \ldots \ldots \ldots \ldots \ldots \ldots \ldots \ldots\end{array}$ & 41 & 12 & 29 & 18 & 9 & 0.9 & * & 0.9 & * & * \\
\hline Complications predominately related to the puerperium $\ldots \ldots \ldots(\mathrm{A} 34,085-092)$ & 93 & 15 & 78 & 35 & 31 & 2.2 & * & 2.4 & 1.5 & 4.9 \\
\hline 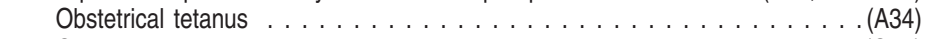 & - & - & - & - & - & * & * & * & * & * \\
\hline Obstetric embolism . . . . . . . . . . . . . . . . . . . . . . . . . . . . . (088) & 33 & 6 & 27 & 12 & 8 & 0.8 & * & 0.8 & * & * \\
\hline $\begin{array}{l}\text { Other complications predominately related to the puerperium }(085-087,089-092) \\
\text { All other direct obstetric }\end{array}$ & 60 & 9 & 51 & 23 & 23 & 1.4 & * & 1.6 & 1.0 & 3.7 \\
\hline$\ldots \ldots \ldots \ldots .(010,012,021-043,047-066,068-071,073-075)$ & 164 & 27 & 137 & 71 & 58 & 3.8 & 2.5 & 4.3 & 3.1 & 9.2 \\
\hline Obstetric death of unspecified cause $\ldots \ldots \ldots$. . . . . . . . . . . . . . . . (095) & 20 & 4 & 16 & 7 & 7 & 0.5 & * & * & * & * \\
\hline Indirect obstetric causes $\ldots \ldots \ldots \ldots \ldots \ldots \ldots \ldots$ (098-099) & 135 & 19 & 116 & 74 & 37 & 3.1 & * & 3.6 & 3.2 & 5.9 \\
\hline 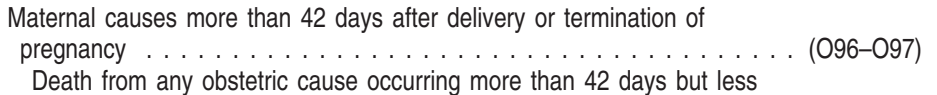 & 221 & 39 & 181 & 92 & 70 & 5.1 & 3.7 & 5.6 & 4.0 & 11.2 \\
\hline than 1 year after delivery . . . . . . . . . . . . . . . . . . . . . . . . . . . . .(096) & 215 & 38 & 176 & 92 & 66 & 5.0 & 3.6 & 5.5 & 4.0 & 10.5 \\
\hline Death from sequelae of direct obstetric causes . . . . . . . . . . . . . . . . . . (097) & 6 & 1 & 5 & - & 4 & * & * & * & * & * \\
\hline
\end{tabular}

"Figure does not meet standards of reliability or precision; see "Technical Notes." - Quantity zero.

${ }^{1}$ All origins includes origin not stated; specified origins exclude origins not stated. ${ }^{2}$ Includes races other than white and black.

${ }^{3}$ Race categories are consistent with the 1977 Office of Management and Budget (OMB) standards. Multiple-race data were reported for deaths by 27 states and the District of Columbia and, for births, by 27 areas; see "Technical Notes." The multiple-race data for these reporting areas were bridged to the single-race categories of the 1977 OMB standards for comparability with other reporting areas; see "Technical Notes." 


\section{Technical Notes}

\section{Nature and sources of data}

Data in this report are based on information from all death certificates filed in the 50 states and the District of Columbia and are processed by the Centers for Disease Control and Prevention's National Center for Health Statistics (NCHS). Data for 2007 are based on records of deaths that occurred during 2007 and were received as of October 28, 2009. Missing from the 2007 data file are approximately 200 deaths that occurred in Allen Parish, Louisiana, but were not registered with the Louisiana state office. The registration problem began in 2006, when approximately 150 deaths that occurred in Allen Parish were not registered with the state office for that year.

The U.S. Standard Certificate of Death-which is used as a model by the states-was revised in 2003 (38). Prior to 2003, the standard certificate of death had not been revised since 1989. This report includes data for the 23 states (California, Connecticut, Delaware, Florida, Idaho, Kansas, Michigan, Montana, Nebraska, New Hampshire, New Jersey, New Mexico, New York, Ohio, Oklahoma, Oregon, Rhode Island, South Carolina, South Dakota, Texas, Utah, Washington, and Wyoming) and the District of Columbia that used the 2003 revision of the U.S. Standard Certificate of Death in 2007, and for the remaining 27 states that collected and reported death data in 2007 based on the 1989 revision of the U.S. Standard Certificate of Death. The 1989 and 2003 revisions are described in detail elsewhere (38-41).

Because most of the items presented in this report appear largely comparable despite changes to item wording and format in the 2003 death certificate revision, data from both groups of states are combined unless otherwise stated. Data for Puerto Rico, Virgin Islands, Guam, American Samoa, and Northern Marianas are included in tables showing data by state but are not included in U.S. totals.

Mortality statistics are based on information coded by the states and provided to NCHS through the Vital Statistics Cooperative Program and from copies of original certificates received by NCHS from state registration offices. In 2007, all states and the District of Columbia participated in this program and submitted part or all of the mortality data for 2007 in electronic data files to NCHS. All areas provided precoded medical (cause-of-death) data to NCHS except Nebraska, New Jersey, and West Virginia. For 2007, all states submitted precoded demographic data for all deaths.

Data for the entire United States refer to events occurring within the United States. Data shown for geographic areas are by place of residence. Beginning with 1970, mortality statistics for the United States exclude deaths of nonresidents of the United States. All data exclude fetal deaths.

Mortality statistics for Puerto Rico, Virgin Islands, American Samoa, and Northern Marianas exclude deaths of nonresidents for each area. For Guam, however, mortality statistics exclude deaths that occurred to a resident of any place other than Guam or the United States.

\section{Cause-of-death classification}

The mortality statistics presented in this report were compiled in accordance with World Health Organization (WHO) regulations, which specify that member nations classify and code causes of death in accordance with the current revision of the International Classification of Diseases (ICD). ICD provides the basic guidance used in virtually all countries to code and classify causes of death. Effective with deaths occurring in 1999, the United States began using the Tenth Revision of this classification (ICD-10) (42). In 2004, the second edition of ICD-10 was adopted (6). For earlier years, causes of death were classified according to the revisions then in use: 1979-1998, Ninth Revision; 1968-1978, Eighth Revision, adapted for use in the United States; 1958-1967, Seventh Revision; and 1949-1957, Sixth Revision.

Changes in classification of causes of death due to these revisions may result in discontinuities in cause-of-death trends. Consequently, cause-of-death comparisons among revisions require consideration of comparability ratios and, where available, estimates of their standard errors. Comparability ratios between the Ninth and Tenth revisions, Eighth and Ninth revisions, Seventh and Eighth revisions, and Sixth and Seventh revisions may be found in other NCHS reports and independent tabulations (23-25,43-45).

Rules for coding a cause or causes of death may sometimes require modification when evidence suggests it will improve the quality of cause-of-death data. Prior to 1999, such modifications were made only when a new ICD revision was implemented. A process for updating the ICD was introduced with ICD-10 that allows for midrevision changes. These changes, however, may affect comparability of data between years for selected causes of death. Minor changes may be implemented every year, whereas major changes may be implemented every 3 years (e.g., 2006 data year). In data year 2007, minor changes were implemented; these are discussed in subsequent sections of this report.

ICD not only details disease classification but also provides definitions, tabulation lists, the format of the death certificate, and the rules for coding cause of death. Cause-of-death data presented in this publication were coded by procedures outlined in annual issues of the NCHS Instruction Manual $(46,47)$. ICD includes rules for selecting the underlying cause of death and regulations on the use of ICD.

Before data year 1968, mortality medical data were based on manual coding of an underlying cause of death for each certificate in accordance with WHO rules. Effective with data year 1968, NCHS converted to computerized coding of the underlying cause and manual coding of all causes (multiple causes) on the death certificate. In this system, called "Automated Classification of Medical Entities" (ACME) (48), multiple-cause codes are inputted to computer software that uses WHO rules to select the underlying cause. All cause-of-death data in this report are coded using ACME.

The ACME system is used to select the underlying cause of death for all death certificates in the United States. In addition, NCHS has developed two computer systems as inputs to ACME. Beginning with 1990 data, the Mortality Medical Indexing, Classification, and Retrieval system (MICAR) $(49,50)$ was introduced to automate the coding of multiple causes of death. In addition, MICAR provides more detailed information on the conditions reported on death certificates than is available through the ICD code structure. Beginning with data year 1993, SuperMICAR, an enhancement of the MICAR system, was introduced, allowing for literal entry of the multiple cause-of-death text as reported by the certifier. This information is then automatically processed by the MICAR and ACME computer systems. Records that cannot be automatically processed by MICAR or SuperMICAR are 
manually multiple-cause coded and then further processed through ACME. In 2007, SuperMICAR was used to process all of the nation's death records.

In this report, tabulations of cause-of-death statistics are based solely on the underlying cause of death. The underlying cause is defined by WHO as "the disease or injury which initiated the train of events leading directly to death, or the circumstances of the accident or violence which produced the fatal injury" (6). The underlying cause is selected from the conditions entered by the physician in the causeof-death section of the death certificate. When more than one cause or condition is entered by the physician, the underlying cause is determined by the sequence of conditions on the certificate, provisions of ICD, and associated selection rules and modifications. Generally, more medical information is reported on death certificates than is directly reflected in the underlying cause of death. This is captured in NCHS multiple cause-of-death statistics (51-53).

\section{Tabulation lists and cause-of-death ranking}

Tabulation lists for ICD-10 are published in the NCHS Instruction Manual, Part 9, "ICD-10 Cause-of-Death Lists for Tabulating Mortality Statistics" (updated October 2007 to include WHO updates to ICD-10 for data year 2007) (54). For this report, two tabulation lists are used: the List of 113 Selected Causes of Death, used for deaths of all ages, and the List of 130 Selected Causes of Infant Death, used for infants. These lists are also used to rank leading causes of death for the two population groups. For the List of 113 Selected Causes of Death, the group titles of Major cardiovascular diseases (ICD-10 codes 100-178), and Symptoms, signs and abnormal clinical and laboratory findings, not elsewhere classified (R00-R99), are not ranked. In addition, category titles that begin with the words "other" and "all other" are not ranked to determine the leading causes of death. When one of the titles that represents a subtotal is ranked-for example, Tuberculosis (A16-A19)-its component parts are not ranked-in this case, Respiratory tuberculosis (A16) and Other tuberculosis (A17-A19). For the List of 130 Selected Causes of Infant Death, the same ranking procedures are used except that the category of major cardiovascular diseases is not on the list. More detail regarding ranking procedures can be found in "Deaths: Leading Causes for 2007" (3).

Leading cause-of-death trends discussed in this report are based on cause-of-death data according to ICD-10 for 1999-2007 and ICD-9 for the most comparable cause-of-death titles for 1979-1998. Tables showing ICD-9 categories that are comparable to ICD-10 titles in the List of 113 Selected Causes of Death may be found in "Comparability of Cause of Death Between ICD-9 and ICD-10: Preliminary Estimates" (25) and "Deaths: Final Data for 1999" (22). Although in some cases categories from the List of 113 Selected Causes of Death are identical to those in the earlier List of 72 Selected Causes of Death used with ICD-9, caution must be used because many of these categories are not comparable even though the cause-of-death titles may be the same.

Trend data for 1979-1998 that are classified by ICD-9 but sorted into the List of 113 Selected Causes of Death developed for ICD-10 can be found on the mortality website at http://www.cdc.gov/nchs/data/ statab/hist001r.pdf.

Revision of ICD and resulting changes in classification and rules for selecting the underlying cause of death have important implications for the analysis of mortality trends by cause of death. For some causes of death, the discontinuity in trend can be substantial $(24,25)$. Therefore, considerable caution should be used in analyzing cause-of-death trends for periods of time that extend across more than one revision of ICD.

\section{Codes added and deleted in 2007}

Effective with data year 2007, four new ICD-10 codes were added as valid, underlying cause-of-death codes. These are: J09, Influenza due to identified avian influenza virus; U04.9, Severe acute respiratory syndrome [SARS], unspecified; X59.0, Exposure to unspecified factor causing fracture; X59.9, Exposure to unspecified factor causing other and unspecified injury.

In 2007, no deaths were assigned to new codes J09 or U04.9. New codes X59.0 and X59.9 provide more detail for code X59, Exposure to unspecified factor. Beginning in 2007, X59 represents the subtotal of new codes X59.0 and X59.9 but is no longer selected as a three-digit underlying cause-of-death code. Deleted from the list of valid underlying cause-of-death codes in 2007 was F10.0, Mental and behavioral disorders due to use of alcohol, acute intoxication.

In 2007, several changes were made to the 113 causes of death list to reflect the addition of the new codes. With the addition of J09, Influenza due to identified avian influenza virus, ICD-10 codes were changed from J10-J18 to J09-J18 for "Influenza and pneumonia" and from J10-J11 to J09-J11 for "Influenza." With the addition of U04.9, Severe acute respiratory syndrome [SARS], ICD-10 codes were changed from J20-J22 to J20-J22,U04 for "Other acute lower respiratory infections." The category title "Unspecified acute lower respiratory infection" also was changed to "Other and unspecified acute lower respiratory infections," and the codes for this cause were changed from J22 to J22,U04 (54).

Changes in 2007 were also made to the list of 130 causes of infant death to reflect the addition of the new codes. With the addition of U04.9, Severe acute respiratory syndrome [SARS], ICD-10 codes were changed from J00-J98 to J00-J98,U04 for "Diseases of the respiratory system," and U04 was added to the ICD-10 codes for "Other and unspecified diseases of respiratory system." With the addition of J09, Influenza due to identified avian influenza virus, ICD-10 codes were changed from J10-J18 to J09-J18 for "Influenza and pneumonia" and from J10-J11 to J09-J11 for "Influenza" (54).

\section{Codes for terrorism}

Beginning with data for 2001, NCHS introduced categories U01-U03 for classifying and coding deaths due to acts of terrorism. The asterisks before the category codes indicate that they are not part of ICD-10. Deaths classified to the terrorism categories are included in the 113 causes of death list in the categories for Assault (homicide) and Intentional self-harm (suicide), and in the 130 causes of death list for infants in the category for Assault (homicide). Additional information on these new categories is available from http://www.cdc.gov/nchs/icd/terrorism_code.htm. No deaths were assigned to the terrorism categories in 2007.

\section{Enterocolitis due to Clostridium difficile}

The number of deaths from Enterocolitis due to Clostridium difficile (C. difficile) (ICD-10 code A04.7) has increased dramatically 
in recent years, from 793 deaths in 1999 to 6,372 deaths in 2007 . Data for C. difficile are included in tables showing data for 113 selected causes of death in "Certain other intestinal infections (A04, A07-A09)" but were previously not identified separately. Because of the increasing importance of this cause of death, beginning with data year 2006, data for $C$. difficile are shown separately at the bottom of tables showing 113 selected causes, and $C$. difficile has been added to the list of rankable causes.

\section{Quality of reporting and processing cause of death}

One index of the quality of reporting causes of death is the proportion of death certificates coded to Chapter XVIII-Symptoms, signs and abnormal clinical and laboratory findings, not elsewhere classified (ICD-10 codes R00-R99). Although deaths occur for which underlying causes are impossible to determine, the proportion coded to R00-R99 indicates the consideration given to the cause-of-death statement by the medical certifier. This proportion also may be used as a rough measure of specificity of medical diagnoses made by the certifier in various areas. In 2007, the percentage of all reported deaths in the United States assigned to Symptoms, signs and abnormal clinical and laboratory findings, not elsewhere classified, was 1.38 percent, up slightly from 1.31 percent in 2006 . Since 2000 , the percentage has ranged from 1.23 to 1.38 , higher than the percentages in the 1990s, which ranged from 1.08 to 1.18 percent.

Rules for coding a cause or causes of death may sometimes require modification when evidence suggests it will improve the quality of cause-of-death data. These changes, however, may affect comparability of data between years for selected causes of death. The implementation of changes in coding rules in 2007 had an impact on several mortality causes-and the comparison of 2007 and 2006 data for these causes-in the following ways:

- The increase in deaths from Anemias (D50-D64) in 2007 from 2006 may largely be due to a coding change in 2007. Specifically, more deaths that would have previously been assigned to Congestive heart failure (150.0) were instead assigned to Anemia unspecified (D64.9).

- The large increase in deaths from Chronic glomerulonephritis, nephritis and nephropathy not specified as acute or chronic, and renal sclerosis unspecified (N02-N03,N05-N07,N26) in 2007 from 2006 may mostly be due to a coding change in 2007 that resulted in fewer deaths being assigned to Chronic renal failure, unspecified (N18.9).

- The increase in Chronic liver disease and cirrhosis (K70,K73-K74) in 2007 from 2006 may largely be due to a coding change that resulted in more deaths being assigned to Alcoholic liver disease (K70).

- In 2007, deaths previously assigned to Mental and behavioral disorders due to use of alcohol, acute intoxication (F10.0) were instead assigned to Accidental poisoning by and exposure to alcohol (X45); Intentional self-poisoning (suicide) by and exposure to alcohol (X65); and Poisoning by and exposure to alcohol, undetermined intent (Y15).

\section{Rare causes of death}

Selected causes of death considered to be of public health concern are routinely confirmed by states according to agreed-upon procedures between state vital statistics programs and NCHS. These causes, termed infrequent and rare causes of death, are listed in the NCHS Instruction Manual, Parts 2a, 11, and $20(47,55,56)$.

For data year 2007, complete confirmation of deaths from infrequent and rare causes was not provided by 12 states: Connecticut, Florida, Indiana, Kentucky, Maryland, North Carolina, Oklahoma, Pennsylvania, Rhode Island, South Carolina, Washington, and West Virginia.

\section{Injury mortality by mechanism and intent}

Injury mortality data are presented using the external cause of injury mortality matrix for ICD-10 (Table 18). In this framework, cause-of-injury deaths are organized principally by mechanism (e.g., firearm or poisoning), and secondarily by manner or intent of death (e.g., unintentional, suicide, or homicide).

The number of deaths for selected causes in this framework may differ from those shown in tables that use the standard mortality tabulation lists. Following WHO conventions, standard mortality tabulations (Table 10) present external causes of death (ICD-10 codes U01-U03 and V01-Y89); in contrast, the matrix (Table 18) excludes deaths classified to Complications of medical and surgical care (Y40-Y84 and Y88). For additional information on injury data presented in this framework, see "Deaths: Injuries, 2002" (57), available from http://www.cdc.gov/nchs/products/nvsr.htm\#vol54. Data for later years are available through CDC's Wonder system at http://wonder.cdc.gov/ or through CDC's Web-based Injury Statistics Query and Reporting System (WISQARS) at http://www.cdc.gov/injury/wisqars/index.html. Implementation of changes to ICD-10 may affect the matrix, requiring modification of codes in selected categories. For information on the latest ICD-10 external cause-of-injury codes included in the matrix, see http://www.cdc.gov/nchs/injury/injury_tools.htm.

\section{Codes for firearm deaths}

Causes of death attributable to firearm mortality include ICD-10 codes U01.4, Terrorism involving firearms (homicide); W32-W34, Accidental discharge of firearms; X72-X74, Intentional self-harm (suicide) by discharge of firearms; X93-X95, Assault (homicide) by discharge of firearms; Y22-Y24, Discharge of firearms, undetermined intent; and Y35.0, Legal intervention involving firearm discharge. Deaths from injury by firearms exclude deaths due to explosives and other causes indirectly related to firearms.

\section{Codes for drug-induced deaths}

Causes of death attributable to drug-induced mortality include ICD-10 codes D52.1, Drug-induced folate deficiency anemia; D59.0, Drug-induced hemolytic anemia; D59.2, Drug-induced nonautoimmune hemolytic anemia; D61.1, Drug-induced aplastic anemia; D64.2, Secondary sideroblastic anemia due to drugs and toxins; E06.4, Drug-induced thyroiditis; E16.0, Drug-induced hypoglycemia without coma; E23.1, Drug-induced hypopituitarism; E24.2, Druginduced Cushing's syndrome; E27.3, Drug-induced adrenocortical insufficiency; E66.1, Drug-induced obesity; selected codes from the 
ICD-10 title of mental and behavioral disorders due to psychoactive substance use, specifically, F11.0-F11.5, F11.7-F11.9, F12.0-F12.5, F12.7-F12.9, F13.0-F13.5, F13.7-F13.9, F14.0-F14.5, F14.7-F14.9, F15.0-F15.5, F15.7-F15.9, F16.0-F16.5, F16.7-F16.9, F17.0, F17.3-F17.5, F17.7-F17.9, F18.0-F18.5, F18.7-F18.9, F19.0-F19.5, and F19.7-F19.9; G21.1, Other drug-induced secondary parkinsonism; G24.0, Drug-induced dystonia; G25.1, Drug-induced tremor; G25.4, Drug-induced chorea; G25.6, Drug-induced tics and other tics of organic origin; G44.4, Drug-induced headache, not elsewhere classified; G62.0, Drug-induced polyneuropathy; G72.0, Drug-induced myopathy; 195.2, Hypotension due to drugs; J70.2, Acute druginduced interstitial lung disorders; J70.3, Chronic drug-induced interstitial lung disorders; J70.4, Drug-induced interstitial lung disorder, unspecified; K85.3, Drug-induced acute pancreatitis; L10.5, Druginduced pemphigus; L27.0, Generalized skin eruption due to drugs and medicaments; L27.1, Localized skin eruption due to drugs and medicaments; M10.2, Drug-induced gout; M32.0, Drug-induced systemic lupus erythematosus; M80.4, Drug-induced osteoporosis with pathological fracture; M81.4, Drug-induced osteoporosis; M83.5, Other drug-induced osteomalacia in adults; M87.1, Osteonecrosis due to drugs; R50.2, Drug-induced fever; R78.1, Finding of opiate drug in blood; R78.2, Finding of cocaine in blood; R78.3, Finding of hallucinogen in blood; R78.4, Finding of other drugs of addictive potential in blood; R78.5, Finding of psychotropic drug in blood; X40-X44, Accidental poisoning by and exposure to drugs, medicaments and biological substances; X60-X64, Intentional self-poisoning (suicide) by and exposure to drugs, medicaments and biological substances; X85, Assault (homicide) by drugs, medicaments and biological substances; and Y10-Y14, Poisoning by and exposure to drugs, medicaments and biological substances, undetermined intent. Drug-induced causes exclude accidents, homicides, and other causes indirectly related to drug use, as well as newborn deaths associated with the mother's drug use.

\section{Codes for alcohol-induced deaths}

Causes of death attributable to alcohol-induced mortality include ICD-10 codes E24.4, Alcohol-induced pseudo-Cushing's syndrome; F10, Mental and behavioral disorders due to alcohol use; G31.2, Degeneration of nervous system due to alcohol; G62.1, Alcoholic polyneuropathy; G72.1, Alcoholic myopathy; 142.6, Alcoholic cardiomyopathy; K29.2, Alcoholic gastritis; K70, Alcoholic liver disease; K85.2, Alcohol-induced acute pancreatitis; K86.0, Alcohol-induced chronic pancreatitis; R78.0, Finding of alcohol in blood; X45, Accidental poisoning by and exposure to alcohol; X65, Intentional self-poisoning by and exposure to alcohol; and Y15, Poisoning by and exposure to alcohol, undetermined intent. Alcohol-induced causes exclude accidents, homicides, and other causes indirectly related to alcohol use, as well as newborn deaths associated with maternal alcohol use.

\section{Race and Hispanic origin}

The 2003 revision of the U.S. Standard Certificate of Death allows the reporting of more than one race (multiple races) (38). This change was implemented to reflect the increasing diversity of the population of the United States and to be consistent with the decennial census. The race and ethnicity items on the revised certificate are compliant with the 1997 "Revision of the Race and Ethnic Standards for Federal Statistics and Administrative Reporting" issued by the Office of Management and Budget (OMB). This revision replaced standards that were issued in 1977 (8). The new standards mandate the collection of more than one race where applicable for federal data (7). In addition, the new certificate is compliant with the OMB-mandated minimum set of five races to be reported for federal data. Multiple race includes any combination of white, black or African American, American Indian or Alaska Native (AIAN), Asian, and Native Hawaiian or Other Pacific Islander (NHOPI). If two or more specific subgroups such as Korean and Chinese are reported, these count as a single race of Asian rather than as multiple races.

The number of states reporting multiple race has increased, from 7 states in 2003 to 27 states and the District of Columbia in 2007:

- In 2003, multiple race was reported on the revised death certificates of California, Idaho, Montana, and New York, as well as on the unrevised certificates of Hawaii, Maine, and Wisconsin.

- In 2004, multiple race was reported for the entire year on the revised death certificates of California, Idaho, Michigan, Montana, New Jersey, New York, Oklahoma, South Dakota, Washington, and Wyoming, as well as on the unrevised certificates of Hawaii, Maine, Minnesota, and Wisconsin. New Hampshire began reporting multiple race in mid-April 2004 upon implementing the revised certificate.

- In 2005, multiple race was reported for the entire year on the revised death certificates of California, Connecticut, Florida, Idaho, Kansas, Michigan, Montana, Nebraska, New Hampshire, New Jersey, New York, Oklahoma, South Carolina, South Dakota, Utah, Washington, and Wyoming as well as on the unrevised certificates of Hawaii, Maine, Minnesota, and Wisconsin. The District of Columbia began reporting multiple race in March 2005 upon implementing the revised certificate.

- In 2006, multiple race was reported on the revised death certificates of California, Connecticut, the District of Columbia, Florida, Idaho, Kansas, Michigan, Montana, Nebraska, New Hampshire, New Jersey, New Mexico, New York, Oklahoma, Oregon, Rhode Island, South Carolina, South Dakota, Texas, Utah, Washington, and Wyoming as well as on the unrevised certificates of Hawaii, Maine, Minnesota, and Wisconsin.

- In 2007, multiple race was reported on the revised death certificates of California, Connecticut, Delaware, the District of Columbia, Florida, Idaho, Kansas, Michigan, Montana, Nebraska, New Hampshire, New Jersey, New Mexico, New York, Ohio, Oklahoma, Oregon, Rhode Island, South Carolina, South Dakota, Texas, Utah, Washington, and Wyoming as well as on the unrevised certificates of Hawaii, Maine, Minnesota, and Wisconsin.

In 2007, more than one race was reported for 0.4 percent of the records in the 27 multiple-race reporting states and the District of Columbia (Table I). Although still uncommon, multiple races were reported more often for younger decedents than for older decedents (2.3 percent of decedents under age 25 years compared with 0.6 percent of decedents aged 25-64 and 0.2 percent of decedents aged 65 and over). No decedent was reported as having more than four races. Of those records where more than one race was reported, the NHOPI category was reported in combination with another race more often ( 46.3 percent) than the other categories (white, 0.4 percent; black, 0.7 percent; Asian, 5.2 percent; and AIAN, 20.0 percent). 
Table I. Deaths by race: California, Connecticut, Delaware, District of Columbia, Florida, Hawaii, Idaho, Kansas, Maine, Michigan, Minnesota, Montana, Nebraska, New Hampshire, New Jersey, New Mexico, New York, Ohio, Oklahoma, Oregon, Rhode Island, South Carolina, South Dakota, Texas, Utah, Washington, Wisconsin, and Wyoming, 2007

[By state of occurrence]

\begin{tabular}{|c|c|c|}
\hline Race & Deaths & $\begin{array}{c}\text { Percent of } \\
\text { deaths }\end{array}$ \\
\hline Total $\ldots \ldots \ldots \ldots \ldots \ldots \ldots \ldots \ldots \ldots \ldots \ldots$ & $1,389,618$ & 100.0 \\
\hline$\ldots \ldots \ldots \ldots \ldots \ldots \ldots \ldots \ldots$ & $1,384,351$ & 99.6 \\
\hline White $\ldots \ldots \ldots \ldots \ldots \ldots \ldots \ldots \ldots$ & $1,189,806$ & 85.6 \\
\hline Black $\ldots \ldots \ldots \ldots \ldots \ldots \ldots \ldots$ & 137,791 & 9.9 \\
\hline Asian . . . . . . . . . . . . . . . . . . . & 34,251 & 2.5 \\
\hline$\ldots \ldots \ldots \ldots \ldots \ldots \ldots$ & 11,865 & 0.9 \\
\hline$\ldots \ldots \ldots \ldots \ldots$ & 8,888 & 0.6 \\
\hline$\ldots \ldots \ldots \ldots \ldots \ldots \ldots$ & 1,750 & 0.1 \\
\hline Two or more races. . . . . . . . . . . . . . . . & 5,267 & 0.4 \\
\hline Two races $\ldots \ldots \ldots \ldots \ldots \ldots \ldots \ldots \ldots \ldots \ldots$ & 4,780 & 0.3 \\
\hline AIAN $^{2}$ and white $\ldots \ldots \ldots \ldots \ldots \ldots \ldots$ & 1,888 & 0.1 \\
\hline Asian and white. $\ldots \ldots \ldots \ldots \ldots \ldots \ldots \ldots$ & 844 & 0.1 \\
\hline$\ldots \ldots \ldots \ldots \ldots \ldots \ldots \ldots$ & 641 & 0.0 \\
\hline $\mathrm{NHOPI}^{3}$ and white $\ldots \ldots \ldots \ldots \ldots \ldots \ldots \ldots$ & 552 & 0.0 \\
\hline$\ldots \ldots \ldots \ldots \ldots$ & 536 & 0.0 \\
\hline$\ldots \ldots \ldots \ldots \ldots$ & 198 & 0.0 \\
\hline Black and Asian $\ldots \ldots \ldots \ldots \ldots \ldots \ldots \ldots \ldots$ & 85 & 0.0 \\
\hline AlAN $^{2}$ and $A s i a n . \ldots \ldots \ldots \ldots \ldots \ldots \ldots$ & 15 & 0.0 \\
\hline Black and $\mathrm{NHOP}^{3} \ldots \ldots \ldots \ldots \ldots \ldots$ & 15 & 0.0 \\
\hline $\mathrm{AIAN}^{2}$ and $\mathrm{NHOP}^{3} \ldots \ldots \ldots \ldots \ldots \ldots \ldots$ & 6 & 0.0 \\
\hline$\ldots \ldots \ldots \ldots \ldots \ldots \ldots \ldots$ & 474 & 0.0 \\
\hline$\ldots \ldots \ldots \ldots \ldots$ & 370 & 0.0 \\
\hline Black, AIAN $^{2}$, and white. . & 57 & 0.0 \\
\hline AIAN $^{2}, \mathrm{NHOP}^{3}$, and white & 15 & 0.0 \\
\hline Black, Asian, and white . . & 13 & 0.0 \\
\hline$\ldots \ldots \ldots \ldots \ldots \ldots$ & 12 & 0.0 \\
\hline$\ldots \ldots \ldots \ldots \ldots$ & 3 & 0.0 \\
\hline Black, NHOP| ${ }^{3}$, and white. . . . . . . . . . . . . & 2 & 0.0 \\
\hline 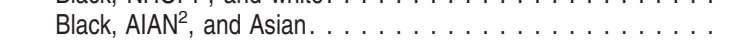 & 1 & 0.0 \\
\hline $\mathrm{AlAN}^{2}, \mathrm{Asian}$, and $\mathrm{NHOPI}^{3} \ldots \ldots \ldots \ldots \ldots \ldots$ & 1 & 0.0 \\
\hline$\ldots \ldots \ldots \ldots \ldots \ldots$ & 13 & 0.0 \\
\hline AlAN $^{2}$, Asian, $\mathrm{NHOP}^{3}$, and white $\ldots \ldots \ldots \ldots \ldots \ldots$ & 8 & 0.0 \\
\hline Black, Asian, $\mathrm{NHOPI}^{3}$, and white. . . . . . . . . . . . & 3 & 0.0 \\
\hline Black, Asian, AlAN ${ }^{2}$, and white. . . . . . . . . . . . . . . . . & 2 & 0.0 \\
\hline
\end{tabular}

0.0 Quantity more than zero but less than 0.05 .

${ }^{1}$ Includes records for which race was reported as "other." Future processing assigns "other" race to one of the recognized categories. "Other" race comprises a wide variety of responses; however, the most common is to check "other" and not provide future specification or to report a Hispanic group as a race.

${ }^{2}$ American Indian or Alaska Native.

${ }^{3}$ Native Hawaiian or Other Pacific Islander.

Data from the vital records of the 23 states based on the 1989 revision of the U.S. Standard Certificate of Death follow the 1977 OMB standard, allowing only a single race to be reported $(8,41)$. In addition, these states report a minimum set of four races as stipulated in the 1977 standard. These are white, black or African American, AIAN, and Asian or Pacific Islander (API).

To provide uniformity and comparability of data during the transition period before all or most of the data become available in the multiple-race format, the responses of those for whom more than one race was reported (multiple race) must be "bridged" to a single race. The bridging procedure is similar to that used to bridge multiracial population estimates $(10,11)$. Multiracial decedents are imputed to a single race (white, black, AIAN, or API) according to their combination of races, Hispanic origin, sex, and age indicated on the death certificate. The imputation procedure is described in detail at http://www.cdc.gov/ nchs/data/dvs/Multiple_race_documentation_5-10-04.pdf. Similarly, when calculating infant and maternal mortality rates, multiracial infants are bridged to a single race. The bridging procedure for multiple-race mothers and fathers is based on the procedure used to bridge the multiple-race population estimates (37); see the following subsection on "Infant and maternal mortality rates."

Race and Hispanic origin are reported separately on the death certificate. Therefore, data shown by race include persons of Hispanic and non-Hispanic origin, and data for Hispanic origin include persons of any race. In this report, unless otherwise specified, deaths of persons of Hispanic origin are included in the totals for each race group-white, black, AIAN, and API-according to the decedent's race as reported on the death certificate. Data shown for Hispanic persons include all persons of Hispanic origin of any race.

Mortality data for the Hispanic-origin population are based on deaths of residents of all 50 states and the District of Columbia. Data year 1997 was the first year in which mortality data for the Hispanic population were available for the entire United States.

Quality of race and Hispanic origin data-Death rates for Hispanic, AIAN, and API persons should be interpreted with caution because of inconsistencies in reporting Hispanic origin or race on the death certificate as compared with censuses, surveys, and birth certificates. Studies have shown underreporting on death certificates of AIAN, API, 
and Hispanic decedents, as well as undercounts of these groups in censuses $(16,18,58,59)$.

A number of studies have been conducted on the reliability of race reported on the death certificate by comparing it with race reported on another data collection instrument, such as the census or a survey $(16,18,58,59)$. Inconsistencies may arise because of differences in who provides race information on the compared records. Race information on the death certificate is reported by a funeral director as provided by an informant or, in the absence of an informant, on the basis of observation. In contrast, race on the census or the Current Population Survey (CPS) is obtained while the person is alive; in these cases, race is self-reported or reported by another member of the household familiar with the person and, therefore, may be considered more valid. A high level of agreement between the death certificate and the census or survey report is essential to assure unbiased death rates by race.

Studies $(16,18,58,59)$ show that a person self-reported as AIAN or API on census or survey records was sometimes reported as white on the death certificate. The net effect of misclassification is an underestimation of deaths and death rates for races other than white and black. In addition, undercoverage of minority groups in the census and resultant population estimates introduces biases into death rates by race $(16,18,58-61)$. Unlike the 1990 census, coverage error in the 2000 census was found to be statistically significant only for the non-Hispanic white population (overcounted by approximately 1.13 percent) and non-Hispanic black population (undercounted by approximately 1.84 percent) (60).

Using the National Longitudinal Mortality Study, Arias et al. examined the reliability of race and Hispanic origin reported on about 250,000 death certificates compared with that reported on a total of 26 CPSs conducted by the U.S. Bureau of the Census for 1979-1998 $(16,18)$. Agreement between the two sources was found to be excellent for the white and black populations, both exhibiting CPS-to-death certificate ratios of 1.00 . On the other hand, substantial differences were found for other race groups. The ratio of CPS to death certificates was found to be 1.30 for the AIAN population and 1.07 for the API population, indicating net underreporting on death certificates of 30 percent for AIAN and 7 percent for API. The ratio of deaths for CPS to death certificates for Hispanics was found to be 1.05, indicating a net underreporting on death certificates for the Hispanic population of 5 percent.

Data on the Central and South American and Other Hispanic-origin populations are affected by whether a state submits literal text to NCHS, thereby making it possible to identify decedents as being of Central and South American origin.

Other races and race not stated-Beginning in 1992, all records coded as "other races" ( 0.36 percent of total deaths in 2007) were assigned to the specified race of the previous record. Records for which race was unknown, not stated, or not classifiable ( 0.17 percent) were assigned the racial designation of the previous record.

Infant and maternal mortality rates-For 1989-2007, as in previous years, infant and maternal deaths continue to be tabulated by the race of the decedent. However, beginning with the 1989 data year, the method of tabulating live births by race was changed from race of parents to race of mother, as stated on the birth certificate. This change affects infant and maternal mortality rates because live births are the denominators of these rates $(40,62)$. To improve continuity and ease of interpretation, trend data by race in this report have been retabulated by race of mother for all years beginning with the 1980 data year.
Quantitatively, the change in the basis for tabulating live births by race of mother results in more white births and fewer black births and births of other races. Consequently, infant and maternal mortality rates under the new tabulating procedure tend to be about 2.0 percent lower for white infants and about 5.0 percent higher for black infants than when they are computed by the previous method of tabulating live births by race of parents. Rates for most other minority races also are higher when computed by race of mother $(63,64)$.

In 2007, multiple race was reported on the revised birth certificates of California, Colorado, Delaware, Florida, Georgia (for births occurring after January 1 only), Idaho, Indiana, lowa, Kansas, Kentucky, Michigan (for births at most facilities), Nebraska, New Hampshire, New York state (excluding New York City), North Dakota, Ohio, Pennsylvania, South Carolina, South Dakota, Tennessee, Texas, Vermont, Washington, and Wyoming, and on the unrevised birth certificates of Hawaii, Minnesota, and Utah (65).

Infant mortality rates for the Hispanic-origin population are based on numbers of resident infant deaths reported to be of Hispanic origin and numbers of resident live births by Hispanic origin of mother for the United States. In computing infant mortality rates, deaths and live births of unknown origin are not distributed among the specified Hispanic and non-Hispanic groups. In the United States in 2007, the percentage of infant deaths of unknown origin was 0.6 percent and the percentage of live births to mothers of unknown origin was 0.7 percent.

Small numbers of infant deaths for specific Hispanic-origin groups result in infant mortality rates subject to relatively large random variation (see following section on "Random variation"). Infant mortality rates by Hispanic origin are less subject to reporting error when based on linked files of infant deaths and live births $(36,37)$.

Infant mortality rates calculated from the general mortality file for specified race and Hispanic origin contain errors because of reporting problems that affect the classification of race and Hispanic origin on the birth and death certificates for the same infant. Infant mortality rates by specified race and Hispanic origin are more accurate when based on the linked file of infant deaths and live births $(36,37)$. The linked file computes infant mortality rates using the race and Hispanic origin of the mother from the birth certificate in both the numerator and denominator of the rate. In addition, the mother's race and Hispanic origin from the birth certificate is considered to be more accurately reported than the infant's race and Hispanic origin from the death certificate-on the birth certificate, race is generally reported by the mother at the time of delivery, whereas on the death certificate, the infant's race and Hispanic origin is reported by an informant, usually the mother but sometimes the funeral director. Estimates of reporting errors have been made by comparing rates based on the linked files with those in which the infant's race is based on information from the death certificate $(36,58)$.

\section{Life tables}

The life table provides a comprehensive measure of the effect of mortality on life expectancy. It is composed of sets of values showing the mortality experience of a hypothetical group of infants born at the same time and subject throughout their lifetime to the age-specific death rates of a particular time period, usually a given year. Prior to data year 1997, U.S. life tables were abridged and constructed by reference to a standard table (63). In addition, the age range for these life tables was limited to 5 -year age groups ending with the age 
group 85 years and over. Beginning with final data reported for 1997 , the life table methodology was changed from previous annual reports, with a revised methodology used for 1997-1999 data and a newly revised methodology for 2000-2007 data.

For data years 1997-1999, complete life tables were constructed by single years of age extending to age 100 (66) using a revised methodology similar to that of the 1989-1991 decennial life tables (67). The revised methodology offers comparability with decennial life table methodology, greater accuracy, and greater age detail. A comparison of the two methods shows small differences in resulting values for life expectancy (66). Although the revised method produces complete life tables (by single years of age), the life table data shown in this report are summarized in 5-year age groupings. To calculate the probability of dying at each age, the revised methodology used vital statistics death rates for ages under 85 years, and mortality data from the Medicare program for ages 85 and over. The Medicare data are shown to be significantly more reliable than vital statistics data when modeling the probability of dying at the oldest ages (68).

Life table data shown in this report for data years 2000-2007 are based on the newly revised methodology and may differ from figures previously published. Complete life tables by single years of age extending to age 100 were constructed using a methodology similar to that developed for the 1999-2001 decennial life tables (69). To calculate the probability of dying at each age, the newly revised methodology used vital statistics death rates for ages under 66 , and modeled probabilities of death for ages 66 to 100 based on blended vital statistics and Medicare probabilities of dying (69). The newly revised methodology, along with a more comprehensive description of the methodology, was published in United States Life Tables, 2005 NVSR Volume 58, Number 10; see http://www.cdc.gov/nchs/data/nvsr/nvsr58/ nvsr58_10.pdf for additional information.

\section{Causes of death contributing to changes in life expectancy}

A life table partitioning technique was used to estimate causes of death contributing to changes in life expectancy in this report. The method partitions changes into component additive parts and identifies the causes of death having the greatest influence, positive or negative, on changes in life expectancy $(17,70,71)$.

\section{Marital status}

Age-specific and age-adjusted death rates by marital status are shown by sex in Table 25 . Mortality data by marital status are generally of high quality. A study of death certificate data using the 1986 National Mortality Followback Survey showed a high level of consistency in reporting marital status (61). Age-adjusted death rates by marital status were computed based on age-specific rates and the standard population for those aged 25 and over. Although agespecific death rates by marital status are shown for the age group 15-24, they are not included in the computation of the age-adjusted rate because of their high variability, particularly for the widowed population. Furthermore, the age groups $75-84$ and 85 and over are combined because of high variability in death rates among those aged 85 and over, particularly for the never-married population.

\section{Educational attainment}

Beginning in 2003, some registration areas adopted the new U.S. Standard Certificate of Death, which includes a revised educational attainment item, replacing the 1989 version, which had focused on highest school grade completed. Neither the new nor old item captures vocational training. The change establishes consistency with U.S. Census Bureau data to improve the ability to identify specific degrees and persons who had completed 12 years of education but did not hold either a high school diploma or General Educational Development (GED) high school equivalency diploma. The previous item also had been used inappropriately and inaccurately to infer degree status. Based on testing by the Census Bureau, the new item identifies about 2 percent more persons with less than a high school diploma or equivalent, 13 percent fewer persons with a high school diploma, and 8 percent more persons with at least some college (72). In 2007, the District of Columbia and 22 states used the revised item: California, Connecticut, Delaware, Florida, Idaho, Kansas, Michigan, Montana, Nebraska, New Hampshire, New Jersey, New Mexico, New York, Ohio, Oklahoma, Oregon, South Carolina, South Dakota, Texas, Utah, Washington, and Wyoming. The unrevised education item continued to be used by 26 states: Alabama, Alaska, Arizona, Arkansas, Colorado, Hawaii, Illinois, Indiana, lowa, Kentucky, Louisiana, Maine, Maryland, Massachusetts, Minnesota, Mississippi, Missouri, Nevada, North Carolina, North Dakota, Pennsylvania, Tennessee, Vermont, Virginia, West Virginia, and Wisconsin.

Table 26 shows mortality data by educational attainment for states using the 2003 version of the standard death certificate and, separately, for states using the 1989 version. Data were approximately 80 percent or more complete on a state-of-occurrence basis. Data for Georgia and Rhode Island were excluded because the educational attainment item was not on their certificates. Age-adjusted death rates by educational attainment were computed based on the age-specific rates and the standard population for those aged 25-64. Data for those aged 65 and over are not shown because reporting quality is poorer at older ages (73).

Rates by educational attainment for states using the unrevised certificate are affected by differences between measurement of education for the numerator, which is based on the number of years of education completed as reported on the 1989 revision of the death certificate, and the denominator, which is based on highest degree completed as reported on the 2000 census and the CPSs $(72,74)$.

Table II shows a 2002-to-2007 comparison of the percent distribution of deaths by measures of educational attainment for areas using the revised certificate in 2007. However, South Dakota is excluded from this table because that state first began reporting education in 2004 and has no comparison data for 2002.

\section{Injury at work}

Information on deaths attributed to injuries at work is derived from a separate item on the death certificate that asks the medical certifier whether the death resulted from an injury sustained at work. This item is on the death certificate of all states. Number of deaths, age-specific death rates, and age-adjusted death rates for injury at work are shown in Tables 27 and 28. Deaths, crude death rates, and 
Table II. Percent distribution of deaths, by education items: California, Connecticut, Delaware, District of Columbia, Florida, Idaho, Kansas, Michigan, Montana, Nebraska, New Hampshire, New Jersey, New Mexico, New York, Ohio, Oklahoma, Oregon, South Carolina, Texas, Utah, Washington, and Wyoming, 2002 and 2007

[By state of occrrence. Excludes nonresidents of the United States. Because of rounding, the sum of the subgroups may not add to the total]

\begin{tabular}{|c|c|c|c|}
\hline \multicolumn{2}{|c|}{2002} & \multicolumn{2}{|l|}{2007} \\
\hline Years of school completed & $\begin{array}{l}\text { Percent } \\
\text { distribution }\end{array}$ & Educational attainment & $\begin{array}{c}\text { Percent } \\
\text { distribution }\end{array}$ \\
\hline Total & 100.0 & Total. & 100.0 \\
\hline Under 12 years & 28.2 & Less than high school diploma or GED. . . . & 26.4 \\
\hline 12 years ... & 41.2 & High school diploma or GED . . . . & 41.0 \\
\hline 13 years or more & 27.5 & Some college or collegiate degree. & 30.6 \\
\hline Not stated & 3.2 & Not stated . . . . . . . . . . . & 2.0 \\
\hline
\end{tabular}

NOTE: GED is General Educational Development high school equivalency diploma.

age-adjusted death rates for injury at work are shown for those aged 15 and over. Age-adjusted death rates for injury at work were computed using age-specific death rates and the 2000 U.S. standard population for those aged 15 and over; see "Computing rates."

\section{Infant mortality}

Infant mortality rates are the most commonly used index for measuring the risk of dying during the first year of life. The rates presented in this report are calculated by dividing the number of infant deaths in a calendar year by the number of live births registered for the same period, and are presented as rates per 1,000 or per 100,000 live births. For final birth figures used in the denominator for infant mortality rates, see "Births: Final Data for 2007" (65). In contrast to infant mortality rates based on live births, infant death rates are based on the estimated population under age 1. Infant death rates that appear in tabulations of age-specific death rates in this report are calculated by dividing the number of infant deaths by the July 1,2007 , population estimate of persons under age 1 , based on 2000 census populations. These rates are presented per 100,000 population in this age group. Because of differences in the denominators, infant death rates may differ from infant mortality rates.

Another data source is available for infant mortality - the linked file of live births and infant deaths. Data from this source differs from the infant mortality data presented in this report because the linked file includes only events in which both the birth and the death occur in the United States, and late-filed births. Processing of the linked file allows for further exclusion of infant records due to duplicates and records with additional information that raise questions about an infant's age. Although the differences are usually minuscule, infant mortality rates based on the linked file tend to be somewhat smaller than those based on data from the general mortality file as presented in this report. The linked file is the preferred source for infant mortality by race because it uses the mother's self-reported race from the child's birth certificate $(36,37)$, which is more reliable than the infant's race listed on the death certificate, and because the numerator and denominator are referring to the same person's race.

\section{Maternal mortality}

Maternal mortality rates are computed on the basis of the number of live births. The maternal mortality rate indicates the likelihood of a pregnant woman dying of maternal causes. The rates are calculated by dividing the number of maternal deaths in a calendar year by the number of live births registered for the same period and are presented as rates per 100,000 live births. The number of live births used in the denominator is an approximation of the population of pregnant women who are at risk of a maternal death.

Maternal deaths are defined by WHO as "the death of a woman while pregnant or within 42 days of termination of pregnancy, irrespective of the duration and the site of the pregnancy, from any cause related to or aggravated by the pregnancy or its management, but not from accidental or incidental causes" (6). Included in these deaths are ICD-10 codes A34, O00-095, and 098-099.

If a state death certificate includes a separate question regarding pregnancy status, a positive response to the question is interpreted as if "pregnant" were reported in Part II of the cause-of-death section of the death certificate. If a specified length of time is not provided by the medical certifier, the pregnancy is assumed to have terminated 42 days or less prior to death. Furthermore, if only indirect maternal causes of death (i.e., a previously existing disease or a disease that developed during pregnancy not due to direct obstetric causes but aggravated by physiological effects of pregnancy) are reported in Part I and pregnancy is reported in either Part I or Part II, the death is classified as a maternal death.

An evaluation study for the 1995-1997 period found that 35 percent more maternal deaths were identified through surveillance efforts than by solely using the death certificate. A number of explanations accounted for the lower ascertainment from death certificates, including lack of information reported in the cause-of-death section, use of fewer sources, and some differences in identification (75). This differential is due, in part, to decreasing changes in the coding of indirect maternal causes under ICD-10 that accounted for a nearly 13 percent increase in maternal deaths compared with ICD-9, and the increasing use of a pregnancy status checkbox on death certificates.

The 2003 revision of the U.S. Standard Certificate of Death introduced a standard question format with categories to take advantage of additional codes available in ICD-10 for deaths with a connection to pregnancy, childbirth, and the puerperium. As states revise their certificates, most are expected to introduce the standard item or replace pre-existing questions with it, allowing for wider adoption of a pregnancy status item nationwide and greater standardization of the particular item used. As of 2007, 34 states and the District of Columbia have a separate question related to pregnancy status of female decedents around the time of their death, and 2 states have a prompt 
encouraging certifiers to report recent pregnancies on the death certificate. However, at least six different questions were used in the 34 states, reflecting the mix of states using the 2003 standard format and states with pre-existing questions.

The number of maternal deaths has increased most years since 2003 as a result of direct and indirect effects of inclusion of a pregnancy status item on the 2003 version of the standard death certificate (76). For states that already had a separate question, additional guidance was provided in 2003 for identifying maternal deaths, resulting in more deaths being identified. For states that adopt the standard item, additional information is available for use in identifying maternal deaths.

\section{Population bases for computing rates}

Populations used for computing death rates and life tables shown in this report represent the population residing in the United States, enumerated as of April 1 for census years and estimated as of July 1 for all other years. Population estimates used to compute death rates for the United States for 2007 are shown by race for 5 -year age groups in Table III and are available by single years of age at http://www.cdc.gov/nchs/nvss/mortality_tables.htm (77).

Population estimates in Table IV for Mexican, Puerto Rican, Cuban, Central and South American, and Other Hispanic populations, and population estimates by marital status in Table V, are based on the CPS adjusted to resident population control totals for the United States (78) and, as such, are subject to sampling variation; see "Random variation." The control totals used are 2000-based population estimates for the United States for July 1, 2007 (77).

Population estimates by educational attainment, shown in Table VI, are also based on the CPS adjusted to resident population control totals (78), and similarly subject to sampling variation (see "Random variation"). The control totals used are 2000-based population estimates for July 1, 2007, for the 22 states and District of Columbia that reported mortality data by educational attainment using the 2003 version of the U.S. Standard Certificate of Death, and for the 26 states that reported it using the 1989 version (77).

Population estimates for each state, shown in Table VII, were estimated from state-level postcensal population estimates based on the 2000 census, estimated as of July 1, 2007 (77). Population estimates for Puerto Rico, Virgin Islands, Guam, American Samoa, and Northern Marianas, also shown in Table VII, are based on the 2000 census, estimated as of July 1, 2007 (79). Population estimates for each state and territory are not subject to sampling variation because the sources used in demographic analysis are complete counts.

Death rates shown in this report for 1991-2007 are based on populations consistent with the 2000 census levels (77-87). These estimates were produced under a collaborative arrangement with the U.S. Census Bureau and are based on the 2000 census counts by age, race, and sex, modified for consistency with OMB race categories as of 1977 and historical categories for death data (8). The modification procedures are described in detail elsewhere $(10,11)$.

\section{Computing rates}

Except for infant and maternal mortality rates, rates are on an annual basis per 100,000 estimated population residing in the specified area. Infant and maternal mortality rates are per 1,000 or per 100,000 live births. Comparisons made among rates in this report, unless otherwise specified, are statistically significant at the 0.05 level of significance. Lack of comment in this report about any two rates does not mean that the difference was tested and found not to be significant at this level.

Age-adjusted rates $\left(R^{\prime}\right)$ are used to compare relative mortality risks among groups and over time. However, they should be viewed as relative indexes rather than as actual measures of mortality risk. They were computed by the direct method-that is, by applying agespecific death rates $\left(R_{i}\right)$ to the U.S. standard population age distribution (Table VIII):

$$
R^{\prime}=\sum_{i} \frac{P_{s i}}{P_{s}} R_{i}
$$

where $P_{s i}$ is the standard population for age group $i$ and $P_{s}$ is the total U.S. standard population (all ages combined).

Beginning with the 1999 data year, a new population standard was adopted by NCHS for use in age-adjusting death rates. Based on the projected year 2000 population of the United States, the new standard replaces the 1940 standard population that had been used for over 50 years. The new population standard affects levels of mortality and, to some extent, trends and group comparisons. Of particular note are the effects on race mortality comparisons. For a detailed discussion, see Age Standardization of Death Rates: Implementation of the Year 2000 Standard (88). Beginning with 2003 data, the traditional standard million population along with corresponding standard weights to six decimal places were replaced by the projected year 2000 population age distribution (see Table VIII). The effect of the change is negligible and does not significantly affect comparability with age-adjusted rates calculated using the previous method.

All age-adjusted rates shown in this report are based on the 2000 U.S. standard population. The 2000 standard population used for computing age-adjusted rates and standard errors, excluding those by marital status, education, injury at work, and the U.S. territories, is shown in Table VIII.

Age-adjusted rates by marital status were computed by applying the age-specific death rates to the U.S. standard population for those aged 25 and over. Although age-specific death rates by marital status are shown for the age group 15-24, they are not included in the calculation of age-adjusted rates because of their high variability, particularly for the widowed population. Age groups $75-84$ and 85 and over are combined because of high variability in death rates in the 85-and-over age group, particularly for the never-married population. The 2000 standard population used for computing age-adjusted rates and standard errors by marital status is shown in Table IX.

Age-adjusted rates by educational attainment were computed by applying the age-specific death rates to the U.S. standard population for those aged 25-64. Data for those aged 65 and over are not shown because reporting quality is poorer for older ages (74). The year 2000 standard population used for computing age-adjusted rates and standard errors by education is shown in Table $\mathrm{X}$.

Age-adjusted rates for injury at work were computed by applying the age-specific death rates to the U.S. standard population for those aged 15 and over. The 2000 standard population used for computing age-adjusted rates and standard errors for injury at work is shown in Table XI.

Age-adjusted rates for Puerto Rico, Virgin Islands, Guam, American Samoa, and Northern Marianas were computed by applying 
Table III. Estimated population by 5-year age groups, specified by race and sex: United States, 2007

[Populations are postcensal estimates based on the 2000 census, estimated as of July 1, 2006; see "Technical Notes"]

\begin{tabular}{|c|c|c|c|c|c|c|c|c|c|c|c|c|c|c|c|}
\hline \multirow[b]{2}{*}{ Age } & \multicolumn{3}{|c|}{ All races } & \multicolumn{3}{|c|}{ White } & \multicolumn{3}{|c|}{ Black } & \multicolumn{3}{|c|}{ American Indian or Alaska Native } & \multicolumn{3}{|c|}{ Asian or Pacific Islander } \\
\hline & Total & Male & Female & Total & Male & Female & Total & Male & Female & Total & Male & Female & Total & Male & Female \\
\hline Total & $301,621,157$ & $148,658,898$ & $152,962,259$ & $243,582,944$ & $120,734,413$ & $122,848,531$ & $40,028,958$ & $19,121,492$ & $20,907,466$ & $3,235,707$ & $1,615,238$ & $1,620,469$ & $14,773,548$ & $7,187,755$ & $7,585,793$ \\
\hline Under 1 year. & $4,257,020$ & $2,178,808$ & $2,078,212$ & $3,278,466$ & $1,678,943$ & $1,599,523$ & 715,507 & 364,946 & 350,561 & 46,869 & 23,864 & 23,005 & 216,178 & 111,055 & 105,123 \\
\hline $1-4$ years & $16,467,105$ & $8,424,049$ & $8,043,056$ & $12,755,623$ & $6,533,079$ & $6,222,544$ & $2,694,527$ & $1,369,860$ & $1,324,667$ & 176,614 & 89,622 & 86,992 & 840,341 & 431,488 & 408,853 \\
\hline $5-9$ years. & $19,849,628$ & $10,148,578$ & $9,701,050$ & $15,455,267$ & $7,918,210$ & $7,537,057$ & $3,200,772$ & $1,625,521$ & $1,575,251$ & 241,317 & 122,624 & 118,693 & 952,272 & 482,223 & 470,049 \\
\hline $10-14$ years & $20,314,309$ & $10,399,927$ & $9,914,382$ & $15,755,855$ & $8,084,214$ & $7,671,641$ & $3,328,041$ & $1,690,973$ & $1,637,068$ & 276,281 & 140,165 & 136,116 & 954,132 & 484,575 & 469,557 \\
\hline $15-19$ years & $21,473,690$ & $11,006,869$ & $10,466,821$ & $16,665,330$ & $8,561,813$ & $8,103,517$ & $3,546,074$ & $1,797,257$ & $1,748,817$ & 306,184 & 155,031 & 151,153 & 956,102 & 492,768 & 463,334 \\
\hline $20-24$ years & $21,032,396$ & $10,852,937$ & $10,179,459$ & $16,532,925$ & $8,567,800$ & $7,965,125$ & $3,206,465$ & $1,624,419$ & $1,582,046$ & 295,896 & 151,839 & 144,057 & 997,110 & 508,879 & 488,231 \\
\hline $25-29$ years & $21,057,706$ & $10,776,189$ & $10,281,517$ & $16,536,906$ & $8,544,726$ & $7,992,180$ & $3,050,545$ & $1,496,400$ & $1,554,145$ & 268,889 & 140,004 & 128,885 & $1,201,366$ & 595,059 & 606,307 \\
\hline $30-34$ years & $19,533,220$ & $9,906,361$ & $9,626,859$ & $15,273,895$ & $7,851,146$ & $7,422,749$ & $2,668,898$ & $1,270,507$ & $1,398,391$ & 229,908 & 119,242 & 110,666 & $1,360,519$ & 665,466 & 695,053 \\
\hline $35-39$ years & $21,176,460$ & $10,654,911$ & $10,521,549$ & $16,783,160$ & $8,550,888$ & $8,232,272$ & $2,800,187$ & $1,321,524$ & $1,478,663$ & 226,516 & 115,500 & 111,016 & $1,366,597$ & 666,999 & 699,598 \\
\hline $40-44$ years & $21,984,829$ & $10,963,823$ & $11,021,006$ & $17,685,779$ & $8,921,462$ & $8,764,317$ & $2,871,828$ & $1,345,570$ & $1,526,258$ & 231,494 & 115,499 & 115,995 & $1,195,728$ & 581,292 & 614,436 \\
\hline $45-49$ years & $22,861,373$ & $11,302,842$ & $11,558,531$ & $18,709,555$ & $9,352,817$ & $9,356,738$ & $2,840,780$ & $1,321,386$ & $1,519,394$ & 229,958 & 112,182 & 117,776 & $1,081,080$ & 516,457 & 564,623 \\
\hline $50-54$ years & $21,013,387$ & $10,292,071$ & $10,721,316$ & $17,391,164$ & $8,616,618$ & $8,774,546$ & $2,463,227$ & $1,130,302$ & $1,332,925$ & 199,090 & 96,020 & 103,070 & 959,906 & 449,131 & 510,775 \\
\hline $55-59$ years & $18,236,259$ & $8,847,222$ & $9,389,037$ & $15,279,051$ & $7,500,803$ & $7,778,248$ & $1,990,314$ & 897,134 & $1,093,180$ & 160,477 & 77,191 & 83,286 & 806,417 & 372,094 & 434,323 \\
\hline $60-64$ years & $14,475,817$ & $6,927,866$ & $7,547,951$ & $12,408,141$ & $6,001,086$ & $6,407,055$ & $1,380,213$ & 606,367 & 773,846 & 115,305 & 55,115 & 60,190 & 572,158 & 265,298 & 306,860 \\
\hline $65-69$ years & $10,752,441$ & $5,019,063$ & $5,733,378$ & $9,213,048$ & $4,345,679$ & $4,867,369$ & $1,030,198$ & 436,678 & 593,520 & 79,620 & 37,601 & 42,019 & 429,575 & 199,105 & 230,470 \\
\hline $70-74$ years & $8,599,708$ & $3,867,910$ & $4,731,798$ & $7,402,758$ & $3,366,096$ & $4,036,662$ & 810,624 & 330,939 & 479,685 & 57,181 & 26,057 & 31,124 & 329,145 & 144,818 & 184,327 \\
\hline $75-79$ years & $7,324,882$ & $3,106,968$ & $4,217,914$ & $6,436,614$ & $2,761,875$ & $3,674,739$ & 600,667 & 225,909 & 374,758 & 40,635 & 17,899 & 22,736 & 246,966 & 101,285 & 145,681 \\
\hline $80-84$ years & $5,698,629$ & $2,205,705$ & $3,492,924$ & $5,074,821$ & $1,979,650$ & $3,095,171$ & 429,538 & 148,134 & 281,404 & 27,072 & 11,051 & 16,021 & 167,198 & 66,870 & 100,328 \\
\hline 85 years and over. & $5,512,298$ & $1,776,799$ & $3,735,499$ & $4,944,586$ & $1,597,508$ & $3,347,078$ & 400,553 & 117,666 & 282,887 & 26,401 & 8,732 & 17,669 & 140,758 & 52,893 & 87,865 \\
\hline
\end{tabular}


Table IV. Estimated population by 5-year age groups, according to specified Hispanic origin, race for non-Hispanic population, and sex: United States,

2007

[Populations for all origins, Hispanic, non-Hispanic, non-Hispanic white, and non-Hispanic black are postcensal estimates based on the 2000 census, estimated as of July 1, 2007; populations for Mexican, Puerto Rican, Cuban, Central and South American, and other and unknown Hispanic are based on the Current Population Survey adjusted to resident population control totals. Due to rounding, population estimates for Hispanic subgroups may not add to Hispanic control totals. The control totals are

\begin{tabular}{|c|c|c|c|c|c|c|c|c|c|c|c|c|c|c|c|c|c|c|c|c|}
\hline $\begin{array}{l}\text { Hispanic origin, } \\
\text { race for } \\
\text { non-Hispanic } \\
\text { population, } \\
\text { and sex }\end{array}$ & Total & $\begin{array}{l}\text { Under } \\
1 \text { year }\end{array}$ & $\begin{array}{c}1-4 \\
\text { years }\end{array}$ & $\begin{array}{l}5-9 \\
\text { years }\end{array}$ & $\begin{array}{l}10-14 \\
\text { years }\end{array}$ & $\begin{array}{l}15-19 \\
\text { years }\end{array}$ & $\begin{array}{l}20-24 \\
\text { years }\end{array}$ & $\begin{array}{l}25-29 \\
\text { years }\end{array}$ & $\begin{array}{l}30-34 \\
\text { years }\end{array}$ & $\begin{array}{l}35-39 \\
\text { years }\end{array}$ & $\begin{array}{l}40-44 \\
\text { years }\end{array}$ & $\begin{array}{l}45-49 \\
\text { years }\end{array}$ & $\begin{array}{l}50-54 \\
\text { years }\end{array}$ & $\begin{array}{l}55-59 \\
\text { years }\end{array}$ & $\begin{array}{l}60-64 \\
\text { years }\end{array}$ & $\begin{array}{l}65-69 \\
\text { years }\end{array}$ & $\begin{array}{l}70-74 \\
\text { years }\end{array}$ & $\begin{array}{l}75-79 \\
\text { years }\end{array}$ & $\begin{array}{l}80-84 \\
\text { years }\end{array}$ & $\begin{array}{l}85 \text { years } \\
\text { and over }\end{array}$ \\
\hline origins & $301,621,157$ & $4,257,020$ & $16,467,105$ & $19,849,628$ & $20,314,309$ & $21,473,690$ & $21,032,396$ & $21,057,706$ & $19,533,220$ & $21,176,460$ & $21,984,829$ & $22,861,373$ & $21,013,387$ & $18,236,259$ & $14,475,817$ & $10,752,441$ & $8,599,708$ & $7,324,882$ & 5,698,629 & $5,512,298$ \\
\hline Male & $148,658,898$ & $2,178,808$ & 3) $8,424,049$ & $10,148,578$ & $10,399,927$ & $11,006,869$ & $10,852,937$ & $10,776,189$ & $9,906,361$ & $10,654,911$ & $10,963,823$ & $11,302,842$ & $10,292,071$ & $8,847,222$ & $6,927,866$ & $5,019,063$ & $3 \quad 3,867,910$ & $3,106,968$ & 3 2,205,705 & $1,776,799$ \\
\hline Female. & $152,962,259$ & $2,078,212$ & 2 $8,043,056$ & $9,701,050$ & $9,914,382$ & $10,466,821$ & $10,179,459$ & $10,281,517$ & $9,626,859$ & $10,521,549$ & $11,021,006$ & $11,558,531$ & $10,721,316$ & $9,389,037$ & $7,547,951$ & $\begin{array}{l}1,733,378 \\
\end{array}$ & 3 $4,731,798$ & $4,217,914$ & 3,492,924 & $3,735,499$ \\
\hline Hispanic . & $45,504,311$ & $1,033,055$ & $3,883,271$ & $4,219,245$ & $3,969,322$ & $3,746,061$ & $3,690,076$ & $4,175,559$ & $3,992,576$ & $3,642,405$ & $3,199,835$ & $2,674,828$ & $2,075,129$ & $1,568,780$ & $1,122,475$ & 809,151 & 621,156 & 479,297 & 322,419 & 279,671 \\
\hline Male & $23,523,580$ & 528,369 & $1,983,457$ & $2,157,265$ & $2,031,123$ & $3 \quad 1,929,122$ & $1,980,450$ & $2,325,944$ & 2,177,384 & $1,946,955$ & $1,683,111$ & $1,374,992$ & $1,038,576$ & 764,820 & 529,804 & 370,316 & $\quad 272,728$ & 201,717 & 129,063 & 98,384 \\
\hline Female. & $21,980,731$ & 504,686 & $1,899,814$ & $2,061,980$ & $1,938,199$ & $1,816,939$ & $1,709,626$ & $1,849,615$ & $1,815,192$ & $1,695,450$ & $1,516,724$ & $1,299,836$ & $1,036,553$ & 803,960 & 592,671 & 438,835 & 348,428 & 277,580 & 193,356 & 181,287 \\
\hline Mexican. & $29,682,376$ & 769,435 & $2,796,952$ & $2,969,479$ & $2,730,598$ & $2,457,086$ & $2,440,469$ & $2,841,146$ & $2,671,787$ & $2,365,202$ & $1,937,013$ & $1,586,257$ & $1,286,346$ & 904,371 & 603,271 & 422,500 & 364,228 & 260,654 & $+151,889$ & 123,693 \\
\hline Male & $15,594,916$ & 405,237 & $1,426,896$ & $1,496,423$ & $1,380,557$ & $1,262,713$ & $1,326,223$ & $1,595,347$ & $1,490,659$ & $1,298,155$ & $1,041,840$ & 824,213 & 676,264 & 467,255 & 300,142 & 207,403 & $3 \quad 169,603$ & 118,607 & 62,301 & 45,078 \\
\hline Female. & $14,087,460$ & 364,198 & $1,370,056$ & $1,473,056$ & $1,350,041$ & $1,194,373$ & $1,114,246$ & $1,245,799$ & $1,181,128$ & $1,067,047$ & 895,173 & 762,044 & 610,082 & 437,116 & 303,129 & 15,097 & 194,625 & 142,047 & 89,588 & 78,615 \\
\hline Puerto Rican. & $3,942,114$ & 68,354 & 293,366 & 382,263 & 350,771 & 354,224 & 279,449 & 323,864 & 305,630 & 257,910 & 288,978 & 274,753 & 180,168 & 171,040 & 125,477 & 100,522 & 62,265 & 48,226 & 39,968 & 34,886 \\
\hline Male. & $1,889,539$ & 30,763 & 54,515 & 206,645 & 165,489 & 181,004 & 137,293 & 165,276 & 138,596 & 125,442 & 132,115 & 128,677 & 85,802 & 71,917 & 48,534 & 43,853 & 26,244 & 22,811 & 13,721 & 10,842 \\
\hline Female. & $2,052,575$ & 37,591 & 138,851 & 175,618 & 185,282 & 173,220 & 142,156 & 158,588 & 167,034 & 132,468 & 156,863 & 146,076 & 94,366 & 99,123 & 76,943 & 56,669 & 36,021 & 25,415 & 26,247 & 24,044 \\
\hline Cuban & $1,660,350$ & 25,785 & 86,096 & 96,603 & 93,623 & 93,556 & 110,169 & 101,183 & 106,740 & 133,270 & 167,010 & 120,300 & 92,377 & 82,162 & 84,947 & 68,427 & 60,914 & 55,975 & 38,316 & 42,897 \\
\hline Male . & 850,103 & 18,603 & 41,640 & 50,820 & 53,790 & 44,151 & 57,635 & 51,233 & 56,756 & 71,353 & 89,144 & 74,282 & 39,221 & 38,048 & 47,365 & 35,042 & 23,944 & 21,518 & 15,802 & 19,756 \\
\hline Female. & 810,247 & 7,182 & 44,456 & 45,783 & 39,833 & 49,405 & 52,534 & 49,950 & 49,984 & 61,917 & 77,866 & 46,018 & 53,156 & 44,114 & 37,582 & 33,385 & 36,970 & 34,457 & 22,514 & 23,141 \\
\hline \multicolumn{21}{|l|}{$\begin{array}{l}\text { Central and } \\
\text { South }\end{array}$} \\
\hline American & $8,051,114$ & 128,335 & 44,473 & 587,857 & 612,063 & 59,166 & 704,741 & 771,590 & 0,551 & 27,705 & 666,226 & 541,966 & 92,884 & 307,290 & 223,411 & 160,253 & 1,288 & 73,624 & 51,259 & 46,432 \\
\hline Male & $4,117,067$ & 58,361 & 277,880 & 303,068 & 337,949 & 346,029 & 378,107 & 448,671 & 414,882 & 366,471 & 349,392 & 274,848 & 181,628 & 130,636 & 99,508 & 59,474 & 33,721 & 23,483 & 21,081 & 11,878 \\
\hline Female. & $3,934,047$ & 69,974 & 266,593 & 284,789 & 274,114 & 313,137 & 326,634 & 322,919 & 345,669 & 361,234 & 316,834 & 267,118 & 211,256 & 176,654 & 123,903 & 100,779 & 57,567 & 50,141 & 30,178 & 34,554 \\
\hline \multicolumn{21}{|l|}{ Other } \\
\hline Hispanic & $2,168,400$ & ,148 & 62,383 & 3,047 & 182,261 & 182,035 & 5,256 & 137,768 & 147,868 & 158,322 & 140,603 & 151,561 & 123,368 & 103,913 & 85,375 & 7,452 & 63 & 40,823 & 40,989 & 31,765 \\
\hline Male . & $1,072,001$ & 15,406 & 82,524 & 100,315 & 93,341 & 95,227 & 81,197 & 65,415 & 76,495 & 85,540 & 70,619 & 72,979 & 55,674 & 56,966 & 34,258 & 24,544 & 19,218 & 15,299 & 16,158 & 10,826 \\
\hline Female. & $1,096,399$ & 25,742 & 79,859 & 82,732 & 88,920 & 86,808 & 74,059 & 72,353 & 71,373 & 72,782 & 69,984 & 78,582 & 67,694 & 46,947 & 51,117 & 32,908 & 23,245 & 25,524 & 24,831 & 20,939 \\
\hline n-Hispanic ${ }^{1}$. & $256,116,846$ & $3,223,965$ & $12,583,834$ & 15,63 & 16 & 629 & 320 & 16,8 & 644 & 055 & 18 & 20 & ,258 & 16 & & ,290 & 7 , & 6,8 & ;210 & \\
\hline Male. & $125,135,318$ & $1,650,439$ & $6,440,592$ & $7,991,313$ & $8,368,804$ & $9,077,747$ & $8,872,487$ & $8,450,245$ & $7,728,977$ & $8,707,956$ & $9,280,712$ & $9,927,850$ & $9,253,495$ & $8,082,402$ & $6,398,062$ & $4,648,747$ & $3,595,182$ & $2,905,251$ & $2,076,642$ & $1,678,415$ \\
\hline Female. . & $130,981,528$ & $1,573,526$ & $6,143,242$ & $7,639,070$ & $7,976,183$ & $8,649,882$ & $8,469,833$ & $8,431,902$ & $7,811,667$ & $8,826,099$ & $9,504,282$ & $10,258,695$ & $9,684,763$ & $8,585,077$ & $6,955,280$ & $5,294,543$ & 3 4,383,370 & $3,940,334$ & 3,299,568 & $3,554,212$ \\
\hline White. & $201,192,088$ & $2,322,476$ & $9,119,428$ & $11,510,172$ & $12,099,624$ & $13,213,933$ & $13,110,538$ & $12,646,303$ & $11,538,854$ & $13,380,351$ & $14,700,986$ & $16,215,851$ & $15,459,460$ & $13,816,279$ & $11,356,774$ & $8,452,418$ & 3 $6,817,597$ & $5,982,983$ & 4,768,812 & $4,679,249$ \\
\hline Male & $98,774,343$ & $1,190,041$ & $4,676,075$ & $5,900,639$ & $6,212,664$ & $6,782,639$ & $6,726,470$ & $6,369,087$ & $5,805,331$ & $6,723,574$ & $7,345,002$ & $8,066,435$ & $7,647,848$ & 3 $\quad 6,786,777$ & $5,504,334$ & $3,997,216$ & $3,108,848$ & $2,570,500$ & $1,856,784$ & $1,504,079$ \\
\hline Female. & $102,417,745$ & $1,132,435$ & $4,443,353$ & $5,609,533$ & $5,886,960$ & $6,431,294$ & $6,384,068$ & $6,277,216$ & $5,733,523$ & $6,656,777$ & $7,355,984$ & $8,149,416$ & $7,811,612$ & $7,029,502$ & $5,852,440$ & $4,455,202$ & $3,708,749$ & $3,412,483$ & 3 2,912,028 & $3,175,170$ \\
\hline Black & $38,147,294$ & 659,172 & $2,521,079$ & $3,031,871$ & $3,142,101$ & $3,373,761$ & $3,054,919$ & $2,886,177$ & $2,518,189$ & $2,657,673$ & $2,744,584$ & $2,735,123$ & $2,378,085$ & $1,926,615$ & $1,336,947$ & 999,595 & 587,415 & 584,108 & $3 \quad 418,787$ & 391,093 \\
\hline Mals & $18,192,572$ & 336,123 & $1,281,030$ & $1,539,424$ & $1,596,170$ & $1,709,634$ & $1,546,015$ & $1,412,079$ & $1,197,198$ & $1,253,127$ & $1,284,267$ & $1,271,210$ & $1,089,922$ & 867,231 & 586,683 & 423,192 & ? $\quad 321,093$ & 219,368 & 144,203 & 114,603 \\
\hline Female. . . & $19,954,722$ & 323,049 & $1,240,049$ & $1,492,447$ & $1,545,931$ & $1,664,127$ & $1,508,904$ & $1,474,098$ & $1,320,991$ & $1,404,546$ & $1,460,317$ & $1,463,913$ & $1,288,163$ & $3 \quad 1,059,384$ & 750,264 & 576,403 & $3 \quad 466,322$ & 364,740 & 274,584 & 276,490 \\
\hline
\end{tabular}

Includes races other than white and black.

SOURCES: CDC/NCHS; population estimates for specified Hispanic subgroups based on unpublished tabulations prepared by the Housing and Household Economic Statistics Division, U.S. Bureau of the Census, 2009; population estimates for all origins,

Hispanic, non-Hispanic, non-Hispanic white, and non-Hispanic black prepared under a collaborative arrangement with the U.S. Census Bureau, 2008. 
Table V. Estimated population for ages 15 years and over by marital status, 10-year age groups, and sex: United States, 2007

[Population estimates are based on the Current Population Survey adjusted to resident population controls for the United States. The control totals used are 2000-based population estimates for the United States for July 1, 2007]

\begin{tabular}{|c|c|c|c|c|c|c|c|c|}
\hline Marital status and sex & $\begin{array}{l}15 \text { years } \\
\text { and over }\end{array}$ & $\begin{array}{l}15-24 \\
\text { years }\end{array}$ & $\begin{array}{l}25-34 \\
\text { years }\end{array}$ & $\begin{array}{l}35-44 \\
\text { years }\end{array}$ & $\begin{array}{l}45-54 \\
\text { years }\end{array}$ & $\begin{array}{l}55-64 \\
\text { years }\end{array}$ & $\begin{array}{l}65-74 \\
\text { years }\end{array}$ & $\begin{array}{l}75 \text { years } \\
\text { and over }\end{array}$ \\
\hline All races. & $240,733,066$ & $42,506,083$ & $40,590,928$ & $43,161,286$ & $43,874,774$ & $32,712,069$ & $19,352,128$ & $18,535,798$ \\
\hline Never married & $71,241,086$ & $38,268,877$ & $16,448,366$ & $7,494,877$ & $5,291,698$ & $2,249,292$ & 809,692 & 678,284 \\
\hline Ever married. & $169,491,980$ & $4,237,206$ & $24,142,562$ & $35,666,409$ & $38,583,076$ & $30,462,777$ & $18,542,436$ & $17,857,514$ \\
\hline Married. & $131,155,048$ & $3,903,625$ & $21,778,655$ & $30,183,745$ & $30,696,158$ & $23,276,717$ & $12,936,228$ & $8,379,920$ \\
\hline Widowed. & $15,076,764$ & 35,798 & 119,611 & 339,914 & 913,589 & $1,861,553$ & $3,368,151$ & $8,438,148$ \\
\hline Divorced . & $23,260,168$ & 297,783 & $2,244,296$ & $5,142,750$ & $6,973,329$ & $5,324,507$ & $2,238,057$ & $1,039,446$ \\
\hline All races, male & $117,507,535$ & $21,859,805$ & $20,682,543$ & $21,618,736$ & $21,594,918$ & $15,775,083$ & $8,886,976$ & $7,089,474$ \\
\hline Never married & $38,990,932$ & $20,266,645$ & $9,538,405$ & $4,418,671$ & $2,999,711$ & $1,117,611$ & 385,005 & 264,884 \\
\hline Ever married. & $78,516,603$ & $1,593,160$ & $11,144,138$ & $17,200,065$ & $18,595,207$ & $14,657,472$ & $8,501,971$ & $6,824,590$ \\
\hline Married. . & $65,747,275$ & $1,468,461$ & $10,178,151$ & $14,836,110$ & $15,241,421$ & $12,105,211$ & $6,987,988$ & $4,929,933$ \\
\hline Widowed. & $2,935,817$ & 13,073 & 21,295 & 91,425 & 224,436 & 359,157 & 665,876 & $1,560,555$ \\
\hline Divorced . & $9,833,511$ & 111,626 & 944,692 & $2,272,530$ & $3,129,350$ & $2,193,104$ & 848,107 & 334,102 \\
\hline All races, female & $123,225,531$ & $20,646,278$ & $19,908,385$ & $21,542,550$ & $22,279,856$ & $16,936,986$ & $10,465,152$ & $11,446,324$ \\
\hline Never married & $32,250,154$ & $18,002,232$ & $6,909,961$ & $3,076,206$ & $2,291,987$ & $1,131,681$ & 424,687 & 413,400 \\
\hline Ever married. & $90,975,377$ & $2,644,046$ & $12,998,424$ & $18,466,344$ & $19,987,869$ & $15,805,305$ & $10,040,465$ & $11,032,924$ \\
\hline Married. & $65,407,773$ & $2,435,164$ & $11,600,504$ & $15,347,635$ & $15,454,737$ & $11,171,506$ & $5,948,240$ & $3,449,987$ \\
\hline Widowed. & $12,140,947$ & 22,725 & 98,316 & 248,489 & 689,153 & $1,502,396$ & $2,702,275$ & $6,877,593$ \\
\hline Divorced. & $13,426,657$ & 186,157 & $1,299,604$ & $2,870,220$ & $3,843,979$ & $3,131,403$ & $1,389,950$ & 705,344 \\
\hline
\end{tabular}

SOURCE: Population estimates based on unpublished tabulations prepared by the Housing and Household Economic Statistics Division of the U.S. Census Bureau, 2009.

Table VI. Estimated population for ages 25-64 years, by educational attainment and sex: Total of 22 reporting states and the District of Columbia using the 2003 version of the U.S. Standard Certificate of Death and total of 26 reporting states using the 1989 version of the U.S. Standard Certificate of Death, 2007

[Population estimates are based on the Current Population Survey adjusted to resident population controls. The control totals used are 2000-based population estimates for reporting states for July 1, 2007; see "Technical Notes"]

\begin{tabular}{|c|c|c|c|c|c|c|c|c|c|c|c|}
\hline \multicolumn{6}{|c|}{$\begin{array}{l}22 \text { reporting states and the District of Columbia }{ }^{1} \text { using } \\
\text { the } 2003 \text { version of the Standard Certificate of Death }\end{array}$} & \multicolumn{6}{|c|}{$\begin{array}{l}26 \text { reporting states }{ }^{2} \text { using the } 1989 \\
\text { version of the Standard Certificate of Death }\end{array}$} \\
\hline Education level and sex & $\begin{array}{l}25-64 \\
\text { years }\end{array}$ & $\begin{array}{l}25-34 \\
\text { years }\end{array}$ & $\begin{array}{l}35-44 \\
\text { years }\end{array}$ & $\begin{array}{l}45-54 \\
\text { years }\end{array}$ & $\begin{array}{l}55-64 \\
\text { years }\end{array}$ & $\begin{array}{l}\text { Years of school } \\
\text { completed and sex }\end{array}$ & $\begin{array}{l}25-64 \\
\text { years }\end{array}$ & $\begin{array}{l}25-34 \\
\text { years }\end{array}$ & $\begin{array}{l}35-44 \\
\text { years }\end{array}$ & $\begin{array}{l}45-54 \\
\text { years }\end{array}$ & $\begin{array}{l}55-64 \\
\text { years }\end{array}$ \\
\hline \multicolumn{6}{|l|}{ All races } & \multicolumn{6}{|l|}{ All races } \\
\hline \multirow{3}{*}{$\begin{array}{l}\text { Both sexes ......... } \\
\text { Less than high school } \\
\text { diploma or GED .... } \\
\text { High school diploma } \\
\text { or GED ......... } \\
\text { Some college or }\end{array}$} & $87,382,054$ & $22,384,308$ & $23,870,966$ & $23,735,821$ & $17,390,959$ & Both sexes . . . & $67,077,697$ & $16,674,345$ & $17,620,711$ & $18,610,198$ & $14,172,443$ \\
\hline & $11,140,785$ & $3,110,670$ & $3,084,400$ & $2,841,294$ & $2,104,421$ & Under 12 years. & $7,153,493$ & $1,799,892$ & $1,766,470$ & $1,863,283$ & $1,723,848$ \\
\hline & $5,072,693$ & $6,101,224$ & $6,770,444$ & $7,234,128$ & $4,966,897$ & 12 years & 58 & 4,9 & 5,4 & $6,358,877$ & $4,762,447$ \\
\hline collegiate degree. . & $51,168,576$ & $13,172,414$ & $14,016,122$ & $13,660,399$ & $10,319,641$ & 13 years or more. & $38,404,546$ & $9,901,123$ & $10,429,237$ & $10,388,038$ & $7,686,148$ \\
\hline \multirow{3}{*}{$\begin{array}{l}\text { Male . . . . . . . . . } \\
\text { Less than high school } \\
\text { diploma or GED . . . } \\
\text { High school diploma } \\
\text { or GED . . . . . . . . }\end{array}$} & 569 & 11,393 & 11,9 & $11,738,301$ & $8,278,682$ & Male & 90 & 8,4 & 8,78 & 9,1 & $6, \varsigma$ \\
\hline & $5,992,226$ & $1,786,456$ & $1,706,433$ & $1,508,477$ & 990,860 & Under 12 years. & $3,982,065$ & $1,051,068$ & $1,025,696$ & $1,006,178$ & 899,123 \\
\hline & $12,886,518$ & $3,445,917$ & $3,594,186$ & $3,701,293$ & $2,145,122$ & 12 years. & $11,101,329$ & $2,815,143$ & $2,895,342$ & $3,265,298$ & $2,125,546$ \\
\hline $\begin{array}{l}\text { Some college or } \\
\text { collegiate degree. }\end{array}$ & $24,518,825$ & $6,161,386$ & $6,686,208$ & $6,528,531$ & $5,142,700$ & 13 years or more. & $18,284,596$ & $4,624,331$ & $4,867,265$ & $4,865,171$ & $3,927,829$ \\
\hline \multirow{4}{*}{$\begin{array}{c}\text { Female. . . . . . . . . } \\
\text { Less than high school } \\
\text { diploma or GED ..... } \\
\text { High school diploma } \\
\text { or GED . . . . . . . } \\
\text { Some college or } \\
\text { collegiate degree. . . . }\end{array}$} & $43,984,485$ & $10,990,549$ & $11,884,139$ & $11,997,520$ & $9,112,277$ & Female & $33,709,707$ & $8,183,803$ & $8,832,408$ & $9,473,551$ & $7,219,945$ \\
\hline & $5,148,559$ & 14 & 67 & $1,332,817$ & 3,561 & Under 12 years. & $3,171,428$ & 748,824 & 740,774 & 857,105 & 824,725 \\
\hline & $12,186,175$ & 07 & 58 & $3,532,835$ & $2,821,775$ & 12 years. & $10,418,329$ & $2,158,187$ & $2,529,662$ & $3,093,579$ & $2,636,901$ \\
\hline & $26,649,751$ & $7,011,028$ & $7,329,914$ & $7,131,868$ & $5,176,941$ & 13 years or more. & $20,119,950$ & $5,276,792$ & $5,561,972$ & $5,522,867$ & $3,758,319$ \\
\hline
\end{tabular}

${ }^{1}$ Includes data for California, Connecticut, Delaware, District of Columbia, Florida, Idaho, Kansas, Michigan, Montana, Nebraska, New Hampshire, New Jersey, New Mexico, New York, Ohio, Oklahoma, Oregon, South Carolina, South Dakota, Texas, Utah, Washington, and Wyoming; see "Technical Notes."

${ }^{2}$ Includes data for Alabama, Alaska, Arizona, Arkansas, Colorado, Hawaii, Illinois, Indiana, lowa, Kentucky, Louisiana, Maine, Maryland, Massachusetts, Minnesota, Mississippi, Missouri, Nevada, North Carolina, North Dakota, Pennsylvania, Tennessee, Vermont, Virginia, Wisconsin, and West Virginia; see "Technical Notes."

SOURCE: Population estimates based on unpublished tabulations prepared by the Housing and Household Economic Statistics Division, U.S. Bureau of the Census, 2009. 
Table VII. Estimated population for the United States, each state, Puerto Rico, Virgin Islands, Guam, American Samoa, and Northern Marianas, 2007

[Populations are postcensal estimates produced in 2008 based on the 2000 census estimated as of July 1, 2007]

\begin{tabular}{|c|c|c|c|}
\hline Area & Total & Area & Total \\
\hline United States & $301,621,157$ & $\begin{array}{l}\text { Nevada . . . . . . . . . . . } \\
\text { New Hampshire }\end{array}$ & $\begin{array}{l}2,565,382 \\
1,315,828\end{array}$ \\
\hline Alabama $\ldots \ldots \ldots \ldots \ldots$ & $4,627,851$ & New Jersey. . . . . . . . . . . . & $8,685,920$ \\
\hline Alaska $\ldots \ldots \ldots \ldots \ldots$ & 683,478 & New Mexico . . . . . . . . . . . & $1,969,915$ \\
\hline$\ldots \ldots \ldots \ldots$ & $6,338,755$ & $\ldots \ldots \ldots \ldots$ & $19,297,729$ \\
\hline$\ldots \ldots \ldots \ldots \ldots$ & $2,834,797$ & North Carolina . . . . . . . . . . & $9,061,032$ \\
\hline$\ldots \ldots \ldots \ldots$ & $36,553,215$ & North Dakota . . . . . . . . . . . . & 639,715 \\
\hline Colorado . & $4,861,515$ & Ohio . . . . . . . . . . & $11,466,917$ \\
\hline Connecticut $\ldots \ldots \ldots \ldots$ & $3,502,309$ & Oklahoma. . . . . . . . . . . . & $3,617,316$ \\
\hline Delaware. . . . . . . . . . . . & 864,764 & Oregon ............... & $3,747,455$ \\
\hline District of Columbia. . . . . . . . . & 588,292 & Pennsylvania . . . . . . . . . . . & $12,432,792$ \\
\hline Florida $\ldots \ldots \ldots \ldots \ldots$ & $18,251,243$ & Rhode Island . . . . . . . . . . . . & $1,057,832$ \\
\hline 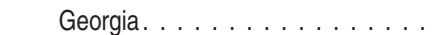 & $9,544,750$ & South Carolina . . . . . . . . . & $4,407,709$ \\
\hline Hawaii & $1,283,388$ & South Dakota. ... & 796,214 \\
\hline Idaho. & $1,499,402$ & Tennessee .... . & $6,156,719$ \\
\hline Illinois. & $12,852,548$ & Texas ......... & $23,904,380$ \\
\hline Indiana $\ldots \ldots \ldots \ldots \ldots$ & $6,345,289$ & $\ldots \ldots \ldots \ldots$ & $2,645,330$ \\
\hline lowa $\ldots \ldots \ldots \ldots \ldots \ldots$ & $2,988,046$ & Vermont. . . . . . . . . . . . . & 621,254 \\
\hline Kansas . . . . . . . . . . . . . & $2,775,997$ & $\ldots \ldots \ldots \ldots \ldots$ & $7,712,091$ \\
\hline 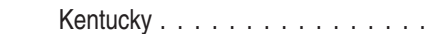 & $4,241,474$ & Washington . . . . . . . . . & $6,468,424$ \\
\hline Louisiana. . & $4,293,204$ & West Virginia . . . . . . . . . . . & $1,812,035$ \\
\hline Maine... & $1,317,207$ & Wisconsin. . . . . . . . . . & $5,601,640$ \\
\hline Maryland. & $5,618,344$ & Wyoming & 522,830 \\
\hline Massachusetts. . . . . & $6,449,755$ & & \\
\hline Michigan . . . . . . . . & $10,071,822$ & & \\
\hline Minnesota . . . . . . . . . . . . . & $5,197,621$ & Puerto Rico. . . . . . . . . . . . . . & $3,942,375$ \\
\hline Mississippi $\ldots \ldots \ldots \ldots \ldots$ & $2,918,785$ & Virgin Islands. . . . . . . . . . & 109,821 \\
\hline Missouri & $5,878,415$ & $\ldots \ldots \ldots \ldots$ & 173,456 \\
\hline Montana . & 957,861 & American Samoa . . . . . . . . . & 64,025 \\
\hline$\ldots \ldots \ldots \ldots$ & $1,774,571$ & Northern Marianas. . . . . . . . . . & 84,546 \\
\hline
\end{tabular}

SOURCES: CDC/NCHS; estimates of the July 1, 2007, U.S. resident population by age, sex, race, and Hispanic origin prepared under a collaborative arrangement with the U.S. Census Bureau, 2008.

Table VIII. United States standard population

\begin{tabular}{|c|c|}
\hline Age & Population \\
\hline All ages $\ldots \ldots \ldots \ldots \ldots \ldots$ & $274,633,642$ \\
\hline 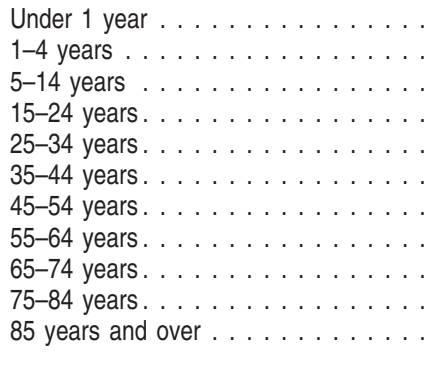 & $\begin{array}{r}3,794,901 \\
15,191,619 \\
39,976,619 \\
38,076,743 \\
37,233,437 \\
44,659,185 \\
37,030,152 \\
23,961,506 \\
18,135,514 \\
12,314,793 \\
4,259,173\end{array}$ \\
\hline
\end{tabular}

the age-specific death rates to the U.S. standard population. Age groups for age 75 and over were combined because population counts were unavailable by age group over 75 years. The 2000 standard population used for computing age-adjusted rates and standard errors for the territories is shown in Table XII.

Using the same standard population, death rates for the total population and for each race-sex group were adjusted separately. The age-adjusted rates were based on 10-year age groups. Age-adjusted death rates are not comparable with crude rates.

Death rates for the Hispanic population are based only on events to persons reported as Hispanic. Rates for non-Hispanic white persons
Table IX. United States standard population for ages 25 years and over

\begin{tabular}{|c|c|}
\hline Age & Population \\
\hline 25 years and over & $177,593,760$ \\
\hline 25-34 years $\ldots \ldots \ldots \ldots \ldots \ldots$ & $37,233,437$ \\
\hline $35-44$ years. . . . . & $44,659,185$ \\
\hline 45-54 years. & $37,030,152$ \\
\hline $55-64$ years. & $23,961,506$ \\
\hline $65-74$ years. & $18,135,514$ \\
\hline 75 years and over & $16,573,966$ \\
\hline
\end{tabular}

Table X. United States standard population for ages 25-64 years

\begin{tabular}{|c|c|}
\hline Age & Population \\
\hline 25-64 years . . . . . . . . . . . . . & $142,884,280$ \\
\hline 25-34 years . . . . . . . . . . . . & $37,233,437$ \\
\hline 35-44 years . . . . . . . . . . . . . . & $44,659,185$ \\
\hline 45-54 years . . . . . . . . . . . . . & $37,030,152$ \\
\hline $55-64$ years . . . . . . . . . . . . . . & $23,961,506$ \\
\hline
\end{tabular}

are based on the sum of all events to white decedents reported as non-Hispanic and white decedents with origin not stated. Hispanic origin is not imputed if it is not reported. 
Table XI. United States standard population for ages 15 years and over

\begin{tabular}{|c|c|}
\hline Age & Population \\
\hline 15 years and over . . . . . . . . . & $215,670,503$ \\
\hline $15-24$ years $\ldots \ldots \ldots \ldots \ldots \ldots$ & $38,076,743$ \\
\hline 25-34 years. . . . . . . . . . . . . & $37,233,437$ \\
\hline $35-44$ years $\ldots \ldots \ldots \ldots \ldots$ & $44,659,185$ \\
\hline $45-54$ years . . . . . . . . . . . . . & $37,030,152$ \\
\hline $55-64$ years. . . . . . . . . . . . . & $23,961,506$ \\
\hline 65 years and over $\ldots \ldots \ldots \ldots$ & $34,709,480$ \\
\hline
\end{tabular}

\section{Random variation}

The mortality data presented in this report, with the exception of data for 1972, are not subject to sampling error. In 1972, mortality data were based on a 50 percent sample of deaths because of resource constraints. Mortality data, even based on complete counts, may be affected by random variation-that is, the number of deaths that actually occurred may be considered as one of a large series of possible results that could have arisen under the same circumstances $(89,90)$. When the number of deaths is small, perhaps fewer than 100 , random variation tends to be relatively large. Therefore, considerable caution must be observed in interpreting statistics based on small numbers of deaths.

Measuring random variability-To quantify the random variation associated with mortality statistics, an assumption must be made regarding the appropriate underlying distribution. Deaths, as infrequent events, can be viewed as deriving from a Poisson probability distribution. The Poisson distribution is simple conceptually and computationally, and provides reasonable, conservative variance estimates for mortality statistics when the probability of dying is relatively low (89). Using the properties of the Poisson distribution, the standard error (SE) associated with the number of deaths $(D)$ is:

$$
\text { 1. } \operatorname{SE}(D)=\sqrt{\operatorname{var}(D)}=\sqrt{D}
$$

where $\operatorname{var}(D)$ denotes the variance of $D$.

The standard error associated with crude and age-specific death rates $(R)$ assumes that the population denominator $(P)$ is a constant and is:

$$
\text { 2. } S E(R)=\sqrt{\operatorname{var}\left(\frac{D}{P}\right)}=\sqrt{\frac{1}{P^{2}} \operatorname{var}(D)}=\sqrt{\frac{D}{P^{2}}}=\frac{R}{\sqrt{D}}
$$

\section{Table XII. United States standard population for the}

\begin{tabular}{|c|c|}
\hline Age & Population \\
\hline All ages $\ldots \ldots \ldots \ldots \ldots$ & $274,633,642$ \\
\hline Under 1 year $\ldots \ldots \ldots \ldots \ldots$ & $3,794,901$ \\
\hline $1-4$ years $\ldots \ldots \ldots \ldots \ldots \ldots$ & $15,191,619$ \\
\hline $5-14$ years $\ldots \ldots \ldots \ldots \ldots \ldots$ & $39,976,619$ \\
\hline 15-24 years . . . . . . . . . . . . . . & $38,076,743$ \\
\hline 25-34 years . . . . . . . . . . . . . & $37,233,437$ \\
\hline 35-44 years . . . . . . . . . . . . . . & $44,659,185$ \\
\hline 45-54 years . . . . . . . . . . . . . . & $37,030,152$ \\
\hline 55-64 years. . . . . . . . . . . . . . & $23,961,506$ \\
\hline $65-74$ years . . . . . . . . . . . . . . & $18,135,514$ \\
\hline 75 years and over . . . . . . . . . . & $16,573,966$ \\
\hline
\end{tabular}
territories
The coefficient of variation or relative standard error (RSE) is a useful measure of relative variation. The RSE is calculated by dividing the statistic (e.g., number of deaths, death rate) into its standard error and multiplying by 100 . For the number of deaths:

$$
\operatorname{RSE}(D)=100 \frac{\operatorname{SE}(D)}{D}=100 \frac{\sqrt{D}}{D}=100 \sqrt{\frac{1}{D}}
$$

For crude and age-specific death rates:

Thus:

$$
\operatorname{RSE}(R)=100 \frac{\operatorname{SE}(R)}{R}=100 \frac{R / \sqrt{D}}{R}=100 \sqrt{\frac{1}{D}}
$$

$$
\text { 3. } \operatorname{RSE}(D)=\operatorname{RSE}(R)=100 \sqrt{\frac{1}{D}}
$$

The standard error of the age-adjusted death rate $\left(R^{\prime}\right)$ is:

$$
\text { 4. } \operatorname{SE}\left(R^{\prime}\right)=\sqrt{\sum_{i}\left(\frac{P_{s i}}{P_{s}}\right)^{2} \operatorname{var}\left(R_{\mathrm{i}}\right)}=\sqrt{\left.\sum_{i} \|\left(\frac{\left.P_{s i}\right|^{2}\left|\frac{R_{i}^{2}}{P_{s}}\right|^{2}}{D_{i}}\right)\right\}}
$$

where:

- $R_{i}$ is the age-specific rate for the $i$ th age group

- $P_{s i}$ is the age-specific standard population for the ith age group from the U.S. standard population age distribution (see Table VIII and age-adjusted death rate under "Definition of terms")

- $P_{s}$ is the total U.S. standard population (all ages combined)

- $D_{i}$ is the number of deaths for the $i$ th age group

The RSE for the age-adjusted rate, $R S E\left(R^{\prime}\right)$, is calculated by dividing $S E\left(R^{\prime}\right)$ from Formula 4 by the age-adjusted death rate, $R^{\prime}$, and multiplying by 100 :

$$
\operatorname{RSE}\left(R^{\prime}\right)=100 \frac{\mathrm{SE}\left(R^{\prime}\right)}{R^{\prime}}
$$

For tables showing infant and maternal mortality rates based on live births $(B)$ in the denominator, calculation of the standard error assumes random variability in both the numerator and denominator. The standard error for the infant mortality rate $(I M R)$ is:

$$
\text { 5. } \quad \operatorname{SE}(I M R)=\sqrt{\frac{\operatorname{var}(D)+I M R \cdot \operatorname{var}(B)}{E(B)^{2}}}=\sqrt{\frac{D}{B^{2}}+\frac{D^{2}}{B^{3}}}
$$

where the number of births, $B$, is also assumed to be distributed according to a Poisson distribution and $E(B)$ is the expectation of $B$. The RSE for the IMR is:

$$
\text { 6. } \operatorname{RSE}(I M R)=100 \frac{\operatorname{SE}(I M R)}{I M R}=100 \sqrt{\frac{1}{D}+\frac{1}{B}}
$$

For maternal mortality rates, Formulas 5 and 6 may be used, substituting the maternal mortality rate for the IMR.

Formulas 1-6 may be used for all tables presented in this report except for death rates and age-adjusted death rates shown in Tables 5 , 25 , and 26, which are calculated using population figures that are subject to sampling error.

Tables 5, 25, and 26-Death rates for Mexican, Puerto Rican, Cuban, and Other Hispanic populations in Table 5, by marital status in Table 25, and by educational attainment in Table 26 are based on population estimates derived from the CPS for 2007 and adjusted to 
resident population control totals. As a result, the rates are subject to sampling variability in the denominator as well as random variability in the numerator.

For crude and age-specific death rates $(R)$, the standard error is calculated as:

$$
\text { 7. } \mathrm{SE}(R)=R \sqrt{\frac{1}{D}+0.67\left(a+\frac{b}{P}\right)}
$$

For age-adjusted death rates $\left(R^{\prime}\right)$

$$
\text { 8. } \operatorname{SE}\left(R^{\prime}\right)=\sqrt{\sum_{i}\left\{\left(\frac{P_{s i}}{P_{s}}\right)^{2} R_{i}^{2}\left[\frac{1}{D_{i}}+0.67\left(a+\frac{b}{P_{i}}\right) \|\right\}\right.}
$$

where $a$ and $b$ in Formulas 7 and 8 represent parameters presented in Table XIII, which are derived from the CPS data for 2007 and 2008 and vary depending on the subgroup of interest $(91,92)$.

Suppression of unreliable rates-Beginning with 1989 data, an asterisk is shown in place of a crude or age-specific death rate based on fewer than 20 deaths, the equivalent of an RSE of 23 percent or more. The limit of 20 deaths is a convenient, if somewhat arbitrary, benchmark, below which rates are considered to be too statistically unreliable for presentation. For infant and maternal mortality rates, the same threshold of fewer than 20 deaths is used to determine whether an asterisk is presented in place of the rate. For age-adjusted death rates, the suppression criterion is based on the sum of age-specific deaths; that is, if the sum of the age-specific deaths is less than 20 , an asterisk replaces the rate. These procedures are used throughout this report except for death rates shown in Tables 5, 25, and 26.

In Tables 5, 25, and 26, sampling variability in the population denominator has a substantial impact on the overall variability in the death rate. Therefore, the number of deaths in the numerator is not used as the sole suppression factor. RSEs for rates shown in Tables 5 , 25 , and 26 are derived from Formulas 7 and 8 by dividing the result of Formula 7 by the crude/age-specific rate, and the result of Formula 8 by the age-adjusted rate, and then multiplying by 100 . Rates are replaced by asterisks if the calculated RSE is 23 percent or more. In some cases, for smaller population subgroups, the estimated sample population from the CPS may be zero, even though deaths are presented for the subgroups. In these cases, the death rate is incalculable and automatically replaced with an asterisk.

Confidence intervals and statistical tests based on 100 deaths or more-When the number of deaths is large, a normal approximation may be used in calculating confidence intervals and statistical tests. How large, in terms of number of deaths, is to some extent subjective. In general, for crude and age-specific death rates and for infant and maternal mortality rates, the normal approximation performs well when the number of deaths is 100 or greater. For age-adjusted rates, the criterion for use of the normal approximation is somewhat more complicated $(63,88,93)$. Formula 9 is used to calculate 95 percent confidence limits for the death rate when the normal approximation is appropriate:

$$
\text { 9. } \quad L(R)=R-1.96(\mathrm{SE}(R)) \text { and } U(R)=R+1.96(\mathrm{SE}(R))
$$

where $L(R)$ and $U(R)$ are the lower and upper limits of the confidence interval, respectively. The resulting 95 percent confidence interval can be interpreted to mean that the chances are 95 in 100 that the "true" death rate falls between $L(R)$ and $U(R)$. For example, suppose that the crude death rate for Malignant neoplasms is 186.6 per 100,000 population based on 562,875 deaths. Lower and upper 95 percent confidence limits using Formula 9 are calculated as:

$L(186.6)=186.6-1.96(.25)=186.1$ and

$U(186.6)=186.6+1.96(.25)=187.1$

Thus, the chances are 95 in 100 that the true death rate for malignant neoplasms is between 186.2 and 187.0. Formula 9 can also be used to calculate 95 percent confidence intervals for the number of deaths, age-adjusted death rates, infant mortality rates, and other mortality statistics when the normal approximation is appropriate by replacing $R$ with $D, R^{\prime}, I M R$, or others.

When testing the difference between two rates, $R_{1}$ and $R_{2}$ (each based on 100 or more deaths), the normal approximation may be used

\begin{tabular}{|c|c|c|c|c|c|c|}
\hline \multirow[b]{2}{*}{ Characteristic } & \multicolumn{2}{|c|}{ Total } & \multicolumn{2}{|c|}{$\begin{array}{l}\text { White, black, non- } \\
\text { Hispanic white, or } \\
\text { non-Hispanic black }\end{array}$} & \multicolumn{2}{|c|}{ Hispanic } \\
\hline & a & $b$ & a & $b$ & a & $b$ \\
\hline \multicolumn{7}{|l|}{ Table 5} \\
\hline $\begin{array}{l}\text { All origins . . . . . . . . . . . . . . . . . . . . . . . } \\
\text { Hispanic subgroups (Mexican, Puerto Rican, Cuban, }\end{array}$ & 0.000000 & 0 & 0.000000 & 0 & 0.000000 & 0 \\
\hline Central and South American, and Other Hispanic). . . . . . . & $\cdots$ & $\cdots$ & $\cdots$ & $\cdots$ & -0.000084 & 3,809 \\
\hline \multicolumn{7}{|l|}{ Tables 25} \\
\hline $\begin{array}{l}\text { All marital status groups combined } \ldots \ldots \ldots \ldots \ldots \ldots \\
\text { Marital status subgroups (never married, ever }\end{array}$ & 0.000000 & 0 & $\cdots$ & $\cdots$ & $\cdots$ & $\cdots$ \\
\hline married, married, widowed, divorced) $\ldots \ldots \ldots \ldots \ldots$ & -0.000009 & 2,652 & $\cdots$ & $\cdots$ & $\cdots$ & $\cdots$ \\
\hline \multicolumn{7}{|l|}{ Table 26} \\
\hline $\begin{array}{l}\text { All education groups . . . . . . . . . . . . . . . . . . } \\
\text { Education subgroups (under } 12 \text { years, } 12 \text { years, }\end{array}$ & 0.000000 & 0 & $\cdots$ & $\cdots$ & $\ldots$ & $\cdots$ \\
\hline 13 years or more) $\ldots \ldots \ldots \ldots \ldots \ldots \ldots$ & -0.000005 & 1,206 & $\cdots$ & $\cdots$ & $\cdots$ & $\cdots$ \\
\hline
\end{tabular}
to calculate a test statistic, $z$, such that:

Table XIII. Current Population Survey standard error parameters for death rates in Tables 5, 25, and 26

... Category not applicable.

SOURCE: The a and b parameters are averages of the 2007 and 2008 Current Population Survey standard error parameters. 
10. $z=\frac{R_{1}-R_{2}}{\sqrt{\mathrm{SE}\left(R_{1}\right)^{2}+\mathrm{SE}\left(R_{2}\right)^{2}}}$

If $|z| \geq 1.96$, then the difference between the rates is statistically significant at the 0.05 level. If $|z|<1.96$, then the difference is not statistically significant. Formula 10 can also be used to perform tests for other mortality statistics when the normal approximation is appropriate (when both statistics being compared meet the normal criteria) by replacing $R_{1}$ and $R_{2}$ with $D_{1}$ and $D_{2}, R_{1}^{\prime}$ and $R_{2}^{\prime}$, or others. For example, suppose that the male age-adjusted death rate for Malignant neoplasms of trachea, bronchus, and lung (lung cancer) is 67.0 per 100,000 U.S. standard population in $2006\left(R_{1}\right)$ and 65.1 per 100,000 U.S. standard population in $2007\left(R_{2}\right)$. The standard error for each of these figures, $\operatorname{SE}\left(R_{1}\right)$ and $\operatorname{SE}\left(R_{2}\right)$, is calculated using Formula 4. A test using Formula 10 can determine if the decrease in the age-adjusted rate is statistically significant:

$$
z=\frac{67.0-65.1}{\sqrt{(0.227)^{2}+(0.222)^{2}}}=5.98
$$

Because $z=5.98>1.96$, the decrease from 2006 to 2007 in the male age-adjusted death rate for lung cancer is statistically significant.

Confidence intervals and statistical tests based on fewer than 100 deaths - When the number of deaths is not large (fewer than 100), the Poisson distribution cannot be approximated by the normal distribution. The normal distribution is symmetrical, with a range from $-\infty$ to $+\infty$. As a result, confidence intervals based on the normal distribution also have this range. The number of deaths or the death rate, however, cannot be less than zero. When the number of deaths is very small, approximating confidence intervals for deaths and death rates using the normal distribution will sometimes produce lower confidence limits that are negative. The Poisson distribution, in contrast, is an asymmetric distribution with zero as a lower bound-confidence limits based on this distribution will never be less than zero. A simple method based on the more general family of gamma distributions, of which the Poisson is a member, can be used to approximate confidence intervals for deaths and death rates when the number of deaths is small $(88,93)$. For more information regarding how the gamma method is derived, see "Derivation of the gamma method" at the end of this section.

Calculations using the gamma method can be made using commonly available spreadsheet programs or statistical software (e.g., Excel, SAS) that include an inverse gamma function. In Excel, the function "gammainv (probability, alpha, beta)" returns values associated with the inverse gamma function for a given probability between 0 and 1 . For 95 percent confidence limits, the probability associated with the lower limit is $.05 / 2=.025$ and with the upper limit, $1-(.05 / 2)$ $=.975$. Alpha and beta are parameters associated with the gamma distribution. For the number of deaths and crude and age-specific death rates, alpha $=D$ (the number of deaths) and beta $=1$. In Excel, the following formulas can be used to calculate lower and upper 95 percent confidence limits for the number of deaths and crude and age-specific death rates:

$$
L(D)=\operatorname{GAMMAINV}(.025, \mathrm{D}, 1) \text { and } U(D)=\operatorname{GAMMAINV}(.975, \mathrm{D}+1,1)
$$

Confidence limits for the death rate are then calculated by dividing $L(D)$ and $U(D)$ by the population $(P)$ at risk of dying (see Formula 17).
Alternatively, 95 percent confidence limits can be estimated using the lower and upper confidence limit factors shown in Table XIV. For the number of deaths, $D$, and the death rate, $R$,

$$
\begin{aligned}
& \text { 11. } L(D)=L \times D \text { and } U(D)=U \times D \\
& \text { 12. } L(R)=L \times R \text { and } U(R)=U \times R
\end{aligned}
$$

where $L$ and $U$ in both formulas are the lower and upper confidence limit factors that correspond to the appropriate number of deaths, $D$, in Table XIV. For example, suppose that the death rate for AIAN females aged 1-4 is 46.0 per 100,000 and based on 40 deaths. Applying Formula 12, values for $L$ and $U$ from Table XIV for 40 deaths are multiplied by the death rate, 46.0 , such that:

$L(R)=L(46.0)=0.714415 \times 46.0=32.9$ and

$U(R)=U(46.0)=1.361716 \times 46.0=62.6$

These confidence limits indicate that the chances are 95 out of 100 that the actual death rate for AIAN females aged 1-4 is between 32.9 and 62.6 per 100,000 .

Although the calculations are similar, confidence intervals based on small numbers for age-adjusted death rates, infant and maternal mortality rates, and rates that are subject to sampling variability in the denominator are somewhat more complicated $(63,88)$. Refer to the most recent version of the Mortality Technical Appendix for more details at http://www.cdc.gov/nchs/products/vsus.htm\#appendices.

When comparing the difference between two rates $\left(R_{1}\right.$ and $\left.R_{2}\right)$, where one or both of the rates are based on fewer than 100 deaths, a comparison of 95 percent confidence intervals may be used as a statistical test. If the 95 percent confidence intervals do not overlap, then the difference can be said to be statistically significant at the 0.05 level. A simple rule of thumb is: If $R_{1}>R_{2}$, then test if $L\left(R_{1}\right)>U\left(R_{2}\right)$, or if $R_{2}>R_{1}$, then test if $L\left(R_{2}\right)>U\left(R_{1}\right)$. Positive tests denote statistical significance at the 0.05 level. For example, suppose that AIAN females aged 1-4 have a death rate $\left(R_{1}\right)$ of 46.0 based on 40 deaths and API females aged 1-4 years have a death rate $\left(R_{2}\right)$ of 17.9 per 100,000 based on 73 deaths. The 95 percent confidence limits for $R_{1}$ and $R_{2}$ calculated using Formula 12 would be:

$$
\begin{aligned}
& L\left(R_{1}\right)=L(46.0)=0.714415 \times 46.0=32.9 \text { and } \\
& U\left(R_{1}\right)=U_{1}(46.0)=1.361716 \times 46.0=62.6 \\
& L\left(R_{2}\right)=L(17.9)=0.783840 \times 17.9=14.0 \text { and } \\
& U\left(R_{2}\right)=U(17.9)=1.257350 \times 17.9=22.5
\end{aligned}
$$

Because $R_{1}>R_{2}$ and $L\left(R_{1}\right)>U\left(R_{2}\right)$, it can be concluded that the difference between the death rates for AIAN females aged 1-4 and API females of the same age is statistically significant at the 0.05 level. That is, taking into account random variability, API females aged 1-4 have a death rate significantly lower than that for AIAN females of the same age.

This test may also be used to perform tests for other statistics when the normal approximation is not appropriate for one or both of the statistics being compared, by replacing $R_{1}$ and $R_{2}$ with $D_{1}$ and $D_{2}$, $R_{1}^{\prime}$ and $R_{2}^{\prime}$, or others.

Users of the method of comparing confidence intervals should be aware that this method is a conservative test for statistical significance-the difference between two rates may, in fact, be statistically significant even though confidence intervals for the two rates 
Table XIV. Lower and upper 95 percent confidence limit factors for the number of deaths and death rate when the number of deaths is less than 100

\begin{tabular}{|c|c|c|c|c|c|}
\hline $\begin{array}{l}\text { Number of deaths } \\
\text { (D) }\end{array}$ & $\begin{array}{l}\text { Lower } \\
\text { confidence } \\
\text { limit } \\
\text { (L) }\end{array}$ & $\begin{array}{l}\text { Upper } \\
\text { confidence } \\
\text { limit } \\
\text { (U) }\end{array}$ & $\begin{array}{l}\text { Number of deaths } \\
\text { (D) }\end{array}$ & $\begin{array}{l}\text { Lower } \\
\text { confidence } \\
\text { limit } \\
\text { (L) }\end{array}$ & $\begin{array}{c}\text { Upper } \\
\text { confidence } \\
\text { limit } \\
\text { (U) }\end{array}$ \\
\hline $1 \ldots \ldots \ldots \ldots$ & 0.025318 & 5.571643 & 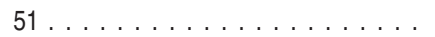 & 0.744566 & 1.314815 \\
\hline $2 \ldots \ldots \ldots \ldots \ldots \ldots \ldots \ldots \ldots \ldots$ & 0.121105 & 3.612344 & 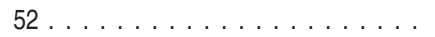 & 0.746848 & 1.311367 \\
\hline 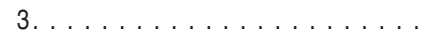 & 0.206224 & 2.922424 & $53 \ldots \ldots \ldots \ldots \ldots \ldots \ldots$ & 0.749069 & 1.308025 \\
\hline $4 \ldots \ldots \ldots \ldots \ldots \ldots \ldots \ldots$ & 0.272466 & 2.560397 & 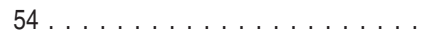 & 0.751231 & 1.304783 \\
\hline $5 \ldots \ldots \ldots \ldots \ldots \ldots \ldots \ldots \ldots \ldots$ & 0.324697 & 2.333666 & 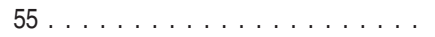 & 0.753337 & 1.301637 \\
\hline $6 \ldots \ldots \ldots \ldots \ldots \ldots$ & 0.366982 & 2.176579 & 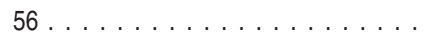 & 0.755389 & 1.298583 \\
\hline 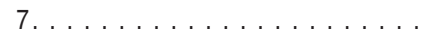 & 0.402052 & 2.060382 & 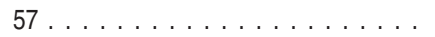 & 0.757390 & 1.295616 \\
\hline $8 \ldots \ldots \ldots \ldots \ldots$ & 0.431729 & 1.970399 & 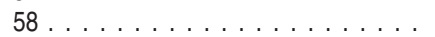 & 0.759342 & 1.292732 \\
\hline 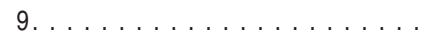 & 0.457264 & 1.898311 & 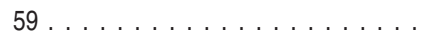 & 0.761246 & 1.289927 \\
\hline 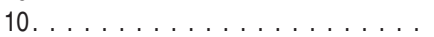 & 0.479539 & 1.839036 & $60 \ldots \ldots \ldots \ldots \ldots \ldots$ & 0.763105 & 1.287198 \\
\hline 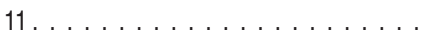 & 0.499196 & 1.789276 & 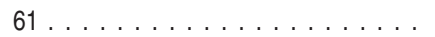 & 0.764921 & 1.284542 \\
\hline 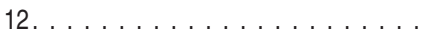 & 0.516715 & 1.746799 & 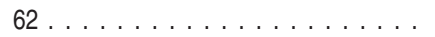 & 0.766694 & 1.281955 \\
\hline $13 \ldots \ldots \ldots \ldots \ldots \ldots \ldots \ldots$ & 0.532458 & 1.710030 & 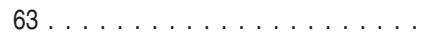 & 0.768427 & 1.279434 \\
\hline $14 \ldots \ldots \ldots \ldots \ldots \ldots$ & 0.546709 & 1.677830 & $64 \ldots \ldots \ldots \ldots \ldots \ldots$ & 0.770122 & 1.276978 \\
\hline $15 \ldots \ldots \ldots \ldots \ldots \ldots \ldots \ldots \ldots \ldots$ & 0.559692 & 1.649348 & $65 \ldots \ldots \ldots \ldots \ldots \ldots \ldots$ & 0.771779 & 1.274582 \\
\hline $16 \ldots \ldots \ldots \ldots \ldots \ldots \ldots \ldots \ldots$ & 0.571586 & 1.623937 & $66 \ldots \ldots \ldots \ldots \ldots \ldots$ & 0.773400 & 1.272245 \\
\hline 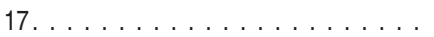 & 0.582537 & 1.601097 & 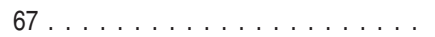 & 0.774986 & 1.269965 \\
\hline 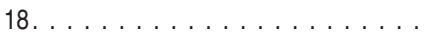 & 0.592663 & 1.580431 & 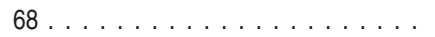 & 0.776539 & 1.267738 \\
\hline 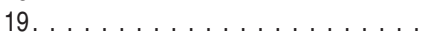 & 0.602065 & 1.561624 & 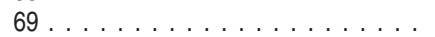 & 0.778060 & 1.265564 \\
\hline 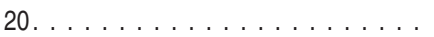 & 0.610826 & 1.544419 & 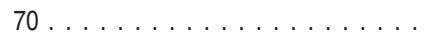 & 0.779549 & 1.263440 \\
\hline $21 \ldots \ldots \ldots \ldots \ldots \ldots \ldots \ldots$ & 0.619016 & 1.528606 & $71 \ldots \ldots \ldots \ldots \ldots$ & 0.781008 & 1.261364 \\
\hline 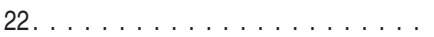 & 0.626695 & 1.514012 & 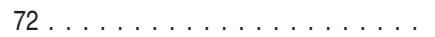 & 0.782438 & 1.259335 \\
\hline $23 \ldots \ldots \ldots \ldots \ldots \ldots \ldots \ldots \ldots$ & 0.633914 & 1.500491 & $73 \ldots \ldots \ldots \ldots \ldots$ & 0.783840 & 1.257350 \\
\hline $24 \ldots \ldots \ldots \ldots \ldots \ldots \ldots$ & 0.640719 & 1.487921 & $74 \ldots \ldots \ldots \ldots \ldots \ldots$ & 0.785215 & 1.255408 \\
\hline $25 \ldots \ldots \ldots \ldots \ldots \ldots \ldots \ldots \ldots$ & 0.647147 & 1.476197 & $75 \ldots \ldots \ldots \ldots \ldots \ldots$ & 0.786563 & 1.253509 \\
\hline $26 \ldots \ldots \ldots \ldots \ldots \ldots \ldots \ldots$ & 0.653233 & 1.465232 & $76 \ldots \ldots \ldots \ldots \ldots$ & 0.787886 & 1.251649 \\
\hline 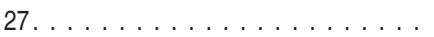 & 0.659006 & 1.454947 & $77 \ldots \ldots \ldots \ldots \ldots \ldots$ & 0.789184 & 1.249828 \\
\hline 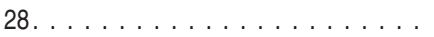 & 0.664493 & 1.445278 & 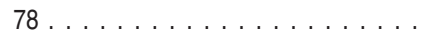 & 0.790459 & 1.248045 \\
\hline 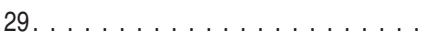 & 0.669716 & 1.436167 & $79 \ldots \ldots \ldots \ldots \ldots$ & 0.791709 & 1.246298 \\
\hline 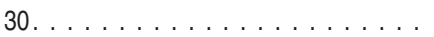 & 0.674696 & 1.427562 & 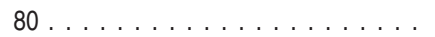 & 0.792938 & 1.244587 \\
\hline 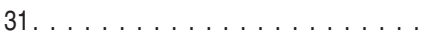 & 0.679451 & 1.419420 & 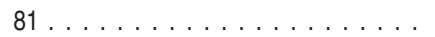 & 0.794144 & 1.242909 \\
\hline 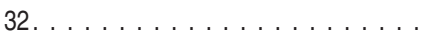 & 0.683999 & 1.411702 & 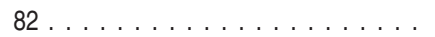 & 0.795330 & 1.241264 \\
\hline $33 \ldots \ldots \ldots \ldots \ldots \ldots \ldots \ldots \ldots \ldots \ldots$ & 0.688354 & 1.404372 & 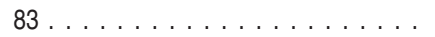 & 0.796494 & 1.239650 \\
\hline $34 \ldots \ldots \ldots \ldots \ldots \ldots \ldots \ldots \ldots$ & 0.692529 & 1.397400 & $84 \ldots \ldots \ldots \ldots \ldots \ldots$ & 0.797639 & 1.238068 \\
\hline $35 \ldots \ldots \ldots \ldots \ldots \ldots \ldots \ldots \ldots$ & 0.696537 & 1.390758 & $85 \ldots \ldots \ldots \ldots \ldots \ldots \ldots \ldots \ldots \ldots$ & 0.798764 & 1.236515 \\
\hline 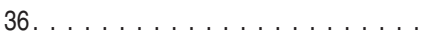 & 0.700388 & 1.384422 & 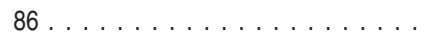 & 0.799871 & 1.234992 \\
\hline $37 \ldots \ldots \ldots \ldots \ldots \ldots \ldots \ldots$ & 0.704092 & 1.378368 & $87 \ldots \ldots \ldots \ldots \ldots \ldots \ldots \ldots \ldots \ldots \ldots \ldots \ldots$ & 0.800959 & 1.233496 \\
\hline 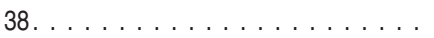 & 0.707660 & 1.372578 & 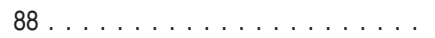 & 0.802029 & 1.232028 \\
\hline 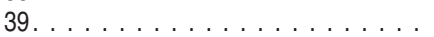 & 0.711098 & 1.367033 & 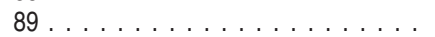 & 0.803082 & 1.230586 \\
\hline 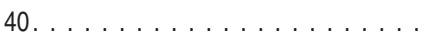 & 0.714415 & 1.361716 & 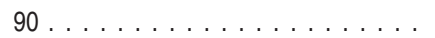 & 0.804118 & 1.229170 \\
\hline $41 \ldots \ldots \ldots \ldots \ldots \ldots \ldots \ldots \ldots$ & 0.717617 & 1.356613 & 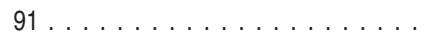 & 0.805138 & 1.227778 \\
\hline $42 \ldots \ldots \ldots \ldots \ldots \ldots \ldots$ & 0.720712 & 1.351709 & 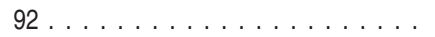 & 0.806141 & 1.226411 \\
\hline $43 \ldots \ldots \ldots \ldots \ldots$ & 0.723705 & 1.346993 & $93 \ldots \ldots \ldots \ldots \ldots$ & 0.807129 & 1.225068 \\
\hline $44 \ldots \ldots \ldots \ldots \ldots \ldots$ & 0.726602 & 1.342453 & $94 \ldots \ldots \ldots \ldots \ldots \ldots$ & 0.808102 & 1.223747 \\
\hline $45 \ldots \ldots \ldots \ldots \ldots \ldots \ldots$ & 0.729407 & 1.338079 & $95 \ldots \ldots \ldots \ldots \ldots \ldots \ldots \ldots \ldots \ldots \ldots \ldots \ldots$ & 0.809060 & 1.222448 \\
\hline 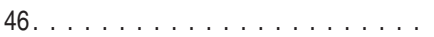 & 0.732126 & 1.333860 & 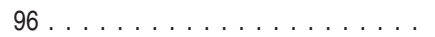 & 0.810003 & 1.221171 \\
\hline $47 \ldots \ldots \ldots \ldots \ldots \ldots \ldots \ldots \ldots$ & 0.734762 & 1.329788 & $97 \ldots \ldots \ldots \ldots \ldots \ldots$ & 0.810933 & 1.219915 \\
\hline $48 \ldots \ldots \ldots \ldots \ldots \ldots$ & 0.737321 & 1.325855 & $98 \ldots \ldots \ldots \ldots \ldots \ldots$ & 0.811848 & 1.218680 \\
\hline $49 \ldots \ldots \ldots \ldots \ldots \ldots \ldots$ & 0.739806 & 1.322053 & 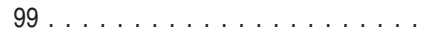 & 0.812751 & 1.217464 \\
\hline 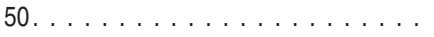 & 0.742219 & 1.318375 & & & \\
\hline
\end{tabular}

overlap (94). Caution should be observed when interpreting a nonsignificant difference between two rates, especially when the lower and upper limits being compared overlap only slightly.

Derivation of the gamma method-For a random variable $X$ that follows a gamma distribution $\Gamma(y, z)$, where $y$ and $z$ are the parameters that determine the shape of the distribution (95), $E(X)=y z$ and $\operatorname{Var}(X)=y z^{2}$. For the number of deaths, $D, E(D)=D$ and $\operatorname{Var}(D)=D$. It follows that $y=D$ and $z=1$, and thus:

$$
\text { 13. } D \sim \Gamma(D, 1)
$$

From Equation 13, it is clear that the shape of the distribution of deaths depends only on the number of deaths.
For the death rate, $R, E(R)=R$ and $\operatorname{Var}(R)=D / P^{2}$. It follows, in this case, that $y=D$ and $z=P^{-1}$, and thus:

$$
\text { 14. } R \sim \Gamma\left(D, P^{-1}\right)
$$

A useful property of the gamma distribution is that for $X \sim \Gamma(y, z), X$ can be divided by $z$ such that $X / Z \sim \Gamma(y, 1)$. This converts the gamma distribution into a simplified, standard form, dependent only on parameter $y$. Expressing Equation 14 in its simplified form gives:

15. $\frac{R}{P^{-1}}=D \sim \Gamma(D, 1)$ 
From Equation 15, it is clear that the shape of the distribution of the death rate is also dependent solely on the number of deaths.

Using the results of Equations 13 and 15, the inverse gamma distribution can be used to to calculate upper and lower confidence limits. Lower and upper $100(1-\alpha)$ percent confidence limits for the number of deaths, $L(D)$ and $U(D)$, are estimated as:

$$
\text { 16. } L(D)=\Gamma_{(D, 1)}^{-1}(\alpha / 2) \text { and } U(D)=\Gamma_{(D+1,1)}^{-1}(1-\alpha / 2)
$$

where $\Gamma^{-1}$ represents the inverse of the gamma distribution and $D+1$ in the formula for $U(D)$ reflects a continuity correction, which is necessary because $D$ is a discrete random variable and the gamma distribution is a continuous distribution. For a 95 percent confidence interval, $\alpha=.05$. For the death rate, it can be shown that:

$$
\text { 17. } L(R)=\frac{L(D)}{P} \text { and } U(R)=\frac{U(D)}{P}
$$

For more detail regarding the derivation of the gamma method and its application to age-adjusted death rates and other mortality statistics, see "References" (63,88,93).

\section{Availability of mortality data}

Mortality data are available in publications, unpublished tables, and electronic products as described on the NCHS mortality website at http://www.cdc.gov/nchs/deaths.htm. More detailed analysis than this report provides can be derived from the mortality public-use data set issued each data year. Since 1968, the data set has been available through NCHS in ASCll format and can now be downloaded from http://www.cdc.gov/nchs/data_access/Vitalstatsonline.htm. Additional resources available from NCHS include Vital Statistics of the United States, Mortality; Vital and Health Statistics, Series 20 reports; and National Vital Statistics Reports.

\section{Definition of terms}

Infant deaths-Deaths of infants under age 1.

Neonatal deaths-Deaths of infants aged 0-27 days.

Postneonatal deaths-Deaths of infants aged 28 days -1 year.

Crude death rate-Total deaths per 100,000 population for a specified period. This rate represents the average chance of dying during a specified period for persons in the entire population.

Age-specific death rate-Deaths per 100,000 population in a specified age group, such as ages $1-4$ or $5-9$, for a specified period.

Age-adjusted death rate-The death rate used to make comparisons of relative mortality risks across groups and over time. This rate should be viewed as a construct or an index rather than a direct or actual measure of mortality risk. Statistically, it is a weighted average of age-specific death rates, where the weights represent the fixed population proportions by age.

\section{Acknowledgments}

This report was prepared in the Division of Vital Statistics under the general direction of Robert N. Anderson, Chief, Mortality Statistics Branch (MSB). Elizabeth Arias of MSB provided content related to life expectancy. Robert N. Anderson and Sherry Murphy of MSB, David W. Justice of the Data Acquisition and Evaluation Branch (DAEB), and Donna E. Glenn of the Mortality Medical Classification Branch (MMCB) contributed to the "Technical Notes." The Systems, Programming, and Statistical Resources Branch (SPSRB) provided computer programming support and produced statistical tables under the general direction of Nicholas F. Pace, Chief, SPSRB. Jaleh Mousavi and Dave Johnson of SPSRB prepared the final mortality file. Candace Cosgrove, Jordan Sacks, Annie Liu, Bonita Gross, Jaleh Mousavi, and John Birken of SPSRB produced statistical tables. Annie Liu managed the population data. Staff of MSB provided content and table review. Vanetta Harrington of SPSRB provided content review. Registration Methods staff and staff of DAEB provided consultation to state vital statistics offices regarding collection of the death certificate data on which this report is based. This report was edited by Jane Sudol, Demarius V. Miller, and Laura Drescher of CDC/NCHM/Division of Creative Services, Writer-Editor Services Branch, and typeset by Jacqueline M. Davis of CDC/OSELS/NCHS/OD/Office of Information Services, Information Design and Publishing Staff. Graphics were produced by Zorica Tomic-Whalen of CDC/OSELS/NCHS/OD/Office of Information Services, Information Design and Publishing Staff, NOVA contractor. 


\section{Contents}

Abstract. ....................................

Highlights. . . . . . . . . . . . . . . . . . 1

Mortality experience in $2007 \ldots \ldots \ldots \ldots \ldots \ldots$

Trends ......................... 2

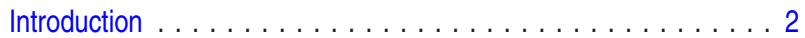

Methods ......................... 2

Results and Discussion ................ 3

Deaths and death rates. . . . . . . . . . . . . . 3

Death rates by age and sex. . . . . . . . . . . 6

Expectation of life at birth and at specified ages . . . . . . 6

Leading causes of death . . . . . . . . . . . . 8

Injury mortality by mechanism and intent $\ldots \ldots \ldots \ldots \ldots$

Drug-induced mortality . . . . . . . . . . . . . . . . . 11

Alcohol-induced mortality . . . . . . . . . . . . . . . . . 11

Marital status . . . . . . . . . . . . . . . . . . 11

Educational attainment. . . . . . . . . . . . . 12

Injury at work. . . . . . . . . . . . . . . . . 12

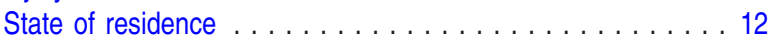

Infant mortality . . . . . . . . . . . . . . . . 12

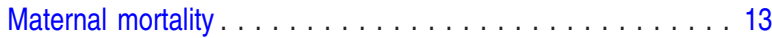

References. . . . . . . . . . . . . . . . . . . . . . . . 14

List of Detailed Tables . . . . . . . . . . . . . . . . . . . . . 17

Technical Notes . . . . . . . . . . . . . . . . . . . . . . 117

\section{Suggested citation}

Xu JQ, Kochanek KD, Murphy SL, Tejada-Vera B. Deaths: Final data for 2007. National vital statistics reports; vol 58 no 19. Hyattsville, MD: National Center for Health Statistics. 2010.

\section{Copyright information}

All material appearing in this report is in the public domain and may be reproduced or copied without permission; citation as to source, however, is appreciated.

\author{
National Center for Health Statistics \\ Edward J. Sondik, Ph.D., Director \\ Jennifer H. Madans, Ph.D., Associate Director for Science
}

Division of Vital Statistics

Charles J. Rothwell, M.S., Director

\section{U.S. DEPARTMENT OF HEALTH \& HUMAN SERVICES}

Centers for Disease Control and Prevention

National Center for Health Statistics

3311 Toledo Road

Hyattsville, MD 20782

\section{OFFICIAL BUSINESS}

PENALTY FOR PRIVATE USE, \$300

To receive this publication regularly, contact the National Center for Health Statistics by calling 1-800-232-4636

E-mail: cdcinfo@cdc.gov

Internet: http://www.cdc.gov/nchs

\section{CS214193}

T37218 (05/2010)

DHHS Publication No. (PHS) 2010-1120
MEDIA MAIL

POSTAGE \& FEES PAID

$\mathrm{CDC} / \mathrm{NCHS}$

PERMIT NO. G-284 